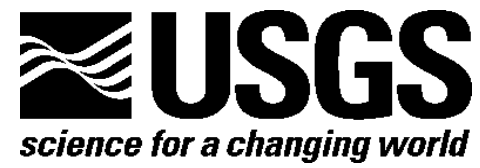

Prepared in collaboration with the Bureau of Ocean Energy Management and the National Oceanic and Atmospheric Administration

\title{
Deepwater Program: Lophelia II, Continuing Ecological Research on Deep-Sea Corals and Deep-Reef Habitats in the Gulf of Mexico
}

By A.W.J. Demopoulos, S.W. Ross, C.A. Kellogg, C.L. Morrison, M. Nizinski, N.G. Prouty, J.R. Bourque, J.P. Galkiewicz, M.A. Gray, M.J. Springmann, D.K. Coykendall, A. Miller, M. Rhode, A. Quattrini, C.L. Ames, S. Brooke, J. McClain-Counts, E.B. Roark, N.A. Buster, R.M. Phillips, and J. Frometa

Open-File Report 2017-1139

OCS Study BOEM 2016-023 


\title{
U.S. Department of the Interior \\ RYAN K. ZINKE, Secretary
}

\author{
U.S. Geological Survey \\ William H. Werkheiser, Deputy Director \\ exercising the authority of the Director \\ U.S. Geological Survey, Reston, Virginia: 2017
}

For more information on the USGS-the Federal source for science about the Earth,

its natural and living resources, natural hazards, and the environment-visit

https://www.usgs.gov or call 1-888-ASK-USGS

For an overview of USGS information products, including maps, imagery, and publications, visit $h$ ttps://store.usgs.gov.

To order this and other USGS information products, visit https://store.usgs.gov.

This study was conducted by the Ecosystems Mission Area of USGS to meet information needs identified by the Bureau of Ocean Energy Management. Any use of trade, firm, or product names is for descriptive purposes only and does not imply endorsement by the U.S. Government. All animal collection and handling was in accordance with Institutional Animal Care and Use Committee (IACUC) guidelines.

Although this information product, for the most part, is in the public domain, it also may contain copyrighted materials as noted in the text. Permission to reproduce copyrighted items must be secured from the copyright owner.

Suggested citation:

Demopoulos, A.W.J., Ross, S.W., Kellogg, C.A., Morrison, C.L., Nizinski, M., Prouty, N.G., Bourque, J.R., Galkiewicz, J.P., Gray, M.A., Springmann, M.J., Coykendall, D.K., Miller, A., Rhode, M., Quattrini, A., Ames, C.L., Brooke, S., McClain-Counts, J., Roark, E.B., Buster, N.A., Phillips, R.M., and Frometa, J., 2017, Deepwater Program: Lophelia II, continuing ecological research on deep-sea corals and deep-reef habitats in the Gulf of Mexico: U.S. Geological Survey Open-File Report 2017-1139, 269 p., https://doi.org/10.3133/ofr20171139.

ISSN 2331-1258 


\section{Preface}

This report presents information and results from the Lophelia II project that examined deep-sea coral habitats in the Gulf of Mexico. Background and details regarding the overall project and scope are presented in the Introductory chapter. The chapters are authored by several scientists from the U.S. Geological Survey, National Oceanic and Atmospheric Administration, University of North Carolina Wilmington, and Florida State University, and cover topics including community ecology (from microbes to fishes), deep-sea coral age, growth, and reproduction, and population connectivity of deep-sea corals and inhabitants. Data from these studies are presented in the chapters and appendices of the report as well as in journal publications. All chapters are published here for the first time except for figures from Chapter 5 entitled "Deep-sea black coral growth rate and age distribution in the Gulf of Mexico" which were previously published in Marine Ecology Progress Series (Prouty and others, 2011). The editors of Marine Ecology Progress Series have granted permission for these figures to be reprinted here. 


\section{Acknowledgments}

We thank our funding agency, USGS Ecosystems Mission Area, which provided support for the research and field endeavors for several of the research cruises. Both Colleen Charles and Dr. Gary Brewer were instrumental in the success of this multi-year study by continuing to support this endeavor. We thank the Bureau of Ocean Energy Management (BOEM), particularly Greg Boland, who provided expert guidance throughout the duration of the project. We thank the captains and ships' crew of the research vessel (R/V) Seward Johnson, R/V Cape Hatteras and the NOAA ships Ronald H. Brown and Nancy Foster. We thank the submersible and remotely operated vehicles from Florida Atlantic University (Johnson Sea Link II), Woods Hole Oceanographic Institution (Jason II), and the University of Connecticut (Kraken II). We are very appreciative of Drs. F. Mienis (Royal Netherlands Institute for Sea Research [NIOZ]), T. van Weering (NIOZ), G. Duineveld (NIOZ), and A. Davies (Bangor University) for their provision of deepsea landers, logistical support before, during, and after the cruises, and analysis of long-term datasets associated with these instruments. Our collaborators on BOEM's companion study led by C. Fisher (Pennsylvania State University) and E. Cordes (Temple University) provided USGS scientists with samples and space on research cruises that enabled some of the research presented in this report. We are very thankful to the numerous graduate and undergraduate students, other personnel, and volunteers who helped with this complex research project. 


\section{Contents}

1 LOPHELIA II INTRODUCTION AND PROGRAM OVERVIEW ....................................................................... 1

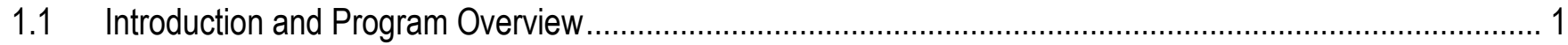

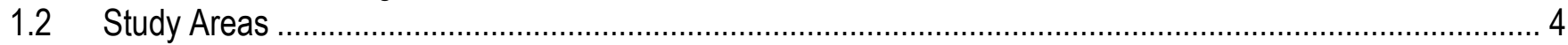

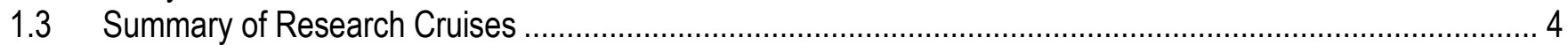

1.3.1 Nancy Foster Cruise .......................................................................................................

1.3.2 Seward Johnson Cruise ......................................................................................................

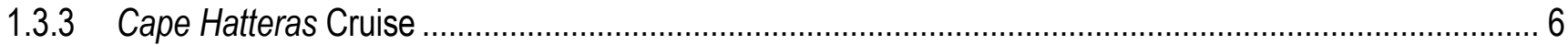

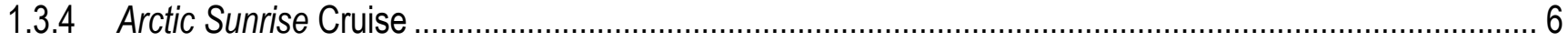

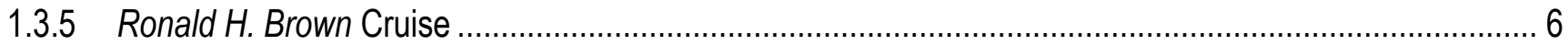

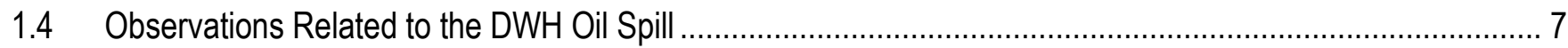

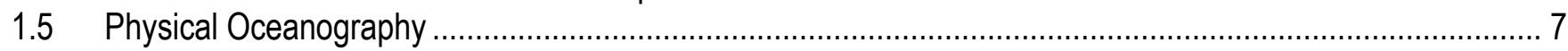

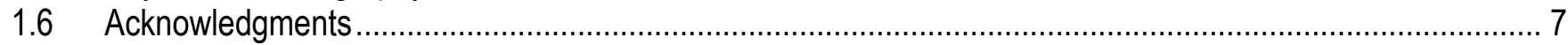

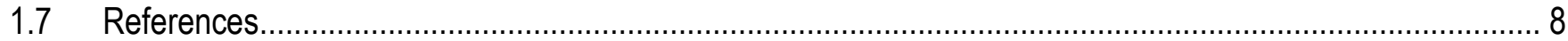

2 MICROBIAL ECOLOGY AND FUNCTIONAL METAGENOMICS OF LOPHELIA PERTUSA IN THE GULF OF MEXICO

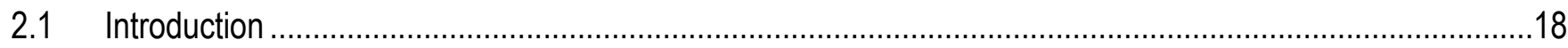

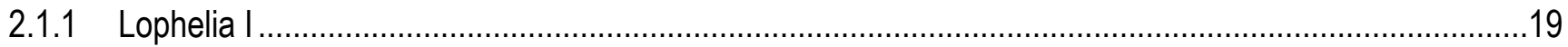

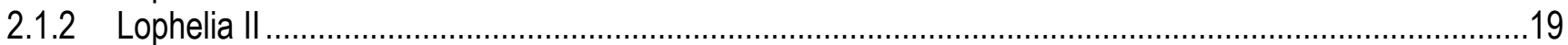

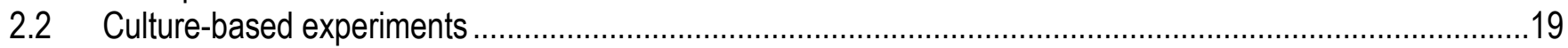

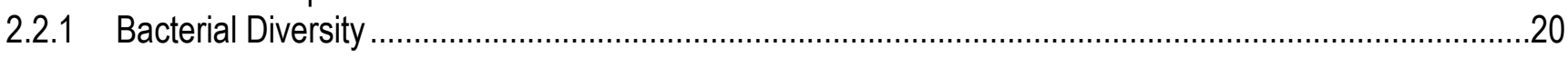

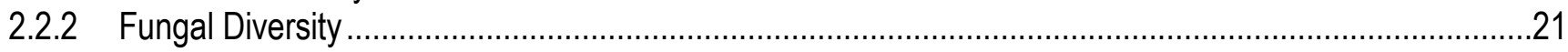

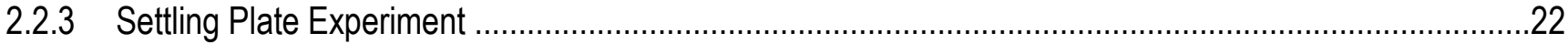

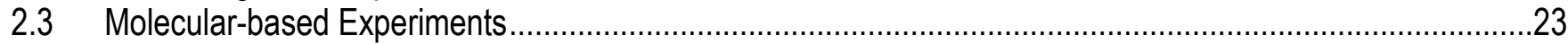

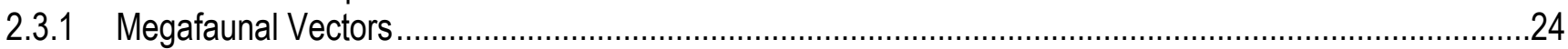

2.3.2 Identifying a Core Microbiome by Examining L. pertusa-associated Bacterial Assemblages ....................24

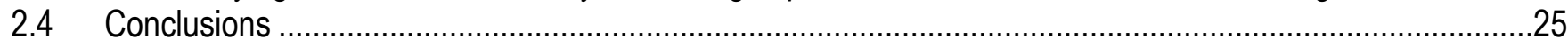

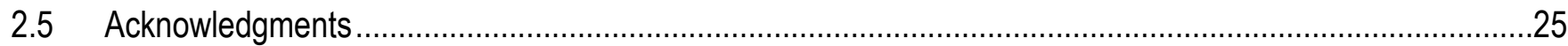

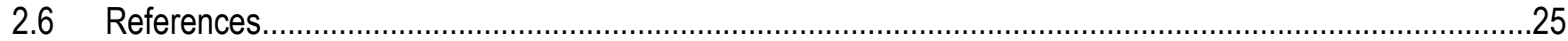

3 GENETIC CONNECTIVITY AMONG NATURAL LOPHELIA PERTUSA REEFS AND SHIPWRECKS IN THE

GULF OF MEXICO

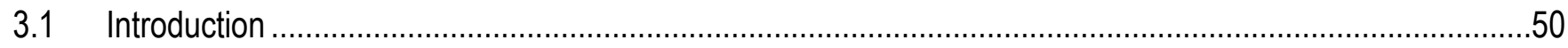

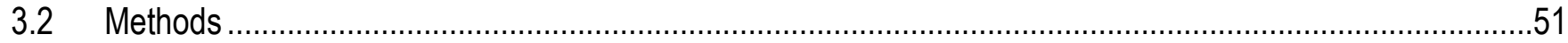

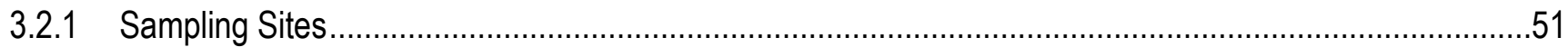

3.2.2 Molecular Methods ……....................................................................................................

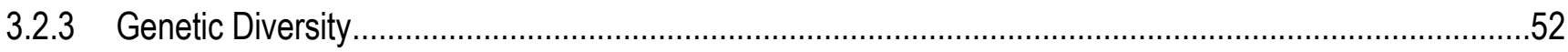

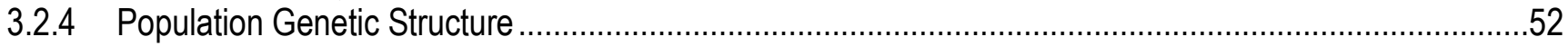

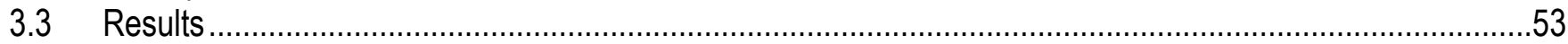

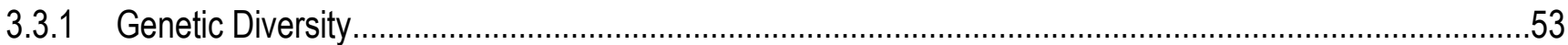

3.3.2 Population Genetic Structure ..................................................................................................54

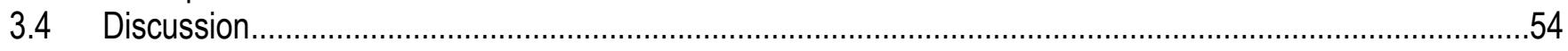

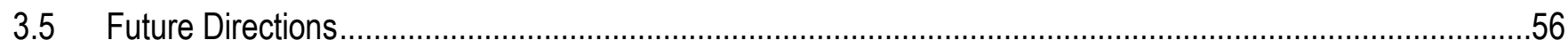

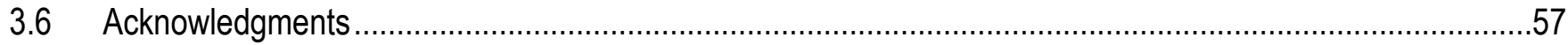

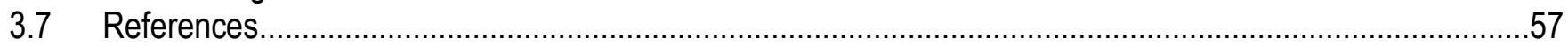

4 GENETIC DIVERSITY WITHIN EUMUNIDA PICTA, A SQUAT LOBSTER ASSOCIATED WITH LOPHELIA

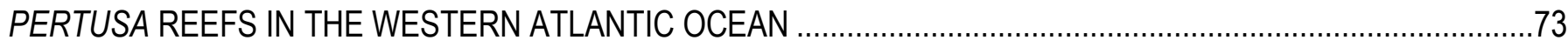

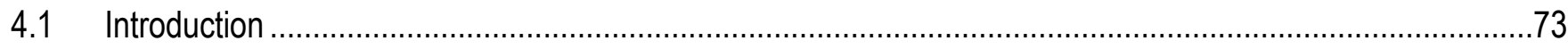




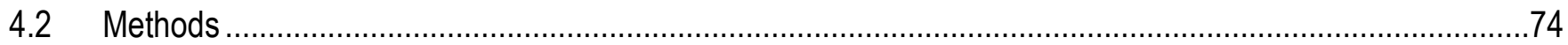

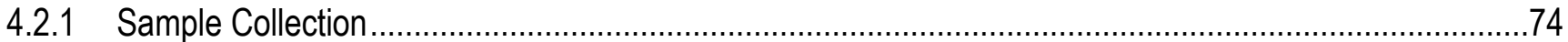

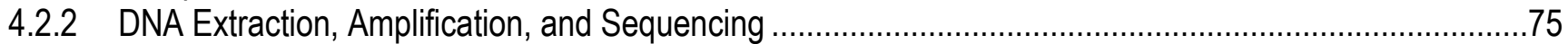

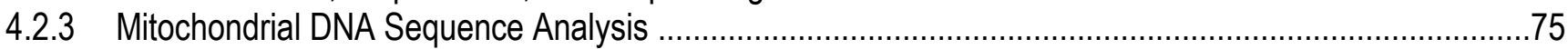

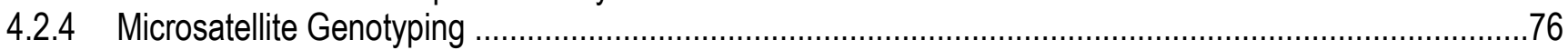

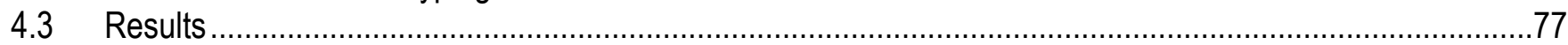

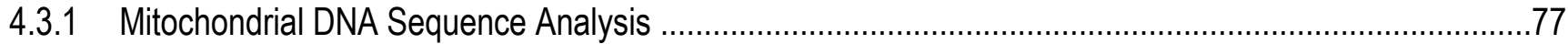

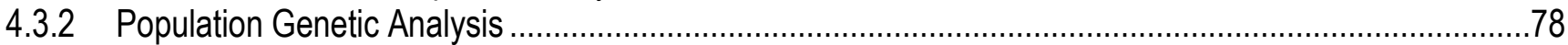

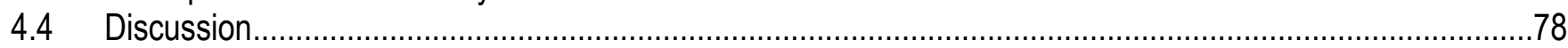

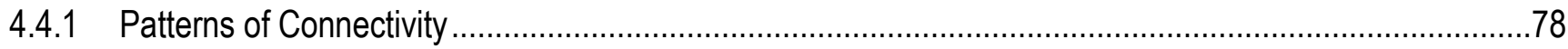

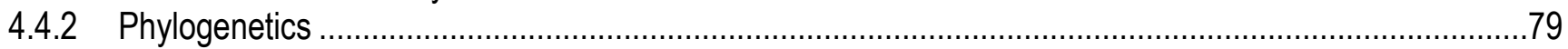

4.4.3 Contrast Between E. picta and L. pertusa Connectivity Patterns …………………….......................79

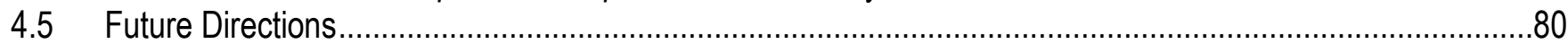

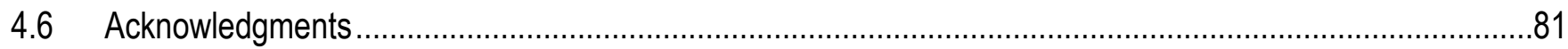

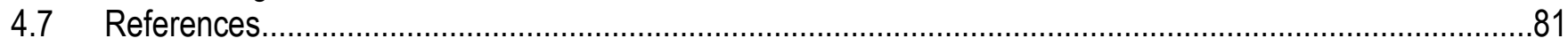

5 DEEP-SEA BLACK CORAL GROWTH RATE AND AGE DISTRIBUTION IN THE GULF OF MEXICO …...........95

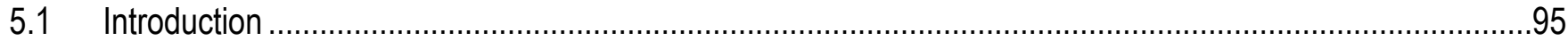

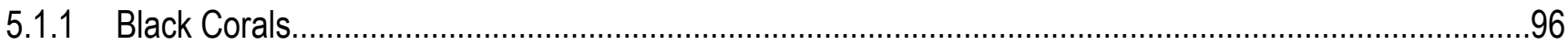

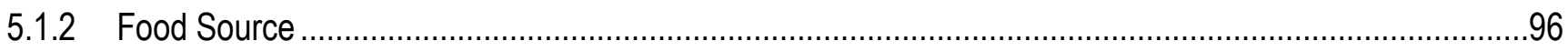

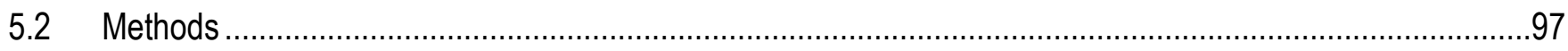

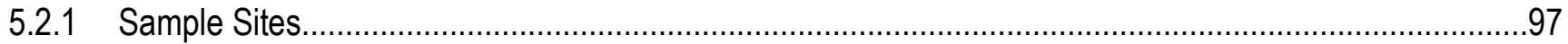

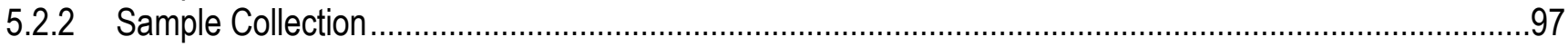

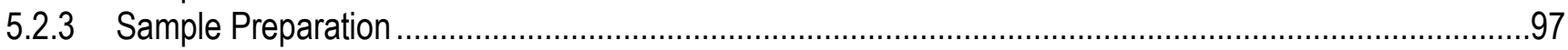

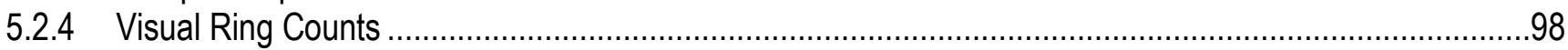

5.2.5 Analytical Measurements.....................................................................................................

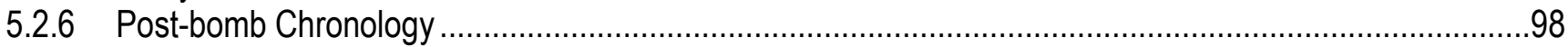

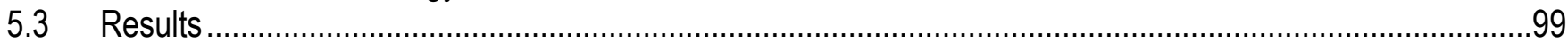

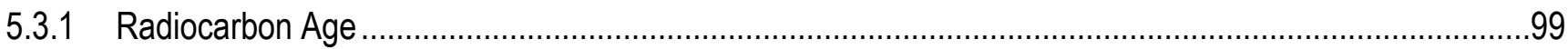

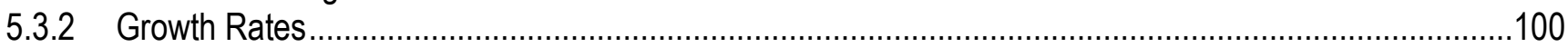

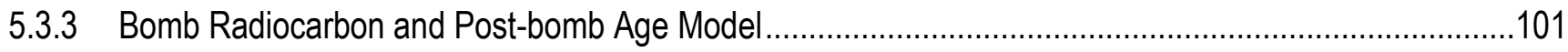

5.3.4 Band Counting ................................................................................................................... 102

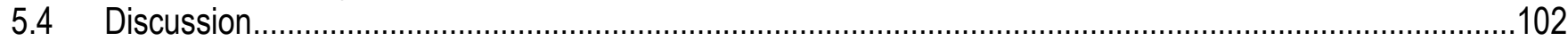

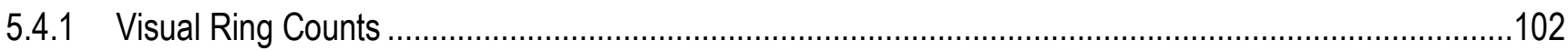

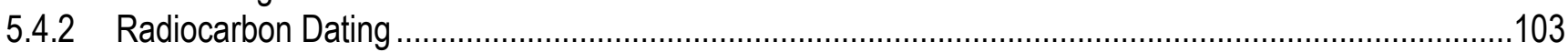

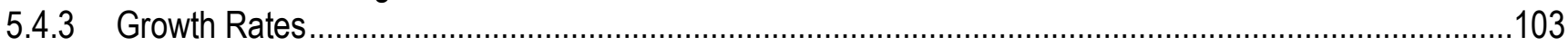

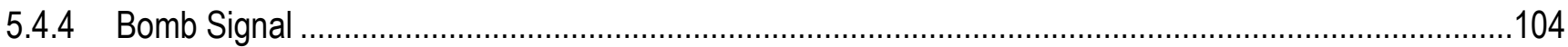

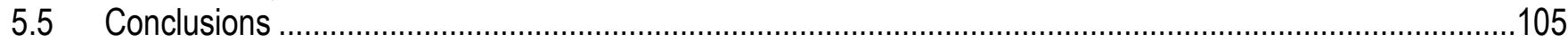

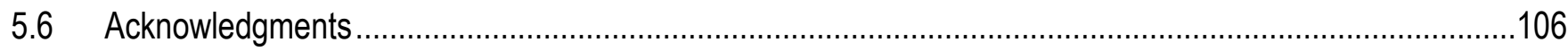

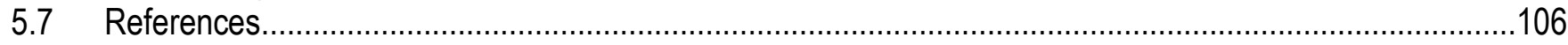

6 MACROFAUNAL COMMUNITY STRUCTURE NEAR LOPHELIA PERTUSA AND SHIPWRECK HABITATS IN

THE GULF OF MEXICO

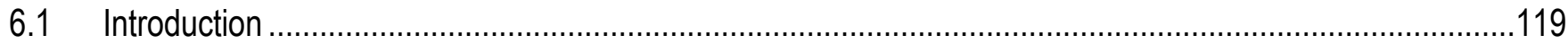

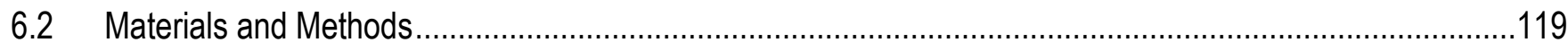

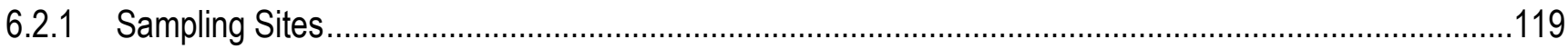

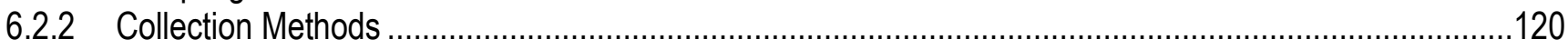

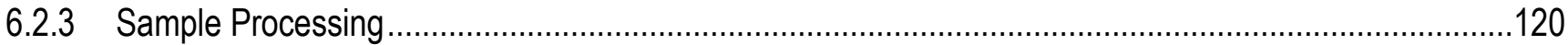

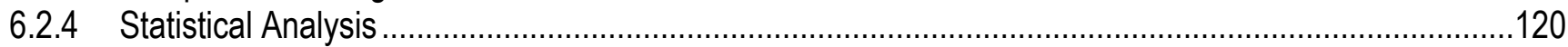

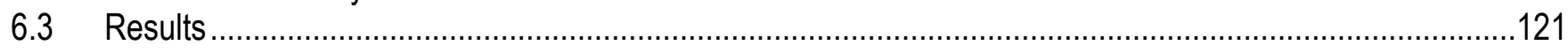




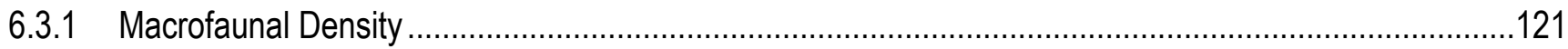

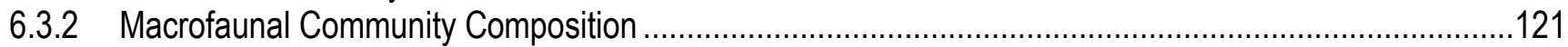

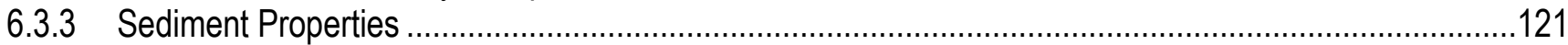

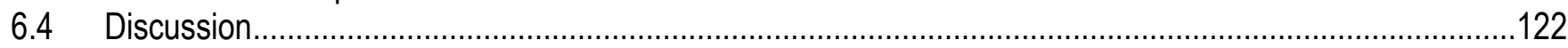

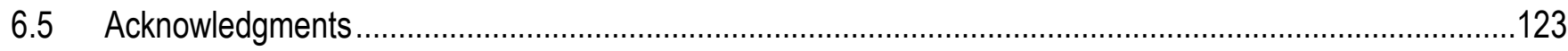

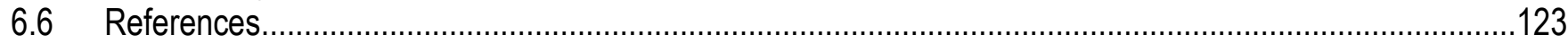

7 TROPHODYNAMICS OF THE FISH COMMUNITY ASSOCIATED WITH DEEP-SEA CORALS IN THE GULF OF

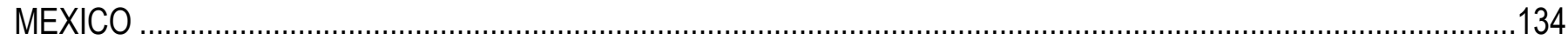

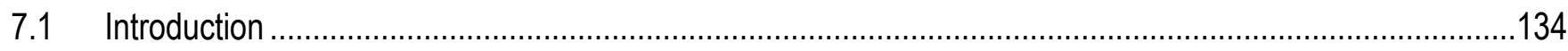

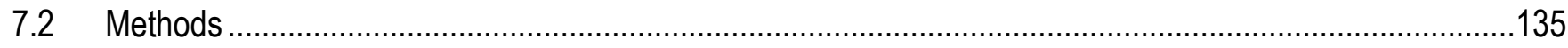

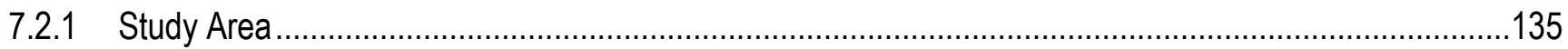

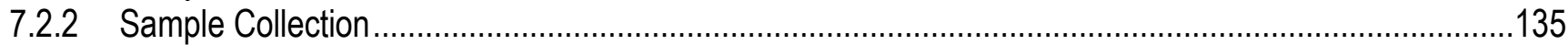

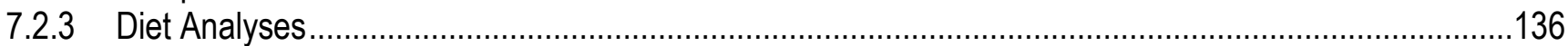

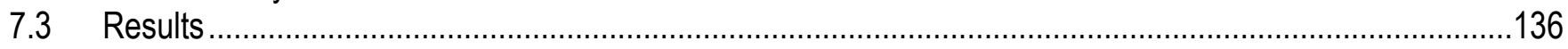

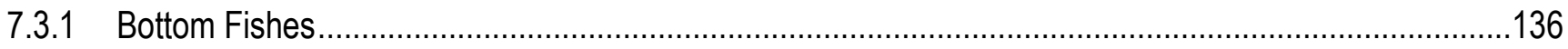

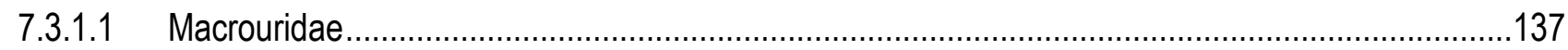

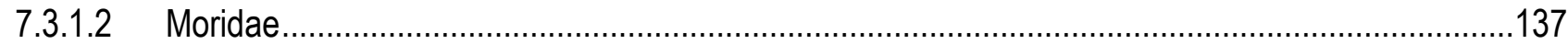

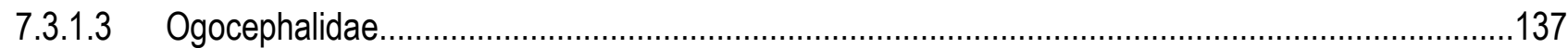

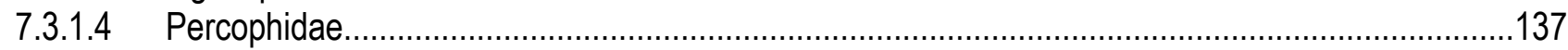

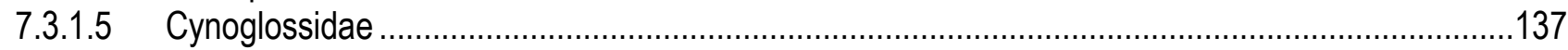

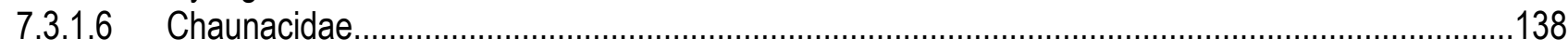

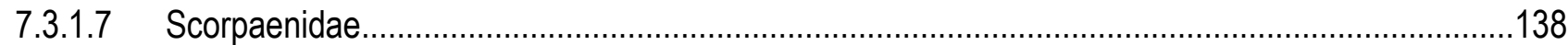

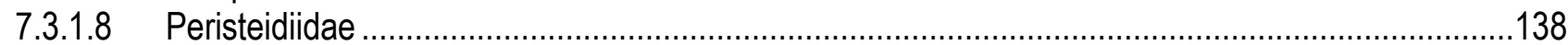

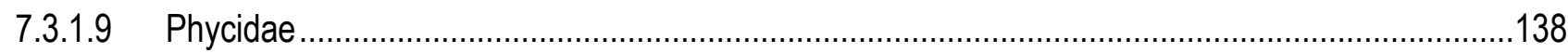

7.3.1.10 Pleuronectidae ........................................................................................................138

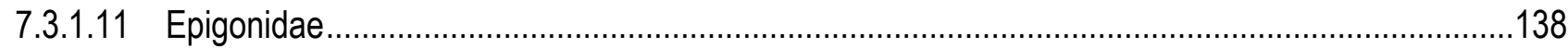

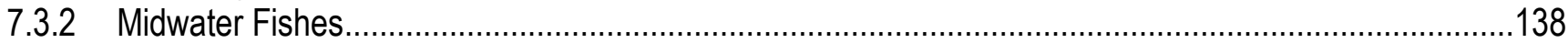

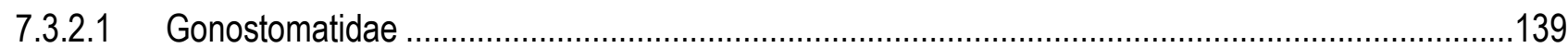

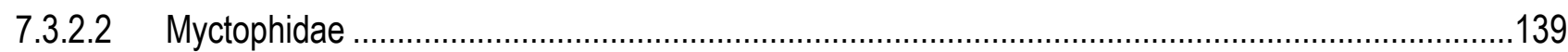

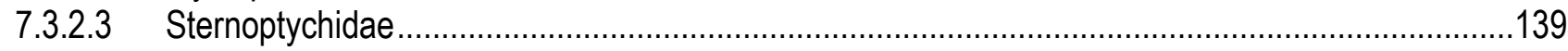

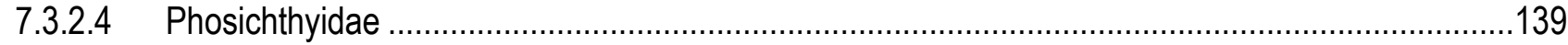

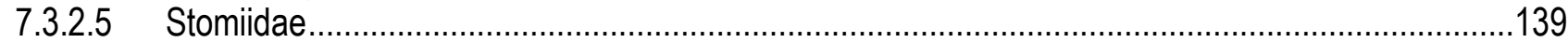

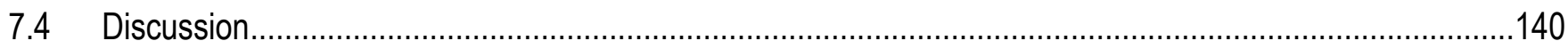

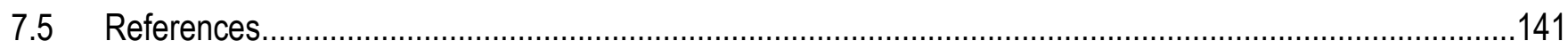

8 FISHES ASSOCIATED WITH DEEP-SEA CORAL HABITATS IN THE GULF OF MEXICO ............................171

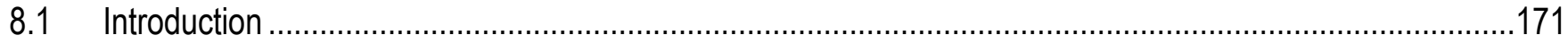

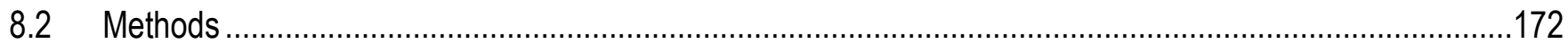

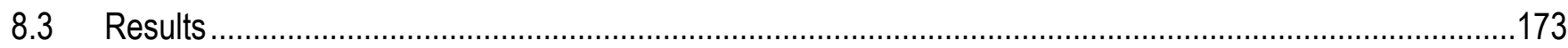

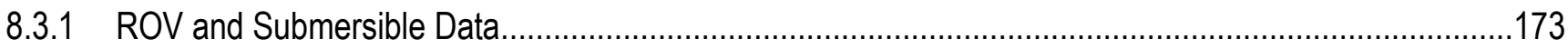

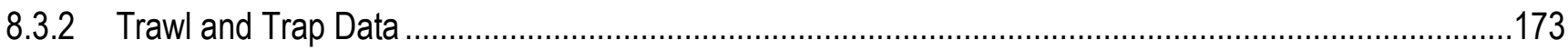

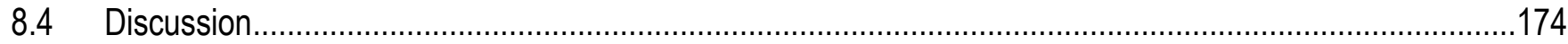

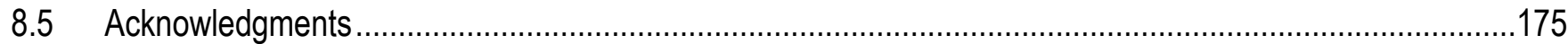

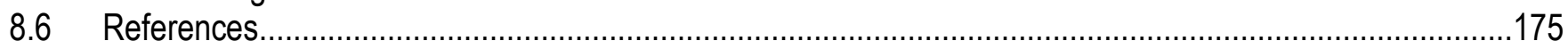

9 DISTRIBUTION, ABUNDANCE, AND SPECIES COMPOSITION OF THE MEGAFAUNAL INVERTEBRATE ASSEMBLAGES ASSOCIATED WITH DEEP-SEA CORAL HABITATS IN THE GULF OF MEXICO ........................185

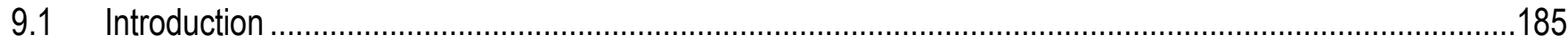

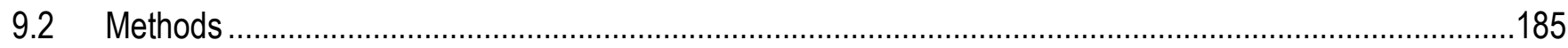

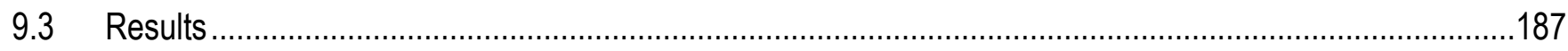


9.3.1 Species Composition and Relative Abundance Among Study Sites ....................................................190

9.3.2 Species Composition and Relative Abundance Among Vertical Zones .............................................191

9.3.3 Species Composition and Habitat Associations ...........................................................................192

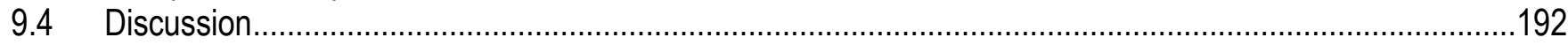

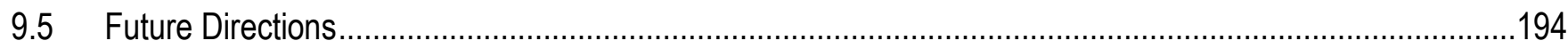

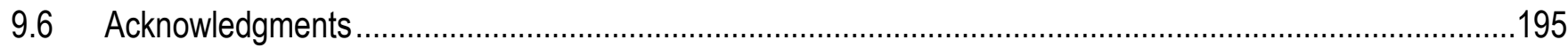

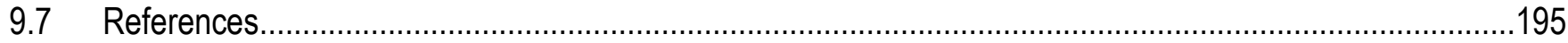

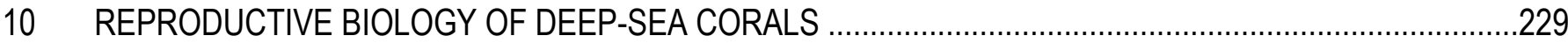

10.1 Introduction to the Reproductive Biology of Deep-sea Corals ...........................................................29

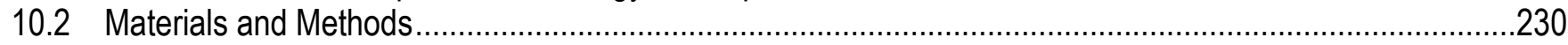

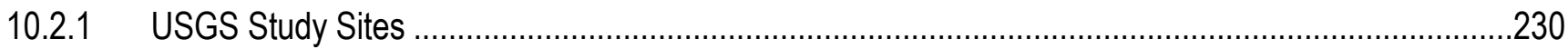

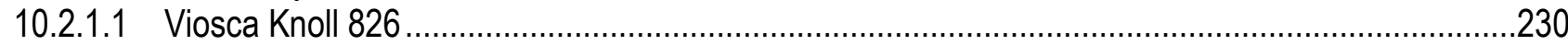

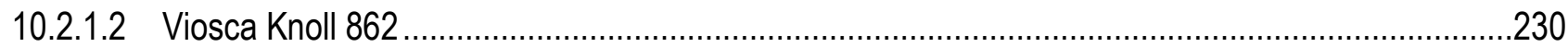

10.2.1.3 West Florida Slope ................................................................................................231

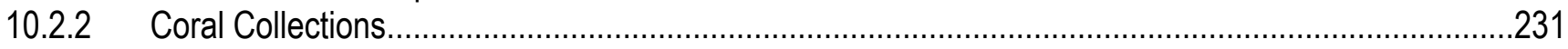

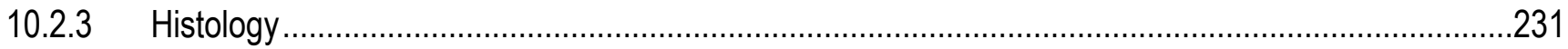

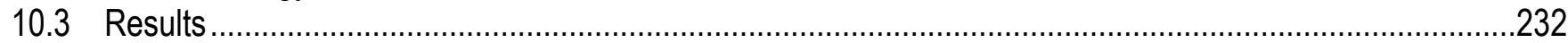

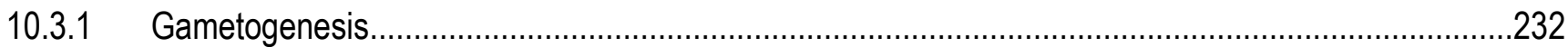

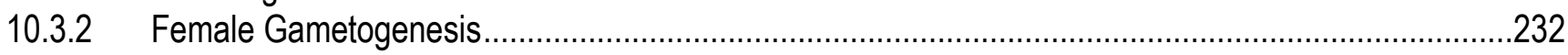

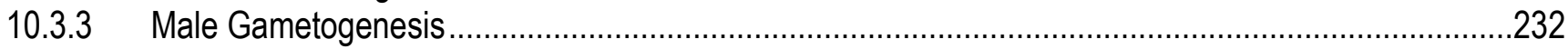

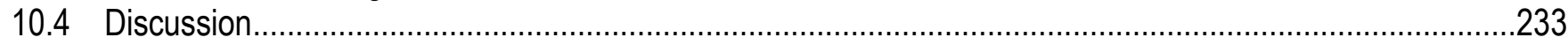

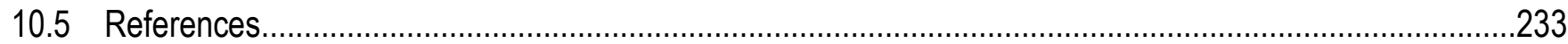

APPENDIX A. LIST OF MANUSCRIPTS PUBLISHED RELATED TO GULF OF MEXICO PROJECTS: LOPHELIA

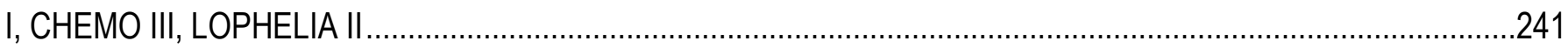

APPENDIX B. STATIONS SAMPLED DURING FIVE LOPHELIA II CRUISES (2008-2010) ….........................245

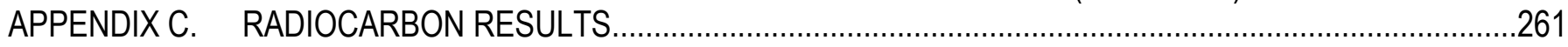

APPENDIX D. PHOTOGRAPHS OF SELECTED BENTHIC FISHES OBSERVED DURING THE LOPHELIA II

PROJECT IN THE GULF OF MEXICO

\section{Figures}

Figure 1.1 Major U.S. Geological Survey/University of North Carolina Wilmington deep-sea study sites in the Gulf of Mexico and off the southeastern United States. Base map data from NOAA. [VK (Viosca Knoll) and WFS (West Florida Slope) were the major study areas for the Lophelia II program. Note the Deepwater Horizon well site (DWH). The Chemo III sites were sampled in 2007.

Figure 1.2 Sampling stations at the two Viosca Knoll deep-sea coral study areas of the Gulf of Mexico during the Lophelia II program, color coded by gear type, 5-13 October 2008. Base map data from NOAA.[TT, Tucker trawl; OT, otter trawl; Phyto, surface phytoplankton sample; PN-1m, 1.0-meter plankton net; CTD, Sea-bird conductivity, temperature, depth instrument; ROV, remotely operated vehicle sampling stations; Albex and BoBo, benthic lander locations.].......

Figure 1.3 Multibeam sonar map of the West Florida Slope resulting from three cruises (Nancy Foster, 20-23 October 2008; Lost Coast Explorer, 7-10 November 2010; Okeanos Explorer, 20-22 March 2012) of the Lophelia II program. Base map data from NOAA. [The bottom of the left panel joins the top of the right panel. CTD, Sea-bird conductivity, temperature, depth instrument; JSL, Johnson Sea Link II stations; JROV, Jason II stations; KROV, Kraken stations; OE, Okeanos Explorer stations. Bathymetric contours represented as depth, in meters. This figure summarizes sampling stations in this region for all cruises, plus Reed and others (2006).] ......

Figure 1.4 Locations of ship-based and Johnson Sea Link II (JSL) submersible stations in four sampling areas in the Gulf of Mexico during the Lophelia II program, sampled 16-24 September 2009. Base map data from NOAA. [PN, 
plankton net; Phyto, surface phytoplankton sample; OD, on deck; Moc, multiple opening/closing net and environmental sensing system; CTD, Sea-bird SBE 911+ CTD; BC, box core.].

Figure 1.5 Stations at the Viosca Knoll 826 (left) and Viosca Knoll 862/906 (right) study sites in the north-central Gulf of Mexico of the Lophelia II program, sampled 21-26 September 2010 by the research vessel Cape Hatteras and Kraken remotely operated vehicle (ROV). Base map data from NOAA. [OT, otter trawl; PN, plankton net; Phyto, surface phytoplankton sample; OD, on deck; TT, Tucker trawl; MC, multi-core; CTD, Sea-bird SBE 911+. For ROV dives, only the bottom start locations are plotted.]....

Figure 1.6 Three areas of the West Florida Slope study site in the eastern Gulf of Mexico of the Lophelia II program, sampled 27 September - 2 October 2010 by the research vessel Cape Hatteras and Kraken II remotely operated vehicle (ROV).Base map data from NOAA. [TT, Tucker trawl; MC, multi-core; CTD, Sea-bird SBE 911+; Phyto, surface phytoplankton sample. For ROV dives, only the bottom start locations are plotted.]

Figure 1.7 Dual DeepWorker (DDW) submersible and benthic lander stations occupied in the Viosca Knoll 862/906 area during the research vessel Arctic Sunrise (AS) cruise (12-22 October 2010) of the Lophelia II program.

Base map data from NOAA. [DDW stations are the bottom starting locations for dives.] 17

Figure 2.1 Map showing Lophelia pertusa collection sites for microbiology during the Lophelia II program. Base map data from Google, SIO, NOAA, U.S. Navy, NGA, GEBCO, U.S. Geological Survey 2010. 46

Figure 2.2 Settling plate stacks being inoculated with bacteria during the Lophelia II program........................47 Figure 2.3 Settling plates mounted on benthic lander, viewed underwater after deployment, October 16, 2010 during the Lophelia II program. Photo courtesy of Steve Ross, University of North Carolina Wilmington, and Sandra Brooke, Florida State University.

Figure 2.4 Swabbing the abdomen of a galatheid crab (Eumunida picta) for microbes during the Lophelia II program

Figure 3.1 Map showing Lophelia pertusa collection localities in the Gulf of Mexico during the Lophelia II program and the western North Atlantic Ocean, and off the southeastern U.S. coast during the Lophelia I program. Site abbreviations given in table 3.1. Base map data from Google, 2016.

Figure 3.2 Mean values of genetic clusters $(K)$ and $\Delta K$ values from STRUCTURE analysis of Lophelia pertusa samples from the Gulf of Mexico during the Lophelia II program.

Figure 3.3 Proportional membership of Lophelia pertusa individuals from the Gulf of Mexico and the northwestern Atlantic Ocean, off the southeastern United States coast during the Lophelia II program, from sequential cluster analysis using the program STRUCTURE. The probability of membership of each individual to the $K=2$ clusters is shown by color coding of a vertical line, with red and green indicating membership in the Gulf of Mexico or western Atlantic clusters, respectively, and proportional membership along the $y$-axis. Proportions of membership by locality are given below the graph and localities are given above, see figure 3.1 for locations.

Figure 3.4 Graphs showing principal component (PC) 1 and PC 2 based upon principal components analysis of genetic distance data from eight microsatellite loci during the Lophelia II program including $A$, Gulf of Mexico (GOM) and northwestern Atlantic Ocean Lophelia pertusa populations and B, GOM L. pertusa populations (site abbreviations given in table 3.1). PC1 captures the inter-population genetic distance between the Garden Banks population and other GOM L. pertusa populations, while PC2 captures the differences between natural reefs and the two shipwrecks, Gulfpenn (MC497) and Gulf Oil (MC796).

Figure 3.5 Isolation-by-distance patterns in Lophelia pertusa during the Lophelia II program. The linear regression $\left(R^{2}\right)$ between spatial (geographic) distance and genetic distance (Nei's $D$, Nei, 1972, 1978) was significant $(P<0.01)$ and explained $A, 44$ percent of the variation across northwestern Atlantic Ocean and Gulf of Mexico sampling localities and $B$, less than 1 percent of the variation in genetic distances among Gulf of Mexico localities $(P>0.05) .72$ Figure 4.1 Map showing Eumunida picta sampling sites in the Gulf of Mexico and western North Atlantic Ocean during the Lophelia I and Lophelia II programs. Base map data from Google, 2016

Figure 4.2 Haplotype network representing 65 different haplotypes in 95 cytochrome oxidase sequences from Eumunida picta from Gulf of Mexico (25), the southeastern United States (67), and the Caribbean (3, obtained from GenBank)...... 
Figure 4.3 Histogram showing pairwise genetic distances between cytochrome oxidase haplotypes of Eumunida picta from the Lophelia II program..................................................................................................... 93

Figure 4.4 A 50-percent majority consensus Bayesian phylogenetic tree constructed with MrBayes within

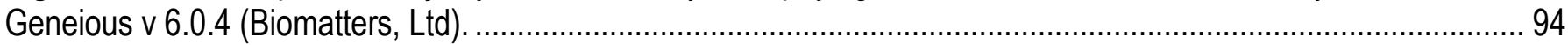
Figure 5.1 Map showing collection sites of Leiopathes sp. between 2003 and 2009 during the Lophelia II program from two different regions: the head of De Soto Canyon and Viosca Knoll (VK), including the BOEM lease blocks VK862 and VK 906/907. The Deepwater Horizon oil wellhead at the Mississippi Canyon, MC252 lease block (star), and the VK826 site are also marked for reference. Base map data from NOAA. Figure reproduced with permission from Prouty and others, 2011.

Figure 5.2 A, Photograph showing petrographic thin section indicating the location and length of 4 orthogonal radii with measured lengths of $7.7,11.2,10.2$, and 9.7 millimeters $(\mathrm{mm})$ with a standard deviation of $\pm 1.5 \mathrm{~mm}$. $B$, Scanning electron microscope (SEM) image of the GOM-JSL04-4734 thin section $(100 \mu \mathrm{m})$. C, Enlarged SEM image of the outer $2.5 \mathrm{~mm}$ of skeletal growth. The optically light bands are growth bands, represented by increased grayscale values, and the optically dark bands are organic cement layers. Figure reproduced with permission from Prouty and others, 2011 .

Figure 5.3 A, Bomb-derived $\Delta^{14} \mathrm{C}(\%)$ from red snapper otoliths $(\mathrm{n}=26)$ in the Gulf of Mexico (Baker and Wilson, 2001 ) and shallow coral $\Delta^{14} \mathrm{C}$ values ( $\mathrm{n}=27$ ) from the Flower Garden Banks (Wagner, 2009), 1943-1996.

Superimposed on the reference curves is the deep-sea antipatharian (Leiopathes sp.) coral $\Delta^{14} \mathrm{C}$ record from the GOM-JSL04-4734-BC1 specimen versus distance from outer edge (in micrometers). $B$, Leiopathes sp. coral bomb${ }^{14} \mathrm{C}$ curve superimposed on the enlarged scanning electron microscope image. Figure reproduced with permission from Prouty and others, 2011.

Figure 5.4 Scatter plots showing distance from outer edge and reservoir-corrected calendar age for the five deepsea black coral samples from the Lophelia II program: A, GOM-JSL05-4876-BC1; B, GOM-TOW-BC2; C, GOMJSL09-3728-BC1; D, GOM-JSL04-4734-BC1; and E, GOM-TOW-BC1. F, Radiocarbon $\left(\Delta \Delta^{14} C\right)$ variability of GOMTOW-BC1 versus and distance from outer edge $(\mathrm{mm})$ and year with an analytical uncertainty of 1.3-3.9 per mil\%o.

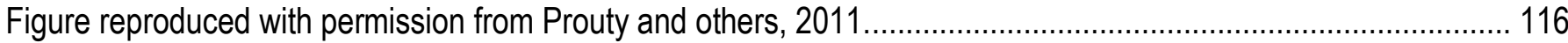
Figure 5.5 Scatter plots of lifespans (defined as the difference between the inner and outer ${ }^{14} \mathrm{C}$ age) versus growth rate (micrometer $[\mu \mathrm{m}]$ year $[y r]^{-1}$ ) calculated from black coral samples collected in the Gulf of Mexico during the Lophelia II program. Figure reproduced with permission from Prouty and others, 2011................................ 117 Figure 5.6 Lifespan and growth rate distribution of Leiopathes sp. collected from sites (about 300 meters deep) in the northern Gulf of Mexico during the Lophelia II program. Figure reproduced with permission from Prouty and others, 2011.

Figure 6.1 Collection sites of sediments in the Gulf of Mexico during the Lophelia II program. Base map data from NOAA 128

Figure 6.2 Mean macrofaunal density near shipwrecks, near coral, and in background soft sediments during the Lophelia II program.

Figure 6.3 Percent composition of major taxonomic groups near shipwrecks, near coral, and in background soft sediments during the Lophelia II program........................................................................................... 130 Figure 6.4 Shannon Index of diversity near shipwrecks, near coral, and in background soft sediments during the Lophelia II program.

Figure 6.5 Non-metric multidimensional scaling plot of all cores collected near wrecks, near corals, and in background soft sediments based on Bray-Curtis similarities of square-root transformed taxa abundance during the Lophelia II program.

Figure 6.6 Sediment grain-size composition for shipwreck, near-coral, and background soft sediments during the Lophelia II program.

Figure 7.1 Map of the study area in the Gulf of Mexico showing sampling sites for bottom and midwater fishes during the Lophelia II program. Base map data from NOAA [VK, Viosca Knoll; MC, Mississippi Canyon] .. 
Figure 7.2 Percent volume and percent frequency of stomach contents for Bathygadus macrops ( $\mathrm{N}=10)$ during

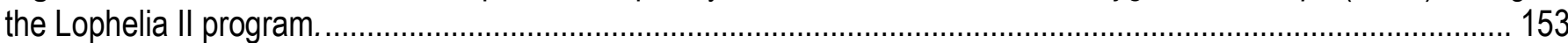

Figure 7.3 Percent volume and percent frequency of stomach contents for Coelorinchus caribbaeus ( $\mathrm{N}=61)$ during the Lophelia II program.

Figure 7.4 Percent volume and percent frequency of stomach contents for Coelorinchus caelorhincus ( $\mathrm{N}=21)$ during the Lophelia II program.

Figure 7.5 Percent volume and percent frequency of stomach contents for Malacocephalus occidentalis $(\mathrm{N}=7)$ during the Lophelia II program.

Figure 7.6 Percent volume and percent frequency of stomach contents for Nezumia aequalis $(\mathrm{N}=28)$ during the Lophelia II program.

Figure 7.7 Percent volume and percent frequency of stomach contents for Laemonema barbatulum ( $\mathrm{N}=4)$ during

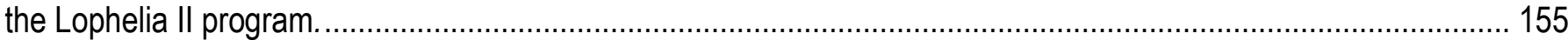

Figure 7.8 Percent volume and percent frequency of stomach contents for Laemonema goodebeanorum $(\mathrm{N}=26)$

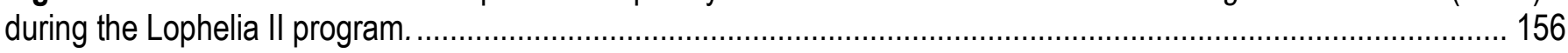

Figure 7.9 Percent volume and percent frequency of stomach contents for Dibranchus atlanticus ( $\mathrm{N}=86)$ during

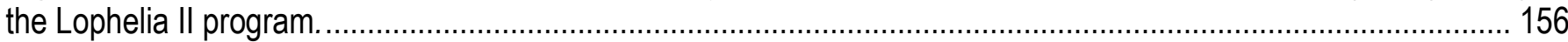
Figure 7.10 Percent volume and percent frequency of stomach contents for Bembrops gobioides $(\mathrm{N}=21)$ during the Lophelia II program.................................................................................................................. 157

Figure 7.11 Percent volume and percent frequency of stomach contents for Symphurus marginatus $(\mathrm{N}=25)$ during

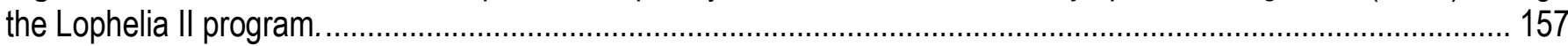
Figure 7.12 Percent volume and percent frequency of stomach contents for Chaunax suttkusi $(\mathrm{N}=17)$ during the Lophelia II program................................................................................................................... 158 Figure 7.13 Percent volume and percent frequency of stomach contents for Helicolenus dactylopterus $(\mathrm{N}=8)$ during

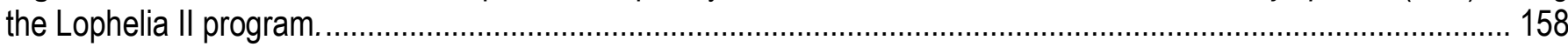
Figure 7.14 Percent volume and percent frequency of stomach contents for Pontinus longispinus $(\mathrm{N}=1)$ during the

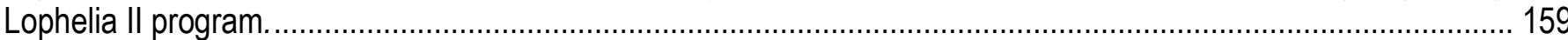

Figure 7.15 Percent volume and percent frequency of stomach contents for Pontinus rathbuni $(\mathrm{N}=1)$ during the

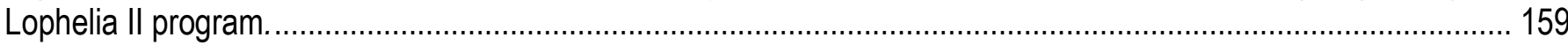
Figure 7.16 Percent volume and percent frequency of stomach contents for Peristedion greyae $(\mathrm{N}=14)$ during the

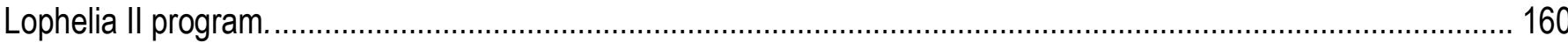

Figure 7.17 Percent volume and percent frequency of stomach contents for Urophycis floridana ( $\mathrm{N}=2$ ) during the

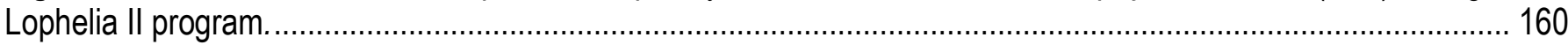

Figure 7.18 Percent volume and percent frequency of stomach contents for Urophycis cirrata $(\mathrm{N}=1)$ during the Lophelia II program......................................................................................................................... 161 Figure 7.19 Percent volume and percent frequency of stomach contents for Poecilopsetta beanii $(\mathrm{N}=12)$ during the

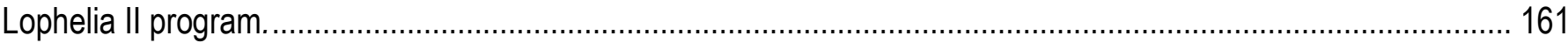

Figure 7.20 Percent volume and percent frequency of stomach contents for Epigonus pandionis ( $N=7)$ during the

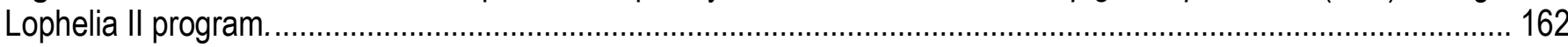

Figure 7.21 Percent volume and percent frequency of stomach contents for Cyclothone alba ( $\mathrm{N}=18)$ during the

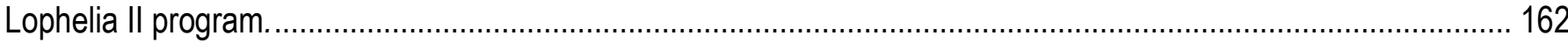
Figure 7.22 Percent volume and percent frequency of stomach contents for Cyclothone braueri $(\mathrm{N}=6)$ during the

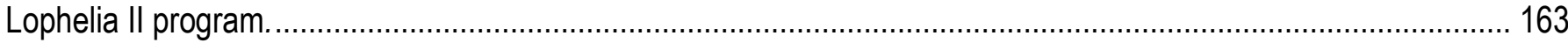

Figure 7.23 Percent volume and percent frequency of stomach contents for Gonostoma atlanticum $(\mathrm{N}=1)$ during

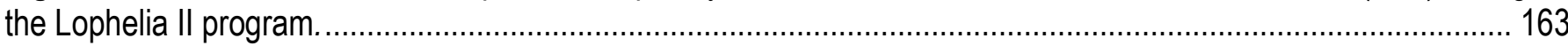

Figure 7.24 Percent volume and percent frequency of stomach contents for Gonostoma elongatum $(\mathrm{N}=3)$ during

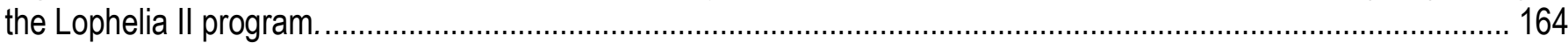
Figure 7.25 Percent volume and percent frequency of stomach contents for Diaphus dumerilii $(\mathrm{N}=15)$ during the Lophelia II program. 
Figure 7.26 Percent volume and percent frequency of stomach contents for Diaphus mollis $(\mathrm{N}=13)$ during the

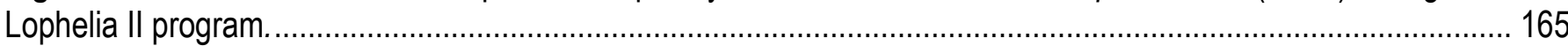

Figure 7.27 Percent volume and percent frequency of stomach contents for Lepidophanes guentheri $(\mathrm{N}=4)$ during

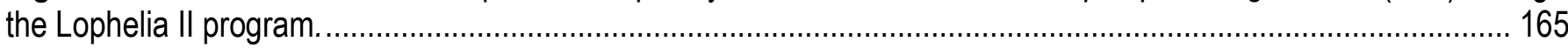

Figure 7.28 Percent volume and percent frequency of stomach contents for Myctophum affine ( $N=30)$ during the

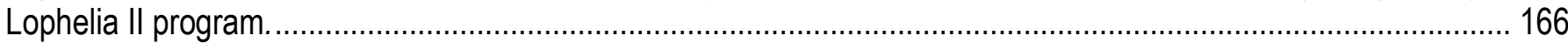

Figure 7.29 Percent volume and percent frequency of stomach contents for Argyropelecus aculeatus ( $N=7)$ during

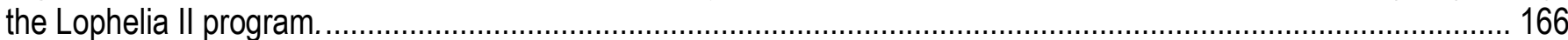

Figure 7.30 Percent volume and percent frequency of stomach contents for Argyropelecus hemigymnus ( $\mathrm{N}=2$ ) during the Lophelia II program............................................................................................................. 167

Figure 7.31 Percent volume and percent frequency of stomach contents for Polyipnus clarus $(\mathrm{N}=2)$ during the

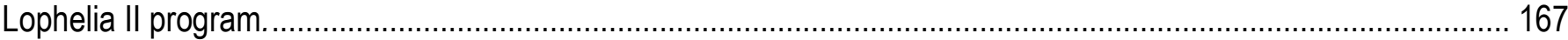

Figure 7.32 Percent volume and percent frequency of stomach contents for Valenciennellus tripunctulatus ( $\mathrm{N}=28$ ) during the Lophelia II program. ........................................................................................................... 168

Figure 7.33 Percent volume and percent frequency of stomach contents for Pollichthys mauli $(\mathrm{N}=16)$ during the

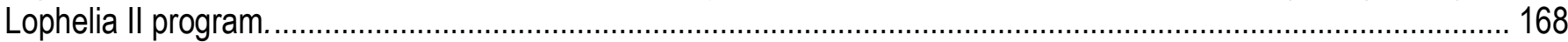
Figure 7.34 Percent volume and percent frequency of stomach contents for Vinciguerria nimbaria $(\mathrm{N}=7)$ during the

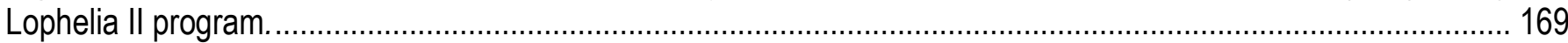

Figure 7.35 Percent volume and percent frequency of stomach contents for Chauliodus sloani $(\mathrm{N}=2)$ during the

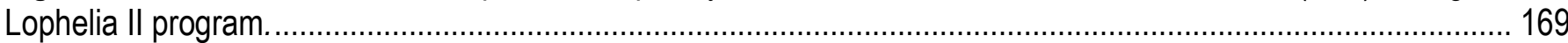

Figure 7.36 Percent volume and percent frequency of stomach contents for Stomias affinis $(\mathrm{N}=1)$ during the

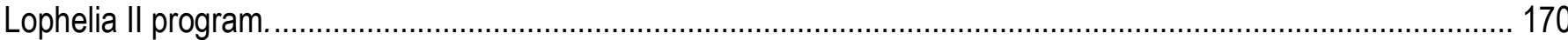

Figure 9.1 Map of study area in the North-central and eastern Gulf of Mexico showing location of sampling sites during the Lophelia II program. Base map data from Google, 2012. [VK906, VK862, VK826, and MC751 refer to Bureau of Ocean Energy Management lease blocks. WFS, West Florida Slope.] ................................................. 218

Figure 9.2 Overall (all sampling sites from all cruises combined) species richness and abundance of major invertebrate taxa during the Lophelia II program. [Number of species is shown in parentheses. Only abundance values greater than 30 are provided in figure.]

Figure 9.3 Percentage of collections represented in each vertical zone (all cruises combined) based on number of specimens collected at each site in surface, midwater, and bottom samples during the Lophelia II program. [ $\mathrm{n}=$ number of stations where invertebrate specimens were collected.].

Figure 9.4. Species accumulation curve of the number of different megafaunal taxa collected over the duration of the project, at all sites during the Lophelia II program. Taxa were cumulatively collected at a total of 86 of the 204 stations sampled in 2008, 2009, and 2010. Many specimens are still unidentified; curve represents a conservative estimate of total number of species collected.

Figure 9.5 Overall species richness and abundance of Decapoda (Crustacea) collected from all sampling sites and cruises during the Lophelia II program. Number of Decapoda specimens collected at each site is shown in pie diagram. [Number of species represented in each taxon is given in parentheses].

Figure 9.6 Species accumulation curve of the number of different crustacean taxa collected during all 3 years, at all sites during the Lophelia II program. [Taxa were cumulatively collected at 55 of the 204 stations sampled in 2008, 2009 and 2010. As many specimens are still unidentified, this curve represents a conservative estimate of total number of species collected.]

Figure 9.7 Abundance and diversity of Cnidaria specimens collected from all sampling sites and cruises during the Lophelia II program. [Number of Cnidaria specimens collected is shown in pie diagram. Number of species represented in each taxon is given in parentheses].

Figure 9.8 Percentage of the total invertebrate catch abundance represented by each major taxon by sampling site during the Lophelia II program. [Number of species is shown in parentheses]. 
Figure 9.9 Total abundance of midwater and bottom invertebrates represented in each major taxon by site during

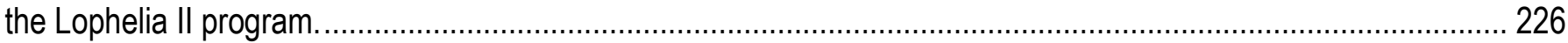

Figure 9.10 Percentage of total midwater invertebrate abundance represented in each major taxon by sampling site during the Lophelia II program. Corresponding abundance listed beside each taxon. Only abundance values

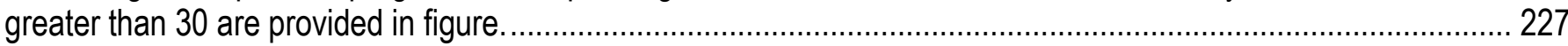
Figure 9.11 Percentage of total bottom invertebrate abundance represented in each major taxon by site during the Lophelia II program. Corresponding abundance listed beside each taxon. Only abundance values greater than 30 are provided in figure.

Figure 10.1 Mean oocyte diameters in micrometers $(\mu \mathrm{m})$ of Lophelia pertusa from different sampling periods showing the progression of the gametogenic cycle from early vitellogenic (July, week 29) to vitellogenic (September, week 44) and mature oocytes (October-November) during the Lophelia II program. Error bars represent 1 standard deviation from the mean.

Figure 10.2 Size-frequency distribution of Lophelia pertusa oocytes by sample period during the Lophelia II program. $A$, the distribution of early vitellogenic oocytes within a single female in July. In September, the median size increases from $B$, early September to $C$, late September as the oocytes mature. $D$, Large mature oocytes with a median size of 110 micrometers $(\mu \mathrm{m})$ and $120 \mu \mathrm{m}$ were measured in October and November, respectively. Error bars represent 1 standard deviation from the mean percent number of oocytes in each size category............................ 238 Figure 10.3 Plates showing stages of Lophelia pertusa oogenesis during the Lophelia II program. A, July: early vitellogenic oocytes; $B-E$, September: mature oocytes with nucleoli near periphery of nucleus; vitellogenic oocytes become more misshapen as they fill the mesenteries; $F-G$, October-November: large prespawning oocytes......... 239 Figure 10.4 Spermatocysts in the mesenteries of male Lophelia pertusa colonies during the Lophelia II program: $A-C$, Stage II spermatocysts, from September samples, and D, samples collected in November showing mature spermatocysts showing primarily spermatozoa with some spermatocytes.

Figure D.1 Photographs of selected benthic fishes observed during the Lophelia II project in the Gulf of Mexico. A, Chaunax pictus at West Florida Slope (WFS); B, Lophiodes beroe at WFS; C, Grammicolepis brachiusculus at WFS; $D$, Gephryoberyx darwini at VK862; E, Hoplostethus occidentalis at VK826; F, Epinephelus niveatus at VK862; G, Cyttopsis rosea at VK862; H, Cirrhigaleus asper at VK826; I, Conger oceanicus at VK826; J, Idiastion kyphos at VK826; K, Trachyscorpia cristulata at VK826; and L, Helicolenus dactylopterus at VK862. Photo credits: Steve Ross, University of North Carolina Wilmington.

\section{Tables}

Table 1.1 Lophelia II program cruises in the Gulf of Mexico (2008-2011) directed toward deep-sea coral reefs.... 10 Table 2.1 Types of samples collected during each research cruise during the Lophelia II program........................ 29 Table 2.2 Nutrient agars tested for bacterial isolation from Lophelia pertusa during the Lophelia II program.......... 30 Table 2.3 Bacterial cultures from various media, 2008-2012, during the Lophelia II program................................ 32 Table 2.4 Nutrient agars tested for fungal isolation from Lophelia pertusa during the Lophelia II program............. 39 Table 2.5 Fungal cultures grown on various media, 2009-2010, during the Lophelia II program........................... 40 Table 2.6 Biolog plate readings for 14 of the fungal isolates showing carbon source usage during the Lophelia II

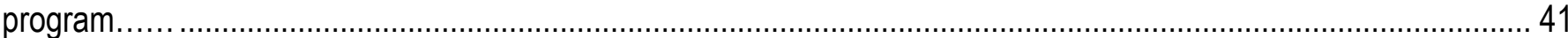

Table 2.7 Components of Lophelia pertusa mucus and the number of fungal isolates during the Lophelia II program.

Components of Lophella pertusa mucus and the number of fungal isolates during the Lophelia II

Table 3.1 Gulf of Mexico localities and southeastern U.S. collection sites with location and sampling depth, number of genets, new samples genotyped during the Lophelia II program, number of new genets (unique individuals) and proportion of clones for new Lophelia pertusa samples analyzed.

Table 3.2 Genetic diversity and differentiation in 10 Gulf of Mexico Lophelia pertusa sampling localities characterized using 8 microsatellite markers during the Lophelia II program.

Table 3.3 Pairwise $F_{\text {ST }}$ (below diagonal) and $D_{\text {EST }}$ (above diagonal) estimates between localities for Lophelia pertusa based on a survey of eight microsatellite loci during the Lophelia II program. 
Table 3.4 Results from Analysis of Molecular Variance (AMOVA) among clusters suggested by STRUCTURE and

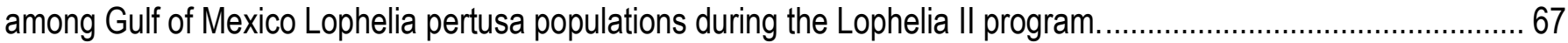

Table 4.1 Sampling localities of Eumunida picta during the Lophelia I and Lophelia II programs.......................... 85

Table 4.2 Summary statistics of cytochrome oxidase (COI) DNA sequence data estimated in DNAsp during the

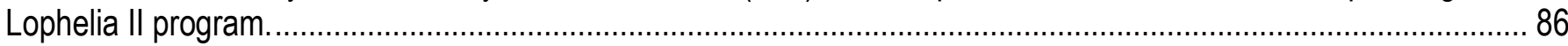

Table 4.3 Summary statistics of nine microsatellite loci in Eumunida picta (CLADE I) computed in GenalEx v4.1 during the Lophelia II program.

Table 4.4 p-values from Hardy-Weinberg Equilibrium exact tests calculated in GENEPOP during the Lophelia II program.

Table 5.1 Sample identification (ID) and location for Leiopathes sp. specimens collected between 2003 and 2009 within the Gulf of Mexico from two different regions: the head of De Soto Canyon (archived at the U.S. National Museum) and Viosca Knoll during the Lophelia II program.

Table 5.2 Lifespans and radiocarbon $\left({ }^{14} \mathrm{C}\right)$ ages of coral samples collected during the Lophelia II program......... 111 Table 5.3 Replicate radiocarbon analysis of adjacent discs collected during the Lophelia II program using different subsampling techniques (microdrilling and chemical band separation) at different AMS laboratories....................... 112

Table 5.4 Bomb-derived ${ }^{14} \mathrm{C}$ values in specimens collected as part of the Lophelia II program. .......................... 112 Table 6.1 Sample locations, sample type, depths, and gear used for all sediment cores collected during the Lophelia II program.

Table 7.1 Number of stomachs analyzed and number of empty or everted stomachs for bottom fishes caught during NF-2008, SJ-2009 and CH-2010 cruises in the Gulf of Mexico near or on deep-sea reef habitats as part of the Lophelia II program.

Table 7.2 Percent frequency (\%F) and percent volume (\%V) of stomach contents for 31 bottom fish species caught during NF-2008, SJ-2009, and CH-2010 cruises at Vioska Knoll 826, Vioska Knoll 862/906, Mississippi Canyon and West Florida Slope during the Lophelia II program.

Table 7.3 Number of stomachs analyzed and number of empty or everted stomachs for midwater fishes caught during $\mathrm{CH}-2007$ and NF-2008 cruises over deep-reef habitats in the Gulf of Mexico during the Lophelia II program. 149 Table 7.4 Percent frequency (\%F) and percent volume (\%V) of stomach contents for 19 midwater fish species caught during $\mathrm{CH}-2007$ and NF-2008 at Viosca Knoll 826, north-central Gulf of Mexico as part of the Lophelia II program

Table 8.1 Lophelia II project summary data on dives and video by general location in the Gulf of Mexico............ 177

Table 8.2 Fishes documented from video analysis from Johnson Sea Link II (2009) and Dual DeepWorker (2010) submersibles and Kraken II remotely operated vehicle (2010) dives in the Gulf of Mexico by major habitat type during the Lophelia II program.

Table 8.3 Fishes caught by bottom otter trawl (OT) and traps during three cruises in the Gulf of Mexico as part of the Lophelia II program.

Table 9.1 Overall diversity and abundance of invertebrates collected (all sampling methods combined) at five deep coral study sites (MC751, VK826, VK862, VK862NK906, West Florida Slope (WFS) in the north-central and eastern Gulf of Mexico, 2008-2010, as part of the Lophelia II program. 197

Table 9.2 Invertebrate species and number of individuals collected at the surface over four deep coral study sites (VK826, VK862, VK862/VK906, and West Florida Slope) in the north-central and eastern Gulf of Mexico, 2008-2010, as part of the Lophelia II program. 206

Table 9.3 Invertebrate species and number of individuals collected in midwater over four deep coral study sites (VK826, VK862, VK862/VK906, and West Florida Slope) in the north-central and eastern Gulf of Mexico, 2008-2010, as part of the Lophelia II program.

Table 9.4 Invertebrate species and number of individuals collected on the bottom at five deep coral study sites (MC751, VK826, VK862, VK862/VK906, and West Florida Slope) in the north-central and eastern Gulf of Mexico, 2008-2010, as part of the Lophelia II program. 
Table 10.1 Summary of cruises that provided samples for reproductive analysis during the Lophelia II

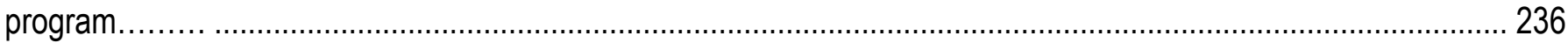

Table 10.2 Collection data for Lophelia pertusa samples from the Gulf of Mexico during 2009 and 2010 during the Lophelia II program. 236

Table B.1 Stations sampled at two deep coral study sites (VK826, VK862/906) in the Gulf of Mexico using the NOAA ship Nancy Foster, October 6-13, 2008, as part of the Lophelia II program. 245

Table B.2 Stations sampled on deep coral areas in the eastern and the north-central Gulf of Mexico using the research vessel (R/V) Seward Johnson, September 16-24, 2009, as part of the Lophelia II program.

Table B.3 Stations sampled in the north-central and eastern Gulf of Mexico using the research vessel (R/V) Cape Hatteras,September 21-October 2, 2010, as part of the Lophelia II program.

Table B.4 Stations sampled on deep coral areas in the north-central Gulf of Mexico using the research vessel (R/V) Arctic Sunrise, October 12-22, 2010, as part of the Lophelia II program.

Table B.5 Stations sampled in the eastern Gulf of Mexico using the NOAA ship Ronald H. Brown November 9-22, 2010, as part of the Lophelia II program. 260

Table C.1 Radiocarbon results from radial transects and radiocarbon calibration results from the Lophelia II program.

\section{Conversion Factors}

SI to Inch/Pound

\begin{tabular}{lcl}
\hline \multicolumn{1}{c}{ Multiply } & By & \multicolumn{1}{c}{ To obtain } \\
\hline centimeter $(\mathrm{cm})$ & 0.3937 & inch (in.) \\
micrometer $(\mu \mathrm{m})$ & 0.00003937 & inch (in.) \\
millimeter $(\mathrm{mm})$ & 0.03937 & inch (in.) \\
meter $(\mathrm{m})$ & 3.281 & foot (ft) \\
kilometer $(\mathrm{km})$ & 0.6214 & mile (mi) \\
kilometer $(\mathrm{km})$ & 0.5400 & mile, nautical $(\mathrm{nmi})$ \\
meter $(\mathrm{m})$ & 1.094 & yard (yd) \\
\hline & Area & \\
\hline square meter $\left(\mathrm{m}^{2}\right)$ & 0.0002471 & acre \\
square centimeter $\left(\mathrm{cm}^{2}\right)$ & 0.001076 & square foot $\left(\mathrm{ft}^{2}\right)$ \\
square meter $\left(\mathrm{m}^{2}\right)$ & 10.76 & square foot $\left(\mathrm{ft}^{2}\right)$ \\
square centimeter $\left(\mathrm{cm}^{2}\right)$ & 0.1550 & square inch $\left(\mathrm{ft}^{2}\right)$ \\
square kilometer $\left(\mathrm{km}^{2}\right)$ & 0.3861 & square mile $\left(\mathrm{mi}^{2}\right)$ \\
\hline & Volume & \\
\hline liter $(\mathrm{L})$ & 33.82 & ounce, fluid $(\mathrm{fl}$. oz) \\
liter $(\mathrm{L})$ & 2.113 & pint (pt) \\
liter $(\mathrm{L})$ & 1.057 & quart $(\mathrm{qt})$ \\
liter $(\mathrm{L})$ & 0.2642 & gallon $(\mathrm{gal})$ \\
liter $(\mathrm{L})$ & 61.02 & cubic inch $\left(\mathrm{in}^{3}\right)$ \\
milliliter $(\mathrm{mL})$ & 0.0338 & ounce, fluid $\left(\mathrm{fl}^{2} . \mathrm{oz}\right)$ \\
& &
\end{tabular}




\begin{tabular}{lll} 
microliter $(\mu \mathrm{L})$ & 0.0000338 & ounce, fluid $(\mathrm{fl} . \mathrm{oz})$ \\
\hline meter per second $(\mathrm{m} / \mathrm{s})$ & Flow rate & \\
meter per minute $(\mathrm{m} / \mathrm{min})$ & 3.281 & foot per second $(\mathrm{ft} / \mathrm{s})$ \\
meter per hour $(\mathrm{m} / \mathrm{hr})$ & 3.281 & foot per minute $(\mathrm{ft} / \mathrm{min})$ \\
liter per second $(\mathrm{L} / \mathrm{s})$ & 3.281 & foot per hour $(\mathrm{ft} / \mathrm{hr})$ \\
kilometer per hour $(\mathrm{km} / \mathrm{h})$ & 15.85 & gallon per minute $(\mathrm{gal} / \mathrm{min})$ \\
& 0.6214 & mile per hour $(\mathrm{mi} / \mathrm{h})$ \\
\hline gram $(\mathrm{g})$ & Mass & \\
milligram $(\mathrm{mg})$ & 0.03527 & ounce, avoirdupois $(\mathrm{oz})$ \\
& 0.0000353 & ounce, avoirdupois $(\mathrm{oz})$ \\
\hline
\end{tabular}

Inch/Pound to SI

\begin{tabular}{lcl}
\hline & \multicolumn{1}{c}{ Multiply } & \multicolumn{1}{c}{ To obtain } \\
\hline inch (in.) & Length & \\
inch (in.) & 2.54 & centimeter (cm) \\
\hline & 25.4 & millimeter (mm) \\
\hline gallon (gal) & Volume & \\
\hline & 3.785 & liter (L) \\
\hline ounce, avoirdupois (oz) & Mass & \\
\hline
\end{tabular}

Temperature in degrees Celsius $\left({ }^{\circ} \mathrm{C}\right)$ may be converted to degrees Fahrenheit $\left({ }^{\circ} \mathrm{F}\right)$ as follows:

${ }^{\circ} \mathrm{F}=\left(1.8 \times{ }^{\circ} \mathrm{C}\right)+32$

Vertical and horizontal coordinate information is referenced to the World Geodetic System of 1984 (WGS 84).

\section{Acronyms}

$\begin{array}{ll}\Delta R & \text { Reservoir correction } \\ \% V & \text { Total prey volume } \\ \% 0 & \text { Per mil } \\ \text { AMOVA } & \text { Analysis of molecular variance } \\ \text { AMS } & \text { Accelerator mass spectrometry } \\ \text { ANOSIM } & \text { Analysis of similarities } \\ \text { ANOVA } & \text { Analysis of variance } \\ \text { AUV } & \text { Autonomous underwater vehicle } \\ \text { BOEM } & \text { Bureau of Ocean Energy Management } \\ \text { BOEMRE } & \text { Bureau of Ocean Energy Management, Regulation, and Enforcement } \\ \text { cal yr BP } & \text { Calendar years before present } \\ \text { CAMS } & \text { The Center for Accelerator Mass Spectrometry, Lawrence Livermore National Laboratory } \\ \text { CITES } & \text { Convention on International Trade in Endangered Species } \\ \text { CLO } & \text { Cape Lookout }\end{array}$




$\begin{array}{ll}\text { COI } & \text { Cytochrome oxidase } \\ \text { CRA } & \text { Conventional radiocarbon age } \\ \text { CTD } & \text { Conductivity, temperature, depth } \\ \text { CWC } & \text { Cold water coral } \\ \text { DDW } & \text { Dual DeepWorker } \\ \text { DIC } & \text { Dissolved inorganic carbon } \\ \text { DISCOVRE } & \text { Diversity, Systematics, and Connectivity of Vulnerable Reef Ecosystems } \\ \text { DNA } & \text { Deoxyribonucleic acid } \\ \text { DSC } & \text { Deep-sea coral } \\ \text { DWH } & \text { Deepwater Horizon } \\ \text { EB } & \text { Ewing } \\ \text { ETOH } & \text { Ethanol } \\ \text { FST } & \text { Fixation index } \\ \text { GASWA } & \text { Glycerol artificial seawater agar } \\ \text { GB } & \text { Garden Banks } \\ \text { GC } & \text { Green Canyon } \\ \text { GCA } & \text { Gut content analysis } \\ \text { GO } & \text { Gulf Oil } \\ \text { GOM } & \text { Gulf of Mexico } \\ \text { GP } & \text { Gulfpenn } \\ \text { HWE } & \text { Hardy-Weinburg equilibrium } \\ \text { IBD } & \text { Isolation by distance } \\ \text { ITS } & \text { Internal transcribed spacer } \\ \text { JSL } & \text { Johnson Sea Link II } \\ \text { KCCAMS } & \text { The Keck Carbon Cycle AMS Laboratory at the University of California Irvine } \\ \text { KOH } & \text { Potassium hydroxide } \\ \text { LIA } & \text { Little ice age } \\ \text { MC } & \text { Mississippi Canyon } \\ \text { MCMC } & \text { Markov chain Monte Carlo } \\ \text { MLG } & \text { Multi-locus genotype } \\ \text { MMS } & \text { Minerals Management Service } \\ \text { MOCNESS } & \text { Multiple opening/closing net and environmental sensing system } \\ \text { MTR } & \text { Miami Terrace } \\ \text { NE } & \text { Northeastern } \\ \text { NIOZ } & \text { Royal Netherland Institute for Sea Research } \\ \text { nMDS } & \text { Non-metric multidimensional scaling } \\ \text { NMFS } & \text { National Marine Fisheries Service } \\ \text { NOAA } & \text { National Oceanic and Atmospheric Administration } \\ \text { NOSAMS } & \text { The National Ocean Sciences Accelerator Mass Spectrometry Facility } \\ \text { NWAO } & \text { Northwest Atlantic Ocean } \\ \text { OT } & \text { Otter trawl } \\ \text { PCA } & \text { Principal components analysis } \\ \text { PCR } & \text { Polymerase chain reaction } \\ \text { PI } & \text { Probability of identity } \\ \text { PLD } & \text { Pelagic larval duration } \\ \text { POM } & \text { Particulate organic matter } \\ \text { PRMT } & \text { Predicted relative metabolic turnover } \\ \text { RNA } & \text { Ribonucleic acid } \\ & \end{array}$




$\begin{array}{ll}\text { ROV } & \text { Remotely operated vehicle } \\ \text { RN } & \text { Research vessel } \\ \text { SD } & \text { Standard deviation } \\ \text { SE } & \text { Standard error of the mean } \\ \text { SEM } & \text { Scanning electron microscope } \\ \text { SEUS } & \text { Southeastern United States } \\ \text { SL } & \text { Standard length } \\ \text { TDR } & \text { Temperature-depth recorder } \\ \text { TL } & \text { Total length } \\ \text { TT } & \text { Tucker trawl } \\ \text { UNCW } & \text { University of North Carolina Wilmington } \\ \text { USGS } & \text { U.S. Geological Survey } \\ \text { USNM } & \text { National Museum of Natural History, Smithsonian Institution } \\ \text { VK } & \text { Viosca Knoll } \\ \text { WFS } & \text { West Florida Slope } \\ \text { WWII } & \text { World War II }\end{array}$




\section{Deepwater Program: Lophelia II Continuing Ecological Research on Deep-Sea Corals and Deep-Reef Habitats in the Gulf of Mexico}

\section{LOPHELIA II INTRODUCTION AND PROGRAM OVERVIEW}

By Steve W. Ross ${ }^{1}$

${ }^{1}$ University of North Carolina Wilmington, Center for Marine Science, Wilmington, NC

\subsection{Introduction and Program Overview}

Knowledge about deep-sea corals (DSC) and deep-reef structures off the southeastern United States (SEUS) and Gulf of Mexico (GOM) has evolved into multi-disciplinary research in a relatively short time. DSC ecosystems in the GOM appear to be more scattered and less abundant (Brooke and Schroeder, 2007; Schroeder and Brooke, 2011) than those in the northeastern Atlantic Ocean (HallSpencer and others, 2007) or off the SEUS (Ross and Nizinski, 2007). Even so, the corals themselves and the communities associated with them are diverse and dynamic. Over the last decade, three large programs funded by the Bureau of Ocean Energy Management (BOEM, formerly Minerals Management Service), the U.S. Geological Survey (USGS), and the National Oceanic and Atmospheric Administration (NOAA) and others (see section 1.6 Acknowledgments) greatly expanded knowledge of DSC ecosystems in the GOM. The first of these programs was called Lophelia I (2004-2006) and was concentrated on 10 deep-reef sites in the north-central GOM (CSA International, Inc., 2007; Sulak and others, 2008). While the second of these programs, Chemo III (2006-2007), was mostly directed toward chemosynthetic communities deeper than 1,000 meters $(\mathrm{m})$, this program also had an emphasis on DSC and deep hardground communities (Roberts, 2010; Ross and others, 2012; Brooks and others, 2014). The third program, which is the subject of this report, was called Lophelia II (2008-2012) and was a joint effort between a USGS/University of North Carolina at Wilmington (UNCW) team and a research team managed by TDI Brooks, Inc. (original study titled "Exploration and Research of Northern Gulf of Mexico Deepwater Natural and Artificial Hard Bottom Habitats with Emphasis on Coral Communities: Reefs, Rigs and Wrecks"). The Brooks team is developing a separate final report for their study components. During these three programs, the USGS/UNCW team developed a list of proposed publications, 37 (includes 1 Master's thesis) of which have been published and some of which are still in progress. The list of published papers is included as Appendix A. The USGS components of these studies were given the acronym DISCOVRE (Diversity, Systematics, and Connectivity of Vulnerable Reef Ecosystems), which also included other programs, and various aspects of these studies were displayed on a USGS website (https://www.usgs.gov/centers/wetland-and-aquatic-research-centerwarc/science-topics/discovre). 
The Lophelia II program concentrated the work around Lophelia pertusa habitats because of its abundance, wide distribution, and structure-forming abilities, but the objectives were applicable to other hard substrata habitats including mixed corals and sponges, as well as artificial substrata (wrecks, oil platforms). Soft bottom areas were also sampled to allow for comparisons between reef and non-reef communities. This project builds upon previous work by the program participants. As an integrated regional exploration of deep-reef physical structure and ecology, this interconnected, multidisciplinary approach helped advance the understanding of critical deep-sea habitats. Using standardized methods throughout this large region will enable comparisons among complex habitats over great depth and latitudinal ranges. By examining deep-reef habitats over a wide geographic range, we can gain an understanding of how these ecosystems function (through comparative analyses) and the degree to which they are interconnected. The USGS/UNCW team originally proposed a six-part series of multidisciplinary projects. Topical areas of the six studies are (1) physical oceanography, (2) trophodynamics, (3) genetics, (4) microbiology, (5) benthic ecology (vertebrate and invertebrate), and (6) geochemistry (paleoecology). A seventh study on the reproductive biology of DSCs was added after the project was underway. Logistics for the cruises (see, section 1.3, Summary of Research Cruises), general data management, and overall study management were overseen by S.W. Ross (UNCW). Lead personnel and general objectives for the seven study parts were as follows.

1. Physical oceanography. Lead Personnel: A. Davies (Bangor University), G. Duineveld (Netherlands Institute for Sea Research, NIOZ), F. Mienis (NIOZ), S.W. Ross (UNCW). The overall goals of this study component were to describe short-term (hours-days) and longer term (weeks-months) variability of benthic physical oceanographic parameters on DSC habitats and provide a platform (benthic landers) for instruments for use in various biological/ecological studies. Objectives were to (A) describe water column and benthic currents around a DSC habitat; (B) describe variability in basic benthic water chemistry; (C) collect data on particle flux and food value of particle rain; (D) conduct long-term observations of habitat and fauna; and (E) examine invertebrate settlement and microbial recruitment via settling plate experiments.

2. Trophodynamics. Lead Personnel: A. Demopoulos (USGS), S.W. Ross (UNCW). The overall study goals were to describe the benthic trophic web, construct an energy flow model, and evaluate effects from potential changes in food supply on and around deep-sea coral habitats in several GOM locations. Objectives were to (A) determine basic feeding patterns of major faunal groups using deep-reef and nearby habitats; (B) determine food sources for deep-reef communities; (C) assess adequacy of the food supply to major faunal groups; (D) assess the impact of seeps on deep-coral trophodynamics; (E) develop a carbon budget for selected deepcoral systems; (F) determine the impact of habitat or micro-habitat characteristics on trophic patterns; and $(\mathrm{G})$ determine the extent of trophic isolation.

3. Genetics. Lead Personnel: C.L. Morrison (USGS). Objectives were to (A) quantify local and regional patterns of genetic variation in L. pertusa through the application of microsatellite markers developed in the project, including assessments of genetic connectivity between natural reefs and humanmade structures, further examination of the relative contributions of clonal (asexual) and sexual reproduction, and inferences regarding larval dispersal patterns; (B) document biodiversity of scleractinian corals by using informative nuclear and mitochondrial DNA markers and appropriate phylogenetic analyses; (C) characterize levels of connectivity between $L$. pertusa-associated mobile fauna (selected invertebrates and fishes); and (D) interpret genetic results in relation to environmental variables and patterns of microbial diversity (collaborating with other study components). 
4. Microbiology. Lead Personnel: C. Kellogg (USGS). Objectives were to (A) identify and characterize the microbial communities associated with live L. pertusa at multiple sites in the GOM; (B) compare the microbial communities associated with live L. pertusa to those associated with dead L. pertusa and surrounding sediments; (C) determine if L. pertusaassociated mobile fauna act as vectors and transport specific bacteria between coral colonies; and (D) identify and characterize the microbial communities associated with other DSC species (for example, Madrepora and Enallopsammia) in conjunction with the genetics task (see above).

5. Benthic ecology. Study objectives were addressed within two major faunal groups: Fishes, Lead Personnel: S.W. Ross (UNCW) and A. Quattrini (Harvey Mudd College) and Invertebrates, Lead Personnel: M. Nizinski (NOAA Fisheries Systematics Lab), C. Ames (UNCW/NOAA), and A. Demopoulos (USGS). The overall study goal was to improve understanding of deep-reef habitat usage and associations through descriptions of deep-reef and off-reef (nearby) fauna and related ecosystem characteristics. Objectives were to (A) identify the fauna observed on and around the study sites and assess habitat usage; (B) assess levels of endemism at deep-reef habitats; (C) examine patterns of species diversity and geographic distribution of the invertebrate and fish fauna between sites and make comparisons with similar habitats in other locations; and (D) assess community structure and basic ecology of the invertebrate and fish fauna.

6. Geochemistry (paleoecology). Lead Personnel: C. Holmes (USGS), later replaced by N. Prouty (USGS). The study goal was to reconstruct past climate, oceanography, environment, and coral feeding history over a time span of hundreds to thousands of years. Objectives were to (A) determine if trace metals and stable isotopes incorporated within the skeletons of black and bamboo corals reflect environmental conditions and (or) climatic events; and (B) determine if annual bands of deep-sea black corals can be used to create a marine radiocarbon $\left({ }^{14} \mathrm{C}\right)$ calibration curve.

7. Coral reproduction. Lead Personnel: S. Brooke (Florida State University). The overall goals of this study component were to provide comprehensive information on the reproductive biology of dominant deep-water, habitat-forming corals in the GOM and to coordinate these results with other study components. Objectives were to (A) add to existing database on reproductive status of L. pertusa and complete description of the gametogenic cycle; (B) assess reproductive status of other dominant cnidarians (including Leiopathes spp., Keratoisis spp., and Callogorgia delta) to determine if reproductive cycles are synchronous across various taxa; (C) correlate physical oceanographic data (temperature, currents, and food supply) with gametogenesis in L. pertusa (and other species if data were sufficient); (D) correlate food supply to the benthos with lipid content and reproductive output; and (E) assess coral distribution relative to physical and biological characteristics (for example, currents, food delivery, sediment levels, temperature, and bathymetry).

These topics and their objectives guided the sampling plan during all cruises. Most of these objectives were met; however, some were modified or omitted as the project developed. Data from these cruises will continue to be analyzed for many years after the project has ended. These study topics are the subjects of separate chapters within this report.

During the Lophelia II project, the GOM experienced the largest oil spill in U.S. history (Ramseur, 2010). This spill resulted from a blowout at the Macondo well site (MC252), 1,522 m deep, on 20 April 2010. This spill is also referred to as the Deepwater Horizon (DWH) blowout or spill. On 15 July 2010, the well was capped, but was not declared "dead" until 19 September 2010. The DWH well 
site was located just 55 kilometers $(\mathrm{km})$ and $80 \mathrm{~km}$ south of the two Viosca Knoll (VK) study sites (fig. 1.1), and during a large period of the oil spill, these sites were underneath the visible surface oil plume. Both during and after the spill, a tremendous effort was organized to study the effects of the oil spill and determine what restoration activities were appropriate. The USGS/UNCW and the Brooks teams were in a unique position of having several years of pre-spill data for several DSC hardground and chemosynthetic sites as well as having planned cruises that served as post-spill comparisons. Sampling and cruise objectives were modified to attempt to determine if these deep-sea ecosystems had been obviously affected by the oil spill. An additional lander deployment was funded to collect post-spill data that could be compared with the pre-spill lander data (Davies and others, 2010; Mienis and others, 2012). Data from that lander are still being analyzed.

\subsection{Study Areas}

The dominant structure-forming DSC in the GOM is L. pertusa, but many other anthozoan species contribute to the deep-reef ecosystems (Brooke and Schroeder, 2007; Schroeder and Brooke, 2011). DSCs in the GOM can form mounds (bioherms) as they do in other places, but they often take advantage of a variety of hard substrata (for example, authigenic carbonates, shipwrecks, oil rigs, and limestone outcrops) for colonization (Brooke and Schroeder, 2007). Upper to middle slope depths (200$1,000 \mathrm{~m}$ ) in the GOM are influenced by the Loop Current which provides strong currents over coral habitats and which facilitates upwelling of nutrient-rich waters.

All target study sites for this project were on the continental slope (approximately $300-1,000 \mathrm{~m}$ ) in the north-central to eastern GOM (fig. 1.1). Some sites (for example, the VK sites, Green Canyon, and Gulfpenn wreck) were visited during previous studies funded by BOEM and USGS (Church and others, 2007; CSA International, Inc., 2007; Cordes and others, 2008; Sulak and others, 2008; Ross and others, 2012). Two primary VK sites were examined during this study: VK826 and VK862/906 (fig. 1.2). The VK826 site (430-520 m) contains some of the most extensive DSC communities known in the GOM (Brooke and Schroeder, 2007) and is probably the most studied site. This site and the neighboring VK 862/906 site were used for intensive studies involving repetitive visits during the project. See the previously listed references as well as Davies and others (2010) and Mienis and others (2012) for descriptions of the VK area. The West Florida Slope (WFS, fig. 1.1) site, first described by Newton and others (1987) and also visited by Reed and others (2006) and Hübscher and others (2010), encompasses hundreds of lithoherms and a rocky escarpment in depths of about $500 \mathrm{~m}$. Prior to this project, the large WFS area was poorly explored and represented an important comparative site for these studies. It now appears that this area may support the most extensive live DSC communities in the GOM.

\subsection{Summary of Research Cruises}

During the Lophelia II program, the USGS/UNCW team executed six research cruises to accomplish the project goals (table 1.1). Some of the USGS scientists also participated in the TDIBrooks cruises. In addition, the project acquired supplemental multibeam sonar mapping data and ROV dive data for the WFS from two cruises (Lost Coast Explorer, 7-10 November 2010, and NOAA ship Okeanos Explorer, 20-22 March 2012). Overall, during the 6 project cruises, 309 stations were occupied (table 1.1; Appendix B) using a wide variety of methods. All methods using a particular sampling gear were standardized to the extent possible for all cruises. Station maps (figs. 1.2-1.7) indicate a wide coverage of the main study areas using a variety of methods. Since the only objective of the research vessel (R/V) Tommy Munroe cruise was to retrieve a benthic lander (see fig. 1.7), a map is not included for that cruise, it is not included in Appendix B, and it is not discussed further. A map is also not included for the single Ronald H. Brown cruise station because it overlapped stations sampled 
previously on the WFS (see fig. 1.3). During most cruises, an extensive outreach effort was made which involved posting daily logs and other data to the USGS DISCOVRE website

(https://www.usgs.gov/centers/wetland-and-aquatic-research-center-warc/science-topics/discovre).

\subsubsection{Nancy Foster Cruise}

This first cruise of the USGS/UNCW program was divided into two legs, each sampling different areas with different methods. During Leg I (5-13 October 2008), all operations were conducted over and on two major DSC ecosystems, VK826 (430-600 m, fig. 1.2) and VK862/906 (300-500 m, fig. 1.2), separated by about $37 \mathrm{~km}$ (20 nautical miles [nmi]). Leg I departed Pascagoula, Miss., on 5 October 2008 (1000) and returned to Pascagoula on 14 October 2008 (about 0200). Sampling started on 5 October with a conductivity, temperature, depth (CTD) transect leading into the VK826 study area, and various sampling (table B.1) continued in that area until about midnight on 8 October. The vessel moved to the VK862/906 study area and sampled there from 8-10 October, after which it returned to VK826 where it remained until inclement weather forced an end to sampling. This leg returned to port 2 days earlier than planned, resulting in lost sampling days, due to deteriorating weather. Leg II (19-23 October 2008) was dedicated to multibeam sonar mapping of a large section of the WFS suspected of having extensive coral mounds. Leg II left Pascagoula on 19 October (1000 hr), and after about a 20-hr transit, arrived at the WFS area (fig. 1.3). This leg ended in Key West, Fla., on 23 October (about $1400 \mathrm{hr}$ ).

Since more detailed methods are included in various chapters of this report and other publications (see Appendix A), they will not be discussed in detail here. During the Leg I cruise, a remotely operated vehicle (ROV; Saab Seaeye Falcon DR) was used to collect video imagery and still photo images, as well as various samples. Six ROV dives were accomplished at the two VK sites: one dive at VK862/906 and five dives at VK826 (table 1.1; fig. 1.2). When the ROV was not being used (generally at night), bottom and water column samples were collected by using an otter trawl and a Tucker trawl, each towed for 30 minutes ( $\mathrm{min})$, only during Leg I. Plankton nets and various traps were also used for some sampling during Leg I (table 1.1). CTD point stations, as well as transects, were sampled during both legs of the cruise. Finally, during Leg I, two benthic landers were deployed at VK826 to collect oceanographic data for a short-term dataset (Davies and others, 2010) and for a 1-year dataset (Mienis and others, 2012). For Leg II, multibeam sonar mapping was conducted by using a Kongsberg-Simrad EM1002 (95 kilohertz [kHz]) mounted on the ship's hull, and an area covering about $47 \mathrm{~km}$ x $4.5 \mathrm{~km}$, or about 222 square kilometers $\left(\mathrm{km}^{2}\right)$, was mapped (fig. 1.3).

\subsubsection{Seward Johnson Cruise}

This GOM cruise had target study sites ( $\geq 300-\mathrm{m}$ depths) on deep coral areas in the eastern and north-central GOM (see figs. 1.3 and 1.4). The R/V Seward Johnson departed the Harbor Branch/FAU port on 13 September 2009 (1430 hr) and steamed to the first study area on the WFS (about $465 \mathrm{nmi}$ ), arriving for the morning human-occupied submersible Johnson Sea Link II (JSL) dive on 16 September. This area was sampled until after the morning dive of 17 September, after which the ship steamed for the Viosca Knoll study sites in the north-central GOM (about $270 \mathrm{nmi}$ ). The remainder of the cruise in the north-central GOM was spent around the two Viosca Knoll sites, except for 1 day spent on a site in Mississippi Canyon. The vessel ended the cruise on the afternoon of 24 September and steamed to Gulfport, Miss., docking late in the evening of 24 September.

As previously noted, more detailed methods are included in various report chapters and

publications (see Appendix A) and will not be repeated here. The human-occupied submersible JSL was used during this cruise to collect video imagery, still photos, and various samples (table 1.1; fig. 1.4). 
Fifteen $J S L$ dives were accomplished during the cruise. JSL samples were supplemented by bottom trawling, midwater multiple opening/closing net and environmental sensing system (MOCNESS) trawls, box coring, plankton net tows, and CTD water column profiles. The two benthic landers deployed in the 2008 cruise were retrieved on this cruise (Mienis and others, 2012).

\subsubsection{Cape Hatteras Cruise}

This cruise complemented the November cruise undertaken by the BOEM contractor (TDI Brooks, Inc.), and where possible, samples were collected for the contractors. The USGS/UNCW cruise had target study sites in the same areas as the previous cruises: deep coral habitats in the eastern and the north-central GOM (fig. 1.5). Considering the DWH oil spill recently preceded this cruise, special attention was paid to the health and status of habitats and communities encountered. Some sampling was directly related to oil impact studies (Fisher and others, 2014; Demopoulos and others, 2016) and represented new objectives for this team.

The R/V Cape Hatteras departed Gulfport, Miss., on 20 September 2010 (about 2000) and steamed for coral banks located at VK826, arriving on station in early morning of 21 September. This area was sampled until early evening of 23 September, after which the vessel moved to the VK862/906 sites (about $20 \mathrm{nmi}$ or $37 \mathrm{~km}$ distance). The VK862/906 coral banks were sampled from the morning of 24 September through late afternoon of 26 September. After the ROV was recovered on 26 September, the vessel steamed to the WFS sites (about $280 \mathrm{nmi}$ or $519 \mathrm{~km}$ distance), arriving late evening of 27 September. This large area was sampled until early evening of 2 October, after which the vessel steamed to St. Petersburg, Fla. (about $150 \mathrm{nmi}$ or $278 \mathrm{~km}$ ), arriving on the morning of 3 October.

The main sampling tool used on this cruise was the Kraken II ROV, which provided video and still imagery and allowed for a variety of sampling. Eleven ROV dives were accomplished (table 1.1; figs. 1.5 and 1.6). As in previous cruises, these samples were supplemented by bottom otter trawling, midwater Tucker trawling, bottom traps, plankton nets, CTD profiles, and bottom coring with a multicore.

\subsubsection{Arctic Sunrise Cruise}

In response to the DWH oil spill, Greenpeace made available their ship (R/V Arctic Sunrise) and human-occupied submersible (Dual DeepWorker, $D D W$ ) to S.W. Ross and S.D. Brooke. Although not originally part of the Lophelia II program, this cruise was used to supplement data for that program. The Arctic Sunrise left Gulfport, Miss., on 12 October 2010, arriving at the first dive site on 13 October. A benthic lander was deployed at VK906 (fig. 1.7). Eight $D D W$ dives were conducted on the VK862/906 coral areas, and two dives were made on shallower inshore reefs (table 1.1; fig. 1.7). The $D D W$ was used to record video imagery as per previous methods and collect limited coral samples. This cruise ended in Gulfport on 22 October.

\subsubsection{Ronald H. Brown Cruise}

The objective of this cruise was to study deep coral communities off the east coast of Florida, but because the cruise originated in the GOM, limited operations were conducted at the WFS study area on the way into the Atlantic. The Ronald H. Brown departed Pensacola, Fla., on 9 November 2010 (about $0900 \mathrm{hr}$ ) and arrived at coral banks stations on the WFS during the morning of 10 November. The Jason II ROV was used on the WFS, and because the Jason II's navigation system required calibration, the first activity in this study area was to deploy an elevator at approximately 600-m depth and conduct calibration transects. This required 6 to $7 \mathrm{hr}$; however, the elevator acoustic release failed, 
and Jason II was launched to recover it. This recovery delayed operations, and the next dive in this area was shortened (just over 5-hr bottom time) and occurred at night (table 1.1). After recovery of the vehicle, the vessel steamed to the Atlantic study sites.

\subsection{Observations Related to the DWH Oil Spill}

Since most of the Lophelia II north-central GOM stations were within the range of the 2010 DWH oil spill, we were particularly vigilant for signs of oil-related damage. Although some samples collected required further analyses to assess potential impacts (for example, reproduction), on gross examination during the three cruises after the spill (Cape Hatteras, Arctic Sunrise, and Ronald $H$. Brown cruises), the surface, water column, and benthic communities appeared to be healthy. At this general overview, macroscopic level, these communities and their habitats appeared to be in similar conditions as observed in pre-oil spill cruises. No overt signs of hydrocarbons of any kind were observed at any stations during the post-spill cruises. Likewise, White and others (2012) also found no oil effects on deep coral sites that were $>20 \mathrm{~km}$ from the DWH well, but signs of stress and environmental damage were observed at a deep coral site about $11 \mathrm{~km}$ southwest of the well. Additional studies are needed at deep-water coral sites in order to assess potential long-term impacts from the DWH spill.

\subsection{Physical Oceanography}

All physical oceanography aspects of this project were addressed through deployments of benthic landers and CTD profiling. Data published from these projects are available as follows: two published papers document oceanographic conditions during a short-term period (6-11 October 2008, Davies and others, 2010) and a 1-year period (October 2008-September 2009, Mienis and others, 2012) in the vicinity of VK826. Some physical oceanographic data relevant to the Lophelia II study sites were included in CSA International, Inc. (2007) and were also published in Ross and others (2010). Additional data from a 1 year study (October 2010-October 2011) lander deployment at VK906 are still being analyzed.

In summary, Davies and others (2010) and Mienis and others (2012) found that the VK $L$. pertusa corals and associated communities existed in a temperature and salinity regime similar to other areas that have been studied. However, dissolved oxygen levels were lower and mass fluxes of particles to the bottom were higher than in the northeastern Atlantic. The particulate load reaching the bottom

appears to have a riverine origin. Both publications noted that the area displayed a dynamic and variable oceanographic regime, similar to the northeastern Atlantic, but not on the scale observed (very large, rapid fluctuations in water properties) under Gulf Stream influence off North Carolina (Brooke and others, 2013). The Loop Current was noted as a major influence on oceanography in the VK area.

\subsection{Acknowledgments}

I thank the U.S. Geological Survey (Outer Continental Shelf Ecosystem Studies Program), especially Dr. G. Brewer, for funding (under Cooperative Agreement number 05HQAG0099, subagreement 5099HS0013) which supported most of these cruises and data analyses. I appreciate the encouragement and support of G. Boland (BOEM) during these studies. The Ronald H. Brown 2010 cruise (1 station in the GOM) was supported by the NOAA Deep-Sea Coral Research and Technology Program. The Nancy Foster 2008 cruise (two legs) was funded by a grant (to S.W. Ross) from the NOAA National Undersea Research Center at UNCW. The UNCW National Undersea Research Center procured the ROV used on the Nancy Foster cruise. I especially thank our European collaborators (Drs. 
T. van Weering, G. Duiniveld, F. Mienis [all of NIOZ], A. Davies [Bangor University], and J.M. Roberts [Heriot-Watt University]) for providing the landers and their equipment and for participating in the cruises as needed. Drs. C. Fisher (Pennsylvania State University) and E. Cordes (Temple University) were very helpful in providing information and multibeam sonar maps that made the cruises more successful. Greenpeace generously donated a cruise and submersible support in 2010 to S.W. Ross and S.D. Brooke, and I thank J. Hocevar (Greenpeace) for much assistance with that cruise. M. Partyka (NOAA) assisted with multibeam operations on the Nancy Foster cruise. I thank all cruise personnel, especially C. Ames, T. Casazza, M. Carlson, J. McClain-Counts, M. Rhode, A. Roa-Varon, and A. Zilg (all formerly of University of North Carolina Wilmington), for their hard work and dedication while at sea and in dealing with logistics and data before and after the cruises. GIS and mapping assistance was provided by M. Rhode. D. Naar (University of South Florida) provided some multibeam sonar data for the WFS. I thank the ship- and shore-based personnel who operated the R/V Seward Johnson, R/V Cape Hatteras, NOAA ship Nancy Foster, NOAA ship Ronald H. Brown, R/V Arctic Sunrise, NOAA ship Okeanos Explorer, and Lost Coast Explorer for their assistance during the cruises. Finally, I thank the personnel of the Johnson Sea Link II, Kraken II, and Jason II for exemplary service during the missions.

\subsection{References}

Brooke, S., Ross, S.W., Bane, J.M., Seim, H.E., and Young, C.M., 2013, Temperature tolerance of the deep-sea coral Lophelia pertusa from the southeastern United States: Deep-Sea Research II, v. 92, p. 240-248.

Brooke, S., and Schroeder, W.W., 2007, State of deep coral ecosystems in the Gulf of Mexico region: Texas to the Florida Straits, in Lumsden, S.E., Hourigan, T.F., Bruckner, A.W., and Dorr, G., eds., The state of deep coral ecosystems of the United States: Silver Spring, Md., National Oceanic and Atmospheric Administration Technical Memorandum CRCP-3, p. 271-306.

Brooks, J.M., Fisher, C., Cordes, E., Roberts, H., Bernard, B., MacDonald, I., Carney, R., Joye, S., Wolff, G., Goehring, E., Girguis, P., and Bright, M., 2014, Investigations of chemosynthetic communities on the lower continental slope of the Gulf of Mexico, v. I: Final Report: New Orleans, La., U.S. Department of the Interior, Bureau of Ocean Energy Management, Gulf of Mexico Outer Continental Slope Region, Outer Continental Slope Study BOEM 2014-650, 560 p.

Church, R., Warren, D., Cullimore, R., Johnston, L., Schroeder, W., Patterson, W., Shirley., T., Kilgour, M., Morris, N., and Moore, J., 2007, Archaeological and biological analysis of World War II shipwrecks in the Gulf of Mexico: Artificial reef effect in deep water: New Orleans, La., U.S. Department of the Interior, Minerals Management Service, Gulf of Mexico Outer Continental Slope Region, Outer Continental Slope Study MMS 2007-015, 387 p.

Cordes, E.E., McGinley, M.P., Podowski, E.L., Becker, E.L., Lessard-Pilon, S., Viada, S.T., and Fisher, C.R., 2008, Coral communities of the deep Gulf of Mexico: Deep-Sea Research I, v. 55, p. 777-787. CSA International, Inc., 2007, Characterization of northern Gulf of Mexico deepwater hard-bottom communities with emphasis on Lophelia coral: U.S. Department of Interior, Minerals Management Service, Gulf of Mexico OCS Region, OCS Study MMS 2007-044, 169 p.

Davies, A.J., Duineveld, G.C.A., van Weering, T.C.E., Mienis, F., Quattrini, A.M., Seim, H.E., Bane, J.M., and Ross, S.W., 2010, Short-term environmental variability in cold-water coral habitat at Viosca Knoll, Gulf of Mexico: Deep-Sea Research I, v. 57, p. 199-212.

Demopoulos, A.W.J., Bourque, J.R., Cordes, E., and Stamler, K.M., 2016, Impacts of the Deepwater Horizon oil spill on deep-sea coral-associated sediment communities: Marine Ecology Progress Series, v. 561, p. 51-68. 
Fisher, C.R., Demopoulos, A.W.J., Cordes, E.E., Baums, I.B., White, H.K., and Bourque, J.R., 2014, Coral communities as indicators of ecosystem-level impacts of the Deepwater Horizon spill:

BioScience, v. 64, no. 9, p. 796-807.

Hall-Spencer, J., Rogers, A., Davies, J., and Foggo, A., 2007, Deep-sea coral distribution on seamounts, oceanic islands, and continental slopes in the northeast Atlantic, in George, R.Y., and Cairns, S.D., eds., Conservation and adaptive management of seamount and deep-sea coral ecosystems: University of Miami, p. 135-146.

Hübscher, C., Dullo, C., Flögel, S., Titschack, J., and Schönfeld, J., 2010, Contourite drift evolution and related coral growth in the eastern Gulf of Mexico and its gateways: International Journal of Earth Science, v. 99, p. 191-206.

Mienis, F., Duineveld, G.C.A., Davies, A.J., Ross, S.W., Seim, H., Bane, J., and van Weering, T.C.E., 2012, The influence of near-bed hydrodynamic conditions on cold-water corals in the Viosca Knoll area, Gulf of Mexico: Deep-Sea Research I, v. 60, p. 32-45.

Newton, C.R., Mullins, H.T., Gardulski, A.F., Hine, A.C., and Dix, G.R., 1987, Coral mounds on the West Florida Slope: Unanswered questions regarding the development of deep-water banks: Palaios, v. 2, p. 359-367.

Ramseur, J.L., 2010, Deepwater horizon oil spill: The fate of the oil: Congressional Research Service, v. 7-5700, $20 \mathrm{p}$.

Reed, J.K., Weaver, D.C., and Pomponi, S.A., 2006, Habitat and fauna of deep-water Lophelia pertusa coral reefs off the southeastern U.S.: Blake Plateau, Straits of Florida, and Gulf of Mexico: Bulletin of Marine Science, v. 78, p. 343-375.

Roberts, H.H., ed., 2010, Gulf of Mexico cold seeps: Deep-Sea Research Part II, v. 57, p. 1835-2060. Ross, S.W., and Nizinski, M.S., 2007, State of deep coral ecosystems in the U.S. southeast region: Cape Hatteras to southeastern Florida, in Lumsden, S.E., Hourigan, T.F., Bruckner, A.W., and Dorr, G., eds., The state of deep coral ecosystems of the United States: Silver Spring, Md., National Oceanic and Atmospheric Administration Technical Memorandum CRCP-3, p. 233-270.

Ross, S.W., Quattrini, A.M., Roa-Varón A.Y., and McClain, J.P., 2010, Species composition and distributions of mesopelagic fishes over the slope of the north-central Gulf of Mexico: Deep-Sea Research II, v. 57, p. 1926-1956.

Ross, S.W., Demopoulos, A.W.J., Kellogg, C.A., Morrison, C.L., Nizinski, M.S., Ames, C.L., Casazza, T.L., Gualtieri, D., Kovacs, K., McClain, J.P., Quattrini, A.M., Roa-Varón, A.Y., and Thaler, A.D., 2012, Deepwater Program: Studies of Gulf of Mexico lower continental slope communities related to chemosynthetic and hard substrate habitats: U.S. Geological Survey Open-File Report 2012-1032, $301 \mathrm{p}$.

Schroeder, W.W., and Brooke, S.D., 2011, Habitat-forming deepwater scleractinian corals in the Gulf of Mexico, in Buster, N.A., and Holmes, C.W., eds., Gulf of Mexico origin, waters, and biota, v. 3, Geology: College Station, Tex., Texas A \& M Press, p. 355-363.

Sulak, K.J., Randall, M.T., Luke, K.E., Norem, A.D., and Miller, J.M., eds., 2008, Characterization of northern Gulf of Mexico deepwater hard bottom communities with emphasis on Lophelia coralLophelia reef megafaunal community structure, biotopes, genetics, microbial ecology, and geology (2004-2006): U.S. Geological Survey Open-File Report 2008-1148; OCS Study MMS 2008-015, accessed April 18, 2017, at http://dx.doi.org/10.3133/ofr20081148.

White, H.K., Hsing, P-Y., Cho, W., Shank, T.M., Cordes, E.E., Quattrini, A.M., Nelson, R.K., Camilli, R., Demopoulos, A.W.J., German, C.R., Brooks, J.M., Roberts, H.H., Shedd, W., Reddy, C.M., and Fisher, C.R., 2012, Impact of the Deepwater Horizon oil spill on a deep-water coral community in the Gulf of Mexico: Proceedings of the National Academy of Sciences, v. 109, p. 20303-20308. 
Table 1.1. Lophelia II program cruises in the Gulf of Mexico (2008-2011) directed toward deep-sea coral reefs.

[Vessels are the ships used in these cruises and vehicles refer to the remotely operated vehicles (ROV) (Falcon, Saab Seaeye Falcon DR; Kraken II; Jason II) or submersibles (JSL, Johnson Sea Link II; DDW, Dual DeepWorker) used. SWR, S.W. Ross; SDB, S.D. Brooke; VK, Viosca Knoll; WFS, West Florida Slope; NURC, National Undersea Research Center (University of North Carolina at Wilmington); USGS, U.S. Geological Survey; NOAA, National Oceanic and Atmospheric Administration]

\begin{tabular}{llccccl}
\hline \multicolumn{1}{c}{ Dates } & \multicolumn{1}{c}{ Vessel/vehicle } & Chief scientist & $\begin{array}{c}\text { Number } \\
\text { of } \\
\text { stations }\end{array}$ & Areas & Funder & Purpose \\
\hline 5-19 Oct 2008 & Nancy Foster/Falcon & SWR & 88 & VK, WFS & NURC & Biology, ecology, mapping, landers \\
14-25 Sep 2009 & Seward Johnson/JSL & SWR & 99 & VK, WFS & USGS & Biology, ecology, landers \\
20 Sep-3 Oct 2010 & Cape Hatteras/Kraken II & SWR & 109 & VK, WFS & USGS & Biology, ecology \\
12-22 Oct 2010 & Arctic Sunrise/DDW & SWR, SDB & 11 & VK & Greenpeace & Biology, ecology, lander \\
10 Nov 2010 & Ronald Brown/Jason II & SWR, SDB & 1 & WFS & NOAA & Biology, ecology \\
31 Oct-2 Nov 2011 & Tommy Munroe & SWR, SDB & 1 & VK & USGS & Lander retrieval \\
\hline
\end{tabular}




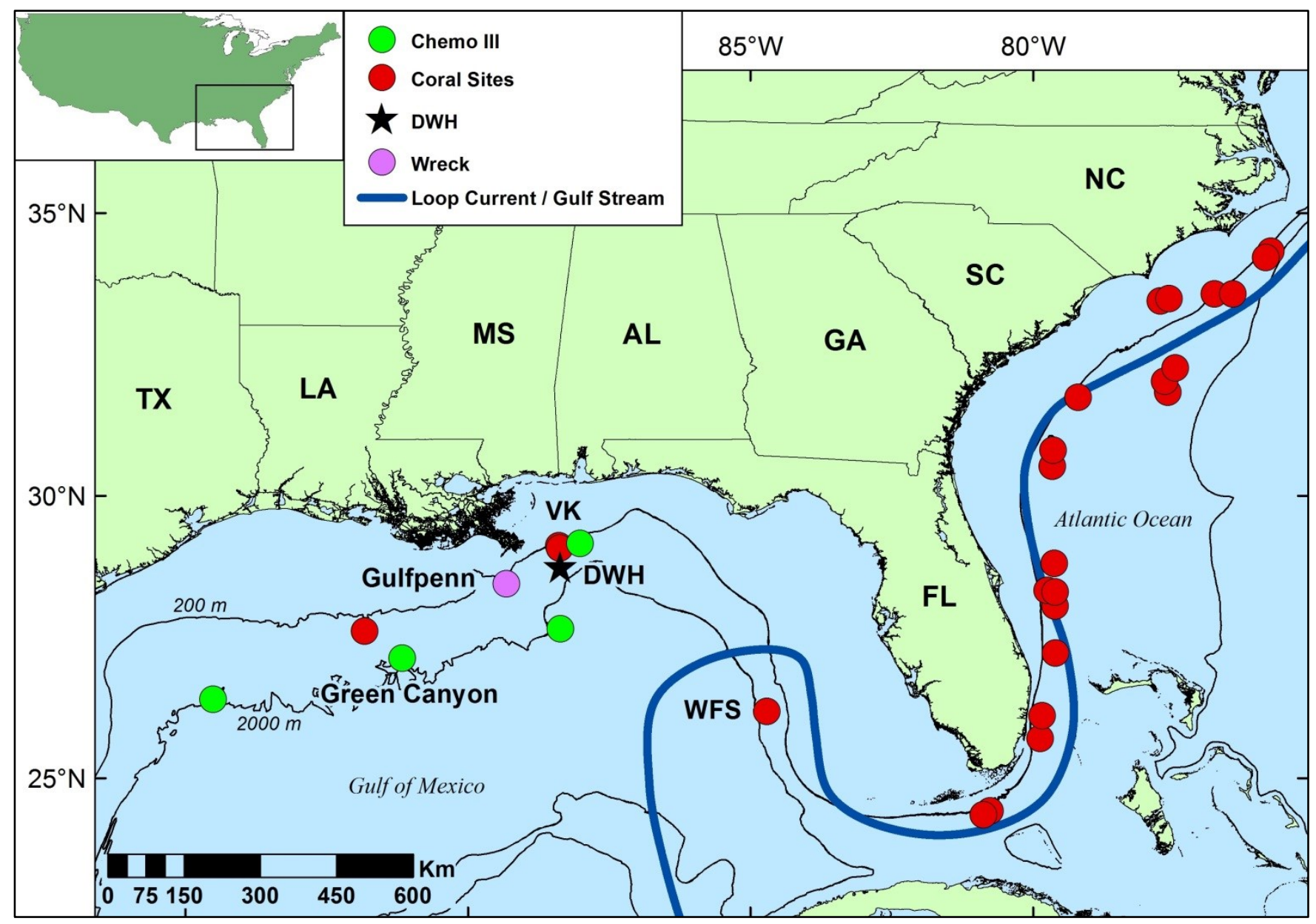

Figure 1.1 Major U.S. Geological Survey/University of North Carolina Wilmington deep-sea study sites in the Gulf of Mexico and off the southeastern United States. Base map data from NOAA. [VK (Viosca Knoll) and WFS (West Florida Slope) were the major study areas for the Lophelia II program. Note the Deepwater Horizon well site (DWH). The Chemo III sites were sampled in 2007. 

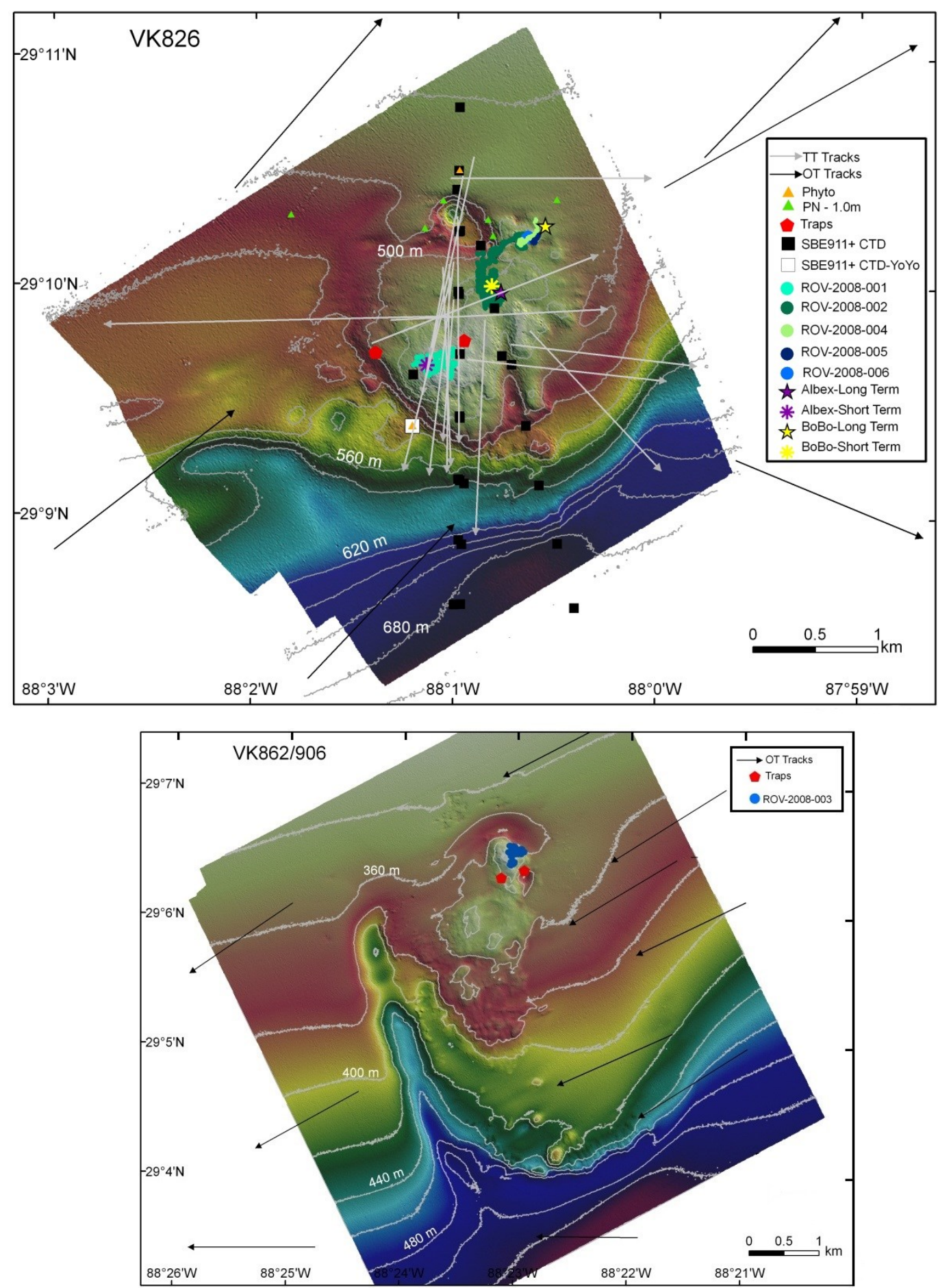

Figure 1.2 Sampling stations at the two Viosca Knoll deep-sea coral study areas of the Gulf of Mexico during the Lophelia II program, color coded by gear type, 5-13 October 2008. Base map data from NOAA.[TT, Tucker trawl; OT, otter trawl; Phyto, surface phytoplankton sample; PN-1m, 1.0-meter plankton net; CTD, Seabird conductivity, temperature, depth instrument; ROV, remotely operated vehicle sampling stations; Albex and BoBo, benthic lander locations.] 


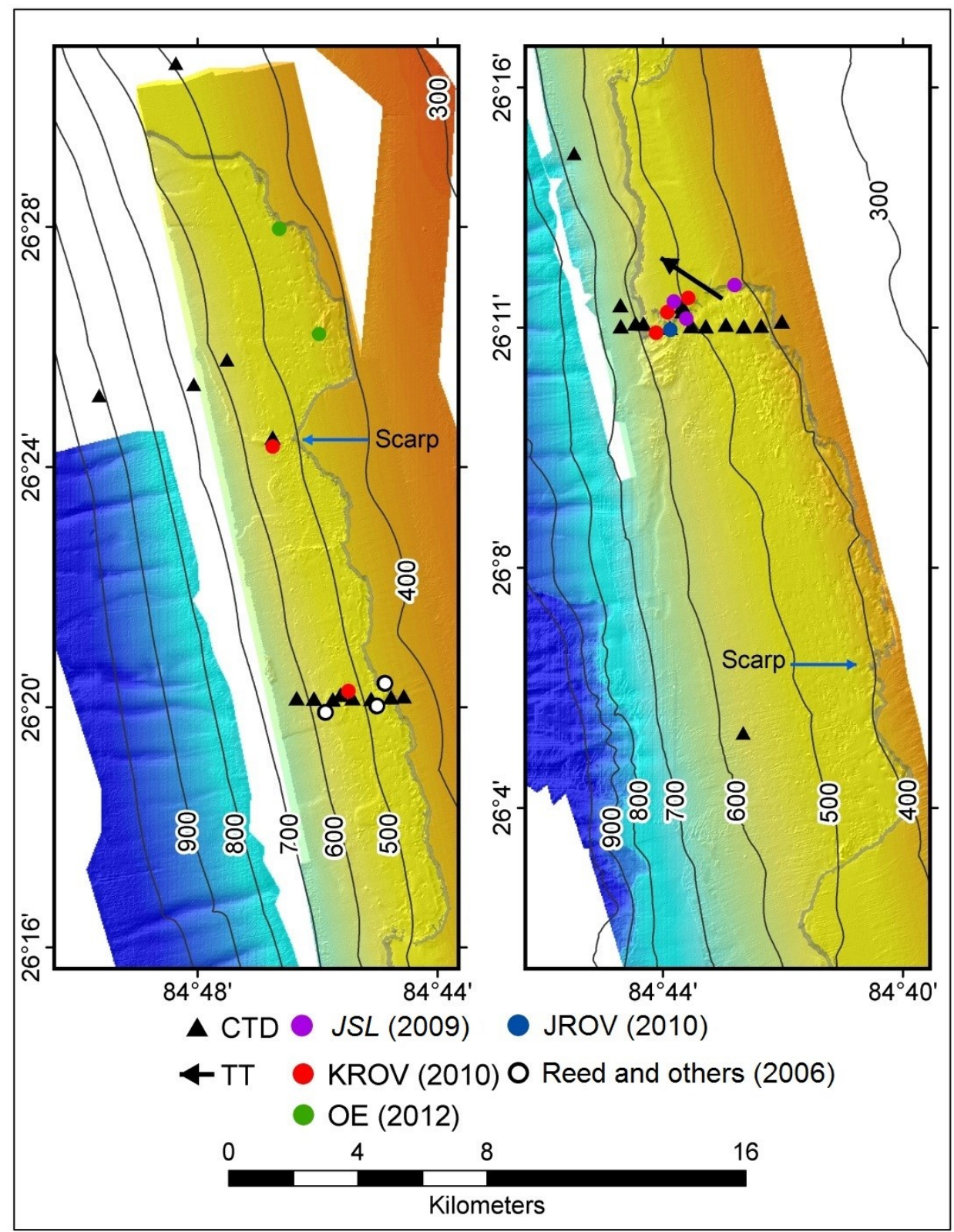

Figure 1.3 Multibeam sonar map of the West Florida Slope resulting from three cruises (Nancy Foster, 20-23 October 2008; Lost Coast Explorer, 7-10 November 2010; Okeanos Explorer, 20-22 March 2012) of the Lophelia II program. Base map data from NOAA. [The bottom of the left panel joins the top of the right panel. CTD, Sea-bird conductivity, temperature, depth instrument; JSL, Johnson Sea Link II stations; JROV, Jason II stations; KROV, Kraken stations; OE, Okeanos Explorer stations. Bathymetric contours represented as depth, in meters. This figure summarizes sampling stations in this region for all cruises, plus Reed and others (2006).] 


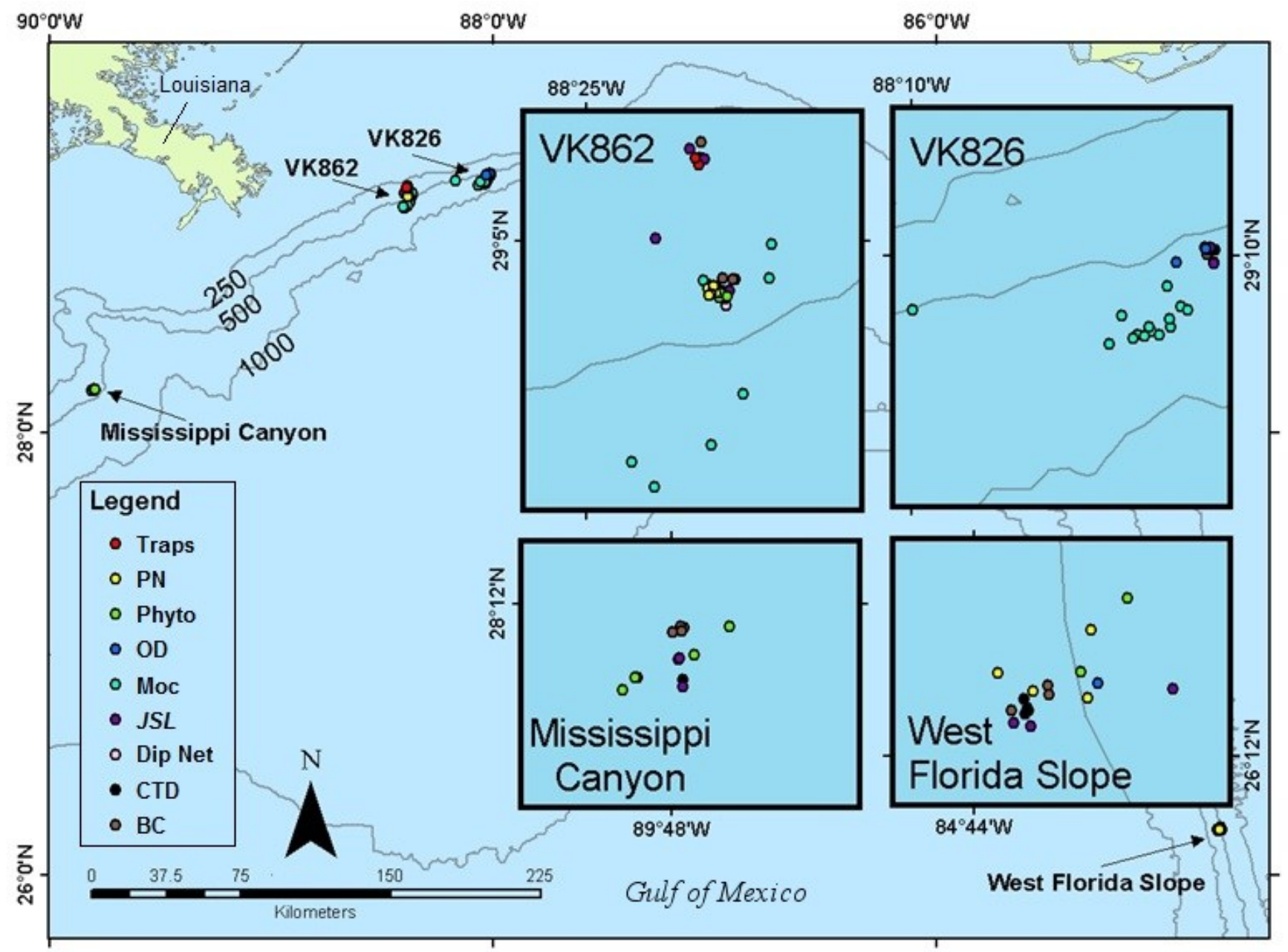

Figure 1.4 Locations of ship-based and Johnson Sea Link II (JSL) submersible stations in four sampling areas in the Gulf of Mexico during the Lophelia II program, sampled 16-24 September 2009. Base map data from NOAA. [PN, plankton net; Phyto, surface phytoplankton sample; OD, on deck; Moc, multiple opening/closing net and environmental sensing system; CTD, Sea-bird SBE 911+ CTD; BC, box core.] 

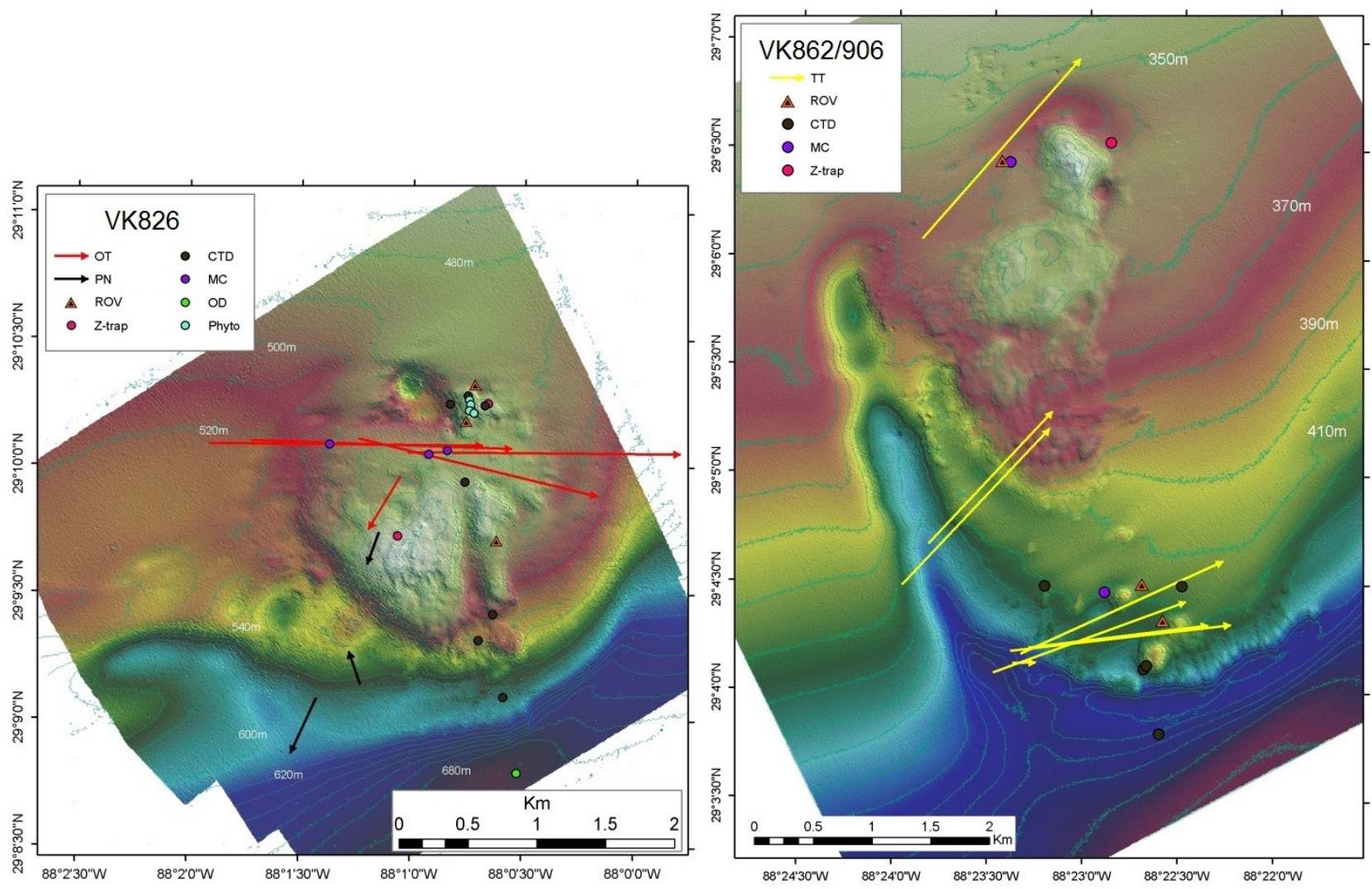

Figure 1.5 Stations at the Viosca Knoll 826 (left) and Viosca Knoll 862/906 (right) study sites in the northcentral Gulf of Mexico of the Lophelia II program, sampled 21-26 September 2010 by the research vessel Cape Hatteras and Kraken remotely operated vehicle (ROV). Base map data from NOAA. [OT, otter trawl; PN, plankton net; Phyto, surface phytoplankton sample; OD, on deck; TT,Tucker trawl; MC, multi-core; CTD, Seabird SBE 911+. For ROV dives, only the bottom start locations are plotted.] 


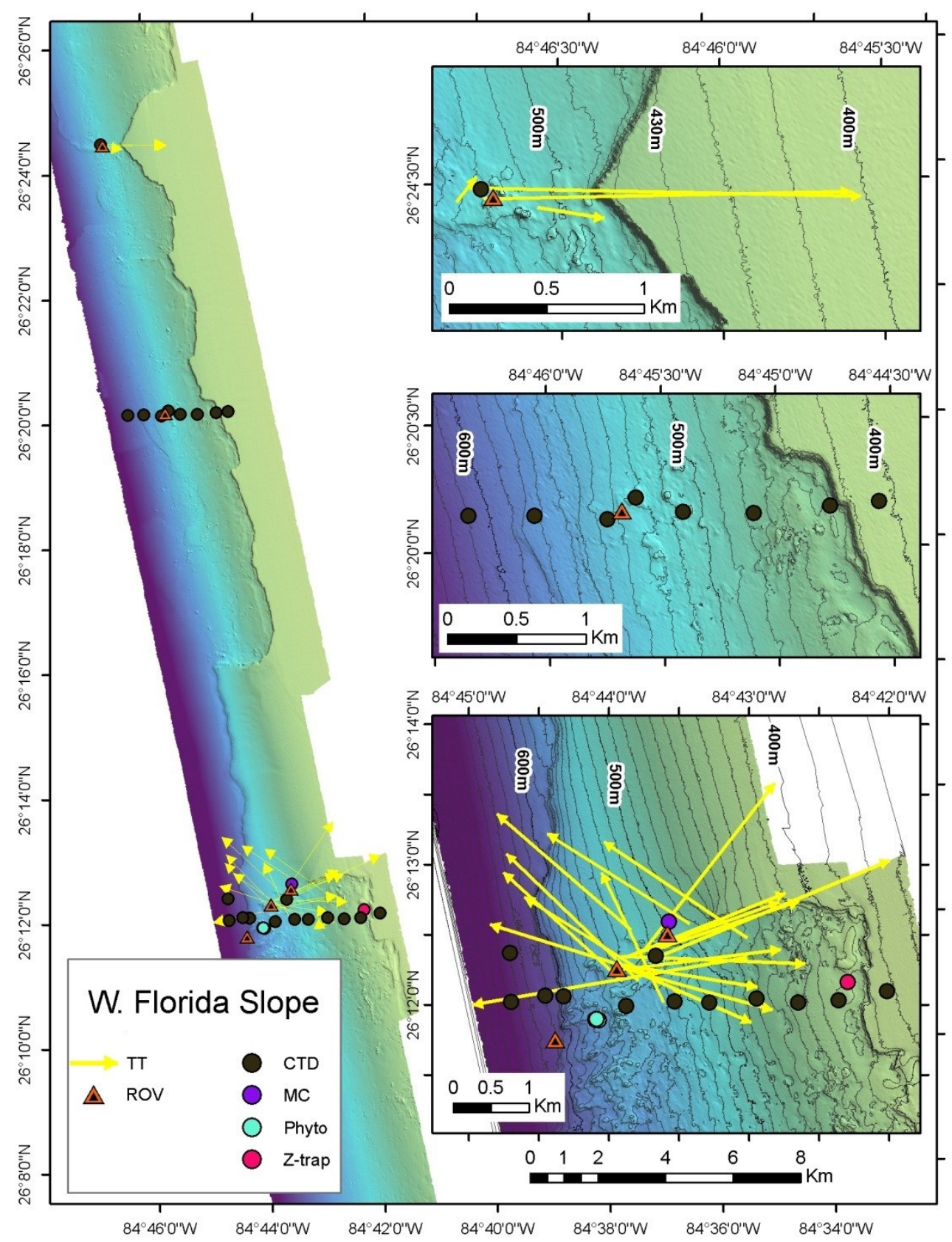

Figure 1.6 Three areas of the West Florida Slope study site in the eastern Gulf of Mexico of the Lophelia II program, sampled 27 September -2 October 2010 by the research vessel Cape Hatteras and Kraken II remotely operated vehicle (ROV).Base map data from NOAA. [TT, Tucker trawl; MC, multi-core; CTD, Seabird SBE 911+; Phyto, surface phytoplankton sample. For ROV dives, only the bottom start locations are plotted.] 


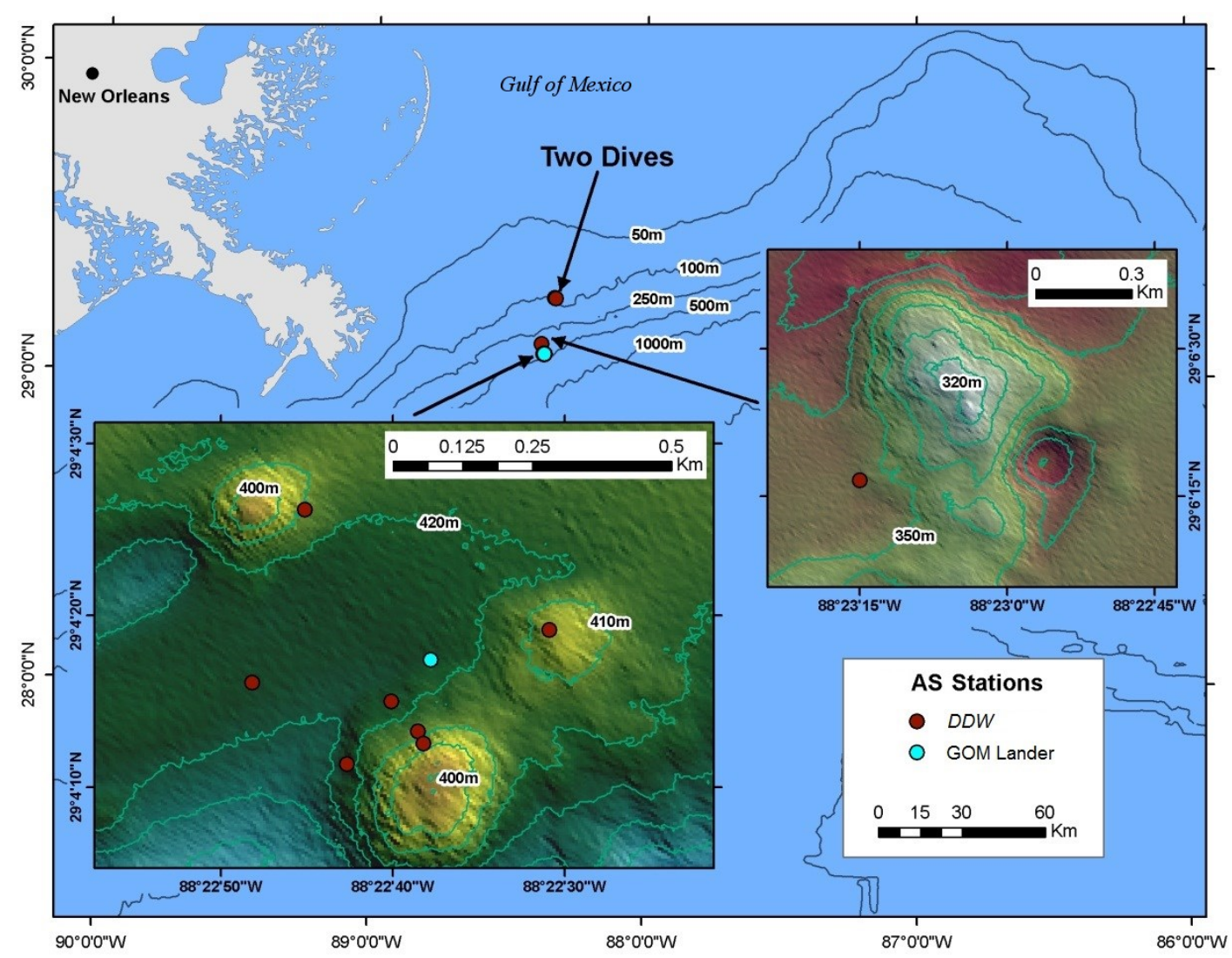

Figure 1.7 Dual DeepWorker (DDW) submersible and benthic lander stations occupied in the Viosca Knoll 862/906 area during the research vessel Arctic Sunrise (AS) cruise (12-22 October 2010) of the Lophelia II program. Base map data from NOAA. [DDW stations are the bottom starting locations for dives.] 


\title{
2 MICROBIAL ECOLOGY AND FUNCTIONAL METAGENOMICS OF LOPHELIA PERTUSA IN THE GULF OF MEXICO
}

\author{
By Christina A. Kellogg, ${ }^{1}$ Julia P. Galkiewicz, ${ }^{2}$ and Michael A. Gray ${ }^{1}$ \\ ${ }^{1}$ U.S. Geological Survey \\ ${ }^{2}$ College of Marine Science, University of South Florida, St. Petersburg, FL
}

\subsection{Introduction}

Lophelia pertusa is a globally distributed species of cold-water scleractinian coral, often found in deep waters ( $>200$ meters $[\mathrm{m}]$ ), that provides important habitat to many invertebrate and fish species and acts as a biodiversity hotspot in the deep sea. Its distribution is determined by factors such as availability of hard substrate, sufficient food supply, and perhaps most important, water temperature between 4 and 12 degrees Celsius $\left({ }^{\circ} \mathrm{C}\right)$ (Freiwald, 2002; Roberts and others, 2006). In the Gulf of Mexico (GOM), these conditions are met at water depths of approximately $300-500 \mathrm{~m}$ when a solid substrate such as authigenic carbonate, shipwreck, or oil platform is also present. These corals can form dense thickets or be present as isolated bushes.

All corals function as consortia of the animals and their associated microorganisms. The scientific term that has been coined to describe this is 'holobiont' (Rohwer and others, 2002), and it describes the coral organism as the sum of the coral animal plus all its associated microorganisms. These coral-associated microorganisms span all three domains of life: bacteria, archaea, and eukarya (for example, fungi and protists) (Rosenberg and others, 2007). Viruses, which are not technically alive, are also present and likely infect all other members of the holobiont (Thurber and Correa, 2011). Microbial associates, which are being found through studies of the human microbiome, are intimately connected to the biology and health of their host. Therefore, in order to answer basic questions we have about $L$. pertusa in the GOM (Is it healthy? Is it being affected by human activities? If damaged, will it regrow? Why is it growing here and not there? How diverse is the population?), we must look to the microbes. The first step is to understand 'who is there' by identifying and characterizing the microbial associates of $L$. pertusa. Then the work shifts toward determining what those microbial associates are doing in an effort to elucidate the roles and pathways by which the microorganisms are providing ecological services to the coral.

A variety of microbiological and molecular methods can be applied to these questions. Classical microbiology techniques are often dependent on culturing the microorganisms first; that is, growing them in isolation such that they can be subjected to any number of tests to determine temperature tolerance, growth rate, antibiotic resistance, and the ability to metabolize different carbon sources, etc. These methods and this information are critical and foundational; however, only about 1-10 percent of environmental bacteria (and even fewer archaea) can be cultured with current methods (for example, Eilers and others, 2000). It is unknown what percentage of microeukaryotes (for example, fungi or protists) can be cultured. To overcome this limitation, many researchers have turned to molecular techniques that rely only on the microbe's DNA (Lau and Liu, 2007). Using bar-code sequences like the ribosomal RNA gene, much previously unknown microbial diversity has been uncovered (Fuhrman and Davis, 1997). However, most researchers agree that the best approach is to use a combination of techniques because all have their inherent limitations and biases. 


\subsubsection{Lophelia I}

Culture-based and culture-independent methods were used during the Lophelia I project (20042007), and the results from that work provided the foundation for the Lophelia II study. During Lophelia I, more than 200 bacterial colonies were cultured on glycerol artificial seawater agar (GASWA) from $L$. pertusa collected at two Viosca Knoll (VK) sites (VK826 and VK906; fig. 2.1) over 2 years (Galkiewicz and others, 2011). This was the first study to culture bacteria from L. pertusa. A total of 196 bacteria were identified based on their 16S rRNA genes, and 76 were screened for antibiotic resistance profiles using the Kirby-Bauer method. The majority was Gammaproteobacteria, and their 16S rRNA genes were very similar (97-100 percent) to previously cultured bacteria, including some found in shallow-water corals. Many of the isolates were resistant to penicillin, and almost all were resistant to clindamycin. This natural resistance is unlikely to be connected to clinical resistance seen in hospitals. More importantly, the antibiotic resistance patterns sometimes differed between isolates that were the same species based on their 16S rRNA gene, illustrating strain-level variability that normally remains undetected when using a single gene for identification.

Molecular techniques were also used during Lophelia I to more fully examine bacterial diversity. By directly extracting microbial community DNA, a number of 16S rRNA gene libraries were assembled from L. pertusa collected in the northeastern GOM (Kellogg and others, 2009). This study revealed the presence of two L. pertusa-specific microbial groups, found in the GOM corals and in corals from Norwegian fjords. One group was Gammaproteobacteria similar to the symbionts found in cold-seep mussels, and the other was a novel group of mycoplasmas (tiny cells that lack a cell wall and are often dependent on their host for some metabolic functions). These mycoplasma sequences dominated L. pertusa from site VK826, but were not detected in corals from VK862/906. Although only two sites were sampled, dramatic differences in the coral-associated bacterial communities were evident between them. This microbial spatial variability seemed to track similar results from the coral genetics studies. Shallow-water studies have found that (1) coral-associated bacterial communities are speciesspecific, that is, the communities varied by coral species not geographic location (Rohwer and others, 2002), and (2) coral-associated bacterial communities shifted in response to coral disease or stress (Pantos and others, 2003; Klaus and others, 2005). These findings potentially link the observed microbial differences in L. pertusa to external factors such as temperature variability or internal factors such as genetic susceptibility. Data from additional sites in the GOM were needed to clarify this issue and also in order to achieve a perspective on basin-scale variability of the L. pertusa microbial community.

\subsubsection{Lophelia II}

This chapter summarizes the microbiological findings of the Lophelia II project, which took place in the GOM during 2008-2012. This work expanded the previous study of $L$. pertusa-associated microbial ecology from two sites in the northeastern GOM to three additional sites in the GOM and two in the western Atlantic Ocean (fig. 2.1) through a number of research cruises (table 2.1). The intention was to conduct further work on the bacterial diversity that had been revealed during the Lophelia I studies, as well as expand the scope of exploration to include the diversity of other microbial associates of $L$. pertusa such as archaea, fungi, and viruses.

\subsection{Culture-based experiments}

As previously noted, while cultivation is currently limited to a small percentage of the total microbial community, it remains critical to groundtruth gene-based studies. Only by examining a 
microorganism can we accurately determine its metabolic and functional capabilities. Relationships that are inferred from bar-coding genes (such as 16S or 18S rRNA) or functional genes are based on what we know from cultivated isolates that have similar genes. For both bacterial and fungal cultivation, small pieces of L. pertusa (1-3 polyps) were cracked open using a flame-sterilized hammer to expose the tissue. Phosphate-buffered saline was added to create a slurry of tissue and mucus that could be spread onto the various types of agar plates. These plates were incubated at $4{ }^{\circ} \mathrm{C}$ to best simulate in situ temperatures. Individual colonies were later isolation streaked to purity.

\subsubsection{Bacterial Diversity}

The bacterial diversity we were able to capture by using GASWA (Smith and Hayasaka, 1982) during Lophelia I was relatively limited in that it was dominated by a few easy-to-grow genera (Pseudoalteromonas, Photobacterium, and Vibrio) (Galkiewicz and others, 2011). To avoid similar low diversity results, we experimented with a lower-nutrient agar and used a variety of selective media containing amendments intended to block the growth of certain bacterial groups in order to allow growth of others (table 2.2). Our main targets with selective media were the two conserved bacterial groups that we had detected by sequence only (Kellogg and others, 2009): mycoplasmas and thiotrophs (sulfur-eaters). Since mycoplasmas lack cell walls, we amended various media with cell-wall poisons (such as thallous acetate) and antibiotics that target cell walls (such as penicillin) to block other bacterial growth. For the thiotrophs, we chose media that included sulfur and had previously been used to isolate these types of bacteria. Unfortunately, to date, none of our efforts have succeeded in cultivating either of these two elusive groups from L. pertusa.

We had a problem multiple times with the Oxoid Mycoplasma medium plus Supplement G. The agar would appear to be solidified in the lab, but after a few days at sea, it liquefied, even when stored at refrigerated temperatures. Although this agar was taken on both the 2009 Atlantic and the 2009 GOM cruises, it was unusable. In 2010, we tried adding extra agar (raising the concentration from 10 percent per liter to 15 percent) to make it firmer. While the increased agar concentration kept the agar plates from liquefying, it may have prevented growth of the target organism because we did not isolate any colonies on that medium. Similarly, most attempts to target mycoplasmas using other marine media (GASWA, 2216, C-mix, 60:40; table 2.2) amended with cell-wall poisons did not yield any isolates or fostered growth of colonies that could not be propagated further. The exceptions were a number of Halomonas species that grew on GASWA with thallous acetate (GASWA+TA; table 2.3) and some Pseudomonas species that grew on GASWA plus Supplement G and Polymixin B.

While we were able to isolate and characterize a number of isolates from the two media containing sulfur (ATCC 295 S8 and MP Agar; table 2.2), none of them were thiotrophs. These included bacteria we had cultured on GASWA in the past such as Pseudoalteromonas, Pseudomonas, and Psychrobacter, but also some types we had not previously cultured: Arthrobacter, Microbacterium, and Sporosarcina (table 2.3).

In spite of additives designed to deter bacterial growth, some bacteria were also cultured on fungal media (table 2.4). These bacteria were all from two genera: Pseudomonas and Curtobacterium (table 2.3). It is not surprising to find Pseudomonas on the fungal media that contained chloramphenicol because some species are intrinsically resistant due to low membrane permeability and active efflux pumping of the antibiotic out of the cell ( $\mathrm{Li}$ and others, 1994). However, it was unusual to find pseudomonads growing on Orange Serum Agar (which has a pH of 5.5) because members of this genus are not known to tolerate acidic conditions and have zero growth at a pH of 4.5 (Palleroni, 2005). In contrast, Curtobacterium is a genus previously shown to colonize acidic environments (Aizawa and others, 2007). 
In spite of our efforts, most of the bacteria we were able to culture were Gram-negative gammaproteobacteria, as we found during Lophelia I. However, 25 percent of our isolates were Grampositive and contained examples of Actinobacteria and Firmicutes (table 2.3). This is at least a start towards uncovering more of the culturable bacterial diversity present in L. pertusa.

\subsubsection{Fungal Diversity}

Since shallow-water corals harbor fungi as part of their holobiont (Bentis and others, 2000; Wegley and others, 2007), we suspected that L. pertusa did also. Fungi have been cultured from a variety of deep-sea locations, including hydrothermal vent fauna (Burgaud and others, 2009, 2010), submarine volcanoes (Connell and others, 2009), and sediments (Singh and others, 2010). Histological investigations have shown fungal bioerosion in dead L. pertusa skeletons attributed to the filamentous fungi Dodgella priscus (Freiwald and others, 1997), which was visually identified but not molecularly confirmed. Fungal hyphae have also been associated with L. pertusa septae, but not identified (Freiwald and Wilson, 1998; Wisshak and others, 2005). Our previous molecular attempts to detect fungi in $L$. pertusa using 18S rRNA genes were swamped by the coral's DNA because both are eukaryotes (Kellogg, 2008), so we attempted to culture fungi using fungal-specific media (table 2.4) during cruises in the Atlantic in 2009 and in the GOM in 2010. Most of the media chosen had low pH to suppress growth of many bacterial species. In addition, most of the agars used antibiotics (such as chloramphenicol) to inhibit bacterial growth in favor of fungi.

Our efforts resulted in the first study to culture fungi from a cold-water scleractinian coral (Galkiewicz and others, 2012). We identified yeast and filamentous forms of fungi from healthy colonies of $L$. pertusa that were capable of metabolizing multiple carbon sources. These fungal isolates were cultured from corals located in the northern GOM, the West Florida Slope, and the western Atlantic Ocean (fig. 2.1) and were phylogenetically closely related, suggesting the possibility of a conserved relationship with the coral. The temperature range for these collections was $7-9{ }^{\circ} \mathrm{C}$, and the salinity range was $34.9-35.5$ practical salinity units (psu).

We identified the fungi by sequencing the 18S rRNA gene and the internal transcribed spacer (ITS) region (table 2.5; for full phylogenetic trees, please see Galkiewicz and others, 2012). Phylogenetic trees based on the $18 \mathrm{~S}$ rDNA phylogenies were used to determine more distant phylogenetic relationships between isolates, while the ITS regions of fungal rDNA were used to determine close relationships between strains (Seifert and others, 1995). These isolates fell into two main fungal groups, basidiomycetes and ascomycetes. The nearest matched 18S and ITS sequences of the L. pertusa-associated fungi are sometimes different (table 2.5), but this is often due to the fact that sexual and asexual life cycles of a single fungus have unique taxonomic names (for example, Nectria/Acremonium; Samuels, 1976).

The limited phylotypes of fungi isolated from the Atlantic L. pertusa reef sites can be attributed to the fact that only one media type was used that is conducive to fungal rather than bacterial growth. With additional fungal media designed to inhibit bacterial growth, many more species of fungi were isolated in the GOM. However, these results represent only a glimpse of fungal diversity associated with L. pertusa because of the limited nature of culturing. The great plate count anomaly has shown that with current culture techniques, between 0.1 and 1 percent of bacteria can be cultured in laboratory conditions (Staley and Konopka, 1985); a similarly sized subset of fungi could conceivably be cultured (Raghukumar, 2006). While culturing these isolates provided valuable insight into their metabolic capabilities, culture-independent techniques should also ultimately be used to determine diversity.

A subset of the fungi also was tested to determine which carbon sources they were capable of oxidizing (table 2.6). This was done by using commercial microtiter plates that have wells containing 
substrates of interest plus a colormetric indicator dye (Biolog FF MicroPlate) designed to be used with environmental fungi. The fungal cultures were grown and inoculated into these plates, and then absorbance readings were used to monitor development of the indicator dye. Of all the averaged Biolog readings for the 14 tested fungal isolates, 4 isolates had no positive wells indicating that they did not metabolize any of the provided carbon sources (ROV09Q2-FVU-01, ROV11Q1-FRB-01, ROV09Q1FVU-02, and ROV02Q2-OSA-02). The other fungal isolates were able to metabolize a variety of carbon sources, with carbohydrates, carboxylic acids, and amino acids being the most common (table 2.6). The remaining carbon source categories were not used by all genera. The Nectria/Acremonium spp. (n=4; a fifth isolate did not grow on any provided substrates) had positive readings in the negative control well (water only). As discussed in Druzhinina and others (2006), positive readings may be attributed either to the fungal cells using nutrient reserves contained within the cells or metabolism of the gel used to stabilize the dye and substrates within the Biolog plate. Evidence of active metabolism in the negative control was only observed for this genus of fungal isolates. Many of the carbon types that could be metabolized are present in the composition of L. pertusa mucus (table 2.7).

The potential function of fungi in association with L. pertusa could fall anywhere along the symbiotic continuum (mutualism, commensalism, or parasitism). Many examples of mutualistic associations between fungi and other eukaryotes can be found, with the fungi performing a range of roles: acting as beneficial saprophytes to break down recalcitrant carbon sources, secreting protective compounds as a defense, or absorbing nutrients that are translocated to the host. The fungi could also have a commensal lifestyle, using the coral as merely a substrate or metabolizing the coral mucus that is continually secreted. A final possibility exists that these fungi represent potential pathogens to the coral. While no disease has ever been documented in L. pertusa (based on direct observations via submersible, remotely operated vehicle (ROV) videos and pictures), it is possible that patchy sampling could miss disease outbreaks. In coral microbiology, it has been demonstrated that bacterial community members found on healthy corals may become pathogenic if conditions shift to increase stress on the coral (for example, Bourne and Munn, 2005; Kline and others, 2006). These pathogenic conditions could extend to fungal members of the microbiota, with fungi acting as pathogens if the balance of the system has been disturbed. While the functional role of fungi in the coral holobiont isn't clear, this study represented a first attempt at understanding potential connections between the eukaryotic members of the L. pertusa holobiont.

\subsubsection{Settling Plate Experiment}

In shallow-water systems, the presence of certain bacteria in biofilms has been shown to trigger settlement or metamorphosis in a variety of invertebrates including corals (Johnson and others, 1991; Johnson and Sutton, 1994; Negri and others, 2001). In particular, Pseudoalteromonas spp. have been shown to induce metamorphosis in Acropora larvae (Negri and others, 2001). However, no settling experiments with $L$. pertusa have been reported. In an effort to determine what cues settlement in $L$. pertusa (in other words, why they are seen on some hard substrates but not others when all other environmental parameters are equal), the following experiment was conducted.

Four different strains of Pseudoalteromonas (4746K6-B8, 4746K6-B13, 4746K8-B4, and 4873K4-B4) that had previously been isolated from L. pertusa (Galkiewicz and others, 2011) were grown in pure culture to high titer. Settling plates were made by cutting 4-inch (in.) by 5-in. by 0.5 -in. (approximately 10 -centimeter [cm] by $12.5-\mathrm{cm}$ by $1.3-\mathrm{cm}$ ) blocks of calcium carbonate coral stone. These plates were threaded on a stainless steel rod through a hole drilled through their centers and spaced apart using 2-in. $(5-\mathrm{cm})$ cylinders of PVC. These stacks were rinsed with water and autoclaved for 10 minutes at $121{ }^{\circ} \mathrm{C}$ to sterilize them, followed by a 25 -minute drying cycle. The stacks were then 
moved to a laminar flow hood to cool. Each stack of five plates was soaked in bacterial culture for 2 weeks to impregnate the coral stone and establish a biofilm on the plates (fig. 2.2). This was accomplished by filling sterilized containers with 7 liters (L) each of GASWA (Smith and Hayasaka, 1982) plus 100 milliliters (mL) of high titer bacterial culture. One control stack did not have any bacteria added. An aliquot of each inoculum was plated as a dilution series in order to estimate the total number of bacterial cells added to each container. For all four bacteria, $10^{11}$ cells were added to each container and then allowed to grow for 2 weeks during their incubation at $4{ }^{\circ} \mathrm{C}$. Note that the containers were sealed with lids and that the liquid was stirred every 2 days to oxygenate the cultures. All inoculated containers had turbid liquid indicating bacterial growth, while the control medium remained clear. After 2 weeks of exposure to bacteria, the stacks were removed from the liquid and each transferred to a 5-gallon (19-L) Ziploc plastic bag for shipment. The stacks were maintained at $4{ }^{\circ} \mathrm{C}$ during shipment and storage.

The five stacks of settling plates were attached to a benthic lander and deployed in October 2010, in the VK lease block (fig. 2.3). L. pertusa in the GOM spawn in late September/early October (CSA International, Inc., 2007), so it was hoped that this timing would place the settling plates in the path of L. pertusa larvae looking to settle. The point of the experiment was to examine the settling plates after a year for evidence of colonization by L. pertusa and to determine if any of the four bacterial cultures enhanced settlement compared to the control (hard substrate alone).

The lander was retrieved in October 2011, and the settling plates were removed from their stacks and preserved in ethanol until they could be examined. The plates were individually inspected using a dissecting microscope in April 2012. Hydroids, foraminifera, and a few barnacles were seen on the plates. None of the treatment plates showed any sign of cnidarian settlement. Three cnidarian spat were identified on the control plates; two on settling plate 4 (second from the bottom of the stack) and one on settling plate 5 (bottom). These juvenile cnidarians were circular and measured approximately 3-5 millimeters $(\mathrm{mm})$ in diameter. They were collected into ethanol and sent to Dr. Stephen Cairns (Smithsonian) for possible morphologic identification. He was unable to identify them further, and they were then sent to Dr. Cheryl Morrison (USGS) for genetic processing. She only received two samples, and it is not known what became of the third. The two samples were confirmed to be cnidarian in origin, but unfortunately, neither sample was L. pertusa. One appeared to be from a complex coral, probably in the family Flabellidae, and the other was identified as an anemone (C.L. Morrison, U.S. Geological Survey, written comm., July 17, 2012).

In summary, none of the bacterial treatments encouraged cnidarian settlement, indicated by the fact that the only cnidarians observed were on the control plates. Moreover, in spite of being deployed soon after L. pertusa spawning time, no L. pertusa recruits were observed. The main colonizers of the settling plates were hydroids and foraminifera. At least three color-morphs of hydroids were observed (colorless, tan, and dark brown). The darkest hydroids were only found inside crevices. Samples of hydroids from each plate were preserved in ethanol and sent to Dr. Leanne Henry (Heriot-Watt University).

\subsection{Molecular-based Experiments}

While our group has been responsible for the only culture-based data on L. pertusa-associated microbes (Galkiewicz and others, 2011, 2012), we are the first to acknowledge that molecular techniques are critical to describing a larger portion of the microbial diversity. For that reason, we

continued to use DNA-based methods such as PCR and have added second-generation sequencing (also referred to as pyrosequencing) to the techniques being used to describe the microbial ecology of $L$.

pertusa. 


\subsubsection{Megafaunal Vectors}

Previous research (Kellogg and others, 2009) revealed the presence of L. pertusa-specific bacterial groups. It was hypothesized that coral-associated mobile fauna, such as crabs or fishes, could be acting as transport vectors for some of these dominant members of the coral microbial community (similar to the hypothesis of Sussman and others, 2003). Could these megafauna be ferrying bacteria from coral to coral, or between seep communities and corals? We sought to answer this question by sampling the bacterial communities of mobile taxa closely associated with corals, particularly species that make physical contact with the corals. In practice, this meant that we sampled two types of galatheid crabs (Eumunida picta and a Munidopsis sp.) and conger eels. The galatheids tend to hold their abdomens curled under, making that the most likely area to be in contact with the coral, and congers have been seen on video burrowing into the coral matrix. The methodology employed was to collect the fauna-associated microbial community by rolling a sterile foam swab across the crab abdomen (fig. 2.4) or the side of the conger eel. The swab tips were then cut off by using sterile scissors and inserted into tubes containing $1.5 \mathrm{~mL}$ of RNALater, kept at $4^{\circ} \mathrm{C}$ overnight, and then stored at $-20^{\circ} \mathrm{C}$ until processed. During the 2009 GOM cruise, a total of 15 Eumunida, 14 Munidopsis, and 4 conger eels were sampled this way. Back in the lab, microbial DNA was extracted from the swabs by using the MOBIO Ultraclean Soil DNA extraction kit, following the alternative protocol for increased yield and with the addition of 400 milligrams $(\mathrm{mg})$ of sterile $0.1-\mathrm{mm}$ zirconia/silica beads to each tube (to physically break open the bacterial cells attached to the swab). Extractions were screened by polymerase chain reaction (PCR) by using $16 \mathrm{~S}$ rRNA primer pairs 8F/1492 R and 63F/1392R (Galkiewicz and Kellogg, 2008). Although amplifications were attempted at a variety of sample dilutions and by using different polymerase enzymes (AmpliTaq Gold and Epicentre's Fail Safe PCR kit), none of the reactions were successful.

We collected additional swab samples during the 2010 GOM cruise from 24 Eumunida, 2 Munidopsis, and 4 conger eels. In an effort to improve sample capture, we tried using sterile nylonflocked swabs (Dalmaso and others, 2008) as well as sterile foam swabs. Back in the laboratory, the samples were extracted by using the Qiagen DNeasy Tissue DNA extraction kit, following the manufacturer's protocol. Extractions were screened by PCR by using primers $8 \mathrm{~F} / 1492 \mathrm{R}$ and Takara polymerase. A total of 12 amplifications were weakly positive. The two strongest amplifications (one from a conger eel and one from Munidopsis) were chosen to generate clone libraries. Unfortunately, the samples cloned poorly, resulting in very few positive clones (3/96 for the conger library and 1/96 for the crab library). This suggested that the quality of the amplicons was poor and that we would be unable to derive quality DNA sequences from these or additional libraries. The experiment was terminated at this point.

\subsubsection{Identifying a Core Microbiome by Examining L. pertusa-associated Bacterial Assemblages}

The presence of identical and similar bacterial sequences on corals from the GOM and Norway (Neulinger and others, 2008; Kellogg and others, 2009) indicates the presence of a conserved core of bacteria in L. pertusa. However, a survey of bacterial-community fingerprints from L. pertusa at three sites in the Trondheim Fjord, Norway, showed significant differences between the locations, which were between 4 and 32 kilometers (km) apart (Neulinger and others, 2008). This suggests that outside the conserved core there is variability in the L. pertusa-associated bacterial community, likely influenced by environmental variables such as temperature, salinity, and food source. In order to conduct a more robust comparison than can be accomplished with the few sequences existing from clone libraries, we designed a 16S rRNA gene pyrosequencing experiment targeting multiple coral samples at a number of geographic locations in the GOM and western Atlantic. We collected triplicate samples (each from a 
separate coral colony) of L. pertusa at two sites in the northeastern GOM (VK906, VK826; fig. 2.1), one site in the eastern GOM (WFS-1; fig. 2.1), and one site in the western Atlantic off the coast of Florida (ATL-1; fig. 2.1). Bacterial community DNA was extracted from each coral sample by using the Sunagawa protocol (Sunagawa and others, 2010), and all 12 samples were subjected to 454 pyrosequencing of the V4-V5 variable region of the 16S rRNA gene (Claesson and others, 2010). The number of sequences returned for each sample ranged from 79,465 to 307,004. These sequences are currently being assessed. They have been sorted to remove low quality and short sequences (Kunin and others, 2010), dereplicated to remove artifacts that can occur during the emulsion PCR step (GomezAlvarez and others, 2009), and denoised prior to analysis by using the bioinformatic pipelines QIIME (Caporaso and others, 2010), mothur (Schloss and others, 2009), and (or) CloVR (Angiuoli and others, 2011).

\subsection{Conclusions}

Studies conducted during the Lophelia II project have expanded our knowledge of L. pertusa microbial ecology, not just for the GOM, but globally. This work included the first look at eukaryotic (fungal) associates from live, healthy L. pertusa. These data provide comparative information for studies in Norway and the United Kingdom and create an important baseline for environmental managers (in terms of what microbial biodiversity exists and what a healthy system looks like) should there later be a disease outbreak, oil spill, or other impacts to these corals.

\subsection{Acknowledgments}

The authors thank Catie Wonders (University of South Florida) and Stephanie Lawler (University of South Florida) for their work extracting DNA from bacterial isolates and amplifying it for 16S rRNA gene sequencing, and Sarah Stellick (University of South Florida) for her work extracting DNA from fungal isolates and amplifying it for $18 \mathrm{~S}$ rRNA gene sequencing.

\subsection{References}

Aizawa, T., Ve, N.B., Kimoto, K.-I., Iwabuchi, N., Sumida, H., Hasegawa, I., Sasaki, S., Tamura, T., Kudo, T., Suzuki, K.-I., Nakajima, M., and Sunairi, M., 2007, Curtobacterium ammoniigenes sp. nov., an ammonia-producing bacterium isolated from plants inhabiting acidic swamps in actual acid sulfate soil areas of Vietnam: International Journal of Systematic and Evolutionary Microbiology, v. 57, p. 1447-1452.

Angiuoli, S.V., Matalka, M., Gussman, A., Galens, K., Vangala, M., Riley, D.R., Arze, C., White, J.R., White, O., and Fricke, W.F., 2011, CloVR: A virtual machine for automated and portable sequence analysis from the desktop using cloud computing: BMC Bioinformatics, v. 12, p. 356.

Atlas, R.M., 2004, Handbook of microbiological media (3d ed.): Boca Raton, CRC Press, p. 1205.

Bentis, C.J., Kaufman, L., and Golubic, S., 2000, Endolithic fungi in reef-building corals (Order: Scleractinia) are common, cosmopolitan, and potentially pathogenic: Biological Bulletin, v. 198, p. 254-260.

Bourne, D.G., and Munn, C.B., 2005, Diversity of bacteria associated with the coral Pocillopora damicornis from the Great Barrier Reef: Environmental Microbiology, v. 7, no. 8, p. 1162-1174.

Burgaud, G., Arzur, D., Durand, L., Cambon-Bonavita, M.-A., and Barbier, G., 2010, Marine culturable yeasts in deep-sea hydrothermal vents: Species richness and association with fauna: FEMS Microbiology Ecology, v. 73, no. 1, p. 121-133. 
Burgaud, G., Le Calvez, T., Arzur, D., Vandenkoornhuyse, P., and Barbler, G., 2009, Diversity of culturable marine filamentous fungi from deep-sea hydrothermal vents: Environmental Microbiology, v. 11 , no. 6 , p. $1588-1600$.

Caporaso, J.G., Kuczynski, J., Stombaugh, J., Bittinger, K., Bushman, F.D., Costello, E.K., Fierer, N., Gonzalez Peña, A., Goodrich, J.K., Gordon, J.I., Huttley, G.A., Kelley, S.T., Knights, D., Koenig, J.E., Ley, R.E., Lozupone, C.A., McDonald, D., Muegge, B.D., Pirrung, M., Reeder, J., Sevinsky, J.R., Turnbaugh, P.J., Walters, W.A., Widmann, J., Yatsunenko, T., Zaneveld, J., and Knight, R., 2010, QIIME allows analysis of high-throughput community sequencing data: Nature Methods, v. 7, p. 335-336.

Claesson, M.J., Wang, Q., O'Sullivan, O., Greene-Diniz, R., Cole, J.R., Ross, R.P., and O'Toole, P.W., 2010, Comparison of two next-generation sequencing technologies for resolving highly complex microbiota composition using tandem variable 16S rRNA gene regions: Nucleic Acids Research, $v$. 38, no. 22, p. e200.

Connell, L., Barrett, A., Templeton, A., and Staudigel, H., 2009, Fungal diversity associated with an active deep sea volcano: Vailulu'u, Samoa: Geomicrobiology Journal v. 26, p. 597-605.

CSA International, Inc., 2007, Characterization of northern Gulf of Mexico deepwater hard-bottom communities with emphasis on Lophelia coral: U.S. Department of Interior, Minerals Management Service, Gulf of Mexico OCS Region, OCS Study MMS 2007-044, 169 p.

Dalmaso, G., Bini, M., Paroni, R., and Ferrari, M., 2008, Qualification of high-recovery, flocked swabs as compared to traditional rayon swabs for microbiological environmental monitoring of surfaces: PDA Journal of Pharmaceutical Science and Technology, v. 62, no. 3, p. 191-199.

Druzhinina, I.S., Schmoll, M., Seiboth, B., and Kubicek, C.P., 2006, Global carbon utilization profiles of wild-type, mutant, and transformant strains of Hypocrea jecorina: Applied and Environmental Microbiology, v. 72, p. 2126-2133.

Eilers, H., Pernthaler, J., Glockner, F.O., and Amann, R.I., 2000, Culturability and in situ abundance of pelagic bacteria from the North Sea: Applied and Environmental Microbiology, v. 66, p. 3044-3051.

Fell, J.W., and van Uden, N., 1963, Yeasts in marine environments, in Oppenheimer, C.H., ed., Symposium on Marine Microbiology: Springfield, Ill., Thomas Publishing Company, p. 329-340.

Freiwald, A., 2002, Reef-forming cold-water corals, in Wefer, G., Billett, D., Hebbeln, D., Jørgensen, B.B., Schlüter, M., and van Weering, T., eds., Ocean margin systems: Berlin, Springer-Verlag, p. 365-385.

Freiwald, A., Reitner, J., and Krutschinna, J., 1997, Microbial alteration of the deep-water coral Lophelia pertusa: Early postmortem processes: Facies, v. 36, p. 223-226.

Freiwald, A., and Wilson, J.B., 1998, Taphonomy of modern deep, cold-temperate water coral reefs: Historical Biology, v. 13, p. 37-52.

Fuhrman, J.A., and Davis, A.A., 1997, Widespread archaea and novel bacteria from the deep sea as shown by $16 \mathrm{~S}$ rRNA gene sequences: Marine Ecology Progress Series, v. 150, p. 275-285.

Galkiewicz, J.P., and Kellogg, C.A., 2008, Cross-kingdom amplification using bacterial-specific primers: coimplications for coral microbial ecology: Applied and Environmental Microbiology, v. 74, no. 24, p. 7828-7831.

Galkiewicz, J.P., Pratte, Z.A., Gray, M.A., and Kellogg, C.A., 2011, Characterization of culturable bacteria isolated from the cold-water coral Lophelia pertusa: FEMS Microbiology Ecology, v. 77, no. 2, p. 333-346.

Galkiewicz, J.P., Stellick, S.H., Gray, M.A., and Kellogg, C.A., 2012, Cultured fungal associates from the deep-sea coral Lophelia pertusa: Deep-Sea Research Part I: Oceanographic Research Papers, v. 67 , p. 12-20. 
Gomez-Alvarez, V., Teal, T.K., and Schmidt, T.M., 2009, Systematic artifacts in metagenomes from complex microbial communities: The ISME Journal, v. 3, p. 1314-1317.

Johnson, C.R., and Sutton, D.C., 1994, Bacteria on the surface of crustose coralline algae induce metamorphosis of the crown-of-thorns starfish Acanthaster planci: Marine Biology, v. 120, p. 305310.

Johnson, C.R., Sutton, D.C., Olson, R.R., and Giddins, R., 1991, Settlement of crown-of-thorns starfish: Role of bacteria on surfaces of coralline algae and a hypothesis for deepwater recruitment: Marine Ecology Progress Series, v. 71, p. 143-162.

Kellogg, C.A., 2008, Microbial ecology of Lophelia pertusa in the northern Gulf of Mexico: U.S. Geological Survey Open-File Report 2008-1148, OCS Study MMS 2008-015, p. 6-1 to 6-42.

Kellogg, C.A., Lisle, J.T., and Galkiewicz, J.P., 2009, Culture-independent characterization of bacterial communities associated with the cold-water coral Lophelia pertusa in the northeastern Gulf of Mexico: Applied and Environmental Microbiology, v. 75, no. 8, p. 2294-2303.

Klaus, J.S., Frias-Lopez, J., Bonheyo, G.T., Heikoop, J.M., and Fouke, B.W., 2005, Bacterial communities inhabiting the healthy tissues of two Caribbean reef corals: Interspecific and spatial variation: Coral Reefs, v. 24, p. 129-137.

Kline, D.I., Kuntz, N.M., Breitbart, M., Knowlton, N., and Rohwer, F., 2006, Role of elevated organic carbon levels and microbial activity in coral mortality: Marine Ecology Progress Series, v. 314, p. 119-125.

Kunin, V., Engelbrektson, A., Ochman, H., and Hugenholtz, P., 2010, Wrinkles in the rare biosphere: Pyrosequencing errors can lead to artificial inflation of diversity estimates: Environmental Microbiology, v. 12, no. 1, p. 118-123.

Larsen, P.E., Collart, F.R., Field, D., Meyer, F., Keegan, K.P., Henry, C.S., McGrath, J., Quinn, J., and Gilbert, J.A., 2011, Predicted relative metabolic turnover (PRMT): Determining metabolic turnover from a coastal marine metagenomic dataset: Microbial Informatics and Experimentation, v. 1, no. 4, doi:10.1186/2042-5783-1-4.

Lau, S.C.K., and Liu, W.-T., 2007, Recent advances in molecular techniques for the detection of phylogenetic markers and functional genes in microbial communities: FEMS Microbiology Letters, v. 275, p. 183-190.

Li, X.-Z., Livermore, D.M., and Nikaido, H., 1994, Role of efflux pump(s) in intrinsic resistance of Pseudomonas aeruginosa: Resistance to tetracycline, chloramphenicol, and norfloxacin:

Antimicrobial Agents and Chemotherapy, v. 38, no. 8, p. 1732-1741.

Negri, A.P., Webster, N.S., Hill, R.T., and Heyward, A.J., 2001, Metamorphosis of broadcast spawning corals in response to bacteria isolated from crustose algae: Marine Ecology Progress Series, v. 223, p. 121-131.

Neulinger, S.C., Järnegren, J., Ludvigsen, M., Lochte, K., and Dullo, W.-C., 2008, Phenotype-specific bacterial communities in the cold-water coral Lophelia pertusa (Scleractinia) and their implications for the coral's nutrition, health, and distribution: Applied and Environmental Microbiology, v. 74, no. 23, p. 7272-7285.

Olson, J.B., Lord, C.C., and McCarthy, P.J., 2000, Improved recoverability of microbial colonies from marine sponge samples: Microbial Ecology, v. 40, p. 139-147.

Palleroni, N.J., 2005, Genus I: Pseudomonas, in Brenner, D.J., Krieg, N.R., and Staley, J.T., ed., Bergey's manual of systematic bacteriology (2d ed.): New York, Springer, p. 323-379.

Pantos, O., Cooney, R.P., Le Tissier, M.D.A., Barer, M.R., O'Donnell, A.G., and Bythell, J.C., 2003, The bacterial ecology of a plague-like disease affecting the Caribbean coral Montastrea annularis: Environmental Microbiology, v. 5, no. 5, p. 370-382. 
Raghukumar, S., 2006, Marine microbial eukaryotic diversity, with particular reference to fungi:

Lessons from prokaryotes: Indian Journal of Marine Sciences, v. 35, p. 388-398.

Roberts, J.M., Wheeler, A.J., and Freiwald, A., 2006, Reefs of the deep: The biology and geology of cold-water coral ecosystems: Science, v. 312, no. 5773, p. 543-547.

Rohwer, F., Seguritan, V., Azam, F., and Knowlton, N., 2002, Diversity and distribution of coralassociated bacteria: Marine Ecology Progress Series, v. 243, p. 1-10.

Rosenberg, E., Kellogg, C.A., and Rohwer, F., 2007, Coral microbiology: Oceanography, v. 20, no. 2, p. 114-122.

Samuels, G.J., 1976, Perfect states of Acremonium: The genera Nectria, Actiniopsis, Ijuhya, Neohenningsia, Ophiodictyon, and Peristomialis: New Zealand Journal of Botany, v. 14, no. 3, p. 231-260.

Schloss, P.D., Westcott, S.L., Ryabin, T., Hall, J.R., Hartmann, M., Hollister, E.B., Lesniewski, R.A., Oakley, B.B., Parks, D.H., Robinson, C.J., Sahl, J.W., Stres, B., Thallinger, G.G., Van Horn, D.J., and Weber, C.F., 2009, Introducing mothur: Open-source, platform-independent, community-supported software for describing and comparing microbial communities: Applied and Environmental Microbiology, v. 75, no. 23, p. 7537-7541.

Seifert, K.A., Wingfield, B.D., and Wingfield, M.J., 1995, A critique of DNA sequence analysis in the taxonomy of filamentous Ascomycetes and ascomycetous anamorphs: Canadian Journal of Botany, v. 73, no. S1, p. S760-S767.

Singh, P., Raghukumar, C., Verma, P., and Shouche, Y., 2010, Phylogenetic diversity of culturable fungi from the deep-sea sediments of the Central Indian Basin and their growth characteristics: Fungal Diversity, v. 40, p. 89-102.

Smith, G.W., and Hayasaka, S.S., 1982, Nitrogenase activity of bacteria associated with Halodule wrightii roots: Applied and Environmental Microbiology, v. 43, no. 6, p. 1244-1248.

Staley, J.T., and Konopka, A., 1985, Measurement of in situ activities of nonphotosynthetic microorganisms in aquatic and terrestrial habitats: Annual Reviews in Microbiology, v. 39, p. 321346.

Sunagawa, S., Woodley, C.M., and Medina, M., 2010, Threatened corals provide underexplored microbial habitats: PLoS One, v. 5, no. 3, p. e9554.

Sussman, M., Loya, Y., Fine, M., and Rosenberg, E., 2003, The marine fireworm Hermodice carunculata is a winter reservoir and spring-summer vector for the coral-bleaching pathogen Vibrio shiloi: Environmental Microbiology, v. 5, no. 4, p. 250-255.

Thurber, R.L.V., and Correa, A.M.S., 2011, Viruses of reef-building scleractinian corals: Journal of Experimental Marine Biology and Ecology, v. 408, p. 102-113.

Wegley, L., Edwards, R., Rodriguez-Brito, B., Liu, H., and Rohwer, F., 2007, Metagenomic analysis of the microbial community associated with the coral Porites astreoides: Environmental Microbiology, v. 9, no. 11, p. 2707-2719.

Wisshak, M., Freiwald, A., Lundälv, T., and Gektidis, M., 2005, The physical niche of the bathyal Lophelia pertusa in a non-bathyal setting: Environmental controls and palaeoecological implications, in Freiwald, A., and Roberts, J.M., eds., Cold-water corals and ecosystems: Berlin, Springer, p. 9791001. 
Table 2.1. Types of samples collected during each research cruise during the Lophelia II program.

[GOM, Gulf of Mexico; ATL, Atlantic; ROV, remotely operated vehicle]

\begin{tabular}{|c|c|c|c|c|c|c|c|}
\hline Dates & $\begin{array}{c}\text { Ocean } \\
\text { basin }\end{array}$ & $\begin{array}{c}\text { Research } \\
\text { vessel }\end{array}$ & $\begin{array}{l}\text { Collection } \\
\text { device }\end{array}$ & $\begin{array}{c}\text { Bacterial } \\
\text { culture }\end{array}$ & $\begin{array}{l}\text { Fungal } \\
\text { culture }\end{array}$ & $\begin{array}{l}\text { Faunal } \\
\text { swabs }\end{array}$ & $\begin{array}{c}\text { Microbial } \\
\text { DNA }\end{array}$ \\
\hline $5-16$ Oct 08 & GOM & $\begin{array}{l}\text { Nancy } \\
\text { Foster }\end{array}$ & $\begin{array}{c}\text { SeaEye } \\
\text { Falcon ROV }\end{array}$ & $X$ & & & \\
\hline 6-17 Aug 09 & ATL & $\begin{array}{l}\text { Seward } \\
\text { Johnson }\end{array}$ & $\begin{array}{c}\text { Johnson Sea } \\
\text { Link II } \\
\text { submersible }\end{array}$ & $\mathrm{X}$ & $\mathrm{X}$ & & $X$ \\
\hline 13-25 Sep 09 & GOM & $\begin{array}{l}\text { Seward } \\
\text { Johnson }\end{array}$ & $\begin{array}{c}\text { Johnson Sea } \\
\text { Link II } \\
\text { submersible }\end{array}$ & $\mathrm{X}$ & & $X$ & $X$ \\
\hline $\begin{array}{c}20 \mathrm{Sep}-3 \text { Oct } \\
10\end{array}$ & GOM & $\begin{array}{c}\text { Cape } \\
\text { Hatteras }\end{array}$ & $\begin{array}{l}\text { Kraken II } \\
\text { ROV }\end{array}$ & $\mathrm{X}$ & $\mathrm{X}$ & $X$ & $\mathrm{X}$ \\
\hline 12-23 Jul 12 & GOM & $\begin{array}{l}\text { Brooks } \\
\text { McCall }\end{array}$ & $\begin{array}{c}\text { Kraken II } \\
\text { ROV }\end{array}$ & $\mathrm{X}$ & & & \\
\hline
\end{tabular}


Table 2.2. Nutrient agars tested for bacterial isolation from Lophelia pertusa during the Lophelia II program.

[GASWA, glycerol artificial seawater agar; g, gram; mL, milliliter; L, liter]

\begin{tabular}{|c|c|c|c|}
\hline Target & Medium & Ingredients per liter or [Manufacturer] if commerci & Ily available \\
\hline Mycoplasmas & $\begin{array}{c}\text { GASWA + Thallous } \\
\text { Acetate }\end{array}$ & $\begin{array}{l}\text { GASWA according to methods of Smith and } \\
\text { Hayasaka (1982), with the following } \\
\text { modification: Substitute Instant Ocean }(2.0 \mathrm{~g}) \text { for } \\
\text { Rila salts }(2.0 \mathrm{~g}) \text {. } \\
\text { Agar } \\
\text { Thallous acetate }\end{array}$ & $\begin{array}{l}15.0 \mathrm{~g} \\
0.25 \mathrm{~g}\end{array}$ \\
\hline Mycoplasmas & GASWA + Supplement G & $\begin{array}{l}\text { GASWA as listed above } \\
\text { Divide into } 80-\mathrm{mL} \text { aliquots. } \\
\text { Add Supplement } \mathrm{G} \text { [Oxoid] } \\
\text { (Makes } 100 \mathrm{~mL} \text { of agar per vial of Supplement } \\
\text { G) }\end{array}$ & $20 \mathrm{~mL}$ \\
\hline Mycoplasmas & $\begin{array}{c}\text { GASWA + Supplement G } \\
+ \text { Polymixin B }\end{array}$ & $\begin{array}{l}\text { GASWA as listed above } \\
\text { Polymixin B } \\
\text { Divide into } 80 \text {-mL aliquots. } \\
\text { Add Supplement G [Oxoid] } \\
\text { (Makes } 100 \mathrm{~mL} \text { of agar per vial of Supplement } \\
\mathrm{G} \text { ) }\end{array}$ & $\begin{array}{l}250,000 \\
\text { units } \\
20 \mathrm{~mL}\end{array}$ \\
\hline Mycoplasmas & $\begin{array}{c}\text { 1\% 2216 Marine Agar + } \\
\text { Thallous Acetate }+ \\
\text { Penicillin }\end{array}$ & $\begin{array}{l}\text { 2216 Marine Agar [Difco] } \\
\text { Thallous Acetate } \\
\text { Penicillin G (sodium) }\end{array}$ & $\begin{array}{l}0.374 \mathrm{~g} \\
0.25 \mathrm{~g} \\
250,000 \\
\text { units } \\
\end{array}$ \\
\hline Mycoplasmas & $\begin{aligned} \text { C-mix } & + \text { Thallous Acetate } \\
& + \text { Penicillin }\end{aligned}$ & $\begin{array}{l}\text { C-mix according to methods of Olson and others } \\
(2000) \\
\text { Thallous Acetate } \\
\text { Penicillin G (sodium) }\end{array}$ & $\begin{array}{l}0.25 \mathrm{~g} \\
250,000 \\
\text { units }\end{array}$ \\
\hline Mycoplasmas & $\begin{aligned} 60: 40 & + \text { Thallous Acetate } \\
& + \text { Penicillin }\end{aligned}$ & $\begin{array}{l}\text { 60:40 according to methods of Olson and others } \\
\text { (2000) } \\
\text { Thallous Acetate } \\
\text { Penicillin G (sodium) }\end{array}$ & $\begin{array}{l}0.25 \mathrm{~g} \\
250,000 \\
\text { units }\end{array}$ \\
\hline Mycoplasmas & $\begin{array}{l}\text { Oxoid Mycoplasma }+ \\
\text { Supplement G }\end{array}$ & $\begin{array}{l}\text { Mycoplasma Agar [Oxoid] } \\
\text { Deionized Water } \\
\text { Divide into } 80 \text {-mL aliquots. } \\
\text { Supplement G [Oxoid] } \\
\text { (Makes } 100 \mathrm{~mL} \text { of agar per vial of Supplement } \\
\text { G) }\end{array}$ & $\begin{array}{l}35.5 \mathrm{~g} \\
1.0 \mathrm{~L} \\
20 \mathrm{~mL}\end{array}$ \\
\hline Thiotrophs & $\begin{array}{c}\text { ATCC } 295 \text { S8 medium for } \\
\text { Thiobacillus }\end{array}$ & $\begin{array}{l}\mathrm{Na}_{2} \mathrm{HPO}_{4} \\
\mathrm{KH}_{2} \mathrm{PO}_{4} \\
\mathrm{MgSO}_{4}-7 \mathrm{H}_{2} \mathrm{O}\end{array}$ & $\begin{array}{l}1.2 \mathrm{~g} \\
1.8 \mathrm{~g} \\
0.1 \mathrm{~g}\end{array}$ \\
\hline
\end{tabular}


Table 2.2. Nutrient agars tested for bacterial isolation from Lophelia pertusa during the Lophelia II program continued.

[GASWA, glycerol artificial seawater agar; g, gram; mL, milliliter; L, liter]

\begin{tabular}{|c|c|c|c|}
\hline Target & Medium & \multicolumn{2}{|c|}{ Ingredients per liter or [Manufacturer] if commercially available } \\
\hline Thiotrophs & $\begin{array}{c}\text { ATCC } 295 \text { S8 medium for } \\
\text { Thiobacillus }\end{array}$ & $\begin{array}{l}\left(\mathrm{NH}_{4}\right)_{2} \mathrm{SO}_{4} \\
\mathrm{CaCl}_{2} \\
\mathrm{FeCl}_{3} \\
\mathrm{MnSO}_{4} \\
\mathrm{Na}_{2} \mathrm{~S}_{2} \mathrm{O}_{3} \\
\mathrm{NaHCO}_{3} \\
\mathrm{KNO}_{3} \\
\text { Agar } \\
\text { Deionized water }\end{array}$ & $\begin{array}{l}0.1 \mathrm{~g} \\
0.03 \mathrm{~g} \\
0.02 \mathrm{~g} \\
0.02 \mathrm{~g} \\
10.0 \mathrm{~g} \\
0.5 \mathrm{~g} \\
5.0 \mathrm{~g} \\
15.0 \mathrm{~g} \\
1.0 \mathrm{~L}\end{array}$ \\
\hline Thiotrophs & MP Agar & MP Agar according to Atlas (2004) & \\
\hline All Bacteria & Salty R2A & $\begin{array}{l}\text { R2A Agar [Remel] } \\
\mathrm{NaCl} \\
\text { Deionized water }\end{array}$ & $\begin{array}{l}18.1 \mathrm{~g} \\
20.8 \mathrm{~g} \\
1.0 \mathrm{~L}\end{array}$ \\
\hline
\end{tabular}


Table 2.3. Bacterial cultures from various media, 2008-2012, during the Lophelia II program.

[Bacteria belonging to the phylum Actinobacteria are highlighted in gray and phylum Firmicutes are highlighted in yellow. All of the unhighlighted bacteria are Proteobacteria.]

\begin{tabular}{|c|c|c|c|c|c|c|c|}
\hline Isolation medium & Bacterial isolate ID & $\begin{array}{c}\text { Top named 16S rRNA } \\
\text { match }\end{array}$ & $\begin{array}{l}\text { Basepair } \\
\text { match (\% } \\
\text { similarity) }\end{array}$ & $\underset{-/+}{\text { Gram }}$ & Year & Sample ID & Collection site \\
\hline ATCC 295 S8 & 3712_K3_LP_ATT_B-01 & Arthrobacter humicola & $\begin{array}{c}745 / 748 \\
(99 \%)\end{array}$ & + & 2009 & $3712 \mathrm{~K} 3$ & ATL-1 \\
\hline ATCC 295 S8 & 3712_K7_LP_ATT_B-01 & Arthrobacter humicola & $\begin{array}{c}793 / 796 \\
(99 \%)\end{array}$ & + & 2009 & $3712 \mathrm{~K} 7$ & ATL-1 \\
\hline ATCC 295 S8 & 3712_K7_LP_ATT_B-02 & Arthrobacter humicola & $\begin{array}{c}744 / 747 \\
(99 \%) \\
\end{array}$ & + & 2009 & $3712 \mathrm{~K} 7$ & ATL-1 \\
\hline ATCC 295 S8 & 3705_K9_LP_ATT_B-05 & $\begin{array}{l}\text { Pseudoalteromonas } \\
\text { sp. } 146 Z 1-2\end{array}$ & $\begin{array}{c}764 / 766 \\
(99 \%)\end{array}$ & - & 2009 & $3705 \mathrm{~K} 9$ & ATL-1 \\
\hline ATCC 295 S8 & 3705_K9_LP_ATT_B-07 & $\begin{array}{l}\text { Pseudoalteromonas } \\
s p .146 Z 1-2\end{array}$ & $\begin{array}{c}756 / 759 \\
(99 \%)\end{array}$ & - & 2009 & $3705 \mathrm{~K} 9$ & ATL-1 \\
\hline ATCC 295 S8 & 3705_K10_LP_ATT_B-02 & $\begin{array}{l}\text { Pseudoalteromonas } \\
\text { sp. } 146 Z 1-2\end{array}$ & $\begin{array}{c}814 / 815 \\
(99 \%)\end{array}$ & - & 2009 & $3705 \mathrm{~K} 10$ & ATL-1 \\
\hline ATCC 295 S8 & 3712_K8_LP_ATT_B-01 & $\begin{array}{l}\text { Pseudoalteromonas } \\
\text { sp. } 146 Z 1-2\end{array}$ & $\begin{array}{c}779 / 780 \\
(99 \%) \\
\end{array}$ & - & 2009 & $3712 \mathrm{~K} 8$ & ATL-1 \\
\hline ATCC 295 S8 & 3712_K8_LP_ATT_B-04 & $\begin{array}{l}\text { Pseudoalteromonas } \\
\text { sp. } 146 Z 1-2\end{array}$ & $\begin{array}{l}737 / 740 \\
(99 \%)\end{array}$ & - & 2009 & $3712 \mathrm{~K} 8$ & ATL-1 \\
\hline ATCC 295 S8 & 3712_K9_LP_ATT_B-02 & $\begin{array}{l}\text { Pseudoalteromonas } \\
\text { sp. } 146 Z 1-2\end{array}$ & $\begin{array}{c}757 / 760 \\
(99 \%)\end{array}$ & - & 2009 & 3712K9 & ATL-1 \\
\hline ATCC 295 S8 & 3712_K8_LP_ATT_B-03 & $\begin{array}{l}\text { Pseudoalteromonas } \\
\text { sp. 199Z-14 }\end{array}$ & $\begin{array}{c}798 / 799 \\
(99 \%)\end{array}$ & - & 2009 & $3712 \mathrm{~K} 8$ & ATL-1 \\
\hline ATCC 295 S8 & 3705_K4_LP_ATT_B-03 & $\begin{array}{l}\text { Pseudoalteromonas } \\
\text { sp. 6-69 }\end{array}$ & $\begin{array}{l}739 / 739 \\
(100 \%) \\
\end{array}$ & - & 2008 & $3705 \mathrm{~K} 4$ & ATL-1 \\
\hline ATCC 295 S8 & 3705_K9_LP_ATT_B-08 & $\begin{array}{l}\text { Pseudoalteromonas } \\
\text { sp. ArcN812K13 }\end{array}$ & $\begin{array}{c}811 / 812 \\
(99 \%) \\
\end{array}$ & - & 2009 & $3705 \mathrm{~K} 9$ & ATL-1 \\
\hline ATCC 295 S8 & 3712_K8_LP_ATT_B-02 & $\begin{array}{l}\text { Pseudoalteromonas } \\
\text { sp. ArcN812K13 }\end{array}$ & $\begin{array}{c}761 / 764 \\
(99 \%)\end{array}$ & - & 2009 & $3712 \mathrm{~K} 8$ & ATL-1 \\
\hline ATCC 295 S8 & 3705_K9_LP_ATT_B-01 & $\begin{array}{l}\text { Pseudoalteromonas } \\
s p . Z 2\end{array}$ & $\begin{array}{l}776 / 776 \\
(100 \%)\end{array}$ & - & 2009 & $3705 \mathrm{~K} 9$ & ATL-1 \\
\hline
\end{tabular}


Table 2.3. Bacterial cultures from various media, 2008-2012, during the Lophelia II program - continued.

[Bacteria belonging to the phylum Actinobacteria are highlighted in gray and phylum Firmicutes are highlighted in yellow. All of the unhighlighted bacteria are Proteobacteria.]

\begin{tabular}{|c|c|c|c|c|c|c|c|}
\hline Isolation medium & Bacterial isolate ID & $\begin{array}{c}\text { Top named 16S rRNA } \\
\text { match }\end{array}$ & $\begin{array}{l}\text { Basepair } \\
\text { match (\% } \\
\text { similarity) }\end{array}$ & $\underset{-/+}{\text { Gram }}$ & Year & Sample ID & Collection site \\
\hline ATCC 295 S8 & 3705_K9_LP_ATT_B-03 & $\begin{array}{l}\text { Pseudoalteromonas } \\
\text { sp. Z2 }\end{array}$ & $\begin{array}{l}782 / 783 \\
(99 \%)\end{array}$ & - & 2009 & 3705K9 & ATL-1 \\
\hline ATCC 295 S8 & 3705_K4_LP_ATT_B-04 & Pseudomonas sp. J465 & $\begin{array}{c}752 / 753 \\
(99 \%) \\
\end{array}$ & - & 2008 & $3705 \mathrm{~K} 4$ & ATL-1 \\
\hline ATCC 295 S8 & 3705_K4_LP_ATT_B-05 & $\begin{array}{l}\text { Psychrobacter sp. } \\
\text { SOZ3-5111 }\end{array}$ & $\begin{array}{l}777 / 777 \\
(100 \%) \\
\end{array}$ & - & 2008 & $3705 \mathrm{~K} 4$ & ATL-1 \\
\hline $\begin{array}{c}\text { Fungal BBL } \\
\text { Mycophil Agar }\end{array}$ & ROV10 - Q1 - FBM - 05 & $\begin{array}{l}\text { Pseudomonas } \\
\text { anguilliseptica }\end{array}$ & $\begin{array}{c}562 / 564 \\
(99 \%)\end{array}$ & - & 2010 & $\begin{array}{l}\text { ROV10 } \\
\text { Quiver } 1\end{array}$ & WFS2 \\
\hline $\begin{array}{l}\text { Fungal Rose } \\
\text { Bengal Agar }\end{array}$ & ROV08 - Q3 - FRB - 01 & $\begin{array}{l}\text { Pseudomonas } \\
\text { anguilliseptica }\end{array}$ & $\begin{array}{c}711 / 715 \\
(99 \%) \\
\end{array}$ & - & 2010 & $\begin{array}{l}\text { ROV08 } \\
\text { Quiver 3 } \\
\end{array}$ & WFS1 \\
\hline $\begin{array}{l}\text { GASWA + Suppl } \\
\text { G + Polymixin B }\end{array}$ & VoD_011_GGPB1 & $\begin{array}{l}\text { Pseudomonas sp. } \\
\text { AMF2745 }\end{array}$ & $\begin{array}{c}860 / 861 \\
(99 \%)\end{array}$ & - & 2012 & Zinc Quiver 8 & $\begin{array}{l}\text { Zinc Subsea } \\
\text { Installation } \\
\text { NAD27 }\end{array}$ \\
\hline $\begin{array}{l}\text { GASWA + Suppl } \\
\text { G + Polymixin B }\end{array}$ & VoD_011_GGPB2 & $\begin{array}{l}\text { Pseudomonas sp. } \\
\text { WB19-24 }\end{array}$ & $\begin{array}{c}885 / 886 \\
(99 \%)\end{array}$ & - & 2012 & Zinc Quiver 8 & $\begin{array}{l}\text { Zinc Subsea } \\
\text { Installation } \\
\text { NAD27 }\end{array}$ \\
\hline $\begin{array}{l}\text { GASWA + Suppl } \\
\text { G + Polymixin B }\end{array}$ & VoD_011_GGPB3 & $\begin{array}{l}\text { Pseudomonas sp. } \\
\text { WB19-24 }\end{array}$ & $\begin{array}{l}920 / 920 \\
(100 \%)\end{array}$ & - & 2012 & Zinc Quiver 8 & $\begin{array}{c}\text { Zinc Subsea } \\
\text { Installation } \\
\text { NAD27 }\end{array}$ \\
\hline $\begin{array}{l}\text { GASWA + Suppl } \\
\text { G + Polymixin B }\end{array}$ & VoD_011_GGPB4 & $\begin{array}{l}\text { Pseudomonas sp. } \\
\text { WB19-24 }\end{array}$ & $\begin{array}{l}845 / 845 \\
(100 \%)\end{array}$ & - & 2012 & Zinc Quiver 8 & $\begin{array}{c}\text { Zinc Subsea } \\
\text { Installation } \\
\text { NAD27 }\end{array}$ \\
\hline GASWA+Chlor & R81DCBW1 & $\begin{array}{l}\text { Pseudomonas } \\
\text { fluorescens }\end{array}$ & $\begin{array}{c}720 / 733 \\
(98 \%) \\
\end{array}$ & - & 2008 & $\begin{array}{l}\text { NFROV08001 } \\
\text { BioboxD }\end{array}$ & VK826 \\
\hline GASWA+Chlor & R81DCBW2 & $\begin{array}{l}\text { Pseudomonas } \\
\text { fluorescens }\end{array}$ & $\begin{array}{l}612 / 612 \\
(100 \%)\end{array}$ & - & 2008 & $\begin{array}{l}\text { NFROV08001 } \\
\text { BioboxD }\end{array}$ & VK826 \\
\hline GASWA+Chlor & R81DCBW3 & $\begin{array}{l}\text { Pseudomonas } \\
\text { fluorescens }\end{array}$ & $\begin{array}{l}543 / 543 \\
(100 \%)\end{array}$ & - & 2008 & $\begin{array}{l}\text { NFROV08001 } \\
\text { BioboxD }\end{array}$ & VK826 \\
\hline
\end{tabular}


Table 2.3. Bacterial cultures from various media, 2008-2012, during the Lophelia II program - continued.

[Bacteria belonging to the phylum Actinobacteria are highlighted in gray and phylum Firmicutes are highlighted in yellow. All of the unhighlighted bacteria are Proteobacteria.]

\begin{tabular}{|c|c|c|c|c|c|c|c|}
\hline Isolation medium & Bacterial isolate ID & $\begin{array}{c}\text { Top named 16S rRNA } \\
\text { match }\end{array}$ & $\begin{array}{l}\text { Basepair } \\
\text { match (\% } \\
\text { similarity) }\end{array}$ & $\underset{-/+}{\text { Gram }}$ & Year & Sample ID & Collection site \\
\hline GASWA+Chlor & R81DCBW5 & $\begin{array}{l}\text { Pseudomonas } \\
\text { fluorescens }\end{array}$ & $\begin{array}{c}608 / 608 \\
(100 \%)\end{array}$ & - & 2008 & $\begin{array}{l}\text { NFROV08001 } \\
\text { BioboxD }\end{array}$ & VK826 \\
\hline GASWA+Chlor & R81DCBW6 & $\begin{array}{l}\text { Pseudomonas } \\
\text { libanensis }\end{array}$ & $\begin{array}{l}715 / 725 \\
(98 \%) \\
\text { Gaps }= \\
2 / 725 \\
(0 \%)\end{array}$ & - & 2008 & $\begin{array}{l}\text { NFROV08001 } \\
\text { BioboxD }\end{array}$ & VK826 \\
\hline GASWA+Chlor & R83ACBW2 & $\begin{array}{l}\text { Pseudomonas sp. } \\
\text { HPC431 }\end{array}$ & $\begin{array}{c}329 / 346 \\
(95 \%)\end{array}$ & - & 2008 & $\begin{array}{l}\text { NFROV08003 } \\
\text { BioboxA }\end{array}$ & VK906 \\
\hline GASWA+Chlor & R81DCBW4 & $\begin{array}{l}\text { Pseudomonas sp. } \\
\text { Zh1N-8 }\end{array}$ & $\begin{array}{c}673 / 678 \\
(99 \%)\end{array}$ & - & 2008 & $\begin{array}{l}\text { NFROV08001 } \\
\text { BioboxD }\end{array}$ & VK826 \\
\hline GASWA+TA & R81BTABW3 & $\begin{array}{l}\text { Halomonas sp. } \\
2 \text { CpOI6 }\end{array}$ & $\begin{array}{l}529 / 529 \\
(100 \%)\end{array}$ & - & 2008 & $\begin{array}{l}\text { NFROV08001 } \\
\text { BioboxB }\end{array}$ & VK826 \\
\hline GASWA+TA & R81ATABW1 & Halomonas sp. EB329 & $\begin{array}{c}596 / 598 \\
(99 \%)\end{array}$ & - & 2008 & $\begin{array}{l}\text { NFROV08001 } \\
\text { BioboxA }\end{array}$ & VK826 \\
\hline GASWA+TA & R81BTABW4 & Halomonas sp. EB359 & $\begin{array}{c}579 / 580 \\
(99 \%)\end{array}$ & - & 2008 & $\begin{array}{l}\text { NFROV08001 } \\
\text { BioboxB }\end{array}$ & VK826 \\
\hline GASWA+TA & R81BTABW2 & Halomonas sp. IW1-1 & $\begin{array}{c}590 / 591 \\
(99 \%)\end{array}$ & - & 2008 & $\begin{array}{l}\text { NFROV08001 } \\
\text { BioboxB }\end{array}$ & VK826 \\
\hline GASWA+TA & R81BTABW1 & $\begin{array}{l}\text { Halomonas sp. RHS- } \\
\text { str. } 203 \mathrm{Cr} B\end{array}$ & $\begin{array}{c}688 / 690 \\
(99 \%)\end{array}$ & - & 2008 & $\begin{array}{l}\text { NFROV08001 } \\
\text { BioboxB }\end{array}$ & VK826 \\
\hline $\begin{array}{l}\text { Orange Serum } \\
\text { Agar }\end{array}$ & 3705_K5_LP_OSA_B-06 & $\begin{array}{l}\text { Curtobacterium } \\
\text { flaccumfaciens }\end{array}$ & $\begin{array}{c}745 / 747 \\
(99 \%) \\
\end{array}$ & + & 2008 & $3705 \mathrm{~K} 5$ & ATL-1 \\
\hline $\begin{array}{l}\text { Orange Serum } \\
\text { Agar }\end{array}$ & 3705_K5_LP_OSA_F-14 & $\begin{array}{l}\text { Curtobacterium } \\
\text { flaccumfaciens }\end{array}$ & $\begin{array}{c}796 / 803 \\
(99 \%)\end{array}$ & + & 2009 & $3705 \mathrm{~K} 5$ & ATL-1 \\
\hline $\begin{array}{l}\text { Orange Serum } \\
\text { Agar }\end{array}$ & 3705_K8_LP_OSA_B-03 & $\begin{array}{l}\text { Curtobacterium } \\
\text { flaccumfaciens }\end{array}$ & $\begin{array}{l}806 / 806 \\
(100 \%)\end{array}$ & + & 2009 & $3705 \mathrm{~K} 8$ & ATL-1 \\
\hline $\begin{array}{l}\text { Orange Serum } \\
\text { Agar }\end{array}$ & 3712_K3_LP_OSA_F-06 & $\begin{array}{l}\text { Curtobacterium } \\
\text { flaccumfaciens }\end{array}$ & $\begin{array}{c}784 / 785 \\
(99 \%)\end{array}$ & + & 2009 & $3712 \mathrm{~K} 3$ & ATL-1 \\
\hline
\end{tabular}


Table 2.3. Bacterial cultures from various media, 2008-2012, during the Lophelia II program - continued.

[Bacteria belonging to the phylum Actinobacteria are highlighted in gray and phylum Firmicutes are highlighted in yellow. All of the unhighlighted bacteria are Proteobacteria.]

\begin{tabular}{|c|c|c|c|c|c|c|c|}
\hline Isolation medium & Bacterial isolate ID & $\begin{array}{c}\text { Top named 16S rRNA } \\
\text { match }\end{array}$ & $\begin{array}{l}\text { Basepair } \\
\text { match (\% } \\
\text { similarity) }\end{array}$ & $\underset{-/+}{\text { Gram }}$ & Year & Sample ID & Collection site \\
\hline $\begin{array}{c}\text { Orange Serum } \\
\text { Agar }\end{array}$ & 3705_K3_LP_OSA_B-01 & $\begin{array}{l}\text { Curtobacterium sp. } \\
1043\end{array}$ & $\begin{array}{l}373 / 373 \\
(100 \%)\end{array}$ & + & 2008 & $3705 \mathrm{~K} 3$ & ATL-1 \\
\hline $\begin{array}{c}\text { Orange Serum } \\
\text { Agar }\end{array}$ & 3705_K5_LP_OSA_B-02 & $\begin{array}{l}\text { Curtobacterium sp. } \\
\text { S78 }\end{array}$ & $\begin{array}{c}758 / 759 \\
(99 \%)\end{array}$ & + & 2008 & $3705 \mathrm{~K} 5$ & ATL-1 \\
\hline $\begin{array}{c}\text { Orange Serum } \\
\text { Agar }\end{array}$ & 3712_K2_LP_OSA_B-07 & $\begin{array}{l}\text { Curtobacterium sp. } \\
\text { S78 }\end{array}$ & $\begin{array}{c}795 / 796 \\
(99 \%)\end{array}$ & + & 2009 & $3712 \mathrm{~K} 2$ & ATL-1 \\
\hline $\begin{array}{c}\text { Orange Serum } \\
\text { Agar }\end{array}$ & 3705_K2_LP_OSA_B-01 & $\begin{array}{l}\text { Curtobacterium sp. } \\
\text { SAP758.3 }\end{array}$ & $\begin{array}{l}736 / 736 \\
(100 \%)\end{array}$ & + & 2008 & $3705 \mathrm{~K} 2$ & ATL-1 \\
\hline $\begin{array}{c}\text { Orange Serum } \\
\text { Agar }\end{array}$ & 3705_K2_LP_OSA_B-02 & $\begin{array}{l}\text { Curtobacterium } s p \text {. } \\
\text { SAP758.3 }\end{array}$ & $\begin{array}{c}795 / 797 \\
(99 \%)\end{array}$ & + & 2008 & $3705 \mathrm{~K} 2$ & ATL-1 \\
\hline $\begin{array}{c}\text { Orange Serum } \\
\text { Agar }\end{array}$ & 3705_K2_LP_OSA_B-08 & $\begin{array}{l}\text { Curtobacterium sp. } \\
\text { SAP758.3 }\end{array}$ & $\begin{array}{l}778 / 778 \\
(100 \%)\end{array}$ & + & 2008 & $3705 \mathrm{~K} 2$ & ATL-1 \\
\hline $\begin{array}{c}\text { Orange Serum } \\
\text { Agar }\end{array}$ & 3705_K4_LP_OSA_B-08 & $\begin{array}{l}\text { Curtobacterium sp. } \\
\text { SAP758.3 }\end{array}$ & $\begin{array}{r}847 / 847 \\
(100 \%) \\
\end{array}$ & + & 2008 & $3705 \mathrm{~K} 4$ & ATL-1 \\
\hline $\begin{array}{c}\text { Orange Serum } \\
\text { Agar }\end{array}$ & 3705_K5_LP_OSA_B-11 & $\begin{array}{l}\text { Curtobacterium sp. } \\
\text { SAP758.3 }\end{array}$ & $\begin{array}{c}772 / 773 \\
(99 \%)\end{array}$ & + & 2008 & $3705 \mathrm{~K} 5$ & ATL-1 \\
\hline $\begin{array}{c}\text { Orange Serum } \\
\text { Agar }\end{array}$ & 3705_K10_LP_OSA_B-05 & $\begin{array}{l}\text { Curtobacterium sp. } \\
\text { SAP758.3 }\end{array}$ & $\begin{array}{c}799 / 801 \\
(99 \%)\end{array}$ & + & 2009 & $3705 \mathrm{~K} 10$ & ATL-1 \\
\hline $\begin{array}{c}\text { Orange Serum } \\
\text { Agar }\end{array}$ & 3712_K2_LP_OSA_B-06 & $\begin{array}{l}\text { Curtobacterium sp. } \\
\text { SAP758.3 }\end{array}$ & $\begin{array}{c}788 / 789 \\
(99 \%)\end{array}$ & + & 2009 & $3712 \mathrm{~K} 2$ & ATL-1 \\
\hline $\begin{array}{c}\text { Orange Serum } \\
\text { Agar }\end{array}$ & 3712_K7_LP_OSA_B-09 & $\begin{array}{l}\text { Curtobacterium sp. } \\
\text { SAP758.3 }\end{array}$ & $\begin{array}{c}745 / 746 \\
(99 \%)\end{array}$ & + & 2009 & $3712 \mathrm{~K} 7$ & ATL-1 \\
\hline $\begin{array}{c}\text { Orange Serum } \\
\text { Agar }\end{array}$ & ROV05 - Q1 - OSA -01 & $\begin{array}{l}\text { Pseudomonas } \\
\text { anguilliseptica }\end{array}$ & $\begin{array}{c}727 / 732 \\
(99 \%)\end{array}$ & - & 2010 & $\begin{array}{l}\text { ROV05 } \\
\text { Quiver 1 }\end{array}$ & VK906 \\
\hline $\begin{array}{c}\text { Orange Serum } \\
\text { Agar }\end{array}$ & ROV08 - Q3 - OSA - 01 & $\begin{array}{l}\text { Pseudomonas } \\
\text { anguilliseptica }\end{array}$ & $\begin{array}{c}696 / 697 \\
(99 \%)\end{array}$ & - & 2010 & $\begin{array}{l}\text { ROV08 } \\
\text { Quiver } 3\end{array}$ & WFS1 \\
\hline $\begin{array}{c}\text { Orange Serum } \\
\text { Agar }\end{array}$ & ROV08 - Q3 - OSA - 02 & $\begin{array}{l}\text { Pseudomonas } \\
\text { anguilliseptica }\end{array}$ & $\begin{array}{c}766 / 772 \\
(99 \%)\end{array}$ & - & 2010 & $\begin{array}{l}\text { ROV08 } \\
\text { Quiver } 3\end{array}$ & WFS1 \\
\hline
\end{tabular}


Table 2.3. Bacterial cultures from various media, 2008-2012, during the Lophelia II program - continued.

[Bacteria belonging to the phylum Actinobacteria are highlighted in gray and phylum Firmicutes are highlighted in yellow. All of the unhighlighted bacteria are Proteobacteria.]

\begin{tabular}{|c|c|c|c|c|c|c|c|}
\hline Isolation medium & Bacterial isolate ID & $\begin{array}{c}\text { Top named 16S rRNA } \\
\text { match }\end{array}$ & $\begin{array}{l}\text { Basepair } \\
\text { match (\% } \\
\text { similarity) }\end{array}$ & $\underset{-/+}{\text { Gram }}$ & Year & Sample ID & Collection site \\
\hline $\begin{array}{c}\text { Orange Serum } \\
\text { Agar }\end{array}$ & ROV08 - Q3 - OSA - 03 & $\begin{array}{l}\text { Pseudomonas } \\
\text { anguilliseptica }\end{array}$ & $\begin{array}{c}678 / 683 \\
(99 \%)\end{array}$ & - & 2010 & $\begin{array}{l}\text { ROV08 } \\
\text { Quiver } 3\end{array}$ & WFS1 \\
\hline $\begin{array}{c}\text { Orange Serum } \\
\text { Agar }\end{array}$ & ROV09 - Q5 - OSA - 01 & $\begin{array}{l}\text { Pseudomonas } \\
\text { anguilliseptica }\end{array}$ & $\begin{array}{c}745 / 754 \\
(98 \%) \\
\end{array}$ & - & 2010 & $\begin{array}{l}\text { ROV09 } \\
\text { Quiver } 5 \\
\end{array}$ & WFS1 \\
\hline $\begin{array}{c}\text { Orange Serum } \\
\text { Agar }\end{array}$ & ROV09 - Q5 - OSA - 02 & $\begin{array}{l}\text { Pseudomonas } \\
\text { anguilliseptica }\end{array}$ & $\begin{array}{c}737 / 740 \\
(99 \%) \\
\end{array}$ & - & 2010 & $\begin{array}{l}\text { ROV09 } \\
\text { Quiver } 5 \\
\end{array}$ & WFS1 \\
\hline $\begin{array}{c}\text { Orange Serum } \\
\text { Agar }\end{array}$ & ROV09 - Q5 - OSA - 03 & $\begin{array}{l}\text { Pseudomonas } \\
\text { anguilliseptica }\end{array}$ & $\begin{array}{c}697 / 702 \\
(99 \%)\end{array}$ & - & 2010 & $\begin{array}{l}\text { ROV09 } \\
\text { Quiver } 5\end{array}$ & WFS1 \\
\hline $\begin{array}{c}\text { Orange Serum } \\
\text { Agar }\end{array}$ & ROV07 - Q1 - OSA - 01 & $\begin{array}{l}\text { Pseudomonas sp. } \\
\text { Wash } 8.2\end{array}$ & $\begin{array}{c}685 / 690 \\
(99 \%) \\
\end{array}$ & - & 2010 & $\begin{array}{l}\text { ROV07 } \\
\text { Quiver 1 }\end{array}$ & WFS1 \\
\hline Salty R2A & VoD_010_SR2A1 & Vibrio splendidus & $\begin{array}{c}839 / 842 \\
(99 \%)\end{array}$ & - & 2012 & Zinc Quiver 7 & $\begin{array}{c}\text { Zinc Subsea } \\
\text { Installation } \\
\text { NAD27 }\end{array}$ \\
\hline Salty R2A & VoD_010_SR2A2 & $\begin{array}{l}\text { Photobacterium } \\
\text { phosphoreum }\end{array}$ & $\begin{array}{c}863 / 865 \\
(99 \%)\end{array}$ & - & 2012 & Zinc Quiver 7 & $\begin{array}{c}\text { Zinc Subsea } \\
\text { Installation } \\
\text { NAD27 }\end{array}$ \\
\hline Salty R2A & VoD_010_SR2A3 & $\begin{array}{l}\text { Pseudomonas } \\
\text { xanthomarina }\end{array}$ & $\begin{array}{l}835 / 835 \\
(100 \%)\end{array}$ & - & 2012 & Zinc Quiver 7 & $\begin{array}{c}\text { Zinc Subsea } \\
\text { Installation } \\
\text { NAD27 }\end{array}$ \\
\hline Salty R2A & VoD_010_SR2A4 & Aliivibrio logei & $\begin{array}{c}906 / 910 \\
(99 \%)\end{array}$ & - & 2012 & Zinc Quiver 7 & $\begin{array}{c}\text { Zinc Subsea } \\
\text { Installation } \\
\text { NAD27 }\end{array}$ \\
\hline Salty R2A & VoD_010_SR2A5 & Aliivibrio wodanis & $\begin{array}{c}937 / 939 \\
(99 \%)\end{array}$ & - & 2012 & Zinc Quiver 7 & $\begin{array}{c}\text { Zinc Subsea } \\
\text { Installation } \\
\text { NAD27 } \\
\end{array}$ \\
\hline Salty R2A & VoD_010_SR2A6 & Aliivibrio wodanis & $\begin{array}{c}923 / 927 \\
(99 \%)\end{array}$ & - & 2012 & Zinc Quiver 7 & $\begin{array}{c}\text { Zinc Subsea } \\
\text { Installation } \\
\text { NAD27 }\end{array}$ \\
\hline
\end{tabular}


Table 2.3. Bacterial cultures from various media, 2008-2012, during the Lophelia II program - continued.

[Bacteria belonging to the phylum Actinobacteria are highlighted in gray and phylum Firmicutes are highlighted in yellow. All of the unhighlighted bacteria are Proteobacteria.]

\begin{tabular}{|c|c|c|c|c|c|c|c|}
\hline Isolation medium & Bacterial isolate ID & $\begin{array}{c}\text { Top named 16S rRNA } \\
\text { match }\end{array}$ & $\begin{array}{l}\text { Basepair } \\
\text { match (\% } \\
\text { similarity) }\end{array}$ & $\underset{-/+}{\text { Gram }}$ & Year & Sample ID & Collection site \\
\hline Salty R2A & VoD_010_SR2A7 & Pseudomonas stutzeri & $\begin{array}{c}932 / 934 \\
(99 \%)\end{array}$ & - & 2012 & Zinc Quiver 7 & $\begin{array}{c}\text { Zinc Subsea } \\
\text { Installation } \\
\text { NAD27 }\end{array}$ \\
\hline Salty R2A & VoD_011_SR2A1 & Moritella sp. & $\begin{array}{c}848 / 851 \\
(99 \%)\end{array}$ & - & 2012 & Zinc Quiver 8 & $\begin{array}{c}\text { Zinc Subsea } \\
\text { Installation } \\
\text { NAD27 }\end{array}$ \\
\hline Salty R2A & VoD_011_SR2A2 & $\begin{array}{l}\text { Photobacterium sp. } \\
\text { PM-GO05 }\end{array}$ & $\begin{array}{l}942 / 944 \\
(99 \%)\end{array}$ & - & 2012 & Zinc Quiver 8 & $\begin{array}{c}\text { Zinc Subsea } \\
\text { Installation } \\
\text { NAD27 }\end{array}$ \\
\hline Salty R2A & VoD_011_SR2A3 & $\begin{array}{l}\text { Photobacterium sp. } \\
\text { PM-GO05 }\end{array}$ & $\begin{array}{c}803 / 804 \\
(99 \%)\end{array}$ & - & 2012 & Zinc Quiver 8 & $\begin{array}{c}\text { Zinc Subsea } \\
\text { Installation } \\
\text { NAD27 }\end{array}$ \\
\hline Salty R2A & VoD_011_SR2A4 & $\begin{array}{l}\text { Pseudomonas sp. } \\
\text { E8.7(2011) }\end{array}$ & $\begin{array}{c}829 / 830 \\
(99 \%)\end{array}$ & - & 2012 & Zinc Quiver 8 & $\begin{array}{c}\text { Zinc Subsea } \\
\text { Installation } \\
\text { NAD27 }\end{array}$ \\
\hline Salty R2A & VoD_011_SR2A5 & $\begin{array}{l}\text { Shewanella woodyi } \\
\text { strain } \mathrm{S}-14\end{array}$ & $\begin{array}{l}908 / 912 \\
(99 \%)\end{array}$ & - & 2012 & Zinc Quiver 8 & $\begin{array}{c}\text { Zinc Subsea } \\
\text { Installation } \\
\text { NAD27 }\end{array}$ \\
\hline Salty R2A & VoD_011_SR2A6 & Moritella sp. & $\begin{array}{l}848 / 851 \\
(99 \%)\end{array}$ & - & 2012 & Zinc Quiver 8 & $\begin{array}{c}\text { Zinc Subsea } \\
\text { Installation } \\
\text { NAD27 }\end{array}$ \\
\hline Salty R2A & VoD_011_SR2A7 & $\begin{array}{l}\text { Photobacterium } \\
\text { kishitanii }\end{array}$ & $\begin{array}{l}941 / 945 \\
(99 \%)\end{array}$ & - & 2012 & Zinc Quiver 8 & $\begin{array}{c}\text { Zinc Subsea } \\
\text { Installation } \\
\text { NAD27 }\end{array}$ \\
\hline Salty R2A & VoD_011_SR2A8 & Vibrio sp. H02C48-48 & $\begin{array}{c}895 / 898 \\
(99 \%)\end{array}$ & - & 2012 & Zinc Quiver 8 & $\begin{array}{c}\text { Zinc Subsea } \\
\text { Installation } \\
\text { NAD27 }\end{array}$ \\
\hline
\end{tabular}


Table 2.3. Bacterial cultures from various media, 2008-2012, during the Lophelia II program - continued.

[Bacteria belonging to the phylum Actinobacteria are highlighted in gray and phylum Firmicutes are highlighted in yellow. All of the unhighlighted bacteria are Proteobacteria.]

\begin{tabular}{|c|c|c|c|c|c|c|c|}
\hline Isolation medium & Bacterial isolate ID & $\begin{array}{c}\text { Top named 16S rRNA } \\
\text { match }\end{array}$ & $\begin{array}{l}\text { Basepair } \\
\text { match (\% } \\
\text { similarity) }\end{array}$ & $\underset{-/+}{\text { Gram }}$ & Year & Sample ID & Collection site \\
\hline Salty R2A & VoD_011_SR2A9 & Vibrio sp. Mj18 & $\begin{array}{c}860 / 862 \\
(99 \%)\end{array}$ & - & 2012 & Zinc Quiver 8 & $\begin{array}{c}\text { Zinc Subsea } \\
\text { Installation } \\
\text { NAD27 }\end{array}$ \\
\hline Salty R2A & VoD_011_SR2A10 & Moritella sp. & $\begin{array}{c}926 / 931 \\
(99 \%)\end{array}$ & - & 2012 & Zinc Quiver 8 & $\begin{array}{c}\text { Zinc Subsea } \\
\text { Installation } \\
\text { NAD27 }\end{array}$ \\
\hline Salty R2A & VoD_011_SR2A11 & Moritella sp. & $\begin{array}{c}926 / 931 \\
(99 \%)\end{array}$ & - & 2012 & Zinc Quiver 8 & $\begin{array}{c}\text { Zinc Subsea } \\
\text { Installation } \\
\text { NAD27 }\end{array}$ \\
\hline Salty R2A & VoD_011_SR2A12 & $\begin{array}{l}\text { Pseudomonas sp. RF- } \\
58\end{array}$ & $\begin{array}{c}835 / 837 \\
(99 \%)\end{array}$ & - & 2012 & Zinc Quiver 8 & $\begin{array}{c}\text { Zinc Subsea } \\
\text { Installation } \\
\text { NAD27 }\end{array}$ \\
\hline Thio MP Agar & ROV02 - Q1 - TMP - 03 & $\begin{array}{l}\text { Microbacterium } \\
\text { aurum }\end{array}$ & $\begin{array}{c}626 / 627 \\
(99 \%)\end{array}$ & + & 2010 & $\begin{array}{l}\text { ROV02 } \\
\text { Quiver } 1\end{array}$ & VK826 \\
\hline Thio MP Agar & ROV03 - Q3 - TMP - 01 & $\begin{array}{l}\text { Sporosarcina sp. KS7- } \\
3\end{array}$ & $\begin{array}{c}714 / 723 \\
(98 \%)\end{array}$ & + & 2010 & $\begin{array}{l}\text { ROV03 } \\
\text { Quiver } 3\end{array}$ & VK826 \\
\hline
\end{tabular}


Table 2.4. Nutrient agars tested for fungal isolation from Lophelia pertusa during the Lophelia II program.

[GASWA, glycerol artificial seawater agar; g, gram; L, liter; mg, milligram; mL, milliliter]

\begin{tabular}{|c|c|c|}
\hline Medium & Ingredients per liter or [Manufacturer] if commercially avai & able \\
\hline \multirow{3}{*}{ GASWA + Chloramphenicol } & \multicolumn{2}{|l|}{$\begin{array}{l}\text { GASWA according to methods of Smith and Hayasaka (1982), } \\
\text { with the following modification: Substitute Instant Ocean }(2.0 \mathrm{~g}) \\
\text { for Rila salts }(2.0 \mathrm{~g}) .\end{array}$} \\
\hline & Agar & $15.0 \mathrm{~g}$ \\
\hline & Chloramphenicol & $0.5 \mathrm{~g}$ \\
\hline \multirow{5}{*}{$\begin{array}{l}\text { Seawater Corn Meal }+ \\
\text { Chloramphenicol }+ \\
\text { Gentamicin }\end{array}$} & Corn Meal Agar [Oxoid] & $17.0 \mathrm{~g}$ \\
\hline & Instant Ocean & $36.0 \mathrm{~g}$ \\
\hline & Deionized water & $1.0 \mathrm{~L}$ \\
\hline & Chloramphenicol & $0.5 \mathrm{~g}$ \\
\hline & Gentamicin & $0.25 \mathrm{~g}$ \\
\hline \multirow{4}{*}{$\begin{array}{l}\text { Saubouraud-Maltose }+ \\
\text { Chloramphenicol }+ \\
\text { Gentamicin }\end{array}$} & Saubouraud-Maltose Agar [Difco] & $65.0 \mathrm{~g}$ \\
\hline & Deionized water & $1.0 \mathrm{~L}$ \\
\hline & Chloramphenicol & $0.5 \mathrm{~g}$ \\
\hline & Gentamicin & $0.25 \mathrm{~g}$ \\
\hline \multirow{3}{*}{ Fell \& van Uden } & $\begin{array}{l}\text { Isolation medium according to methods of Fell and van Uden } \\
\text { (1963) }\end{array}$ & \\
\hline & $\mathrm{pH}$ to 4.5 with lactic acid & \\
\hline & $\begin{array}{l}\text { Solution containing } 10 \mathrm{mg} \text { Chlortetracycline HCL, } 2 \mathrm{mg} \\
\text { Chloramphenicol, and } 2 \mathrm{mg} \text { Streptomycin Sulfate }\end{array}$ & \\
\hline \multirow{2}{*}{ BBL Mycophil } & BBL Mycophyil Agar [BD] & $38 \mathrm{~g}$ \\
\hline & Deionized water & $1.0 \mathrm{~L}$ \\
\hline \multirow{2}{*}{ Orange Serum } & Orange Serum Agar [Alpha Biosciences] & $45 \mathrm{~g}$ \\
\hline & Deionized water & $1.0 \mathrm{~L}$ \\
\hline \multirow{3}{*}{$\begin{array}{l}\text { Rose Bengal } \\
\text { Chloramphenicol }\end{array}$} & Rose Bengal Agar [Oxoid] & $16 \mathrm{~g}$ \\
\hline & Deionized water & $500 \mathrm{~mL}$ \\
\hline & Chloramphenicol Supplement [Oxoid] & 1 vial \\
\hline
\end{tabular}


Table 2.5. Fungal cultures grown on various media, 2009-2010, during the Lophelia II program.

[VK826, Viosca Knoll; WFS, West Florida Slope; ITS, internal transcribed spacer; m, meter. Atlantic collections were made in 2009 and Gulf of Mexico collections in 2010. The Biolog column indicates isolates further characterized in table 2.6. See figure 2.1 for locations]

\begin{tabular}{|c|c|c|c|c|c|c|c|}
\hline \multirow[t]{2}{*}{ Site } & \multirow[t]{2}{*}{ Isolate } & \multicolumn{2}{|c|}{ Top GenBank matches } & \multicolumn{2}{|c|}{$\begin{array}{l}\text { Accession numbers for } \\
\text { our sequences }\end{array}$} & \multirow[t]{2}{*}{ Depth (m) } & \multirow[t]{2}{*}{ Biolog } \\
\hline & & $18 S$ & ITS & $18 S$ & ITS & & \\
\hline $\begin{array}{l}\text { ATL-1 } \\
\text { (Atlantic) }\end{array}$ & 3705k9Lp-OSAB-02 & Cryptococcus albidus & Cryptococcus saitoi & JN571453 & JN383903 & 777 & $\mathrm{X}$ \\
\hline $\begin{array}{l}\text { ATL-2 } \\
\text { (Atlantic) }\end{array}$ & 3712K3Lp-OSAB-01 & Sporidiobolus johnsoni & Sporidiobolus salmonicolor & JN571439 & JN383898 & 438 & $\mathrm{X}$ \\
\hline VK826 (Gulf) & ROV02Q2OSA-02 & Epicoccum nigrum & Fungal sp. AB21 & JN571446 & JN383892 & 490 & $\mathrm{X}$ \\
\hline VK826 (Gulf) & ROV02Q2OSA-03 & Epicoccum nigrum & Fungal sp. AB21 & JN571457 & JN383891 & 490 & \\
\hline WFS1 (Gulf) & ROV07Q1FBM-04 & Rhodosporidium diobovatum & Rhodosporidium diobovatum & JN571462 & JN383910 & 504 & $\mathrm{X}$ \\
\hline WFS1(Gulf) & ROV07Q1OSA-02 & Rhodosporidium diobovatum & Rhodosporidium diobovatum & JN571450 & JN383904 & 504 & \\
\hline WFS1 (Gulf) & ROV07Q3FBM-02 & Rhodosporidium diobovatum & Rhodosporidium diobovatum & JN571444 & JN383907 & 504 & \\
\hline WFS1 (Gulf) & ROV07Q3FBM-03 & Rhodotorula mucilaginosa & Rhodotorula sp. & JN571463 & JN383908 & 504 & \\
\hline WFS1 (Gulf) & ROV07Q3FVU-04 & Paecilomyces sp. 080834 & Uncultured Nectria & JN571443 & JN383889 & 504 & $\mathrm{X}$ \\
\hline WFS1 (Gulf) & ROV08Q3FBM-03 & $\begin{array}{l}\text { Rhodosporidium } \\
\text { sphaerocarpum }\end{array}$ & $\begin{array}{l}\text { Rhodosporidium } \\
\text { sphaerocarpum }\end{array}$ & JN571460 & JN383901 & 537 & \\
\hline WFS1 (Gulf) & ROV08Q4FRB-03 & Paecilomyces sp. & Uncultured Nectria & JN571442 & JN383888 & 537 & $\mathrm{X}$ \\
\hline WFS1 (Gulf) & ROV09Q1FVU-02 & Kondoa malvinella & Kondoa aeria & JN571449 & JN383900 & 543 & $\mathrm{X}$ \\
\hline WFS1 (Gulf) & ROV09Q2FVU-01 & Uncultured Dikarya & Uncultured fungus genes & JN571440 & JN383896 & 543 & $\mathrm{X}$ \\
\hline WFS1 (Gulf) & ROV09Q2FVU-02 & Ascomycete sp. & Glomerella sp. & JN571455 & JN383895 & 543 & \\
\hline WFS1 (Gulf) & ROV09Q2FVU-03 & Uncultured Dikarya & Glomerella sp. & JN571456 & JN383897 & 543 & \\
\hline WFS2 (Gulf) & ROV10Q2FRB-03 & Paecilomyces sp. & Uncultured fungus & JN571459 & JN383886 & 524 & $\mathrm{X}$ \\
\hline WFS2 (Gulf) & ROV10Q4FRB-01 & Rhodosporidium diobovatum & Rhodosporidium diobovatum & JN571441 & JN383899 & 524 & \\
\hline WFS2 (Gulf) & ROV10Q4FVU-05 & Uncultured Basidiomycota & Rhodosporidium diobovatum & JN571448 & JN383906 & 524 & $\mathrm{X}$ \\
\hline WFS2 (Gulf) & ROV10Q4FVU-06 & Rhodosporidium diobovatum & Rhodosporidium diobovatum & JN571447 & JN383909 & 524 & \\
\hline WFS2 (Gulf) & ROV10Q4FVU-07 & Rhodosporidium diobovatum & Rhodosporidium diobovatum & JN571454 & JN383905 & 524 & \\
\hline WFS3 (Gulf) & ROV11Q1FRB-01 & Geomyces destructans & Geomyces sp. & JN571445 & JN383894 & 509 & $\mathrm{X}$ \\
\hline WFS3 (Gulf) & ROV11Q1FRB-02 & Sporidiobolus johnsoni & Sporidiobolus johnsonii & JN571452 & JN383902 & 509 & $\mathrm{X}$ \\
\hline WFS3 (Gulf) & ROV11Q1FRB-04 & Penicillium sp. & Uncultured fungus & JN571458 & JN383893 & 509 & \\
\hline WFS3 (Gulf) & ROV11Q2FVU-01 & Paecilomyces sp. & Uncultured Nectria & JN571461 & JN383887 & 509 & $\mathrm{X}$ \\
\hline WFS3 (Gulf) & ROV11Q2FVU-02 & Paecilomyces sp. & Acremonium strictum & JN571451 & JN383890 & 509 & $\mathrm{X}$ \\
\hline
\end{tabular}


Table 2.6. Biolog plate readings for 14 of the fungal isolates showing carbon source usage during the Lophelia II program.

[Initial readings at time zero were subtracted from the final readings ( 144 hours), and any absorbance above 0.100 was considered positive (highlighted in yellow)]

\begin{tabular}{|c|c|c|c|c|c|c|c|c|c|c|c|c|c|c|c|}
\hline Carbor & Carbo $>$ & 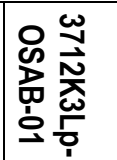 & 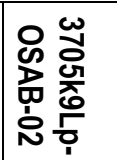 & 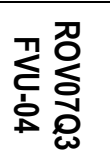 & 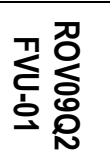 & 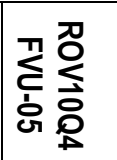 & 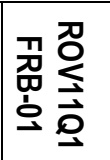 & 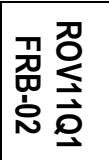 & 꽁 & 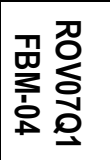 & 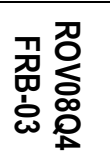 & 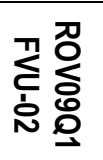 & 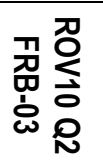 & 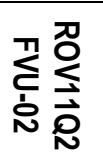 & 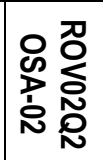 \\
\hline & & 016 & .041 & 144 & 038 & 042 & 015 & 012 & 165 & 037 & 085 & 011 & 139 & 111 & .022 \\
\hline & Cyclodex & 34 & 047 & 061 & 049 & 055 & 061 & 049 & 061 & 037 & 065 & 032 & 057 & 103 & .066 \\
\hline $\begin{array}{r}\text { Phosphory } \\
\text { chemic }\end{array}$ & pha & 02 & 06 & 181 & 046 & 083 & 023 & 103 & .204 & .043 & 168 & .072 & .175 & .183 & 0.025 \\
\hline Carbohyc & & 375 & 137 & 192 & .048 & .094 & 015 & 102 & 174 & 0.037 & 075 & .033 & .106 & .096 & 0.026 \\
\hline Carb & & 76 & 87 & 029 & 028 & 044 & 002 & 016 & 032 & 009 & 012 & 008 & 03 & 017 & 02 \\
\hline Amino Acid & (G & 0.029 & 0.193 & 0.039 & 0.032 & 0.056 & 0.011 & 0.005 & 0.027 & 0.089 & 0.025 & 0.015 & .014 & .023 & 0.029 \\
\hline Carbox & Acid & 44 & 4 & 088 & 037 & 027 & 15 & 12 & 83 & 24 & 34 & 22 & 43 & 57 & 023 \\
\hline Amino Acid & $A$ & 0.015 & 0.045 & 0.07 & 0.032 & 0.022 & 0.008 & 0.004 & 0.061 & 0.02 & 0.02 & 0.011 & 0.033 & 0.035 & 0.018 \\
\hline & & 0.043 & 0.183 & 0.203 & 0.086 & 0.156 & 022 & 036 & 0.235 & 0.197 & 176 & 067 & .21 & 234 & 0.059 \\
\hline Polymer & $\beta-\mathrm{Cly}$ & 0.03 & \begin{tabular}{|l|}
0.054 \\
\end{tabular} & \begin{tabular}{|l|}
0.053 \\
\end{tabular} & 0.025 & 0.037 & \begin{tabular}{|l|}
0.039 \\
\end{tabular} & 0.018 & 0.05 & \begin{tabular}{|l|}
0.034 \\
\end{tabular} & 066 & 017 & 055 & .068 & 0.034 \\
\hline Amide & & 02 & 0.031 & 0.032 & 0.007 & 0.008 & 0.014 & 0.006 & 0.016 & 0.006 & .019 & .013 & .017 & .044 & 0.021 \\
\hline Carl & & 387 & & 0.235 & 0.041 & 0.089 & & & & & & & & 209 & 0.063 \\
\hline & Salicin & 034 & 3 & 0112 & 004 & 0.04 & 0.019 & 027 & 107 & 0.026 & 133 & .029 & .107 & .121 & 0.026 \\
\hline $\begin{array}{r}\text { Brom } \\
\text { chem }\end{array}$ & Brom & 029 & 1 & 0.028 & 02 & 0.012 & 016 & 005 & 11 & 048 & 15 & 061 & .01 & .019 & 0.028 \\
\hline Carboxylic aci & & 035 & 0.057 & 0.025 & 0.01 & 0.024 & 0.013 & 0.008 & \begin{tabular}{|l|}
0.013 \\
\end{tabular} & 0.042 & 0.019 & .009 & .015 & .017 & 0.026 \\
\hline Amino Acid & 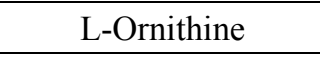 & .022 & 0.043 & 0.154 & 0.034 & 0.038 & 0.013 & 0.016 & 0.148 & 0.04 & 0.054 & .017 & .089 & .097 & 0.025 \\
\hline Carbohydrate & $\Lambda$ & 0.032 & 0.055 & 0.1 & 0.034 & 0.025 & 0.016 & 0.007 & 3 & 0.03 & (505 & 008 & 087 & 086 & .019 \\
\hline Polymer & Dextrin & 0.039 & 0.296 & 0.069 & 0.03 & 0.066 & 0.02 & 0.046 & \begin{tabular}{|l|}
0.053 \\
\end{tabular} & 0.041 & 0.048 & 0.043 & 056 & 071 & 0.055 \\
\hline Carboxylic acid & D-Giluc & 0.016 & 0.388 & 0.104 & 0.023 & 0.012 & 0.012 & 0.007 & 0.078 & 0.02 & 0.034 & 0.009 & .051 & .084 & 0.02 \\
\hline Carbohydrate & D-Melezitose & 0.016 & 0.373 & 0.218 & 0.043 & 0.028 & 0.01 & 0.008 & 0.234 & 0.03 & 0.133 & 0.012 & 0.194 & 0.136 & 0.034 \\
\hline Carbohydrate & . & 0.013 & 0.037 & 0.177 & 0.029 & 0.018 & 0.008 & 0.008 & 0.185 & \begin{tabular}{|l|}
0.019 \\
\end{tabular} & 0.109 & 0.003 & .159 & .136 & 0.058 \\
\hline Carboxylic acid & Fumaric Acid & 0.058 & 0.52 & 0.037 & 0.022 & 0.013 & 0.009 & 0.006 & 0.015 & \begin{tabular}{|l|}
0.137 \\
\end{tabular} & 0.016 & 0.016 & 0.012 & 0.024 & 0.028 \\
\hline Amide & Succinamic Acid & 0.066 & 0.083 & 0.027 & 0.013 & 0.014 & 0.014 & 0.005 & 0.009 & 0.05 & 0.021 & 0.008 & 0.011 & 0.022 & 0.045 \\
\hline
\end{tabular}


Table 2.6. Biolog plate readings for 14 of the fungal isolates showing carbon source usage during the Lophelia II program - continued.

[Initial readings at time zero were subtracted from the final readings (144 hours), and any absorbance above 0.100 was considered positive (highlighted in yellow)]

\begin{tabular}{|c|c|c|c|c|c|c|c|c|c|c|c|c|c|c|c|}
\hline Carbon Type & Carbon Source & 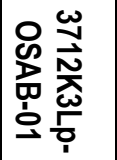 & 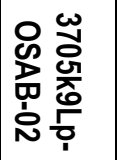 & 꽁이 & 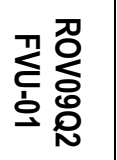 & 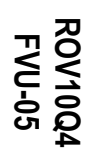 & 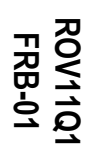 & 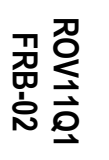 & 突员 & 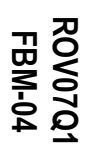 & 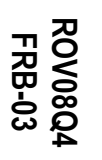 & 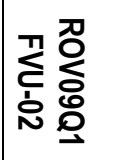 & 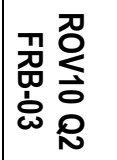 & 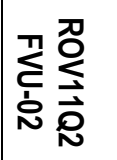 & 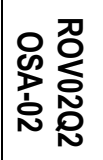 \\
\hline Ami & L-Phenylalanine & 0.026 & 048 & 78 & 035 & 0.107 & 2 & 85 & 158 & 95 & 15 & 0.019 & 143 & 46 & .025 \\
\hline Carbohydrate & & 0.015 & 048 & 0.217 & 0.034 & 024 & 0.018 & 007 & 211 & 028 & 138 & .011 & 0.252 & .146 & 0.025 \\
\hline Carbohydrate & -Erythritol & 0.027 & 0.048 & 0.213 & .028 & 0.035 & 0.009 & .008 & 187 & .028 & 0.08 & 0.007 & 0.143 & 0.22 & 0.037 \\
\hline Alcohol & Glycerol & 0.051 & 0.067 & 0.782 & 0.04 & 0.091 & 0.016 & 0.01 & 0.93 & 0.111 & 0.76 & 0.073 & 1.088 & .998 & 0.071 \\
\hline Carbohy & se & 0.013 & 0.043 & 0.151 & 04 & 019 & 0.012 & .005 & 125 & .02 & 085 & .007 & 0.113 & .121 & 0.016 \\
\hline Carbohydrate & D-Sorbitol & 0.2 & 0.259 & 0.146 & 0.027 & 0.057 & 0.009 & 0.073 & 0.147 & 0.028 & 0.079 & 0.023 & 0.143 & .152 & 0.024 \\
\hline Carboxylic acid & Hydroxybutyric Acid & 0.027 & 0.049 & 0.022 & 0.008 & .014 & 0.004 & 0.007 & 007 & 026 & 0.013 & 0.008 & 0.008 & .016 & 0.03 \\
\hline Carboxylic acid & Succinic Acid & 0.088 & 0.432 & 0.081 & 0.025 & 0.025 & 0.013 & 0.005 & 0.067 & 0.166 & 0.013 & 0.031 & 0.062 & 0.072 & 0.044 \\
\hline Amino Acid & & 016 & .253 & 0.049 & .032 & 109 & 0.012 & 005 & 071 & 285 & 02 & 0.019 & .039 & .036 & 0.025 \\
\hline Amino Acid & & 011 & 0042 & 0128 & 0031 & 0027 & 0016 & 0006 & 0.134 & 0.028 & 071 & 012 & 0.132 & 0.098 & 0.021 \\
\hline Carbol & & 381 & 337 & 196 & .046 & .096 & 0.002 & 111 & 169 & 172 & 117 & .036 & 0.193 & 0.164 & 0.037 \\
\hline & & 29 & 72 & 0.223 & 0.041 & 032 & 01 & 08 & 232 & 29 & 83 & 23 & 182 & 06 & 027 \\
\hline Carbohydrate & lethyl-D-Galact & 0.009 & 0.041 & 0.148 & 0.04 & 0.028 & 0.004 & 0.005 & 127 & .026 & .097 & 0.008 & 0.13 & 0.113 & 0.013 \\
\hline Carboh & & & 41 & 3 & & & & 22 & & & & 011 & 128 & 121 & .048 \\
\hline Carhorl & Hydroxy & 147 & 0.054 & 129 & 18 & 0.064 & 0.013 & .006 & 124 & 083 & 138 & .027 & .104 & .125 & 0.041 \\
\hline Ester & $\begin{array}{c}\text { Succinic Acid } \\
\text { Monomethyl Ester }\end{array}$ & 0.195 & 0.205 & 0.018 & 0.003 & 02 & 0.007 & 0.059 & 007 & .06 & 0.009 & .005 & 0.008 & 0.013 & 0.029 \\
\hline Amino Acid & L-Pyroglutamic Acid & 0.075 & 0.036 & 0.022 & 0.019 & 0.018 & 0.013 & 0.01 & 0.023 & 0.039 & .015 & 0.014 & 0.013 & .017 & 0.029 \\
\hline Carbohydrate & & 0.088 & 0.044 & 0.148 & 0.03 & 0.077 & 0.016 & 0.013 & 0.18 & 0.151 & 0.066 & 0.03 & 0.147 & 0.127 & 0.027 \\
\hline Carbohydrate & L-Fucose & 0.017 & 0.049 & 0.083 & 0.016 & 0.036 & 0.008 & 0.007 & 0.066 & 0.034 & 0.059 & 0.007 & 0.084 & 0.079 & 0.02 \\
\hline Carbohydrate & Inositol & 0.023 & 0.19 & 0.138 & 0.032 & 0.021 & 0.005 & 0.005 & 0.123 & 0.03 & 0.097 & 0.009 & 0.148 & 0.104 & 0.016 \\
\hline $\begin{array}{l}\text { Aromatic } \\
\text { chemical }\end{array}$ & $\beta$-Methyl-D-Galactoside & 0.014 & 0.047 & 0.146 & 0.036 & 0.036 & 0.005 & 0.004 & 0.123 & 034 & 0.116 & 0.013 & 0.158 & 0.118 & 0.017 \\
\hline Carbohydrate & & & 051 & 5 & 46 & 086 & 006 & 004 & 9 & 47 & 2 & 01 & 72 & .161 & 0.011 \\
\hline Carboxylic acid & $\begin{array}{c}\text { p-Hydroxyphenylacetic } \\
\text { Acid }\end{array}$ & 0.051 & 0.04 & 0.172 & 0.013 & 0.163 & 0.002 & 0.047 & 0.183 & 0.103 & 0.094 & 0.01 & 0.172 & 0.238 & 0.027 \\
\hline Carboxylic acid & $\mathrm{N}$-Acetyl-Glutamic Acid & 0.016 & 0.04 & 0.035 & 0.019 & 0.006 & 0 & 0.006 & 0.023 & 0 & 0.013 & 0.006 & 0.015 & 0.025 & 0.022 \\
\hline
\end{tabular}


Table 2.6. Biolog plate readings for 14 of the fungal isolates showing carbon source usage during the Lophelia II program - continued.

[Initial readings at time zero were subtracted from the final readings (144 hours), and any absorbance above 0.100 was considered positive (highlighted in yellow)]

\begin{tabular}{|c|c|c|c|c|c|c|c|c|c|c|c|c|c|c|c|}
\hline C & Carho & 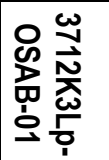 & 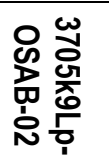 & 꽁ㅇㅀ & 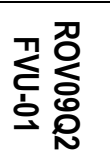 & 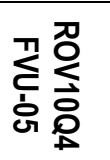 & 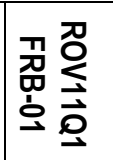 & 㞧 号 & 꽁ํㅇ & 囬 & 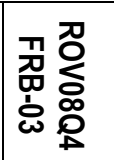 & 꽁ㅇㅀ & 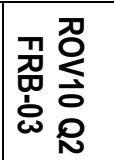 & 꽁ํㅇ & 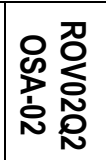 \\
\hline & & 18 & 056 & 168 & 943 & 951 & 015 & 009 & 163 & 058 & 047 & 017 & 117 & 115 & .024 \\
\hline & 1 & 019 & 101 & 177 & .042 & 077 & 018 & 042 & 0.2 & 031 & 136 & 019 & 284 & .271 & .032 \\
\hline 1 & & 05 & 064 & 133 & 044 & 046 & 006 & .02 & 122 & 089 & 075 & 023 & 124 & .11 & .024 \\
\hline Car & $\mathrm{a}$ & 03 & 0.454 & .048 & 0.029 & 0.112 & .006 & .003 & 0.03 & 0.525 & .032 & 0.01 & .022 & 0.046 & 0.034 \\
\hline & Mothyl D & 14 & 57 & 141 & 0.033 & 035 & 005 & 005 & 125 & 936 & 27 & 11 & 162 & 143 & .016 \\
\hline te & & 45 & & 266 & .06 & 097 & 006 & 007 & 279 & 177 & 89 & 63 & 299 & 255 & 057 \\
\hline 年 & 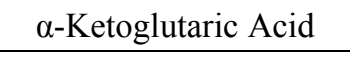 & 288 & 211 & 0.139 & .048 & 0.1 & 015 & 0.094 & 128 & 0.075 & 015 & 021 & .04 & .068 & .024 \\
\hline & & & & 172 & 037 & 223 & 03 & & 65 & 01 & 68 & 07 & 61 & 73 & 023 \\
\hline Amino & & 018 & 06 & 0.142 & 0.03 & 0.045 & 0.017 & 0.012 & 0.129 & 0.046 & 074 & 021 & 081 & 0.068 & 0.025 \\
\hline & & 44 & 64 & 06 & 033 & .05 & 011 & 11 & 942 & 047 & 19 & 13 & 17 & 038 & 024 \\
\hline Car & $\mathrm{Gol}$ & 013 & 0.068 & 0.017 & 0.004 & 0.007 & 003 & .004 & 004 & 0.07 & 005 & .009 & 003 & 0.005 & 0.011 \\
\hline & & 018 & 0.054 & 0.128 & 0.045 & 0.017 & 003 & 0 & 107 & .017 & 083 & 009 & 116 & .105 & 0.011 \\
\hline & & 013 & 0.268 & 0.128 & 0.046 & 0.058 & .007 & 0.001 & 0.138 & 161 & 138 & 057 & 169 & 125 & 0.02 \\
\hline ate & & 024 & 0.051 & 0.103 & 0.038 & 0.021 & .003 & 0.008 & 0.091 & 0.021 & 058 & .007 & 062 & .079 & .011 \\
\hline $\mathrm{Ca}$ & $\mathrm{E}$ & 0.028 & 0.038 & 0.174 & 0.03 & 0.026 & 0.000 & 0.01 & 0.134 & 0.024 & 0.156 & 0.012 & 0.1 & 0.113 & 16 \\
\hline 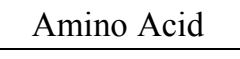 & & 0.034 & 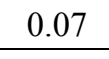 & 0.171 & 0.038 & 0.043 & 0.005 & 0.006 & 0.148 & 0.06 & 211 & 018 & .29 & .145 & 0.024 \\
\hline Amine & $-5+2>$ & \begin{tabular}{|l|}
0.027 \\
\end{tabular} & 039 & 0.086 & 0.02 & 0.036 & \begin{tabular}{|l|}
0.012 \\
\end{tabular} & 0.019 & 0.067 & 0.026 & 018 & .014 & 055 & .077 & 0.032 \\
\hline Carbohy & & 0.049 & 0.212 & 0.068 & 0.042 & 0.059 & 0.009 & 0.019 & 0.053 & 0.048 & 028 & 0.02 & 023 & 0.042 & 0.036 \\
\hline Carbohydrate & & .011 & 0.299 & 0.152 & 0.034 & 0.022 & .004 & 0.007 & 0.15 & \begin{tabular}{|l|}
0.038 \\
\end{tabular} & 062 & .054 & 095 & 0.094 & 0.023 \\
\hline Carbohydrate & & .013 & 0.049 & 0.112 & 0.047 & 0.013 & 0.005 & 0.004 & 0.105 & 0.019 & .063 & .009 & .095 & 0.102 & 0.014 \\
\hline Carbohydrate & & \begin{tabular}{|l|}
0.022 \\
\end{tabular} & 0.313 & 0.131 & 0.044 & 0.029 & \begin{tabular}{|l|}
0.005 \\
\end{tabular} & 0.005 & 0.112 & 0.022 & 089 & 012 & 0.12 & 0.119 & 0.013 \\
\hline & & 0.319 & 0.328 & 0.183 & 0.036 & 0.062 & 0.004 & 0.131 & 0.215 & 0.143 & 0.148 & 0.053 & 0.236 & 0.18 & 0.051 \\
\hline Carboxylic acid & -Lactic Acid & \begin{tabular}{|l|}
0.035 \\
\end{tabular} & 0.053 & 0.036 & 0.015 & 0.013 & 0.005 & 0.005 & \begin{tabular}{|l|}
0.017 \\
\end{tabular} & \begin{tabular}{|l|}
0.011 \\
\end{tabular} & 0.013 & 0.012 & 0.01 & 0.015 & 0.018 \\
\hline Amino Ac & Alanyl-Glycine & 0.027 & & 0.13 & 0.035 & 0.029 & 0.005 & 0.006 & 0.129 & 0.059 & 0.085 & 0.045 & 0.133 & 0.121 & 0.027 \\
\hline Amine & Putrescine & 0.02 & 0.125 & .171 & 0.033 & 0.03 & 0.018 & 0.027 & 0.149 & 0.022 & 0.152 & 0.01 & 0.14 & 0.082 & 0.034 \\
\hline
\end{tabular}


Table 2.6. Biolog plate readings for 14 of the fungal isolates showing carbon source usage during the Lophelia II program - continued.

[Initial readings at time zero were subtracted from the final readings (144 hours), and any absorbance above 0.100 was considered positive (highlighted in yellow)]

\begin{tabular}{|c|c|c|c|c|c|c|c|c|c|c|c|c|c|c|c|}
\hline Carbon Type & Carbon Source & 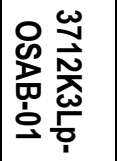 & 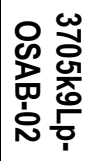 & 꽁 잉 & 꽁 ঠo & 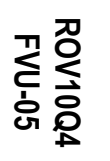 & 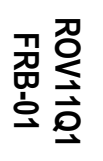 & 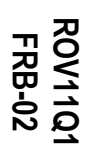 & 突㤎 & 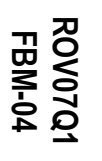 & 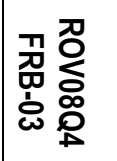 & 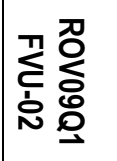 & 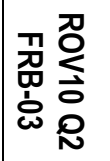 & 꽁 옹 & 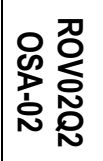 \\
\hline Carb & & 0.213 & 0.063 & 0.185 & 0.03 & 0.065 & .016 & 094 & 0.16 & 053 & 0.085 & 0.026 & 0.143 & 0.113 & 0.024 \\
\hline Carboxylic acid & -Gluconic Ac & 0.059 & 0.068 & 0.066 & 0.023 & 0.032 & 0.011 & .008 & 0.048 & 0.04 & 0.016 & 0.03 & 0.038 & 0.056 & 0.024 \\
\hline Alcohol & Maltitol & 0.014 & 0.237 & 0.115 & 0.026 & 0.019 & 0.008 & 0.008 & 0.091 & 0.015 & 0.059 & 0.013 & 0.084 & 0.089 & 0.015 \\
\hline Carbohydrate & Psicose & 0.057 & 0.117 & 0.199 & 0.039 & 0.052 & 0.009 & 0.038 & 0.183 & 0.064 & 0.15 & 0.009 & 0.206 & 0.153 & 0.027 \\
\hline Carbol & & 0.019 & 0.288 & 0.149 & 0.028 & 0.016 & 0.008 & 0.007 & 0.176 & 0.016 & 0.098 & 0.004 & 0.132 & 0.105 & 0.017 \\
\hline Carboxylic acid & -Malic Acid & 0.032 & 0.062 & 0.036 & 0.016 & 0.012 & 0.009 & 0.007 & 0.018 & 0.02 & 0.012 & 0.013 & 0.011 & 0.023 & 0.016 \\
\hline Amino Acid & & .025 & 0.122 & 138 & 0.034 & .039 & 0.012 & 007 & 0.117 & 101 & 031 & 0.01 & 0.088 & .112 & 0.025 \\
\hline & e & 009 & .046 & 172 & . 024 & 018 & 0 & 01 & 0.141 & .013 & 0.092 & 0 & .12 & .06 & 0 \\
\hline & Arbutin & 0.078 & 0.248 & 0.075 & 0.028 & 0.091 & 0.019 & 0.088 & 0.038 & 0.257 & 0.064 & 0.024 & 0.061 & 0.066 & 0.024 \\
\hline Amino Acid & Cllupcrin & 22 & .026 & 156 & 0.017 & 0.023 & 016 & 005 & 109 & 001 & 099 & 009 & 153 & 094 & .023 \\
\hline Carbohydrate & Malt & 0.013 & 0.301 & 0.118 & 0.044 & 0.028 & 0.012 & 0.008 & 0.106 & 0.023 & 0.061 & 0.032 & 0.094 & 0.083 & 0.023 \\
\hline Carbohydrate & Raffinose & 0.14 & 0.175 & 0.231 & 0.057 & 0.116 & 0.009 & 0.006 & 0.225 & 0.142 & 0.152 & 0.012 & 0.2 & 0.146 & 0.031 \\
\hline Carbohydrate & Xylitol & 0.013 & 0.048 & 0.15 & 0.045 & 0.027 & 0.012 & 0.006 & 0.16 & 0.019 & 0.075 & 0.006 & 0.098 & 0.096 & 0.03 \\
\hline Carboxylic acid & Malic Acid & 0.047 & 0.104 & 0.042 & 0.027 & 0.021 & 0.024 & 0.007 & 0.035 & 0.13 & 0.014 & 0.021 & 0.012 & 0.024 & 0.022 \\
\hline Amino Acid & -Aspartic Acid & 0.024 & 0.228 & 0.051 & 0.023 & 0.044 & 0.015 & 0.008 & 0.034 & 0.149 & \begin{tabular}{|l|}
0.009 \\
\end{tabular} & 0.011 & 0.026 & 0.039 & 0.023 \\
\hline $\begin{array}{l}\text { Aromatic } \\
\text { chemicals }\end{array}$ & Iridine & 0.019 & 0.043 & 0.182 & 0.03 & 0.038 & 0.016 & 0.015 & 0.15 & 0.031 & 0.114 & 0.012 & 0.127 & 0.111 & 0.022 \\
\hline Carbohydrate & D-C & 0.015 & 0.248 & 0.197 & 0.036 & 0.046 & 0.014 & 0.013 & 0.175 & 0.031 & 0.075 & 0.041 & 0.124 & 0.106 & 0.03 \\
\hline Carbohydrate & $\alpha$-D-Glucose & 0.484 & 0.366 & 0.242 & 0.056 & 0.108 & 0.015 & 0.125 & 0.198 & 0.155 & 0.152 & 0.062 & 0.213 & 0.155 & 0.056 \\
\hline Carbohydrate & Viditudutus & 0.025 & 0.314 & 0.191 & 0.05 & 0.064 & 0.016 & 0.011 & 0.166 & 0.061 & \begin{tabular}{|l|}
0.124 \\
\end{tabular} & 0.036 & 0.159 & 0.136 & 0.037 \\
\hline Carbohydrate & L-Rhamnose & 0.013 & 0.177 & \begin{tabular}{|l|}
0.149 \\
\end{tabular} & 0.043 & 0.036 & 0.014 & 0.01 & 0.142 & 0.024 & 0.101 & 0.014 & 0.082 & 0.072 & 0.029 \\
\hline Carbohydrate & D-Xylose & 0.103 & \begin{tabular}{|l|}
0.329 \\
\end{tabular} & \begin{tabular}{|l|}
0.097 \\
\end{tabular} & 0.042 & 0.071 & 0.01 & 0.037 & 0.081 & 0.032 & \begin{tabular}{|l|}
0.063 \\
\end{tabular} & 0.022 & 0.043 & 0.051 & 0.05 \\
\hline Carboxylic acid & uinic Acid & 0.04 & 0.235 & 0.11 & 0.035 & 0.037 & 0.013 & 0.104 & 0.076 & 0.467 & 0.041 & 0.059 & 0.074 & 0.1 & 0.03 \\
\hline Amino Acid & & 0024 & 0.26 & 0.071 & 0.026 & 0.058 & 0.013 & 0.008 & 0.046 & 0.123 & 0.024 & 0.018 & 0.033 & 0.05 & 0.024 \\
\hline $\begin{array}{l}\text { Aromatic } \\
\text { chemical }\end{array}$ & $\begin{array}{c}\text { Adenosine-5'- } \\
\text { Monophosphate }\end{array}$ & 0.052 & 0.081 & 0.127 & 0.031 & 0.051 & 0.016 & 0.017 & 0.113 & 0.019 & 0.02 & 0.012 & 0.049 & 0.055 & 0.024 \\
\hline
\end{tabular}


Table 2.7. Components of Lophelia pertusa mucus and the number of fungal isolates during the Lophelia II program.

\begin{tabular}{|c|c|c|c|}
\hline & $\begin{array}{l}\text { Lophelia mucus } \\
\text { components }\end{array}$ & $\begin{array}{c}\text { Number of isolates that } \\
\text { metabolized this carbon source }\end{array}$ & $\begin{array}{c}\text { Fungal genera that metabolized this } \\
\text { carbon source }\end{array}$ \\
\hline \multirow{7}{*}{ Amino Acids } & Phenylalanine & $7(50 \%)$ & $\begin{array}{c}\text { Nectria/Acremonium, } \\
\text { Sporidiobolus, Rhodosporidium }\end{array}$ \\
\hline & Alanine & $5(36 \%)$ & Nectria/Acremonium \\
\hline & Serine & $4(29 \%)$ & Nectria/Acremonium \\
\hline & Aspartic acid & $2(14 \%)$ & Cryptococcus, Rhodosporidium \\
\hline & Glutamic acid & $2(14 \%)$ & Cryptococcus, Rhodosporidium \\
\hline & Threonine & $2(14 \%)$ & Nectria/Acremonium \\
\hline & $\begin{array}{c}\gamma \text {-Aminobutyric } \\
\text { Acid (GABA) }\end{array}$ & $1(7 \%)$ & Cryptococcus \\
\hline \multirow{6}{*}{$\begin{array}{c}\text { C6 sugars, } \\
\text { Deoxysugars, } \\
\text { Amino sugars, C5 } \\
\text { sugars }\end{array}$} & Glucose & $10(71 \%)$ & $\begin{array}{c}\text { Nectria/Acremonium, } \\
\text { Sporidiobolus, Cryptococcus, } \\
\text { Rhodosporidium }\end{array}$ \\
\hline & Mannose & $9(64 \%)$ & $\begin{array}{c}\text { Nectria/Acremonium, } \\
\text { Sporidiobolus, Cryptococcus, } \\
\text { Rhodosporidium } \\
\end{array}$ \\
\hline & $\begin{array}{c}N \text {-acetyl } \\
\text { glucosamine }\end{array}$ & $5(36 \%)$ & Nectria/Acremonium \\
\hline & Galactose & $4(29 \%)$ & Nectria/Acremonium \\
\hline & Xylose & $2(14 \%)$ & Cryptococcus, Rhodosporidium \\
\hline & Fucose & $0(0 \%)$ & None \\
\hline
\end{tabular}




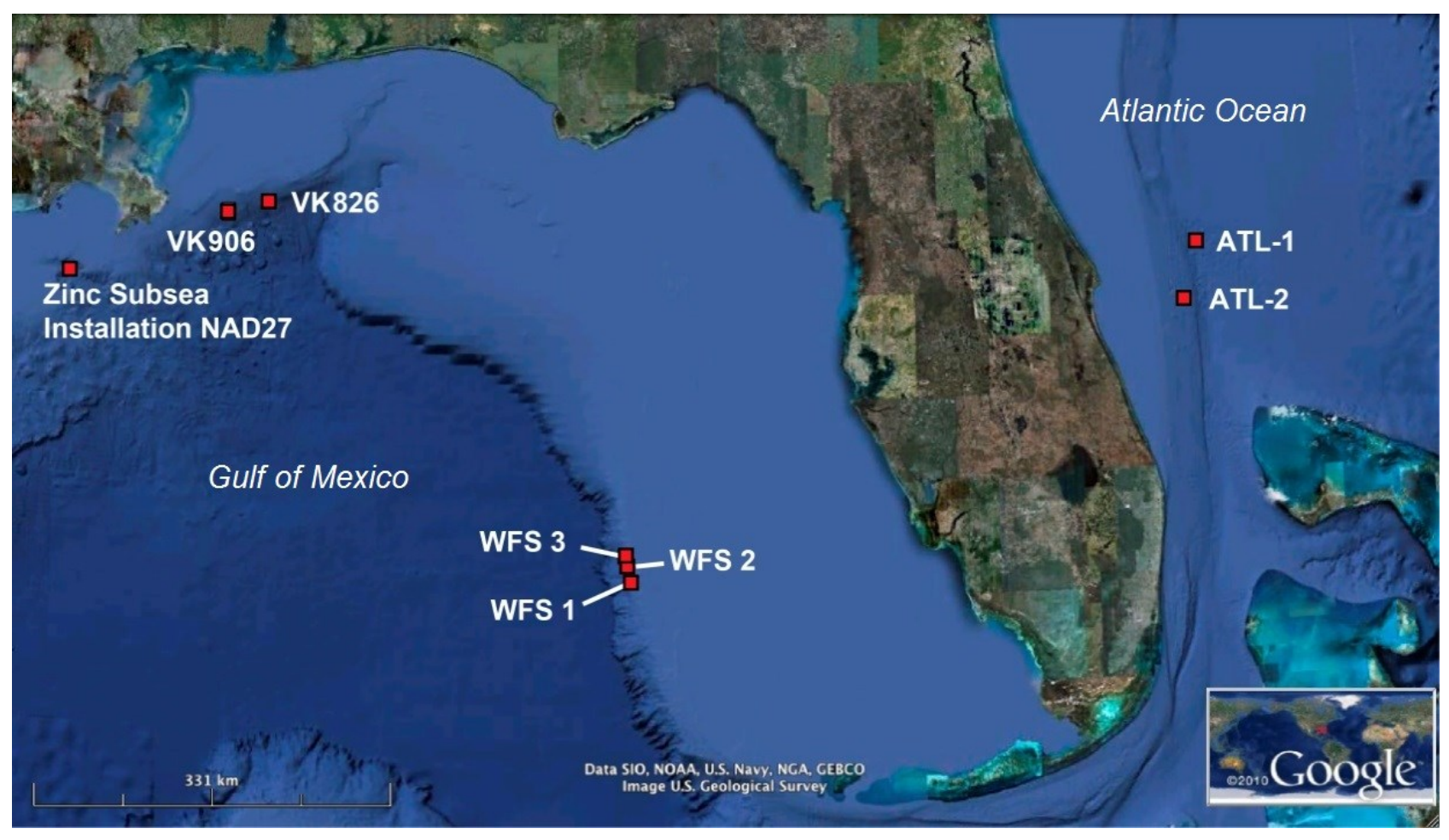

Figure 2.1 Map showing Lophelia pertusa collection sites for microbiology during the Lophelia II program. Base map data from Google, SIO, NOAA, U.S. Navy, NGA, GEBCO, U.S. Geological Survey 2010. 


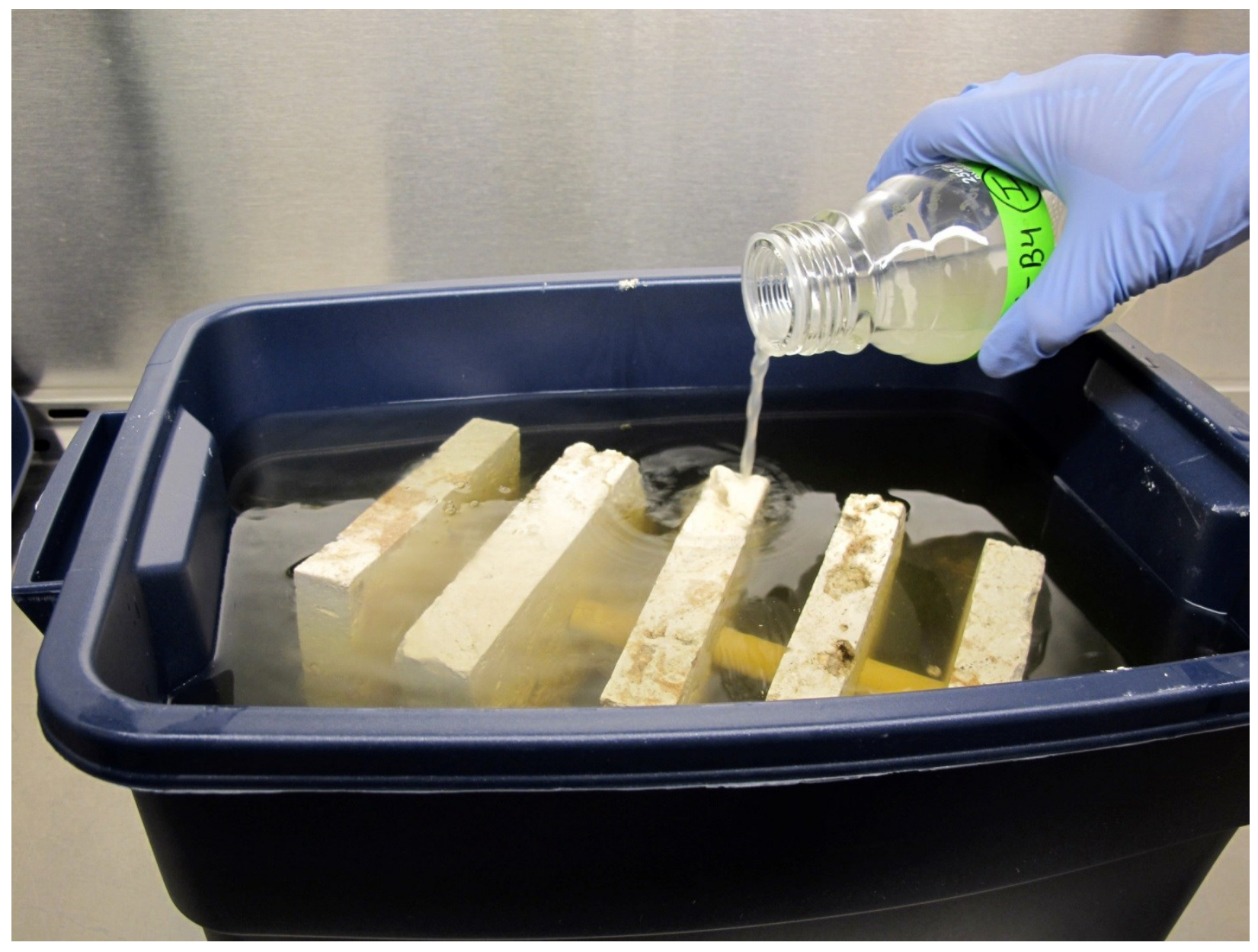

Figure 2.2 Settling plate stacks being inoculated with bacteria during the Lophelia II program. 


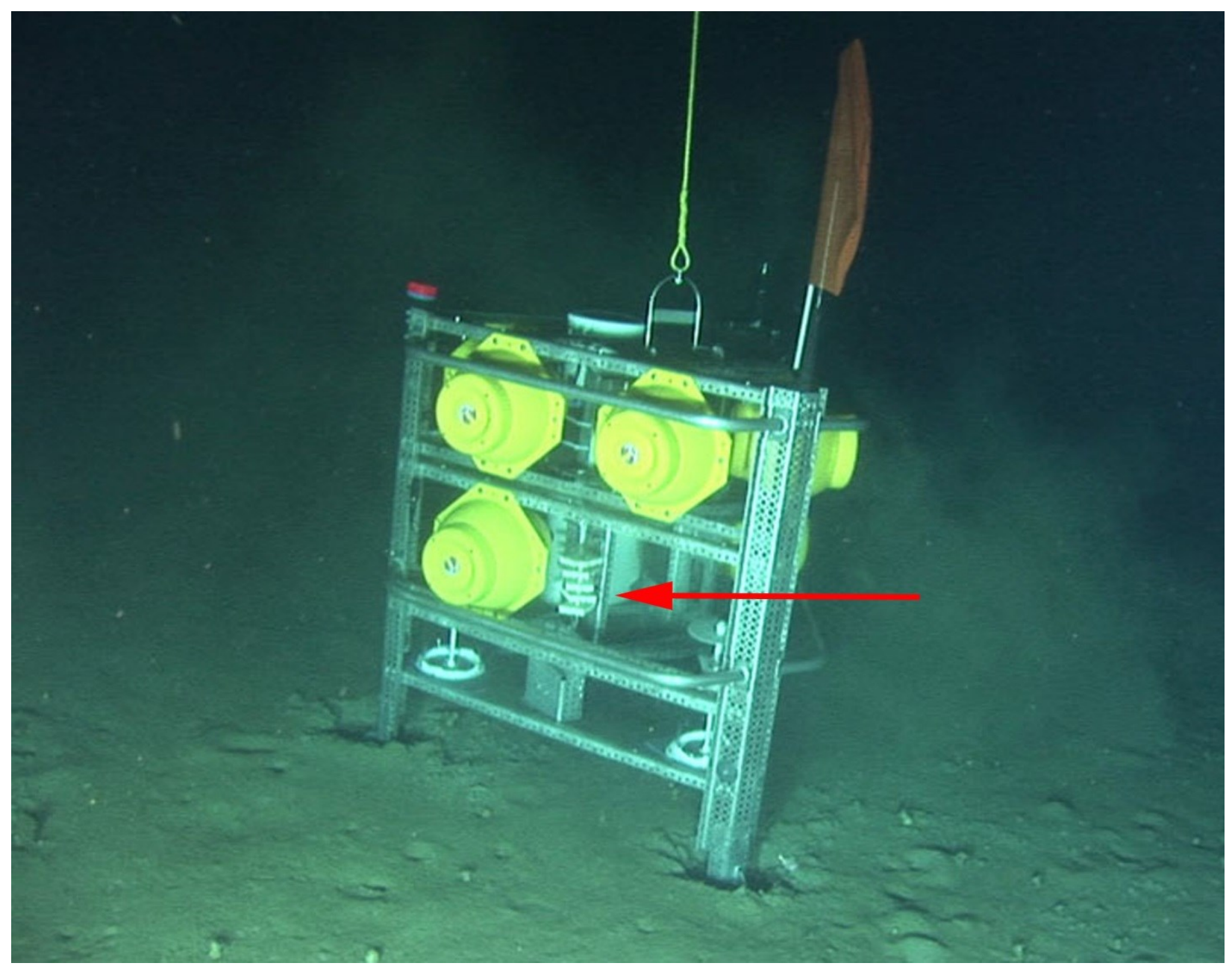

Figure 2.3 Settling plates mounted on benthic lander, viewed underwater after deployment, October 16, 2010 during the Lophelia II program. Photo courtesy of Steve Ross, University of North Carolina Wilmington, and Sandra Brooke, Florida State University. 


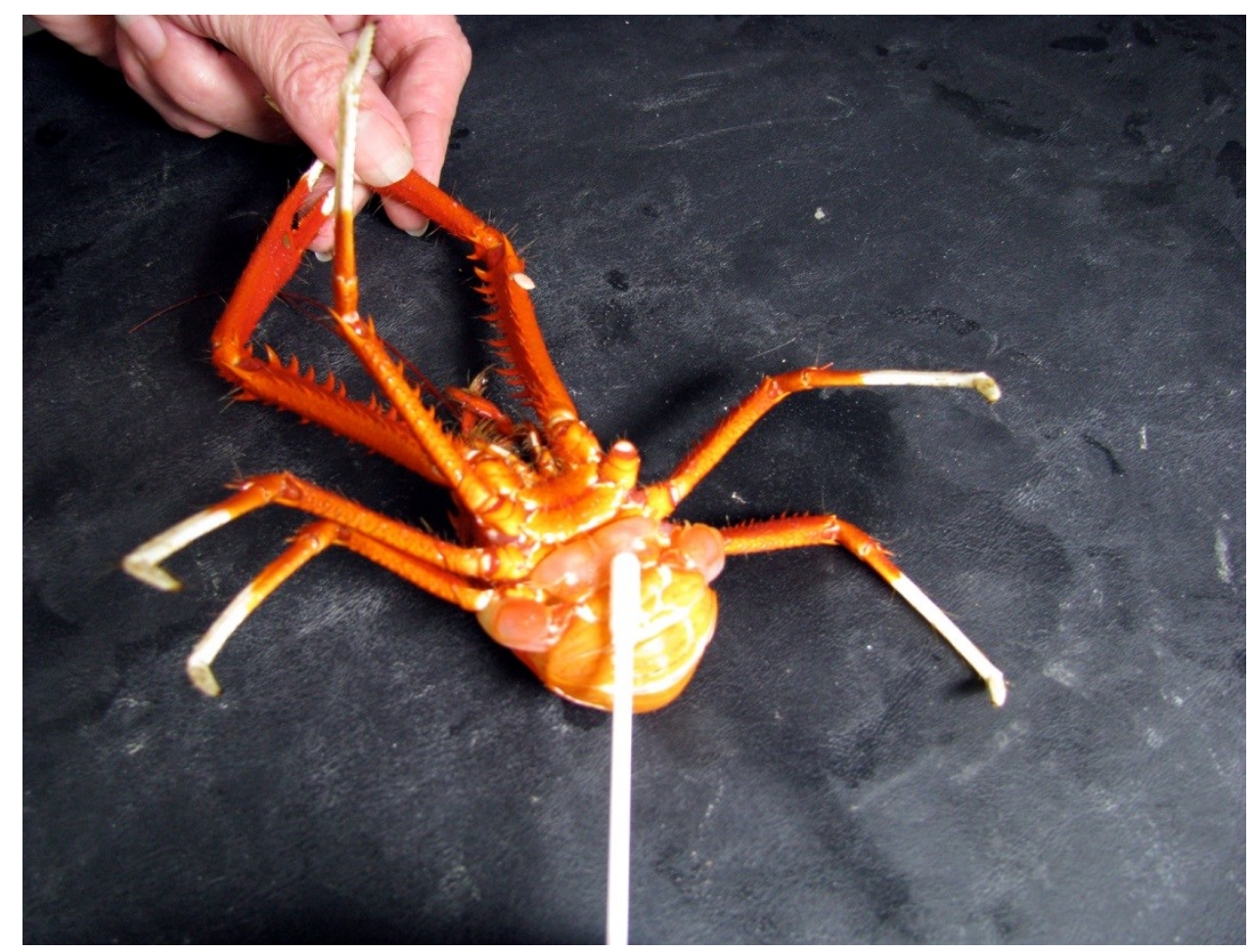

Figure 2.4 Swabbing the abdomen of a galatheid crab (Eumunida picta) for microbes during the Lophelia II program. 


\title{
3 GENETIC CONNECTIVITY AMONG NATURAL LOPHELIA PERTUSA REEFS AND SHIPWRECKS IN THE GULF OF MEXICO
}

\author{
By Cheryl L. Morrison ${ }^{1}$ and Marcus J. Springmann ${ }^{1}$ \\ ${ }^{1}$ U.S. Geological Survey
}

\subsection{Introduction}

In the Gulf of Mexico (GOM), the cold-water coral Lophelia pertusa forms extensive thickets at relict cold-seep sites where authigenic carbonates serve as a substrate for larval settlement (Brooke and Schroeder, 2007; Cordes and others, 2008; Becker and others, 2009). Reef or mound structures, more common along the southeastern coast of the United States (Ross and Nizinski, 2007), are more rare in the GOM, except in the Viosca Knoll (VK) area (the most extensive L. pertusa habitat known in the GOM) and the West Florida Slope (Brooke and Schroeder, 2007; Davies and others, 2010). Thickets and reefs in the GOM formed by L. pertusa support distinct and biologically diverse communities (Cordes and others, 2008) that are important to fisheries and perform critical ecological services, such as nutrient cycling, carbon sequestration, and nursery habitats (Levin and Sibuet, 2012). Along with naturally occurring L. pertusa reefs, artificial structures, such as platforms and shipwrecks on the sea floor, may be inhabited by corals, creating artificial deep-sea reefs. Given the close proximity between hydrocarbon seeps, oil reserves, and known locations of L. pertusa populations in the GOM, deep reefs may be especially vulnerable to oil industry activities. In order to avoid long-term negative effects on deep L. pertusa reefs from anthropogenic activities, additional information regarding coral larval dispersal, recruitment, and survival is necessary as a means to predict potential vulnerability or resilience to disturbance of reef habitats.

Identifying the scale of dispersal has been a challenge in marine ecology for decades (see Cowen and others, 2006; Hixon, 2011). Successful dispersal, settlement, growth, and reproduction of new migrants link and maintain the genetic connectivity between spatially separated populations (Hedgecock and others, 2007). Since connectivity influences genetic composition and diversity, demographic stability may be enhanced by the frequent exchange of juveniles or adults among neighboring populations. Alternatively, isolation of populations from new recruits may lead to extinction following severe impacts (Cowan and others, 2007). As such, to effectively manage marine species, knowledge of the spatial scale at which populations are connected is valuable (Palumbi, 2003; Fogarty and Botsford, 2007).

Benthic marine species such as corals move between populations by dispersal of larvae. Both biological and physical processes influence larval dispersal (Cowen and Sponaugle, 2009), each of which may vary across the range of a species (Ayre and Hughes, 2004; Sotka and others, 2004; Selkoe and others, 2008). Biological attributes such as timing of reproduction, larval behavior (such as swimming instead of passive movement in currents), and feeding behavior may influence dispersal distances. For some marine organisms, pelagic larval duration (PLD) is positively correlated with dispersal distance (Bohonak, 1999; Shanks and others, 2003; Selkoe and Toonen, 2011). Following expectations for short PLDs, several coral species with brooded larvae exhibit higher levels of genetic structuring than spawning species with longer PLDs (Hellberg, 1996; Ayre and Hughes, 2000; 
Whitaker, 2004; Underwood and others, 2009). Many broadcast spawning corals, however, exhibit evidence of limited dispersal between reefs or regions (Ayre and Hughes, 2004; Underwood and others, 2007; Miller and Ayre, 2008; Underwood and others, 2009). For most cold-water coral (CWC) species, biological aspects of larvae are poorly understood (Waller, 2005; Waller and Tyler, 2005). Lophelia pertusa, like most CWC species surveyed, produces lecithotrophic (non-feeding) larvae (Waller and Tyler, 2005), and the length of PLD is unknown.

Due to the small sizes of coral larvae and the large volume of ocean into which they disperse, larval dispersal distances remain difficult to directly measure. However, an indirect measure of connectivity can be ascertained from molecular markers, such as microsatellites. Genetic estimates of connectivity result from examining the variance in allele frequencies between populations that will accumulate when connectivity is low or non-existent. For example, through the use of molecular techniques, it has been demonstrated that the scale over which populations of shallow-water scleractinian corals are differentiated can range from as little as 25 kilometers (km; among reefs) to $7,500 \mathrm{~km}$ (across ocean basins) (Baums, 2008). Understanding of the physical and biological processes that underlie the spatial connections among populations can be inferred from the correlated patterns of genetic differentiation.

An initial study identified genetic structuring at a broad geographic scale (the North Atlantic Ocean), with GOM populations distinct from western and eastern North Atlantic and New England Seamount populations (Morrison and others, 2011). In the present study, improved sampling of GOM $L$. pertusa populations has allowed for a more thorough examination of connectivity patterns. Using 8 microsatellite DNA markers and more than 400 samples, we assessed the spatial scale and pattern of genetic connectivity across 8 natural L. pertusa reef localities that span $900 \mathrm{~km}$ in the GOM, as well as colonies inhabiting 3 anthropogenic structures, the shipwrecks Gulfpenn, Gulf Oil, and Ewing.

\subsection{Methods}

\subsubsection{Sampling Sites}

Small samples (partial branches, approximately 4-6 centimeters [cm]) from 305 L. pertusa colonies were collected during five cruises between August 2009 and November 2010 (table 3.1; fig. 3.1). Samples originated from 8 deep-reef localities: Garden Banks (GB535), Green Canyon (GC354 and GC234), Mississippi Canyon (MC751), Viosca Knoll (VK862, VK906, VK826), and West Florida Slope (WFS), 317-527 meters (m) deep. Samples were also collected from three shipwrecks: Gulfpenn, (GP, MC497), Gulf Oil (GO, MC796), and Ewing (EB, EB1008), 533-620 m deep in the GOM (table 3.1; fig. 3.1).

Sampling occurred during August and September 2009 and October and November 2010 aboard the R/V Ronald H. Brown (National Oceanic and Atmospheric Administration [NOAA]) using the remotely operated vehicle (ROV) Jason II (Woods Hole Oceanographic Institute). Additionally, samples were obtained while aboard the research vessel (R/V) Seward Johnson by using the Johnson Sea Link II (Harbor Branch Oceanographic Institute) submersible in September 2009, as well as aboard the R/V Cape Hatteras by using the Kraken II ROV (University of Connecticut) in September-October 2010. Once on board the vessel, small tissue samples were preserved in 95 percent ethanol (ETOH) and FTA Technology Classic card (Whatman).

\subsubsection{Molecular Methods}

Total DNA was isolated from preserved coral tissue and (or) FTA cards by using the tissue protocol from the PureGene DNA extraction kit (Gentra Systems Inc., Minneapolis, Minnesota). DNA 
concentrations were determined by fluorescence assay (Labarca and Paigen, 1980), and integrity of the DNA was visualized on 1-percent agarose gels (Sambrook and others, 1989).

PCR conditions for amplification of eight microsatellite loci designed for L. pertusa (LpeA5, LpeC44, LpeC52, LpeC61, LpeC142, LpeC151, LpeD3, LpeD5) followed (Morrison and others, 2008) and were carried out in 20-microliter $(\mu \mathrm{L})$ reactions. Fluorescent DNA fragments were analyzed by using an ABI 3130xl Genetic Analyzer (Applied Biosystems) with GeneScan-500 ROX or LIZ internal size standards. GeneMapper v.4.1 fragment analysis software (Applied Biosystems) was used to score, bin, and output allelic data. Additionally, multi-locus genotype (MLG) data from 195 L. pertusa individuals collected at 7 localities in the northwestern Atlantic Ocean, off the southeastern United States (SEUS) (Morrison and others, 2011), were included for inter-regional comparisons of connectivity patterns.

\subsubsection{Genetic Diversity}

Individuals with identical MLGs were identified by using the program GenAlEx v. $6.5 \mathrm{~b} 4$ (Peakall and Smouse, 2006, 2012). Unique MLGs were used in subsequent analyses. The program MICROCHECKER v. 2.2.3 (Van Oosterhout and others, 2004) was used to check for scoring errors and large allele dropout. The probability of identity (PI, the probability of two unrelated individuals sharing the same genotype) was calculated for increasing locus combinations by using GenAlEx.

Loci were tested for fit to statistical assumptions of population genetic analyses. Observed and expected heterozygosities under Hardy-Weinberg equilibrium (HWE), and fixation indices per locus and locality were calculated by using GenAlEx. Exact tests for HWE and linkage disequilibrium were performed by using Genepop on the Web (Raymond and Rousset, 1995). To assess levels of inbreeding, Weir and Cockerham's (Weir and Cockerham, 1984) estimators $f$ and $F$, analogous to Wright's (1951) $F_{I S}$ and $F_{I T}$, respectively, were calculated for each locality and tested for statistical significance by 1,000 permutations with FSTAT ver. 2.9.3.2 (Goudet, 1995). Because observed allelic diversity can be proportional to sample size (Leberg, 2002), allelic richness, or the number of alleles per locality, and private allelic richness were calculated by rarefaction in FSTAT. Sequential Bonferroni adjustments for multiple tests (Rice, 1989) were used on these and other multiple tests.

\subsubsection{Population Genetic Structure}

Several techniques were used to describe genetic relationships between populations as estimated from microsatellite data. First, a Bayesian model-based clustering approach (Pritchard and others, 2000) was implemented in STRUCTURE v. 2.3.2 (Hubisz and others, 2009). This method infers the number of genetic clusters $(K)$ from MLG data by minimizing Hardy-Weinburg and linkage disequilibrium among loci within groups, assigning individuals (probabilistically) to each cluster. Because models utilizing collection location information as priors are useful for small datasets and weak structuring (Hubisz and others, 2009), locality designations were included as priors. Settings for all runs also included an admixture model (where individuals may have mixed ancestry), correlated allele frequencies (Falush and others, 2003), and 20,000 Markov chain Monte Carlo (MCMC) iterations after a burn-in of 10,000 iterations. Ten independent chains were run to test each value of $K$ from $K=1-15$. The optimum number of clusters was determined by evaluating the values of $K$ as the highest mean likelihood of the probability of the number of clusters given the data observed, $\ln \operatorname{Pr}(\mathrm{X} \mid K)$ (Pritchard and others, 2000), and $\Delta K$ (Evanno and others, 2005). This information was compiled and graphed by using STRUCTURE Harvester v. 0.56.1 (Earl and vonHoldt, 2012). Each cluster identified in the initial STRUCTURE run was analyzed separately by using the same settings to identify potential withincluster structure (Evanno and others, 2005). 
We further explored genetic differentiation among localities by examining pairwise distance measures and principal components analysis (PCA), as well as analysis of molecular variance (AMOVA; Excoffier and others, 1992). Two pairwise genetic distances were calculated among localities: $F_{\mathrm{ST}}$ (Weir and Cockerham, 1984), or the proportion of variance among populations relative to the total variance; and Jost's actual measure of differentiation ( $D_{\text {est }}$; Jost, 2008). These pairwise distances were computed and tested for significance (difference from zero) by 9,999 permutations by using GenAlEx. PCA, a technique used to find the major patterns within a multivariate dataset (for example, multiple microsatellite loci and localities), was performed on a matrix of covariance values from allele frequencies in GenAlEx. AMOVA was used to partition genetic variation among the clusters suggested by STRUCTURE, and among localities within clusters, by using GenAlEx.

Lastly, isolation by distance (IBD; Wright, 1943) was examined by testing the correlation between genetic and marine geographical distances between locality pairs at two geographic scales. The regression of Nei's standard genetic distance (Nei, 1972, 1978) versus geographic distance was tested by using Mantel tests for matrix correspondence (Mantel, 1967), for the entire dataset, as well as for the GOM populations that had sufficient sampling. Nei's genetic distance performs well in IBD tests due to low variance, high linearity, and a slope that approaches zero at small geographic distances (Peatkau and others, 1997). Straight-line marine geographic distances between populations were estimated by using ARCGIS v. 9.2 (Esri Inc.). Mantel tests were implemented in GenAlEx by using 9,999 permutations to test for significance of the relationship between distance matrices.

\subsection{Results}

\subsubsection{Genetic Diversity}

Genotypes at eight microsatellite loci were determined for 305 L. pertusa colonies representing 10 GOM localities (table 3.1). No evidence of scoring errors or large allele dropout was found when using the program MICROCHECKER. Additionally, there were no multi-locus genotypes differing by only one or two alleles, which is often indicative of scoring errors.

Of the new L. pertusa genotyped, 15 percent had non-unique multi-locus genotypes (proportion of clones, table 3.1), and all identical MLGs were from the same locality. Given the low probability that two unrelated individuals share identical MLGs $\left(\mathrm{PI}_{\text {sibs }}=9.9 \times 10^{-4}\right)$, these individuals were considered clonemates, and only one of each MLG was included in further analyses, for a total of 255 unique genets. These new data were combined with previously published L. pertusa data from four GOM localities (Morrison and others, 2011) for a total of 351 GOM genets.

The 8 microsatellite loci were highly polymorphic, with 247 alleles detected in the GOM populations. The number of alleles ranged from 12 (LpeA5) to 54 (LpeD3) and averaged 31 alleles per locus (table 3.2). The mean number of alleles per locus (Na, table 3.2) ranged from 2.88 (EB) to 25 (VK826). Private alleles were detected at all loci and at all GOM sites except for the EB wreck. VK826 had the highest number of private alleles (33; P, table 3.2). When rarified to smallest sample size (16 alleles; Ar, table 3.2), estimates of numbers of private alleles were similar among sites, with GP having the highest (8.6) and GO having the lowest (7.7). Observed heterozygosities were high in the majority of locus-by-population comparisons, ranging from $7.7(\mathrm{GO})$ to $8.6\left(\mathrm{GP} ; H_{O}\right.$, table 3.2). Randomization tests showed that the 351 unique GOM MLGs were consistent with HWE for the majority of locus-bypopulation comparisons $\left(F_{I S}\right.$, table 3.2). However, significant heterozygote deficits (positive $f$ values, table 3.2) were detected in most populations, with the exceptions of the EB and GO wrecks. 


\subsubsection{Population Genetic Structure}

Plots of $\Delta K$ (Evanno and others, 2005) from STRUCTURE analysis indicate that two genetic groupings is the most likely number of population clusters in the full dataset ( $K=2$; fig. 3.2). Ten independent runs at $K=2$ produced the same clustering solution as illustrated in figure 3.3, with clusters corresponding to oceanic region of origin (GOM, northwestern Atlantic; fig. 3.3). Additional STRUCTURE runs performed on each of the two clusters separately did not detect hierarchical structuring within these clusters. For most localities, the majority of individuals assigned to their cluster of origin. However, admixture was apparent within the Miami Terrace (MTR) locality, as 37 percent of assignment was to the GOM cluster and 63 percent was to the Atlantic.

Fixation index $\left(F_{\mathrm{ST}}\right)$ estimates between GOM populations (table 3.3, below diagonal) ranged between 0.003 and 0.030 , and none of the $F_{\text {ST }}$ estimates were significantly different from zero. Estimates of $F_{\text {ST }}$ were generally higher between GOM and northwestern Atlantic Ocean (NWAO) localities (range $0.012-0.046$ ), and about 40 percent of these pairwise estimates were significant. Similarly, $D_{\text {est }}$ estimates were generally low and non-significant between GOM localities (range -0.049 - 0.042; table 3.3, above diagonal), and inter-regional comparisons were higher (range $-0.010-0.239$, approximately 44 percent of comparisons significant). None of the inter-regional pairwise estimates of differentiation that included MTR were significant.

Quantitative estimates of hierarchical gene diversity (AMOVA) indicated significant genetic population structure at all levels tested, with greatest variance observed within localities (table 3.4). Variation was maximally distributed when localities were defined as regional clusters, as suggested by the STRUCTURE analysis (fig. 3.3), with approximately 2 percent of the variance among clusters $(P$ $<0.0001$; table 3.4). Although less than 1 percent of the variance was observed among localities in the GOM, this weak structuring was still significant $(P<0.0001$; table 3.4$)$.

The PCA analysis separated the GOM localities from the NWAO (fig. 3.4A). The MTR occupied an intermediate position between the regional groupings. When the genetic distances among GOM localities were analyzed (fig. 3.4B), PC1 captured the inter-population genetic distance between GB535 and other GOM localities, while PC2 reflects differentiation between the shipwrecks Gulfpenn and Gulf Oil from natural L. pertusa populations.

The result of Mantel's test (fig. 3.5A) revealed a significant pattern of IBD when GOM and WNA populations were considered, with geographic distance explaining 44 percent of the variation in genetic distance. When the GOM localities were considered separately, however, no relationship was detected between geographic and genetic distances (fig. 3.5B).

\subsection{Discussion}

The Lophelia II study has allowed for the most comprehensive analysis of L. pertusa connectivity in the GOM, and for any ocean basin, to date. Several new L. pertusa reefs were discovered, explored, and sampled for our genetic analyses during this study, including GB535, MC751, VK906, and WFS. Inclusion of the WFS population (the most eastern/southern) and the GB535 population (the most western) greatly expanded geographic coverage of GOM L. pertusa, allowing for more robust estimates of gene flow and increased confidence in the biological meaning of results.

Population genetic analyses indicate weak structuring and moderate gene flow among sampled L. pertusa populations in the GOM. These results confirm an initial analysis of L. pertusa gene flow in the GOM (Morrison and others, 2011). Several analyses presented here suggest that long-distance dispersal occurs with enough frequency to produce regional cohesion. For example, the STRUCTURE analysis indicated that all GOM populations formed a single cluster, including those populations that are geographically distant (for example, GB535 to WFS, approximately $900 \mathrm{~km}$ ). The WFS population was 
more genetically similar to GOM populations than to the western Atlantic populations that were closest in proximity (fig. 3.3).

Circulation patterns in the GOM are complicated, but are generally dominated by the Loop Current and its associated rings and eddies and should be adequate to transport organic material (such as larvae) throughout the GOM (Jochens and DiMarco, 2008). The Loop Current enters the GOM through the Yucatan Channel, extends north towards the Mississippi Delta, and then exits through the Florida Straits, eventually becoming the Gulf Stream (fig 1.1). The VK region is usually bathed in Loop Current waters (Jochens and DiMarco, 2008; Davies and others, 2010), and currents generally follow isobaths (Mienis and others, 2012). However, both short- (Davies and others, 2010) and long-term (Mienis and others, 2012) current measurements suggest local recurrent events in which current flow direction changes (East-West shifts), along with particulate load and temperature changes, emphasizing the variable nature of environmental conditions. Such shifts in prevailing currents may increase chances for dispersal in different directions, resulting in enhanced mixing among GOM L. pertusa larvae.

Despite the potential for the Loop Current/Florida Current/Gulf Stream to connect L. pertusa populations between the GOM and the northwestern Atlantic Ocean, restricted gene flow between these neighboring ocean regions, originally noted by Morrison and others (2011), was confirmed in the present analysis. Analyses presented here help to define the boundaries of the discontinuity in gene flow between the oceanic regions. The WFS L. pertusa population is geographically proximate to the SEUS, yet clustered with the other GOM populations in the STRUCTURE analysis, with little admixture detected (fig. 3.4). Therefore, regular gene flow appears restricted between the WFS population and MTR, which has an admixed genetic structure (fig. 3.4).

A recent study was the first to estimate potential dispersal pathways of several deep-sea invertebrates with known PLDs by using ocean circulation and a Lagrangian larval transport model (Young and others, 2012). Several of the species were cold-seep inhabitants of the Louisiana slope in the northern GOM. Although absolute dispersal distances were highly variable, data indicated that the majority of larvae were retained in the same geographic area where spawned, and those that dispersed followed a unidirectional trajectory. In the GOM, their data suggested that populations in the western GOM were more likely to be sources of larvae, whereas populations in the northern and eastern GOM were more likely to be sinks for larval recruitment. Another interesting finding was that maximal dispersal distances in the deep-sea species examined were strongly related to PLD for these deep-sea organisms. A pattern of dispersal similar to that of the sipunculid Phascolosoma tumerae (fig. 1, Young and others, 2012), which has a 390-day PLD with larvae that remain below the thermocline, would explain the genetic structuring between the GOM and SEUS well. For P. tumerae, models suggested that the majority of larvae would be retained within the ocean basin of origin, with a small potential for some dispersal from the GOM to the SEUS. The addition of genotypic data from L. pertusa samples from the Caribbean would help complete the picture of gene flow in the western Atlantic Ocean.

Although gene flow in the GOM appears adequate to maintain connectivity among sampled populations, results from several analyses suggest complexity in patterns of gene flow. Although less than 1 percent of the variance in allele frequencies was attributed among GOM populations (AMOVA, table 3.4), the analysis was significant, indicating weak structuring that may be biologically meaningful. For example, the westernmost L. pertusa population, GB535, was also the most genetically differentiated based upon PCA (fig. 3.4) and pairwise estimates of population divergence (table 3.3). Additionally, the shipwrecks were unique compared to other natural localities based upon the PCA analysis (fig. 3.4). While the genetic signatures of these two shipwrecks indicate high genetic diversity, likely reflecting recruitment from multiple natural populations, the Gulfpenn L. pertusa samples (MC497) were more admixed than the Gulf Oil population (MC794) in the STRUCTURE analysis (fig. 
3.4). An interesting aspect of the shipwreck L. pertusa populations is the known age of the structures, which sets a maximum age boundary on the corals. Since natural reefs are likely hundreds to thousands of years old (Neumann and others, 1977; Wilson, 1979; Mikkelsen and others, 1982; Mortensen and Rapp, 1998) and may vary in the degree of clonality present, population structuring may differ substantially between natural and artificial reefs. Essentially, these wreck populations reflect a natural experiment of ecologically recent dispersal, whereas natural populations may harbor genotypes that are thousands of years old, therefore creating a more complex genetic signature. A slight difference in depth also exists between the natural reefs (average depth $474 \mathrm{~m}$ ) and shipwrecks (average depth $564 \mathrm{~m}$ ). Given that many environmental variables change rapidly with depth (for example, pressure, temperature, and levels of dissolved oxygen), selective regimes may vary over small vertical distances, promoting adaptive divergence (Levin and Sibuet, 2012; Jennings and others, 2013). Until sample sizes can be increased for the shipwreck populations, conclusions regarding their genetic structure remain tentative.

In most of GOM L. pertusa populations surveyed, a deficit of heterozygotes was detected. Similar results were obtained previously for L. pertusa populations in the northeastern (Le Goff-Vitry and others, 2004) and northwestern (Morrison and others, 2011) Atlantic Ocean, therefore, the GOM results confirm the generality of such local genetic patterns. Clonal organisms exhibit population dynamics that depart from the null hypothesis of population genetic theory (Orive, 1993; Yonezawa and others, 2004; Rozenfeld and others, 2007). Basic tenets such as random mating, equilibrium, and nonoverlapping generations, that are assumed when interpreting statistics regarding population composition and structure, are often violated (Hey and Machado, 2003). Even the basic unit on which evolutionary forces are acting (the genetic individual) are complicated in clonal species (Orive, 1995; Fischer and Van Kleunen, 2001). This in turn limits the interpretation of some of the general summary statistics and analyses that rely on genetic distances between individuals. A clearer picture of structuring within populations is obtained when demographic assessments are also made through analyses such as kinship detection, spatial autocorrelation, and demographic assessments that do not rely on a priori assignment to groups, such as network analyses (Rozenfeld and others, 2007). Such analyses are planned and will help quantify the structure of each population, as well as the roles that each population plays in the GOM system. Utilizing L. pertusa samples obtained with accurate bottom locations that allow estimates of geographic distance between samples, such as those obtained using the Jason II ROV, will improve the quality of demographic analyses.

\subsection{Future Directions}

Population genetic analyses indicate weak structuring and moderate gene flow among sampled L. pertusa populations in the GOM. It follows that the natural L. pertusa communities sampled are important larval sources and may replenish affected areas with new recruits. Human-made structures such as shipwrecks likely enhance connectivity among natural $L$. pertusa populations. Continued exploration and sampling throughout the GOM should enhance our knowledge of connectivity among natural and human-made reef sites. In order to methodically study these sensitive communities, continued access to precision deep-sea sampling equipment mounted on robotic vehicles such as ROVs and autonomous underwater vehicles (AUVs) will be necessary. Expanding the scope of connectivity studies to organisms associated with structure-forming corals could improve understanding of the functioning and potential sensitivities to disturbance of these ecosystems. The development of molecular tools that allow for assessment of functional portions of genomes may reveal adaptive differences between populations and may allow for development of tools to assess coral health. 
Molecular tools, such as microsatellite markers, perform best when integrated with other data (Selkoe and others, 2008; White and others, 2010; Young and others, 2012). Additional research on the reproductive biology of $L$. pertusa, including better estimation of PLD and estimates of general larval behavior (for example, swimming instead of benthic) and temperature tolerances of larvae, will improve estimates of dispersal and connectivity. These biological data, coupled with long-term data describing hydrodynamics at reef- and basin-wide scales, should allow for accurate larval dispersal models for $L$. pertusa in the GOM.

\subsection{Acknowledgments}

This work was financially supported by the U.S. Geological Survey (USGS) Outer Continental Shelf Environments Program and was sponsored by the Bureau of Ocean Energy Management (BOEM). G. Brewer, C. Charles, and A. Demopoulos of the USGS provided guidance throughout the project, along with members of the USGS Diversity, Systematics, and Connectivity of Vulnerable Reef Ecosystems (DISCOVRE) team. The authors wish to thank the captains and crews of the ships, ROVs, and submersibles used during the Lophelia II research cruises. TDI-Brooks International, Inc., C. Fisher (Pennsylvania State University), and E. Cordes (Temple University) facilitated the participation of one author (C. Morrison) on their R/V Ron Brown Lophelia II research cruises. Thanks to S.W. Ross (University of North Carolina, Wilmington) for leading cruises on the R/V Seward Johnson, the R/V Cape Hatteras, and R/V Ron Brown. Thanks to A. Quattrini (Harvey Mudd College), J. Lunden (Lock Haven University), W. Cho (Point Loma Nazarene University), T. Shank (Woods Hole Oceanographic Institution), M. Nizinski (National Marine Fisheries Service National Systematics Laboratory), J.

McClain-Counts (USGS Wetlands and Aquatic Research Center), and J. Galkiewicz (NOAA) who helped with sample preservation at sea. T. King, R. Johnson, B. Lubinski, and D.K. Coykendall of the USGS Leetown Science Center provided help in the laboratory. J. McClain Counts created the sampling map. D.K. Coykendall provided helpful comments on an earlier draft of this report.

\subsection{References}

Ayre, D.J., and Hughes, T.P., 2000, Genotypic diversity and gene flow in brooding and spawning corals along the Great Barrier Reef, Australia: Evolution, v. 54, no. 5, p. 1590-1605.

Ayre, D.J., and Hughes, T.P., 2004, Climate change, genotypic diversity and gene flow in reef-building corals: Ecology Letters, v. 7, no. 4, p. 273.

Baums, I.B., 2008, A restoration genetics guide for coral reef conservation: Molecular Ecology, v. 17, p. 2796-2811.

Becker, E.L., Cordes, E.E., Macko, S.A., and Fisher, C.R., 2009, Importance of seep primary production to Lophelia pertusa and associated fauna in the Gulf of Mexico: Deep-Sea Research I, v. 56, p. 786800.

Bohonak, A.J., 1999, Dispersal, gene flow, and population structure: The Quarterly Review of Biology, v. 74, no. 1, p. 21-45.

Brooke, S.B., and Schroeder, W.W., 2007, State of the U.S. deep coral ecosystems in the northern Gulf of Mexico region: Florida Straits to Texas, in Lumsden, S.E., Hourigan, T.F., Bruckner, A.W., and Dorr, G., eds., The state of deep coral ecosystems of the United States: Silver Spring, Md., National Oceanic and Atmospheric Administration Technical Memorandum, p. 271-306.

Cordes, E.E., McGinley, M.P., Podowski, E.L., Becker, E.L., Lessard-Pilon, S., Viada, S.T., and Fisher, C.R., 2008, Coral communities of the deep Gulf of Mexico: Deep-Sea Research Part I: Oceanographic Research Papers, v. 55, no. 6, p. 777-787. 
Cowen, R.K., Paris, C.B., and Srinivasan, A., 2006, Scaling of connectivity in marine populations: Science, v. 311, p. 522-527.

Cowen, R.K., Gawarkiewicz, G., Pineda, J., Thorrold, S.R., and Werner, F.E., 2007, Population connectivity in marine systems: An overview: Oceanography, v. 20, no. 3, p. 14-21.

Cowen, R.K., and Sponaugle, S., 2009, Larval dispersal and marine population connectivity: Annual Review of Marine Science, v. 1, p. 443-466.

Davies, A.J., Duineveld, G.C.A., van Weering, T.C.E., Mienis, F., Quattrini, A.M., Seim, H.E., Bane, J.M., and Ross, S.W., 2010, Short-term environmental variability in cold-water coral habitat at Viosca Knoll, Gulf of Mexico: Deep-Sea Research I, v. 57, p. 199-212.

Earl, D.A., and vonHoldt, B.M., 2012, STRUCTURE HARVESTER: A website and program for visualizing STRUCTURE output and implementing the Evanno method: Conservation Genetics Resources, v. 4, no. 2, p. 359-361. doi: 10.1007/s12686-011-9548-7.

Evanno, G., Regnaut, S., and Goudet, J., 2005, Detecting the number of clusters of individuals using the software STRUCTURE: A simulation study: Molecular Ecology, v. 14, p. 2611-2620.

Excoffier, L., Smouse, P.E., and Quattro, J.M., 1992, Analysis of molecular variance inferred from metric distances among DNA haplotypes: Application to human mitochondrial DNA restriction data: Genetics, v. 131, p. 479-491.

Falush, D., Stephens, M., and Pritchard, J.K., 2003, Inference of population structure using multilocus genotype data: Linked loci and correlated allele frequencies: Genetics, v. 164, p. 1567-1587.

Fischer, M., and Van Kleunen, M., 2001, On the evolution of clonal plant life histories: Evolutionary Ecology, v. 15, no. 4-6, p. 565-582.

Fogarty, M. J., and Botsford, L. W., 2007, Population connectivity and spatial management of marine fisheries: Oceanography, v. 20, no. 3, p. 112-123.

Goudet, J., 1995, FSTAT (Version 1.2): A computer program to calculate F-statistics: Journal of Heredity, v. 86, p. 485-486.

Hedgecock, D., Barber, P.H., and Edmands, S., 2007, Genetic approaches to measuring connectivity: Oceanography, v. 20, no. 3, p. 70-79.

Hellberg, M.E., 1996, Dependence of gene flow on geographic distance in two solitary corals with different larval dispersal: Evolution, v. 50, no. 3, p. 1167-1175.

Hey, J., and Machado, C.A., 2003, The study of structured populations-New hope for a difficult and divided science: Nature Review Genetics, v. 4, no. 7, p. 535-543.

Hixon, M.A., 2011, 60 years of coral reef fish ecology: Past, present, future: Bulletin of Marine Science, v. 87 , no. 4 , p. $727-765$.

Hubisz, M.J., Falush, D., Stephens, M., and Pritchard, J.K., 2009, Inferring weak population structure with the assistance of sample group information: Molecular Ecology Resources, v. 9, no. 5, p. 13221332.

Jennings, R.M., Etter, R.J., and Ficarra, L., 2013, Population differentiation and species formation in the deep sea: the potential role of environmental gradients and depth: PLoS ONE v. 8, no. 10, p. e77594.

Jochens, A.E., and DiMarco, S.F., 2008, Physical oceanographic conditions in the deepwater Gulf of Mexico in summer 2000-2002: Deep-Sea Research Part II: Topical Studies in Oceanography, v. 55, no. 24-26, p. 2541-2554.

Jost, L.O.U., 2008, GST and its relatives do not measure differentiation: Molecular Ecology, v. 17, no. 18 , p. 4015-4026.

Labarca, C., and Paigen, K., 1980, A simple, rapid and sensitive DNA assay procedure: Analytical Biochemistry, v. 102, p. 344-352. 
Leberg, P.L., 2002, Estimating allelic richness: Effects of sample size and bottlenecks: Molecular Ecology, v. 11, p. 2445-2449.

Le Goff-Vitry, M.C., Pybus, O.G., and Rogers, A.D., 2004, Genetic structure of the deep-sea coral Lophelia pertusa in the northeast Atlantic revealed by microsatellites and internal transcribed spacer sequences: Molecular Ecology, v. 13, no. 3, p. 537-549.

Levin, L.A., and Sibuet, M., 2012, Understanding continental margin biodiversity: A new imperative: Annual Review of Marine Science, v. 4, p. 79-112.

Mantel, N., 1967, The detection of disease clustering and a generalized regression approach: Cancer Research, v. 27, p. 209-220.

Mienis, F., Duineveld, G.C.A., Davies, A.J., Ross, S.W., Seim, H., Bane, J., and van Weering, T.C.E., 2012, The influence of near-bed hydroynamic conditions on cold-water corals in the Viosca Knoll area, Gulf of Mexico: Deep-Sea Research I, v. 60, p. 32-45.

Mikkelsen, N., Erlenkeuser, H., Killingley, J.S., and Berger, W.H., 1982, Norwegian corals: Radiocarbon and stable isotopes in Lophelia pertusa: Boreas, v. 11, p. 163-171.

Miller, K., and Ayre, D., 2008, Population structure is not a simple function of reproductive mode and larval type: insights from tropical corals: Journal of Animal Ecology, v. 77, no. 4, p. 713-724.

Morrison, C.L., Eackles, M.S., Johnson, R.L., and King, T.L., 2008, Characterization of 13 microsatellite loci for the deep-sea coral, Lophelia pertusa (Linnaeus 1758), from the western North Atlantic Ocean and Gulf of Mexico: Molecular Ecology Resources, v. 8, p. 1037-1039.

Morrison, C.L., Ross, S.W., Nizinski, M.S., Brooke, S.B., Jarnegren, J., Waller, R.G., Johnson, R.L., and King, T.L., 2011, Genetic discontinuity among regional populations of Lophelia pertusa in the North Atlantic Ocean: Conservation Genetics, v. 12, p. 713-729.

Mortensen, P.B., and Rapp, H.T., 1998, Oxygen and carbon isotope ratios related to growth line patterns in skeletons of Lophelia pertusa (L) (Anthozoa, Scleractinia): Implications for determination of linear extension rates: Sarsia, v. 83, p. 433-446.

Nei, M., 1972, Genetic distance between populations: The American Naturalist, v. 106, no. 949, p. 283292.

Nei, M., 1978, Estimation of average heterozygosity and genetic distance from a small number of individuals: Genetics, v. 89, p. 583-590.

Neumann, A.C., Kofoed, J.W., and Keller, G., 1977, Lithoherms in the Straits of Florida: Geology, v. 5, p. $4-10$.

Orive, M.E., 1993, Effective population size in organisms with complex life-histories: Theoretical Population Biology, v. 44, no. 3, p. 316-340.

Orive, M.E., 1995, Senescence in organisms with clonal reproduction and complex life histories: The American Naturalist, v. 145, no. 1, p. 90-108.

Palumbi, S.R., 2003, Population genetics, demographic connectivity, and the design of marine reserves: Ecological Applications, v. 13, no. 1, p. S146-S158.

Peakall, R., and Smouse, P.E., 2006, GENALEX 6: Genetic analysis in Excel. Population genetic software for teaching and research: Molecular Ecology Notes, v. 6, p. 288-295.

Peakall, R., and Smouse, P., 2012, GenAlEx 6.5: Genetic analysis in Excel. Population genetic software for teaching and research-An update: Bioinformatics, v. 28, no. 19, p. 2537-2539.

Peatkau, D., Waits, L.P., Clarkson, P.L., Craighead, L., and Strobeck, C., 1997, An empirical evaluation of genetic distance statistics using microsatellite data from bear (Ursidae) populations: Genetics, $\mathrm{v}$. 147, p. 1945-1957.

Pritchard, J.K., Stephens, M., and Donnelly, P., 2000, Inference of population structure using multilocus genotype data: Genetics, v. 155, p. 945-959. 
Raymond, M., and Rousset, F., 1995, GENEPOP (Version 1.2): Population genetics software for exact tests and ecumenicism: Journal of Heredity, v. 86, no. 3, p. 248-249.

Rice, W.R., 1989, Analyzing tables of statistical tests: Evolution, v. 43, no. 1, p. 223-225.

Ross, S.W., and Nizinski, M.S., 2007, State of deep coral ecosystems in the southeastern U.S. region, in Lumsden, S.E., Hourigan, T.F., Bruckner, A.W., and Dorr, G., eds., The state of deep coral ecosystems of the United States: Silver Spring, Md., National Oceanic and Atmospheric Administration Technical Memorandum, p. 233-269.

Rozenfeld, A.F., Arnaud-Haond, S., Hernández-García, E., Eguíluz, V.M., Matías, M.A., Serrão, E., and Duarte, C.M., 2007, Spectrum of genetic diversity and networks of clonal organisms: Journal of The Royal Society Interface, v. 4, no. 17, p. 1093-1102.

Sambrook, J., Fritsch, E.F., and Maniatis, T., 1989, Molecular cloning: A laboratory manual (2d ed.): Cold Spring Harbor, N.Y., Cold Spring Harbor Laboratory Press.

Selkoe, K.A., Henzler, C.M., and Gaines, S.D., 2008, Seascape genetics and the spatial ecology of marine populations: Fish and Fisheries, v. 9, no. 4, p. 363-377.

Selkoe, K., and Toonen, R., 2011, Marine connectivity: A new look at pelagic larval duration and genetic metrics of dispersal: Marine Ecology Progress Series, v. 436, p. 291-305.

Shanks, A.L., Grantham, B.A., and Carr, M.H., 2003, Propagule dispersal distance and the size and spacing of marine reserves: Ecological Applications, v. 13, no. 1, supplement, p. S159-S169.

Sotka, E.E., Wares, J.P., Barth, J.A., Grosberg, R.K., and Palumbi, S.R., 2004, Strong genetic clines and geographical variation in gene flow in the rocky intertidal barnacle Balanus glandula: Molecular Ecology, v. 13, p. 2143-2156.

Underwood, J.N., Smith, L.D., van Oppen, M.J.H., and Gilmour, J.P., 2007, Multiple scales of genetic connectivity in a brooding coral on isolated reefs following catastrophic bleaching: Molecular Ecology, v. 16, no. 4, p. 771-784.

Underwood, J.N., Smith, L.D., van Oppen, M.J.H., and Gilmour, J.P., 2009, Ecologically relevant dispersal of corals on isolated reefs: Implications for managing resilience: Ecological Applications, $v$. 19, no. 1, p. 18-29.

Van Oosterhout, C., Hutchinson, W.F., Wills, D.P.M., and Shipley, P., 2004, Micro-checker: Software for identifying and correcting genotyping errors in microsatellite data: Molecular Ecology Notes, v. 4, no. 3 , p. 535-538.

Waller, R., 2005, Deep-water Scleractinia (Cnidaria: Anthozoa): Current knowledge of reproductive processes, in Freiwald, A., and Roberts, J.M., eds., Cold-water corals and ecosystems: Springer, p. 691-700.

Waller, R.G., and Tyler, P.A., 2005, The reproductive biology of two deep-water, reef-building scleractinians from the NE Atlantic Ocean: Coral Reefs, v. 24, p. 514-522.

Weir, B.S., and Cockerham, C.C., 1984, Estimating F-Statistics for the analysis of population structure: Evolution, v. 38, no. 6, p. 1358-1370.

Whitaker, K., 2004, Non-random mating and population genetic subdivision of two broadcasting corals at Ningaloo Reef, Western Australia: Marine Biology, v. 144, p. 593-603.

White, C., Selkoe, K.A., Watson, J., Siegel, D.A., Zacherl, D.C., and Toonen, R.J., 2010, Ocean currents help explain population genetic structure: Proceedings of the Royal Society B-Biological Sciences, v. 277, p. 1685-1694.

Wilson, J.B., 1979, "Patch" development of the deep-water coral Lophelia pertusa (L.) on Rockall

Bank: Journal of the Marine Biological Association of the United Kingdom, v. 59, p. 165-177.

Wright, S., 1943, Isolation by distance: Genetics, v. 28, p. 114-138.

Wright, S., 1951, The genetical structure of populations: The Annals of Eugenics, v. 15, p. 323-354. 
Yonezawa, K., Ishii, T., and Nagamine, T., 2004, The effective size of mixed sexually and asexually reproducing populations: Genetics, v. 166, no. 3, p. 1529-1539.

Young, C.M., He, R., Emlet, R.B., Li, Y., Qian, H., Arellano, S.M., Van Gaest, A., Bennett, K.C., Wolf, M., Smart, T.I., and Rice, M.E., 2012, Dispersal of deep-sea larvae from the Intra-American Seas:

Simulations of trajectories using ocean models: Integrative and Comparative Biology, v. 52, no. 4, p. 483-496. 
Table 3.1. Gulf of Mexico localities and southeastern U.S. collection sites with location and sampling depth, number of genets, new samples genotyped during the Lophelia II program, number of new genets (unique individuals) and proportion of clones for new Lophelia pertusa samples analyzed.

$\left[{ }^{\circ}\right.$, degrees; m, meter; n.a., not applicable. Localities are ordered east to west for the Gulf of Mexico and south to north for the southeastern United States. Location, and previous genet data, and genet data from southeaster United States are from Morrison and others (2011)]

\begin{tabular}{|c|c|c|c|c|c|c|c|c|c|c|}
\hline Locality & $\begin{array}{l}\text { Collection } \\
\text { site }\end{array}$ & $\begin{array}{l}\text { Latitude } \\
\text { ('North) }\end{array}$ & $\begin{array}{c}\text { Longitude } \\
\text { ( }{ }^{\circ} \text { West) }\end{array}$ & $\begin{array}{l}\text { Avg. } \\
\text { depth } \\
\text { (m) }\end{array}$ & $\begin{array}{c}\text { Previous } \\
\text { genets } \\
\text { (number) }\end{array}$ & $\begin{array}{c}\text { New } \\
\text { samples } \\
\text { (number) }\end{array}$ & $\begin{array}{l}\text { Genets } \\
\text { (number) }\end{array}$ & $\begin{array}{l}\text { Clones } \\
\text { (number) }\end{array}$ & $\begin{array}{l}\text { Proportion } \\
\text { of clones }\end{array}$ & $\begin{array}{l}\text { Total } \\
\text { genets }\end{array}$ \\
\hline \multicolumn{11}{|l|}{ Gulf of Mexico } \\
\hline Garden Banks (GB) & GB535 & 27.42 & -93.6 & 525 & 0 & 25 & 19 & 6 & 0.24 & 19 \\
\hline \multirow{2}{*}{ Green Canyon (GC) } & GC354 & 27.6 & -91.83 & 650 & 2 & 7 & 5 & 2 & 0.286 & 7 \\
\hline & GC234 & 27.45 & -91.83 & 507 & 5 & 0 & 0 & 0 & 0 & 5 \\
\hline Ewing Bank wreck (EB) & EB1008 & 27.98 & -90.08 & 620 & 0 & 3 & 3 & 0 & 0 & 3 \\
\hline Tanker Gulf Oil (GO) & MC796 & 28.16 & -89.75 & 533 & 0 & 9 & 9 & 0 & 0 & 9 \\
\hline Mississippi Canyon (MC) & MC751 & 28.19 & -89.8 & 438 & 0 & 31 & 25 & 6 & 0.194 & 25 \\
\hline Tanker Gulfpenn (GP) & MC497 & 28.44 & -89.32 & 540 & 6 & 11 & 10 & 1 & 0.091 & 16 \\
\hline \multirow[t]{3}{*}{ Viosca Knoll (VK) } & VK862 & 29.11 & -88.39 & 360 & 13 & 4 & 4 & 0 & 0 & 17 \\
\hline & VK906 & 29.07 & -88.38 & 390 & 0 & 76 & 64 & 12 & 0.158 & 64 \\
\hline & VK826 & 29.15 & -88.02 & 450 & 70 & 76 & 65 & 11 & 0.145 & 135 \\
\hline West Florida (WFS) & WFS & 26.18 & -84.71 & 468 & 0 & 55 & 51 & 4 & 0.073 & 51 \\
\hline Gulf of Mexico Totals & & & & & 96 & 305 & 255 & 46 & 0.151 & 351 \\
\hline \multicolumn{11}{|l|}{$\begin{array}{l}\text { Southeastern United } \\
\text { States }\end{array}$} \\
\hline Miami Terrace & MTR & 25.7 & -79.87 & 344 & n.a. & n.a. & 15 & 4 & 0.211 & 15 \\
\hline Cape Canaveral & $\mathrm{CCN}$ & 28.29 & -79.61 & 699 & n.a. & n.a. & 24 & 5 & 0.143 & 24 \\
\hline Jacksonville & JAX & 30.8 & -79.66 & 557 & n.a. & n.a. & 24 & 2 & 0.077 & 24 \\
\hline Savannah Banks & SAV & 31.74 & -79.2 & 511 & n.a. & n.a. & 16 & 0 & 0 & 16 \\
\hline Stetson & STS & 32.02 & -77.48 & 626 & n.a. & $\mathrm{n} . \mathrm{a}$ & 38 & 1 & 0.026 & 38 \\
\hline Cape Fear & CFR & 33.57 & -76.79 & 394 & n.a. & n.a. & 20 & 3 & 0.13 & 20 \\
\hline Cape Lookout & CLO & 34.21 & -75.88 & 398 & n.a. & n.a. & 58 & 14 & 0.194 & 58 \\
\hline Southeastern U.S. Totals & & & & & & & 195 & 29 & 0.114 & 195 \\
\hline
\end{tabular}


Table 3.2. Genetic diversity and differentiation in 10 Gulf of Mexico Lophelia pertusa sampling localities characterized using 8 microsatellite markers during the Lophelia II program.

[Bold values indicate significant deviations from Hardy-Weinberg equilibrium after sequential Bonferroni corrections $(\alpha=0.05, P<0.0004)$ for each locality and locus. GB, Garden Banks; GC, Green Canyon; EB, Ewing Bank wreck; MC, Mississippi Canyon; GO, Tanker Gulf Oil; GP, Tanker Gulfpenn; VK, Viosca Knoll; WFS, West Florida Slope; Na, number of observed alleles; P, number of private alleles; Ar, allelic richness per locus and population standardized to smallest sample size (16 alleles) using the rarefaction method of FSTAT 2.9.3 (Goudet, 1995); $H_{\mathrm{O}}$, proportion of observed heterozygotes per locus and site; $H_{\mathrm{E}}$, proportions of expected heterozygotes per locus and site; $F$ and $f$, Weir and Cockerham's (1984) estimators of inbreeding calculated and tested for significance in FSTAT.]

\begin{tabular}{|c|c|c|c|c|c|c|c|c|c|c|c|}
\hline Locality & Locus & LpeA5 & LpeC44 & LpeC52 & LpeC61 & LpeC142 & LpeC151 & LpeD3 & LpeD5 & Mean $(P)$ & $f$ \\
\hline \multirow[t]{6}{*}{ GB (19) } & $\mathrm{N}$ & 19 & 18 & 19 & 19 & 18 & 17 & 17 & 19 & 18.25 & \\
\hline & $\mathrm{Na}(\mathrm{P})$ & 8 & $17(1)$ & $12(1)$ & $24(2)$ & $15(1)$ & $8(2)$ & 10 & $10(2)$ & $13.00(9)$ & \\
\hline & $\operatorname{Ar}(16)$ & 5.507 & 10.314 & 7.739 & 12.431 & 9.897 & 6.242 & 6.278 & 7.147 & 8.194 & \\
\hline & $H_{O}$ & 0.842 & 0.611 & 0.842 & 0.947 & 1 & 0.176 & 0.588 & 0.474 & 0.685 & \\
\hline & $H_{E}$ & 0.726 & 0.883 & 0.783 & 0.934 & 0.887 & 0.761 & 0.63 & 0.828 & 0.804 & \\
\hline & $F_{I S}$ & -0.16 & 0.308 & -0.076 & -0.015 & -0.127 & 0.768 & 0.066 & 0.428 & & 0.175 \\
\hline \multirow[t]{6}{*}{ GC (12) } & $\mathrm{N}$ & 12 & 12 & 11 & 12 & 12 & 11 & 12 & 12 & 11.75 & \\
\hline & $\mathrm{Na}(\mathrm{P})$ & 7 (1) & 14 & 9 & 16 & 10 & 4 & $11(1)$ & 7 & $9.75(2)$ & \\
\hline & $\operatorname{Ar}(16)$ & 6.307 & 10.899 & 8.013 & 11.837 & 8.422 & 3.933 & 8.51 & 6.176 & 8.012 & \\
\hline & $H_{O}$ & 0.75 & 0.667 & 0.455 & 0.833 & 0.917 & 0.091 & 0.833 & 0.417 & 0.62 & \\
\hline & $H_{E}$ & 0.736 & 0.899 & 0.847 & 0.913 & 0.865 & 0.657 & 0.809 & 0.785 & 0.814 & \\
\hline & $F_{I S}$ & -0.019 & 0.259 & 0.463 & 0.087 & -0.06 & 0.862 & -0.03 & 0.469 & & 0.280 \\
\hline \multirow[t]{6}{*}{ EB (3) } & $\mathrm{N}$ & 3 & 3 & 3 & 3 & 3 & 3 & 3 & 2 & 2.88 & \\
\hline & $\mathrm{Na}(\mathrm{P})$ & 4 & 5 & 2 & 5 & 4 & 2 & 3 & 2 & 3.36 & \\
\hline & $\operatorname{Ar}(16)$ & N/A & & & & & & & & & \\
\hline & $H_{O}$ & 1 & 0.667 & 0.333 & 0.667 & 0.667 & 0 & 0.667 & 0.5 & 0.563 & \\
\hline & $H_{E}$ & 0.722 & 0.778 & 0.278 & 0.778 & 0.667 & 0.444 & 0.611 & 0.375 & 0.582 & \\
\hline & $F_{I S}$ & -0.385 & 0.143 & -0.2 & 0.143 & 0 & 1 & -0.091 & -0.333 & & 0.239 \\
\hline \multirow[t]{6}{*}{ MC751 (25) } & $\mathrm{N}$ & 24 & 25 & 24 & 24 & 25 & 25 & 25 & 25 & 24.63 & \\
\hline & $\mathrm{Na}(\mathrm{P})$ & 7 & 21 & 12 & $24(1)$ & $14(1)$ & 6 & $14(1)$ & $10(2)$ & $13.50(5)$ & \\
\hline & $\operatorname{Ar}(16)$ & 5.03 & 11.46 & 7.678 & 12.125 & 8.924 & 5.008 & 8.095 & 6.178 & 8.062 & \\
\hline & $H_{O}$ & 0.625 & 0.8 & 0.75 & 1 & 0.84 & 0.6 & 0.52 & 0.52 & 0.707 & \\
\hline & $H_{E}$ & 0.748 & 0.93 & 0.775 & 0.939 & 0.889 & 0.7 & 0.778 & 0.767 & 0.816 & \\
\hline & $F_{I S}$ & 0.165 & 0.14 & 0.032 & -0.065 & 0.055 & 0.143 & 0.332 & 0.322 & & 0.154 \\
\hline
\end{tabular}


Table 3.2. Genetic diversity and differentiation in 10 Gulf of Mexico Lophelia pertusa sampling localities characterized using 8 microsatellite markers during the Lophelia II program - continued.

[Bold values indicate significant deviations from Hardy-Weinberg equilibrium after sequential Bonferroni corrections $(\alpha=0.05, P<0.0004)$ for each locality and locus. GB, Garden Banks; GC, Green Canyon; EB, Ewing Bank wreck; MC, Mississippi Canyon; GO, Tanker Gulf Oil; GP, Tanker Gulfpenn; VK, Viosca Knoll; WFS, West Florida Slope; Na, number of observed alleles; P, number of private alleles; Ar, allelic richness per locus and population standardized to smallest sample size (16 alleles) using the rarefaction method of FSTAT 2.9.3 (Goudet, 1995); $H_{\mathrm{O}}$, proportion of observed heterozygotes per locus and site; $H_{\mathrm{E}}$, proportions of expected heterozygotes per locus and site; $F$ and $f$, Weir and Cockerham's (1984) estimators of inbreeding calculated and tested for significance in FSTAT.]

\begin{tabular}{|c|c|c|c|c|c|c|c|c|c|c|c|}
\hline Locality & Locus & LpeA5 & LpeC44 & LpeC52 & LpeC61 & LpeC142 & LpeC151 & LpeD3 & LpeD5 & Mean $(P)$ & $f$ \\
\hline \multirow[t]{6}{*}{ GO (9) } & $\mathrm{N}$ & 9 & 8 & 8 & 9 & 9 & 9 & 9 & 9 & 8.75 & \\
\hline & $\mathrm{Na}(\mathrm{P})$ & $7(1)$ & 11 & 8 & 13 & 9 & 5 & 4 & 7 & $8.00(1)$ & \\
\hline & $\operatorname{Ar}(16)$ & 6.66 & 11 & 8 & 11.771 & 8.529 & 4.987 & 3.771 & 6.752 & 7.683 & \\
\hline & $H_{O}$ & 0.889 & 0.75 & 0.75 & 0.889 & 1 & 0.556 & 0.444 & 0.556 & 0.729 & \\
\hline & $H_{E}$ & 0.79 & 0.891 & 0.758 & 0.877 & 0.827 & 0.747 & 0.377 & 0.747 & 0.752 & \\
\hline & $F_{I S}$ & -0.125 & 0.158 & 0.01 & -0.014 & -0.209 & 0.256 & -0.18 & 0.256 & & 0.091 \\
\hline \multirow[t]{6}{*}{ GP (16) } & $\mathrm{N}$ & 16 & 14 & 16 & 16 & 16 & 15 & 16 & 16 & 15.63 & \\
\hline & $\mathrm{Na}(\mathrm{P})$ & 5 & 18 & 10 & 20 & 15 & $6(1)$ & 16 & $9(1)$ & $12.38(2)$ & \\
\hline & $\operatorname{Ar}(16)$ & 3.996 & 12.386 & 7.369 & 12.839 & 10.492 & 4.939 & 10.306 & 6.457 & 8.598 & \\
\hline & $H_{O}$ & 0.813 & 0.571 & 0.563 & 1 & 0.875 & 0.4 & 0.75 & 0.563 & 0.692 & \\
\hline & $H_{E}$ & 0.688 & 0.931 & 0.766 & 0.941 & 0.91 & 0.698 & 0.879 & 0.746 & 0.82 & \\
\hline & $F_{I S}$ & -0.182 & 0.386 & 0.265 & -0.062 & 0.039 & 0.427 & 0.147 & 0.246 & & 0.189 \\
\hline \multirow[t]{6}{*}{ VK862 (17) } & $\mathrm{N}$ & 16 & 15 & 17 & 17 & 17 & 16 & 17 & 16 & 16.38 & \\
\hline & $\mathrm{Na}(\mathrm{P})$ & 8 & $16(1)$ & 10 & 18 & $15(1)$ & 5 & $15(1)$ & 6 & $11.63(3)$ & \\
\hline & $\operatorname{Ar}(16)$ & 6.029 & 11.52 & 7.275 & 11.014 & 10.065 & 4.365 & 9.144 & 4.645 & 8.007 & \\
\hline & $H_{O}$ & 0.75 & 0.667 & 0.588 & 0.941 & 0.824 & 0.375 & 0.706 & 0.625 & 0.684 & \\
\hline & $H_{E}$ & 0.736 & 0.924 & 0.798 & 0.908 & 0.898 & 0.695 & 0.82 & 0.668 & 0.806 & \\
\hline & $F_{I S}$ & -0.019 & 0.279 & 0.262 & -0.036 & 0.083 & 0.461 & 0.139 & 0.064 & & 0.182 \\
\hline \multirow[t]{6}{*}{ VK906 (64) } & $\mathrm{N}$ & 63 & 61 & 63 & 64 & 64 & 62 & 63 & 59 & 62.38 & \\
\hline & $\mathrm{Na}(\mathrm{P})$ & 9 & $30(2)$ & 12 & 32 & $18(1)$ & 8 & $19(3)$ & $12(1)$ & $17.5(7)$ & \\
\hline & $\operatorname{Ar}(16)$ & 5.654 & 11.379 & 7.889 & 12.118 & 9.318 & 5.115 & 7.197 & 6.493 & 8.145 & \\
\hline & $H_{O}$ & 0.746 & 0.721 & 0.27 & 0.938 & 0.875 & 0.484 & 0.349 & 0.458 & 0.605 & \\
\hline & $H_{E}$ & 0.766 & 0.931 & 0.862 & 0.951 & 0.908 & 0.725 & 0.768 & 0.811 & 0.84 & \\
\hline & $F_{I S}$ & 0.026 & 0.226 & 0.687 & 0.014 & 0.036 & 0.332 & 0.545 & 0.436 & & 0.287 \\
\hline
\end{tabular}


Table 3.2. Genetic diversity and differentiation in 10 Gulf of Mexico Lophelia pertusa sampling localities characterized using 8 microsatellite markers during the Lophelia II program - continued.

[Bold values indicate significant deviations from Hardy-Weinberg equilibrium after sequential Bonferroni corrections $(\alpha=0.05, P<0.0004)$ for each locality and locus. GB, Garden Banks; GC, Green Canyon; EB, Ewing Bank wreck; MC, Mississippi Canyon; GO, Tanker Gulf Oil; GP, Tanker Gulfpenn; VK, Viosca Knoll; WFS, West Florida Slope; Na, number of observed alleles; P, number of private alleles; Ar, allelic richness per locus and population standardized to smallest sample size (16 alleles) using the rarefaction method of FSTAT 2.9.3 (Goudet, 1995); $H_{\mathrm{O}}$, proportion of observed heterozygotes per locus and site; $H_{\mathrm{E}}$, proportions of expected heterozygotes per locus and site; $F$ and $f$, Weir and Cockerham's (1984) estimators of inbreeding calculated and tested for significance in FSTAT.]

\begin{tabular}{lccccccccccc}
\hline Locality & Locus & LpeA5 & LpeC44 & LpeC52 & LpeC61 & LpeC142 & LpeC151 & LpeD3 & LpeD5 & Mean $(P)$ & $f$ \\
\hline VK826 (135) & $\mathrm{N}$ & 130 & 127 & 132 & 132 & 134 & 130 & 134 & 125 & 130.5 \\
& $\mathrm{Na}(\mathrm{P})$ & $10(1)$ & $38(5)$ & $16(3)$ & $41(3)$ & $26(5)$ & $11(1)$ & $41(9)$ & $17(6)$ & $25.00(33)$ \\
& $\mathrm{Ar}(16)$ & 5.418 & 11.714 & 7.455 & 12.214 & 9.552 & 5.188 & 8.874 & 6.107 & 8.315 \\
& $H_{O}$ & 0.838 & 0.78 & 0.545 & 0.947 & 0.843 & 0.469 & 0.694 & 0.4 & 0.69 \\
& $H_{E}$ & 0.774 & 0.946 & 0.823 & 0.955 & 0.912 & 0.717 & 0.814 & 0.743 & 0.836 & \\
& $F_{I S}$ & -0.083 & $\mathbf{0 . 1 7 6}$ & $\mathbf{0 . 3 3 7}$ & 0.009 & $\mathbf{0 . 0 7 6}$ & $\mathbf{0 . 3 4 6}$ & 0.148 & $\mathbf{0 . 4 6 2}$ & & $\mathbf{0 . 1 7 8}$ \\
\hline WFS (51) & $\mathrm{N}$ & 49 & 50 & 51 & 51 & 51 & 51 & 50 & 45 & 49.75 & \\
& $\mathrm{Na}(\mathrm{P})$ & 8 & $27(1)$ & $13(1)$ & $32(1)$ & $21(1)$ & $9(1)$ & $30(4)$ & 9 & $18.63(9)$ & 8.295 \\
& $\mathrm{Ar}(16)$ & 5.408 & 11.375 & 7.555 & 11.917 & 9.884 & 5.021 & 9.825 & 5.371 & 0.669 \\
& $H_{O}$ & 0.694 & 0.68 & 0.667 & 0.882 & 0.941 & 0.49 & 0.62 & 0.378 & 0.823 & $\mathbf{0 . 1 9 7}$ \\
& $H_{E}$ & 0.741 & 0.936 & 0.829 & 0.945 & 0.916 & 0.697 & 0.861 & 0.66 & 0.428 & \\
\hline Sample & $F_{I S}$ & 0.064 & $\mathbf{0 . 2 7 4}$ & $\mathbf{0 . 1 9 6}$ & 0.066 & -0.028 & $\mathbf{0 . 2 9 7}$ & $\mathbf{0 . 2 8}$ & $\mathbf{0 . 4 2 8}$ & \\
& $\mathrm{N}$ & 34.1 & 33.3 & 34.4 & 34.7 & 34.9 & 33.9 & 34.6 & 32.8 & & \\
& $\mathrm{Na}(\mathrm{P})$ & $12(3)$ & $44(10)$ & $18(5)$ & $47(7)$ & $33(10)$ & $15(5)$ & $54(19)$ & $24(12)$ & $30.86(71)$ \\
\hline
\end{tabular}


Table 3.3. Pairwise $F_{S T}$ (below diagonal) and $D_{\text {EST }}$ (above diagonal) estimates between localities for Lophelia pertusa based on a survey of eight microsatellite loci during the Lophelia II program.

[Estimates in bold italics indicate significance based on 9,999 permutations after sequential Bonferroni correction $(\alpha=0.05, P<0.0006)$. Site abbreviations given in table 3.1]

\begin{tabular}{|c|c|c|c|c|c|c|c|c|c|c|c|c|c|c|c|c|}
\hline Site & GB & GC & MC & GO & GP & VK862 & VK906 & VK826 & WFL & MTR & $\mathrm{CCN}$ & JAX & SAV & STS & CFR & CLO \\
\hline GB & 0 & 0.025 & 0.033 & -0.006 & 0.036 & 0.052 & -0.013 & 0.023 & 0.042 & 0.116 & 0.208 & 0.218 & 0.239 & 0.19 & 0.209 & 0.179 \\
\hline GC & 0.024 & 0 & -0.038 & 0.008 & -0.049 & -0.034 & -0.035 & -0.032 & -0.024 & 0.077 & 0.141 & 0.113 & 0.115 & 0.118 & 0.118 & 0.096 \\
\hline MC751 & 0.017 & 0.016 & 0 & -0.018 & -0.041 & -0.011 & -0.014 & -0.017 & 0.002 & 0.023 & 0.066 & 0.077 & 0.09 & 0.097 & 0.086 & 0.066 \\
\hline GO & 0.024 & 0.031 & 0.02 & 0 & 0.032 & -0.001 & -0.003 & -0.002 & 0.007 & 0.133 & 0.166 & 0.151 & 0.206 & 0.202 & 0.178 & 0.148 \\
\hline GP & 0.021 & 0.019 & 0.012 & 0.029 & 0 & -0.03 & -0.007 & -0.017 & -0.019 & -0.01 & 0.022 & 0.028 & 0.044 & 0.021 & 0.055 & 0.019 \\
\hline VK862 & 0.023 & 0.02 & 0.014 & 0.025 & 0.016 & 0 & 0.012 & 0.004 & -0.009 & 0.037 & 0.078 & 0.11 & 0.115 & 0.059 & 0.087 & 0.078 \\
\hline VK906 & 0.01 & 0.013 & 0.007 & 0.019 & 0.012 & 0.013 & 0 & 0.002 & 0.007 & 0.087 & 0.172 & 0.187 & 0.166 & 0.157 & 0.127 & 0.144 \\
\hline VK826 & 0.012 & 0.012 & 0.005 & 0.017 & 0.009 & 0.011 & 0.004 & 0 & -0.008 & 0.072 & 0.103 & 0.107 & 0.111 & 0.099 & 0.106 & 0.086 \\
\hline WFS & 0.015 & 0.014 & 0.009 & 0.02 & 0.011 & 0.011 & 0.006 & 0.003 & 0 & 0.066 & 0.088 & 0.11 & 0.087 & 0.073 & 0.097 & 0.068 \\
\hline MTR & 0.03 & 0.031 & 0.019 & 0.041 & 0.019 & 0.023 & 0.021 & 0.018 & 0.02 & 0 & 0.054 & 0.06 & 0.076 & 0.046 & 0.094 & 0.04 \\
\hline $\mathrm{CCN}$ & 0.037 & 0.034 & 0.019 & 0.042 & 0.018 & 0.024 & 0.027 & 0.018 & 0.019 & 0.023 & 0 & -0.008 & -0.015 & 0.004 & 0.05 & 0.013 \\
\hline JAX & 0.036 & 0.03 & 0.02 & 0.039 & 0.019 & 0.026 & 0.027 & 0.018 & 0.02 & 0.023 & 0.012 & 0 & 0.02 & 0.01 & 0.025 & 0.001 \\
\hline SAV & 0.039 & 0.033 & 0.024 & 0.046 & 0.023 & 0.029 & 0.026 & 0.02 & 0.021 & 0.027 & 0.015 & 0.018 & 0 & -0.025 & -0.037 & -0.028 \\
\hline STS & 0.03 & 0.027 & 0.019 & 0.041 & 0.015 & 0.019 & 0.02 & 0.014 & 0.014 & 0.019 & 0.011 & 0.011 & 0.012 & 0 & -0.011 & -0.017 \\
\hline CFR & 0.032 & 0.029 & 0.02 & 0.039 & 0.021 & 0.023 & 0.02 & 0.017 & 0.018 & 0.025 & 0.018 & 0.016 & 0.014 & 0.011 & 0 & -0.009 \\
\hline CLO & 0.028 & 0.024 & 0.015 & 0.034 & 0.014 & 0.019 & 0.018 & 0.012 & 0.012 & 0.017 & 0.01 & 0.009 & 0.01 & 0.005 & 0.009 & 0 \\
\hline
\end{tabular}


Table 3.4. Results from Analysis of Molecular Variance (AMOVA) among clusters suggested by STRUCTURE and among Gulf of Mexico Lophelia pertusa populations during the Lophelia Il program.

[df, degrees of freedom; $F_{\mathrm{ST}}$, fixation index]

\begin{tabular}{|l|c|c|c|c|}
\hline Variation & df & $\begin{array}{c}\text { Percent } \\
\text { variation }\end{array}$ & $\boldsymbol{F}_{\text {ST }}$ & $\boldsymbol{P}$ \\
\hline STRUCTURE grouping, 2 clusters & & & & \\
Among clusters & 1 & 1.81 & & \\
Among localities within clusters & 14 & 0.33 & & \\
Among individuals within localities & 1070 & 97.86 & & \\
Total & 1085 & 100 & 0.021 & $<0.0001$ \\
\hline Gulf of Mexico & & & & \\
Among localities & 8 & 0.27 & & \\
Among individuals within localities & 687 & 99.73 & & \\
Total & 695 & 100 & 0.003 & $<0.0001$ \\
\hline
\end{tabular}




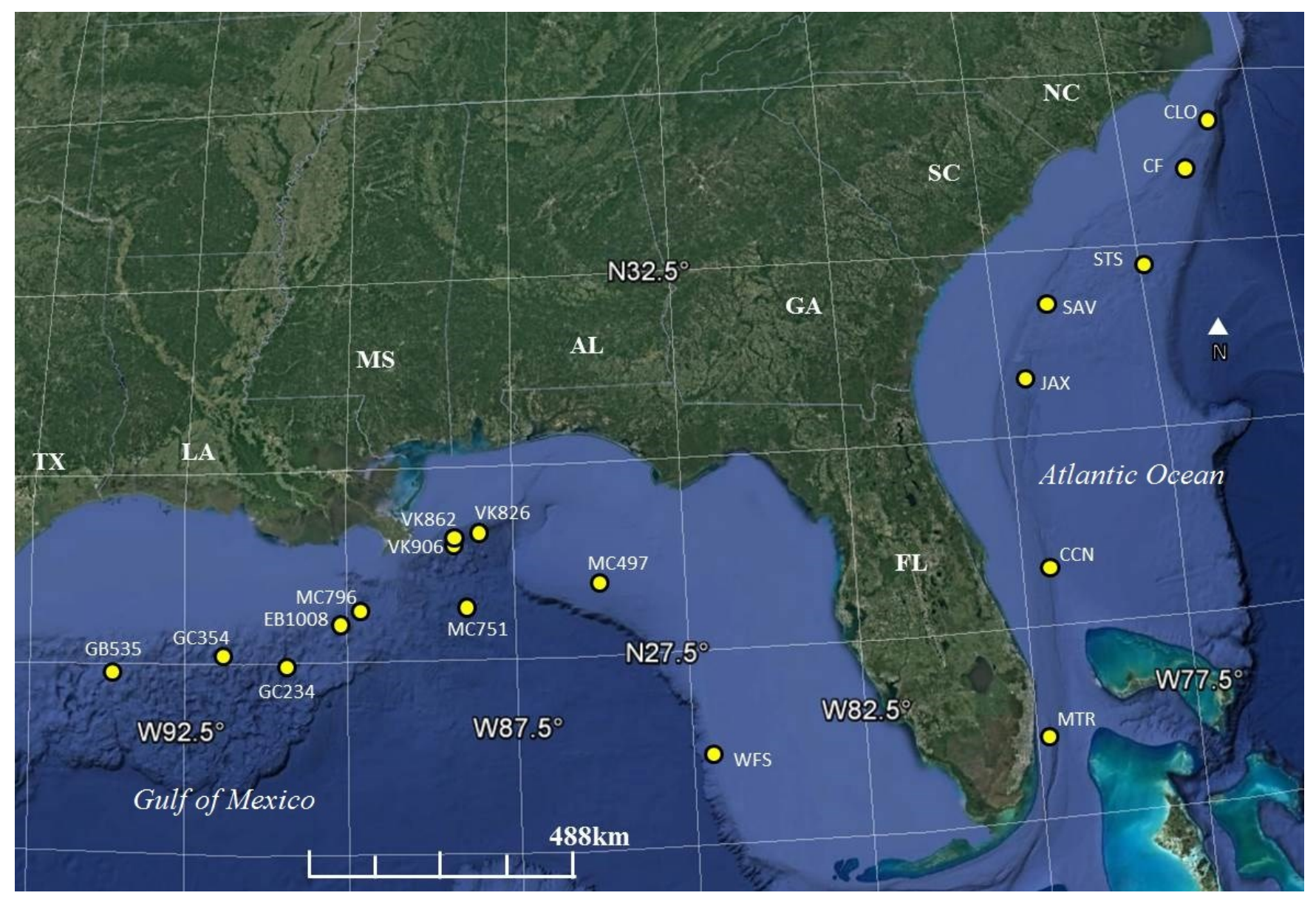

Figure 3.1 Map showing Lophelia pertusa collection localities in the Gulf of Mexico during the Lophelia II program and the western North Atlantic Ocean, and off the southeastern U.S. coast during the Lophelia I program. Site abbreviations given in table 3.1. Base map data from Google, 2016. 


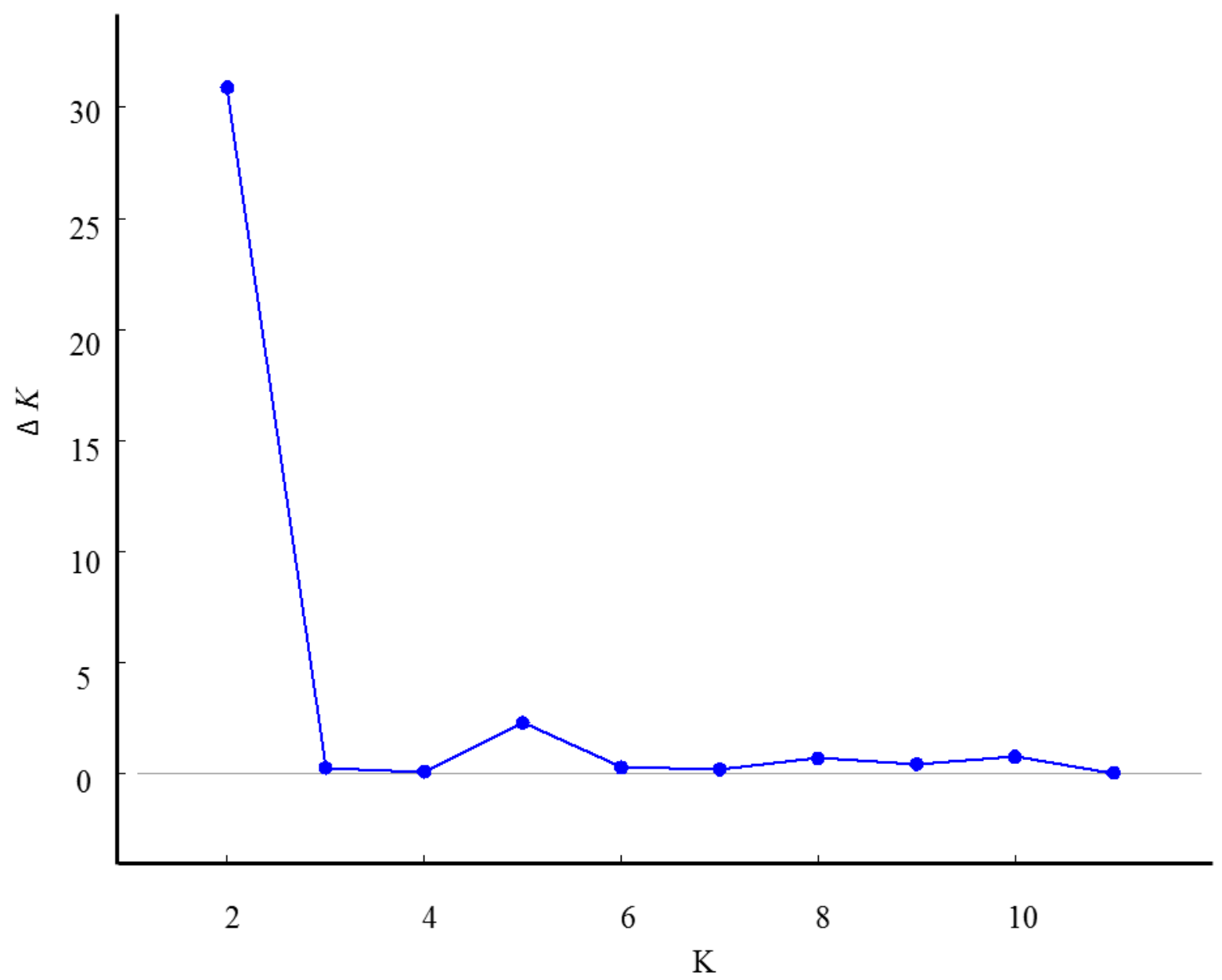

Figure 3.2 Mean values of genetic clusters $(K)$ and $\Delta K$ values from STRUCTURE analysis of Lophelia pertusa samples from the Gulf of Mexico during the Lophelia II program. 


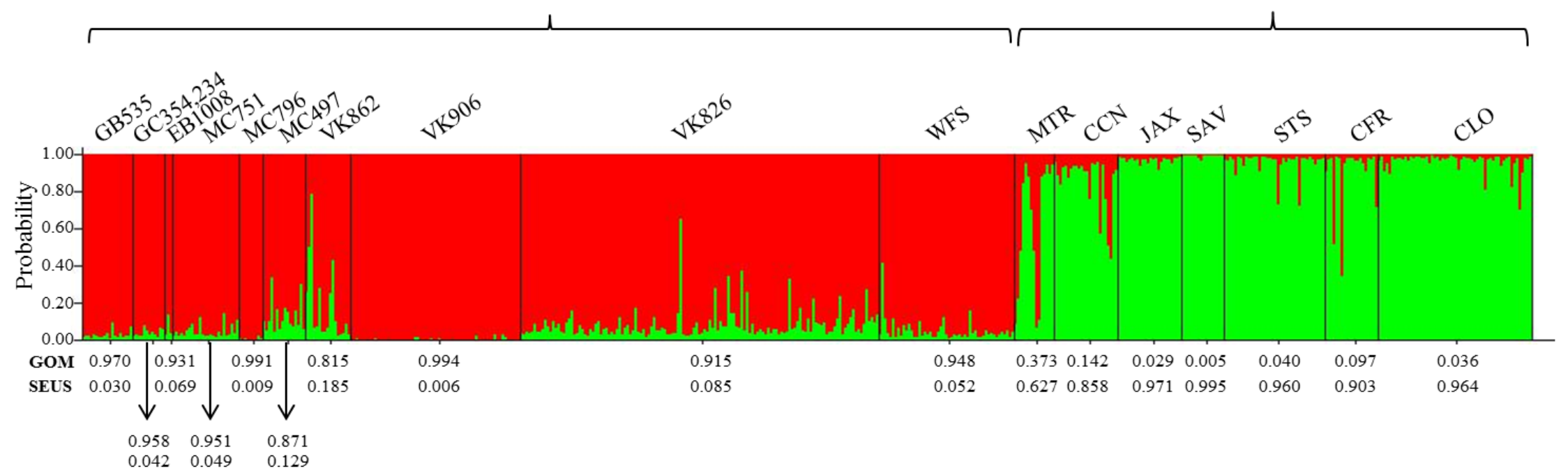

Figure 3.3 Proportional membership of Lophelia pertusa individuals from the Gulf of Mexico and the northwestern Atlantic Ocean, off the southeastern United States coast during the Lophelia II program, from sequential cluster analysis using the program STRUCTURE. The probability of membership of each individual to the $K=2$ clusters is shown by color coding of a vertical line, with red and green indicating membership in the Gulf of Mexico or western Atlantic clusters, respectively, and proportional membership along the y-axis. Proportions of membership by locality are given below the graph and localities are given above, see figure 3.1 for locations. 


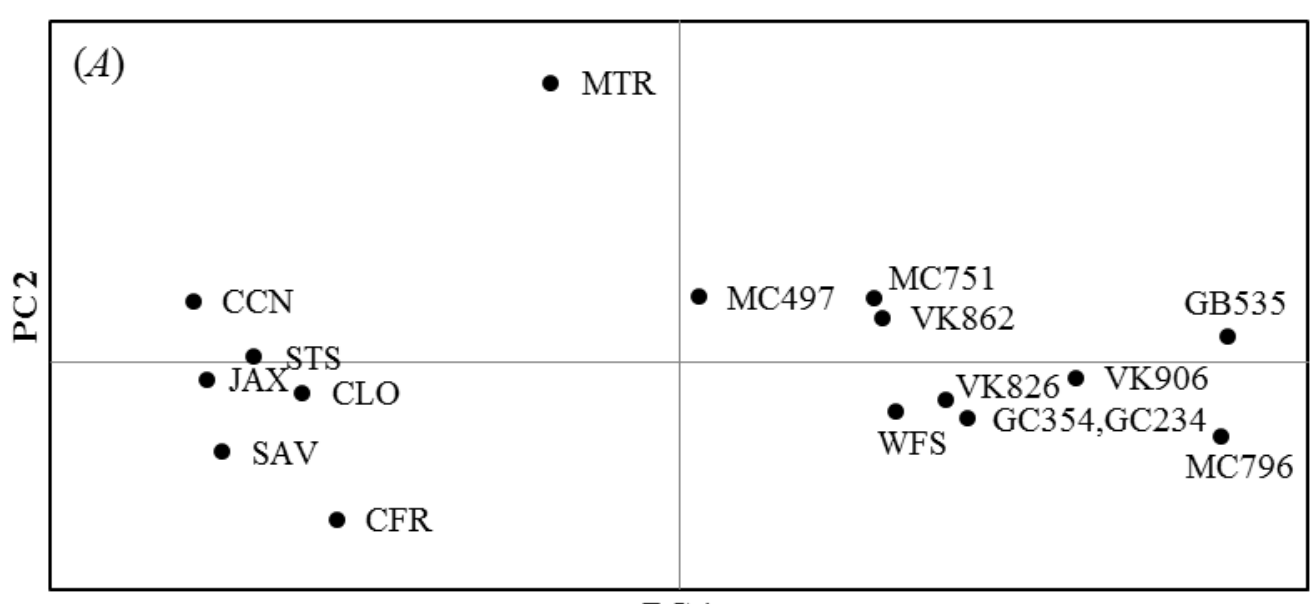

PC 1

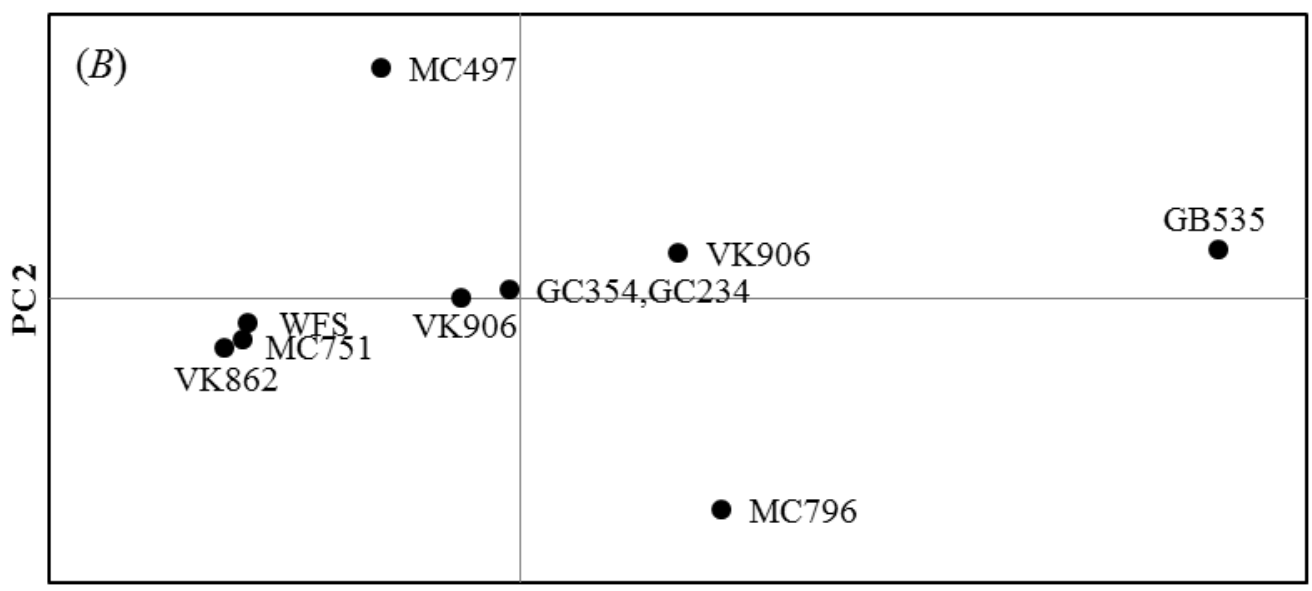

PC1

Figure 3.4 Graphs showing principal component (PC) 1 and PC 2 based upon principal components analysis of genetic distance data from eight microsatellite loci during the Lophelia II program including $A$, Gulf of Mexico (GOM) and northwestern Atlantic Ocean Lophelia pertusa populations and B, GOM L. pertusa populations (site abbreviations given in table 3.1). PC1 captures the inter-population genetic distance between the Garden Banks population and other GOM L. pertusa populations, while PC2 captures the differences between natural reefs and the two shipwrecks, Gulfpenn (MC497) and Gulf Oil (MC796). 

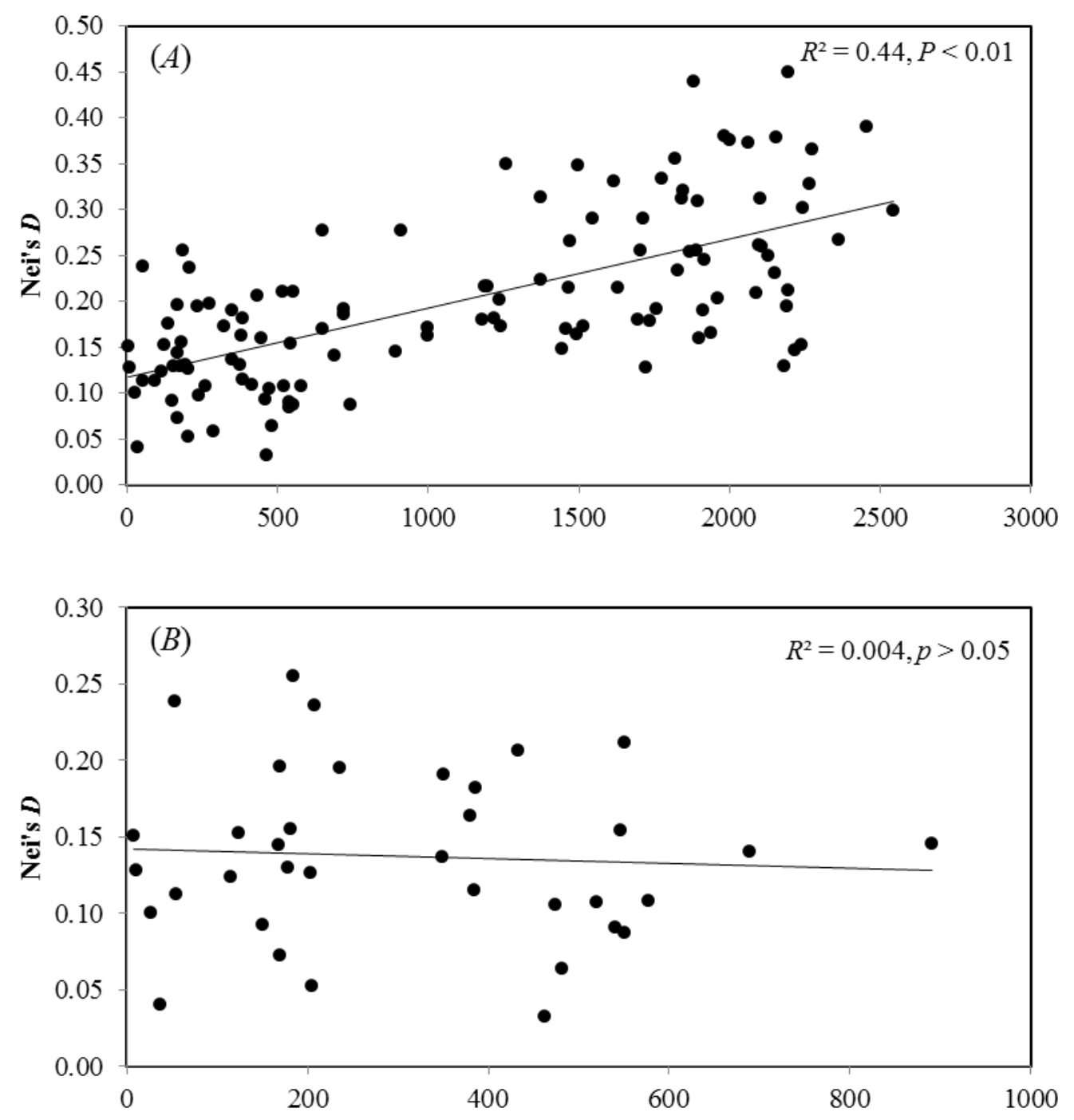

Geographic Distance (km)

Figure 3.5 Isolation-by-distance patterns in Lophelia pertusa during the Lophelia II program. The linear regression $\left(R^{2}\right)$ between spatial (geographic) distance and genetic distance (Nei's $D$, Nei, 1972, 1978) was significant $(P<0.01)$ and explained $A, 44$ percent of the variation across northwestern Atlantic Ocean and Gulf of Mexico sampling localities and $B$, less than 1 percent of the variation in genetic distances among Gulf of Mexico localities $(P>0.05)$. 


\title{
4 GENETIC DIVERSITY WITHIN EUMUNIDA PICTA, A SQUAT LOBSTER ASSOCIATED WITH LOPHELIA PERTUSA REEFS IN THE WESTERN ATLANTIC OCEAN
}

\author{
By D. Katharine Coykendall, ${ }^{1}$ Cheryl L. Morrison, ${ }^{1}$ and Martha S. Nizinski²
}

${ }^{1}$ U.S. Geological Survey
${ }^{2}$ NOAA, National Marine Fisheries Service National Systematics Laboratory, Smithsonian Institution, Washington, DC

\subsection{Introduction}

Understanding processes that affect population dynamics such as dispersal, migration (both within and between ocean basins), larval settlement, and survival is vitally important to understanding the short- and long-term viability of ecosystems. These processes can contribute to populations becoming isolated and to possible, subsequent speciation or extinction. Thus, patterns of connectivity between populations within ecosystems are important to consider when planning for the protection of biodiversity and the design of marine reserves. In remote places, such as the deep sea, ecological processes are difficult and costly to measure. Fortunately, genetic information provides an indirect measure of population dynamics. Genetic markers act like identification tags when direct tagging and observation of individuals or larvae is impossible. Analysis of patterns in DNA sequences or in simple, tandem, repeat motifs (microsatellites) can be used for broad-scale and fine-scale approaches to questions of species diversity, genetic diversity, and population connectivity in environments where demographic parameters are difficult to measure, such as the deep sea. On a broad scale, DNA sequence data may serve to clarify taxonomic issues, including evolutionary relationships, identification of cryptic species, and hybrid zones. On a finer scale, microsatellites can be used as tags to identify population substructure as well as to discern directional migration patterns to identify possible larval sources that warrant conservation and protection. Genetic approaches also provide an independent assessment of species diversity, an important parameter to measure from an ecosystems management standpoint, from that of morphology and may reveal underlying diversity not evident in taxa featuring morphological conservatism.

Recent studies (Le Goff-Vitry and others, 2004a; Morrison and others, 2011) examining regional and basinwide patterns of genetic connectivity in Lophelia pertusa, the foundation species of the coldwater coral (CWC) communities off the coast of the southeastern United States (SEUS) and the Gulf of Mexico (GOM), have improved our understanding of gene flow in patchy habitats below 200 meters (m). However, our knowledge is limited to very few species, particularly sessile invertebrates in discrete environments such as chemosynthetic communities (Black and others, 1994; Vrijenhoek, 1997; Won and others, 2003a, b; Hurtado and others, 2004; Johnson and others, 2006; Cordes and others, 2007; Young and others, 2008; Coykendall and others, 2011; Quattrini and others, 2013). Additional methodologies and examination of additional taxa are necessary to adequately assess connectivity patterns across large spatial (geographic and bathymetric) and temporal scales. Are observed patterns of connectivity species- or taxon-specific, or are patterns more general in nature? Examination of gene flow of members of the faunal assemblage associated with CWCs is key to our understanding of 
connectivity and to testing the hypothesis that patterns of connectivity are similar between associated species within a deep-sea community.

Examination of patterns of genetic diversity and patterns of connectivity of closely associated invertebrates that use L. pertusa as habitat is of interest to test the hypothesis of similarity among organisms within the same CWC community. We chose the squat lobster Eumunida picta (family Eumunididae), which is 1 of 29 species presently recognized in the genus Eumunida (Baba and others, 2008; Macpherson and Baba, 2011; also see Puillandre and others, 2011; fig. 2.4). This species occurs in the western Atlantic Ocean from off the coast of Massachusetts to Florida, in the GOM, and in the Caribbean Sea (de Saint Laurent and Macpherson, 1990) and is a numerically dominant megafaunal invertebrate associated with L. pertusa reefs (Ross and Nizinski, 2007). In the GOM, a close association between the squat lobster, Eumunida picta, and L. pertusa has been observed (Brooke and Schroeder, 2007; Cordes and others, 2008; Kilgour and Shirley, 2008). Data from video transects conducted in the northern GOM indicated that 81 percent of E. picta were in direct contact with L. pertusa or were observed less than $1 \mathrm{~m}$ away from the coral (Kilgour and Shirley, 2008). This species has also been observed at cold-seep environments off the coast of Louisiana (Carney, 1994; Bergquist and others, 2003; Cordes and others, 2007) and in association with the soft coral Primnoa resedaeformis in the northwest Atlantic Ocean (Buhl-Mortensen and Mortensen, 2004). A recent phylogenetic study of a subset of the genus Eumunida (Puillandre and others, 2011), used two genes (mitochondrial cytochrome oxidase [COI] and nuclear ribosomal 28S) to illustrate that although several species formed a single clade (that is, likely one species), cryptic species diversity was also present in the genus. Thus, the taxonomy of Eumunida appears to be unresolved.

Genetic comparisons among fauna across latitudes, oceans, and evolutionary timeframes provide a unique ecological perspective on broad patterns of linkages andconnectivity in deep-reef areas. Preliminary data suggest that E. picta are more abundant on L. pertusa reefs off North Carolina than Florida, suggesting that abundance of E. picta off the coast of the SEUS decreases with decreasing latitude. Thus, there appears to be regional differences in abundance and distribution of this species. Similar patterns of genetic differentiation (such as barriers to gene flow between the SEUS and GOM) among fauna associated with CWCs and the corals themselves would indicate that large-scale, oceanographic parameters such as bathymetry, geography, oceanography, and (or) other physical and chemical influences driving dispersal, settlement, and (or) retention affect the coral host and its associates similarly. If, however, the genetic patterns between the species are discordant, this would suggest that different life histories influence patterns of connectivity of CWC hosts versus associated fauna.

Our objectives for this study were to (1) characterize levels of connectivity among populations of an L. pertusa associate, Eumunida picta, and examine levels of genetic diversity and patterns of connectivity within E. picta; (2) examine the relationship between western Atlantic E. picta and other Eumunida species; and (3) compare connectivity patterns between E. picta with its coral associate, $L$. pertusa.

\subsection{Methods}

\subsubsection{Sample Collection}

During 10 cruises conducted from July 2004 to November 2010 (table 4.1; fig. 4.1), 92 E. picta were collected from 7 SEUS and 4 GOM sampling sites. Individuals were collected either by humanoccupied submersible (Johnson Sea Link I \& II, Harbor Branch Oceanographic Institute), remotely operated vehicle (ROV) (Kraken II, University of Connecticut; Jason II, Woods Hole Oceanographic 
Institution), or otter trawl. A small portion of tissue (leg, abdomen, or egg) was dissected from each individual collected and placed in 95 percent ethanol (ETOH) for subsequent DNA analysis. Voucher specimens were preserved in 70 percent ETOH and were deposited at the National Museum of Natural History, Smithsonian Institution (USNM).

\subsubsection{DNA Extraction, Amplification, and Sequencing}

DNA was extracted from preserved tissue by using the PureGene DNA extraction kit (Gentra Systems, Inc.) according to the manufacturer's protocol with twice the amounts of cell lysis solution and protein precipitation reagents. The isolated and purified genomic DNA was resuspended in 50 microliters $(\mu \mathrm{L})$ of molecular grade water.

A portion of the cytochrome oxidase mitochondrial gene was sequenced in 92 individuals. The polymerase chain reactions (PCRs) included 0.5 micromolar $(\mu \mathrm{M})$ of the forward primer, LCO1490 (5' - GGTCAACAAATCATAAAGATATTGG - 3') (Folmer and others, 1994) and reverse primer, COI-H (5' - TCAGGGTGACCAAAAAATCA - 3') (Machordom and Macpherson, 2004), 0.025 units per microliter (units $/ \mu \mathrm{L}$ ) of GoTaq Flexi, $1 \mathrm{X}$ GoTaq Flexi buffer, 2 millimolar $(\mathrm{mM})$ of magnesium chloride $\left(\mathrm{MgCl}_{2}\right)$ (Promega), 0.4 milligram per milliliter $(\mathrm{mg} / \mathrm{mL})$ of Bovine Serum Albumin (New England Biolabs), and molecular grade water to a final volume of $20 \mu \mathrm{L}$. PCRs were run on a MJ thermalcycler under the following conditions: initial denaturation of 95 degrees Celsius $\left({ }^{\circ} \mathrm{C}\right)$ for 3 minutes (min), 35 cycles of $94^{\circ} \mathrm{C}$ for 30 seconds (s), $50{ }^{\circ} \mathrm{C}$ for $45 \mathrm{~s}$, and $72^{\circ} \mathrm{C}$ for $90 \mathrm{~s}$, and a final extension of $72^{\circ} \mathrm{C}$ for $10 \mathrm{~min}$. The PCR reactions were checked by running on a 2-percent agarose gel. Clean products with a single band were purified by using a Qiaquick PCR purification kit (Qiagen). PCR products with more than one band were gel-purified with a Qiaquick gel extraction kit (Qiagen). Cycle sequencing reactions were conducted with BigDye terminator v3.1 chemistry (Applied Biosystems) by using $1 \mu \mathrm{L}$ of the purified reactions as a template for sequencing reactions along with $0.5 \mathrm{X}$ of $\mathrm{ABI}$ cycle sequencing buffer, $1.0 \mu \mathrm{M}$ of primer, $1.0 \mu \mathrm{L}$ of Big Dye, and molecular grade water to a final volume of $10 \mu \mathrm{L}$. Sequencing reactions were performed in both directions with the forward and reverse primers. Cycle sequencing reactions were run on a Peltier thermal cycler (BioRad) under the following conditions: initial denaturation of $95^{\circ} \mathrm{C}$ for $3 \mathrm{~min}, 35$ cycles of $94^{\circ} \mathrm{C}$ for $30 \mathrm{~s}, 52^{\circ} \mathrm{C}$ for $45 \mathrm{~s}$, and $72{ }^{\circ} \mathrm{C}$ for $90 \mathrm{~s}$, and a final extension of $72^{\circ} \mathrm{C}$ for $10 \mathrm{~min}$.

Sequencing reactions were purified by adding $10 \mu \mathrm{L}$ of AMPure XP magnetic beads (Agencourt) and $42 \mu \mathrm{L}$ of 85 percent ethanol to the sequencing reaction, immobilizing the beads magnetically, washing with $100 \mu \mathrm{L}$ of 85 percent ETOH, and resuspending in $20 \mu \mathrm{L}$ of molecular grade water. Reactions were sequenced bi-directionally on an ABI 3130XL. The sequence data was converted to chromatograms, visualized, and edited in the software Sequencher v. 4.10.1 (Gene Codes Corporation).

\subsubsection{Mitochondrial DNA Sequence Analysis}

For quality-control measures, using the BLASTN algorithm, each COI sequence that was generated was queried to the Genbank nucleotide database. Sequences from this study as well as three E. picta COI sequences from individuals collected off the Caribbean island of Guadaloupe from GenBank (accession numbers EU243556 - EU243558) were aligned using ClustalW (Thompson and others, 1994) within the program MEGA v5 (Tamura and others, 2011). The resulting alignment was translated into amino acid sequences, assuming the Drosophila mitochondrial codon table, and the consensus protein sequence was used to query the Universal Protein Resource (UniProt) database with the BLASTP searching algorithm to ensure the integrity of the alignment. Statistics summarizing the data were computed in DnaSP v5 (Librado and Rozas, 2009). A haplotype network was constructed in 
the program TCS (Clement and others, 2000) by using the default setting of a 95 percent parsimony cutoff (as calculated in Templeton and others [1992]). Pairwise genetic distances assuming a Kimura 2Parameter (K2P) DNA substitution model (Kimura, 1980) were calculated in MEGA v5 in order to directly compare results with those in Puillandre and others (2011).

A phylogeny was constructed using MrBayes v3.2.1 (Huelsenbeck and Ronquist, 2001). COI sequences from western Atlantic individuals (this study) and 19 Eumunida taxa in GenBank were included in the phylogenetic construction: E. annulosa (EU243354), E. aff. annulosa (EU243353), E. bispinata (EU243544, EU243545), E. capillata (EU243341, EU24342), E. karubar (EU243347, EU243348, EU243528, EU243529), E. keijii (EU24337, EU24338), E. laevimana (EU243497, EU243508), E. marginata (EU243543), E. minor (EU243502, EU243547), E. pacifica (EU243499), E. parva (EU243345, EU24346, EU243518, EU243521), E. picta (EU243556-558), E. similior (EU243498), E. smithii (EU243350, EU243351), E. spinosa (EU243500, EU243501, EU243513, EU243533, EU243534), E. squamifera (EU243559, EU243560), E. sternomaculata (EU243384-86), E. treguieri (EU243352, EU243358, EU243359), and E. multineata (EU243546). Based on the phylogeny presented in Puillandre and others (2011), E. multineata was used as an outgroup.

MrBayes employs Markov chain Monte Carlo (MCMC) algorithms to sample the posterior probability distribution of various parameters. A general time-reversible model of evolution was assumed with a proportion of invariant sites and a gamma-shaped distribution of mutation rates across sites with six possible rate categories: branch lengths, topologies, and stationary frequencies of each of four nucleotide. Following a burn-in of 800,000 steps, 2 million MCMC generations were run, sampling every 1,000 th generation. To improve mixing (sampling of the posterior distribution), three heated chains were used with the temperature parameter that controls how often chains swap set to 0.2. A 50percent majority consensus tree, illustrating all clusters occurring in more than half of the sampled phylogenies, was reported.

\subsubsection{Microsatellite Genotyping}

Microsatellite markers were isolated, screened, and run as in Coykendall and Morrison (2013). Loci were genotyped in Genemapper v. 4.1 (Applied Biosystems). Microsatellite genotype data were checked for excessive non-amplification, high allele drop-out, and possible scoring errors in MICROCHECKER (Van Oosterhout and others, 2004). Expected and observed heterozygosity $\left(H_{E}\right.$ and $H_{O}$, respectively), number of alleles per locus $(\mathrm{Na})$, number of alleles found in a single population $(\mathrm{Np})$, fixation index $\left(F_{S T}\right)$, and Analysis of Molecular Variance (AMOVA) were calculated in GenalEx v. 6.41 (Peakall and Smouse, 2006, 2012). The $F_{S T}$-based AMOVA was performed by using allelic information with 9,999 permutations of the data and suppressing within individual variance. Exact tests for HardyWeinberg equilibrium were executed in Genepop (Raymond and Rousset, 1995; Rousset, 2008) by using the following parameter values: dememorization of 10,000,1,000 batches, and 10,000 iterations per batch. Populations with sample sizes less than three were excluded in AMOVA and pairwise population genetic distances.

Various pooling strategies were employed to examine polymorphism patterns within the data. Based on our findings of cryptic diversity within our dataset (see Results), we separated the two groups we uncovered (fig. 4.2), and minimal analyses were performed on the CLADE II individuals due to small sample sizes per sampling locality. Within the larger group (CLADE I), samples were first clustered into two major geographic regions, SEUS $(\mathrm{N}=63)$ and GOM $(\mathrm{N}=20)$. In order to avoid confounding results due to the large geographic range between sampling sites from SEUS (978 kilometers $[\mathrm{km}])$ and GOM $(550 \mathrm{~km})$ resulting in possible subpopulation structure coupled with very small samples sizes from some sites, a single sampling site from each region was also chosen for 
comparison of patterns of polymorphism. From SEUS, CLO was chosen $(\mathrm{N}=26)$ and from GOM, VK826 and VK862 samples were pooled into a single Viosca Knoll (VK) group (N=18).

\subsection{Results}

\subsubsection{Mitochondrial DNA Sequence Analysis}

Ninety-two E. picta individuals from 7 sampling sites from SEUS and 4 sampling sites from GOM (table 4.1) were sequenced at the mitochondrial COI locus. Samples from SEUS and GOM are new to the phylogenetic consideration of Eumunida. The consensus sequence from our data contained 628 nucleotides (nt). When querying GenBank with our sequences, the highest percent identity (99 percent) occurred between nine of our samples and the three E. picta specimens collected off Guadaloupe (EU243556-EU243558). The remaining 83 individuals in our dataset also matched most closely (maximum identity of 96 percent) with the Guadaloupe E. picta individuals. In all queries of our dataset with GenBank Eumunida sequences, overlap between the query and subject sequence was 98100 percent, and E-values $=0.0$.

The nucleotide alignment of all western Atlantic E. picta sequences contained no insertions or deletions and translated into 200 amino acids (excluding missing data) without stop codons. The UniProt database query resulted in many equally likely (identity $=100$ percent; E-value $=1.0 \times 10^{-148}$ ) identities between E. picta and other Eumunida species. Nucleotide positions (sites) that contained missing data in any individual were excluded from further analyses, reducing the total number of sites used to 492. The new DNA dataset composed of our 92 sequences and the 3 GenBank E. picta, contained 55 variable nucleotides with twice as many Parsimony Informative Sites $(P I S)$ as singleton mutations (table 4.2). Of the 95 sequences, 58 were unique haplotypes. The most common haplotype was shared by 10 individuals (fig. 4.2). The average pairwise difference between all sequences, $k$, was 7.16 (table 4.2). Fifty-nine total mutations were observed in the data. Of these, five resulted in a change at the amino acid level (nonsynonymous); two of these nonsynonymous changes occurred in a single individual (EU243557).

The TCS haplotype network (Fig 4.2) estimated from 95 E. picta mitochondrial COI sequences, including sites with missing data, consisted of 65 unique haplotypes. Two distinct (95-percent parsimony level), unconnected clades (I and II) were revealed. Thirteen mutational steps separate the 2 clades. CLADE I contained 56 unique haplotypes and 83 individuals. The 2 most divergent haplotypes within this clade were separated by 22 mutational changes. CLADE II included 12 individuals and 9 unique haplotypes including the 3-voucher E. picta collected from the Caribbean Sea. Eleven mutational steps separate the most divergent haplotypes within CLADE II.

Within CLADE I, geographic regional comparisons between SEUS (7 sampling locations; $\mathrm{N}=63$ ) and GOM (4 sampling locations; $\mathrm{N}=20$ ) showed that increased sample size corresponded to increased values in polymorphism indices in most cases. Only average number of nucleotide differences between sequences, $k$, showed a different pattern (table 4.2). The increase in polymorphism indices was most pronounced with total number of segregating sites, $S$, and total number of haplotypes, $H$ and less pronounced with PIS. The $k$ values within each of the defined groups were very similar (average $=3.86$; not including "all"; table 4.2). The $k$ values between SEUS and GOM and between Cape Lookout and VK were very similar to each other and to $k$ values within each geographic region or sampling site. Between CLADE I and CLADE II, however, $k=18.9$, which is almost five times higher than the average $k$ value within and between other groups. Within both clades, however, individuals did not group by region (Fig 4.2). Haplotypes from both geographic regions were found scattered throughout 
both clades. Of the 11 haplotypes with frequencies greater than 1, 5 were found in both the SEUS and the GOM.

Pairwise genetic distances between all unique sequences followed a bimodal distribution pattern (Fig 4.3). Distance values were binned into 0.001 intervals. The first peak extended from 0.000 to 0.021 with a mean of 0.009 . The second peak extended from 0.029 to 0.053 with a mean of 0.041 . The overall average of all pairwise genetic distances was 0.017. All comparisons from the first peak were between individuals within the same clade (Fig 4.2), and all comparisons from the second peak were comparisons between individuals from different clades.

In the Bayesian phylogenetic tree, all E. picta individuals grouped more closely to each other than to other species within Eumunida (fig. 4.4). Individuals from the TCS haplotype network CLADE II also formed a distinct group in the tree (posterior probability $=100$ percent). These 12 CLADE II individuals had more derived (as opposed to ancestral) haplotypes than those from CLADE I. The closest relative to E. picta, according to this topology, was E. squamifera, collected from the southwestern coast of Africa.

\subsubsection{Population Genetic Analysis}

Ninety individuals were genotyped at 14 nuclear microsatellite loci: Epic002, Epic005, Epic014, Epic019, Epic020, Epic027, Epic035, Epic043, Epic214, Epic1379, Epic1714, Epic1950, Epic2348, and Epic2874. Due to the small number of individuals available from mitochondrial CLADE II, only individuals $(\mathrm{N}=81)$ from CLADE I were analyzed. MICROCHECKER did not detect any scoring errors or large allele drop-out at any loci, although there was significant lack of amplification at five loci (Epic002, Epic027, Epic2348, Epic214, and Epic2874). Thus, these loci were not included in further analyses. The number of alleles per locus ranged from 5 at Epic035 to 28 at Epic1379 with an average of 15 alleles per locus across all loci (table 4.3). Across all sampling sites, differences between observed heterozygosity $\left(H_{O}\right)$ and expected heterozygosity $\left(H_{E}\right)$ were highest at Epic1379, indicating heterozygosities higher than expected at that locus. Expected heterozygosities were lowest at Epic1950. Across populations, average $H_{O}-H_{E}$ was close to zero (0.04), suggesting that no population exhibited extreme $H_{O}$ values.

When all loci were examined simultaneously, a significant departure from null expectations of Hardy Weinberg equilibrium (HWE) was found, with three loci, Epic019, Epic020, and Epic1950, contributing to the disequilibrium $(\alpha=0.006$ after Bonferroni correction for multiple tests; Rice, 1989; table 4.4). The SEUS loci exhibited disequilibrium, but GOM loci were in HWE when data were parsed by geographic region. CLO $(\mathrm{N}=26)$, the SEUS group with the largest sample size, was also in HWE. The remaining SEUS populations had sample sizes too small to test.

No variation in the microsatellite data was attributed to geographic region (AMOVA; $F_{S T}=$ $0.018 ; P=0.001$, not shown). However, 2 percent of the variation was explained by sampling site differences. The remaining 98 percent was explained by variation within populations.

\subsection{Discussion}

\subsubsection{Patterns of Connectivity}

This is the first study to quantify patterns of genetic differentiation within a western North Atlantic squat lobster species. Our analyses of COI revealed two distinctive groupings, or clades, of $E$. picta sequences. Within each clade, however, genetic differentiation among sampling sites was low, suggesting little differentiation among geographically distant locations. For example, average pairwise distances between two geographically disparate (SEUS/CLO vs. GOM/VK) sites were low $(k=3.56-$ 
3.58) and similar to the overall $k$ values. In fact, the most common haplotypes were shared between GOM and SEUS populations, and, in many cases, both lineages were present at the same collecting site or in close geographic proximity. Similarly, Samadi and others (2006) reported high genetic connectivity in E. annulosa and E. sternomaculata between seamounts on the Norfolk Ridge in the South Pacific, suggesting that biogeography may not have been a significant force influencing connectivity in Eumunida over evolutionary time.

At least 13 mutational steps separated CLADEs I and II, and the average pairwise distance between sequences from these two groups was five times higher than among-clade comparisons. This level of genetic divergence could be indicative of the presence of a cryptic species. In fact, Puillandre and others (2011) provided a quantitative measure to determine species breaks in genetic data via pairwise comparisons of K2P genetic distances between Eumunida individuals. Our genetic distance data follow the same bimodal distribution pattern, and the distance separating the two peaks is similar to that presented by Puillandre and others (2011) (fig. 4.3). The peak located nearer the origin contains all genetic distance comparisons between individuals belonging to the same clade. The second peak is created by the distances observed between individuals of different clades. Comparisons of pairwise differences in sequences from individuals belonging to a single species would have a unimodal distribution pattern.

Molecular studies of marine invertebrates often reveal the presence of cryptic species where morphology is conservative. In fact, uncovering cryptic species within marine invertebrate taxa is a common phenomenon (Knowlton, 1993). Within CWC ecosystems in the Atlantic, Le Goff-Vitry and others (2004b) found evidence of possible cryptic speciation between $L$. pertusa collected from the northwest Atlantic and Brazil. Puillandre and others (2011) revealed possible species-level differences within E. annulosa based on a Bayesian analysis of $C O I$ and nuclear $28 \mathrm{~S}$ sequence data. Given that cryptic taxa are more likely to be discovered when sample sizes are larger (as seen in the Puillandre and others [2011] study), increased sampling of species within this genus of squat lobsters may uncover more diversity and differentiation than previously thought. Expanding the sampling range to the northwestern GOM and SEUS revealed that the most common taxon, CLADE I, is not the same as the E. picta collected from Guadaloupe and vouchered at the Museum National d'Histoire Naturelle in Paris, France, which is from CLADE II. Whether or not the abundances change as latitude decreases remains to be seen.

\subsubsection{Phylogenetics}

Eumunida contains 29 recognized species (Macpherson and Baba, 2011), 3 of which occur in the Atlantic Ocean, and only 1 in the western Atlantic (de Saint Laurent and Macpherson, 1990). We have put E. picta, the only western Atlantic Eumunida species, into a phylogenetic context with approximately 17 other species in the genus. Confirming results from the TCS analysis, the Bayesian phylogenetic tree that includes E. picta and other Eumunida taxa showed high support (posterior probability $>99$ percent) of CLADE II within a larger clade containing the other E. picta individuals. Moreover, CLADE I and CLADE II E. picta are more closely related to each other than to any other Eumunida species, with the latter representing a more recent lineage stemming from CLADE I. In conclusion, the E. picta COI sequence data suggest two unique genetic lineages within our samples that may represent distinct taxa of Eumunida that are geographically co-distributed in the SEUS and GOM.

\subsubsection{Contrast Between E. picta and L. pertusa Connectivity Patterns}

With regard to E. picta's coral "host," L. pertusa, Morrison and others (2011) detected a barrier to gene flow between GOM and SEUS. This geographic break in connectivity was not observed in our 
E. picta COI data. Thus, different mechanisms may be driving dispersal of $L$. pertusa and that of $E$. picta. Little is known about L. pertusa and E. picta larval development and dispersal, though Eumunida have small eggs (Van Dover and Williams, 1991), which might result in higher dispersal, and the larvae have well-developed mouth parts upon hatching, indicating a planktotrophic larval stage (de Saint Laurent and Macpherson, 1990; Guerao and others, 2006). Larvae capable of feeding may be able to stay in the water column longer, thereby traveling farther distances. Invertebrates with planktotrophic larvae sampled at the Norfolk Ridge displayed high connectivity, whereas the gastropod Nassaria problematica, a species with limited larval dispersal and nonplanktotrophic larvae, exhibited striking population structure (Samadi and others, 2006). Nevertheless, some benthic, deep-sea invertebrates, such as asteroids and echinoids, with lecithotrophic (non-feeding) larvae exhibit large geographic and bathymetric ranges (Pearse, 1994; Young and others, 1997). If spawn timing, larval feeding, morphology, and (or) development are markedly different between L. pertusa and E. picta, discordant patterns of genetic connectivity between the two species could occur, even when dispersing in the same environment. Using genetic tools to characterize and classify cryptic species could help provide better estimates of the number of species utilizing a particular habitat. As more biological diversity is uncovered, the importance of CWC ecosystems as an oasis of life becomes clearer.

\subsection{Future Directions}

The results of this study illustrate the importance of collecting and maintaining voucher specimens, even those species assumed or considered to be well known. Voucher specimens from those included in our molecular analyses can now be re-examined for subtle morphological differences.

Where possible, examination of the habitat type from video or images taken where each specimen was collected may reveal differences in behaviors that may drive differentiation. Members of the invertebrate assemblage associated with deep-sea corals are often collected opportunistically. Although trawling increases the number of samples attained, discrete sampling with ROVs and submersibles ensures that each sample is georeferenced. Additionally, the ability to observe behavioral characteristics such as habitat/coral association of sampled individuals, as is possible when using submersibles and ROVs, may lead to conclusions about potential ecological speciation.

While both clades of E. picta appear to be widely distributed throughout the GOM and SEUS, additional samples that fill geographic sampling gaps may help define geographic differences in abundance. For example, the E. picta collected from Guadaloupe in the Caribbean belonged only to CLADE II, suggesting that this clade may be more prevalent in this location. Samples from parts of the range of E. picta not included thus far, such as the mid-Atlantic canyons off the U.S. east coast, will also help define ranges of each clade.

Depth can be another important physical factor that drives differentiation in deep-sea species as seen in a chemosynthetic seep mussel genus, Bathymodiolus (Cordes and others, 2007), rockfish (Ingram, 2011), and Caribbean candelabrum coral (Prada and Hellberg, 2013), though often a semipermeable barrier to gene flow (McClain and Hardy, 2010). The average sampling depth in our study ranged from 317 to $642 \mathrm{~m}$. Testing differentiation between samples from their depth range extremes could be useful. Unfortunately, our data contain only seven specimens from "shallow" sites (317-319 $\mathrm{m}$ ) and 22 from "deep" sites (632-642 m; table 4.1). Additional sampling at the bathymetric extremes of the species' range would need to be collected to fully test this hypothesis. Apart from biogeography and depth, other mechanisms of divergence could be sex-specific migratory patterns or type of coral host (for example, octocoral or stony coral).

Due to different mutational time scales, microsatellites can offer insight into more recent, finer scale population differentiation. High levels of polymorphism were found in all E. picta populations 
sampled, and hierarchical analysis of SEUS and GOM samples did not suggest that a significant geneflow barrier exists between the GOM and SEUS oceanic regions. Unfortunately, the small sample sizes reported allow only preliminary conclusions to be drawn at this time. Additional sampling of E. picta from both regions, plus the inclusion of intermediate locations such as the eastern GOM and Miami Terrace, may uncover a signal of genetic differentiation, if such a signal exists. The small sample size of CLADE II individuals $(\mathrm{N}=9)$ prevented the analysis of genetic differentiation between the two clades using microsatellite genotype information. However, some microsatellite alleles could likely be diagnostic for CLADE II individuals, considering three private alleles were found at three loci (data not shown). Acquiring more samples of CLADE II individuals would help clarify this matter. Thus, determining ecological and (or) morphological differences between individuals in the two clades identified in this study may help uncover the biological and ecological processes that lead to genetic differentiation, as well as directing further sampling efforts in the field.

\subsection{Acknowledgments}

This work was financially supported by the U.S. Geological Survey (USGS) Outer Continental Shelf Ecosystem Program and was sponsored by the Bureau of Ocean Energy Management (BOEM). G. Brewer, C. Charles, and A. Demopoulos of the USGS provided guidance throughout the project, along with members of the USGS Diversity, Systematics, and Connectivity of Vulnerable Reef Ecosystems (DISCOVRE) team. The authors wish to thank the captains and crews of the ships, remotely operated vehicles, and submersible used on the Lophelia II research cruises. TDI-Brooks International, Inc., C. Fisher (Pennsylvania State University), and E. Cordes (Temple University) facilitated the participation of one author (C. Morrison) on their research vessel (R/V) Ron Brown Lophelia II research cruises. Thanks to S.W. Ross (University of North Carolina, Wilmington) for leading cruises on the R/V Seward Johnson, the R/V Cape Hatteras, and the R/V Ron Brown. Thanks to Jana Thoma (University of Louisiana, Lafayette) and J. McClain-Counts (USGS Wetland and Aquatic Research Center) who helped with sample preservation at sea. T. King, R. Johnson, B. Lubinski, D. Iwanowicz, and M. Springmann (USGS) provided help in the laboratory.

\subsection{References}

Baba, K., Macpherson, E., Poore, G.C.B., Ahyong, S., Bermudez, A., Cabezas, P., Lin, C.-W., Nizinski, M., Rodrigues, C., and Schnabel, K.E., 2008, Catalogue of squat lobsters of the world (Crusteacea: Decapoda: Anomura - families Chirostylidae, Galatheidae and Kiwaidae): Zootaxa, v. 1905, p. 1220.

Bergquist, D.C., Ward, T., Cordes, E.E., McNelis, T., Howlett, S., Kosoff, R., Hourdez, S., Carney, R., and Fisher, C.R., 2003, Community structure of vestimentiferan-generated habitat islands from Gulf of Mexico cold seeps: Journal of Experimental Marine Biology and Ecology, v. 289, no. 2, p. 197222.

Black, M.B., Lutz, R.A., and Vrijenhoek, R.C., 1994, Gene flow among vestimentiferan tube worm (Riftia Pachyptila) populations from hydrothermal vents of the eastern Pacific: Marine Biology, v. 120, no. 1, p. 33-39.

Brooke, S.B., and Schroeder, W.W., 2007, State of the U.S. deep coral ecosystems in the northern Gulf of Mexico region: Florida Straits to Texas, in Lumsden, S.E., Hourigan, T.F., Bruckner, A.W., and Dorr, G., eds., The state of deep coral ecosystems of the United States: Silver Spring, Md., National Oceanic and Atmospheric Administration Technical Memorandum CRCP-3, p. 271-306.

Buhl-Mortensen, L., and Mortensen, P.B., 2004, Symbiosis in deep-water corals: Symbiosis, v. 37, p. $33-61$. 
Carney, R.S., 1994, Consideration of the oasis analogy for the chemosynthetic communities at Gulf of Mexico hydrocarbon vents: Geo-Marine Letters, v. 14, p. 149-159.

Clement, M., Posada, D., and Crandall, K.A., 2000, TCS: A computer program to estimate gene genealogies: Molecular Ecology, v. 9, no. 10, p. 1657-1659.

Cordes, E.E., Carney, S.L., Hourdez, S., Carney, R.S., Brooks, J.M., and Fisher, C.R., 2007, Cold seeps of the deep Gulf of Mexico: Community structure and biogeographic comparisons to Atlantic equatorial belt seep communities: Deep-Sea Research Part I-Oceanographic Research Papers, v. 54, no. 4, p. 637-653.

Cordes, E.E., McGinley, M.P., Podowski, E.L., Becker, E.L., Lessard-Pilon, S., Viada, S.T., and Fisher, C.R., 2008, Coral communities of the deep Gulf of Mexico: Deep-Sea Research Part IOceanographic Research Papers, v. 55, no. 6, p. 777-787.

Coykendall, D.K., Johnson, S.B., Karl, S.A., Lutz, R.A., and Vrijenhoek, R.C., 2011, Genetic diversity and demographic instability in Riftia pachyptila tubeworms from eastern Pacific hydrothermal vents: BMC Evolutionary Biology, v. 11.

Coykendall, D.K., and Morrison, C.L., 2013, Polymorphic microsatellite markers developed from 454 pyro-sequencing in the cold water coral-associated squat lobster species, Eumunida picta (Chirostylidae: Eumunididae): Conservation Genetics Resources, v. 5, no. 2., p. 495-498.

de Saint Laurent, M., and Macpherson, E., 1990, Crustacea Decapoda: Le genre Eumunida Smith, 1883 (Chirostylidae) dans les eaux neo-caledoniennes, in Crosnier, A., ed., Resultats des campagnes MUSORSTOM: Paris, Memoires du Museum national d'Histoire naturelle, p. 227-288.

Folmer, O., Black, M., Hoeh, W., Lutz, R.A., and Vrijenhoek, R.C., 1994, DNA primers for amplification of mitochondrial cytochrome c oxidase subunit I from diverse metazoan invertebrates: Molecular Marine Biology and Biotechnology, v. 3, no. 5, p. 294-299.

Guerao, G., Macpherson, E., Samadi, S., De Forges, B.R., and Boisselier, M.C., 2006, First stage zoeal descriptions of five Galatheoidea species from Western Pacific (Crustacea: Decapoda: Anomura): Zootaxa, no. 1227, p. 1-29.

Huelsenbeck, J.P., and Ronquist, F., 2001, MRBAYES: Bayesian inference of phylogenetic trees: Bioinformatics, v. 17, no. 8, p. 754-755.

Hurtado, L.A., Lutz, R.A., and Vrijenhoek, R.C., 2004, Distinct patterns of genetic differentiation among annelids of eastern Pacific hydrothermal vents: Molecular Ecology, v. 13, no. 9, p. 2603-2615.

Ingram, T., 2011, Speciation along a depth gradient in a marine adaptive radiation: Proceedings of the Royal Society B-Biological Sciences, v. 278, no. 1705, p. 613-618.

Johnson, S.B., Young, C.R., Jones, W.J., Waren, A., and Vrijenhoek, R.C., 2006, Migration, isolation, and speciation of hydrothermal vent limpets (Gastropoda; Lepetodrilidae) across the Blanco Transform Fault: Biological Bulletin, v. 210, no. 2, p. 140-157.

Kilgour, M.J., and Shirley, T.C., 2008, Eumunida picta S. I. Smith, 1883, and Lophelia pertusa (Linnaeus, 1758): A relationship or just good friends?: Crustaceana, v. 81, no. 5, p. 587-593.

Kimura, M., 1980, A simple method for estimating evolutionary rates of base substitutions through comparative studies of nucleotide sequences: Journal of Molecular Evolution, v. 16, no. 2, p. 111120.

Knowlton, N., 1993, Sibling species in the sea: Annual Review of Ecology and Systematics, v. 24, p. 189-216.

Le Goff-Vitry, M.C., Pybus, O.G., and Rogers, A.D., 2004a, Genetic structure of the deep-sea coral Lophelia pertusa in the northeast Atlantic revealed by microsatellites and internal transcribed spacer sequences: Molecular Ecology, v. 13, no. 3, p. 537-549. 
Le Goff-Vitry, M.C., Rogers, A.D., and Baglow, D., 2004b, A deep-sea slant on the molecular phylogeny of the Scleractinia: Molecular Phylogenetics and Evolution, v. 30, no. 1, p. 167-177.

Librado, P., and Rozas, J., 2009, DnaSP v5: A software for comprehensive analysis of DNA polymorphism data: Bioinformatics, v. 25, no. 11, p. 1451-1452.

Machordom, A., and Macpherson, E., 2004, Rapid radiation and cryptic speciation in squat lobsters of the genus Munida (Crustacea, Decapoda) and related genera in the South West Pacific: molecular and morphological evidence: Molecular Phylogenetics and Evolution, v. 33, no. 2, p. 259-279.

Macpherson, E., and Baba, K., 2011, Taxonomy of squat lobsters, in Poore, G.C.B., Ahyong, S.T., and Taylor, J., eds., The biology of squat lobsters: Boca Raton, Fla., CRC Press, p. 39-72.

McClain, C.R., and Hardy, S.M., 2010, The dynamics of biogeographic ranges in the deep sea: Proceedings of the Royal Society B-Biological Sciences, v. 277, no. 1700, p. 3533-3546.

Morrison, C.L., Ross, S.W., Nizinski, M.S., Brooke, S., Jarnegren, J., Waller, R.G., Johnson, R.L., and King, T.L., 2011, Genetic discontinuity among regional populations of Lophelia pertusa in the North Atlantic Ocean: Conservation Genetics, v. 12, no. 3, p. 713-729.

Peakall, R., and Smouse, P.E., 2006, GENALEX 6: genetic analysis in Excel. Population genetic software for teaching and research: Molecular Ecology Notes, v. 6, no. 1, p. 288-295.

Peakall, R., and Smouse, P.E., 2012, GenAlEx 6.5: genetic analysis in Excel. Population genetic software for teaching and research—an update: Bioinformatics, v. 28, no. 19, p. 2537-2539.

Pearse, J.S., 1994, Cold-water echinoderms break "Thorson's rule," in Young, C.M., and Eckelbarger, K.J., eds., Reproduction, larval biology, and recruitment of the deep-sea benthos: New York, N.Y., Columbia University Press, p. 26-35.

Prada, C., and Hellberg, M.E., 2013, Long prereproductive selection and divergence by depth in a Caribbean candelabrum coral: Proceedings of the National Academy of Sciences of the United States of America, v. 110, no. 10, p. 3961-3966.

Puillandre, N., Macpherson, E., Lambourdiere, J., Cruaud, C., Boisselier-Dubayle, M.-C., and Samadi, S., 2011, Barcoding type specimens helps to identify synonyms and an unnamed new species in the Eumunida Smith, 1883 (Decapoda: Eumunidae): Invertebrate Systematics, v. 25, p. 322-333.

Quattrini, A.M., Georgian, S.E., Byrnes, L., Stevens, A., Falco, R., and Cordes, E.E., 2013, Niche divergence by deep-sea octocorals in the genus Callogorgia across the continental slope of the Gulf of Mexico: Molecular Ecology, p. 4123-4140.

Raymond, M., and Rousset, F., 1995, GENEPOP (Version 1.2): Population genetics software for exact tests and ecumenicism: Journal of Heredity, v. 86, no. 3, p. 248-249.

Rice, W.R., 1989, Analyzing tables of statistical tests: Evolution, v. 43, no. 1, p. 223-225.

Ross, S.W., and Nizinski, M.S., 2007, State of deep coral ecosystems in the southeastern U.S. region, in Lumsden, S.E., Hourigan, T.F., Bruckner, A.W., and Dorr, G., eds., The state of deep coral ecosystems of the United States: Silver Spring, Md., National Oceanic and Atmospheric Administration Technical Memorandum CRCP-3, p. 223-225.

Rousset, F., 2008, GENEPOP ' 007: a complete re-implementation of the GENEPOP software for Windows and Linux: Molecular Ecology Resources, v. 8, no. 1, p. 103-106.

Samadi, S., Bottan, L., Macpherson, E., Forges, B., and Boisselier, M.C., 2006, Seamount endemism questioned by the geographic distribution and population genetic structure of marine invertebrates: Marine Biology, v. 149, no. 6, p. 1463-1475.

Tamura, K., Peterson, D., Peterson, N., Stecher, G., Nei, M., and Kumar, S., 2011, MEGA5: Molecular Evolutionary Genetics Analysis using maximum likelihood, evolutionary distance, and maximum parsimony methods: Molecular Biology and Evolution, v. 28, no. 10, p. 2731-2739. 
Templeton, A.R., Crandall, K.A., and Sing, C.F., 1992, A cladistic-analysis of phenotypic associations with haplotypes inferred from restriction endonuclease mapping and DNA-sequence data. III. Cladogram estimation: Genetics, v. 132, no. 2, p. 619-633.

Thompson, J.D., Higgins, D.G., and Gibson, T.J., 1994, CLUSTAL W: Improving the sensitivity of progressive multiple sequence alignment through sequence weighting, position-specific gap penalties and weight matrix choice: Nucleic Acids Research, v. 22, no. 22, p. 4673-4680.

Van Dover, C., and Williams, A., 1991, Egg size in squat lobsters (Galatheoidea): constraint and freedom, in Wenner, A., and Kuris, A., eds., Crustacean issues, v. 7., Crustacean egg production: Rotterdam, A.A. Balkema, p. 143-155.

Van Oosterhout, C., Hutchinson, W.F., Wills, D.P.M., and Shipley, P., 2004, MICRO-CHECKER: software for identifying and correcting genotyping errors in microsatellite data: Molecular Ecology Notes, v. 4, no. 3, p. 535-538.

Vrijenhoek, R.C., 1997, Gene flow and genetic diversity in naturally fragmented metapopulations of deep-sea hydrothermal vent animals: Journal of Heredity, v. 88, no. 4, p. 285-293.

Won, Y., Hallam, S.J., O'Mullan, G.D., and Vrijenhoek, R.C., 2003a, Cytonuclear disequilibrium in a hybrid zone involving deep-sea hydrothermal vent mussels of the genus Bathymodiolus: Molecular Ecology, v. 12, no. 11, p. 3185-3190.

Won, Y., Young, C.R., Lutz, R.A., and Vrijenhoek, R.C., 2003b, Dispersal barriers and isolation among deep-sea mussel populations (Mytilidae: Bathymodiolus) from eastern Pacific hydrothermal vents: Molecular Ecology, v. 12, no. 1, p. 169-184.

Young, C.M., Sewell, M.A., Tyler, P.A., and Metataxas, A., 1997, Biogeographic and bathymetric ranges of Atlantic deep-sea echinoderms and ascidians: the role of larval dispersal: Biodiversity and Conservation, v. 6, no. 11, p. 1507-1522.

Young, C.R., Fujio, S., and Vrijenhoek, R.C., 2008, Directional dispersal between mid-ocean ridges: deep-ocean circulation and gene flow in Ridgeia piscesae: Molecular Ecology, v. 17, no. 7, p. 17181731. 
Table 4.1. Sampling localities of Eumunida picta during the Lophelia I and Lophelia II programs.

[Southeastern U.S. (SEUS) samples are ordered north to south, and Gulf of Mexico (GOM) samples are ordered east to west. $\mathrm{m}$, meter; COI, number sequences for cytochrome oxidase (COI); $\mu$ sats, and the number of individuals genotyped at up to 14 microsatellite loci]

\begin{tabular}{|c|c|c|c|c|c|c|c|c|}
\hline & Locality & $\begin{array}{l}\text { Collection } \\
\text { site }\end{array}$ & Latitude & Longitude & $\begin{array}{c}\text { Avg. } \\
\text { depth }(m)\end{array}$ & Date & $\mathrm{COI}$ & $\mu$ sats \\
\hline \multirow[t]{7}{*}{ SEUS } & Cape Lookout & CLO & $34.21^{\circ} \mathrm{N}$ & $75.88^{\circ} \mathrm{W}$ & 392 & Oct 05 ; Sep 06 & 27 & 27 \\
\hline & Cape Fear & CFR & $33.57^{\circ} \mathrm{N}$ & $76.46^{\circ} \mathrm{W}$ & 394 & Oct 05 & 7 & 6 \\
\hline & Stetson & STS & $32.26^{\circ} \mathrm{N}$ & $77.48^{\circ} \mathrm{W}$ & 632 & Oct 05 & 5 & 5 \\
\hline & Savannah & SAV & $31.74^{\circ} \mathrm{N}$ & $79.20^{\circ} \mathrm{W}$ & 511 & Oct 05 & 1 & 1 \\
\hline & Jacksonville & JAX & $30.80^{\circ} \mathrm{N}$ & $79.64^{\circ} \mathrm{W}$ & 574 & Nov $05 ;$ Nov 10 & 8 & 7 \\
\hline & Cape Canaveral & $\mathrm{CCN}$ & $28.29^{\circ} \mathrm{N}$ & $79.61^{\circ} \mathrm{W}$ & 642 & Aug 09 & 17 & 17 \\
\hline & Miami Terrace & MTR & $26.10^{\circ} \mathrm{N}$ & $79.84^{\circ} \mathrm{W}$ & 319 & Nov 05 & 2 & 2 \\
\hline \multirow[t]{7}{*}{ GOM } & West Florida & WFL & $26.18^{\circ} \mathrm{N}$ & $84.71^{\circ} \mathrm{W}$ & 468 & Sep 10 & 1 & 1 \\
\hline & Viosca Knoll 826 & VK826 & $29.15^{\circ} \mathrm{N}$ & $88.02^{\circ} \mathrm{W}$ & 455 & $\begin{array}{c}\text { Aug 04; Sep } 05 \\
\text { Aug 09; Sep-Nov } 10\end{array}$ & 17 & 17 \\
\hline & Viosca Knoll 862 & VK862 & $29.11^{\circ} \mathrm{N}$ & $88.39^{\circ} \mathrm{W}$ & 317 & Jul 04; Sep 05 & 6 & 6 \\
\hline & Mississippi Canyon & MC751 & $28.19^{\circ} \mathrm{N}$ & $89.80^{\circ} \mathrm{W}$ & 441 & Sep 09 & 1 & 1 \\
\hline & Total GOM & & & & & & 25 & 25 \\
\hline & Total SEUS & & & & & & 67 & 65 \\
\hline & Grand total & & & & & & 92 & 90 \\
\hline
\end{tabular}


Table 4.2. Summary statistics of cytochrome oxidase (COI) DNA sequence data estimated in DNAsp during the Lophelia II program.

[All, all samples collected for this study as well as three E. picta sequences from Genbank. CLADE I and CLADE II are groups in TCS haplotype network (see figure 4.2). SEUS, southeastern United States; GOM, Gulf of Mexico; CLO, Cape Lookout; VK, Viosca Knoll (contains samples from VK826 and VK862); $N$, number of sequences in each comparison; \#nt, number of nucleotides in the analysis out of a total of $628 ; S$, total number of segregating sites; PIS, parsimony informative sites; singletons, number of mutations that only occur once; $H$, number of unique haplotypes in the group; $k$, the average number of pairwise differences between all sequences within the group]

\begin{tabular}{|c|c|c|c|c|c|c|c|}
\hline & $N$ & $\# n t$ & $S$ & PIS & Singletons & $H$ & $k$ \\
\hline All & 95 & 492 & 55 & 37 & 18 & 58 & 7.16 \\
\hline SEUS & 63 & 499 & 39 & 22 & 17 & 42 & 4.26 \\
\hline GOM & 20 & 516 & 17 & 8 & 9 & 15 & 3.45 \\
\hline $\begin{array}{l}\text { SEUS vs } \\
\text { GOM }\end{array}$ & & & & & & & 3.58 \\
\hline CLO & 26 & 603 & 25 & 13 & 12 & 20 & 4.46 \\
\hline VK & 18 & 517 & 16 & 7 & 9 & 13 & 3.29 \\
\hline CLO vs VK & & & & & & & 3.56 \\
\hline CLADE I & 83 & 492 & 40 & 24 & 16 & 50 & 3.8 \\
\hline CLADE II & 12 & 547 & 16 & 5 & 11 & 9 & 3.92 \\
\hline $\begin{array}{l}\text { CLADE I vs } \\
\text { CLADE II }\end{array}$ & & & & & & & 18.9 \\
\hline
\end{tabular}


Table 4.3. Summary statistics of nine microsatellite loci in Eumunida picta (CLADE I) computed in GenalEx v4.1 during the Lophelia II program.

[Data were parsed into different sampling sites. Names of loci are the headings of the columns. $N$, number of individuals sampled per locus; $N a$, number of alleles per locus; $N p$, number of private alleles; $H_{O}$, observed heterozygosity; $H_{E}$, expected heterozygosity; $F$, Wright's

Fixation index or the inbreeding coefficient. $F$ is not calculated from populations where $\mathrm{N}=1 . H_{O}, H_{E}$, and $F$ are averages across populations not including ones where $\mathrm{N}<3 . F_{\mathrm{ST}}$ is a measure of differences between populations averaged across populations where $\mathrm{N}>3$. See table 4.1 for locations]

\begin{tabular}{|c|c|c|c|c|c|c|c|c|c|c|c|}
\hline Pop & & Epic005 & Epic014 & Epic019 & Epic020 & Epic035 & Epic043 & Epic1379 & Epic1950 & Epic1714 & Avg \\
\hline \multirow[t]{6}{*}{$\mathrm{CCN}$} & $N$ & 15 & 15 & 15 & 17 & 15 & 12 & 14 & 9 & 17 & 14 \\
\hline & $\mathrm{Na}$ & 4 & 6 & 15 & 3 & 4 & 5 & 16 & 8 & 5 & \\
\hline & $N p$ & 0 & 0 & 2 & 1 & 0 & 1 & 1 & 1 & 0 & \\
\hline & $H_{O}$ & 0.4 & 0.87 & 0.87 & 0.65 & 0.33 & 0.75 & 0.93 & 0.78 & 0.82 & 0.71 \\
\hline & $H_{E}$ & 0.34 & 0.78 & 0.9 & 0.47 & 0.34 & 0.57 & 0.9 & 0.84 & 0.6 & 0.64 \\
\hline & $F$ & -0.16 & -0.11 & 0.04 & -0.38 & 0.02 & -0.32 & -0.03 & 0.07 & -0.37 & -0.14 \\
\hline \multirow[t]{6}{*}{ CFR } & $N$ & 6 & 6 & 5 & 6 & 6 & 5 & 6 & 5 & 6 & 6 \\
\hline & $\mathrm{Na}$ & 5 & 5 & 7 & 4 & 4 & 5 & 8 & 6 & 5 & \\
\hline & $N p$ & 2 & 0 & 0 & 1 & 0 & 0 & 0 & 2 & 0 & \\
\hline & $H_{O}$ & 0.83 & 1 & 0.8 & 0.67 & 1 & 0.8 & 1 & 0.4 & 0.83 & 0.81 \\
\hline & $H_{E}$ & 0.67 & 0.76 & 0.78 & 0.51 & 0.68 & 0.78 & 0.82 & 0.76 & 0.75 & 0.72 \\
\hline & $F$ & -0.25 & -0.31 & -0.03 & -0.3 & -0.47 & -0.03 & -0.22 & 0.47 & -0.11 & -0.14 \\
\hline \multirow[t]{6}{*}{ CLO } & $N$ & 26 & 26 & 25 & 26 & 21 & 23 & 22 & 22 & 23 & 24 \\
\hline & $\mathrm{Na}$ & 8 & 10 & 20 & 3 & 5 & 9 & 20 & 12 & 9 & \\
\hline & $N p$ & 0 & 2 & 2 & 0 & 0 & 1 & 4 & 2 & 1 & \\
\hline & $H_{O}$ & 0.5 & 0.85 & 0.84 & 0.39 & 0.48 & 0.61 & 0.91 & 0.86 & 0.78 & 0.69 \\
\hline & $H_{E}$ & 0.55 & 0.81 & 0.94 & 0.4 & 0.47 & 0.68 & 0.93 & 0.89 & 0.75 & 0.71 \\
\hline & $F$ & 0.09 & -0.04 & 0.1 & 0.05 & -0.02 & 0.11 & 0.02 & 0.03 & -0.04 & 0.03 \\
\hline \multirow[t]{6}{*}{ JAX } & $N$ & 4 & 5 & 4 & 4 & 5 & 5 & 4 & 3 & 5 & 4 \\
\hline & $\mathrm{Na}$ & 5 & 5 & 5 & 2 & 2 & 6 & 7 & 4 & 3 & \\
\hline & $N p$ & 0 & 0 & 0 & 0 & 0 & 0 & 1 & 0 & 0 & \\
\hline & $H_{O}$ & 0.75 & 0.6 & 0.75 & 0.5 & 0.4 & 0.6 & 1 & 0.67 & 0.2 & 0.61 \\
\hline & $H_{E}$ & 0.75 & 0.76 & 0.75 & 0.38 & 0.48 & 0.8 & 0.84 & 0.72 & 0.46 & 0.66 \\
\hline & $F$ & 0 & 0.21 & 0 & -0.33 & 0.17 & 0.25 & -0.19 & 0.08 & 0.57 & 0.08 \\
\hline
\end{tabular}


Table 4.3. Summary statistics of nine microsatellite loci in Eumunida picta (Clade I) computed in GenalEx v4.1 during the Lophelia II program continued.

[Data were parsed into different sampling sites. Names of loci are the headings of the columns. $N$, number of individuals sampled per locus; $N a$, number of alleles per locus; $N p$, number of private alleles; $H_{O}$, observed heterozygosity; $H_{E}$, expected heterozygosity; $F$, Wright's Fixation index or the inbreeding coefficient. $F$ is not calculated from populations where $\mathrm{N}=1 . H_{O}, H_{E}$, and $F$ are averages across populations not including ones where $\mathrm{N}<3 . F_{\mathrm{ST}}$ is a measure of differences between populations averaged across populations where $\mathrm{N}>3$. See table 4.1 for locations]

\begin{tabular}{|c|c|c|c|c|c|c|c|c|c|c|c|}
\hline Pop & & Epic005 & Epic014 & Epic019 & Epic020 & Epic035 & Epic043 & Epic1379 & Epic1950 & Epic1714 & Avg \\
\hline \multirow[t]{6}{*}{ MTR } & $N$ & 2 & 2 & 2 & 2 & 2 & 2 & 1 & 0 & 2 & 2 \\
\hline & $\mathrm{Na}$ & 3 & 4 & 4 & 2 & 3 & 2 & 2 & 0 & 3 & \\
\hline & $N p$ & 0 & 0 & 0 & 0 & 0 & 0 & 0 & 0 & 0 & \\
\hline & $H_{O}$ & 1 & 1 & 1 & 0.5 & 1 & 0.5 & 1 & 0 & 0.5 & 0.72 \\
\hline & $H_{E}$ & 0.63 & 0.75 & 0.75 & 0.38 & 0.63 & 0.38 & 0.5 & 0 & 0.63 & 0.51 \\
\hline & $F$ & -0.6 & -0.33 & -0.33 & -0.33 & -0.6 & -0.33 & -1 & $\mathrm{~N} / \mathrm{A}$ & 0.2 & -0.42 \\
\hline \multirow[t]{6}{*}{ STS } & $N$ & 5 & 5 & 4 & 2 & 5 & 5 & 1 & 3 & 4 & 4 \\
\hline & $\mathrm{Na}$ & 4 & 4 & 6 & 1 & 2 & 4 & 2 & 5 & 3 & \\
\hline & $N p$ & 0 & 0 & 0 & 0 & 0 & 0 & 0 & 0 & 0 & \\
\hline & $H_{O}$ & 0.8 & 1 & 1 & 0 & 0.4 & 0.8 & 1 & 0.67 & 0.75 & 0.71 \\
\hline & $H_{E}$ & 0.64 & 0.66 & 0.81 & 0 & 0.48 & 0.58 & 0.5 & 0.78 & 0.53 & 0.55 \\
\hline & $F$ & -0.25 & -0.52 & -0.23 & N/A & 0.17 & -0.38 & -1 & 0.14 & -0.41 & -0.31 \\
\hline \multirow[t]{5}{*}{ MC751 } & $N$ & 1 & 1 & 0 & 1 & 1 & 0 & 1 & 0 & 1 & 1 \\
\hline & $\mathrm{Na}$ & 2 & 2 & 0 & 1 & 1 & 0 & 2 & 0 & 1 & \\
\hline & $N p$ & 0 & 0 & 0 & 0 & 0 & 0 & 0 & 0 & 0 & \\
\hline & $H_{O}$ & 1 & 1 & 0 & 0 & 0 & 0 & 1 & 0 & 0 & 0.33 \\
\hline & $H_{E}$ & 0.5 & 0.5 & 0 & 0 & 0 & 0 & 0.5 & 0 & 0 & 0.17 \\
\hline \multirow[t]{6}{*}{ VK826 } & $N$ & 14 & 15 & 14 & 15 & 11 & 12 & 15 & 15 & 14 & 14 \\
\hline & $\mathrm{Na}$ & 7 & 8 & 15 & 6 & 4 & 7 & 17 & 12 & 7 & \\
\hline & $N p$ & 0 & 1 & 1 & 0 & 0 & 2 & 1 & 3 & 1 & \\
\hline & $H_{O}$ & 0.57 & 0.73 & 0.93 & 0.53 & 0.36 & 0.67 & 0.93 & 0.87 & 1 & 0.73 \\
\hline & $H_{E}$ & 0.56 & 0.81 & 0.9 & 0.66 & 0.38 & 0.76 & 0.92 & 0.87 & 0.79 & 0.74 \\
\hline & $F$ & -0.02 & 0.09 & -0.03 & 0.2 & 0.04 & 0.13 & -0.01 & 0.01 & -0.27 & 0.01 \\
\hline
\end{tabular}


Table 4.3. Summary statistics of nine microsatellite loci in Eumunida picta (Clade I) computer in GenalEx v4.1 during the Lophelia II program continued.

[Data were parsed into different sampling sites. Names of loci are the headings of the columns. $N$, number of individuals sampled per locus; $N a$, number of alleles per locus; $N p$, number of private alleles; $H_{O}$, observed heterozygosity; $H_{E}$, expected heterozygosity; $F$, Wright's Fixation index or the inbreeding coefficient. $F$ is not calculated from populations where $\mathrm{N}=1 . H_{O}, H_{E}$, and $F$ are averages across populations not including ones where $\mathrm{N}<3 . F_{\mathrm{ST}}$ is a measure of differences between populations averaged across populations where $\mathrm{N}>3$. See table 4.1 for locations]

\begin{tabular}{cccccccccccc}
\hline Pop & & Epic005 & Epic014 & Epic019 & Epic020 & Epic035 & Epic043 & Epic1379 & Epic1950 & Epic1714 & Avg \\
\hline VK862 & $N$ & 3 & 2 & 2 & 3 & 0 & 3 & 0 & 2 & 3 & $\mathbf{2}$ \\
& $N a$ & 4 & 2 & 4 & 3 & 0 & 4 & 0 & 2 & 3 & \\
& $N p$ & 0 & 0 & 0 & 1 & 0 & 0 & 0 & 1 & 0 & \\
& $H_{O}$ & 1 & 0.5 & 1 & 1 & 0 & 1 & 0 & 0 & 0.67 & $\mathbf{0 . 5 7}$ \\
& $H_{E}$ & 0.67 & 0.38 & 0.75 & 0.61 & 0 & 0.67 & 0 & 0.5 & 0.61 & $\mathbf{0 . 4 6}$ \\
& $F$ & -0.5 & -0.33 & -0.33 & -0.64 & N/A & -0.5 & N/A & 1 & -0.09 & $\mathbf{- 0 . 2}$ \\
\hline WFL & $N$ & 0 & 0 & 0 & 1 & 1 & 0 & 0 & 0 & 1 & $\mathbf{0}$ \\
& $N a$ & 0 & 0 & 0 & 2 & 2 & 0 & 0 & 0 & 2 & \\
& $N p$ & 0 & 0 & 0 & 1 & 0 & 0 & 0 & 1 & 0 & \\
& $H_{O}$ & 0 & 0 & 0 & 1 & 1 & 0 & 0 & 0 & 1 & $\mathbf{0 . 3 3}$ \\
& $H_{E}$ & 0 & 0 & 0 & 0.5 & 0.5 & 0 & 0 & 0 & 0.5 & $\mathbf{0 . 1 7}$ \\
\hline${ }^{1}$ Total & $N$ & 76 & 77 & 71 & 77 & 67 & 67 & 64 & 59 & 76 & $\mathbf{7 0}$ \\
& $N a$ & 12 & 11 & 25 & 9 & 5 & 13 & 28 & 19 & 10 & $\mathbf{1 5}$ \\
& $H_{O}$ & 0.66 & 0.84 & 0.87 & 0.47 & 0.5 & 0.72 & 0.96 & 0.69 & 0.72 & $\mathbf{0 . 7 1}$ \\
& $H_{E}$ & 0.59 & 0.76 & 0.85 & 0.41 & 0.47 & 0.69 & 0.82 & 0.81 & 0.64 & $\mathbf{0 . 6 7}$ \\
& $F$ & -0.11 & -0.11 & -0.03 & -0.17 & -0.02 & -0.06 & -0.24 & 0.16 & -0.1 & $\mathbf{- 0 . 0 7}$ \\
& $F_{\text {ST }}$ & 0.08 & 0.05 & 0.08 & 0.09 & 0.08 & 0.09 & 0.12 & 0.09 & 0.12 & $\mathbf{0 . 0 9}$ \\
\hline
\end{tabular}

${ }^{1}$ Totals of $\mathrm{N}$ and $\mathrm{Na}$ are within entire dataset. 
Table 4.4. p-values from Hardy-Weinberg Equilibrium exact tests calculated in GENEPOP during the Lophelia II program.

[Values in bold indicate significance at the $\alpha=0.05 / 9$ level after Bonferroni correction for multiple tests. GOM, Gulf of Mexico; SEUS, southeastern United States; CLO, Cape Lookout; CFR, Cape Fear; hs, highly significant result of a $\chi 2$ test across all loci.]

\begin{tabular}{cccccc}
\hline Microsatellite loci & CLADE I & GOM & SEUS & CLO & CLO and CFR \\
\hline Epic005 & 0.547 & 0.776 & 0.349 & 0.185 & 0.209 \\
Epic014 & 0.517 & 0.375 & 0.252 & 0.537 & 0.342 \\
Epic019 & $\mathbf{0 . 0 0 3}$ & 0.663 & $\mathbf{0}$ & 0.014 & 0.031 \\
Epic020 & $\mathbf{0 . 0 0 2}$ & $\mathbf{0 . 0 0 2}$ & 0.817 & 0.732 & 0.883 \\
Epic035 & 0.428 & 0.345 & 0.672 & 0.724 & 0.655 \\
Epic043 & 0.014 & 0.25 & 0.025 & 0.449 & 0.098 \\
Epic1379 & 0.022 & 0.404 & 0.081 & 0.35 & 0.425 \\
Epic1714 & 0.144 & 0.419 & 0.351 & 0.89 & 0.618 \\
Epic1950 & $\mathbf{0}$ & 0.123 & $\mathbf{0}$ & 0.045 & $\mathbf{0}$ \\
Total & hs & 0.062 & hs & 0.136 & hs \\
\hline
\end{tabular}




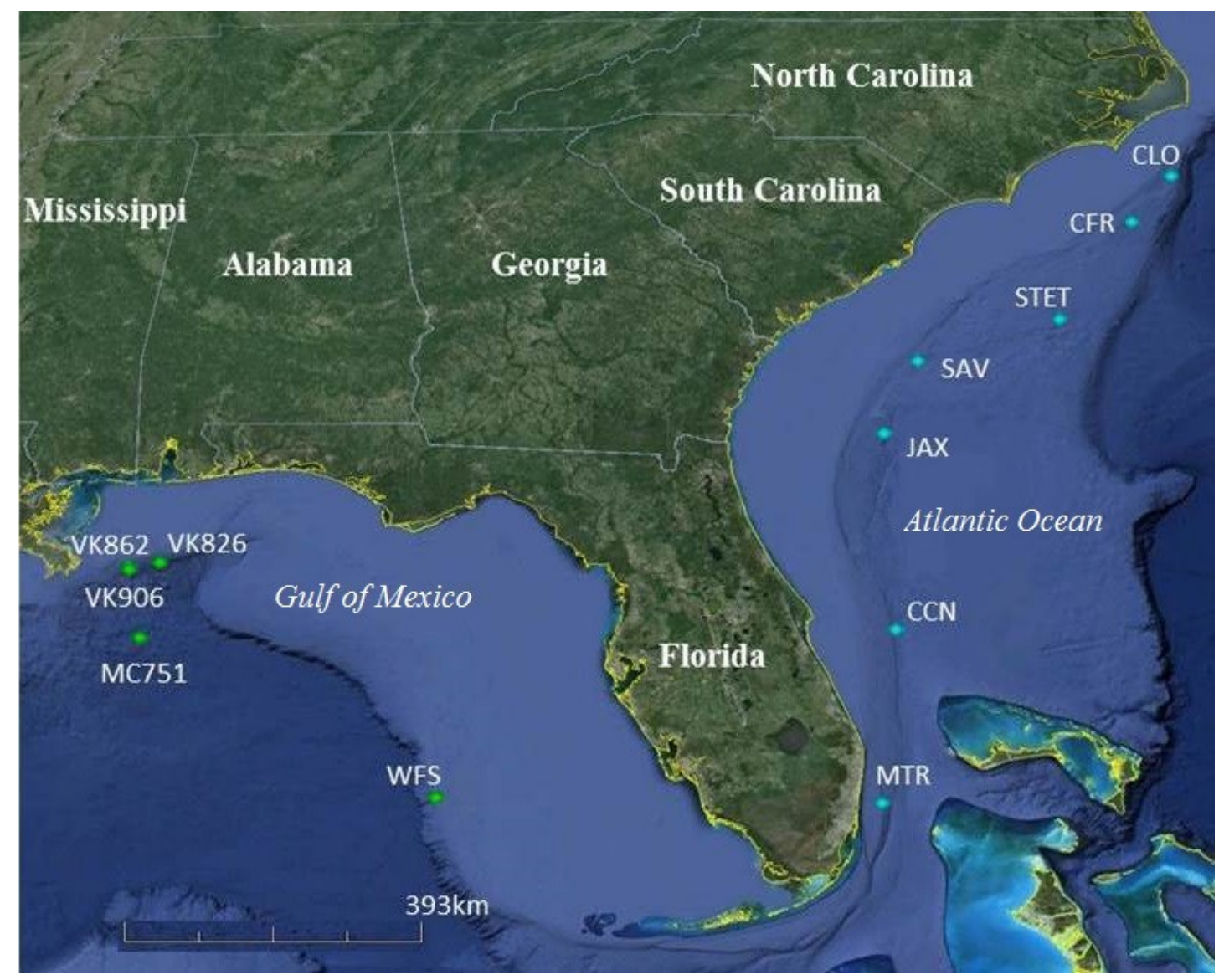

Figure 4.1 Map showing Eumunida picta sampling sites in the Gulf of Mexico and western North Atlantic Ocean during the Lophelia I and Lophelia II programs. Base map data from Google, 2016 


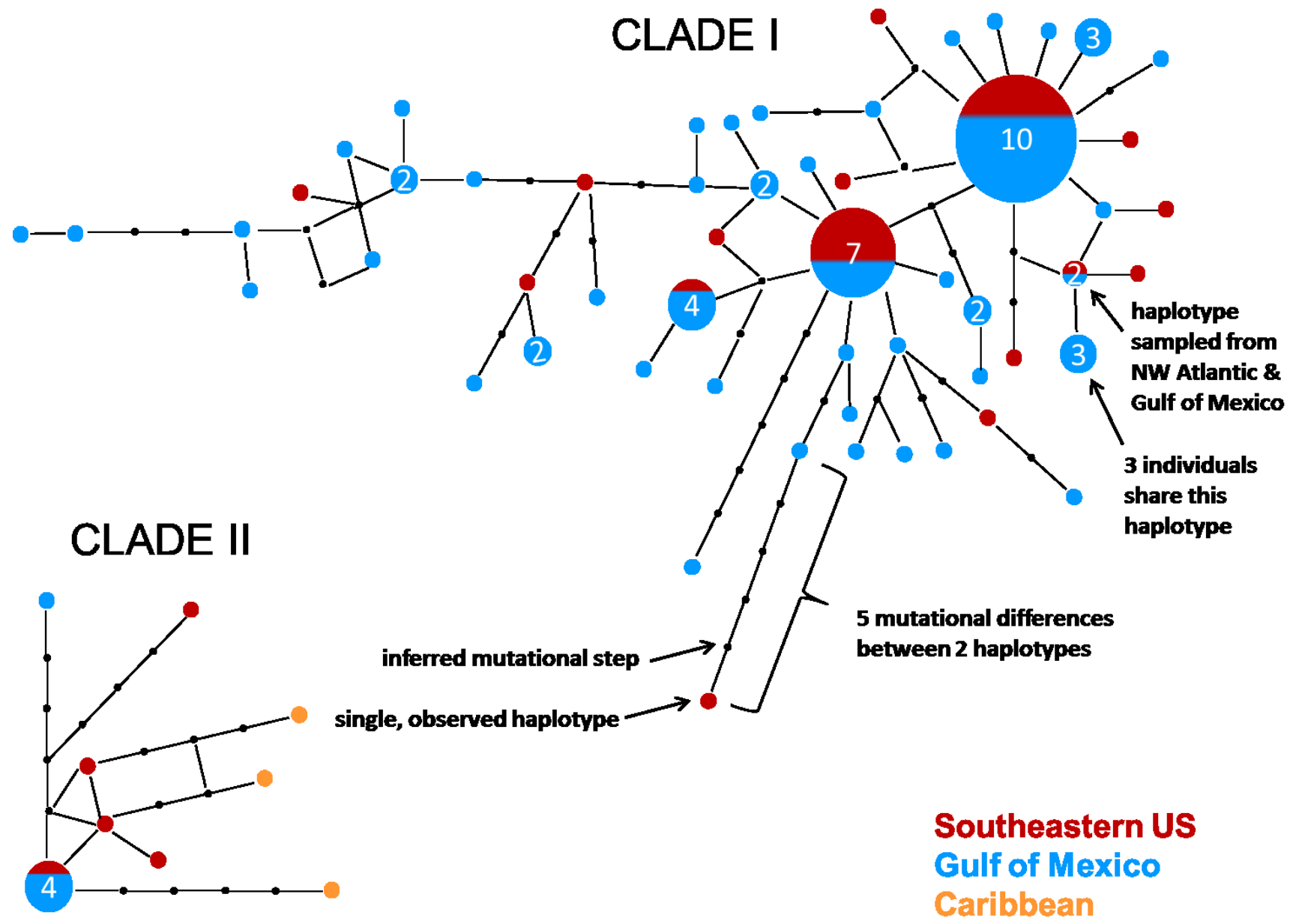

Figure 4.2 Haplotype network representing 65 different haplotypes in 95 cytochrome oxidase sequences from Eumunida picta from Gulf of Mexico (25), the southeastern United States (67), and the Caribbean (3, obtained from GenBank). 


\section{Eumunida picta genetic distances}

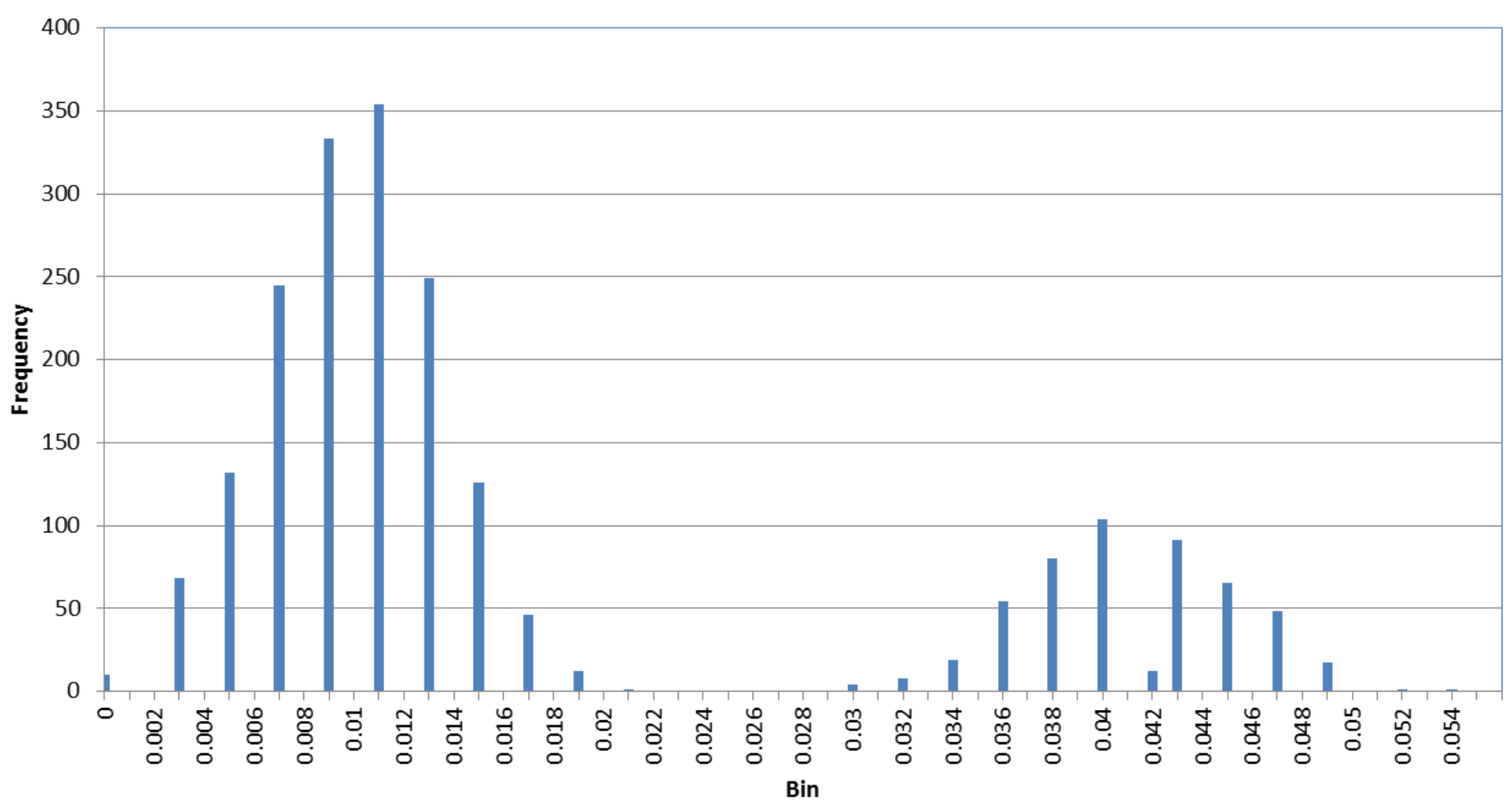

Figure 4.3 Histogram showing pairwise genetic distances between cytochrome oxidase haplotypes of Eumunida picta from the Lophelia II program. 


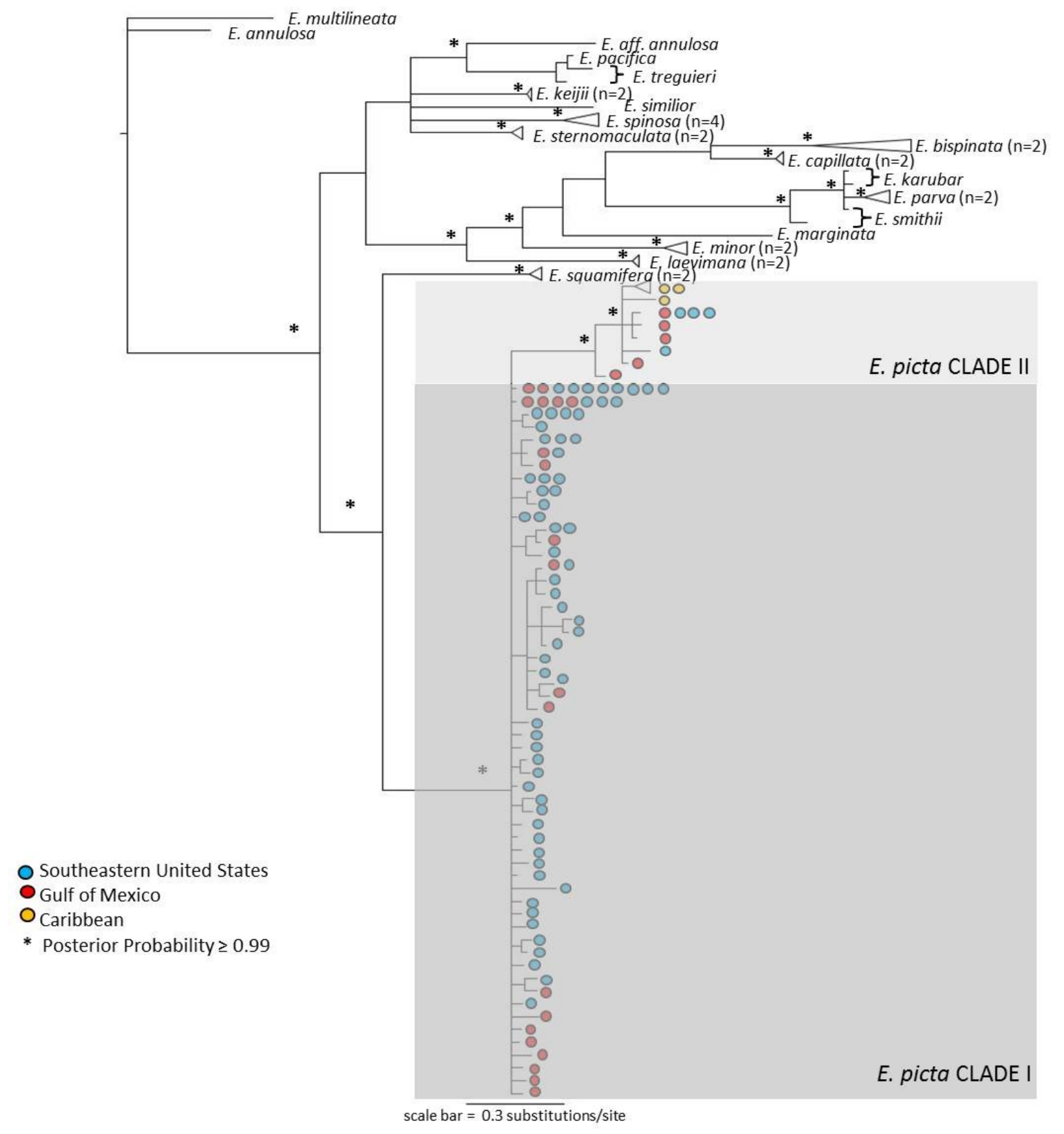

Figure 4.4 A 50-percent majority consensus Bayesian phylogenetic tree constructed with MrBayes within Geneious v 6.0.4 (Biomatters, Ltd). 


\section{DEEP-SEA BLACK CORAL GROWTH RATE AND AGE DISTRIBUTION IN THE GULF OF MEXICO}

A version of this paper was published as and reprinted here with permission:

Prouty, N., Roark, E., Buster, N., and Ross, S., 2011, Growth rate and age distribution of deep-sea black corals in the Gulf of Mexico: Marine Ecology Progress Series, v. 423, p. 101-115.

By Nancy G. Prouty, ${ }^{1}$ E. Brendan Roark, ${ }^{2}$ Noreen A. Buster, ${ }^{1}$ and Steve W. Ross ${ }^{3}$

${ }^{1}$ U.S. Geological Survey

${ }^{2}$ Department of Geography, Texas A\&M University, College Station, TX

${ }^{3}$ University of North Carolina at Wilmington, Center for Marine Sciences, Wilmington, NC

\subsection{Introduction}

Deep-sea coral (DSC) ecosystems are now widely recognized as biodiversity hotspots consisting of a large number of megafauna and macrofauna species (Roberts and others, 2009). These ecosystems provide habitat and reproductive grounds for certain commercially important fish species (Lumsden and others, 2007). Recent research expeditions conducted over the last decade have provided considerable new information on the distribution, habitat, and biodiversity of the DSC communities in the Gulf of Mexico (GOM; see review by Brooke and Schroeder, 2007). The most extensive Lophelia pertusa habitat in the GOM is situated within the Bureau of Ocean Energy Management, Regulation and Enforcement (BOEMRE) lease block Viosca Knoll 826 (VK826) and has been the focus of several recent studies (Becker and others, 2009; Schroeder and others, 2005; CSA International, Inc., 2007; Cordes and others, 2008; Sulak and others, 2008).

We present radiocarbon ages and growth rates from deep-water black corals (Leiopathes sp.) collected in the GOM along the continental slope at approximately 300-meter (m) water depth (fig. 5.1). Information on growth rates and life spans of DSCs is essential for conservation and management and for assessing the vulnerability of these organisms to natural and anthropogenic disturbances. Black corals, in particular Leiopathes sp., are among the slowest growing DSCs (Newton and Bak, 1979; Oakley, 1988) and exhibit extreme longevities. For example, Roark and others (2009) dated a Leiopathes sp. specimen from Hawaii to be 4,265 \pm 44 calendar years (cal yr) with a radial growth rate of less than 5 micrometers $(\mu \mathrm{m})$ per year $\left(\mathrm{yr}^{-1}\right)$. A combination of factors such as low frequency of recruitment events, delayed first reproduction, limited larval dispersal, and the demonstrated longevity and slow growth rates suggest that it may take centuries for these species to recover from negative effects. Longevity seems to be the key factor for population maintenance given the limited and complex genetic flow among black coral populations over long distances, as inferred by molecular markers (Miller, 1997, 1998; Miller and others, 2010). Consequently, overexploitation of black corals without proper management could easily lead to local population extinction.

Previous age dating in the GOM focused on extrapolated ages and growth rates based on skeletal ${ }^{210} \mathrm{~Pb}$ dating from a limited set of samples (Williams and others, 2006). Using "bomb"-produced radiocarbon over the last approximately 60 years and conventional radiocarbon $\left({ }^{14} \mathrm{C}\right)$ ages (based on the known radioactive decay rate) calibrated with reservoir corrections into calendar ages, robust and 
reliable ages, as well as growth rates, can be calculated (Roark and others, 2005, 2006, 2009; Sherwood and others, 2005). In this study, radiocarbon measurements were performed on samples selected from two distinct sites, Viosca Knoll and the head of De Soto Canyon (fig. 5.1). In addition, multiple discs were cut from the base of each specimen and analyzed to investigate inter- and intra-sample reproducibility.

\subsubsection{Black Corals}

Black corals are colonial cnidarians in the order Antipatharia and are found in all oceans, usually in waters greater than $30 \mathrm{~m}$ deep (Grigg, 1965). Black corals are long-lived, habitat-forming, sessile, benthic, suspension feeders (Grigg, 1965; Lewis, 1978; Parrish and others, 2002). Dense populations of these corals have been found in the tropical western Atlantic and southwestern Pacific (Warner, 1981; Grange, 1985; Sánchez, 1999). Six families are recognized (Opresko, 2001, 2002, 2003, 2004), and at least 20 species of antipatharians have been documented within the GOM region (Brooke and Schroeder, 2007). At the VK862 and VK906/907 sites, at least four species of antipatharians were identified, making up one of the most diverse megafaunal taxon at the sites (Sulak and others, 2008). Black corals are protected by international treaties and are listed in Appendix II of the Convention on International Trade in Endangered Species (CITES), restricting their exportation/importation.

Skeletons of black corals are composed mainly of organic matter, consisting of successive layers of chitin and protein microlayers glued together by an organic cement layer (Goldberg, 1991). The protein content accounts for almost 50 percent, while the chitin content is about 10-15 percent of the skeletal weight but can vary according to species (Goldberg and others, 1994). More recently, Nowak and others (2009) found the chitin content to contribute between 6 and 18 percent of the skeletal weight, with proteins and carbohydrates accounting for the remaining mass. The black coral skeleton is hard and sturdy and stands upright off the ocean floor. The architectural and mechanical properties of the black coral skeleton contribute to its stiffness (Kim and others, 1992).

\subsubsection{Food Source}

Earlier studies have suggested that L. pertusa, the framework reef-building scleractinian corals, are in part feeding on a food chain supported by chemosynthetic bacteria reliant on gas seeps (Hovland, 1990). However, more recent isotopic studies have shown that methanotrophy is not a significant source of carbon to deep-sea coral reefs (Becker and others, 2009; Duineveld and others, 2004; Kiriakoulakis and others, 2004). Instead, these organisms acquire their carbon from surface-water organic matter after rapid transport to depth. The various organic carbon sources (sedimentary, particulate, and dissolved organic carbon) used by proteinaceous corals such as black corals, were first investigated by Druffel and others (1995) in Gerardia sp. samples from the Straits of Florida using radiocarbon measured in the skeletal tips. According to isotopic mass balance calculations, the elevated post-bomb $\Delta^{14} \mathrm{C}$ values $(105$ per mil [\%o]) strongly suggested a surface-derived food source (for example, particulate organic carbon) rather than sedimentary or dissolved organic carbon. More recently, studies have shown similarities between surface water $\Delta^{14} \mathrm{C}$ derived from shallow-water coral $\Delta^{14} \mathrm{C}$ records and deep-water black coral

$\Delta^{14} \mathrm{C}$ records (Roark and others, 2006; 2009; this study), highlighting the fact that these animals are acquiring their carbon from surface-water particulate organic matter (POM). These studies also show that the ${ }^{14} \mathrm{C}$-derived age estimates of black corals are unaffected by feeding upon old resuspended sedimentary carbon (Roark and others, 2009). 


\subsection{Methods}

\subsubsection{Sample Sites}

The samples for this study were collected from the GOM in 2003, 2004, 2005, and 2009 as part of several ongoing DSC ecosystem studies (fig. 5.1; CSA International, Inc., 2007; Sulak and others, 2008). Corals were collected from two different locations: the head of De Soto Canyon and Viosca Knoll, both east of the Mississippi Delta (fig. 5.1; table 5.1). In the Viosca Knoll region, two mound features were identified in lease blocks VK862 and VK906/907. These sites were of interest because of the extensive development of the coral L. pertusa in the Viosca Knoll region (Brooke and Schroeder, 2007; Sulak and others, 2008). As described in Sulak and others (2008), the substrate capping the topographic mound features at these sites consisted of extensive deposits of authigenic goethite, a ferric oxide mineral, providing a substrate for settlement. The samples from the head of De Soto Canyon were inadvertently collected by bottom trawl, resulting in little information regarding their exact location. In comparison, Davies and others (2010) provide a more detailed description of Viosca Knoll (VK826), which is located approximately $37 \mathrm{~km}$ east-northwest of VK862 and VK906/907. Several key findings of their study include documentation of relatively low dissolved oxygen concentrations (27-28 milliliter $[\mathrm{mL}]$ liter $[\mathrm{L}]^{-1}$ ) and a 24-hour (h) diel vertical migration of zooplankton that was suggested to form part of the food chain for the corals. Sediment trap data consisted of fine-grained aggregates of riverine origin with particles of high organic carbon content (Davies and others, 2010).

\subsubsection{Sample Collection}

A bottom trawl inadvertently collected (15 November 2003) three specimens of living black coral, which are archived at the U.S. National Museum (USNM), from the head of DeSoto Canyon at a depth of $304 \mathrm{~m}$ (table 5.1). Two of these specimens were used in this study. The human-occupied submersible Johnson Sea Link II (JSL, Harbor Branch Oceanographic Institute) was used to collect live black coral colonies from the Viosca Knoll sites at depths of 310-317 m (table 5.1). Specimens archived at the USNM were tentatively identified based on branch pattern and size (D. Opresko, Smithsonian Institution, written comm., 2011) as Leiopathes sp. Species identification of the remaining samples was done at the time of collection and confirmed by comparisons with previously identified black corals. After collection, tissues were removed with forceps, and the specimens were rinsed in freshwater and air-dried on deck. The objective of the project was to calculate long-term growth rates and ages; therefore, samples with basal diameters greater than $(>) 2$ centimeters $(\mathrm{cm})$ were selected for radiocarbon analysis. The age range of specimens discussed in the following sections is a function of the variability in samples collected and the natural distribution of ages at a given site.

\subsubsection{Sample Preparation}

Cross-sectional discs about 0.3 to $0.5 \mathrm{~cm}$ thick were cut from the basal portion of the corals by using a diamond band saw. Thin sections approximately 30 and 100 micrometers $(\mu \mathrm{m})$ thick were prepared commercially for scanning electron and petrographic imaging. Following Williams and others (2006, 2007), growth bands were separated by placing the discs in a solution of 4 grams (g) of potassium hydroxide $(\mathrm{KOH})$ in $50 \mathrm{~mL}$ of water for about 1 week. The $\mathrm{KOH}$ acts as an effective protein denaturant and solvent causing the skeleton to swell and growth band laminae to separate, in a similar fashion to concentrated formic acid treatment (Goldberg, 1991). This treatment does not appear to have any effect on the isotopic composition of the skeleton (Williams, 2009) and was confirmed by replicate radiocarbon dates from samples treated with $\mathrm{KOH}$ versus samples that were microdrilled without any 
chemical band separation. Using forceps and a reflected-light microscope, individual growth bands were separated, rinsed with Milli-Q water, and dried overnight. Depending on the size of the disc and the ease of growth band separation, this yielded a maximum of 325 and a minimum of 66 subsamples. Typically, individual bands were between 100 and $200 \mu \mathrm{m}$ thick as measured by a dial and digital caliber. A small subset of samples was also drilled from the center (inner), middle, and outer portions across the radial transects by using a hand-held microdrill.

\subsubsection{Visual Ring Counts}

In preparation for scanning electron microscope (SEM) analysis, samples were impregnated with epoxy, and 100- $\mu \mathrm{m}$-thin sections were prepared commercially and imaged at the SEM facility at the University of South Florida, St. Petersburg, Florida (fig. 5.2A). Visual ring counts were conducted on the SEM images by counting the optically visible light lines (fig. $5.2 B, C$ ). The dark bands visible in figure 5.2 represent organic cement layers that serve to "glue" the growth laminae together (Goldberg, 1991; Nowak and others, 2009). In contrast to Goldberg's (1991) observation that the skeleton of Antipathes fiordensis is composed of concentric laminae interspersed by radially oriented spines, we did not observe spines in the SEM images of the Leiopathes sp. specimens; therefore, they did not interfere with the visual growth-ring counts. Visual ring counts were also determined by creating a grayscale series using the SEM image and ImageJ software (Abramoff and others, 2004). Each visible light band was counted regardless of the band's thickness. For each method, the reported ring counts were determined by taking the average and standard deviation of five independent traverses.

\subsubsection{Analytical Measurements}

Subsamples between 1 and 6 milligrams (mg), depending on laboratory requirements, were prepared for accelerator mass spectrometry (AMS) ${ }^{14} \mathrm{C}$ dating at the following centers: (1) The Center for Accelerator Mass Spectrometry, Lawrence Livermore National Laboratory (CAMS); (2) The Keck Carbon Cycle AMS Laboratory at the University of California Irvine (KCCAMS); (3) The National Ocean Sciences Accelerator Mass Spectrometry Facility (NOSAMS) at Woods Hole Oceanographic Institution; and (4) Beta Analytic in Miami, Fla. Samples were pretreated either with a deionized water (DI) rinse three times or a weak hydrochloric acid $(1 \mathrm{~N})$ rinse, placed on a hot plate for 20 minutes, and then rinsed three times with DI. Excess liquid was pipetted off prior to drying on the hot plate. The carbon in the samples was converted to carbon dioxide $\left(\mathrm{CO}_{2}\right)$ via sealed-tube combustion with silver and copper(II) oxide $(\mathrm{CuO})$, and upon cryogenic purification, the $\mathrm{CO}_{2}$ was reduced to graphite in the presence of iron catalyst and a stoichiometric excess of hydrogen. Process blanks were either a coal or calcite blank.

\subsubsection{Post-bomb Chronology}

The $\Delta^{14} \mathrm{C}$ values (radiocarbon values without age correction) were age corrected to account for decay that took place between collection (or death) and the time of measurement by using the following equation: $\Delta^{14} \mathrm{C}=([\mathrm{Fm} \times$ age correction $]-1) \times 1,000$, where age correction is defined as $\exp (1950-$ year of measurement)/8,267) (Stuiver and Polach, 1977). Radiocarbon results are reported as $\Delta^{14} \mathrm{C}(\%)$ and conventional radiocarbon age (CRA) after applying a stable carbon isotope $\left(\delta^{13} \mathrm{C}\right)$ correction (Stuiver and Polach, 1977). Analytical uncertainty was 1.3-3.9\%o, and age errors ranged between \pm 15 and 40 years. CRAs were converted to calendar years by using a reservoir correction $(\Delta R)$ of $240 \pm 13$ years $\left(\Delta \mathrm{R}\right.$ of $-30 \pm 26{ }^{14} \mathrm{C}$ years) based on pre-bomb surface-water radiocarbon measurements derived from a coral record from the Flower Garden Banks, northern GOM (Wagner and others, 2009) and the Calib 
6.0 program (Stuiver and Reimer, 1993). The reported radiocarbon and calibrated age uncertainties are reported at the 1 sigma level.

For those samples too young to apply a CRA, the chronology was developed by using the bombderived ${ }^{14} \mathrm{C}$ age model based on otolith and shallow coral $\Delta^{14} \mathrm{C}$ values. In the GOM, the bomb-derived ${ }^{14} \mathrm{C}$ was constructed by using the bomb $\Delta{ }^{14} \mathrm{C}$ curve from the otolith and shallow coral $\Delta{ }^{14} \mathrm{C}$ curves (Baker and Wilson, 2001; Wagner, 2009). The rising limb of the bomb- ${ }^{14} \mathrm{C}$ curve from these datasets represents $\Delta^{14} \mathrm{C}$ data from 1955 to $1973(\mathrm{n}=25)$, and the descending limb represents $\Delta^{14} \mathrm{C}$ data from 1975 to $1996(\mathrm{n}=12)$. The age models prior and subsequent to the bomb peak are linear, with regression coefficients of 0.92 and 0.97 , respectively (fig. 5.3). The bomb- $\Delta{ }^{14} \mathrm{C}$ curve is a reflection of artificially produced ${ }^{14} \mathrm{C}$ from detonation of nuclear test weapons in the atmosphere in the late 1950 s and early 1960s. The peak in surface ocean ${ }^{14} \mathrm{C}$ occurs about 10 years after the atmospheric peak due to result of air-sea ${ }^{14} \mathrm{C}$ exchange and the approximate decadal time delay for isotopic equilibration (Broecker and Peng, 1982). From the atmospheric peak in 1963, the level of ${ }^{14} \mathrm{CO}_{2}$ has decreased exponentially with a mean life of about 16 years, not due to radioactive decay, but due to mixing with large marine and terrestrial carbon reservoirs (Levin and Kromer, 2004).

\subsection{Results}

\subsubsection{Radiocarbon Age}

CRAs were determined from five different individual samples (table 5.2), plus replicate samples on adjacent discs in two of the samples GOM-TOW-BC1 and GOM-TOW-BC2. Conventional ${ }^{14} \mathrm{C}$ ages were converted to calendar years before present (cal yr BP), and these results are presented in table 5.2 and reported as the median probability age. The 1 sigma calibrated age ranges are reported in appendix C. The oldest specimen (GOM-JSL09-3728-BC1) is from Viosca Knoll (VK862). The inner ${ }^{14} \mathrm{C}$ age of this specimen is $2,380 \pm 30{ }^{14} \mathrm{C}$ years; the corresponding calibrated age is $2040 \pm 40 \mathrm{cal}$ yr BP (table 5.2). Since this specimen was collected alive, the date of collection (2009) was used to calculate the life span, yielding 2,100 \pm 40 years. As discussed in the following text, the bomb-derived ${ }^{14} \mathrm{C}$ age model based on otolith and shallow coral $\Delta^{14} \mathrm{C}$ values and the bomb-derived ${ }^{14} \mathrm{C}$ detected in the outermost portion of this specimen can be used to estimate an age for the outermost portion of the skeleton. This is useful when it is not known if the specimen was collected alive. In this specimen, the outermost $\Delta^{14} \mathrm{C}$ value (53\%) corresponds to an age of 2,003 years common era (C.E), yielding a life span of 2,090 \pm 40 years (center age of $2,040 \pm 40$ years BP or 90 years before common era [B.C.E.]).

The second oldest specimen was collected from the head of De Soto Canyon (GOM-TOWBC2), with maximum center age dates between $2,100 \pm 40$ and $2,030 \pm 15{ }^{14} \mathrm{C}$ years, reflecting radiocarbon analysis from discreetly milled and delaminated samples, respectively (table 5.3). The reservoir-corrected calibrated ages are 1,720 $\pm 50 \mathrm{cal}$ yr BP and 1,630 $\pm 30 \mathrm{cal} \mathrm{yr} \mathrm{BP}$. Accounting for the ${ }^{14} \mathrm{C}$ age errors ( $\pm 30-50$ years), the minimum age differences from these replicate analyses, which were conducted at different laboratories on two adjacent discs using different subsampling techniques, are within 10 years of the ${ }^{14} \mathrm{C}$ age. The outermost sample from the GOM-TOW-BC2 disc that was discreetly drilled did not indicate the presence of bomb- ${ }^{14} \mathrm{C}(-49 \%)$. This was because the outermost layer, sampled at approximately 0.6 -millimeter $(\mathrm{mm})$ resolution, integrated approximately 75 years of growth, including years in which no bomb carbon was produced. In comparison, the outermost layer (about $200 \mu \mathrm{m}$ thick) from an adjacent disc that was delaminated yielded bomb- ${ }^{14} \mathrm{C}$ (47 \%o) (table 5.3). Using the bomb-derived ${ }^{14} \mathrm{C}$ age model based on otolith and shallow coral $\Delta^{14} \mathrm{C}$ values (see Discussion section), we determined that the outermost $\Delta^{14} \mathrm{C}$ values corresponded to an age of 1,964 years C.E., and the resultant life span of this sample was approximately 1,640 \pm 30 years. In comparison, using the 
difference between the date of collection (2003) and the inner ${ }^{14} \mathrm{C}$ age, we determined that the life span is $1,680 \pm 30$ years.

Specimen GOM-TOW-BC1 collected from the De Soto Canyon was age dated between 1,090 \pm 35 and $1,080 \pm 25{ }^{14} \mathrm{C}$ years, reflecting radiocarbon analysis from discreetly milled and delaminated samples, respectively (table 5.3). Reservoir-corrected age dates yielded center ages between $2040 \pm 40$ and $620 \pm 40 \mathrm{cal}$ yr BP (table 5.2). Unlike specimen GOM-TOW-BC2, there was no bomb- ${ }^{14} \mathrm{C}$ detected in the outermost skeletal samples in either disc from the GOM-TOW-BC1 specimen (tables 5.3 and 5.4). The outermost replicated samples yielded equivalent ages, $550 \pm 35$ and $550 \pm 60{ }^{14} \mathrm{C}$ years. The reservoir-corrected calibrated ages are $210 \pm 45$ and $205 \pm 65$ cal yr BP According to the inner and outer ${ }^{14} \mathrm{C}$ ages from the delaminated samples, this specimen had a life span of approximately $530 \pm 60$ years.

The two remaining samples were collected from separate sites on Viosca Knoll, were approximately 1,100 $\mathrm{m}$ apart, and occupied the same depth horizon, centered at $325 \mathrm{~m}$ (Sulak and others, 2007). The center age of GOM-JSL04-4734-BC1 was dated at $1,020 \pm 30{ }^{14} \mathrm{C}$ yrs $(620 \pm 40 \mathrm{cal}$ yr BP; table 5.2). The outermost $1.5 \mathrm{~mm}$ contained bomb-derived ${ }^{14} \mathrm{C}$ (72 \%o) (table 5.4). The postbomb age model suggests that the lifespan of this sample from Viosca Knoll is approximately $670 \pm 40$ years, with the outermost sample representing growth in the year 1997 (fig. 5.3). Incorporating the date of collection (2004) yielded a lifespan of $670 \pm 40$ years. In comparison, radiocarbon analysis of GOMJSL05-4876-BC1 yielded a center age of $1,260 \pm 30{ }^{14} \mathrm{C}$ years $(830 \pm 40 \mathrm{cal} \mathrm{yr} \mathrm{BP})$ (table 5.2). The outermost sample contained bomb-derived ${ }^{14} \mathrm{C}(69 \pm 3.6 \%$ o (table 5.4), corresponding to an age of 1998 C.E., according to the post-bomb age model, and yielding a life span of approximately $870 \pm 40$ years, or $890 \pm 40$ years given that the specimen was collected alive in 2005 .

\subsubsection{Growth Rates}

Radial growth rates were calculated by plotting reservoir-corrected ${ }^{14} \mathrm{C}$ age (year C.E.) versus sample distance from the outer edge (micrometers) (fig. 5.4A-E). Samples from the head of De Soto Canyon exhibited linear growth rates of 8-22 $\mu \mathrm{m} \mathrm{yr}^{-1}$, with the older sample (GOM-TOW-BC2) exhibiting a slower growth rate (table 5.2). Average radii from GOM-TOW-BC2 adjacent discs were 15 $\mathrm{mm}$ and $11.1 \mathrm{~mm}$ and yielded equivalent growth rates of $8 \mu \mathrm{m} \mathrm{yr}^{-1}$. Likewise, radii from GOM-TOWBC1 discs were $9.7 \mathrm{~mm}$ and $7.6 \mathrm{~mm}$ and yielded growth rates of $21 \mu \mathrm{m} \mathrm{yr}^{-1}$ and $22 \mu \mathrm{m} \mathrm{yr}^{-1}$, respectively. The growth rates were strongly linear, with $\mathrm{R}^{2}$ values between 0.85 and 0.97 (table 5.2). One exception was the specimen GOM-TOW-BC1, with an $\mathrm{R}^{2}$ value of $0.46(\mathrm{n}=29)$. Approximately 30 percent of the data (10 samples) were outside the age error window with equal distribution above and below this window (fig. 5.4e), and there was no systematic pattern in the distribution of these outliers in this specimen (GOM-TOW-BC1). In summary, five samples yielded ages that were younger than expected, and five samples yielded ages older than expected according to the linear-age growth model. Replicate radiocarbon analysis was conducted on two of the outliers, one sample each from above and below the regression line. Both replicates yielded similar ${ }^{14} \mathrm{C}$ ages, $635 \pm 30$ and $595 \pm 30$, and $480 \pm 30$ and $480 \pm$ 30 years at sample distances of $578 \mu \mathrm{m}$ and 3,048 $\mu \mathrm{m}$ from the outer edge, respectively.

The growth rates for the Viosca Knoll samples ranged from 8 to $17 \mu \mathrm{m} \mathrm{yr}^{-1}$ (table 5.2). At Viosca Knoll, the sample (GOM-JSL04-4734-BC1) with the shortest lifespan (670 \pm 40 cal yr BP) also displayed the fastest growth rate $17 \mu \mathrm{m} \mathrm{yr}^{-1}\left(\mathrm{R}^{2}=0.85\right.$; fig. $\left.5.4 D\right)$. With a radius of $14.3 \mathrm{~mm}$, the GOMJSL05-4876-BC1 sample from VK906/907 yielded a growth rate of $14 \mu \mathrm{m} \mathrm{yr}^{-1}\left(\mathrm{R}^{2}=0.96\right)$. In comparison, the older and larger sample from VK862 (GOM-JSL09-3728-BC1) with a radius of 18.1 $\mathrm{mm}$, yielded a slower growth rate $\left(8 \mu \mathrm{m} \mathrm{yr}^{-1} ; \mathrm{R}^{2}=0.87\right)$. There was an inverse relationship between coral ages (defined as lifespan) versus growth rate at both sites $\left(\mathrm{R}^{2}=0.88\right)$ (fig. 5.5). 
The uncertainty of the estimated lifespans and growth rates goes beyond just the uncertainty of the calibrated radiocarbon ages. By way of example, we calculated the uncertainty of the innermost calibrated radiocarbon age for the oldest specimen (GOM-JSL09-3728-BC1) by calculating the age differences of the median probability age from the minimum (55 years) and maximum ages (60 years) of the calibrated age range (cal BP; see Appendix C). For all samples, the largest age difference between the minimum and maximum ages or the median probability age was approximately 120 years, while the average of all such age differences for all calibrated radiocarbon results was approximately 60 years. For the age uncertainty of the outermost age, we presumed the worst-case scenario of not knowing whether the specimen was collected alive or dead and assigned a qualitative age uncertainty of \pm 5 years based the correlation of bomb-derived $\Delta^{14} \mathrm{C}$ in the specimen to an age model constructed using the bomb $\Delta^{14} \mathrm{C}$ curve from the otolith and shallow coral $\Delta^{14} \mathrm{C}$ curves (Baker and Wilson, 2001; Wagner, 2009; fig. 5.3). Thus, using an uncertainty of \pm 5 years for the outermost age and \pm 60 years (or worst case \pm 120 years for all the samples) and calculating the root mean squared error (RMSE) resulted in an uncertainty estimate of \pm 60 years (worst case \pm 120 years) for the life span. In calculating the uncertainty of the estimated growth rates, one must consider the estimated lifespan uncertainty along with the uncertainty of the radial distance measurement, which we qualitatively estimated at $\pm 1 \mathrm{~mm}$ for the GOM-JSL09-3728-BC1 specimen with a radial distance of $18.1 \mathrm{~mm}$. The estimated uncertainty of radial distance is purposely large in order to account for the asymmetry of the specimens and measurement error. By calculating the growth rate using minimum radial distance and maximum life span $\left(17.1 \mathrm{~mm} / 2,160 \mathrm{yr}=7.9 \mu \mathrm{m} \mathrm{yr}^{-1}\right)$ and comparing those results to growth rates calculated using maximum radial distance and minimum lifespan $\left(19.1 \mathrm{~mm} / 2,040 \mathrm{yr}=9.4 \mu \mathrm{m} \mathrm{yr}^{-1}\right)$, the resulting average growth rate estimate uncertainty will be on the order of approximately $\pm 1.5 \mu \mathrm{m} \mathrm{yr}^{-1}$. The results of this exercise show that lifespan differences greater than \pm 60 years on average and growth rate difference greater than $\pm 1.5 \mu \mathrm{m} \mathrm{yr}^{-1}$ are significant at the 1 sigma level.

\subsubsection{Bomb Radiocarbon and Post-bomb Age Model}

Bomb radiocarbon (values above a pre-bomb $\Delta^{14} \mathrm{C}$ mean of $-50 \%$ ) was found in all the specimens except the GOM-TOW-BC1 sample (table 5.4). The bomb- $\Delta^{14} \mathrm{C}$ curve was captured in the GOM-JSL04-4734-BC1 $\Delta^{14} \mathrm{C}$ record (fig. 5.3). Pre-bomb baseline values centered at $-56 \%$ with the first influx of bomb-derived ${ }^{14} \mathrm{C}$ at $1,504 \mu \mathrm{m}$. Post-bomb $\Delta{ }^{14} \mathrm{C}$ values increased from $-40 \%$ to $68 \%$ o within $238 \mu \mathrm{m}$. Pre-bomb averages (1943-1955) from red snapper (Lutjanus campechanus) otoliths $(n=26)$ in the GOM (Baker and Wilson, 2001) and shallow coral (Montastraea faveolata) $\Delta^{14} \mathrm{C}$ values from the Flower Garden Banks ( $\mathrm{n}=27$, Wagner and others, 2009; see GB535 on fig. 3.1) were -60 and $-53 \%$, respectively, representative of pre-bomb surface ocean $\Delta^{14} \mathrm{C}$ values. Bomb- ${ }^{14} \mathrm{C}$ values peaked $\left(119 \pm 59 \%\right.$ ) in the GOM-JSL04-4734-BC1 specimen at $827.25 \mu \mathrm{m}$ (fig. 5.3). In comparison, $\Delta^{14} \mathrm{C}$ values from the otolith data peaked at $177 \pm 91 \%$ in 1973 (Baker and Wilson, 2001), and shallow coral $\Delta^{14} \mathrm{C}$ values peaked at $158 \pm 35 \%$ in 1975 (Wagner, 2009). The deep-sea coral peak of $119 \%$ can be age dated between 1973 and 1975. In the remaining outer skeletal samples $(n=5)$, representing the outer $600 \mu \mathrm{m}, \Delta^{14} \mathrm{C}$ values decreased with the outermost sample yielding a $\Delta^{14} \mathrm{C}$ value of $72 \pm 36 \%$ at 125.3 $\mu \mathrm{m}$. The growth rate during the bomb spike can be calculated by using the local radiocarbon inflection point that is assigned a calendar year of 1957 and the bomb peak that is assigned a calendar year of 1975. With a collection date of 2003, growth rates were calculated over the following time intervals 1957-1975, 1975-2003, and 1957-2003, yielding linear $\left(\mathrm{R}^{2}=0.99-0.98\right)$ growth rates of 41, 24, and 33

$\mu \mathrm{m} \mathrm{yr}^{-1}$, respectively. These growth rates over the outermost $1.5 \mathrm{~mm}$ are on average twice as fast as the growth rate calculated over the entire radius of the specimen $\left(17 \mu \mathrm{m} \mathrm{yr}^{-1}\right)$. Despite displaying a faster 
growth rate over the outermost portion of growth, the average growth rate over the entire lifespan remained relatively linear $\left(\mathrm{R}^{2}=0.85\right.$; fig. 5.4D; table 5.2).

Bomb-derived ${ }^{14} \mathrm{C}$ from GOM-TOW-BC2 was only measured in the outermost delaminated sample (at a distance of $191 \mu \mathrm{m}$ from the outer edge) with a $\Delta^{14} \mathrm{C}$ value of $47 \pm 1.8 \%$. According to the age model, this yielded an approximate date of 1,964 yr C.E. Although sampled at less than a 200- $\mu \mathrm{m}$ interval in the outer $1 \mathrm{~mm}$, the remaining samples yielded pre-bomb $\Delta^{14} \mathrm{C}$ values, averaging $-60 \%$. After approximately $1.5 \mathrm{~mm}$ from the outer edge, $\Delta^{14} \mathrm{C}$ values decreased linearly with sample distance, indicative of radioactive decay. In contrast to the delaminated samples, the microdrilled samples did not yield bomb-derived ${ }^{14} \mathrm{C}$. Instead, the outermost drilled sample yielded a $\Delta^{14} \mathrm{C}$ value of $-49 \pm 33 \%$.

The outermost approximate $150 \mu \mathrm{m}$ for the samples GOM-JSL05-4876-BC1 and GOM-JSL09$3728-B C 1$ were subsampled at a higher resolution $(27-40-\mu \mathrm{m}$ increments) for radiocarbon analysis in order to capture the bomb peak. For both samples, $\Delta^{14} \mathrm{C}$ values decreased towards the outer edge; therefore, the age model based on the descending limb of the reference bomb- ${ }^{14} \mathrm{C}$ chronology was used to assign calendar ages. Accordingly, the outermost approximate $150 \mu \mathrm{m}$ for the samples GOM-JSL054876-BC1 and GOM-JSL09-3728-BC1 represented growth since 1996 and 1990 with dates of collection at 2005 and 2009, respectively. Accordingly, the growth rates over this period were 18 and 9 $\mu \mathrm{m}^{-1}$. In comparison to post-bomb growth rates calculated from the GOM-JSL04-4734-BC1 specimen, the latter two samples from Viosca Knoll did not exhibit a doubling in growth rate during the most recent decades.

\subsubsection{Band Counting}

Layer counting of sample GOM-JSL04-4734-BC1 based on an SEM image yielded an average age of $576 \pm 37$ years compared to the calculated life span of $670 \pm 40$ years, calculated as the difference between the reservoir-corrected inner layer and date of collection. Taking into account the

${ }^{14} \mathrm{C}$-age error (37 to 40 years), there is a minimum age difference of 21 years based on these two dating techniques. Visual ring counts in the outermost $1.77 \mathrm{~mm}$ (fig. 5.2C) were also compared to the high resolution chronology developed from the bomb- ${ }^{14} \mathrm{C}$ curve derived from otolith and shallow coral $\Delta^{14} \mathrm{C}$ values, as discussed below. According to this age model, the outermost skeletal growth in this specimen covered a time period of 49 years (1955 to 2004) (fig. 5.3B). Excluding the outer $300 \mu \mathrm{m}$ where the visual rings were indistinct, visual ring counts in the outermost $1.77 \mathrm{~mm}$ revealed approximately $55 \pm 3$ distinct bands (fig. 5.2C). Therefore, the estimated age error between these two age estimates is between 3 and 9 years.

\subsection{Discussion}

\subsubsection{Visual Ring Counts}

Previous studies of growth-ring structure in black corals indicated that the skeleton is formed of concentric coeval rings (Grange and Goldberg, 1993), such that visually counting the rings provides a means of developing radial growth chronologies. For example, in shallow-water black corals from the Red Sea, Risk and others (2009) found good correspondence between the number of visually counted rings and ages calculated using a bomb radiocarbon analysis compared to a local independent bomb curve. This approach has also been applied to gorgonian corals (Risk and others, 2002; Sherwood and others, 2005). In our current study, visual ring counts of GOM-JSL04-4734-BC1 based on SEM images yielded an average age of $576 \pm 37$ years compared to the calculated life span of $670 \pm 40$ years. The minimum age difference between these two age calculations is 17 years. Given a growth rate of $17 \mu \mathrm{m}$ $\mathrm{yr}^{-1}$, this age difference represents approximately $0.30 \mathrm{~mm}$ of skeletal growth. While black corals 
display radial growth, the growth is not necessarily symmetrical. In other words, the core can be offcenter. For example, the standard deviation on orthogonal radii was $\pm 1.5 \mathrm{~mm}$ for the GOM-JSL044734-BC1 specimen (fig. 5.2A). Therefore, the distance equivalent to calculated age difference $(0.30$ $\mathrm{mm}$ ) is within the variance associated with the growth asymmetry. Visual ring counting therefore appears to be a viable approach for aging black corals from the GOM. This observation is further validated by a strong similarity in ages based on visual ring counts in this specimen to the bomb-derived ${ }^{14} \mathrm{C}$ age models. The estimated age error between the two approaches, 3-9 years, suggests annual ring formation. Annual variations in food supply and surface productivity may be factors influencing annual skeletal growth layers (Sherwood, 2002); the exact mechanism to explain annual growth-ring formation remains unclear. While results from this present study support the notion that annual growth rings are formed by black corals as suggested by Williams and others (2007), further studies are required to validate this assumption.

\subsubsection{Radiocarbon Dating}

The reliability of ${ }^{14} \mathrm{C}$ age dating was investigated by conducting radiocarbon analysis on adjacent discs as well as running replicates of individually delaminated samples. The high degree of reproducibility found between discs as well as within duplicate subsamples highlights the robustness of ${ }^{14} \mathrm{C}$-derived chronologies and also demonstrates that exposure to $\mathrm{KOH}$ did not contaminate the geochemical signal. Williams (2009) also noted that exposure to $\mathrm{KOH}$ did not alter the stable isotope (carbon and nitrogen) signatures. Thus, delaminating the samples with $\mathrm{KOH}$ remains a reliable and effective approach to subsampling black corals (Leiopathes sp.) at high resolution (less than $200 \mu \mathrm{m}$ ).

Reservoir age-corrected ${ }^{14} \mathrm{C}$ ages suggested that deep-sea black corals in this region have grown continuously for at least the past 2 millennia (fig. 5.6). These results are consistent with previous work illustrating that black corals exhibit extreme longevities (Roark and others, 2009). As mentioned earlier, previous age dating of black corals in the GOM focused on extrapolated ages and growth rates based on skeletal lead-210 $\left({ }^{210} \mathrm{~Pb}\right)$ dating from limited samples (Williams and others, 2006, 2007). According to this work, their sample from Viosca Knoll yielded a life span of 386 years with an extrapolated growth rate of $14.5 \mu \mathrm{m} \mathrm{yr}^{-1}$. This sample was collected alive, and therefore, its skeletal growth extended into the early $17^{\text {th }}$ century (Williams, 2009). Results from our current study greatly expand the age and growth rate distribution of deep-sea black corals from the GOM. The difference in the results between the two studies reflects the temporal coverage of the two dating methods as well as the application of measured ages rather than extrapolated ages. Only the last 200 years or so can be fully resolved by ${ }^{210} \mathrm{~Pb}$ dating, beyond which growth rates and lifespan calculations have to be extrapolated. In contrast, radiocarbon dating is capable of dating specimens over the last approximately 50,000 years with uncertainties ranging from about \pm 20 to 400 years or greater depending on the age of the specimen. Thus, choosing the appropriate dating technique capable of resolving the time period in question is critical to obtaining accurate and precise age and growth rates.

\subsubsection{Growth Rates}

Long-term radial growth rates were calculated on black corals collected from the GOM, yielding growth rates ranging between 8 and $22 \mu \mathrm{m} \mathrm{yr}^{-1}$. These results are consistent with growth rates from Leiopathes sp. specimens from Hawaii (Roark and others, 2009), and clearly confirm that Leiopathes $\mathrm{sp}$. are the slowest growing deep-sea corals relative to other known deep-sea species. For example, antipatharian Stauropathes arctica collected off Newfoundland yielded growth rates of 33 to $66 \mu \mathrm{m} \mathrm{yr}^{-1}$ (Sherwood and Edinger, 2009). In comparison, growth rates measured in bamboo corals off California were estimated at 50-110 $\mathrm{m} \mathrm{yr}^{-1}$ (Andrews and others, 2005), 50-60 $\mu \mathrm{m} \mathrm{yr}^{-1}$ from the Gulf of Alaska 
(Roark and others, 2005), and $180 \mu \mathrm{m} \mathrm{yr}^{-1}$ from New Zealand (Tracey and others, 2007). Gerardia sp. from Hawaii, with average life spans of 950 years $(\mathrm{n}=23)$, yielded an overall average radial growth of $41 \mu \mathrm{m} \mathrm{yr}^{-1}$ (Parrish and Roark, 2009), while Druffel and others (1995) measured growth rates in Gerardia sp. from the Straits of Florida that were more consistent with Leiopathes sp. growth rates of about $5 \mu \mathrm{m} \mathrm{yr}^{-1}$ (Roark and others, 2009). This information is essential for assessing the vulnerability of these organisms to both natural and anthropogenic perturbations.

In most cases, radial growth rates were linear, except in one specimen (GOM-TOW-BC1) where the linear-age model yielded approximately 30 percent of the samples outside the age error window (fig. $5.4 E$ ). The lack of pattern in these outliers suggested no contamination or influx of older ${ }^{14} \mathrm{C}$, and replicate radiocarbon analyses did not indicate sampling contamination. This variance could represent natural ${ }^{14} \mathrm{C}$ variation (Druffel and others, 2008), which was possibly not captured in the other samples from the same location (GOM-TOW-BC2), due to a lower sample resolution (950- versus $220-\mu \mathrm{m}$ increments). These variations are most likely related to changes in atmospheric ${ }^{14} \mathrm{C}$ concentration that are only captured at relatively high sampling resolution for ${ }^{14} \mathrm{C}$ analysis. Changes in the ${ }^{14} \mathrm{C}$ production rate due to solar variability are believed to be the major causal factor for the atmospheric $\Delta^{14} \mathrm{C}$ variations during the Little Ice Age (LIA) (Stuiver and Quay, 1980; Bard and others, 1997).

Atmospheric ${ }^{14} \mathrm{C}$ variation derived from tree rings during the LIA (approximately $1300-1850$ C.E.) indicate $\Delta^{14} \mathrm{C}$ variability with amplitudes of about 20 to $28 \%$ (Reimer and others, 2004). This is consistent with the range of $\Delta^{14} \mathrm{C}$ variability observed in the GOM-TOW-BC1 record (fig. 5.4f). To fully explore the idea that ${ }^{14} \mathrm{C}$ records in Leiopathes sp. can be used as a water-mass tracer will require the development of independent chronologies (maybe growth band counts) as well as replication studies using higher sampling frequencies for radiocarbon analysis.

Earlier work with Gerardia sp. (Druffel and others, 1995) suggested that DSCs exhibit high rates of growth early in life. Likewise, Roark and others (2009) also found faster radial growth rates over the initial 400 years of a 2,370-year-old Leiopathes sp. from Hawaii. While results from our study did not find faster initial growth rates within individual specimens, as concluded from calculating linear growth rates $\left(\mathrm{R}^{2}\right.$ values between 0.85 and 0.97$)$, there is evidence of an inverse relationship between age (defined as lifespan) and growth rate at both sites (fig. 5.6). Based on this relationship, growth rates decreased as the lifespan of the coral increased. For example, the growth rate of the oldest specimen, GOM-JSL09-3728-BC1 during the first 600 years is $16 \mu \mathrm{m} \mathrm{yr}^{-1}\left(\mathrm{R}^{2}=0.67\right)$, which is similar to the growth rate measured in GOM-JSL04-4734-BC1 that has a lifespan of 670 years. Over the course of the specimen's lifespan, however, the average growth rate is slower, $8 \mu \mathrm{m} \mathrm{yr}^{-1}\left(\mathrm{R}^{2}=0.87\right)$. Therefore, older specimens may be more vulnerable to disturbance as a result of slower average growth rates over the entire lifespan of the specimen. Given the extremely long lifespans and very slow growth rates documented (even in the younger corals) in the GOM from our study and in the Pacific (Roark and others, 2006, 2009), it is unlikely that these species are renewable within the context of fishery management or even within a human lifespan. Any bottom-disturbing activity (for example, bottom trawling, energy exploitation, anchoring) could pose a serious threat to these organisms. Damage to black corals in the GOM will have long-term implications to the biodiversity in these deep-water reefs, requiring management efforts to protect sea-floor habitats (National Marine Fisheries Service and National Oceanic and Atmospheric Adminstration, 2002).

\subsubsection{Bomb Signal}

Given the reference bomb- ${ }^{14} \mathrm{C}$ chronology derived from otolith and shallow coral $\Delta{ }^{14} \mathrm{C}$ records, bomb- ${ }^{14} \mathrm{C}$ measurements in deep-sea black corals can be used to date these specimens (Roark and others, 2005; Sherwood and others, 2005; Sherwood and Edinger, 2009). While the date of collection 
can be used to calculate lifespans and growth rates for specimens collected alive, samples where the tissue layer has decayed and (or) there is uncertainty as to whether the samples were collected alive (as with archived samples), an alternative approach is required. Therefore, as demonstrated in this study, age models constructed from the rising and descending limbs of the bomb curves can be used to reliably age date the outermost ring samples.

The outermost 1.25-mm layers of black coral contain bomb-derived carbon. The exception was the microdrilled GOM-TOW-BC2 sample which yielded a $\Delta^{14} \mathrm{C}$ value of $-49 \pm 3.3 \%$. This is in contrast to the outermost delaminated GOM-TOW-BC2 sample that yielded a $\Delta^{14} \mathrm{C}$ value of $47 \pm 1.8$ \%. Because the outermost microdrilled sample incorporates skeletal material over a distance of about $600 \mu \mathrm{m}$, the sample integrates over 75 years of growth, including years in which no bomb carbon was produced. Thus, at sufficient sample resolution, the proteinaceous skeleton from black corals can capture the surface-water $\Delta^{14} \mathrm{C}$ history. The ability of the black corals to capture the surface-water $\Delta^{14} \mathrm{C}$ history is a function of the animal's organic carbon source, surface-derived POM. If this were not the case, then the outer skeletal material would yield more depleted $\Delta^{14} \mathrm{C}$ values. However, given the enriched $\Delta^{14} \mathrm{C}$ values measured in the outer skeletal material (table 5.4), there is no evidence indicating that the corals were feeding on old carbon. This in contrast to coral calcite from bamboo corals, for example, where the carbonate material reflects the $\Delta^{14} \mathrm{C}$ of the dissolved inorganic carbon (DIC) pool. For example, Roark and others (2005) showed the coral calcite from a bamboo coral reflected seawater $\Delta^{14} \mathrm{C}$ at approximately $700 \mathrm{~m}$. The deep-sea coral record indicates a dramatic increase in $\Delta^{14} \mathrm{C}$. Within a distance of $112 \mu \mathrm{m}, \Delta^{14} \mathrm{C}$ values increased from $-40 \%$ to $68 \%$. The $40-\%$ offset between the surface and deep-sea coral $\Delta^{14} \mathrm{C}$ peak could reflect mixing with deep water, or more likely, that the subsampling resolution for ${ }^{14} \mathrm{C}$ analysis in the coral disc was not sufficient to capture the peak.

Radiocarbon time series from scleractinian corals provide information about surface and shallow circulation that can be used to test ocean dynamics in circulation models. For example, Grumet and others (2005) conducted a model-data comparison by using surface radiocarbon time series from coral records from the coasts of Kenya and Sumatra and a suite of dynamic three-dimensional ocean models to test parameterizations of mixing and air-sea gas exchange between the ocean and atmosphere. The similarity of the $\Delta^{14} \mathrm{C}$ history between the Flower Garden Banks shallow coral record and the deep-sea VK862 record (GOM-JSL04-4734-BC1) demonstrates that $\Delta^{14} \mathrm{C}$ deep-sea coral records with independent chronologies can expand the temporal and spatial records used to document ocean $\Delta^{14} \mathrm{C}$ variability.

\subsection{Conclusions}

Results from our study represent the first comprehensive investigation of growth rates and age distributions of black coral in the GOM and provide the quantitative background to assess negative impacts. Results from this work highlight the fact that black corals in particular are the slowest growing deep-sea corals and exhibit extreme longevities. Radiocarbon analysis of specimens from the GOM indicated that these animals have been growing continuously for at least the last 2 millennia, with growth rates ranging from 8 to $22 \mu \mathrm{m} \mathrm{yr}^{-1}$. Furthermore, the high degree of reproducibility found between discs, as well as within duplicate subsamples, validates the robustness of ${ }^{14} \mathrm{C}$-derived chronologies. Reliable age models can be applied to multi-decadal paleoclimate reconstructions derived from the skeletal geochemistry. The presence of bomb-derived ${ }^{14} \mathrm{C}$ in the outermost ring samples of black coral skeletons confirms sinking POM as the dominant carbon source. Additionally, deprivation of surface-derived food sources could have adverse indirect effects to the deep-reef community given its dependence on sinking POM. We anticipate that the recovery of deep-sea corals, particularly black corals, would take at least decades to centuries. These reefs are unique components of the GOM, and 
monitoring their growth rates, age distribution, and other ecological characteristics will provide scientific data that can be used to protect them.

\subsection{Acknowledgments}

The author wishes to thank S. Griffin and E. Druffel (University of California, Irvine) and T. Guilderson (Lawrence Livermore National Laboratory) for assistance with radiocarbon analysis; K.

Sulak (USGS) and S. Cairns (Smithsonian Institution National Museum of Natural History) for donating samples; D. Opresko (Smithsonian) for species identification of some samples; and C. Holmes (USGS) for providing supplementary radiocarbon data. Gratitude is also extended to the entire USGS Diversity, Systematics, and Connectivity of Vulnerable Reef Ecosystems (DISCOVRE) team for their support and collaboration.

\subsection{References}

Abramoff, M.D., Magelhae, P.J., and Ram, S.J., 2004, Image processing with ImageJ: Biophotonics International, v. 11, no. 7, p. 36-42.

Andrews, A.H., Cailliet, G.M., Kerr, L.A., Coale, K.H., Lundstrom, C., and DeVogelaere, A., 2005 , Investigations of age and growth for three species of deep-sea coral from the Davidson Seamount off central California, in Freiwald, A.J.M.R., ed., Cold-water corals and ecosystems: Berlin, SpringerVerlag, p. 965-982.

Baker, M.S., and Wilson, C.A., 2001, Use of bomb radiocarbon to validate otolith section ages of Red Snapper Lutjanus campechanus from the Northern Gulf of Mexico: Limnology Oceanography, v. 46, no. 7, p. 1819-1824.

Bard, E., Raisbeck, G.M., Yiou, F., and Jouzel, J., 1997, Solar modulation of cosmogenic nuclide production over the last millennium: comparison between ${ }^{14} \mathrm{C}$ and ${ }^{10} \mathrm{Be}$ records: Earth and Planetary Science Letters, v. 150, p. 453-462.

Becker, E.L., Cordes, E.E., Macko, S.A., and Fisher, C.R., 2009, Importance of seep primary production to Lophelia pertusa and associated fauna in the Gulf of Mexico: Deep-Sea Research Part I, v. 56, p. 786-800.

Broecker, W.S., and Peng, T.S., 1982, Tracers in the sea: Palisades, New York, Eldigio Press, 690 p. Brooke, S., and Schroeder, W.W., 2007, State of deep coral ecosystems in the Gulf of Mexico Region: Texas to the Florida Straits, in Lumsden, S.E., Hourigan, T.F., Bruckner, A.W., and Dorr, G., eds., The state of deep coral ecosystems of the United States: Silver Spring, Md., National Oceanic and Atmospheric Administration Technical Memorandum CRCP-3, p. 271-306.

Cordes, E.E., McGinley, M.P., Podowski, E.L., Becker, E.L., Lessard-Pilon, S., Viada, S.T., and Fisher, C.R., 2008, Coral communities of the deep Gulf of Mexico: Deep-Sea Research Part I, v. 55, no. 6, p. 777-787.

CSA International, Inc., 2007, Characterization of northern Gulf of Mexico deepwater hard-bottom communities with emphasis on Lophelia coral: U.S. Department of Interior, Minerals Management Service, Gulf of Mexico OCS Region, OCS Study MMS 2007-044, 169 p.

Davies, A.J., Duineveld, G.C.A., van Weering, T.C.E., Mienis, F., Quattrini, A.M., Seim, H.E., Bane, J.M., and Ross, S.W., 2010 Short-term environmental variability in cold-water coral habitat at Viosca Knoll, Gulf of Mexico: Deep-Sea Research Part I, v. 57, no. 2, p. 199-212.

Druffel, E.R.M., Griffin, S., Witter, A., Nelson, E., Southon, J., Kashgarian, M., and Vogel, J., 1995 , Gerardia: Bristlecone pine of the deep-sea?: Geochimica et Cosmochimica Acta, v. 59, no. 23, p. 5031-5036. 
Druffel, E.R.M., Robinson, L.F., Griffin, S., Halley, R.B., Southon, J.R., and Adkins, J.F., 2008, Low reservoir ages for the surface ocean from mid-Holocene Florida corals: Paleoceanography, v. 23, PA2209, doi:101029/2007PA001527.

Duineveld, G.C.A., Lavaleye, M.S.S., and Berghuis, E.M., 2004, Particle flux and food supply to a seamount cold-water coral community (Galicia Bank, NW Spain): Marine Ecology Progress Series, v. 277 , p. 13-23.

Goldberg, W., Hopkins, T.L., Holl, S.M., Schaefer, J., Kramer, K.J., Morgan, T.D., and Kim, K., 1994, Chemical composition of the sclerotized black coral skeleton (Coelenterata: Antipatharia): A comparison of two species: Comparative Biochemistry and Physiology Part B, v. 107B, p. 633-643. Goldberg, W.M., 1991, Chemistry and structure of skeletal growth rings in the black coral Antipathes fiordensis (Cnidaria, Antipathatia): Hydrobiologia, v. 216, p. 403-409.

Grange, K.R., 1985, Distribution, standing crop, population structure, and growth rates of black corals in the Southern fiord of New Zealand: New Zealand Journal of Marine and Freshwater Research, v. 19 , no. 4., p. 467-475.

Grange, K.R., and Goldberg, W.M., 1993, Chronology of black coral growth bands: 300 years of environmental history?: in Battershill, C., ed., Proceedings of the Second International Temperature Reef Symposium: Auckland, New Zealand, NIWA Marine Wellington, p. 169-174.

Grigg, R.W., 1965, Ecological studies on black coral in Hawaii: Pacific Science, v. 19, p. 244-260.

Grumet, N.S., Duffy, P.B., Wickett, M.E., Caldeira, K., and Dunbar, R.B., 2005, Intrabasin comparison of surface radiocarbon levels in the Indian Ocean between coral records and three-dimensional global ocean models: Global Biogeochemical Cycles, v. 19, no. 2, doi:10.1029/2004GB002289.

Hovland, M., 1990, Do carbonate reefs form due to fluid seepage?: Terra Nova, v. 2, p. 8-18.

Hughen, K., Kaiser, K., McCormac, F., Mannings, W., Reimer, R., Richards, D., Southon, J., Talamo, S., Turney, C., and Van der Plicht, J., 2009, IntCa109 and Marine09 calibration curves: Radiocarbon, v. 51, p. 1111-1150.Kim, K., Goldberg, W.M., and Taylor, G.T., 1992, Architectural and mechanical properties of the black coral skeleton (Coelenterata: Antipatharia): A comparison of two species: Biological Bulletin, v. 182, no. 2, p. 195-209.

Kiriakoulakis, K., Bett, B.J., White, M., and Wolff, G.A., 2004, Organic biogeochemistry of the Darwin Mounds, a deep-water coral ecosystem, of the NE Atlantic: Deep-Sea Research Part I, v. 51, no. 12, p. 1937-1954.

Levin, I., and Kromer, B., 2004, The tropospheric ${ }^{14} \mathrm{CO}_{2}$ level in mid-latitudes of the Northern Hemisphere (1959-2003): Radiocarbon, v. 46, p. 1261-1272.

Lewis, J.B., 1978, Feeding mechanisms in black corals (Antipatharia): Journal of Zoology, v. 186, p. 393-396.

Lumsden, S.E., Hourigan, T.F., Bruckner, A.W., and Dorr, G., eds., 2007, The state of deep coral ecosystems of the United States: Silver Spring, Md., National Oceanic and Atmospheric Administration Technical Memorandum CRCP-3.

Miller, K., Williams, A., Rowden, A.A., Knowles, C., and Dunshea, G., 2010, Conflicting estimates of connectivity among deep-sea coral populations: Marine Ecology, v. 31, p. 144-157.

Miller, K.J., 1997, Genetic structure of black coral populations in New Zealand's fiord: Marine Ecology Progress Series, v. 161, p. 123-132.

Miller, K.J., 1998, Short-distance dispersal of black coral larvae: Inference from spatial analysis of colony genotypes: Marine Ecology Progress Series, v. 163, p. 225-233.

National Marine Fisheries Service and National Oceanic and Atmospheric Adminstration, 2002, Fisheries off west coast states and the western Pacific; precious corals fisheries; harvest quotas, 
definitions, size limits, gear restrictions, and bed classifications: Federal Register, v. 67, no. 52, p. 11941-11945.

Newton, E.D., and Bak, R.P.M., 1979, Ecological aspects of Antipatharia (black corals) in Curacao:

Proceedings of the Association of Island Marine Laboratories of the Caribbean, v. 14, p. 14.

Nowak, D., Florek, M, Nowak, J., Kwiatek, W., Lekki, J., Chevallier, P., Hacura, A., and Wrzalik, R., Ben-Nissan, B., Van Grieken, R., and Kuczumow, A., 2009, Morphology and the chemical make-up of the inorganic components of black corals: Materials Science and Engineering C, v. 29, no. 3, p. 1029-1038.

Oakley, S.G., 1988, Settlement and growth of Antipathes pennacea on a shipwreck: Coral Reefs, v. 7, p. 77-79.

Opresko, D.M., 2001, Revision of the Antipatharia (Cnidaria: Anthozoa). Part I. Establishment of a new family, Myriopathidae: Zoologische Mededelingen, v. 75, p. 147-174.

Opresko, D.M., 2002, Revision of the Antipatharia (Cnidaria: Anthozoa). Part II. Schizopathidae: Zoologische Mededelingen, v. 76, p. 411-442.

Opresko, D.M., 2003, Revision of the Antipatharia (Cnidaria: Anthozoa). Part III. Cladopathidae: Zoologische Mededelingen, v. 77, p. 495-536.

Opresko, D.M., 2004, Revision of the Antipatharia (Cnidaria: Anthozoa). Part IV. Establishment of a new family, Aphanipathidae: Zoologische Mededelingen, v. 78, p. 209-240.

Parrish, F.A., Abernathy, K., Marshall, G.J., and Buhleier, B.M., 2002, Hawaiian monk seals (Monachus schauinslandi) foraging in deep-water coral beds: Marine Mammal Science, v. 18, p. 244258.

Parrish, F.A., and Roark, E.B., 2009, Growth validation of gold coral Gerardia sp. in the Hawaiian Archipelago: Marine Ecology Progress Series, v. 397, p. 163-172.

Prouty, N.G., Roark, E.B., Buster, N.A., and Ross, S.W., 2011, Growth rate and age distribution of deep-sea black corals in the Gulf of Mexico: Marine Ecology Progress Series, v. 423, p. 101-115.

Reimer, P.J., Baillie, M.G.L., Bard, E., Bayliss, A., Beck, J.W., Bertrand, C.J.H., Blackwell, P.G., Buck, C.E., Burr, G.S., Cutler, K.B., Damon, P.E., Edwards, R.L., Fairbanks, R.G., Friedrich, M., Guilderson, T.P., Hogg, A.G., Hughen, K.A., Kromer, B., McCormac, G., Manning, S., Bronk Ramsey, C., Reimer, R.W., Remmele, S., Southon, J.R., Stuiver, M., Talamo, S., Taylor, F.W., van der Plicht, J., and Weyhenmeyer, C.E., 2004, IntCal04 terrestrial radiocarbon age calibration, 0-26 kyr BP: Radiocarbon, v. 46, p. 1029-1058.

Risk, M., Heikoop, J., Snow, M., and Beukens, R., 2002, Lifespans and growth patterns of two deep-sea corals: Primnoa resedaeformis and Desmophyllum cristagalli: Hydrobiologia, v. 471, p. 125-131.

Risk, M.J., Sherwood, O.A., Nairn, R., and Gibbons, C., 2009, Tracking the record of sewage discharge off Jeddah, Saudi Arabia, since 1950, using stable isotope records from antipatharians: Marine Ecology Progress Series, v. 397, p. 219-226.

Roark, E.B., Guilderson, T.P., Dunbar, R.B., Fallon, S.J., and Mucciarone, D.A., 2009, Extreme longevity in proteinaceous deep-sea corals: Proceedings of the National Academy of Sciences, v. 106, no. 13, p. 5204-5208.

Roark, E.B., Guilderson, T.P., Flood-Page, S., Dunbar, R.B., Ingram, B.L., Fallon, S.J., and McCulloch, M., 2005, Radiocarbon-based ages and growth rates of bamboo corals from the Gulf of Alaska: Geophysical Research Letters, v. 32, L04606, doi:10.1029/2004GL021919.

Roark, E.B., Thomas, P.G., Robert, B.D., and Ingram, B.L., 2006, Radiocarbon-based ages and growth rates of Hawaiian deep-sea corals: Marine Ecology Progress Series, v. 327, p. 1-14.

Roberts, J.M., Wheeler, A., Freiwald, A., and Cairns, S., 2009, Cold-water corals: The biology and geology of deep-sea coral habitats: Cambridge, Cambridge University Press, 367 p. 
Sánchez, J.A., 1999, Black coral—octocoral distribution patterns on Imelda Bank, a deep-water reef, Colombia, Caribbean Sea: Bulletin of Marine Science, v. 65, p. 215-225.

Schroeder, W.W., Brooke, S.D., Olson, J.B., Phaneuf, B., McDonough, J.J., and Etnoyer, P., 2005, Occurrence of deep-water Lophelia pertusa and Madrepora oculata in the Gulf of Mexico, in Freiwald, A., and Roberts, J.M., eds., Cold-water corals and ecosystems spring: Berlin, Heidelberg, p. 297-307.

Sherwood, O., 2002, The deep-sea gorgonian coral Primnoa resedaeformis as an oceanographic monitor: Hamilton, Ontario, McMaster University, Master's Thesis, 65 p.

Sherwood, O.A., and Edinger, E.N., 2009, Ages and growth rates of some deep-sea gorgonian and antipatharian corals of Newfoundland and Labrador: Canadian Journal of Fisheries and Aquatic Sciences, v. 66, no. 1, p. 142-152.

Sherwood, O.A., Scott, D.B., Risk, M.J., and Guilderson, T.P., 2005, Radiocarbon evidence for annual growth rings in the deep-sea octocoral Primnoa resedaeformis: Marine Ecology Progress Series, v. 301, p. 129-134.

Stuiver, M., and Polach, H.A., 1977, Discussion: reporting of ${ }^{14} \mathrm{C}$ data: Radiocarbon, v. 19, no. 3, p. 355-363.

Stuiver, M., and Quay, P.D., 1980, Changes in atmospheric carbon-14 attributed to a variable sun: Science, v. 207, p. 11-19.

Stuiver, M., and Reimer, P.J., 1993, Extended ${ }^{14} \mathrm{C}$ database and revised CALIB radiocarbon calibration program: Radiocarbon, v. 35, p. 215-230.

Sulak, K.J., Brooks, R.A., Luke, K.E., Norem, A.D., Randall, M., Quaid, A.J., Yeargin, G.E., Miller, J.M., Harden, W.M., Caruso, J.H., and Ross, S.W., 2007, Demersal fishes associated with Lophelia pertusa coral and hard-substrate biotopes on the continental slope, northern Gulf of Mexico, in George, R.Y., and Cairns, S.D., eds., Conservation and adaptive management of seamount and deepsea coral ecosystems: University of Miami, p. 65-92.

Sulak, K.J., Randall, M.T., Luke, K.E., Norem, A.D., and Miller, J.M., 2008, Characterization of Northern Gulf of Mexico deepwater hard bottom communities with emphasis on Lophelia coralLophelia reef megafaunal community structure, biotopes, genetics, microbial ecology, and geology (2004-2006): U.S. Geological Survey Open-File Report 2008-1148; OCS Study MMS 2008-015, accessed May 2, 2017, at http://dx.doi.org/10.3133/ofr20081148.

Tracey, D.M., Neil, H., Marriott, P., Andrews, A.H., Cailliet, G.M., and Sánchez, J.A., 2007, Age and growth of two genera of deep-sea bamboo corals (family Isididae) in New Zealand waters: Bulletin of Marine Science, v. 81, no. 3, p. 393-408.

Wagner, A.J., 2009, Oxygen and carbon isotopes and coral growth in the Gulf of Mexico and Caribbean Sea as environmental and climate indicators: College Station, Tex., Texas A\&M University, Ph.D. dissertation, $117 \mathrm{p}$.

Wagner, A.J., Guilderson, T.P., Slowey, N., and Cole, J.E., 2009, Pre-bomb surface water radiocarbon of the Gulf of Mexico and Caribbean as recorded in hermatypic corals: Radiocarbon, v. 51, no. 3, p. 947-954.

Warner, G.F., 1981, Species descriptions and ecological observations of black corals (Antipatharia) from Trinidad: Bulletin of Marine Science, v. 31, no. 1, p. 147-163.

Williams, B., 2009, Biogeochemistry of soft corals and black corals, and implication for paleoceanography in the western tropical Pacific: Columbus, Ohio, The Ohio State University, Ph.D. thesis, $209 \mathrm{p}$.

Williams, B., Risk, M.J., Ross, S.W., and Sulak, K.J., 2006, Deep-water antipatharians: Proxies of environmental change: Geology, v. 34, p. 773-776. 
Williams, B., Risk, M.J., Ross, S.W., and Sulak, K.J., 2007, Stable isotope data from deep-water antipatharians: 400-year records from the southeastern coast of the United States of America: Bulletin of Marine Science, v. 81, p. 437-447. 
Table 5.1. Sample identification (ID) and location for Leiopathes sp. specimens collected between 2003 and 2009 within the Gulf of Mexico from two different regions: the head of De Soto Canyon (archived at the U.S. National Museum) and Viosca Knoll during the Lophelia II program.

[Samples IDs include information about whether the samples were collected by trawl (TOW) or manned submersible, Johnson Sea Link II (JSL); m, meter]

\begin{tabular}{lccccc}
\hline \multicolumn{1}{c}{ Sample ID } & Collection date & Site & Latitude & Longitude & $\begin{array}{c}\text { Water } \\
\text { Depth (m) }\end{array}$ \\
\hline GOM-TOW-BC1 & 15 Nov 03 & De Soto Canyon & $29^{\circ} 32.24^{\prime} \mathrm{N}$ & $86^{\circ} 52.19^{\prime} \mathrm{W}$ & 304 \\
GOM-TOW-BC2 & 15 Nov 03 & De Soto Canyon & $29^{\circ} 32.24^{\prime} \mathrm{N}$ & $86^{\circ} 52.19^{\prime} \mathrm{W}$ & 304 \\
GOM-JSL05-4876-BC1 & 17 Sep 05 & Viosca Knoll 906/907 & $29^{\circ} 06.42^{\prime} \mathrm{N}$ & $88^{\circ} 23.08^{\prime} \mathrm{W}$ & 312 \\
GOM-JSL09-3728-BC1 & 20 Sep 09 & Viosca Knoll 862 & $29^{\circ} 06.41^{\prime} \mathrm{N}$ & $88^{\circ} 23.10^{\prime} \mathrm{W}$ & 317 \\
GOM-JSL04-4734-BC1 & 23 Jul 04 & Viosca Knoll 862 & $29^{\circ} 06.22^{\prime} \mathrm{N}$ & $88^{\circ} 23.05^{\prime} \mathrm{W}$ & 310 \\
\hline
\end{tabular}

Table 5.2. Lifespans and radiocarbon $\left({ }^{14} \mathrm{C}\right)$ ages of coral samples collected during the Lophelia II program.

[Cal yr BP, calendar years before present; $\mu \mathrm{m} \mathrm{yr}^{-1}$, micrometers per year; $\mathrm{yr}$, year, ${ }^{14} \mathrm{C}$, radiocarbon. Calendar years $\mathrm{BP}$ are reported as median probability age. See Appendix $\mathrm{C}$ for 1 sigma calibrated age ranges. Age errors ranged between \pm 30 and 65 years. The life spans were calculated as the difference between the inner sample calendar year and the date of collection, unless the outer sample did not contain modern $14 \mathrm{C}$, then the difference in ${ }^{14} \mathrm{C}$ age was used. Growth rates in parentheses indicate rates calculated on adjacent discs from microdrilling an outer, middle, and inner sample. Regression coefficients are listed for the linear-age models.]

\begin{tabular}{lcccccc}
\hline \multicolumn{1}{c}{ Sample } & $\begin{array}{c}{ }^{14} \mathbf{C} \text { age } \\
\text { inner }\end{array}$ & Cal yr BP & $\begin{array}{c}{ }^{14} \mathbf{C} \text { age } \\
\text { Outer }\end{array}$ & $\begin{array}{c}\text { Growth rate } \\
(\boldsymbol{\mu} \mathbf{m} \text { yr-1) }\end{array}$ & $\mathbf{R}^{2}$ & Life span (yr) \\
\hline GOM-TOW-BC1 & $1,080 \pm 25$ & $670 \pm 36$ & $550 \pm 60$ & $22(21)$ & 0.46 & 530 \\
GOM-TOW-BC2 & $2,030 \pm 15$ & $1,630 \pm 30$ & modern & $8(8)$ & 0.97 & 1,680 \\
GOM-JSL04-4734-BC1 & $1,020 \pm 30$ & $620 \pm 40$ & modern & 17 & 0.85 & 670 \\
GOM-JSL05-4876-BC1 & $1,260 \pm 30$ & $830 \pm 40$ & modern & 14 & 0.96 & 890 \\
GOM-JSL09-3728-BC1 & $2,380 \pm 30$ & $2,040 \pm 40$ & modern & 8 & 0.87 & 2,100 \\
\hline
\end{tabular}


Table 5.3. Replicate radiocarbon analysis of adjacent discs collected during the Lophelia II program using different subsampling techniques (microdrilling and chemical band separation) at different AMS laboratories.

[\%o, per mil, ${ }^{14} \mathrm{C}$, radiocarbon. Analytical uncertainty for $\Delta{ }^{14} \mathrm{C}$ was 1.3 to 3.9 per mil. Those samples analyzed at LLNL were subsampled by microdrilling an inner, middle, and outer sample, whereas the remaining samples were chemically separated using potassium hydroxide $(\mathrm{KOH})]$

\begin{tabular}{cccccc}
\hline Sample & ${ }^{14} \mathrm{C}$ age (inner) & ${ }^{14} \mathrm{C}$ age (outer) & $\begin{array}{c}\text { Outer } \Delta^{14} \mathrm{C} \\
(\%)\end{array}$ & Inner $\Delta^{14} \mathrm{C}(\%)$ & $\begin{array}{c}\text { Number of } \\
\text { samples }\end{array}$ \\
\hline GOM-TOW-BC1 ${ }^{1}$ & $1,090 \pm 35$ & $550 \pm 35$ & $-73 \pm 3.9$ & $-133 \pm 3.4$ & 3 \\
GOM-TOW-BC1 $^{2}$ & $1,080 \pm 25$ & $550 \pm 60$ & $-73 \pm 3.5$ & $-132 \pm 2.7$ & 35 \\
GOM-TOW-BC2 $^{3}$ & $2,030 \pm 15$ & modern & $47 \pm 1.8$ & $-229 \pm 1.3$ & 12 \\
GOM-TOW-BC2 $^{1}$ & $2,100 \pm 40$ & $350 \pm 30$ & $-49 \pm 3.3$ & $-235 \pm 3.5$ & 3 \\
\hline
\end{tabular}

${ }^{1}$ The Center for Accelerator Mass Spectrometry at Lawrence Livermore National Laboratory;

${ }^{2}$ BetaAnalytic; ${ }^{3}$ The Keck Carbon Cycle AMS Laboratory at the University of California Irvine

Table 5.4. Bomb-derived ${ }^{14} \mathrm{C}$ values in specimens collected as part of the Lophelia II program.

[Carbon-14 values are defined as those values above a pre-bomb mean of -50 per mil (\%o) that were analyzed in all the specimens except the GOM-TOW-BC1 sample. Analytical uncertainty for $\Delta^{14} \mathrm{C}$ was \pm 1.3 to $3.9 \%$. The reported radius represents the mean of four orthogonal radii measured on each disc with a standard deviation of between 1 to 2 millimeters $(\mathrm{mm})$ ]

\begin{tabular}{lcccc}
\hline \multicolumn{1}{c}{ Sample } & Outer $\Delta^{14} \mathrm{C}(\%)$ & Inner $\Delta^{14} \mathrm{C}(\%)$ & $\begin{array}{c}\text { Number of } \\
\text { samples }\end{array}$ & Radius (mm) \\
\hline GOM-TOW-BC1 ${ }^{2}$ & $-73 \pm 3.5$ & $-132 \pm 2.7$ & 35 & 7.6 \\
GOM-TOW-BC2 ${ }^{1}$ & $47 \pm 1.8$ & $-229 \pm 1.3$ & 12 & 11.4 \\
GOM-JSL04-4734-BC1 ${ }^{2,3}$ & $72 \pm 3.6$ & $-126 \pm 3.1$ & 48 & 8.8 \\
GOM-JSL05-4876-BC1 ${ }^{1}$ & $69 \pm 3.6$ & $-151 \pm 2.8$ & 10 & 14.3 \\
GOM-JSL09-3728-BC1 ${ }^{1}$ & $53 \pm 3.5$ & $-261 \pm 2.5$ & 11 & 18.1 \\
\hline
\end{tabular}

${ }^{1}$ The Keck Carbon Cycle AMS Laboratory at the University of California Irvine; ${ }^{2}$ BetaAnalytic; ${ }^{3}$ The National Ocean Sciences Accelerator Mass Spectrometry Facility at Woods Hole Oceanographic Institution 


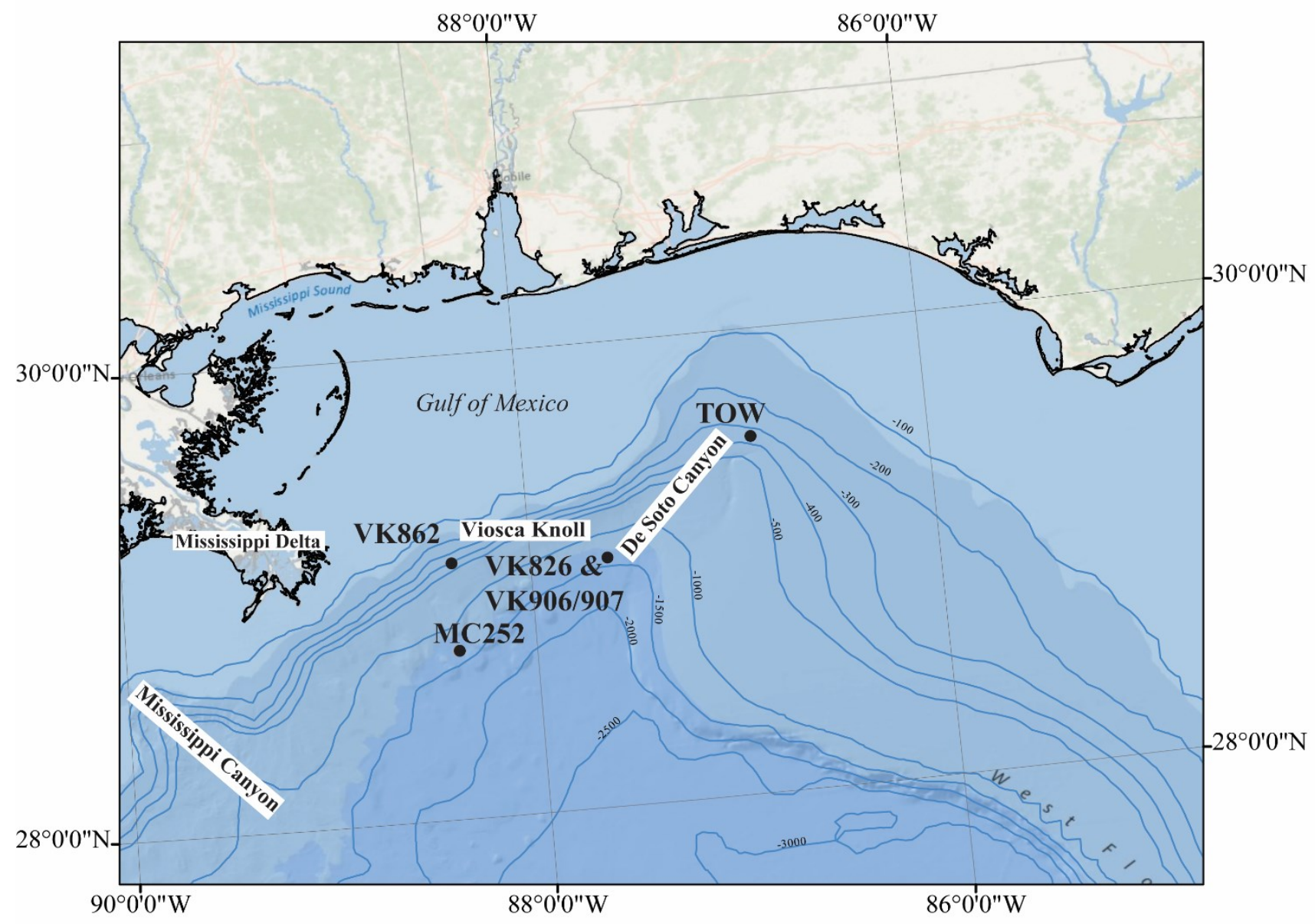

Figure 5.1 Map showing collection sites of Leiopathes sp. between 2003 and 2009 during the Lophelia II program from two different regions: the head of De Soto Canyon (TOW) and Viosca Knoll (VK), including the BOEM lease blocks VK862 and VK 906/907. The Deepwater Horizon oil wellhead at the Mississippi Canyon, MC252 lease block, and the VK826 site are also marked for reference. Base map data from NOAA. Figure reproduced with permission from Prouty and others, 2011. 

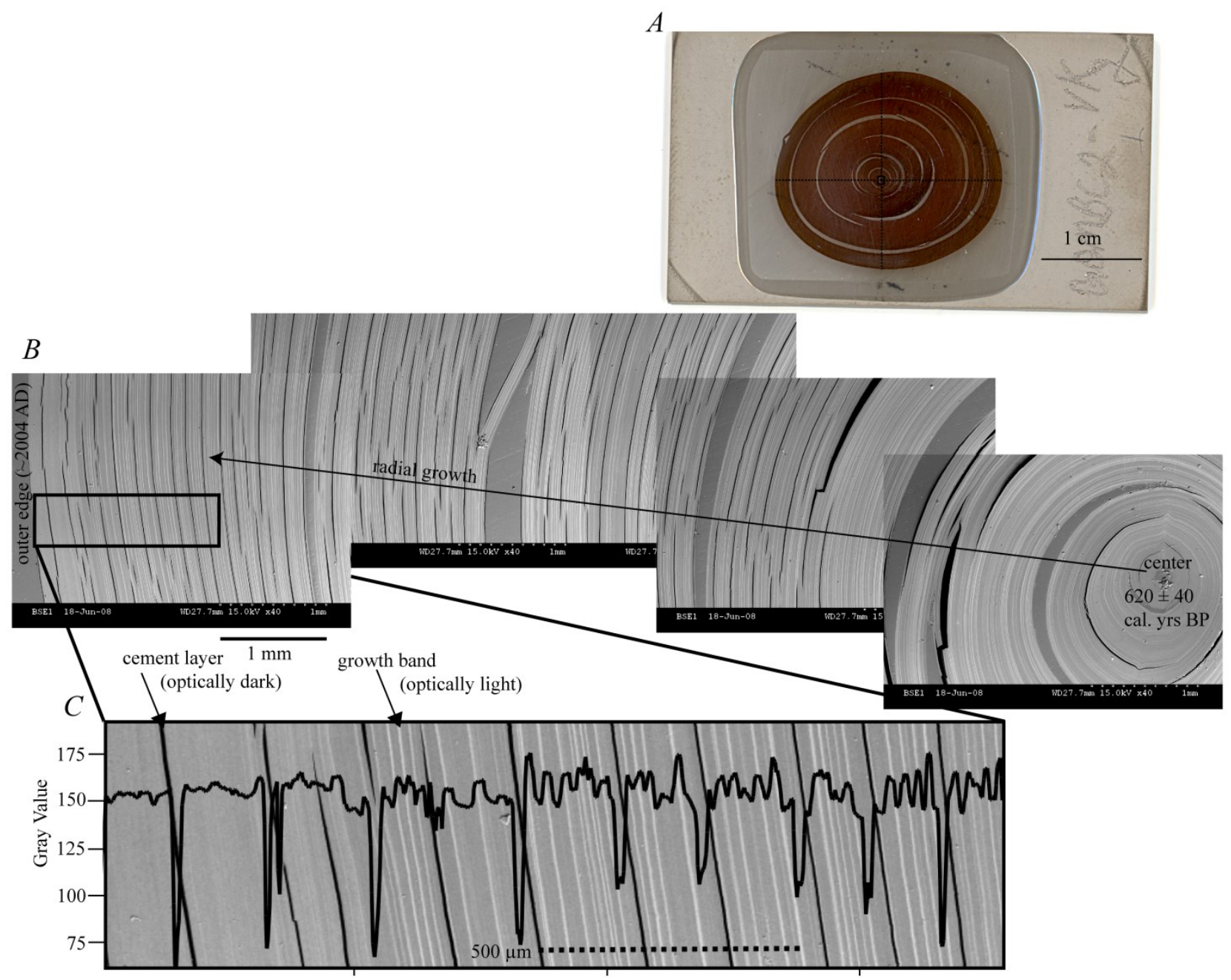

Figure 5.2 A, Photograph showing petrographic thin section indicating the location and length of 4 orthogonal radii with measured lengths of $7.7,11.2,10.2$, and 9.7 millimeters $(\mathrm{mm})$ with a standard deviation of $\pm 1.5 \mathrm{~mm}$. $B$, Scanning electron microscope (SEM) image of the GOM-JSL04-4734 thin section (100 $\mu \mathrm{m})$. C, Enlarged SEM image of the outer $2.5 \mathrm{~mm}$ of skeletal growth. The optically light bands are growth bands, represented by increased gray-scale values, and the optically dark bands are organic cement layers. Figure reproduced with permission from Prouty and others, 2011. 


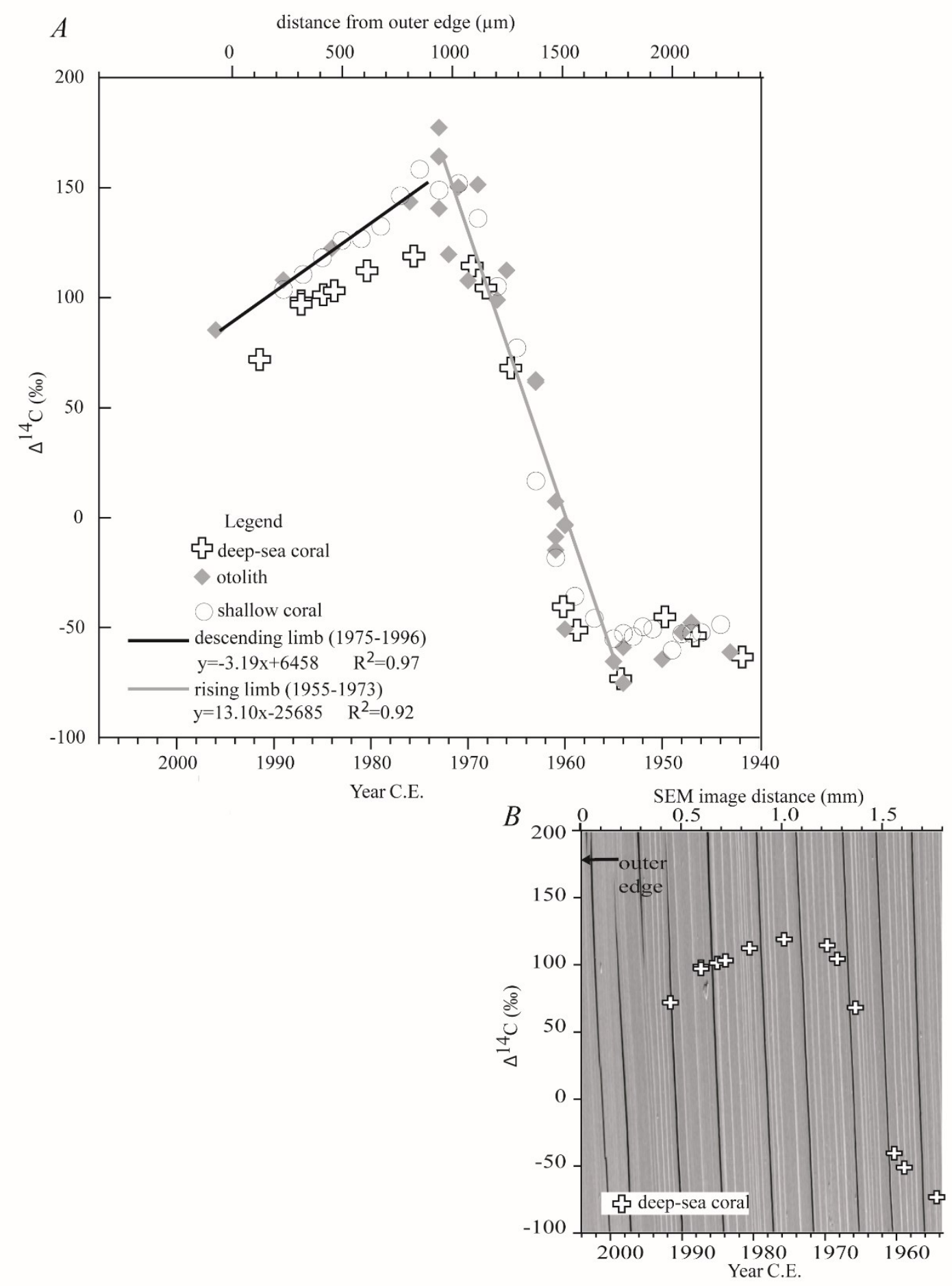

Figure 5.3 A, Bomb-derived $\Delta^{14} \mathrm{C}(\%)$ from red snapper otoliths $(\mathrm{n}=26)$ in the Gulf of Mexico (Baker and Wilson, 2001) and shallow coral $\Delta^{14} \mathrm{C}$ values ( $\left.\mathrm{n}=27\right)$ from the Flower Garden Banks (Wagner, 2009), 19431996. Superimposed on the reference curves is the deep-sea antipatharian (Leiopathes sp.) coral $\Delta^{14} \mathrm{C}$ record from the GOM-JSL04-4734-BC1 specimen versus distance from outer edge (in micrometers). B, Leiopathes $\mathrm{sp}$. coral bomb ${ }^{14} \mathrm{C}$ curve superimposed on the enlarged scanning electron microscope image. Figure reproduced with permission from Prouty and others, 2011. 

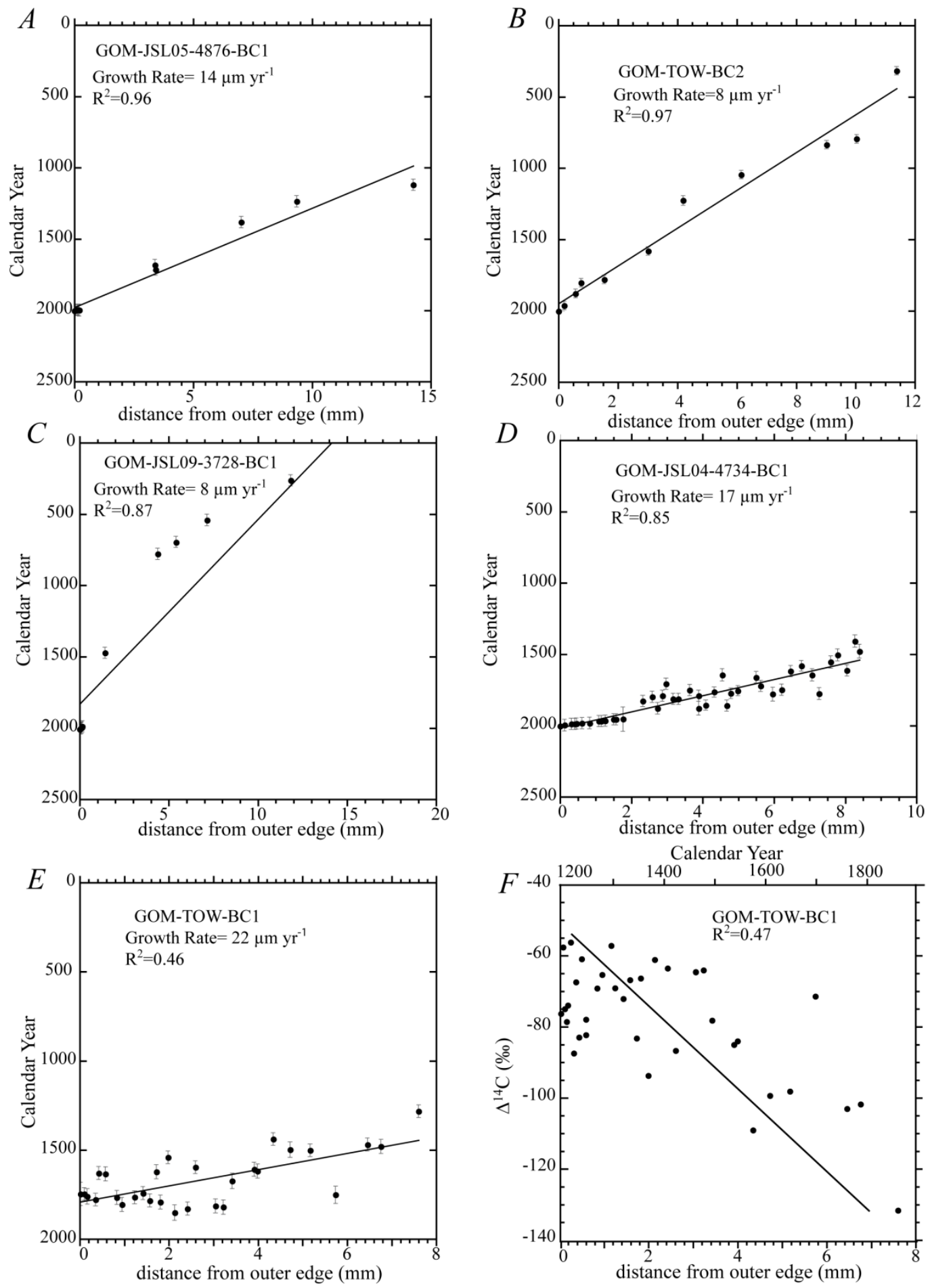

Figure 5.4 Scatter plots showing distance from outer edge and reservoir-corrected calendar age for the five deep-sea black coral samples from the Lophelia II program: A, GOM-JSL05-4876-BC1; B, GOM-TOW-BC2; C, GOM-JSL09-3728-BC1; D, GOM-JSL04-4734-BC1; and E, GOM-TOW-BC1. F, Radiocarbon $\left(\Delta^{14} \mathrm{C}\right)$ variability of GOM-TOW-BC1 versus and distance from outer edge $(\mathrm{mm})$ and year with an analytical uncertainty of 1.3--3.9 per mil\%o. Figure reproduced with permission from Prouty and others, 2011. 


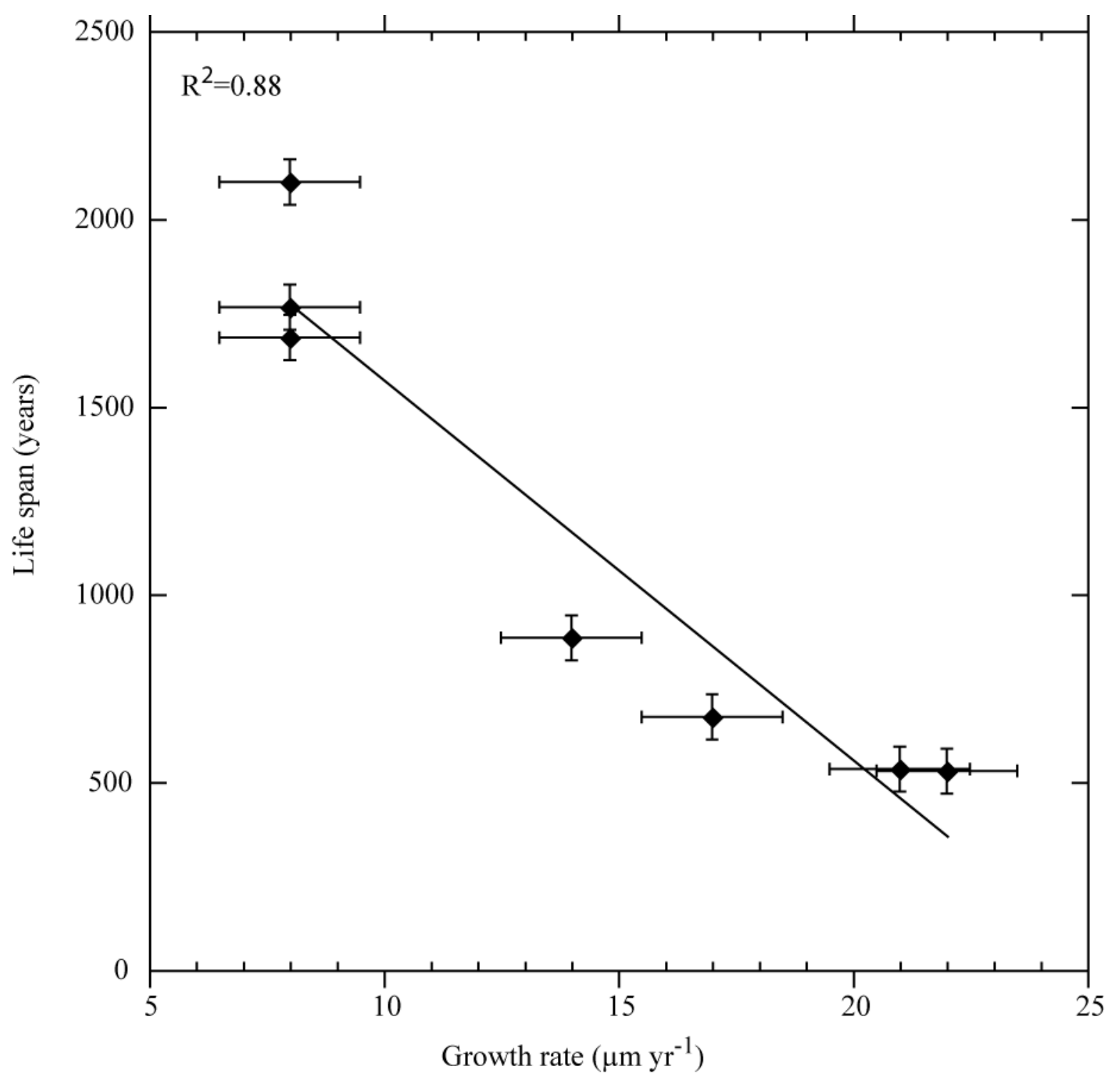

Figure 5.5 Scatter plots of lifespans (defined as the difference between the inner and outer ${ }^{14} \mathrm{C}$ age) versus growth rate (micrometer $[\mu \mathrm{m}]$ year $[\mathrm{yr}]^{-1}$ ) calculated from black coral samples collected in the Gulf of Mexico during the Lophelia II program. Figure reproduced with permission from Prouty and others, 2011. 


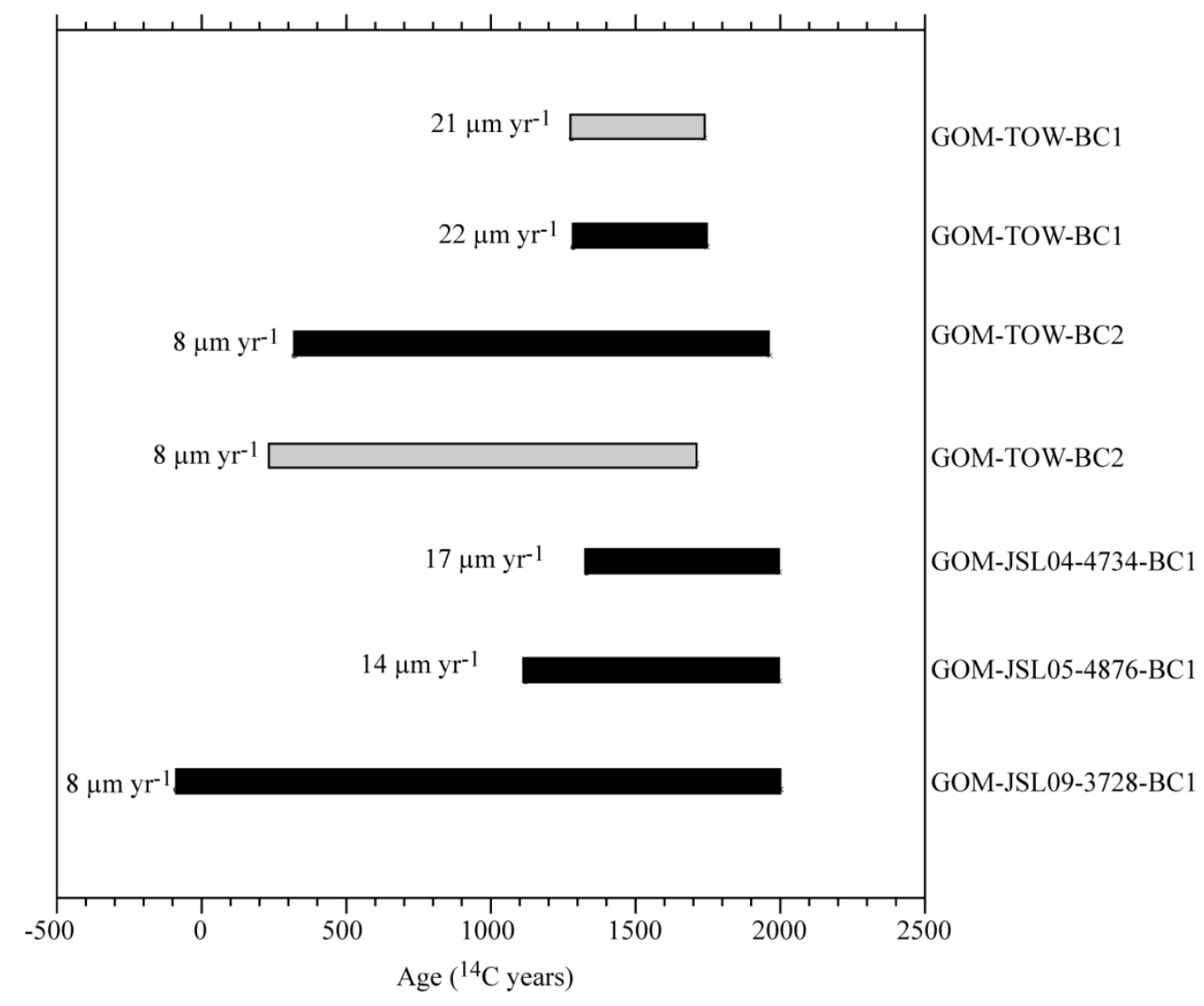

Figure 5.6 Lifespan and growth rate distribution of Leiopathes sp. collected from sites (about 300 meters deep) in the northern Gulf of Mexico during the Lophelia II program. Figure reproduced with permission from Prouty and others, 2011. 


\section{MACROFAUNAL COMMUNITY STRUCTURE NEAR LOPHELIA PERTUSA AND SHIPWRECK HABITATS IN THE GULF OF MEXICO}

By Amanda W.J. Demopoulos, ${ }^{1}$ Jill R. Bourque, ${ }^{1}$ Ryan M. Phillips, ${ }^{1}$ and Janessy Frometa ${ }^{2}$

${ }^{1}$ U.S. Geological Survey

${ }^{2}$ National Oceanic and Atmosphere Administration National Centers for Coastal Ocean Science, Stennis, MS

\subsection{Introduction}

The scleractinian coral Lophelia pertusa is the most common reef-building species in the deep sea worldwide (Freiwald and others, 2004). Lophelia pertusa reefs create biodiversity hot spots by providing a structurally complex three-dimensional substrate known to support abundant and diverse fish and benthic assemblages (Jensen and Frederiksen, 1992; Jonsson and others, 2004; Costello and others, 2005; Reed and others, 2006; Henry and Roberts, 2007). In the Gulf of Mexico (GOM), $L$. pertusa occupies hard substrata in areas of increased hydrodynamic flow (Davies and others, 2010), facilitating sediment and organic matter accumulation in adjacent benthos. While the importance of deep-sea corals is well documented for macrofauna and megafauna occurring within the coral matrix, few studies have addressed their relationship to adjacent soft-sediment habitats. Sediment macrofauna (greater than $[>] 300$ micrometers $[\mu \mathrm{m}]$ ) provide important ecosystem services, including serving as a food source for both resident and transient fish and larger invertebrates, yet their relative abundance and general composition is poorly understood.

In addition to deep-sea corals, shipwrecks also provide a three-dimensional structure capable of supporting abundant and diverse fish and invertebrate populations (Bohnsack, 1989; Mallefet and others, 2008; Church and others, 2009). Often considered artificial reefs, shipwrecks in the GOM have previously been studied as surrogates for deep-sea drilling structures and natural reefs (Kilgour and Shirley, 2008), and also are capable of supporting large colonies of L. pertusa (Church and others, 2009). While the importance of shipwrecks in providing artificial reef habitat is beginning to be understood, their relationship with and effect on adjacent soft-sediment macrofauna are unknown.

Here we describe macrofaunal community composition in sediments adjacent to three natural $L$. pertusa habitats, three shipwreck habitats containing L. pertusa, and background soft sediments at all six sites. We examined whether GOM coral and shipwreck communities were similar in terms of density, diversity, and taxonomic composition and how they compared to background soft-sediment communities. The description of infaunal communities adjacent to structural habitat adds to the existing knowledge of small macrofauna from coral and shipwreck habitats and provides information for future comparisons across biogeographical regions and ecosystems.

\subsection{Materials and Methods}

\subsubsection{Sampling Sites}

Investigations were conducted at three natural L. pertusa habitats and at three shipwrecks containing L. pertusa colonies in the GOM (table 6.1; fig. 6.1). Data from natural L. pertusa habitats 
from Demopoulos and others, 2014. The natural L. pertusa habitats included one site in Mississippi Canyon (MC751) and two sites in Viosca Knoll (VK906 and VK826). VK906 and VK826 were described and characterized in Schroeder (2002), Schroeder and others (2005), and Schroeder (2007). The shipwreck habitats included Ewing Bank (Ewing), the Gulfpenn (Gulfpenn), and Viosca Knoll 786 (VK786). The Ewing wreck is of wooden construction with copper sheathing from the $19^{\text {th }}$ century at 620 meters (m) depth. The Gulfpenn wreck is a World War II (WWII) era oil tanker of steel construction at $560 \mathrm{~m}$ depth. The VK786 wreck is of wooden construction with copper sheathing from the $19^{\text {th }}$ century at $615 \mathrm{~m}$ depth.

\subsubsection{Collection Methods}

Sampling at coral, shipwreck, and background soft-sediment habitats was conducted during September 2009 aboard the National Oceanic and Atmospheric Administration (NOAA) ship Ronald H. Brown and the research vessel (R/V) Seward Johnson using the remotely operated vehicle (ROV) Jason II and the Johnson Sea Link II submersible. Sampling locations were based on proximity to known locations of $L$. pertusa reefs and shipwrecks. Near-coral and near-wreck sampling locations were less than $(<) 1 \mathrm{~m}$ from the structure, and the sediment infauna were sampled in situ by using push cores $(32$ square centimeters $\left[\mathrm{cm}^{2}\right]$ x 10 centimeters $[\mathrm{cm}]$ depth). Background soft sediments were located greater than $100 \mathrm{~m}$ but less than 1,000 $\mathrm{m}$ away from corals or wrecks and were sampled in situ by using push cores $\left(32 \mathrm{~cm}^{2} \times 10 \mathrm{~cm}\right.$ depth or $54 \mathrm{~cm}^{2} \times 10 \mathrm{~cm}$ depth) or by using an Ocean Instruments Mark III (50 $\mathrm{cm} \times 50 \mathrm{~cm})$ box core sub-sampled by push cores $\left(32 \mathrm{~cm}^{2} \times 10 \mathrm{~cm}\right.$ depth).

\subsubsection{Sample Processing}

Sediment cores were sectioned vertically $(0-2,2-5$, and 5-10 $\mathrm{cm})$ immediately after recovery. Not all cores penetrated to $10 \mathrm{~cm}$, so we only present data from the $0-5-\mathrm{cm}$ fractions. Sediment fractions were preserved in the field unsieved in an 8-percent buffered formalin solution for macrofauna analysis (table 6.1, Fauna) or frozen for sediment grain-size analysis (table 6.1, SC). Due to sampling limitations, cores for sediment grain-size analysis were not collected at MC751 and VK906. In the laboratory, a portion of sediment for grain-size analysis was digested with hydrogen peroxide to remove organic material and subsequently was wet sieved through $2,000-\mu \mathrm{m}$ (to separate rubble/large grain sizes) and 63- $\mu \mathrm{m}$ sieves. Size fractions $(>2,000 \mu \mathrm{m}, 63-2,000 \mu \mathrm{m}$, and $<63 \mu \mathrm{m})$ were dried at 60 degrees Celsius $\left({ }^{\circ} \mathrm{C}\right)$ for $1-2$ days and then weighed, and percent rubble/shell $(>2,000 \mu \mathrm{m})$, sand $(63-$ $2,000 \mu \mathrm{m})$, and silt/clay $(<63 \mu \mathrm{m})$ was calculated. In order to characterize sediment macrofauna, preserved sediment fractions were washed through a 300- $\mu \mathrm{m}$ sieve. Animals retained on the sieve were sorted under a dissecting microscope, identified to major taxonomic group, and transferred to 80 percent ethanol for storage. Further taxonomic identification was performed under dissecting and compound microscopes. Polychaetes and peracarid crustaceans were identified to family level.

\subsubsection{Statistical Analysis}

All data are expressed as mean \pm 1 standard error (SE) unless indicated otherwise. All statistical tests were performed with $\alpha=0.05$. Density of individuals, diversity (Shannon Index H' $\log _{e}$ ), and community composition were tested for habitat effects. Univariate measures were analyzed using oneway analysis of variance (ANOVA) in R statistical software. Multivariate community composition was analyzed using non-metric multidimensional scaling (nMDS) and analysis of similarities (ANOSIM)

using Bray-Curtis similarities on square-root transformed data in Primer statistical software. 


\subsection{Results}

\subsubsection{Macrofaunal Density}

Total macrofaunal densities ranged from 5,687 individuals $\mathrm{m}^{-2}$ in background soft sediments at Ewing to 39,494 individuals $\mathrm{m}^{-2}$ in near-coral sediments at VK826. Mean density (fig. 6.2) was highest in near-coral sediments at VK826, followed by near-coral sediments at VK906, and near-wreck sediments at VK786. Macrofaunal density was significantly greater near structure than in background sediments at Ewing (ANOVA, $\mathrm{F}_{1,4}=12.30, \mathrm{p}=0.025$ ), VK786 (ANOVA, $\mathrm{F}_{1,3}=21.52, \mathrm{p}=0.019$ ), and VK826 (ANOVA, log-transformed, $\mathrm{F}_{1,9}=7.94, \mathrm{p}=0.02$ ).

\subsubsection{Macrofaunal Community Composition}

Macrofaunal community composition varied between sites and habitats. At the major taxonomic grouping level (fig. 6.3), all sites and habitats were dominated by polychaetes ( $>50$ percent). Oligochaetes were absent from all shipwreck sites, but were present in near-coral and background soft sediments at natural L. pertusa habitats. All sites had proportionately more crustaceans, including amphipods, isopods, cumaceans, and tanaids, in background soft sediments (16-40 percent) than in near-wreck or near-coral soft sediments (7-16 percent). Mollusca, including bivalves, gastropods, and aplacophorans, represented only $2-15$ percent of sediment communities. The group "other" included echinoderms, cnidarians, sipunculids, nemerteans, echiurans, halacarids, and pycnogonids, and composed 1-15 percent of sediment communities. Diversity of macrofaunal communities (fig. 6.4), as measured by the Shannon Index $\left(H^{\prime} \log _{e}\right)$, was overall greater in near-structure sediments at all wreck sites and MC751, although only significantly greater at Ewing (ANOVA, $\mathrm{F}_{1,4}=13.85, \mathrm{p}=0.02$ ). Diversity at VK906 and VK826 was generally higher in background soft sediments although not statistically significant.

Community composition was distinctly different among near-coral, near-wreck, and background soft sediments (fig. 6.5). A significant habitat effect (L. pertusa vs. shipwrecks) was observed in multivariate community structure allowing for differences in proximity (two-way ANOSIM; $\mathrm{R}=0.551$, $\mathrm{p}=0.001$ ). Near-structure and background sediments were also significantly different from each other (Two-way ANOSIM; $\mathrm{R}=0.271, \mathrm{p}=0.005$ ). In addition, community composition was significantly different among sites (Two-way ANOSIM; $\mathrm{R}=0.692, \mathrm{p}=0.001$ ), also allowing for differences in proximity. In near-coral habitats, five taxa accounted for 56 percent of the similarity between samples: the polychaete families Oweniidae (19 percent), Spionidae (15 percent), Syllidae ( 8 percent), and Maldanidae (7 percent) and bivalves (7 percent). In near-wreck habitats, four polychaete families accounted for 50 percent of the similarity between samples: Spionidae (23 percent), Syllidae (11 percent), Paraonidae (10 percent), and Opheliidae ( 8 percent). Three polychaete families, Spionidae, Syllidae, and Paraonidae, accounted for 44 and 56 percent of the similarity at both L. pertusa and wreck sites, respectively.

\subsubsection{Sediment Properties}

Particle size varied between wreck and natural $L$. pertusa sites (fig. 6.6). Sediments at wreck sites were dominated by silt/clay (fig. 6.6), with near and background sediments at Ewing, Gulfpenn, and VK786 all containing greater than 88 percent silt/clay. Ewing differed from the other two wreck sites in containing 2 percent rubble/shell material near the wreck, while the other near-wrecks had less than 0.2 percent rubble/shell. The natural L. pertusa reef at VK826 had a different sediment composition 
from the wreck sites, with a larger proportion of rubble/shell (46 and 16 percent) and sand (12 and 43 percent) in near and background habitats, respectively.

\subsection{Discussion}

Our results suggest that L. pertusa and shipwrecks are supporting distinct and abundant macrofaunal communities in adjacent sediments. The GOM is generally defined as a low productivity water mass, with macrofaunal densities in the deep GOM generally less than 6,000 individuals $\mathrm{m}^{-2}$ (Pequegnat and others, 1990; Tyler, 2003; Wei, 2006). Macrofaunal densities in near-coral habitat presented here are comparable to enhanced densities reported near other deep-sea communities such as cold seeps (Ross and others, 2012) and microbial mats (Levin and Mendoza, 2007), as well as soft sediments at the head of Mississippi Canyon (Wei, 2006). There are currently no comparisons for sediment macrofauna at other deep-sea coral locations. Background soft-sediment cores collected up to 1 kilometer $(\mathrm{km})$ away from physical structure in this study contained greater than 5,000 individuals $\mathrm{m}^{-}$ ${ }^{2}$, suggesting the area of structural effect may be more widespread than previously indicated. High particle flux of fine-grained aggregates has been recorded at VK826 with short-term variability in flow direction and velocity with an Eckman layer extending $70 \mathrm{~m}$ above the bottom (Davies and others, 2010).

The effect of physical structure on adjacent macrofaunal communities is site specific. Differences among sites could be explained by multiple factors, including habitat size, age, physical composition, depth, and geographical location. For natural L. pertusa habitats, the size of the colonies or reef may play a role in the level of effect imparted onto the adjacent sediment. Our sampling sites represent a range of $L$. pertusa habitat sizes, with VK826 containing the largest reef, VK906 a somewhat smaller reef (Schroeder, 2007), and MC751 composed of a small collection of colonies. In addition, the shipwrecks contained attached L. pertusa colonies, but the physical size of the wreck may be more important for the effect imparted to the adjacent benthos. Increases in habitat complexity have been shown to correspond to increased macrofaunal densities in vestimentiferan cold-seep communities in the GOM (Bergquist and others, 2003) and sponge spicule communities in the North Atlantic (Bett and Rice, 1992); however, diversity at the L. pertusa reef sites VK906 and VK826 was lower than in background soft sediments. Henry and Roberts (2007) reported enhanced diversity on L. pertusa mounds versus off-mound in the North Atlantic, but their sampling included taxa living both within the coral matrix and underlying sediments.

Depth differences between our sites may play a significant role in structuring site differences. Macrofaunal density is known to decrease with depth in the GOM (Pequegnat and others, 1990; Wei, 2006) and is likely a function of food availability (Levin and Gooday, 2003; Wei and others, 2010). This pattern is consistent with the densities observed in the background soft-sediment cores; however, this pattern was not observed near structure. At the depths sampled in this study, macrofaunal diversity is expected to increase with depth (Rex, 1981; Wei and others, 2010), but this pattern is not observed in near-structure or in background sediments.

Although the benthic macrofaunal communities adjacent to shipwrecks exhibited similar patterns in dominant taxa as those near L. pertusa, they were still significantly different from natural reefs. While the age of natural L. pertusa reefs are estimated at $>1,000$ years, the age of shipwrecks in this study are known to be much younger (70-200 years). In shallow systems, shipwrecks are known to exhibit similar epifaunal species composition as natural reefs in as little as 20 years (Perkol-Finkel and others, 2005). In previous studies, the Gulfpenn contained higher species richness of invertebrates than other WWII-era shipwrecks (Church and others, 2009), suggesting a similar rate of colonization on deep-sea wrecks. Grain-size characteristics at wreck and L. pertusa reef sites may also play a significant 
role in the structuring of the community differences between the two habitats. The natural L. pertusa reefs exhibited greater sediment heterogeneity than the wreck sites, which has previously been associated with structuring communities that are different from areas of more homogeneous sediments (Levin and others, 2001). The Ewing and VK786 wrecks exhibited enhanced macrofaunal abundance near-wreck, both approximately 200 years old with similar structural composition (for example, wood and copper sheathing), while the younger Gulfpenn wreck (70 years old) exhibited no enhancement. Despite the similar physical characteristics of the Ewing and VK786 wrecks, however, the multivariate community at Ewing was more similar to that at Gulfpenn than to the community at VK786.

Geographic separation by the Mississippi Canyon may explain this observation; a similar pattern was observed in the natural L. pertusa habitats, with VK906 and VK826 separated from MC751.

This study represents the first to quantify and compare near-structure sediment macrofauna from deep-sea corals and shipwrecks to background soft sediments. Both $L$. pertusa reefs and shipwrecks are capable of supporting distinct benthic communities with enhanced density but variable diversity. Although overall community differences are habitat driven (near versus background), site-specific factors such as hydrodynamics and sediment properties play a significant role in structuring benthic macrofaunal communities.

\subsection{Acknowledgments}

We thank the captains and crews of the NOAA ship Ronald H. Brown, R/V Seward Johnson, ROV Jason II, and Johnson Sea Link II submersible. We also thank the following individuals and groups for their help at sea, site selection, and helpful suggestions: C. Fisher (Pennsylvania State University), E. Cordes (Temple University), Ross Lab (University of North Carolina Wilmington), R. Church (C \& C Technologies), D. Warren (C \& C Technologies), and J. McClain-Counts (USGS).

\subsection{References}

Bergquist, D.C., Ward, T., Cordes, E.E., McNelis, T., Howlett, S., Kosoff, R., Hourdez, S., Carney, R., and Fisher, C.R., 2003, Community structure of vestimentiferan-generated habitat islands from Gulf of Mexico cold seeps: Journal of Experimental Marine Biology and Ecology, v. 289, no. 2, p. 197222.

Bett, B.J., and Rice, A.L., 1992, The influence of hexactinellid sponge (Pheronema carpenteri) spicules on the patchy distribution of macrobenthos in the Porcupine Seabight (bathyal NE Atlantic): Ophelia, v. 36, no. 3, p. 217-226.

Bohnsack, J.A., 1989, Are high densities of fishes at artificial reefs the result of habitat limitation or behavioral preference?: Bulletin of Marine Science, v. 44, no. 2, p. 631-645.

Church, R.A., Warren, D.J., and Irion, J.B., 2009, Analysis of deepwater shipwrecks in the Gulf of Mexico: Artificial reef effect of six World War II shipwrecks: Oceanography, v. 22, no. 2, p. 50-63.

Costello, M., McCrea, M., Freiwald, A., Lundälv, T., Jonsson, L., Bett, B., Weering, T., Haas, H., Roberts, J., Allen, D., Freiwald, A., and Roberts, J.M., 2005, Role of cold-water Lophelia pertusa coral reefs as fish habitat in the NE Atlantic, in Freiwald, A., and Roberts, J.M., eds., Cold-water corals and ecosystems: Berlin, Springer-Verlag, p. 771-805.

Davies, A.J., Duineveld, G.C.A., van Weering, T.C.E., Mienis, F., Quattrini, A.M., Seim, H.E., Bane, J.M., and Ross, S.W., 2010, Short-term environmental variability in cold-water coral habitat at Viosca Knoll, Gulf of Mexico: Deep-Sea Research Part I: Oceanographic Research Papers, v. 57, no. 2, p. 199-212. 
Demopoulos, A.W.J., Bourque, J.R., and Frometa, J., 2014, Biodiversity and community composition of sediment macrofauna associated with deep-sea Lophelia pertusa habitats in the Gulf of Mexico:

Deep-Sea Research Part I: Oceanographic Research Papers, v. 93, p. 91-103.

Freiwald, A., Fosså, J.H., Grehan, A., Koslow, T., and Roberts, J.M., 2004, Cold-water coral reefs: Cambridge, UK, UNEP-WCMC, v. 84.

Henry, L., and Roberts, J.M., 2007, Biodiversity and ecological composition of macrobenthos on coldwater coral mounds and adjacent off-mound habitat in the bathyal Porcupine Seabight, NE Atlantic: Deep-Sea Research Part I, v. 54, p. 654-672.

Jensen, A., and Frederiksen, R., 1992, The fauna associated with the bank-forming deep-water coral Lophelia pertusa (Scleractinaria) on the Faroe Shelf: Sarsia, v. 77, no. 1, p. 53-69.

Jonsson, L.G., Nilsson, P.G., Floruta, F., and Lundälv, T., 2004, Distributional patterns of macro- and megafauna associated with a reef of the cold-water coral Lophelia pertusa on the Swedish west coast: Marine Ecology Progress Series, v. 284, p. 163-171.

Kilgour, M.J., and Shirley, T.C., 2008, Bathymetric and spatial distribution of decapod crustaceans on deep-water shipwrecks in the Gulf of Mexico: Bulletin of Marine Science, v. 82, no. 3, p. 333-344.

Levin, L., Etter, R.J., Rex, M.A., Gooday, A.J., Smith, C.R., Pineda, J., Stuart, C.T., Hessler, R.R., and Pawson, D., 2001, Environmental influences on regional deep-sea species diversity: Annual Revue of Ecology, Evolution, and Systematics, v. 32, p. 51-93.

Levin, L., and Gooday, A.J., 2003, The deep Atlantic Ocean, in Tyler, P.A., ed., Ecosystems of the world, volume 28: Ecosystems of the deep ocean: Amsterdam, Elsevier, p. 111-178.

Levin, L.A., and Mendoza, G.F., 2007, Community structure and nutrition of deep methane-seep macrobenthos from the North Pacific (Aleutian) margin and the Gulf of Mexico (Florida Escarpment): Marine Ecology, v. 28, p. 131-151.

Mallefet, J., Zintzen, V., Massin, C., Norro, A., Vincx, M., DeMaersschalck, V., Steyaert, M., Degraer, S., and Cattrijsse, A., 2008, Belgian shipwreck: hotspots for marine biodiversity: Belgian Science Policy, $155 \mathrm{p}$.

Pequegnat, W.E., Gallaway, B.J., and Pequegnat, L.H., 1990, Aspects of the ecology of the deep-water fauna of the Gulf of Mexico: American Zoologist, v. 30, no. 1, p. 45-64.

Perkol-Finkel, S., Shashar, N., Barneah, O., Ben-David-Zaslow, R., Oren, U., Reichert, T., Yacobovich, T., Yahel, G., Yahel, R., and Benayahu, Y., 2005, Fouling reefal communities on artifical reefs: does age matter?: Biofouling, v. 21, no. 2, p. 127-140.

Reed, J.K., Weaver, D.C., and Pomponi, S.A., 2006, Habitat and fauna of deep-water Lophelia pertusa coral reefs off the southeastern U.S.: Blake Plateau, Straits of Florida, and Gulf of Mexico: Bulletin of Marine Science, v. 78, no. 2, p. 343-375.

Rex, M.A., 1981, Community structure in the deep-sea benthos: Annual Review of Ecology and Systematics, v. 12, p. 331-353.

Ross, S.W., Demopoulos, A.W.J., Kellogg, C.A., Morrison, C.L., Nizinkski, M.S., Ames, C.L., Casazza, T.L., Gualtieri, D., Kovacs, K.M., McClain, J.P., Quattrini, A.M., Roa-Varon, A.Y., and Thaler, A.D., 2012, Deepwater Program: Studies of Gulf of Mexico lower continental slope communities related to chemosynthetic and hard substrate habitats: U.S. Geological Survey Open-File Report 2012-1032, 301 p.

Schroeder, W.W., 2002, Observations of Lophelia pertusa and the surficial geology at a deep-water site in the northeastern Gulf of Mexico: Hydrobiologia, v. 471, no. 1, p. 29-33.

Schroeder, W.W., 2007, Seafloor characteristics and distribution patterns of Lophelia pertusa and other sessile megafauna at two upper-slope sites in the northeastern Gulf of Mexico: Gulf of Mexico OCS 
Region, New Orleans, La., U.S. Department of the Interior, Minerals Management Service, OCS Study MMS 2007-035, $49 \mathrm{p}$.

Schroeder, W.W., Brooke, S.D., Olson, J.B., Phaneuf, B., McDonough, J.J., III, and Etnoyer, P., 2005, Occurrence of the deep-water Lophelia pertusa and Madrepora oculata in the Gulf of Mexico, in Freiwald, A., and Roberts, J.M., eds., Cold-water corals and ecosystems: Berlin, Springer-Verlag, p. 297-307.

Tyler, P.A., 2003, The peripheral seas, in Tyler, P.A., ed., Ecosystems of the world: The deep sea: Amsterdam, Elsevier, p. 261-293.

Wei, C.L., 2006, The bathymetric zonation and community structure of deep-sea macrobenthos in the northern Gulf of Mexico: Texas A\&M University, Ph.D. dissertation, $101 \mathrm{p}$.

Wei, C.L., Rowe, G.T., Fain Hubbard, G., Scheltema, A.H., Wilson, G.D.F., Petrescu, I., Foster, J.M., Wicksten, M.K., Chen, M., Davenport, R., Soliman, Y., and Wang, Y., 2010, Bathymetric zonation of deep-sea macrofauna in relation to export of surface phytoplankton production: Marine Ecology Progress Series, v. 399, p. 1-14. 
Table 6.1. Sample locations, sample type, depths, and gear used for all sediment cores collected during the Lophelia Il program.

[SC, sediment grain-size analysis; PC, push cores (6.35-cm diameter); SPC, large push cores $(8.26-\mathrm{cm}$ diameter); $\mathrm{BC}$, box cores subsampled with push cores $(6.35-\mathrm{cm}$ diameter $) ; \mathrm{cm}$, centimeter; $\mathrm{m}$, meter]

\begin{tabular}{|c|c|c|c|c|c|c|c|}
\hline Site & Habitat & Type & Gear & Dive/Station & Depth (m) & Latitude & Longitude \\
\hline MC751 & Near-coral & Fauna & $\mathrm{PC}$ & 464 & 440 & $28.19373^{\circ} \mathrm{N}$ & $89.79889^{\circ} \mathrm{W}$ \\
\hline MC751 & Near-coral & Fauna & $\mathrm{PC}$ & 464 & 438 & $28.19368^{\circ} \mathrm{N}$ & $89.79886^{\circ} \mathrm{W}$ \\
\hline MC751 & Near-coral & Fauna & $\mathrm{PC}$ & 464 & 440 & $28.19368^{\circ} \mathrm{N}$ & $89.79884^{\circ} \mathrm{W}$ \\
\hline MC751 & Background & Fauna & $\mathrm{PC}$ & 464 & 431 & $28.19872^{\circ} \mathrm{N}$ & $89.80106^{\circ} \mathrm{W}$ \\
\hline MC751 & Background & Fauna & $\mathrm{PC}$ & 464 & 431 & $28.19872^{\circ} \mathrm{N}$ & $89.80105^{\circ} \mathrm{W}$ \\
\hline MC751 & Background & Fauna & $\mathrm{BC}$ & $\operatorname{Stn} 27$ & 429 & $28.19688^{\circ} \mathrm{N}$ & $89.79877^{\circ} \mathrm{W}$ \\
\hline MC751 & Background & Fauna & $\mathrm{BC}$ & Stn 28 & 427 & $28.19675^{\circ} \mathrm{N}$ & $89.79985^{\circ} \mathrm{W}$ \\
\hline VK906 & Near-coral & Fauna & $\mathrm{PC}$ & 465 & 388 & $29.06961^{\circ} \mathrm{N}$ & $88.37707^{\circ} \mathrm{W}$ \\
\hline VK906 & Near-coral & Fauna & $\mathrm{PC}$ & 465 & 388 & $29.06965^{\circ} \mathrm{N}$ & $88.37710^{\circ} \mathrm{W}$ \\
\hline VK906 & Near-coral & Fauna & $\mathrm{PC}$ & 465 & 388 & $29.06966^{\circ} \mathrm{N}$ & $88.37710^{\circ} \mathrm{W}$ \\
\hline VK906 & Near-coral & Fauna & $\mathrm{PC}$ & 465 & 393 & $29.06918^{\circ} \mathrm{N}$ & $88.37759^{\circ} \mathrm{W}$ \\
\hline VK906 & Near-coral & Fauna & $\mathrm{PC}$ & 465 & 393 & $29.06919^{\circ} \mathrm{N}$ & $88.37760^{\circ} \mathrm{W}$ \\
\hline VK906 & Near-coral & Fauna & $\mathrm{PC}$ & 465 & 394 & $29.06917^{\circ} \mathrm{N}$ & $88.37760^{\circ} \mathrm{W}$ \\
\hline VK906 & Near-coral & Fauna & $\mathrm{PC}$ & 473 & 392 & $29.06905^{\circ} \mathrm{N}$ & $88.37712^{\circ} \mathrm{W}$ \\
\hline VK906 & Near-coral & Fauna & $\mathrm{PC}$ & 473 & 392 & $29.06904^{\circ} \mathrm{N}$ & $88.37712^{\circ} \mathrm{W}$ \\
\hline VK906 & Near-coral & Fauna & $\mathrm{PC}$ & 473 & 394 & $29.06904^{\circ} \mathrm{N}$ & $88.37712^{\circ} \mathrm{W}$ \\
\hline VK906 & Background & Fauna & $\mathrm{PC}$ & 465 & 432 & $29.06725^{\circ} \mathrm{N}$ & $88.38018^{\circ} \mathrm{W}$ \\
\hline VK906 & Background & Fauna & $\mathrm{PC}$ & 465 & 432 & $29.06724^{\circ} \mathrm{N}$ & $88.38018^{\circ} \mathrm{W}$ \\
\hline VK906 & Background & Fauna & $\mathrm{PC}$ & 465 & 432 & $29.06725^{\circ} \mathrm{N}$ & $88.38019^{\circ} \mathrm{W}$ \\
\hline VK906 & Background & Fauna & SPC & 3725 & 393 & $29.06910^{\circ} \mathrm{N}$ & $88.37612^{\circ} \mathrm{W}$ \\
\hline VK906 & Background & Fauna & $\mathrm{BC}$ & Stn 33 & 418 & $29.0727^{\circ} \mathrm{N}$ & $88.37808^{\circ} \mathrm{W}$ \\
\hline VK826 & Near-coral & Fauna & $\mathrm{PC}$ & 466 & 477 & $29.15777^{\circ} \mathrm{N}$ & $88.01615^{\circ} \mathrm{W}$ \\
\hline VK826 & Near-coral & Fauna & $\mathrm{PC}$ & 466 & 475 & $29.15779^{\circ} \mathrm{N}$ & $88.01615^{\circ} \mathrm{W}$ \\
\hline VK826 & Near-coral & Fauna & $\mathrm{PC}$ & 466 & 475 & $29.15779^{\circ} \mathrm{N}$ & $88.01615^{\circ} \mathrm{W}$ \\
\hline VK826 & Near-coral & Fauna & $\mathrm{PC}$ & 466 & 470 & $29.15817^{\circ} \mathrm{N}$ & $88.01682^{\circ} \mathrm{W}$ \\
\hline VK826 & Near-coral & Fauna & $\mathrm{PC}$ & 466 & 470 & $29.15817^{\circ} \mathrm{N}$ & $88.01681^{\circ} \mathrm{W}$ \\
\hline VK826 & Near-coral & Fauna & $\mathrm{PC}$ & 467 & 480 & $29.15869^{\circ} \mathrm{N}$ & $88.01043^{\circ} \mathrm{W}$ \\
\hline VK826 & Near-coral & Fauna & $\mathrm{PC}$ & 467 & 479 & $29.15872^{\circ} \mathrm{N}$ & $88.01039^{\circ} \mathrm{W}$ \\
\hline VK826 & Near-coral & $\mathrm{SC}$ & $\mathrm{PC}$ & 3735 & 471 & $29.17099^{\circ} \mathrm{N}$ & $88.01039^{\circ} \mathrm{W}$ \\
\hline VK826 & Background & Fauna & $\mathrm{BC}$ & Stn 62 & 470 & $29.17012^{\circ} \mathrm{N}$ & $88.01330^{\circ} \mathrm{W}$ \\
\hline VK826 & Background & Fauna & $\mathrm{BC}$ & Stn 63 & 461 & $29.17068^{\circ} \mathrm{N}$ & $88.01233^{\circ} \mathrm{W}$ \\
\hline VK826 & Background & Fauna & $\mathrm{BC}$ & Stn 72 & 472 & $29.16772^{\circ} \mathrm{N}$ & $88.01315^{\circ} \mathrm{W}$ \\
\hline VK826 & Background & Fauna & $\mathrm{BC}$ & Stn 74 & 458 & $29.17077^{\circ} \mathrm{N}$ & $88.01133^{\circ} \mathrm{W}$ \\
\hline VK826 & Background & $\mathrm{SC}$ & $\mathrm{PC}$ & 474 & 514 & $29.15776^{\circ} \mathrm{N}$ & $88.01970^{\circ} \mathrm{W}$ \\
\hline Ewing & Near-wreck & Fauna & $\mathrm{PC}$ & 470 & 622 & $27.97^{\circ} \mathrm{N}$ & $90.08^{\circ} \mathrm{W}$ \\
\hline Ewing & Near-wreck & Fauna & $\mathrm{PC}$ & 470 & 622 & $27.97^{\circ} \mathrm{N}$ & $90.08^{\circ} \mathrm{W}$ \\
\hline Ewing & Near-wreck & Fauna & $\mathrm{PC}$ & 470 & 622 & $27.97^{\circ} \mathrm{N}$ & $90.08^{\circ} \mathrm{W}$ \\
\hline Ewing & Near-wreck & $\mathrm{SC}$ & $\mathrm{PC}$ & 470 & 622 & $27.97^{\circ} \mathrm{N}$ & $90.08^{\circ} \mathrm{W}$ \\
\hline Ewing & Background & Fauna & $\mathrm{PC}$ & 470 & 622 & $27.97^{\circ} \mathrm{N}$ & $90.08^{\circ} \mathrm{W}$ \\
\hline
\end{tabular}


Table 6.1. Sample locations, sample type, depths, and gear used for all sediment cores collected during the Lophelia II program - continued.

[SC, sediment grain-size analysis; PC, push cores (6.35-cm diameter); SPC, large push cores $(8.26-\mathrm{cm}$ diameter); $\mathrm{BC}$, box cores subsampled with push cores $(6.35-\mathrm{cm}$ diameter); $\mathrm{cm}$, centimeter; m, meter]

\begin{tabular}{lccccccc}
\hline Site & Habitat & Type & Gear & Dive/Station & Depth $(\mathrm{m})$ & Latitude & Longitude \\
\hline Ewing & Background & Fauna & PC & 470 & 621 & $27.97^{\circ} \mathrm{N}$ & $90.08^{\circ} \mathrm{W}$ \\
Ewing & Background & Fauna & PC & 470 & 620 & $27.97^{\circ} \mathrm{N}$ & $90.08^{\circ} \mathrm{W}$ \\
Ewing & Background & SC & PC & 470 & 620 & $27.97^{\circ} \mathrm{N}$ & $90.08^{\circ} \mathrm{W}$ \\
\hline Gulfpenn & Near-wreck & Fauna & PC & 472 & 557 & $28.44^{\circ} \mathrm{N}$ & $89.32^{\circ} \mathrm{W}$ \\
Gulfpenn & Near-wreck & Fauna & PC & 472 & 557 & $28.44^{\circ} \mathrm{N}$ & $89.32^{\circ} \mathrm{W}$ \\
Gulfpenn & Near-wreck & Fauna & PC & 472 & 557 & $28.44^{\circ} \mathrm{N}$ & $89.32^{\circ} \mathrm{W}$ \\
Gulfpenn & Near-wreck & SC & PC & 472 & 557 & $28.44^{\circ} \mathrm{N}$ & $89.32^{\circ} \mathrm{W}$ \\
Gulfpenn & Background & Fauna & PC & 472 & 561 & $28.44^{\circ} \mathrm{N}$ & $89.32^{\circ} \mathrm{W}$ \\
Gulfpenn & Background & Fauna & PC & 472 & 562 & $28.44^{\circ} \mathrm{N}$ & $89.32^{\circ} \mathrm{W}$ \\
Gulfpenn & Background & Fauna & PC & 472 & 562 & $28.44^{\circ} \mathrm{N}$ & $89.32^{\circ} \mathrm{W}$ \\
Gulfpenn & Background & SC & PC & 472 & 561 & $28.44^{\circ} \mathrm{N}$ & $89.32^{\circ} \mathrm{W}$ \\
\hline VK786 & Near-wreck & Fauna & PC & 468 & 613 & $29.22^{\circ} \mathrm{N}$ & $87.77^{\circ} \mathrm{W}$ \\
VK786 & Near-wreck & Fauna & PC & 468 & 613 & $29.22^{\circ} \mathrm{N}$ & $87.77^{\circ} \mathrm{W}$ \\
VK786 & Near-wreck & Fauna & PC & 468 & 613 & $29.22^{\circ} \mathrm{N}$ & $87.77^{\circ} \mathrm{W}$ \\
VK786 & Near-wreck & SC & PC & 468 & 613 & $29.22^{\circ} \mathrm{N}$ & $87.77^{\circ} \mathrm{W}$ \\
VK786 & Background & Fauna & PC & 468 & 619 & $29.22^{\circ} \mathrm{N}$ & $87.77^{\circ} \mathrm{W}$ \\
VK786 & Background & Fauna & PC & 468 & 618 & $29.22^{\circ} \mathrm{N}$ & $87.77^{\circ} \mathrm{W}$ \\
VK786 & Background & SC & PC & 468 & 619 & $29.22^{\circ} \mathrm{N}$ & $87.77^{\circ} \mathrm{W}$ \\
\hline
\end{tabular}




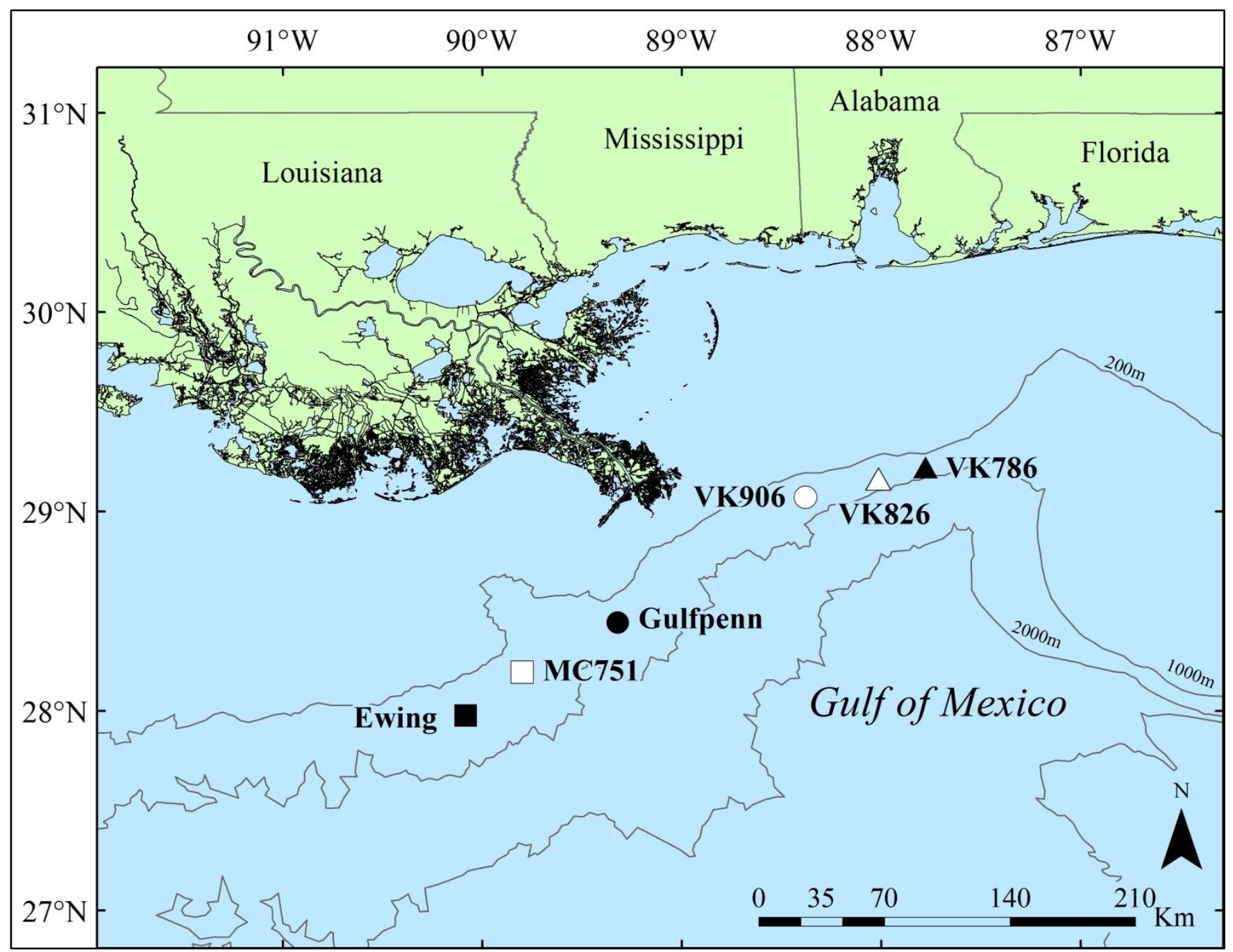

Figure 6.1 Collection sites of sediments in the Gulf of Mexico during the Lophelia II program. Base map data from NOAA. 


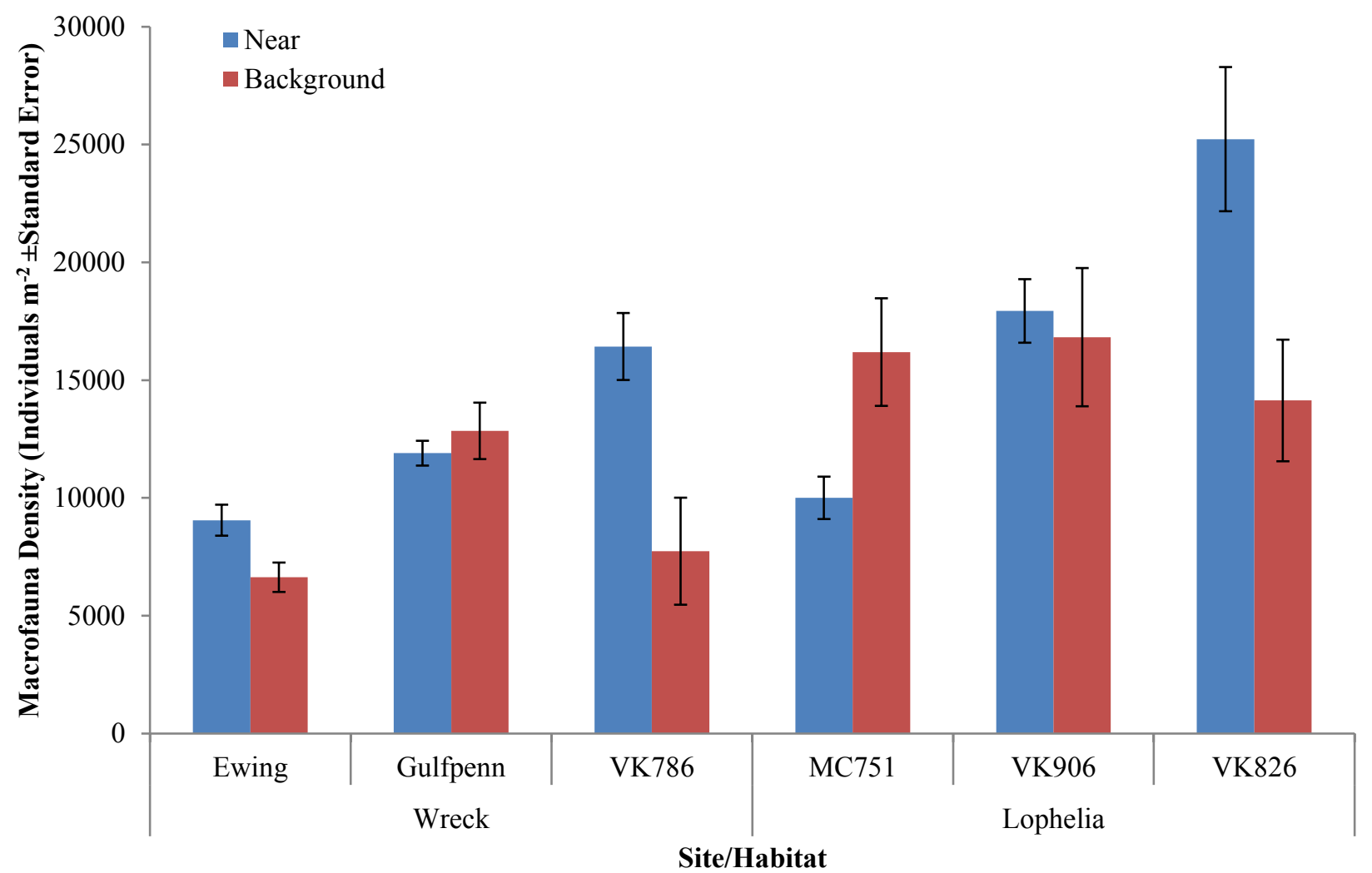

Figure 6.2 Mean macrofaunal density near shipwrecks, near coral, and in background soft sediments during the Lophelia II program. 


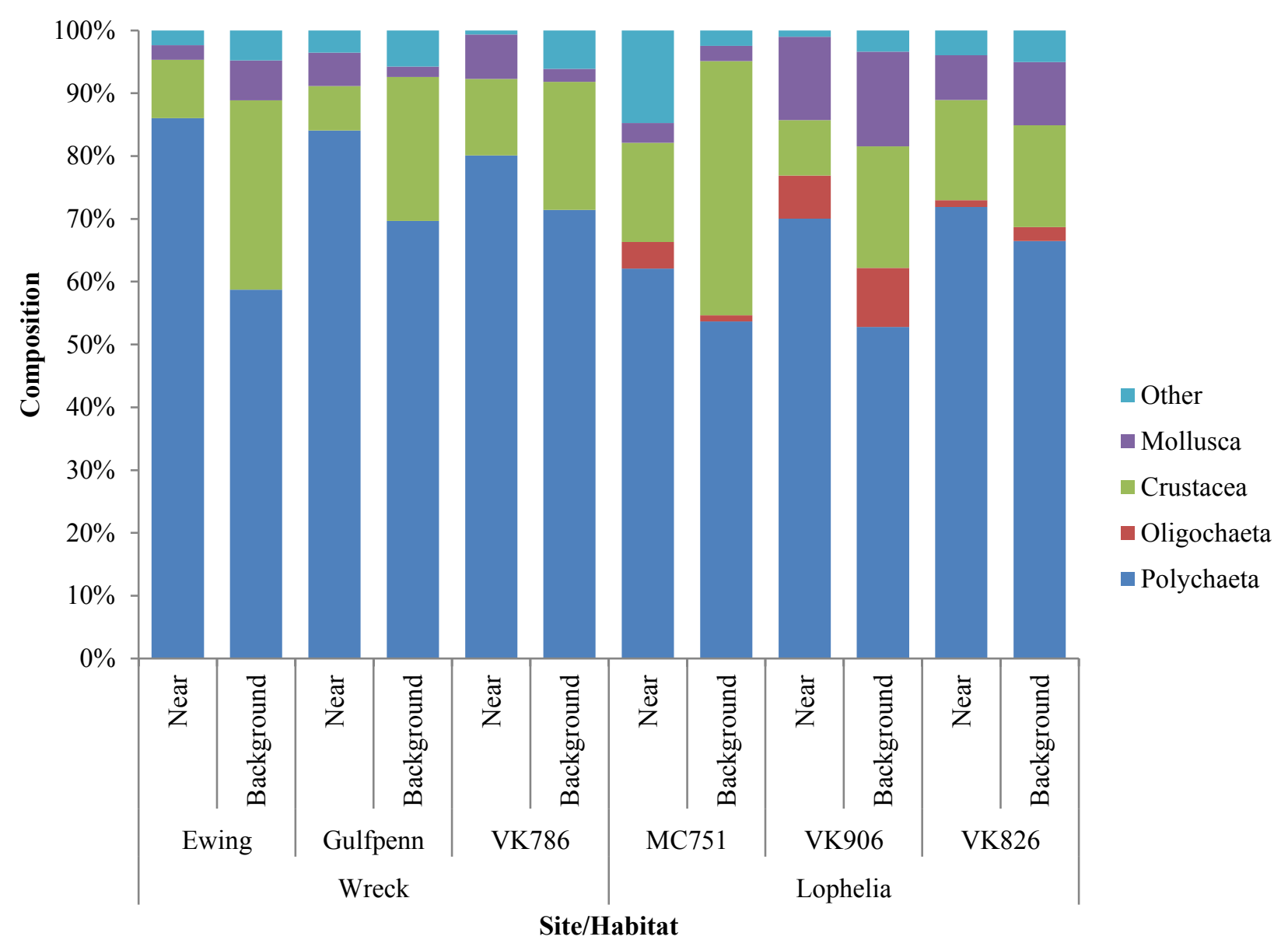

Figure 6.3 Percent composition of major taxonomic groups near shipwrecks, near coral, and in background soft sediments during the Lophelia II program. 


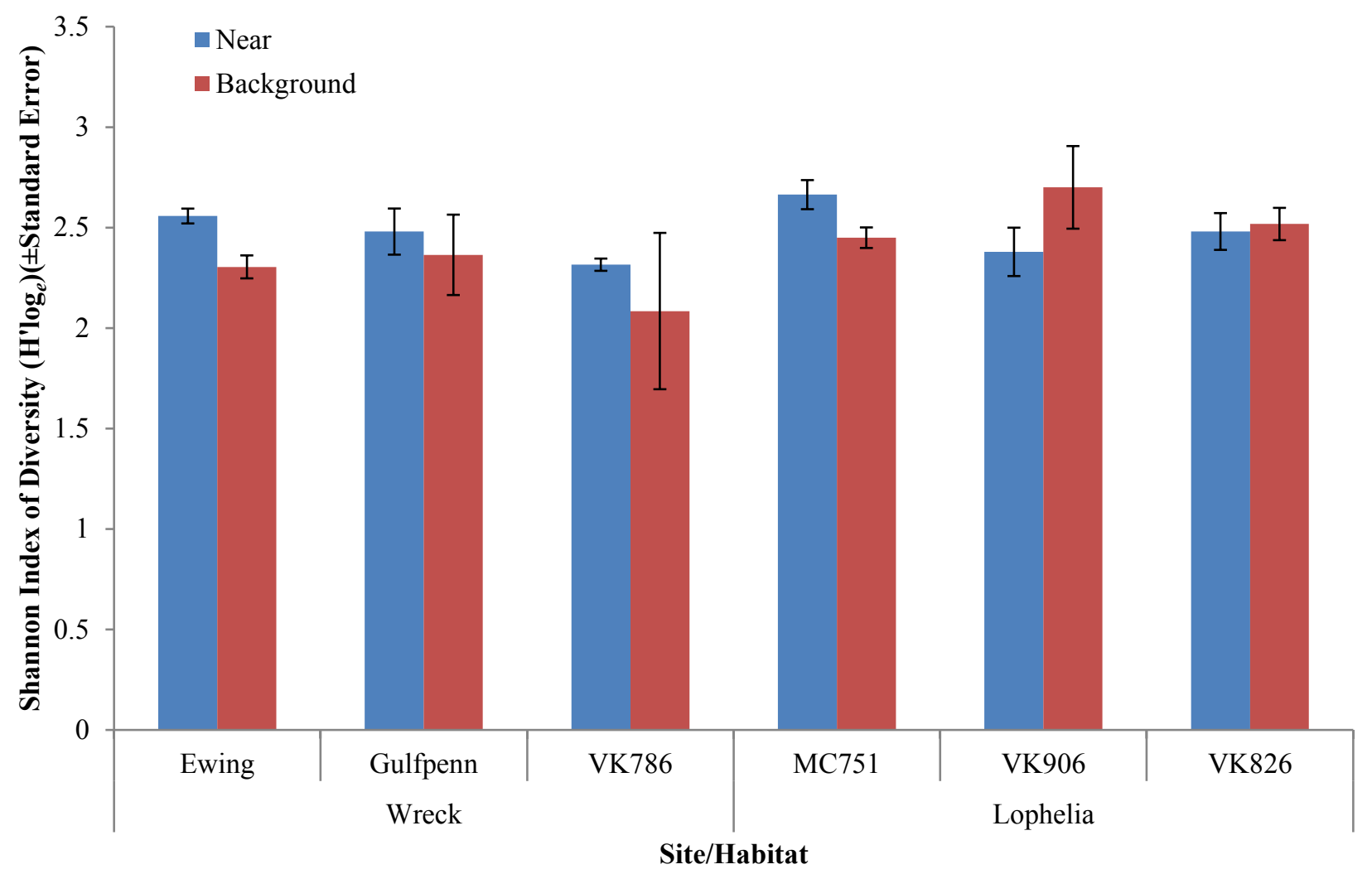

Figure 6.4 Shannon Index of diversity near shipwrecks, near coral, and in background soft sediments during the Lophelia II program. 


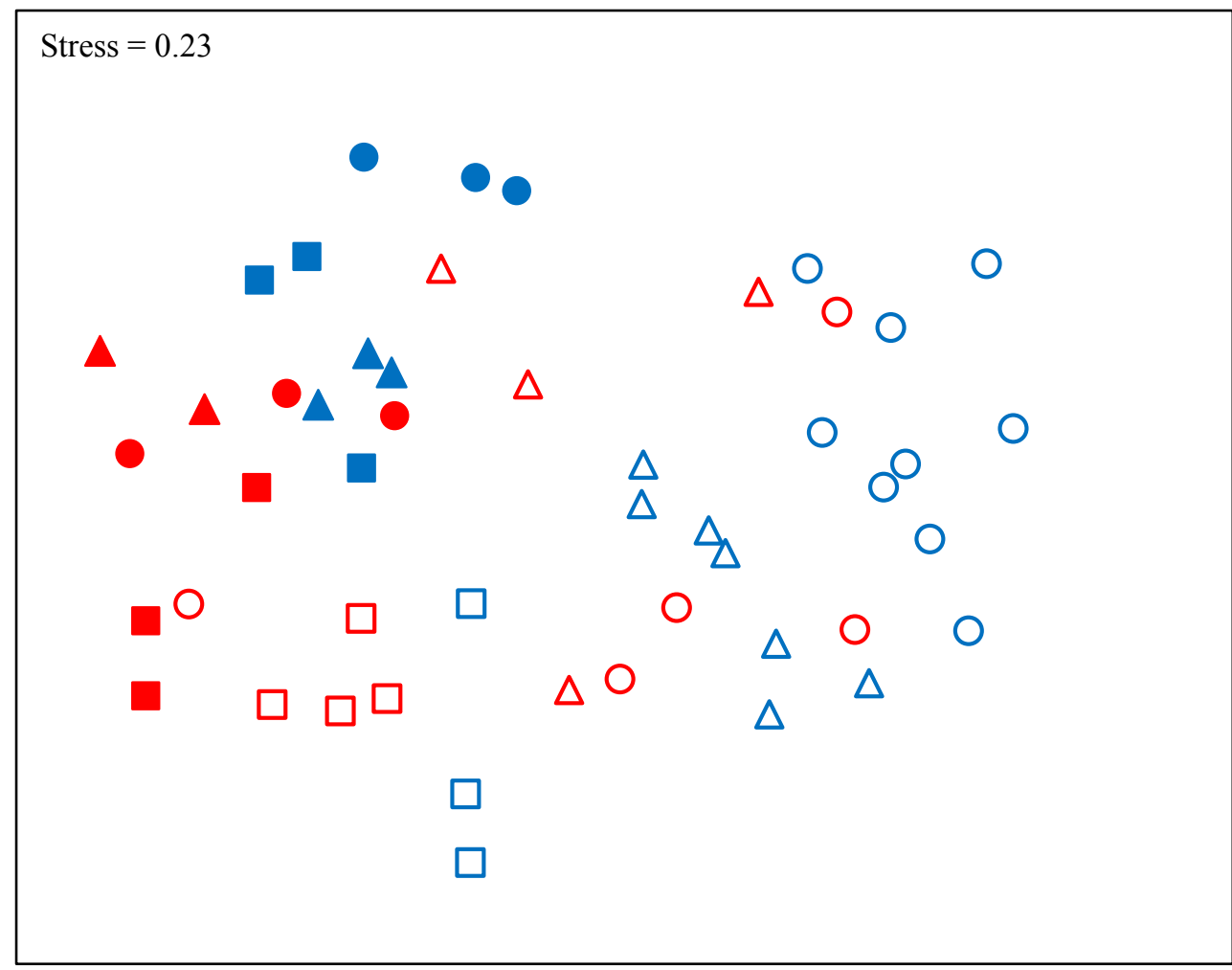

\section{Wrecks}

- Ewing Near

- Ewing Background

- Gulfpenn Near

- Gulfpenn Background

\ VK786 Near

A VK786 Background

\section{Lophelia}

$\square$ MC751 Near
$\square$ MC751 Background
$\circ$ VK906 Near
$\circ$ VK906 Background
$\Delta$ VK826 Near
$\Delta$ VK826 Background

Figure 6.5 Non-metric multidimensional scaling plot of all cores collected near wrecks, near corals, and in background soft sediments based on Bray-Curtis similarities of square-root transformed taxa abundance during the Lophelia II program. 


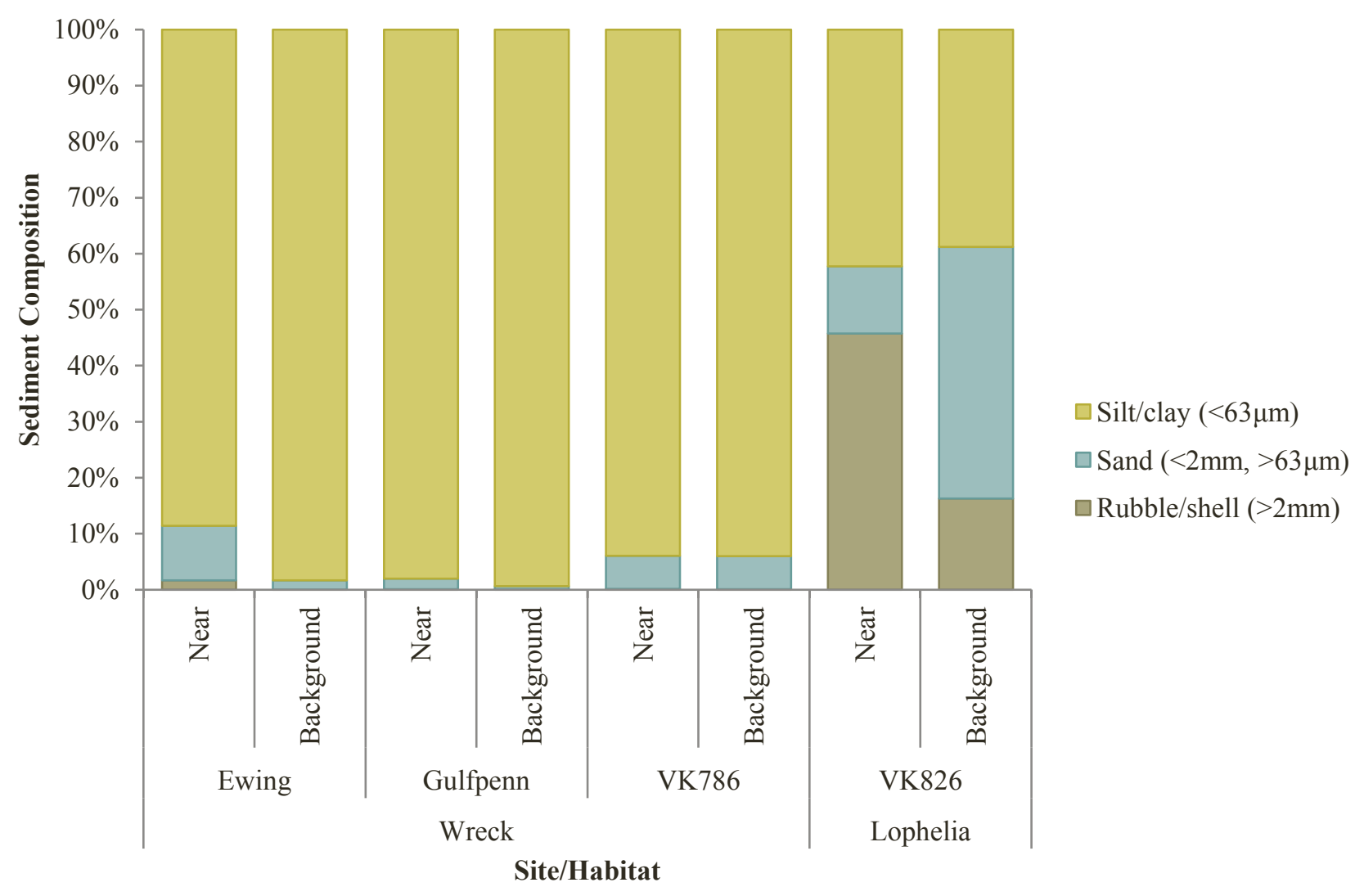

Figure 6.6 Sediment grain-size composition for shipwreck, near-coral, and background soft sediments during the Lophelia II program. 


\title{
7 TROPHODYNAMICS OF THE FISH COMMUNITY ASSOCIATED WITH DEEP. SEA CORALS IN THE GULF OF MEXICO
}

\author{
By Andrew Miller, ${ }^{1}$ Steve W. Ross, ${ }^{1}$ and Mike Rhode ${ }^{1}$ \\ ${ }^{1}$ University of North Carolina Wilmington, Center for Marine Science, Wilmington, NC
}

\subsection{Introduction}

Structure-forming cold-water corals provide habitat for many diverse organisms (Roberts and others, 2006), and these reef ecosystems are considered to be important "hot-spots" of biodiversity. These ecosystems may include scleractinian corals, octocorals, antipatharians, echinoderms, sponges, soft corals, anemones, crustaceans, and fishes (Freiwald and others, 2004). In addition to structureforming invertebrates, rocky substrata form considerable deep-reef habitat which in turn is usually colonized by many invertebrate taxa. Although research on deep-sea coral ecosystems has expanded considerably in the past 10 years, information on the biology and ecology, including trophodynamics, is still lacking.

Gartner and others (1997) organized deep-sea fishes into 10 demersal trophic guilds and 3 pelagic trophic guilds based on their diets. The demersal guilds are piscivores, macronekton foragers, micronekton predators, benthivorous infaunal predators, microphagous epifaunal browsers, megafaunal croppers and browsers, macroplanktonivores, specialist necrophages, necrophagivores, and detritivores. The pelagic guilds are micronektonivores, zooplanktivores, and generalists. Each guild includes dissimilar taxa and is not based on behavior or morphology. While generally useful, the data presented here did not allow such trophic classifications.

Very few studies have examined the feeding habits and diets of deep-sea fishes, despite the claim that food limitation may be the most important limiting factor in deep-sea ecology (Gage and Tyler, 1991). This lack of information is due to a number of factors, such as the financial and physical limitations of deep-sea work, problematic nature of in situ and laboratory observation or experimentation, everted stomachs that are common in some species brought to the surface quickly from the deep sea, and empty stomachs due to irregular feeding intervals (Gartner and others, 1997; Merrett and Haedrich, 1997). As a result, the diets and feeding behavior of bottom and midwater fishes associated with deep-sea coral reefs are relatively poorly understood. In fact, we are not aware of any fish diet studies based on stomach content analysis from deep-sea coral ecosystems.

Considering the lack of diet data and the importance of deep-sea reef ecosystems, we initiated a fish diet study as a component of the multidisciplinary Lophelia II project. This study, which is part of a larger, comprehensive trophodynamics study, is based on fish feeding as revealed through stomach analysis. Other components of the trophodynamics study are ongoing and will examine the trophic roles of the invertebrate community, elucidate the basis of the food web, and estimate food sources mostly via stable isotope analysis. A better understanding of the feeding habits of midwater and bottom fishes living on or adjacent to deep-sea coral reefs could provide important information regarding the flow of energy from the benthos up through the water column and could lead to a broader comprehension of the energy flow within deep-water coral reef ecosystems. The objectives of this study were to illustrate 
feeding patterns among bottom and midwater fishes associated with deep-sea coral reefs and attempt to determine the degree to which these fishes rely on the reef ecosystem.

\subsection{Methods}

\subsubsection{Study Area}

All study sites for this project were on the continental slope ( $\geq 300$-meter $[\mathrm{m}]$ depths) in the eastern and north-central Gulf of Mexico (GOM; fig. 7.1). Sites where bottom fishes were collected were Viosca Knoll 826 (VK826), Viosca Knoll 862/906 (VK862/906), Mississippi Canyon (MC751), and West Florida Slope (WFS; fig. 7.1). Midwater fishes were collected from VK826. See Chapter 1 of this report for additional details about these sites.

\subsubsection{Sample Collection}

Bottom fishes were collected during three cruises: National Oceanic and Atmospheric Administration (NOAA) ship Nancy Foster (NF-2008) during 5-13 October 2008; research vessel (R/V) Seward Johnson (SJ-2009) during 14-25 September 2009; and R/V Cape Hatteras (CH-2010) during 20 September-3 October 2010. Midwater fishes were collected during two cruises: R/V Cape Hatteras (CH-2007) during 9-25 August 2007; and Nancy Foster (NF-2008) during 5-13 October 2008. All sampling was conducted during 24-hour (h) operations, but most of the fishes were collected during nighttime operations. See table 1.1 in Chapter 1 of this report for additional station details. See Ross and others (2012) for details of the CH-2007 cruise.

Bottom fishes were collected by using an otter trawl (3.5-m-width, 3.8-centimeter [cm] mesh) and benthic traps. The otter trawl was deployed open, and it was assumed no significant fishing occurred during deployment due to the rapid lowering, steep wire angle, and minimal forward movement of the vessel. Upon reaching the estimated bottom, the trawl was towed for approximately 30 minutes $(\mathrm{min})$ at a 2-knot $(3.7$ kilometers per hour $[\mathrm{km} / \mathrm{h}])$ ground speed and was then retrieved. Baited deep-sea traps consisted of Z-traps and benthic trap arrays. Z-traps were of the Antillean-type design (approximately $1.8 \times 0.66 \times 1.6 \mathrm{~m}$ ). Benthic trap arrays consisted of three small mesh wire traps held in an aluminum frame. Both types of trap were set on reefs by surface deployment. Bottom fishes were also collected opportunistically with manipulator claw and suction devices by using the Johnson Sea Link II (JSL) submersible.

Midwater fishes were collected by using a discrete-depth sampling Tucker trawl (2- by 2-m, 0.16-cm mesh) containing a plankton net $(0.05$-m-diameter, 335-micrometer $[\mu \mathrm{m}] \mathrm{mesh})$ placed inside the Tucker trawl to sample smaller members of the midwater community. The trawl was deployed open, and as with the otter trawl, it was assumed no significant fishing occurred during deployment. Upon reaching the designated depth, the net was towed for $30 \mathrm{~min}$ at a 2-knot ground speed and was triggered closed by using a double-trip mechanism. The Tucker trawl was equipped with a Sea-Bird SBE39 temperature-depth recorder (TDR) attached to the upper frame bar to record time, depth, and temperature during deployment. Actual time and depth fished for each trawl were determined post-trawl by using data from the TDR, and these data were used throughout the cruise to adjust fishing strategies to achieve desired sampling depths. Midwater fishes were also collected to a lesser by extent using plankton net tows. See Ross and others (2010) for additional details on collection of the midwater fishes.

All fishes collected were preserved at sea in 10-percent formalin in seawater solution and later transferred to 50 percent isopropyl alcohol for storage until dietary analyses. All specimens were sorted, identified to the lowest possible taxa, and measured to the nearest millimeter standard length (SL) or 
total length (TL), depending on species. The life history stage of fishes was also recorded based on the condition of gonads. A fish was classified as juvenile when either no gonads or immature gonads were documented. Fish measurements and life history stages are not reported in this chapter.

\subsubsection{Diet Analyses}

Gut content analyses (GCA) were conducted using methods outlined in Ross and Moser (1995). Fish specimens were subsampled from each site to include all sizes and depths sampled, and the stomachs were removed for GCA. Stomach contents were placed on a Petri dish and identified to the lowest possible taxa. Similar prey species were then piled together on a grid of 1-millimeter (mm) squares and flattened to a uniform height, which was measured. The height multiplied by the number of squares occupied by the food item yielded volume in cubic millimeters. The sum of all prey volumes equaled the total volume of food in the stomach, and the volume of each prey item was converted to a percentage of the total prey volume $(\% \mathrm{~V})$. The frequency of occurrence for a prey item equaled the number of times a prey item occurred in the fish species examined divided by the total number of stomachs with food analyzed for that species.

Food items found during GCA were grouped into 18 general food types: Amphipoda, Tunicates (Chordata), Cnidaria, Copepoda, Crustacea, Cumacea, Euphausiacea, fish, Foraminifera, Isopoda, Mollusca, Mysida, Ostracoda, other, parasite, Polychaeta, Radiolaria, and Tanaidacea. If food items were not present, stomach contents were described as empty or everted. Everted stomachs were eliminated from GCA. The percentage of empty stomachs was reported separately. The food type Crustacea consisted of crustaceans other than amphipods, copepods, cumacids, euphausids, isopods, mysids, ostracods, and tanaidacids. The food group other consisted of unidentifiable organic and inorganic material. The results of GCA for families with $n \geq 10$ individuals are presented in the next section.

\subsection{Results}

\subsubsection{Bottom Fishes}

During NF-2008, SJ-2009, and CH-2010 cruises, 926 bottom or demersal fishes were caught. Of that total, 634 fishes were caught by otter trawl during NF-2008; 35 fishes were caught during SJ-2009, with 15 captured by submersible and 20 caught in benthic traps; and 90 fishes were caught during $\mathrm{CH}-$ 2010, with 82 caught by otter trawl and 8 by benthic traps. In total, 18 families were collected by bottom-fishing gear: Macrouridae (30.4 percent of the total), Moridae (22.0 percent), Ogcocephalidae (18.1 percent), Percophidae (5.9 percent), Cynoglossidae (4.6 percent), Chaunacidae (3.3 percent), Scorpaenidae (3.0 percent), Peristeidiidae (2.5 percent), Phycidae (2.5 percent), Pleuronectidae (2.4 percent), Epigonidae (1.3 percent), Congridae (1.1 percent), Trachichtyhidae (0.9 percent), Squalidae (0.8 percent), Myxinidae (0.4 percent), Lophiidae (0.3 percent), Ophiididae (0.3 percent), and Synaphobranchidae (0.3 percent).

GCA was conducted for 759 individual bottom fish from the 3 cruises (table 7.1), including species $(n=30)$ from all families caught during all 3 cruises. The 30 species of bottom fishes subjected to GCA included (in order of decreasing abundance): Laemonema goodebeanorum, Dibranchus atlanticus, Nezumia aequalis, Coelorinchus caribbaeus, Bembrops gobioides, Symphurus marginatus, Malacocephalus occidentalis, Coelorinchus coelorinchus, Bathygadus macrops, Chaunax suttkusi, Peristedion greyae, Poecilopsetta beanii, Helicolenus dactylopterus, Urophycis cirrata, Epigonus

pandionis, Conger oceanicus, Laemonema barbatulum, Hoplostethus occidentalis, Urophycis floridana, Pontinus rathbuni, Eptatretus minor, Etmopterus schultzi, Benthocometes robustus, Dysommina rugosa, 
Etmopterus virens, Lophiodes beroe, Physiculus fulvus, Pontinus longispinus, Chaunax pictus, and Etmopterus bigelowi. Stomach content data for these bottom fishes are provided in table 7.2, except for four species (Etmopterus bigelowi, Dysommina rugosa, Chaunax pictus, and Physiculus fulvus) for which there were no food data due to either empty or everted stomachs. A summary of diets is provided below for 19 species for which there were the most data (table 7.2).

\subsubsection{Macrouridae}

GCA were conducted on five species of Macrouridae (B. macrops, C. carribaeus, C. caelorinchus, $M$. occidentalis, and $N$. aequalis). The diets of macrourids were diverse, ranging from 5 prey categories (B. macrops) to 13 prey categories (C. carribaeus) (Figs. 7.2-7.6). Crustaceans were documented in the stomachs of all macrourid species (table 7.2), and it was the dominant prey type based on food volume in four of the five macrourid species: B. macrops (91.2 percent of total volume), C. caelorinchus (52.0 percent), M. occidentalis ( 94.7 percent), and $N$. aequalis (46.8 percent). The dominant prey category in $C$. carribaeus was Polychaeta (58.5 percent) based on stomach content volume. "Other" was the most frequently observed category of prey item found in the stomachs of $C$. caelorinchus.

\subsubsection{Moridae}

GCA were conducted on two species of Moridae (L. barbatulum and L. goodebeanorum). The diets of these 2 morids were fairly diverse, ranging from 5 prey categories (L. barbatulum) to 10 prey categories (L. goodebeanorum) (Figs. 7.7 and 7.8). Mysids were the dominant prey type found in the stomachs of $L$. barbatulum, composing 65.0 percent of the stomach contents volumetrically. Crustaceans were the dominant prey type found in the stomachs of L. goodebeanorum, composing 63.9 percent of the stomach contents volumetrically. Crustaceans other than Amphipoda, Copepoda, and Mysida were absent from the stomach contents of all L. barbatulum individuals (table 7.2).

\subsubsection{Ogocephalidae}

GCA were conducted on one species of Ogocephalidae (D. atlanticus). The diet of this batfish was quite diverse, consisting of 15 prey categories (fig. 7.9). Mollusks were the dominant prey type found in the stomachs of $D$. atlanticus, composing 51.9 percent of the stomach contents volumetrically (table 7.2).

\subsubsection{Percophidae}

GCA were conducted on one species of Percophidae (B. gobioides). The diet of this species consisted of five prey categories (fig. 7.10), the dominant prey being euphausids, which composed 48.1 percent of the stomach contents volumetrically (table 7.2).

\subsubsection{Cynoglossidae}

GCA were conducted on one species of Cynoglossidae (S. marginatus). The diet of this species was relatively diverse (fig. 7.11), consisting of 10 prey categories. Mollusks were the dominant prey type found in the stomachs of $S$. marginatus, composing 49.2 percent of the stomach contents volumetrically (table 7.2). 


\subsubsection{Chaunacidae}

GCA were conducted on one species of Chaunacidae (C. suttkusi). The diet of this species was relatively diverse, consisting of 10 prey categories (fig. 7.12). Other crustaceans were overwhelmingly the dominant prey type found in the stomachs of $C$. suttkusi, composing 97.0 percent of the stomach contents volumetrically (table 7.2).

\subsubsection{Scorpaenidae}

GCA were conducted on three species of Scorpaenidae (H. dactylopterus, $P$. longispinus, and $P$. rathbuni). The diets of these species ranged from consisting of only two prey categories (for $P$. longispinus and P. rathbuni) to seven prey categories (for H. dactylopterus) (Figs. 7.13-7.15). Volumetrically, the dominant prey item for H. dactylopterus (50.4 percent) and P. rathbuni (98.3 percent) was tunicates. The dominant prey item by volume found in the stomach contents of $P$. longispinus was Tanaidacea (58.7 percent) (table 7.2).

\subsubsection{Peristeidiidae}

GCA were conducted on one species of Peristeidiidae (P. greyae). The diet of this species consisted of eight prey categories (fig. 7.16), though the dominant prey category was other crustaceans, composing 54.0 percent of the stomach contents volumetrically (table 7.2).

\subsubsection{Phycidae}

GCA were conducted on two species of Phycidae (U. cirrata and U. floridana). The stomach contents of $U$. floridana consisted of only fish (fig. 7.17), a prey type not found in the stomach contents of $U$. cirrata (fig. 7.18). The stomach contents of $U$. cirrata consisted of five prey types, and crustaceans (42.6 percent) and mollusks (38.7 percent) were the two dominant prey types found in the stomach contents of this species by volume (table 7.2).

\subsubsection{Pleuronectidae}

GCA were conducted on one species of Pleuronectidae (P. beanii). The diet of this species consisted of eight prey types (fig. 7.19), the dominant being mollusks, which composed 55.1 percent of the stomach contents by volume (table 7.2).

\subsubsection{Epigonidae}

GCA were conducted on one species of Epigonidae (E. pandionis). The diet of this species consisted of five prey categories (fig. 7.20). The dominant prey type was "other" (42.1 percent), followed by crustaceans (29.0 percent) and amphipods (18.9 percent) by volume (table 7.2).

\subsubsection{Midwater Fishes}

During CH-2007 and NF-2008 cruises, 1,149 midwater fishes were caught at VK826. Of that total, GCA was conducted for 316 individual midwater fishes from the two cruises (table 7.3): 303 fishes were caught during CH-2007, 287 caught by Tucker trawl and 16 by plankton net; 13 fishes were caught during NF-2008, 12 by Tucker trawl and 1 by plankton net. Overall, seven families were collected by midwater sampling: Gonostomatidae (29.9 percent of total), Myctophidae (27.5 percent), Sternoptychidae (24.4 percent), Phosichthyidae (11.1 percent), Stomiidae (5.1 percent), Bregmacerotidae (1.9 percent), and Melamphaidae (0.6 percent). 
A total of 18 midwater fish species were subjected to GCA (in order of decreasing abundance): Valenciennellus tripunctulatus, Cyclothone braueri, Myctophum affine, Cyclothone alba, Diaphus dumerilii, Pollichthys mauli, Diaphus mollis, Argyropelecus aculeatus, Chauliodus sloani, Argyropelecus hemigymnus, Vinciguerria nimbaria, Gonostoma elongatum, Bregmaceros atlanticus, Lepidophanes guentheri, Stomias affinis, Polyipnus clarus, Melamphaes simus, and Gonostoma atlanticum. Stomach content data are provided in table 7.4. A summary of diets is provided below for 16 species for which there were the most data.

\subsubsection{Gonostomatidae}

GCA were conducted on four Gonostomatidae species (C. alba, C. braueri, G. atlanticum, and G. elongatum). The diets of these species ranged from one prey category (C. braueri and G. atlanticum) to four prey categories (G. elongatum) (Figs. 7.21-7.24). The dominant prey type in three of the four species, C. alba, C. braueri, and G. elongatum, was Copepoda, with each of those species' diets consisting of greater than 50 percent copepods by volume. The dominant prey type in G. atlanticum was Euphausiacea, composing 100 percent of the stomach contents by volume (table 7.4).

\subsubsection{Myctophidae}

GCA were conducted on four Myctophidae species (D. dumerilii, D. mollis, L. guentheri, and M. affine). The diets of these myctophids ranged from four prey categories (D. dumerilii, D. mollis, and L.guentheri) to seven prey categories (M. affine) (Figs. 7.25-7.28). Copepoda was the dominant prey type in three of the four myctophid species, D. dumerilii (86.3 percent), D. mollis (73.0 percent), and $M$. affine (43.4 percent), based on the volumes of stomach contents. The dominant prey category in $L$. guentheri was Euphausiacea, composing 57.6 percent of the stomach contents by volume; however, copepods were the most frequent prey type found in the stomachs of L. guentheri (table 7.4).

\subsubsection{Sternoptychidae}

GCA were conducted on four sternoptychid species (A. aculeatus, A. hemigymnus, $P$. clarus, and $V$. tripunctulatus). The diets of these species ranged from one (A. hemigymnus and $V$. tripunctulatus) to five prey categories (A. aculeatus) (Figs. 7.29-7.32). The dominant prey category by volume in three of the four species of sternoptychid (A. hemigymnus, $P$. clarus, and $V$. tripunctulatus) was Copepoda (\%V $>64$ percent in each of the three species). The dominant prey category in the fourth species, $A$. aculeatus, was "other" (table 7.4).

\subsubsection{Phosichthyidae}

GCA were conducted on two phosichthyid species ( $P$. mauli and $V$. nimbaria). The diets of both of these species were composed of six prey categories (Figs. 7.33 and 7.34). The dominant prey category found in the stomachs of $P$. mauli was Copepoda (62.6 percent) by volume. The dominant prey item by volume in $V$. nimbaria was Euphausiacea (49.1 percent). Copepods and euphausids were found in both $P$. mauli and $V$. nimbaria (table 7.4).

\subsubsection{Stomiidae}

GCA were conducted on two stomiid species (C. sloani and S. affinis). The diets of these two stomiid species were very different (Figs. 7.35 and 7.36). The stomach contents of $C$. sloani contained 97.4 percent euphausids and 2.6 percent "other" by volume, and S. affinis stomachs contained 100 percent other crustaceans by volume (table 7.4). 


\subsection{Discussion}

Since there is little primary production below the photic zone in the deep sea, nearly all energy is ultimately derived from primary production in surface waters, some of which (for example, phytodetritus) eventually reaches the deep sea. The only exception to this are the chemosynthetic trophic pathways that exist for seep and vent communities. Although seep communities are common in the GOM and often in close proximity to the deep-reef ecosystems, current data indicate that coral and deep-reef communities are not energetically dependent on them (Cordes and others, 2008; Becker and others, 2009). Despite evidence that some bottom (Jeffreys and others, 2011) and midwater (Robison, 1984) fishes may directly use phytodetritus or diatoms as food, most deep-sea fishes are carnivorous. Organic matter that fuels the deep benthic communities reaches the bottom via downward drift, active current transport, and transport by the migrating midwater fauna. The continental slopes of the GOM where deep corals occur are in the vicinity of riverine and upwelling sources of nutrients which support surface productivity that eventually reaches the bottom in seasonal waves (Mienis and others, 2012). This seasonal delivery of food resources or nutrients has been widely noted in slope depths elsewhere (see review in Beaulieu, 2002).

Fishes in the GOM have been relatively well studied for feeding habits, except that the diets of fishes in the deep-sea (including the slope) and mesopelagic regions are still poorly known (Simmons and others, 2013), and there are no diet-based studies for fishes in the vicinity of deep-reef ecosystems, thus limiting our ability to make comparisons. Three bottom fishes (D. atlanticus, S. marginatus, and $P$. beanii) that are generally regarded as being restricted to soft-sediment habitat (Ross and Quattrini, 2007; S.W. Ross, personal observations) fed predominantly on Mollusca, which was a noticeable difference from most other species examined. These three species also exhibited some of the most diverse diets, perhaps reflecting their use of the diverse benthic or infaunal invertebrate community. Most other fishes relied on various Crustacea for food; however, in some species, polychaetes and fishes contributed noticeably to diets. High abundance and diversity of invertebrates have been observed on GOM reefs (Cordes and others, 2008; Lessard-Pilon and others, 2010; Nizinski, Chapter 9 of this report), indicating a rich food resource for benthic fishes. In addition, Davies and others (2010) and Mienis and others (2012) noted large aggregations of small benthic fauna (likely crustaceans), a potential food resource, undergoing nightly migrations off the bottom at the VK826 site. The high proportion of fish remains in the diets of the hagfish $E$. minor could reflect that this species is more of an active predator than previously thought, an observation also recently noted for a congener (Zintzen and others, 2011) and for Atlantic hagfish, Myxine glutinosa (Auster and Barber, 2006). Bright (1970) also noted the preponderance of small Crustacea in the diets of GOM deep-sea fishes, but he did not present individual fish data. In addition, most of his sampling was deeper than for this study and only 8 of his 81 fish species overlapped with this study.

As with the bottom fishes, the diets of the mesopelagic fishes examined were dominated by small Crustacea, particularly copepods and euphausids. As mesopelagic fishes were often observed near the bottom and were observed being preyed upon by some benthic fishes (for example, $H$.

dactylopterus), they may serve an important role in the transfer of energy through the water column as noted by Gartner and others $(1997,2008)$. The most extensive studies of mesopelagic fishes trophodynamics in the GOM was conducted over several years in the eastern GOM near our WFS study site. Data from that effort (Hopkins and Baird, 1981; Hopkins and Baird, 1985; Hopkins and others, 1996; Sutton and Hopkins, 1996) as well as other studies on mesopelagic feeding (Merrett and Roe, 1974; Champalbert and others, 2008) agree closely with the diet data presented here. Although deeper waters of the central GOM were sampled, our data agree closely with results of McClain-Counts (2010), who also reported copepods to be the dominant prey item for most mesopelagic fishes. 
At the general level of food categories summarized here and considering that most of these fishes were collected by trawl (non-habitat specific), it was not possible to distinguish habitat impacts on fish diets. In addition, there were not enough data for any species to compare diets between general study areas, years, or seasons. Other limits imposed on this study were the inability to collect any or sufficient numbers of some fishes that are closely associated with reef habitats (such as Beryx spp., $C$. oceanicus, $H$. perciformis, and G. brachiusculus; see Chapter 8 of this report). Diets of two of these taxa (B. decadactylus and H. perciformis), however, were examined by Goldman and Sedberry (2010) on the Charleston Bump (southeastern United States).

\subsection{References}

Auster, P.J., and Barber, K., 2006, Atlantic hagfish exploit prey captured by other taxa: Journal of Fish Biology, v. 68, p. 618-621.

Beaulieu, S.E., 2002, Accumulation and fate of phytodetritus on the sea floor: Oceanography and Marine Biology: an Annual Review, v. 40, p. 171-232.

Becker, E.L., Cordes, E.E., Macko, S.A., and Fisher, C.R., 2009, Importance of seep primary production to Lophelia pertusa and associated fauna in the Gulf of Mexico: Deep-Sea Research Part I, v. 56, p. 786-800.

Bright, T.J., 1970, Food of deep-sea bottom fishes, Chapter 9, in Pequegnat, W.E., and Chace, F.A., eds., Contributions to the biology of the Gulf of Mexico, v. 1, p. 245-252.

Champalbert, G.A., Kouamé, B., Pagano, M., and Marchal, E., 2008, Feeding behavior of adult Vinciguerria nimbaria (Phosichthyidae), in the tropical Atlantic $\left(0^{\circ}-4^{\circ} \mathrm{N}, 15^{\circ} \mathrm{W}\right)$ : Marine Biology, $\mathrm{v}$. 156, p. 79-95.

Cordes, E.E., McGinley, M.P., Podowski, E.L., Becker, E.L., Lessard-Pilon, S., Viada, S.T., and Fisher, C.R., 2008, Coral communities of the deep Gulf of Mexico: Deep-Sea Research Part I, v. 55, p. 777787.

Davies, A.J., Duineveld, G.C.A., van Weering, T.C.E., Mienis, F., Quattrini, A.M., Seim, H.E., Bane J.M., and Ross, S.W., 2010, Short-term environmental variability in cold-water coral habitat at Viosca Knoll, Gulf of Mexico: Deep-Sea Research Part I, v. 57, p. 199-212.

Friewald, A., Fossa, J.H., Grehan, A., Koslow, T., and Roberts, J.M., 2004, Cold-water coral reefs: Cambridge, UK, United Nations Environment Programme, World Conservation Monitoring Centre.

Gage, J.D., and Tyler, P.A., 1991, Deep-sea biology: a natural history of organisms at the deep-sea floor: Cambridge University Press, 504 p.

Gartner, J.V., Jr., Crabtree, R.E., and Sulak, K.J., 1997, Feeding at depth, in Randall, D.J., and

Farrell, A.P., eds., Deep-sea fishes: San Diego, Academic Press, p. 115-193.

Gartner, J.V., Jr., Sulak, K.J., Ross, S.W., and Necaise, A-M., 2008, Persistent near-bottom aggregations of mesopelagic animals along the North Carolina and Virginia continental slopes: Marine Biology, v. 153, p. 825-841.

Goldman, S.F., and Sedberry, G.R., 2010, Feeding habits of some demersal fish on the Charleston Bump off the southeastern United States: ICES Journal of Marine Science, v. 68, p. 390-398.

Hopkins, T.L., and Baird, R.C., 1981, Trophodynamics of the fish Valenciennellus tripunctulatus. I. Vertical distribution, diet, and feeding chronology: Marine Ecology Progress Series, v. 5, p. 1-10. Hopkins, T.L., and Baird, R.C., 1985, Feeding ecology of four hatchetfishes (Sternoptychidae) in the eastern Gulf of Mexico: Bulletin of Marine Science, v. 36, p. 260-277.

Hopkins, T.L., Sutton, T.T., and Lancraft, T.M., 1996, The trophic structure and predation impact of a low latitude midwater fish assemblage: Progress in Oceanography, v. 38, p. 205-239. 
Jeffreys, R.M., Lavaleye, M.S.S., Bergman, J.N., Duineveld, G.C.A., and Witbaard, R., 2011, Do abyssal scavengers use phytodetritus as a food resource? Video and biochemical evidence from the Atlantic and Mediterranean: Deep-Sea Research Part I, v. 58, p. 415-428.

Lessard-Pilon, S.A., Podowski, E.L., Cordes, E.E., and Fisher, C.R., 2010, Megafauna community composition associated with Lophelia pertusa colonies in the Gulf of Mexico: Deep-Sea Research Part II, v. 57, p. 1882-1890.

McClain-Counts, J.P., 2010, Trophic structure of midwater fishes over cold seeps in the north central Gulf of Mexico: Masters Thesis, University of North Carolina at Wilmington, $115 \mathrm{p}$.

Merrett, N.R., and Haedrich, R.L., 1997, Deep-sea demersal fish and fisheries: London, Chapman and Hall, $282 \mathrm{p}$.

Merrett, N.R., and Roe, H.S.J., 1974, Patterns and selectivity in the feeding of certain mesopelagic fishes: Marine Biology, v. 28, p. 115-126.

Mienis, F., Duineveld, G.C.A., Davies, A.J., Ross, S.W., Seim, H., Bane J., and van Weering, T.C.E., 2012, The influence of near-bed hydrodynamic conditions on cold-water corals in the Viosca Knoll area, Gulf of Mexico: Deep-Sea Research Part I, v. 60, p. 32-45.

Roberts, J.M., Wheeler, A.J., and Freiwald, A., 2006, Reefs of the deep: The biology and geology of cold-water coral ecosystems: Science, v. 312, p. 534-547.

Robison, B.H., 1984, Herbivory by the myctophid fish Ceratoscopelus warmingii: Marine Biology, v. 84, p. 119-123.

Ross, S.W., Demopoulos, A.W.J., Kellogg, C.A., Morrison, C.L., Nizinski, M.S., Ames, C.L., Casazza, T.L., Gualtieri, D., Kovacs, K., McClain, J.P., Quattrini, A.M., Roa-Varón, A.Y., and Thaler, A.D., 2012, Deepwater Program: Studies of Gulf of Mexico lower continental slope communities related to chemosynthetic and hard substrate habitats: U.S. Geological Survey Open-File Report 2012-1032, $301 \mathrm{p}$.

Ross, S.W., and Moser, M.L., 1995, Life history of juvenile gag, Mycteroperca microlepis, in North Carolina estuaries: Bulletin of Marine Science v. 56, p. 222-237.

Ross, S.W., and Quattrini, A.M., 2007, The fish fauna associated with deep coral banks off the southeastern United States: Deep-Sea Research Part I, v. 54, p. 975-1007.

Ross, S.W., Quattrini, A.M., Roa-Varón, A.Y., and McClain, J.P., 2010, Species composition and distributions of mesopelagic fishes over the slope of the north-central Gulf of Mexico: Deep-Sea Research Part II, v. 57, p. 1926-1956.

Simmons, J.D., Yuan, M., Carollo, C., Vega-Cendejas, M., Shirley, T., Palomares, M.L.D., Roopnarine, P., Arenas, L., Ibanez, A., Holmes, J., Schnoonard, C.M., Hertog, R., Reed, D., and Poelen, J., 2013, Building a fisheries trophic interaction database for management and modeling research in the Gulf of Mexico large marine ecosystem: Bulletin of Marine Science, v. 89, p. 135-160.

Sutton, T.T., and Hopkins, T.L., 1996, Trophic ecology of the stomiid (Pisces: Stomiidae) fish assemblage of the eastern Gulf of Mexico: Strategies, selectivity and impact of a top mesopelagic predator group: Marine Biology, v. 127, p. 179-192.

Zintzen, V., Roberts, C.D., Anderson, M.J., Stewart, A.L., and Struthers, C.D., 2011, Hagfish predatory behaviour and slime defence mechanism: Scientific Reports, v. 1, Article 131, 6 p. 
Table 7.1. Number of stomachs analyzed and number of empty or everted stomachs for bottom fishes caught during NF-2008, SJ-2009 and CH-2010 cruises in the Gulf of Mexico near or on deep-sea reef habitats as part of the Lophelia II program.

\begin{tabular}{|c|c|c|c|}
\hline Family/species & $\begin{array}{c}\text { Stomachs } \\
\text { analyzed }\end{array}$ & $\begin{array}{c}\text { Empty } \\
\text { stomachs }\end{array}$ & $\begin{array}{c}\text { Everted } \\
\text { stomachs }\end{array}$ \\
\hline Myxinidae & 3 & & \\
\hline Eptatretus minor & 3 & 1 & \\
\hline Squalidae & 6 & & \\
\hline Etmopterus bigelowi & 1 & 1 & \\
\hline Etmopterus schultzi & 3 & 1 & \\
\hline Etmopterus virens & 2 & 1 & \\
\hline Synaphobranchidae & 2 & & \\
\hline Dysommina rugosa & 2 & 2 & \\
\hline Congridae & 8 & & \\
\hline Conger oceanicus & 8 & 6 & \\
\hline Ophiididae & 3 & & \\
\hline Benthocometes robustus & 3 & & \\
\hline Macrouridae & 231 & & \\
\hline Bathygadus macrops & 24 & 1 & 13 \\
\hline Coelorinchus caribbaeus & 71 & 1 & 9 \\
\hline Coelorinchus caelorhincus & 27 & & 6 \\
\hline Malacocephalus occidentalis & 30 & 2 & 21 \\
\hline Nezumia aequalis & 79 & 1 & 50 \\
\hline Moridae & 167 & & \\
\hline Laemonema barbatulum & 8 & & 4 \\
\hline Laemonema goodebeanorum & 157 & & 131 \\
\hline Physiculus fulvus & 2 & 1 & 1 \\
\hline Phycidae & 19 & & \\
\hline Urophycis cirrata & 14 & 1 & 12 \\
\hline Urophycis floridana & 5 & 3 & \\
\hline Lophiidae & 2 & & \\
\hline Lophiodes beroe & 2 & & 1 \\
\hline Ogcocephalidae & 137 & & \\
\hline Dibranchus atlanticus & 137 & 51 & \\
\hline Chaunacidae & 25 & & \\
\hline Chaunax pictus & 1 & 1 & \\
\hline Chaunax suttkusi & 24 & 7 & \\
\hline Trachichthyidae & 7 & & \\
\hline Hoplostethus occidentalis & 7 & 1 & 1 \\
\hline Scorpaenidae & 23 & & \\
\hline Helicolenus dactylopterus & 17 & 9 & \\
\hline Pontinus longispinus & 2 & 1 & \\
\hline Pontinus rathbuni & 4 & 1 & 2 \\
\hline
\end{tabular}


Table 7.1. Number of stomachs analyzed and number of empty or everted stomachs for bottom fishes cause during NF-2008, SJ-2009, and CH-2010 cruises in the Gulf of Mexico near or on deep-sea reef habitats as part of the Lophelia II program - continued.

\begin{tabular}{lccc}
\hline \multicolumn{1}{c}{ Family/species } & $\begin{array}{c}\text { Stomachs } \\
\text { analyzed }\end{array}$ & $\begin{array}{c}\text { Empty } \\
\text { stomachs }\end{array}$ & $\begin{array}{c}\text { Everted } \\
\text { stomachs }\end{array}$ \\
\hline Peristeidiidae & 19 & & \\
$\quad$ Peristedion greyae & 19 & 3 & 2 \\
Epigonidae & 10 & & 1 \\
$\quad$ Epigonus pandionis & 10 & 2 & \\
Percophidae & 45 & & \\
$\quad$ Bembrops gobioides & 45 & 24 & \\
Pleuronectidae & 18 & & \\
$\quad$ Poecilopsetta beanii & 18 & 6 & \\
Cynoglossidae & 35 & & \\
$\quad$ Symphurus marginatus & 35 & 9 & \\
\hline
\end{tabular}


Table 7.2. Percent frequency $(\% \mathrm{~F})$ and percent volume $(\% \mathrm{~V})$ of stomach contents for 31 bottom fish species caught during NF-2008, SJ-2009, and $\mathrm{CH}-2010$ cruises at Vioska Knoll 826, Vioska Knoll 862/906, Mississippi Canyon and West Florida Slope during the Lophelia II program.

[See figure 7.1 for site locations. Amphi, Amphipoda; Tuni, Tunicata; Cnid, Cnidaria; Cope, Copepoda; Crust, Crustacea; Cuma, Cumacea; Euph,

Euphausiacea; Fish, Fish; Isop, Isopoda; Moll, Mollusca; Mysi, Mysida; Other, Other; Ostr, Ostracoda; Para, Parasite; Poly, Polychaeta; Radi, Radiolaria; Tana,

Tanaidacea; n, number; <, less than]

\begin{tabular}{|c|c|c|c|c|c|c|c|c|c|c|c|c|c|c|c|c|c|c|}
\hline Species & Amphi & Tuni & Cnid & Cope & Crust & Cuma & Euph & Fish & Fora & Isop & Moll & Mysi & Ostr & Other & Para & Poly & Radi & Tana \\
\hline \multicolumn{19}{|c|}{ Bathygadus macrops $(\mathrm{n}=10)$} \\
\hline$\% \mathrm{~F}$ & 10 & & & 40 & 60 & 10 & & & & & & & & 40 & & & & \\
\hline$\% \mathrm{~V}$ & 0.2 & & & 3 & 91.2 & $<0.1$ & & & & & & & & 5.6 & & & & \\
\hline \multicolumn{19}{|c|}{ Bembrops gobioides $(\mathrm{n}=21)$} \\
\hline$\% \mathrm{~F}$ & & 4.8 & & 9.5 & 42.9 & & 9.5 & & 42.9 & & & & & 47.6 & & & & \\
\hline$\% \mathrm{~V}$ & & 0.6 & & 0.5 & 34.3 & & 48.1 & & 0.3 & & & & & 16.2 & & & & \\
\hline \multicolumn{19}{|c|}{ Benthocometes robustus $(\mathrm{n}=3)$} \\
\hline$\% \mathrm{~F}$ & & & & 100 & & & & & & 33 & & & 33 & 66 & & & & \\
\hline$\% \mathrm{~V}$ & & & & 92.3 & & & & & & 11.8 & & & 1.9 & 5.8 & & & & \\
\hline \multicolumn{19}{|c|}{ Chaunax suttkusi $(\mathrm{n}=17)$} \\
\hline$\% \mathrm{~F}$ & 11.8 & & & 17.6 & 52.9 & & & 11.8 & 17.6 & & 5.9 & 17.6 & & 17.6 & 5.9 & 5.9 & & \\
\hline$\% \mathrm{~V}$ & 0.2 & & & 0.1 & 97 & & & 0.9 & 0.1 & & 0.5 & 0.6 & & 0.6 & $<0.1$ & 0.1 & & \\
\hline \multicolumn{19}{|c|}{ Coelorinchus caribbaeus $(\mathrm{n}=61)$} \\
\hline$\% \mathrm{~F}$ & 65.6 & & & 93.4 & 52.5 & & 1.6 & 18 & 42.6 & & 26.2 & 29.5 & 11.5 & 88.5 & 8.2 & 96.7 & & 21.3 \\
\hline$\% \mathrm{~V}$ & 1.3 & & & 1.3 & 4.7 & & 0.1 & 6 & 0.1 & & 2.3 & 0.3 & 0.1 & 25.2 & $<0.1$ & 58.5 & & 0.2 \\
\hline \multicolumn{19}{|c|}{ Coelorinchus caelorhincus $(\mathrm{n}=21)$} \\
\hline$\% \mathrm{~F}$ & 66.7 & & & 42.9 & 66.7 & & & 14.3 & 28.6 & & 19 & 19 & & 76.2 & 14.3 & 71.4 & & 4.8 \\
\hline$\% \mathrm{~V}$ & 2 & & & 0.3 & 52 & & & 0.3 & 0.4 & & 1 & 0.4 & & 18.3 & $<0.1$ & 25.2 & & $<0.1$ \\
\hline \multicolumn{19}{|c|}{ Conger oceanicus $(\mathrm{n}=2)$} \\
\hline$\% \mathrm{~F}$ & & & & & 50 & & & & & & & & & 50 & 50 & & & \\
\hline$\% \mathrm{~V}$ & & & & & 97.4 & & & & & & & & & 1.9 & 0.8 & & & \\
\hline \multicolumn{19}{|c|}{ Dibranchus atlanticus $(\mathrm{n}=86)$} \\
\hline$\% \mathrm{~F}$ & 18.6 & & & 18.6 & 5.8 & & 1.2 & 1.2 & 19.8 & 1.2 & 54.7 & 17.4 & 1.2 & 29.1 & 3.5 & 15.1 & 1.2 & 2.3 \\
\hline$\% \mathrm{~V}$ & 3.7 & & & 1.2 & 3.6 & & 6 & $<0.1$ & 2.6 & $<0.1$ & 51.9 & 9.6 & 0.1 & 6 & 0.5 & 14.6 & $<0.1$ & $<0.1$ \\
\hline
\end{tabular}


Table 7.2. Percent frequency (\%F) and percent volume (\%V) of stomach contents for 31 bottom fish species caught during NF-2008, SJ-2009, $\mathrm{CH}-2010$ cruises at Viosca Knoll 826, Viosca Knoll 862/906, Mississippi Canyon, and West Florida Slope during the Lophelia II program continued.

[See figure 7.1 for site locations. Amphi, Amphipoda; Tuni, Tunicata; Cnid, Cnidaria; Cope, Copepoda; Crust, Crustacea; Cuma, Cumacea; Euph,

Euphausiacea; Fish, Fish; Isop, Isopoda; Moll, Mollusca; Mysi, Mysida; Other, Other; Ostr, Ostracoda; Para, Parasite; Poly, Polychaeta; Radi, Radiolaria; Tana,

Tanaidacea; n, number; <, less than]

\begin{tabular}{|c|c|c|c|c|c|c|c|c|c|c|c|c|c|c|c|c|c|c|}
\hline Species & Amphi & Tuni & Cnid & Cope & Crust & Cuma & Euph & Fish & Fora & Isop & Moll & Mysi & Ostr & Other & Para & Poly & Radi & Tana \\
\hline \multicolumn{19}{|c|}{ Epigonus pandionis $(\mathrm{n}=7)$} \\
\hline$\% \mathrm{~F}$ & 14.3 & & & 42.9 & 42.9 & & & & & & & & & 85.7 & 14.3 & & & \\
\hline$\% \mathrm{~V}$ & 18.9 & & & 10 & 29 & & & & & & & & & 42.1 & 0.1 & & & \\
\hline \multicolumn{19}{|c|}{ Eptatretus minor $(\mathrm{n}=2)$} \\
\hline$\% \mathrm{~F}$ & & & & & & & & 50 & & & & & & 50 & & & & \\
\hline$\% \mathrm{~V}$ & & & & & & & & 83.8 & & & & & & 16.2 & & & & \\
\hline \multicolumn{19}{|c|}{ Etmopterus schultzi $(\mathrm{n}=2)$} \\
\hline$\% \mathrm{~F}$ & & & & & 50 & & & & & & 50 & & & & & & & \\
\hline$\% \mathrm{~V}$ & & & & & 93.9 & & & & & & 6.1 & & & & & & & \\
\hline \multicolumn{19}{|c|}{ Etmopterus virens $(\mathrm{n}=1)$} \\
\hline$\% \mathrm{~F}$ & & & & & & & & & & & 100 & & & & & & & \\
\hline$\% \mathrm{~V}$ & & & & & & & & & & & 100 & & & & & & & \\
\hline \multicolumn{19}{|c|}{ Helicolenus dactylopterus $(\mathrm{n}=8)$} \\
\hline$\% \mathrm{~F}$ & & 75 & 12.5 & & 25 & & & 25 & 12.5 & & 25 & & & 25 & & & & \\
\hline$\% \mathrm{~V}$ & & 50.4 & 0.5 & & 4.2 & & & 21.1 & 0.1 & & 22.7 & & & 1 & & & & \\
\hline \multicolumn{19}{|c|}{ Hoplostethus occidentalis $(\mathrm{n}=5)$} \\
\hline$\% \mathrm{~F}$ & & & & & 100 & & & & & & & & & 20 & & & & \\
\hline$\% \mathrm{~V}$ & & & & & 99.8 & & & & & & & & & 0.2 & & & & \\
\hline \multicolumn{19}{|c|}{ Laemonema barbatulum $(\mathrm{n}=4)$} \\
\hline$\% \mathrm{~F}$ & 25 & & & 50 & & & & 50 & & & & 100 & & 25 & & & & \\
\hline$\% \mathrm{~V}$ & 1.4 & & & 0.3 & & & & 19.2 & & & & 65 & & 14.2 & & & & \\
\hline \multicolumn{19}{|c|}{ Laemonema goodebeanorum $(\mathrm{n}=26)$} \\
\hline$\% \mathrm{~F}$ & 46.2 & & & 53.8 & 38.5 & & & 3.8 & 11.5 & & & 57.7 & 3.8 & 65.4 & & 15.4 & & 3.8 \\
\hline$\% \mathrm{~V}$ & 1.7 & & & 1.8 & 63.9 & & & 4 & 0.1 & & & 15.3 & 0.3 & 7.3 & & 3.9 & & 1.7 \\
\hline
\end{tabular}


Table 7.2. Percent frequency (\%F) and percent volume (\%V) of stomach contents for 31 bottom fishes caught during NF-2008, SJ-2009, and $\mathrm{CH}-2010$ cruises at Viosca Knoll 826, Viosca Knoll 862/906, Mississippi Canyon, and West Florida Slope during the Lophelia II program continued.

[See figure 7.1 for site locations. Amphi, Amphipoda; Tuni, Tunicata; Cnid, Cnidaria; Cope, Copepoda; Crust, Crustacea; Cuma, Cumacea; Euph,

Euphausiacea; Fish, Fish; Isop, Isopoda; Moll, Mollusca; Mysi, Mysida; Other, Other; Ostr, Ostracoda; Para, Parasite; Poly, Polychaeta; Radi, Radiolaria; Tana,

Tanaidacea; n, number; <, less than]

\begin{tabular}{|c|c|c|c|c|c|c|c|c|c|c|c|c|c|c|c|c|c|c|}
\hline Species & Amphi & Tuni & Cnid & Cope & Crust & Cuma & Euph & Fish & Fora & Isop & Moll & Mysi & Ostr & Other & Para & Poly & Radi & Tana \\
\hline \multicolumn{19}{|c|}{ Lophiodes beroe $(\mathrm{n}=1)$} \\
\hline$\% \mathrm{~F}$ & & & & & & & & 100 & & & 100 & & & & 100 & & & \\
\hline$\% \mathrm{~V}$ & & & & & & & & 100 & & & $<0.1$ & & & & $<0.1$ & & & \\
\hline \multicolumn{19}{|c|}{ Malacocephalus occidentalis $(\mathrm{n}=7)$} \\
\hline$\% \mathrm{~F}$ & 42.9 & & 14.3 & 42.9 & 71.4 & & & & 28.6 & & & 28.6 & & 85.7 & 14.3 & & & \\
\hline$\% \mathrm{~V}$ & 1 & & 0.2 & 0.3 & 94.7 & & & & $<0.1$ & & & 0.2 & & 3.7 & $<0.1$ & & & \\
\hline \multicolumn{19}{|c|}{ Nezumia aequalis $(\mathrm{n}=28)$} \\
\hline$\% \mathrm{~F}$ & 32.1 & & & 35.7 & 50 & & 3.6 & & 10.7 & & 10.7 & 14.3 & 3.6 & 35.7 & & 17.9 & & 21.4 \\
\hline$\% \mathrm{~V}$ & 21.1 & & & 1.7 & 46.8 & & 2.5 & & $<0.1$ & & 0.6 & 11.3 & $<0.1$ & 10.7 & & 5.3 & & $<0.1$ \\
\hline \multicolumn{19}{|c|}{ Peristedion greyae $(\mathrm{n}=14)$} \\
\hline$\% \mathrm{~F}$ & 64.3 & & & 57.1 & 64.3 & & & 14.3 & & & & 14.3 & & 71.4 & 14.3 & 7.1 & & \\
\hline$\% \mathrm{~V}$ & 15.5 & & & 2.4 & 54 & & & 7.1 & & & & 6.8 & & 12.1 & 1.1 & 1 & & \\
\hline \multicolumn{19}{|c|}{ Poecilopsetta beanii $(\mathrm{n}=12)$} \\
\hline$\% \mathrm{~F}$ & 8.3 & & & 16.7 & & & & & 16.7 & 8.3 & 33.3 & & & 25 & & 25 & 8.3 & \\
\hline$\% \mathrm{~V}$ & 3.9 & & & 2.9 & & & & & 2 & 1.2 & 55.1 & & & 12.4 & & 21.5 & 1 & \\
\hline \multicolumn{19}{|c|}{ Pontinus longispinus $(\mathrm{n}=1)$} \\
\hline$\% \mathrm{~F}$ & & & & & & & & & & & & & & & & 100 & & 100 \\
\hline$\% \mathrm{~V}$ & & & & & & & & & & & & & & & & 41.3 & & 58.7 \\
\hline \multicolumn{19}{|c|}{ Pontinus rathbuni $(\mathrm{n}=1)$} \\
\hline$\% \mathrm{~F}$ & & 100 & & & & & & & & & & & & 100 & & & & \\
\hline$\% \mathrm{~V}$ & & 98.3 & & & & & & & & & & & & 1.7 & & & & \\
\hline \multicolumn{19}{|c|}{ Symphurus marginatus $(\mathrm{n}=25)$} \\
\hline$\% \mathrm{~F}$ & 28 & & & 16 & 4 & & & & 24 & & 48 & 4 & & 40 & 4 & 4 & & 4 \\
\hline$\% \mathrm{~V}$ & 16.5 & & & 2.8 & 1.1 & & & & 0.6 & & 49.2 & 5.6 & & 18.7 & $<0.1$ & 5.3 & & 0.2 \\
\hline
\end{tabular}


Table 7.2. Percent frequency (\%F) and percent volume (\%V) of stomach contents for 31 bottom fishes caught during NF-2008, SJ-2009, and $\mathrm{CH}-2010$ cruises at Viosca Knoll 826, Viosca Knoll 862/906, Mississippi Canyon, and West Florida Slope during the Lophelia II program continued.

[See figure 7.1 for site locations. Amphi, Amphipoda; Tuni, Tunicata; Cnid, Cnidaria; Cope, Copepoda; Crust, Crustacea; Cuma, Cumacea; Euph, Euphausiacea; Fish, Fish; Isop, Isopoda; Moll, Mollusca; Mysi, Mysida; Other, Other; Ostr, Ostracoda; Para, Parasite; Poly, Polychaeta; Radi, Radiolaria; Tana,

Tanaidacea; n, number; <, less than]

\begin{tabular}{|c|c|c|c|c|c|c|c|c|c|c|c|c|c|c|c|c|c|c|}
\hline Species & Amphi & Tuni & Cnid & Cope & Crust & Cuma & Euph & Fish & Fora & Isop & Moll & Mysi & Ostr & Other & Para & Poly & Radi & Tana \\
\hline \multicolumn{19}{|c|}{ Urophycis cirrata $(\mathrm{n}=1)$} \\
\hline$\% \mathrm{~F}$ & & & & 100 & 100 & & & & & & 100 & 100 & & & & 100 & & \\
\hline$\% \mathrm{~V}$ & & & & 1.6 & 42.6 & & & & & & 38.7 & 13.8 & & & & 3.4 & & \\
\hline \multicolumn{19}{|c|}{ Urophycis floridana $(\mathrm{n}=2)$} \\
\hline$\% \mathrm{~F}$ & & & & & & & & 100 & & & & & & & & & & \\
\hline$\% \mathrm{~V}$ & & & & & & & & 100 & & & & & & & & & & \\
\hline
\end{tabular}


Table 7.3. Number of stomachs analyzed and number of empty or everted stomachs for midwater fishes caught during $\mathrm{CH}-2007$ and NF-2008 cruises over deep-reef habitats in the Gulf of Mexico during the Lophelia II program.

\begin{tabular}{lccc}
\hline \multicolumn{1}{c}{ Family/ Species } & $\begin{array}{c}\text { Stomachs } \\
\text { analyzed }\end{array}$ & $\begin{array}{c}\text { Empty } \\
\text { stomachs }\end{array}$ & $\begin{array}{c}\text { Everted } \\
\text { stomachs }\end{array}$ \\
\hline Gonostomatidae & 92 & & \\
Cyclothone alba & 38 & 20 & \\
Cyclothone braueri & 43 & 37 & \\
Gonostoma atlanticum & 1 & & \\
Gonostoma elongatum & 10 & 7 & \\
Sternoptychidae & 77 & & \\
Argyropelecus aculeatus & 12 & 5 & \\
Argyropelecus hemigymmus & 11 & 1 & \\
Polyipnus clarus & 3 & & \\
Valenciennellus tripunctulatus & 51 & 23 & \\
Phosichthyidae & 35 & & \\
Pollichthys mauli & 24 & 8 & \\
Vinciguerria nimbaria & 11 & 4 & \\
Stomiidae & 16 & & \\
Chauliodus sloani & 12 & 10 & \\
Stomias affinis & 4 & 3 & \\
Myctophidae & 87 & & \\
Diaphus dumerilii & 27 & 12 & \\
Diaphus mollis & 13 & & \\
Lepidophanes guentheri & 5 & 1 & \\
Myctophum affine & 42 & 12 & \\
Bregmacerotidae & 6 & & \\
Bregmaceros atlanticus & 6 & 3 & \\
Melamphaidae & 2 & & \\
Melamphaes simus & 2 & & \\
\hline
\end{tabular}


Table 7.4. Percent frequency $(\% \mathrm{~F})$ and percent volume $(\% \mathrm{~V})$ of stomach contents for 19 midwater fish species caught during $\mathrm{CH}-2007$ and NF-2008 at Viosca Knoll 826, north-central Gulf of Mexico as part of the Lophelia II program.

[Amphi, Amphipoda; Cope, Copepoda; Crust, Crustacea; Euph, Euphausiacea; Fish, Fish; Isop, Isopoda; Mysi, Mysida; Other, Other; Ostr, Ostracoda; n, number]

\begin{tabular}{|c|c|c|c|c|c|c|c|c|c|}
\hline Species & Amphi & Cope & Crust & Euph & Fish & Isop & Mysi & Ostra & Other \\
\hline \multicolumn{10}{|c|}{ Argyropelecus aculeatus $(\mathrm{n}=7)$} \\
\hline$\% \mathrm{~F}$ & 14.3 & 57.1 & & 14.3 & & & & 57.1 & 57.1 \\
\hline$\% \mathrm{~V}$ & 1 & 7.9 & & 13.5 & & & & 17.8 & 59.9 \\
\hline \multicolumn{10}{|c|}{ Argyropelecus hemigymnus $(\mathrm{n}=2)$} \\
\hline$\% \mathrm{~F}$ & & 100 & & & & & & & \\
\hline$\% \mathrm{~V}$ & & 100 & & & & & & & \\
\hline \multicolumn{10}{|c|}{ Bregmaceros atlanticus $(\mathrm{n}=3)$} \\
\hline$\% \mathrm{~F}$ & & 100 & & 33.3 & & & & 33.3 & \\
\hline$\% \mathrm{~V}$ & & 75.1 & & 23.5 & & & & 1.5 & \\
\hline \multicolumn{10}{|c|}{ Chauliodus sloani $(\mathrm{n}=2)$} \\
\hline$\% \mathrm{~F}$ & & & & 50 & & & & & 50 \\
\hline$\% \mathrm{~V}$ & & & & 97.4 & & & & & 2.6 \\
\hline \multicolumn{10}{|c|}{ Cyclothone alba $(\mathrm{n}=18)$} \\
\hline$\% \mathrm{~F}$ & & 88.9 & & & & & & 5.6 & 5.6 \\
\hline$\% \mathrm{~V}$ & & 97.9 & & & & & & 1 & 1.1 \\
\hline \multicolumn{10}{|c|}{ Cyclothone braueri $(\mathrm{n}=6)$} \\
\hline$\% \mathrm{~F}$ & & 100 & & & & & & & \\
\hline$\% \mathrm{~V}$ & & 100 & & & & & & & \\
\hline \multicolumn{10}{|c|}{ Diaphus dumerilii $(\mathrm{n}=15)$} \\
\hline$\% \mathrm{~F}$ & 6.7 & 100 & & & & & & 33.3 & 6.7 \\
\hline$\% \mathrm{~V}$ & 0.7 & 86.3 & & & & & & 11.1 & 1.8 \\
\hline \multicolumn{10}{|c|}{ Diaphus mollis $(\mathrm{n}=13)$} \\
\hline$\% \mathrm{~F}$ & & 100 & & 7.7 & & & & 23.1 & 38.5 \\
\hline$\% \mathrm{~V}$ & & 73 & & 6.2 & & & & 2.6 & 18.1 \\
\hline \multicolumn{10}{|c|}{ Gonostoma atlanticum $(\mathrm{n}=1)$} \\
\hline$\% \mathrm{~F}$ & & & & 100 & & & & & \\
\hline$\% \mathrm{~V}$ & & & & 100 & & & & & \\
\hline \multicolumn{10}{|c|}{ Gonostoma elongatum $(\mathrm{n}=3)$} \\
\hline$\% \mathrm{~F}$ & & 100 & 33.3 & 33.3 & & & & 33.3 & \\
\hline$\% \mathrm{~V}$ & & 52.9 & 9.1 & 37 & & & & 1 & \\
\hline \multicolumn{10}{|c|}{ Lepidophanes guentheri $(\mathrm{n}=4)$} \\
\hline$\% \mathrm{~F}$ & & 75 & 25 & 25 & & & & 25 & \\
\hline$\% \mathrm{~V}$ & & 27.6 & 12.7 & 57.6 & & & & 2.2 & \\
\hline \multicolumn{10}{|c|}{ Melamphaes simus $(\mathrm{n}=2)$} \\
\hline$\% \mathrm{~F}$ & & 50 & & & & & & & 100 \\
\hline$\% \mathrm{~V}$ & & 31.2 & & & & & & & 68.8 \\
\hline
\end{tabular}


Table 7.4. Percent frequency (\%F) and percent volume (\%V) of stomach contents for 19 midwater fish species caught during $\mathrm{CH}-2007$ and NF-2008 at Viosca Knoll 826, north-central Gulf of Mexico - continued.

[Amphi, Amphipoda; Cope, Copepoda; Crust, Crustacea; Euph, Euphausiacea; Fish, Fish; Isop, Isopoda; Mysi, Mysida; Other, Other; Ostr, Ostracoda; n, number]

\begin{tabular}{|c|c|c|c|c|c|c|c|c|c|}
\hline Species & Amphi & Cope & Crust & Euph & Fish & Isop & Mysi & Ostra & Other \\
\hline \multicolumn{10}{|c|}{ Myctophum affine $(\mathrm{n}=30)$} \\
\hline$\% \mathrm{~F}$ & 26.7 & 86.7 & 10 & & 3.3 & & 3.3 & 6.7 & 63.3 \\
\hline$\% \mathrm{~V}$ & 15.4 & 43.4 & 18.8 & & 0.4 & & 0.3 & 0.6 & 21 \\
\hline \multicolumn{10}{|c|}{ Pollichthys mauli $(\mathrm{n}=16)$} \\
\hline$\% \mathrm{~F}$ & & 100 & 13.3 & 6.7 & & & 6.7 & 33.3 & 13.3 \\
\hline$\% \mathrm{~V}$ & & 62.6 & 15.7 & 10.6 & & & 6.6 & 2.4 & 2.2 \\
\hline \multicolumn{10}{|c|}{ Polyipnus clarus $(\mathrm{n}=2)$} \\
\hline$\% \mathrm{~F}$ & & 100 & & & & & & 50 & \\
\hline$\% \mathrm{~V}$ & & 64.5 & & & & & & 35.5 & \\
\hline \multicolumn{10}{|c|}{ Stomias affinis $(\mathrm{n}=1)$} \\
\hline$\% \mathrm{~F}$ & & & 100 & & & & & & \\
\hline$\% \mathrm{~V}$ & & & 100 & & & & & & \\
\hline \multicolumn{10}{|c|}{ Valenciennellus tripunctulatus $(\mathrm{n}=28)$} \\
\hline$\% \mathrm{~F}$ & & 100 & & & & & & & \\
\hline$\% \mathrm{~V}$ & & 100 & & & & & & & \\
\hline \multicolumn{10}{|c|}{ Vinciguerria nimbaria $(\mathrm{n}=7)$} \\
\hline$\% \mathrm{~F}$ & 28.6 & 71.4 & & 42.9 & 28.6 & & & 28.6 & 14.3 \\
\hline$\% \mathrm{~V}$ & 2.9 & 30.8 & & 49.1 & 12.8 & & & 2.6 & 1.8 \\
\hline
\end{tabular}




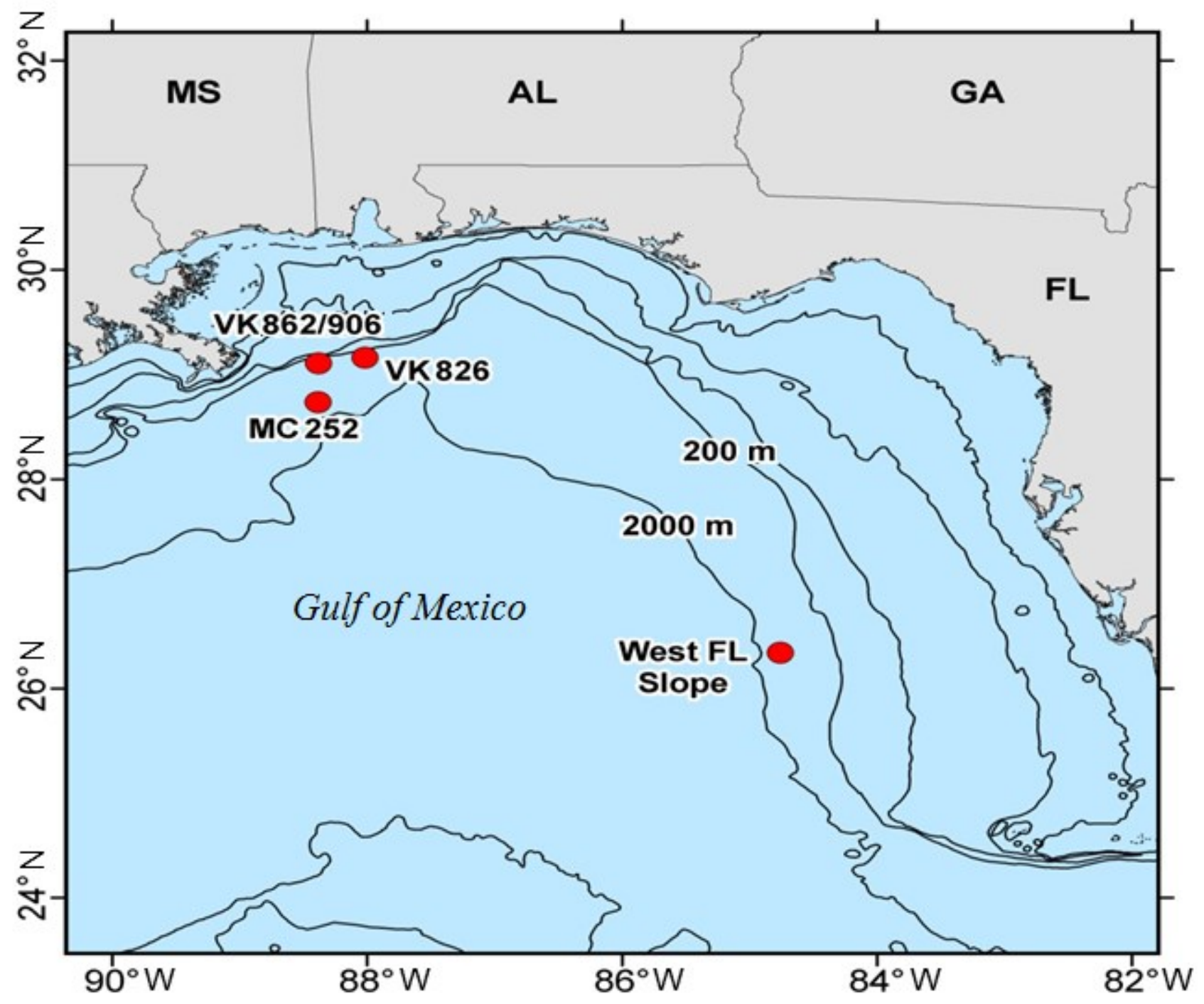

Figure 7.1 Map of the study area in the Gulf of Mexico showing sampling sites for bottom and midwater fishes during the Lophelia II program. Base map data from NOAA [VK, Viosca Knoll; MC, Mississippi Canyon] 


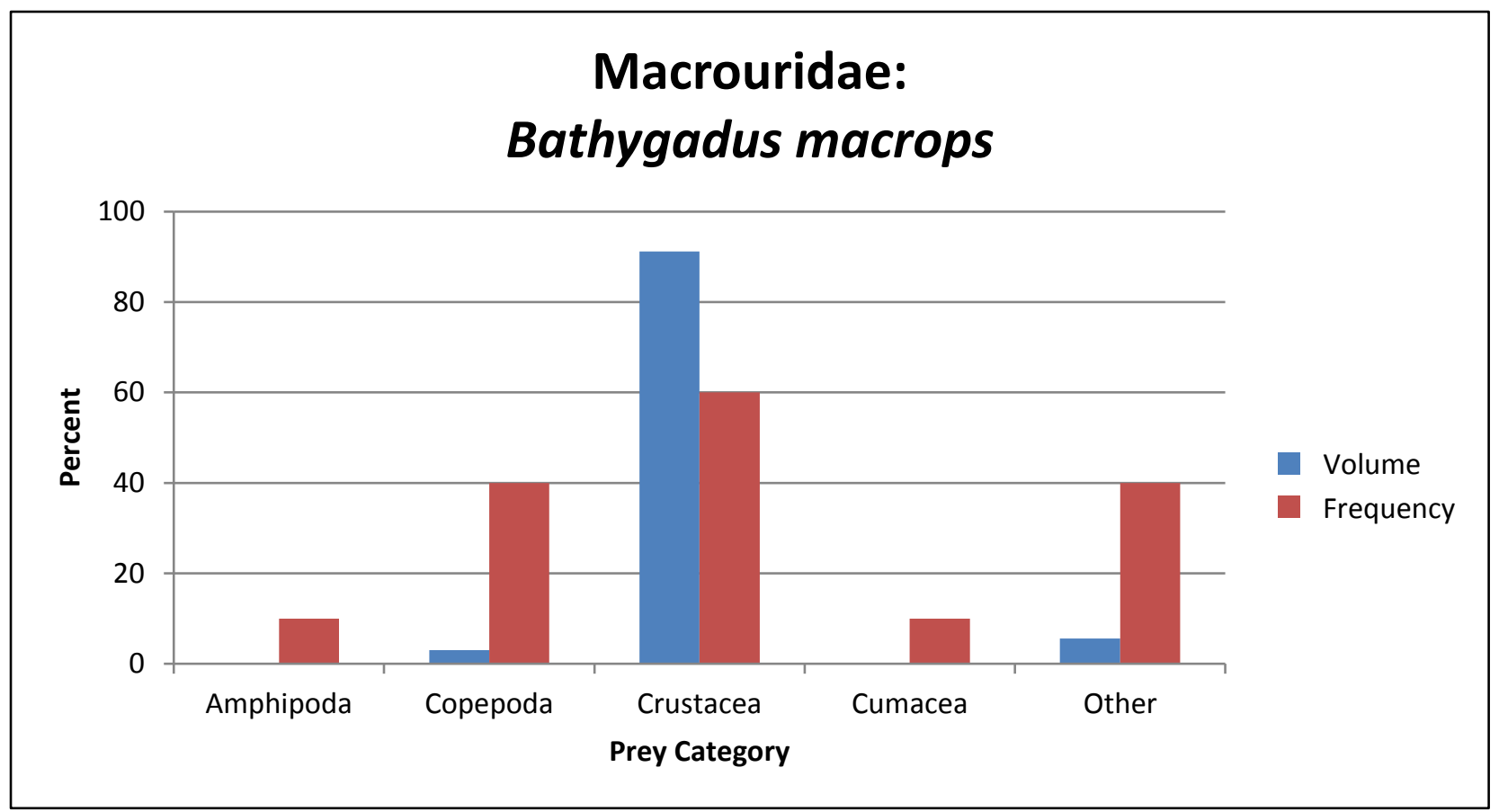

Figure 7.2 Percent volume and percent frequency of stomach contents for Bathygadus macrops $(\mathrm{N}=10)$ during the Lophelia II program.

\section{Macrouridae: Coelorinchus caribbaeus}

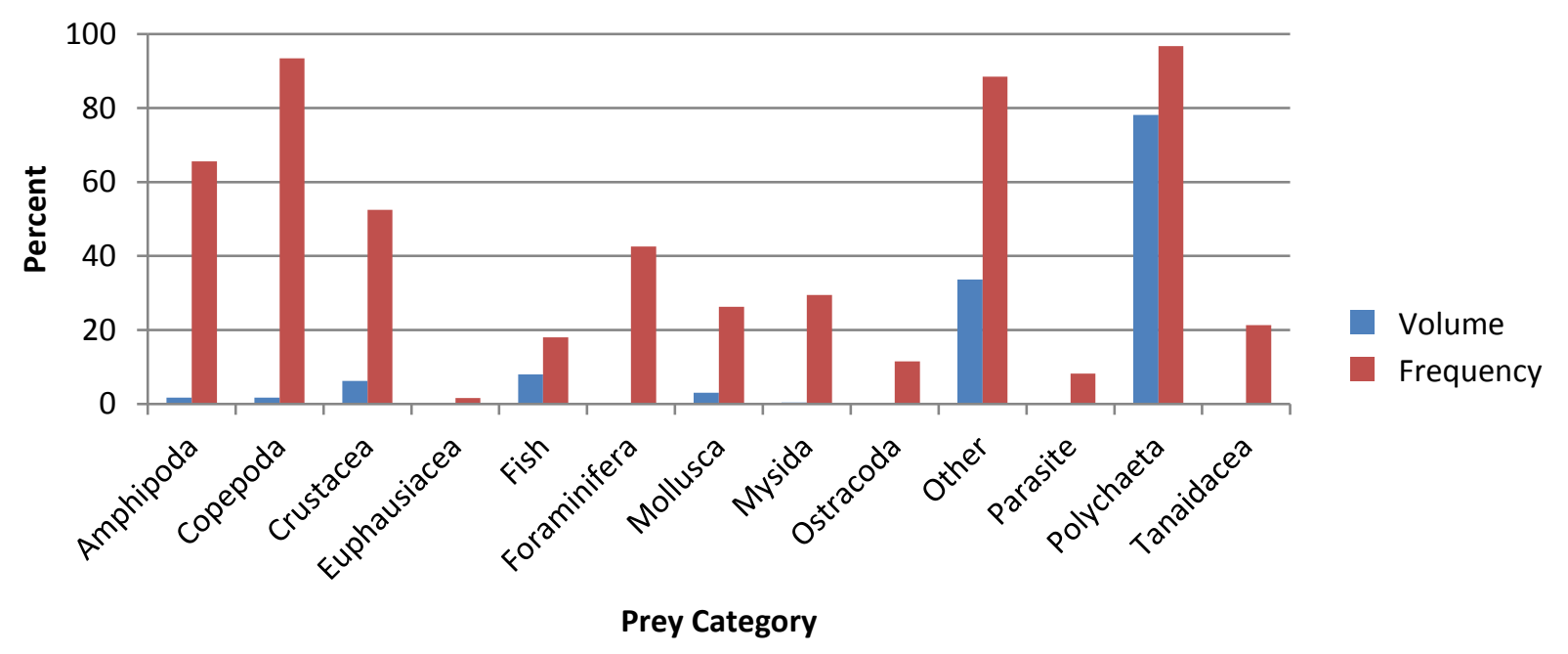

Figure 7.3 Percent volume and percent frequency of stomach contents for Coelorinchus caribbaeus $(\mathrm{N}=61)$ during the Lophelia II program. 


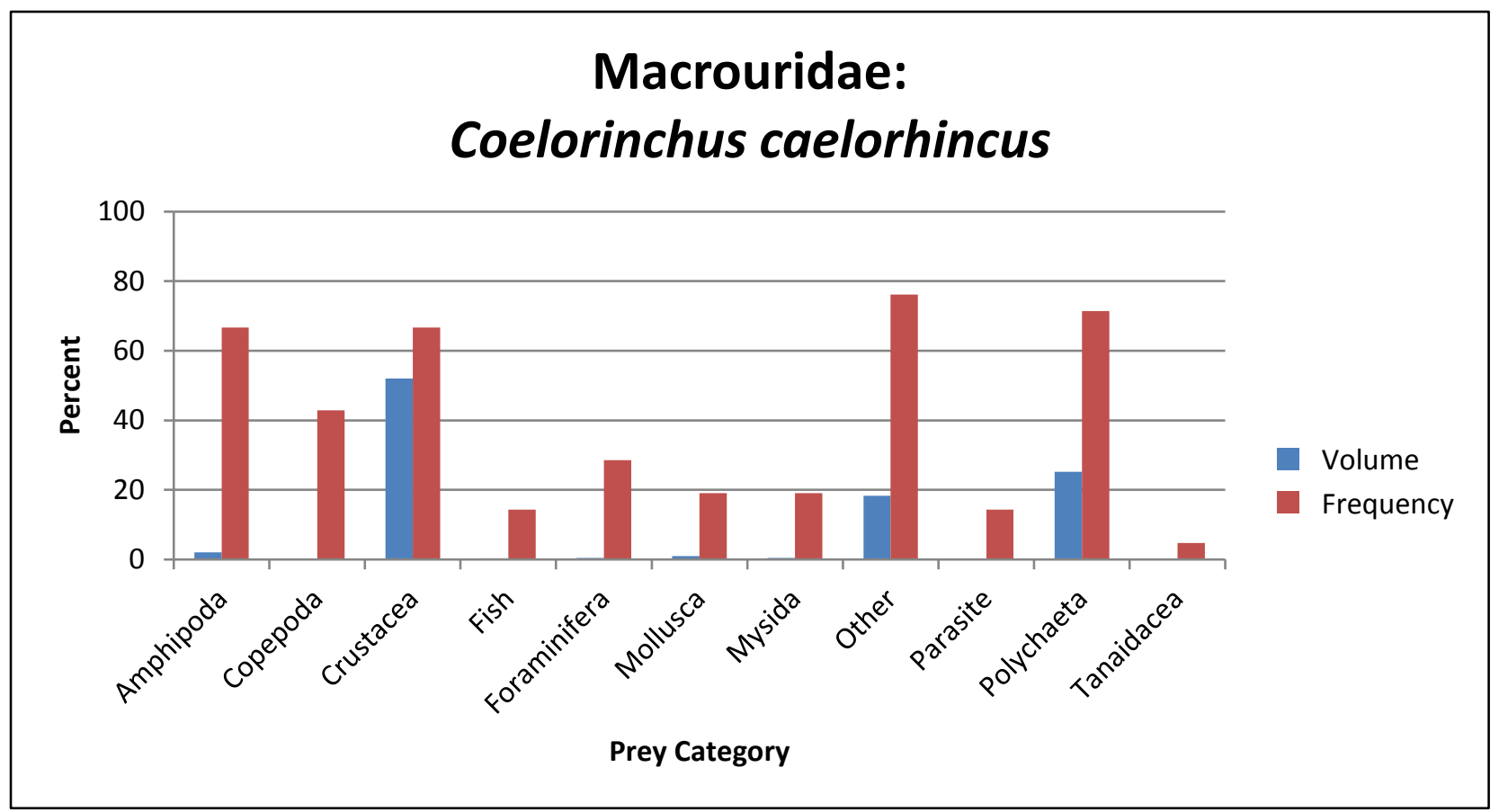

Figure 7.4 Percent volume and percent frequency of stomach contents for Coelorinchus caelorhincus ( $\mathrm{N}=21)$ during the Lophelia II program.

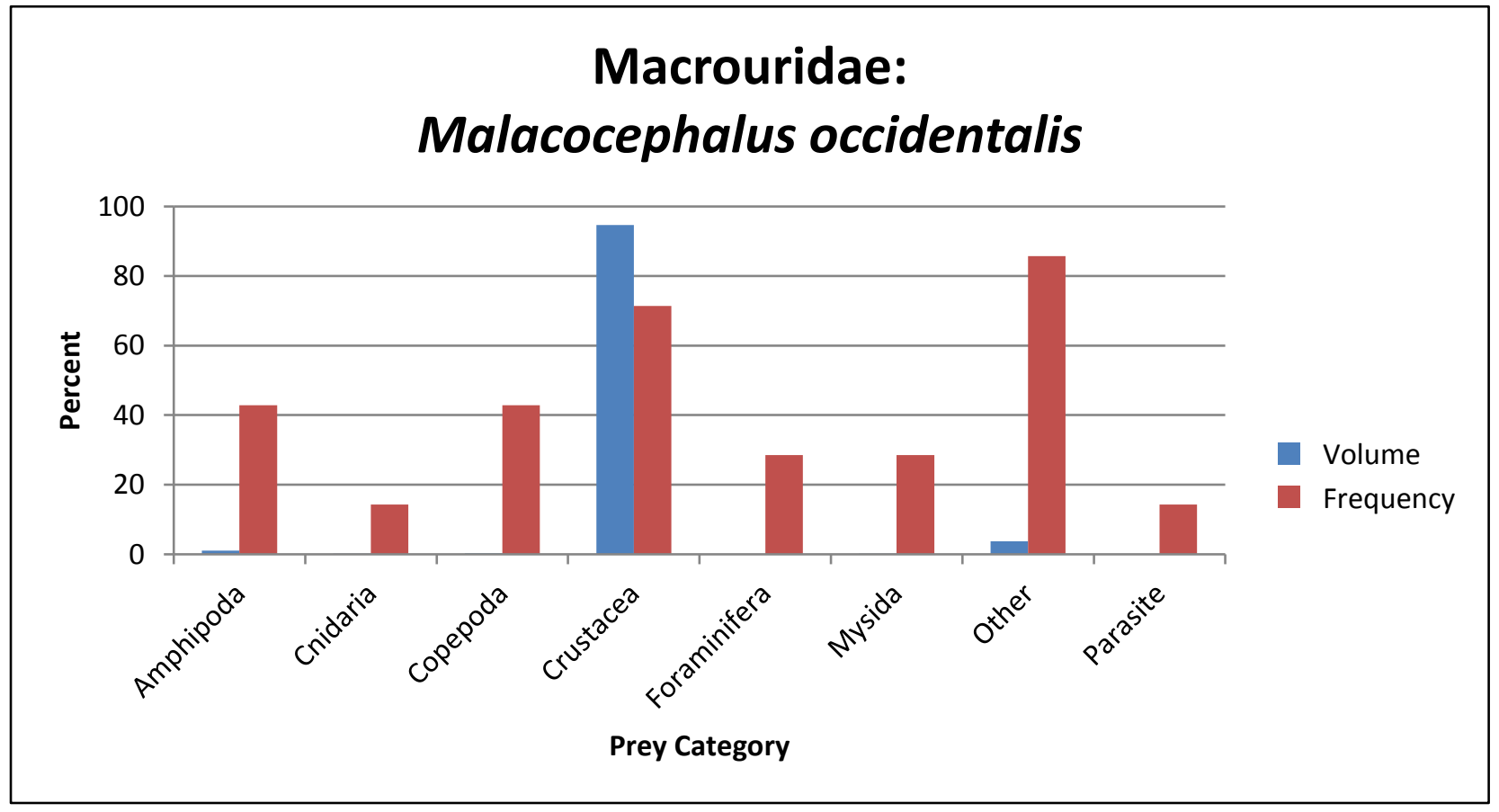

Figure 7.5 Percent volume and percent frequency of stomach contents for Malacocephalus occidentalis $(\mathrm{N}=7)$ during the Lophelia II program. 


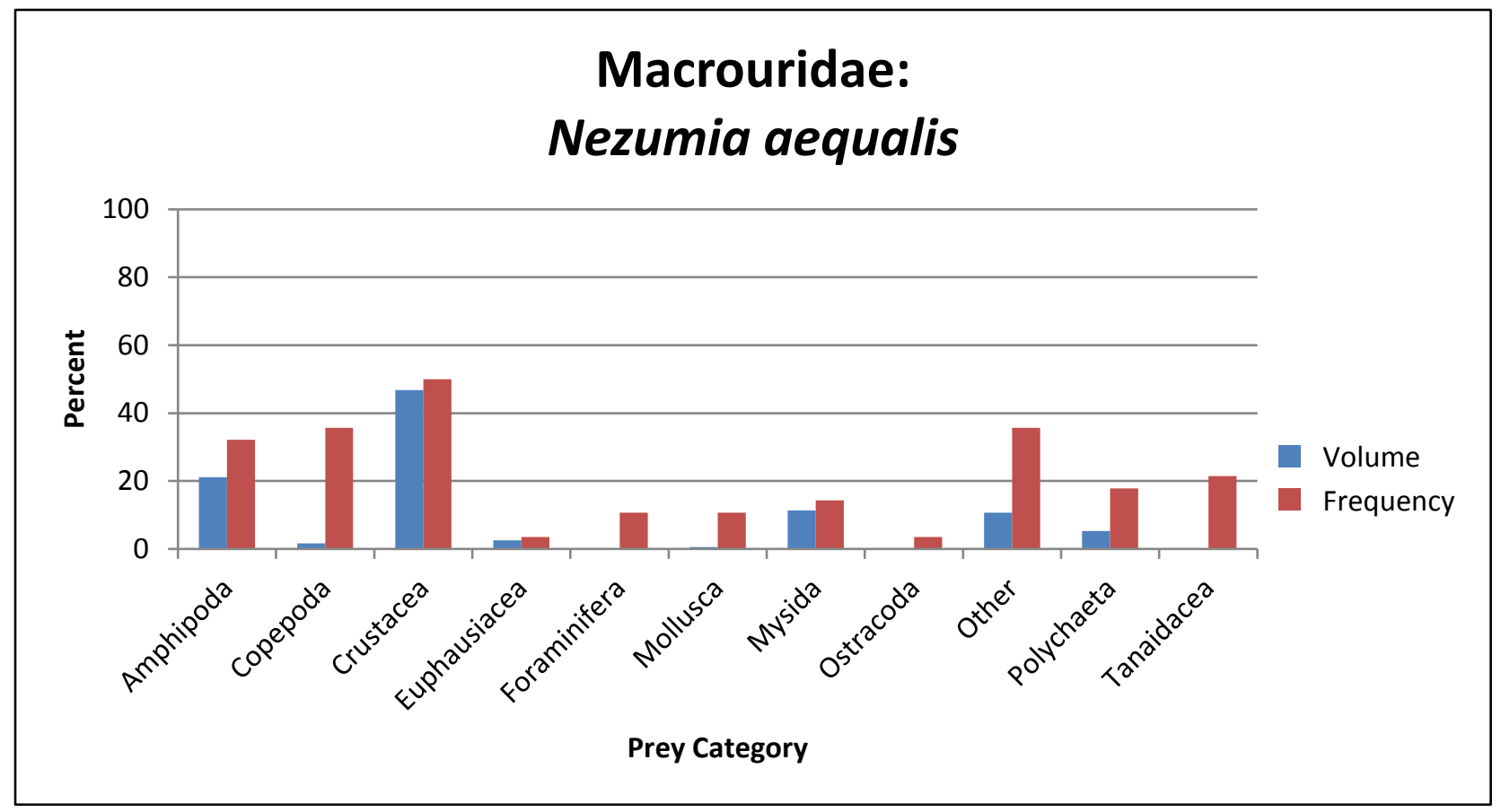

Figure 7.6 Percent volume and percent frequency of stomach contents for Nezumia aequalis $(\mathrm{N}=28)$ during the Lophelia II program.

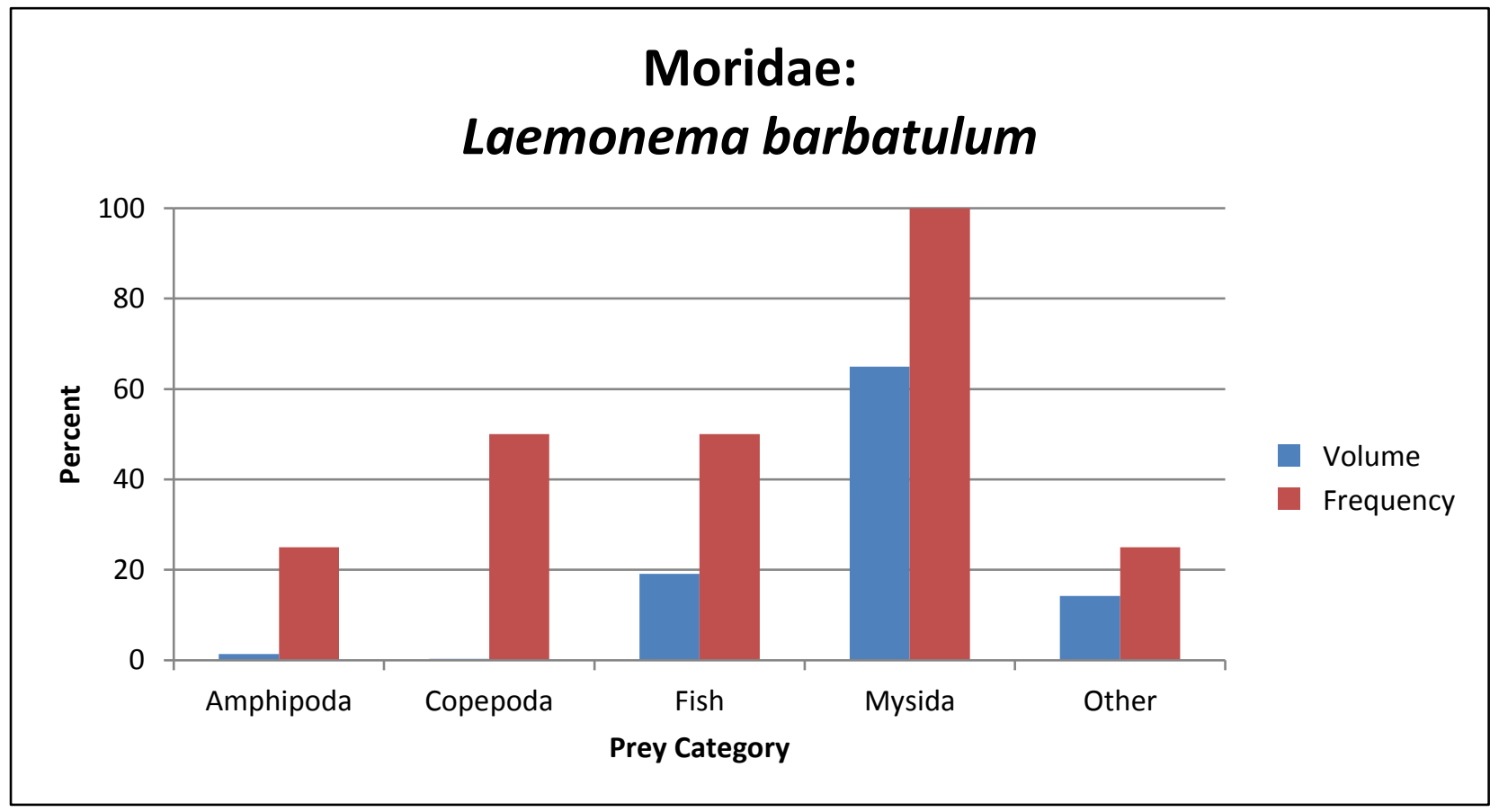

Figure 7.7 Percent volume and percent frequency of stomach contents for Laemonema barbatulum $(\mathrm{N}=4)$ during the Lophelia II program. 


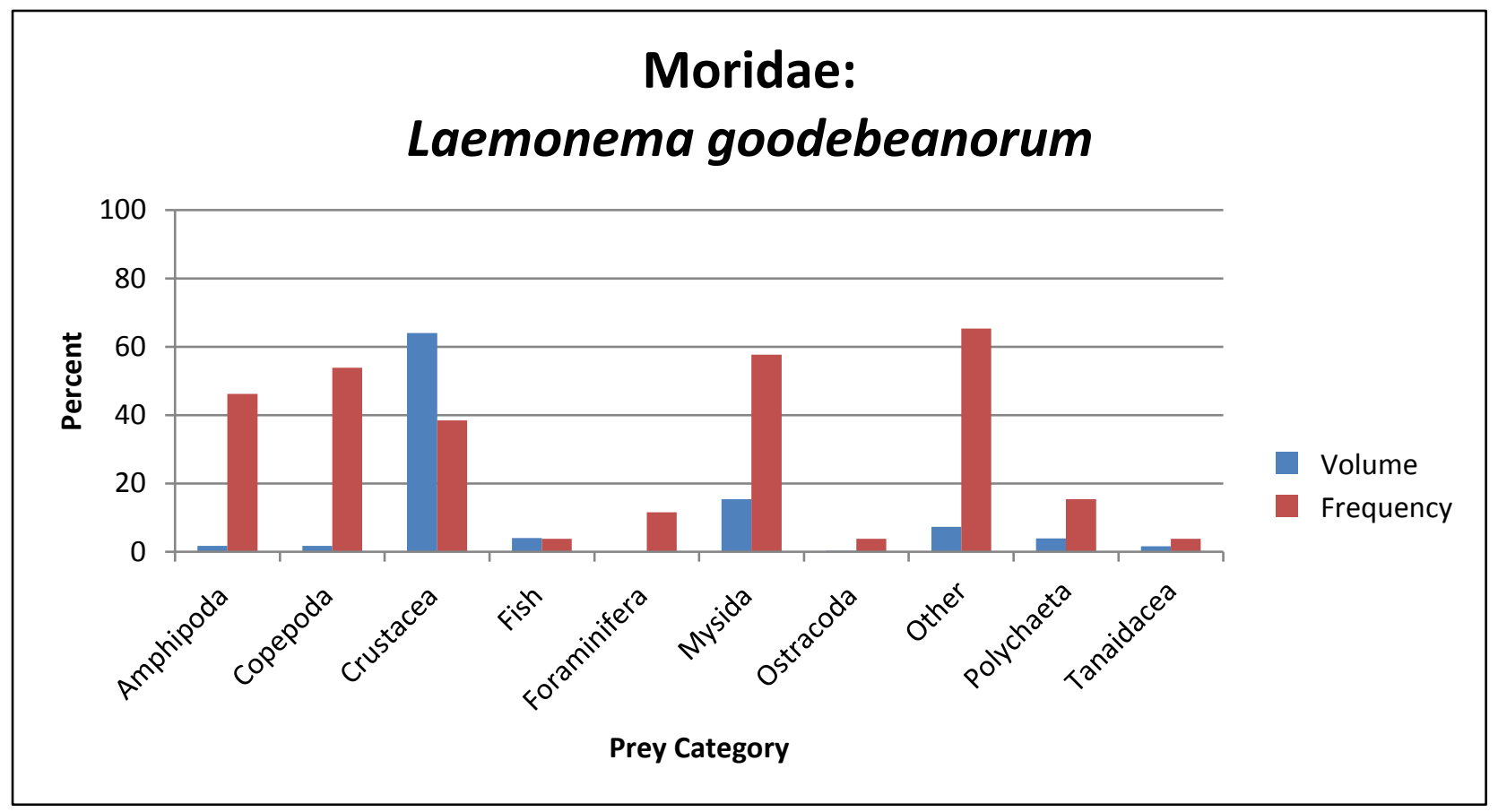

Figure 7.8 Percent volume and percent frequency of stomach contents for Laemonema goodebeanorum $(\mathrm{N}=26)$ during the Lophelia II program.

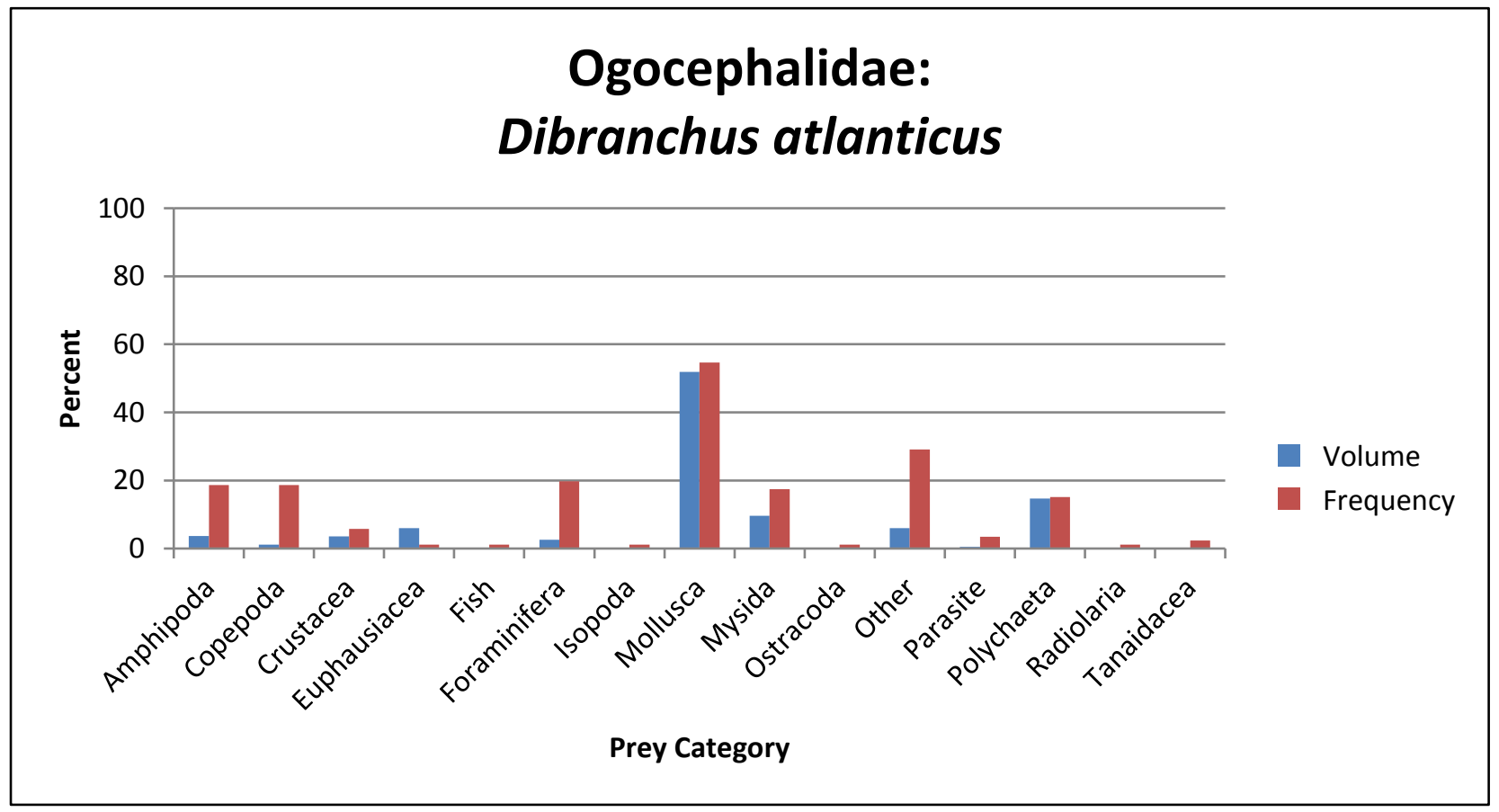

Figure 7.9 Percent volume and percent frequency of stomach contents for Dibranchus atlanticus ( $\mathrm{N}=86)$ during the Lophelia II program. 


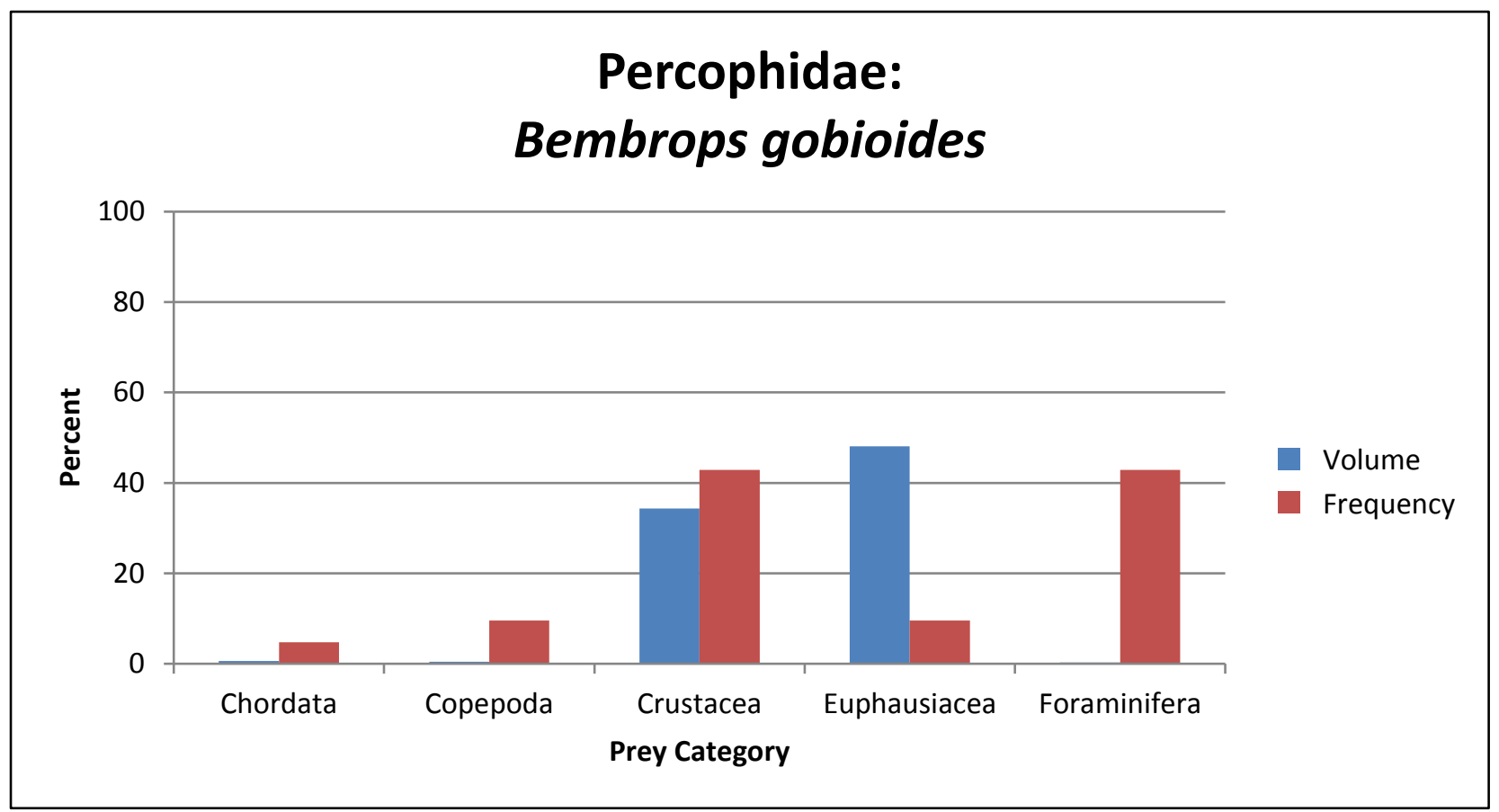

Figure 7.10 Percent volume and percent frequency of stomach contents for Bembrops gobioides (N=21) during the Lophelia II program.

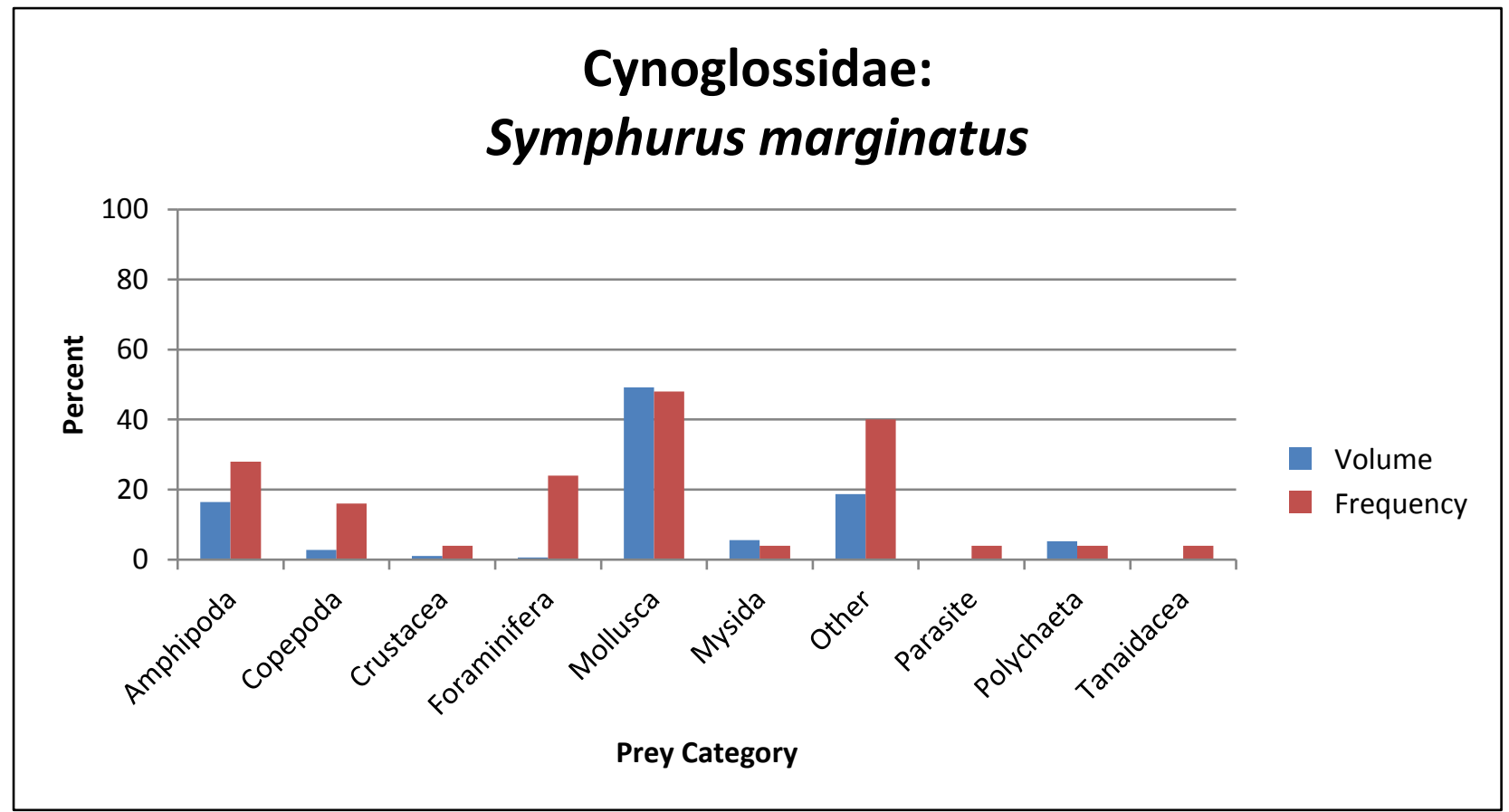

Figure 7.11 Percent volume and percent frequency of stomach contents for Symphurus marginatus $(\mathrm{N}=25)$ during the Lophelia II program. 


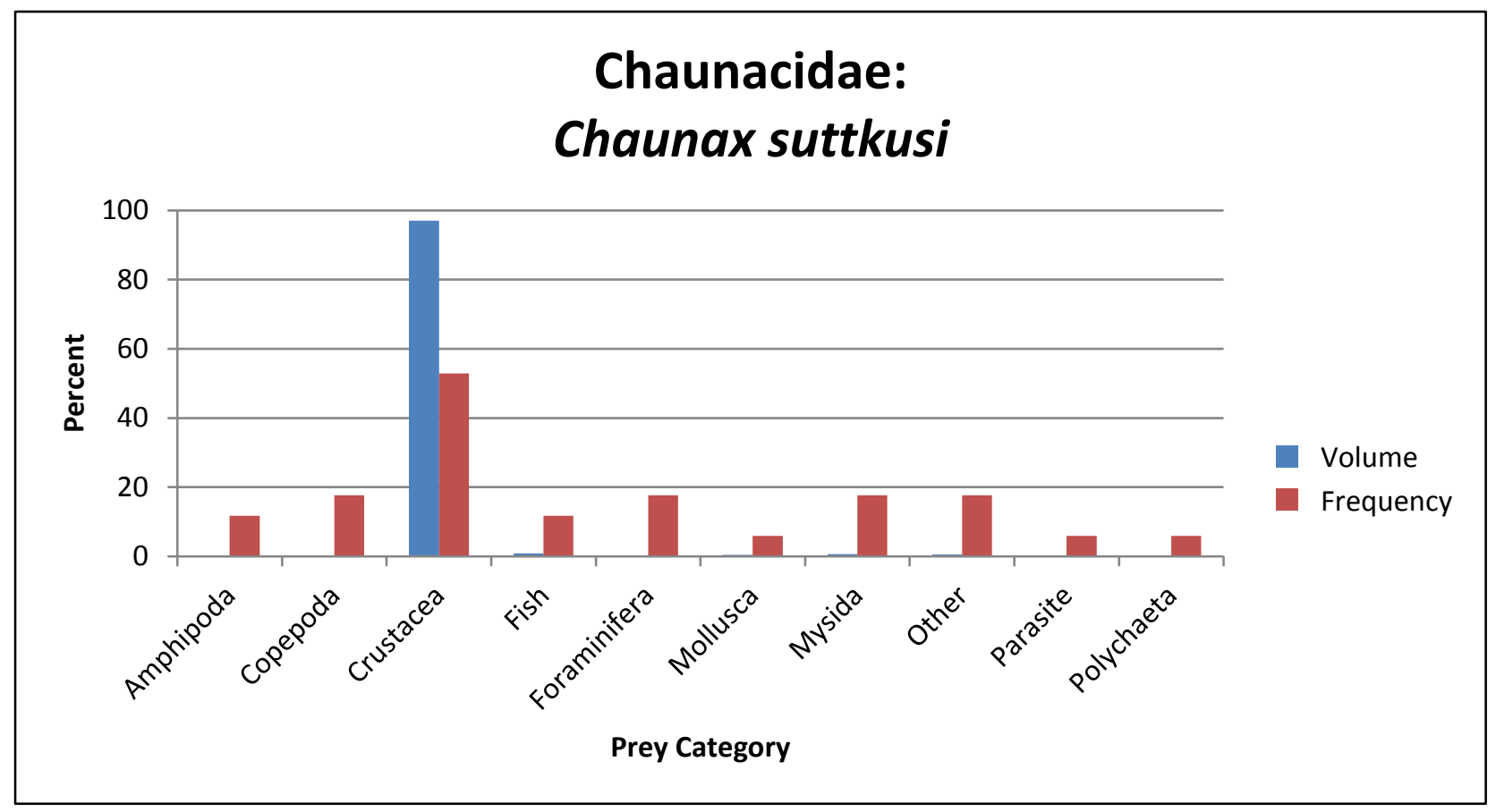

Figure 7.12 Percent volume and percent frequency of stomach contents for Chaunax suttkusi ( $\mathrm{N}=17)$ during the Lophelia II program.

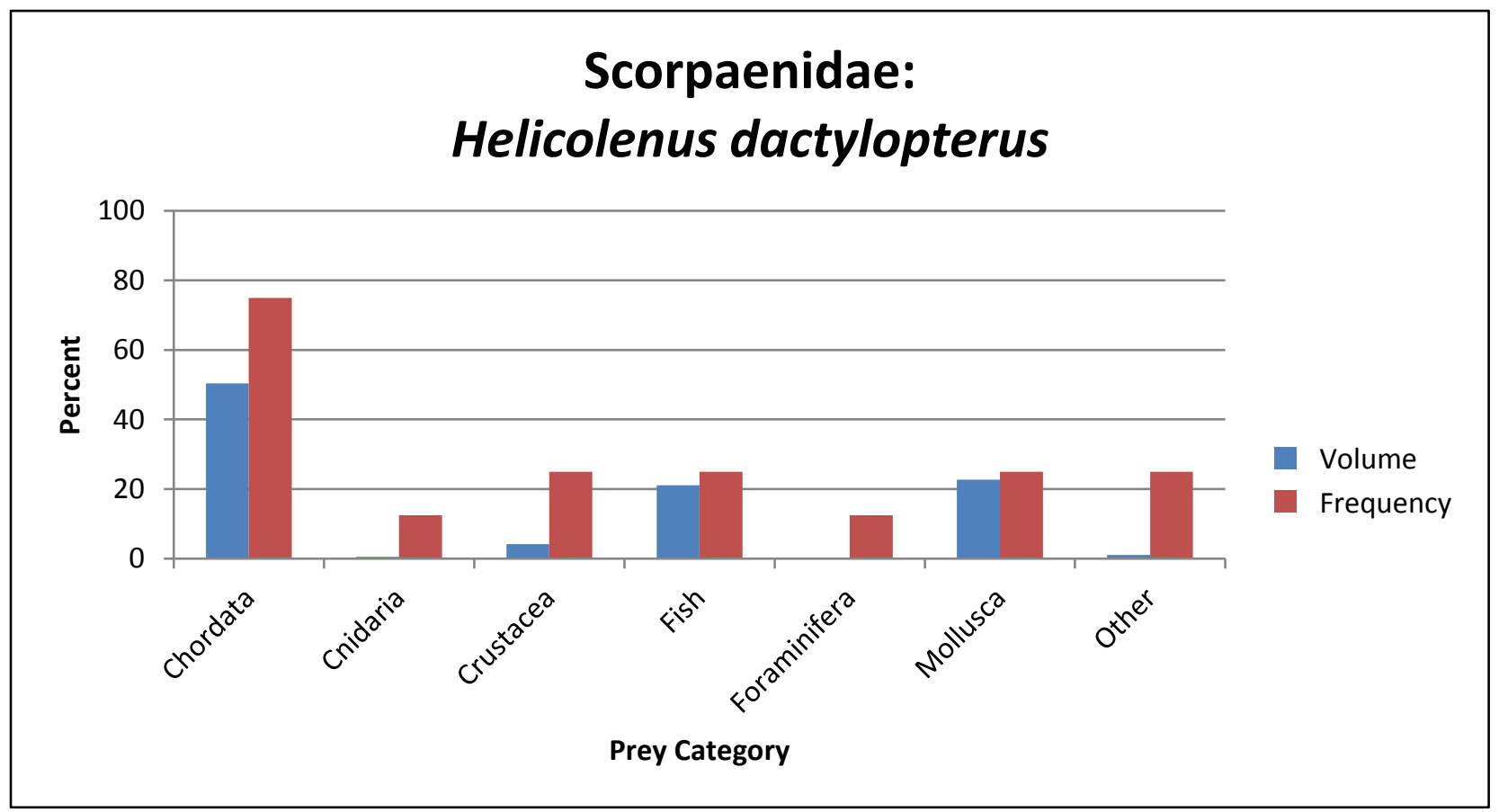

Figure 7.13 Percent volume and percent frequency of stomach contents for Helicolenus dactylopterus ( $\mathrm{N}=8)$ during the Lophelia II program. 


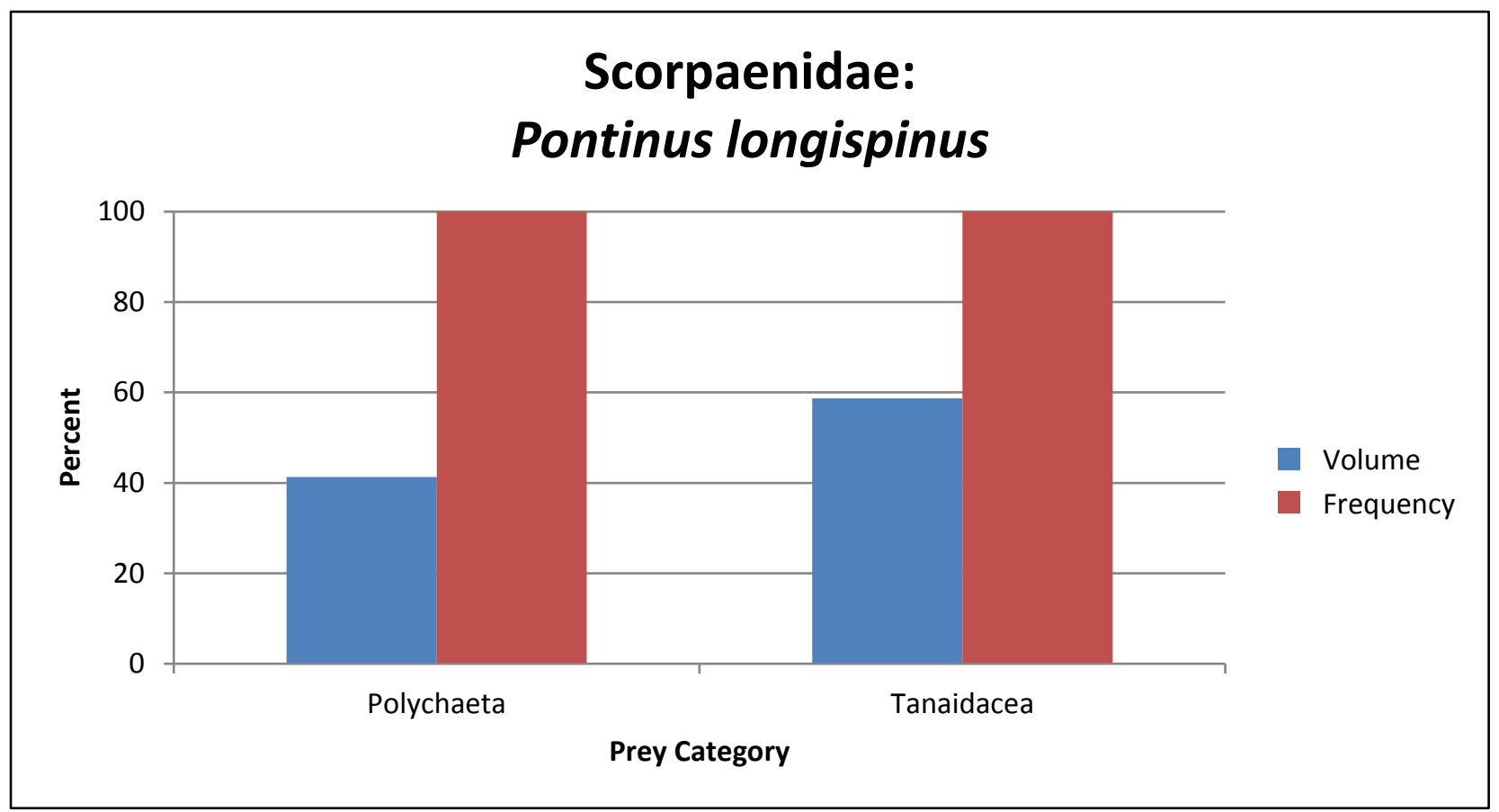

Figure 7.14 Percent volume and percent frequency of stomach contents for Pontinus longispinus ( $N=1)$ during the Lophelia II program.

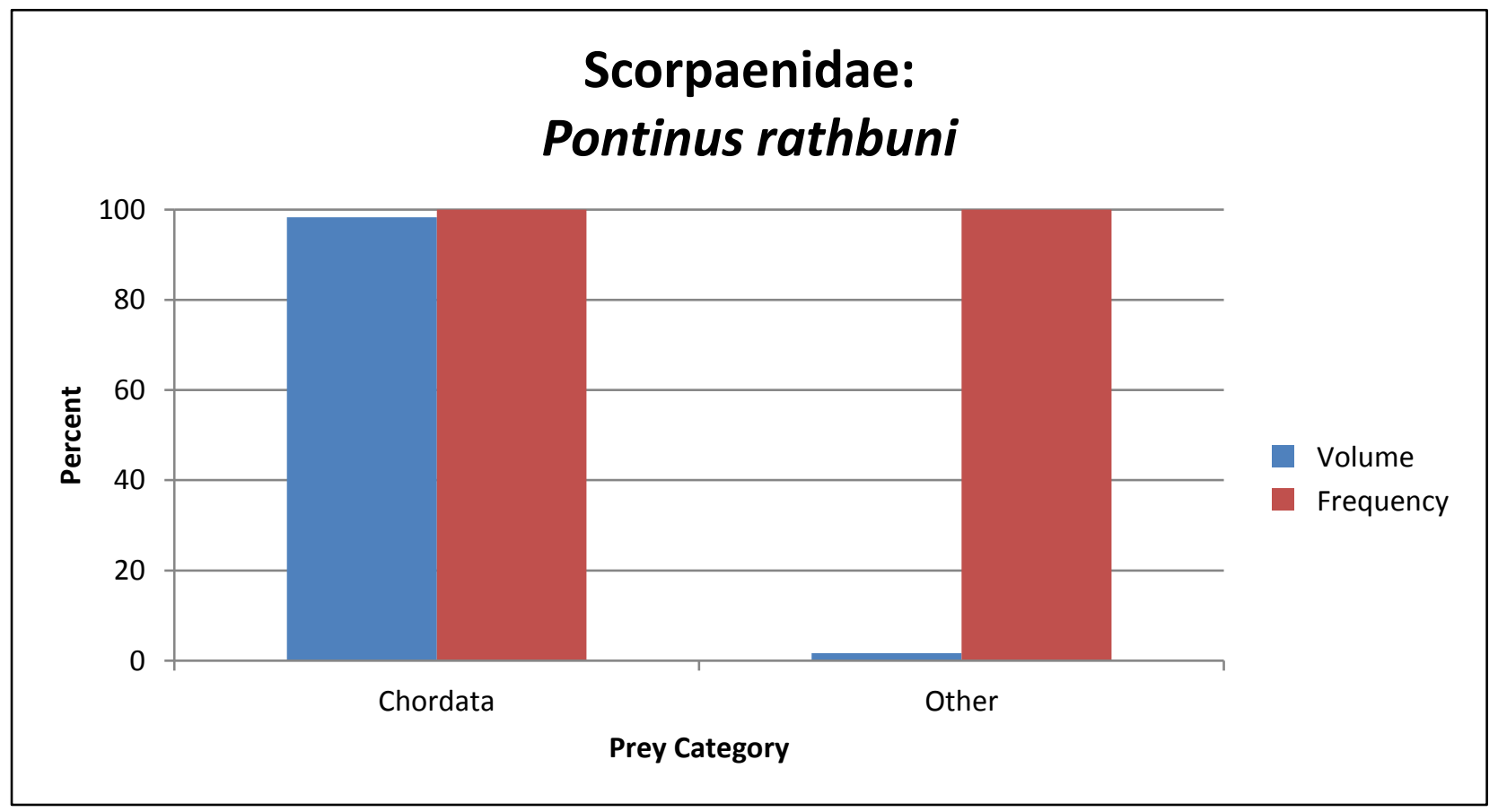

Figure 7.15 Percent volume and percent frequency of stomach contents for Pontinus rathbuni $(\mathrm{N}=1)$ during the Lophelia II program. 


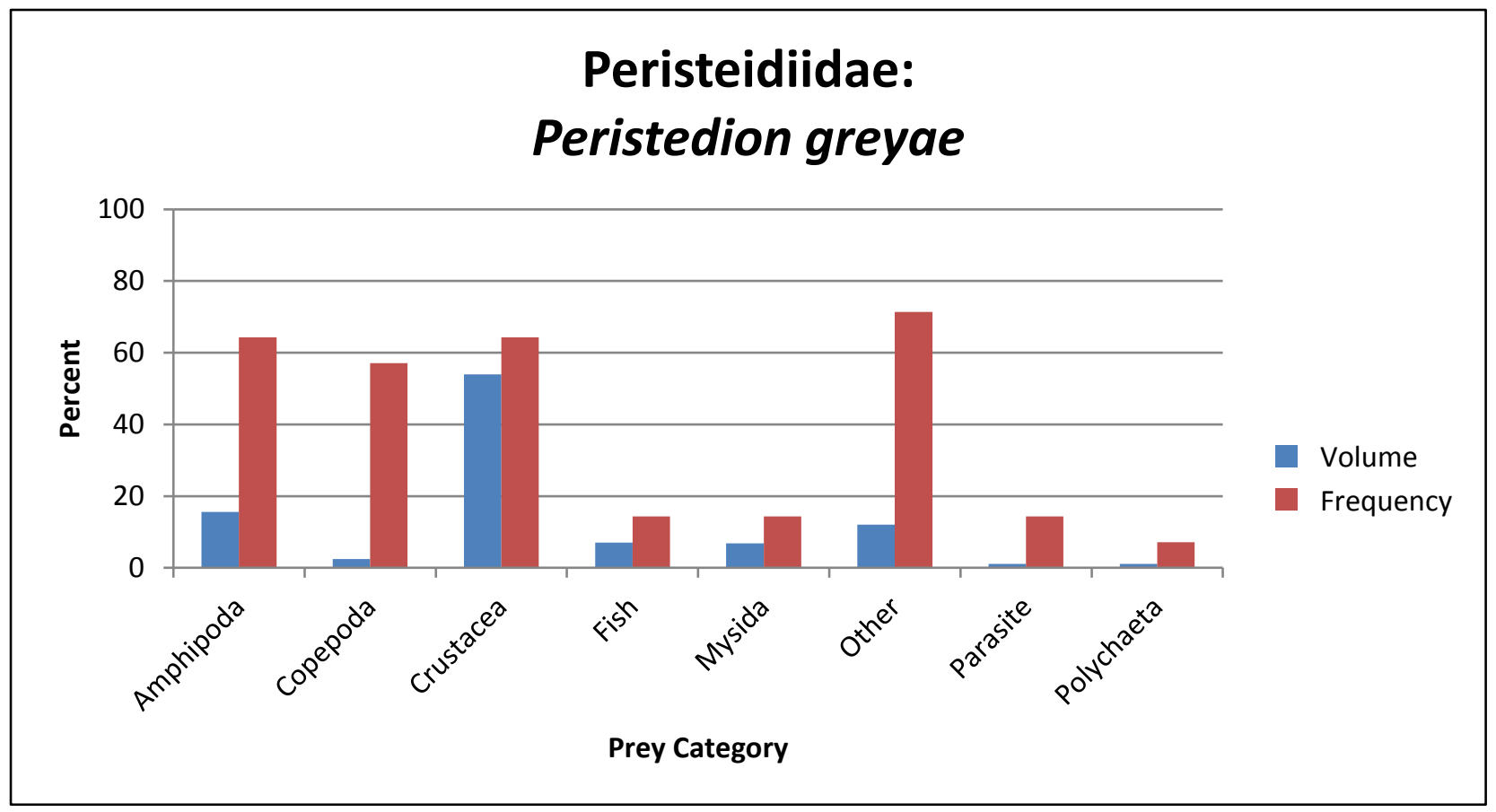

Figure 7.16 Percent volume and percent frequency of stomach contents for Peristedion greyae $(\mathrm{N}=14)$ during the Lophelia II program.

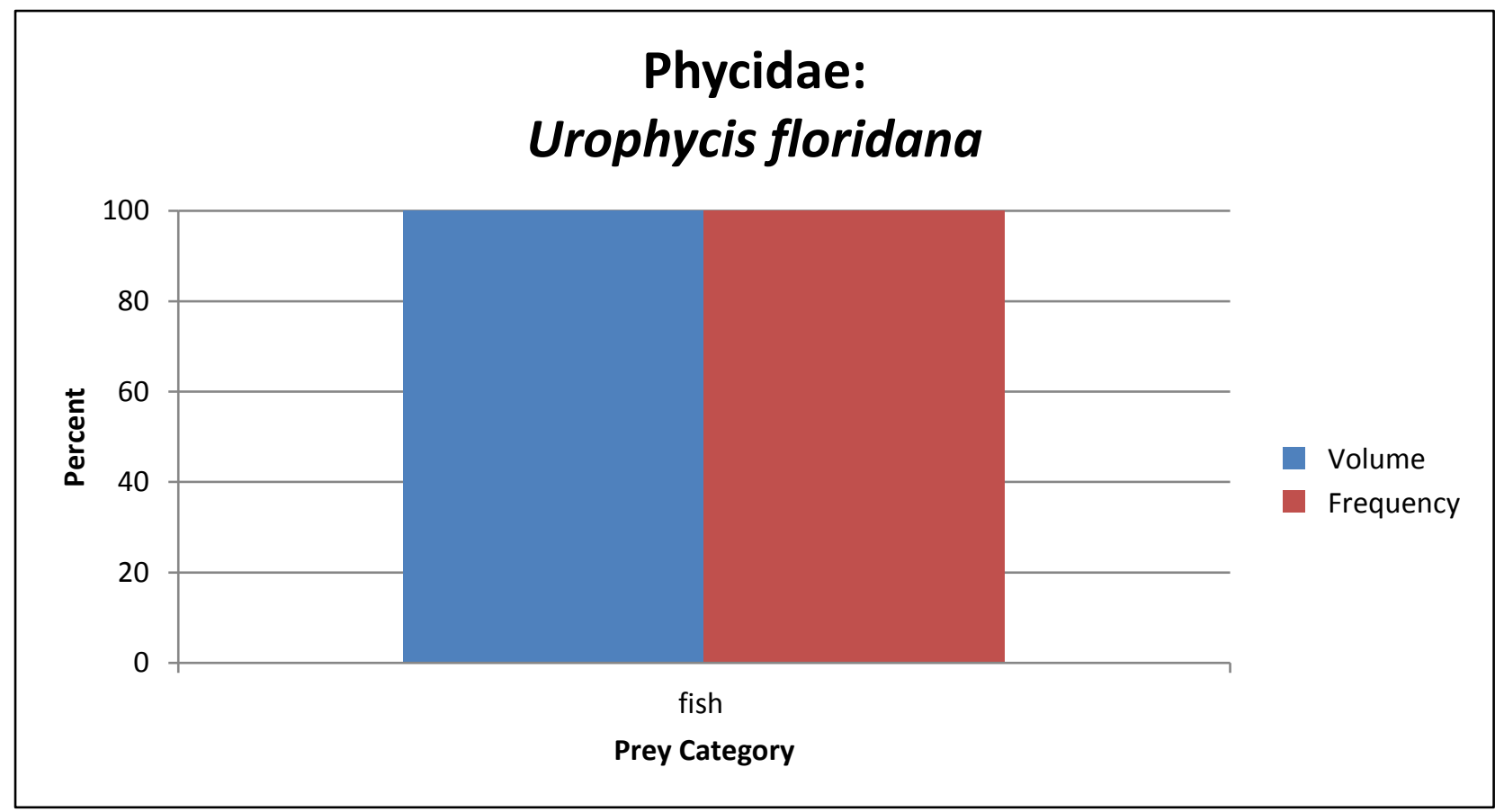

Figure 7.17 Percent volume and percent frequency of stomach contents for Urophycis floridana (N=2) during the Lophelia II program. 


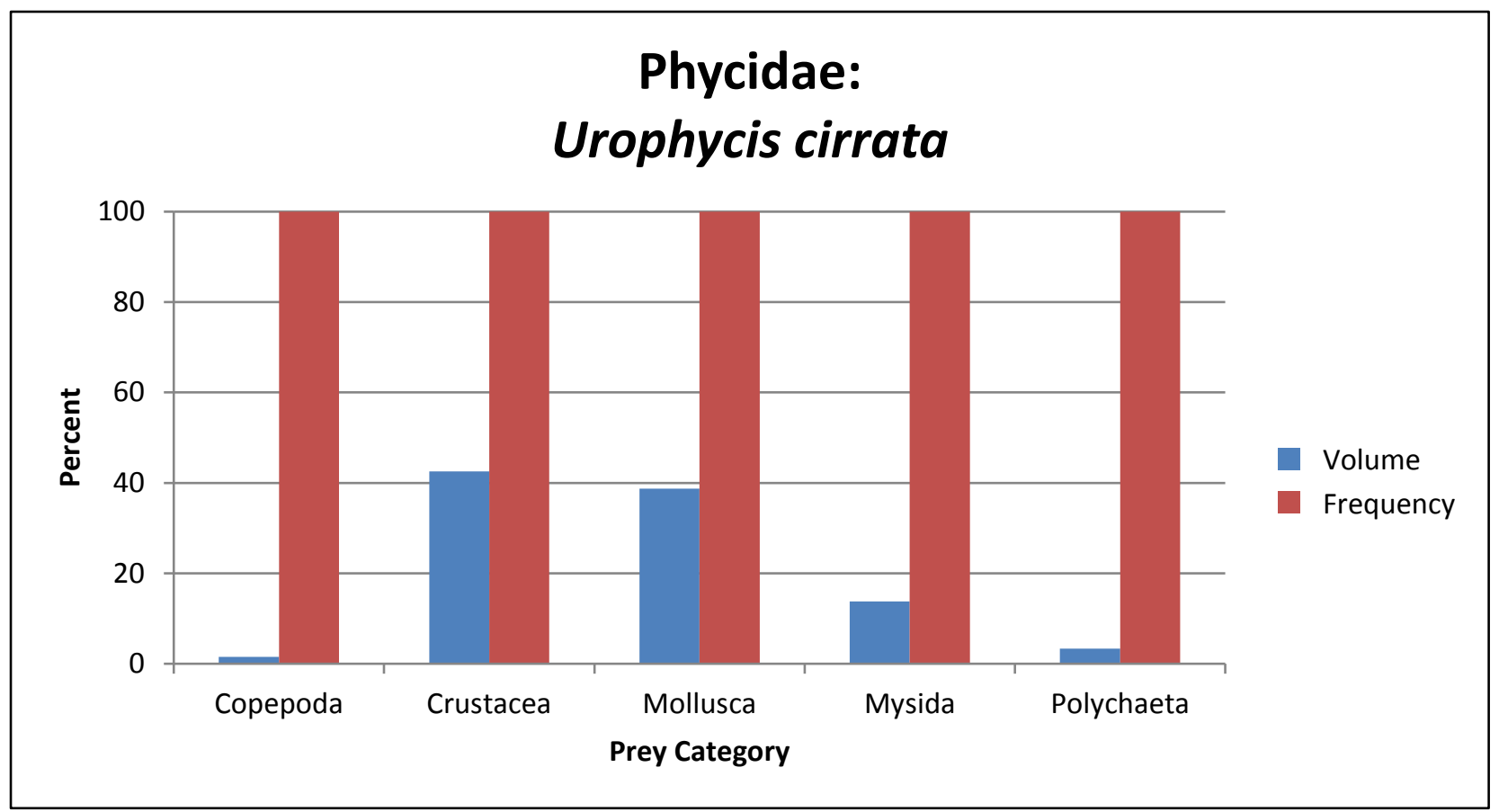

Figure 7.18 Percent volume and percent frequency of stomach contents for Urophycis cirrata $(\mathrm{N}=1)$ during the Lophelia II program.

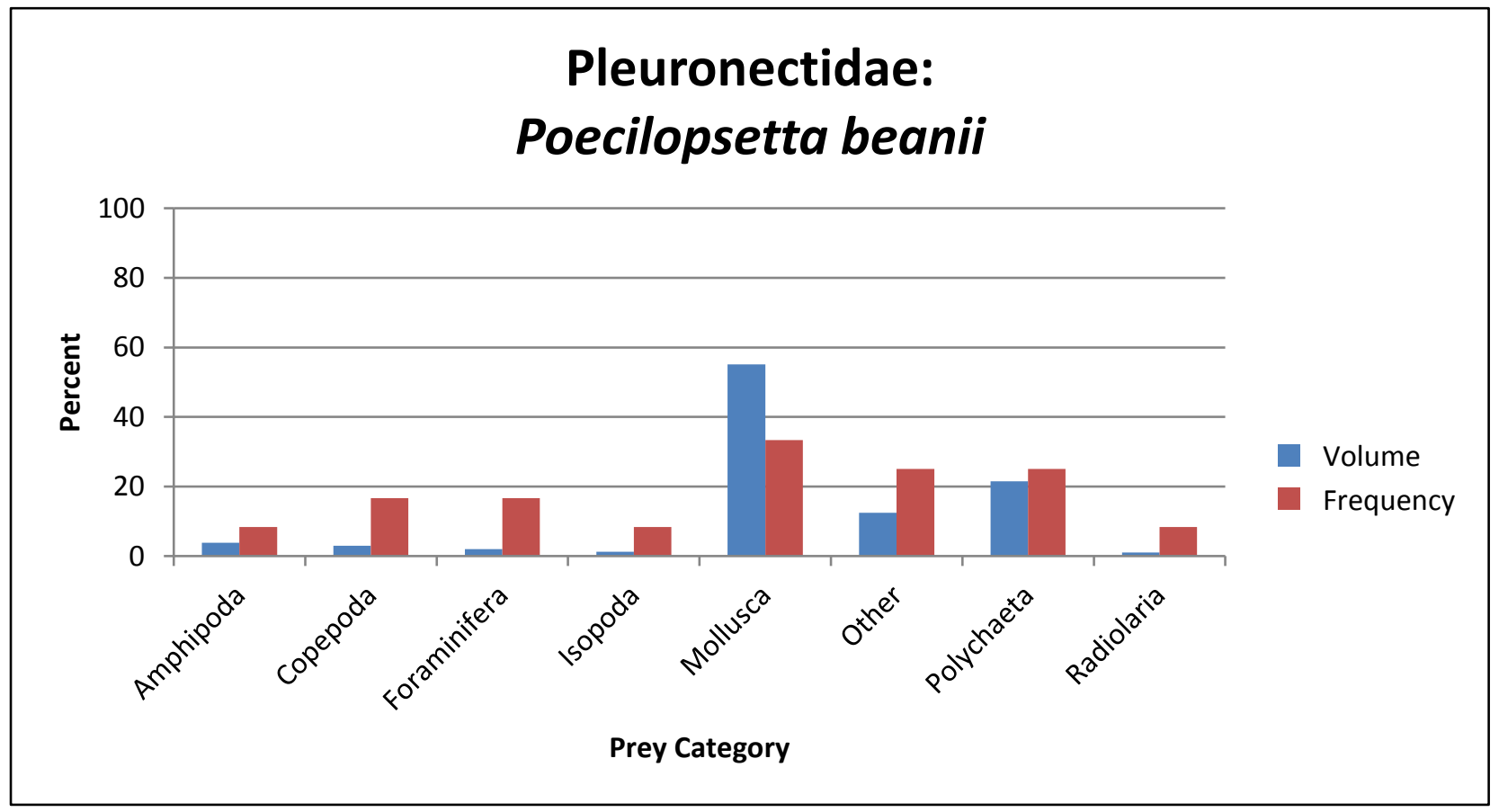

Figure 7.19 Percent volume and percent frequency of stomach contents for Poecilopsetta beanii $(\mathrm{N}=12)$ during the Lophelia II program. 


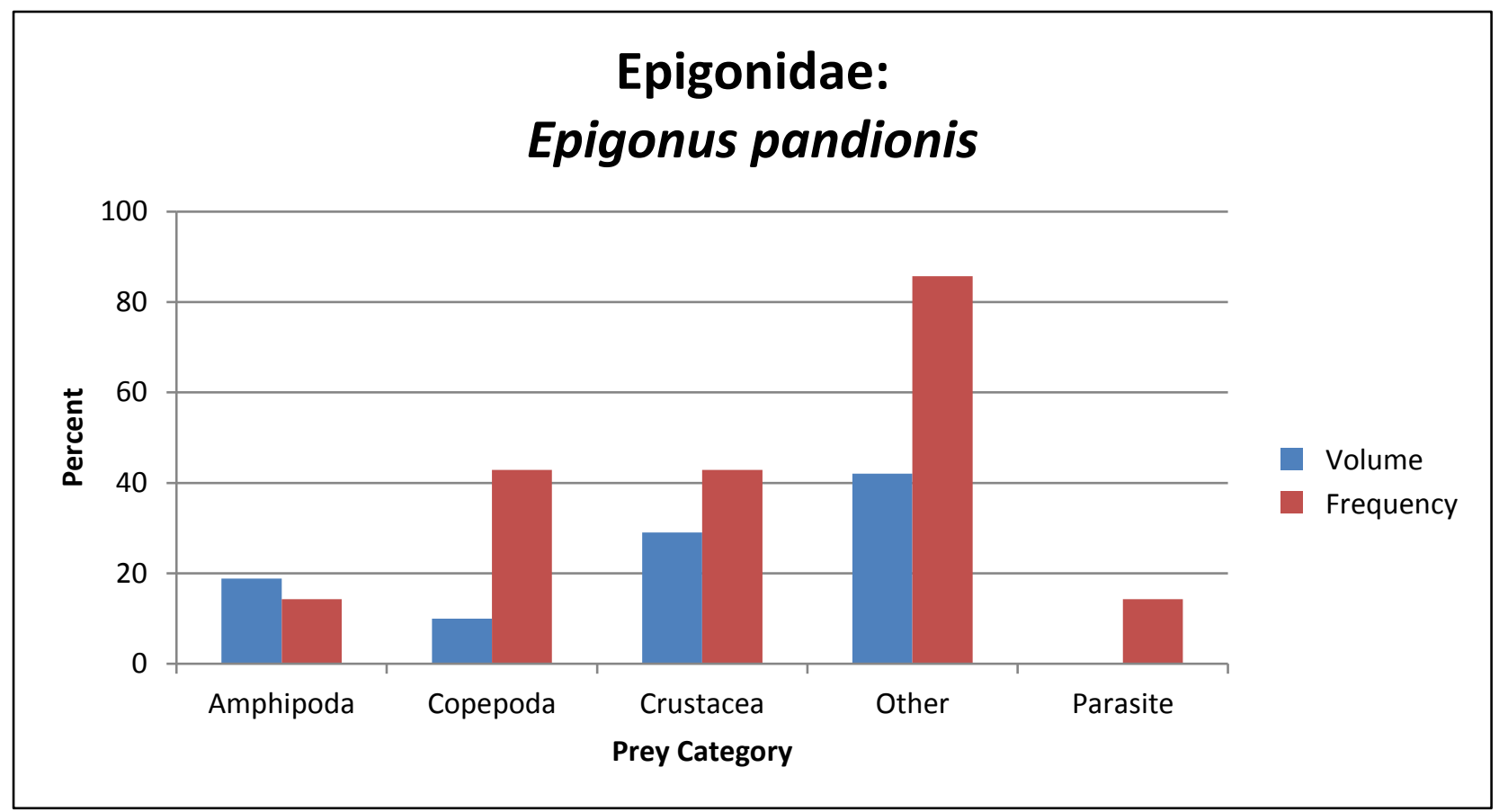

Figure 7.20 Percent volume and percent frequency of stomach contents for Epigonus pandionis ( $\mathrm{N}=7$ ) during the Lophelia II program.

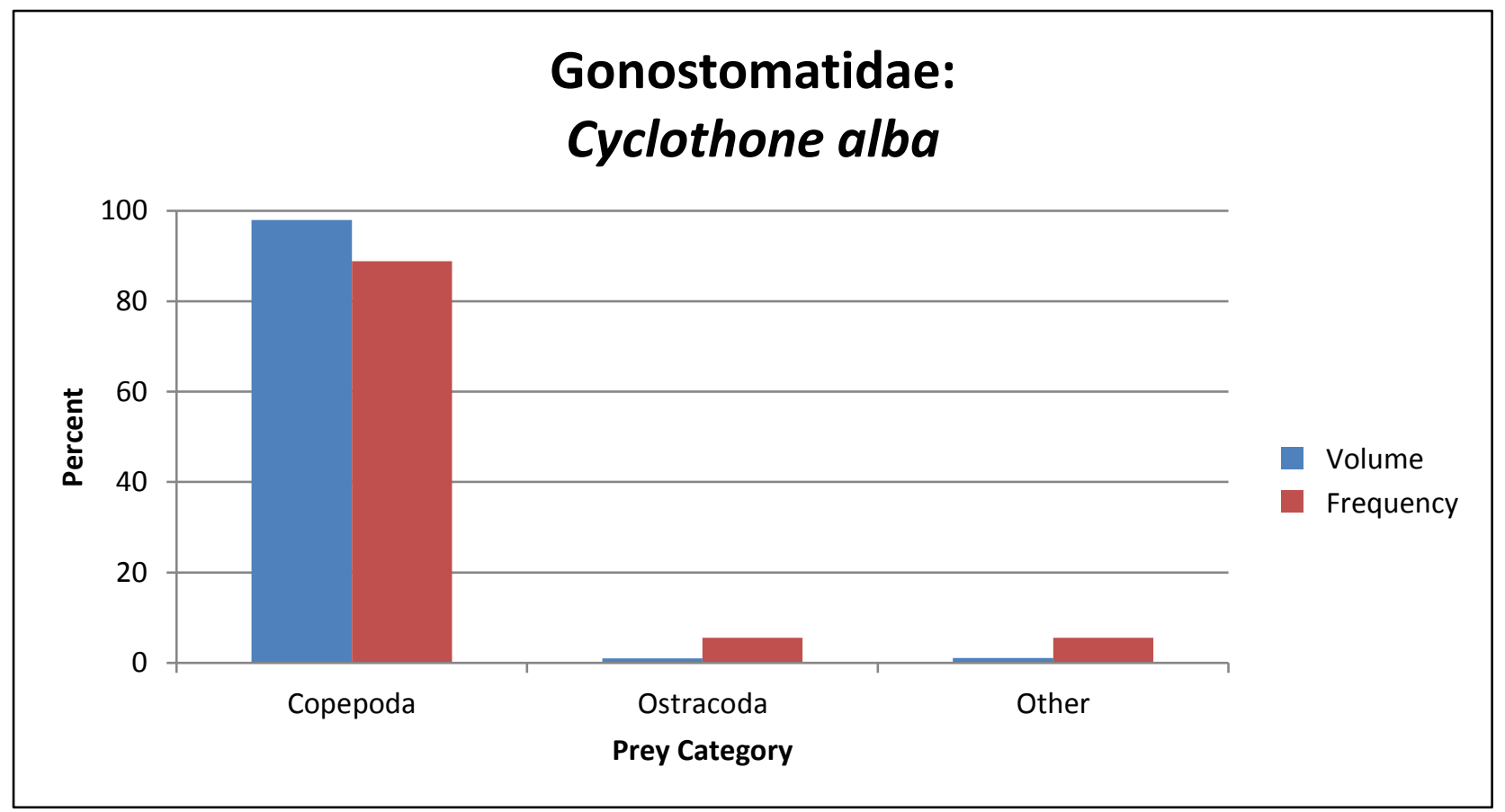

Figure 7.21 Percent volume and percent frequency of stomach contents for Cyclothone alba $(\mathrm{N}=18)$ during the Lophelia II program. 


\section{Gonostomatidae: \\ Cyclothone braueri}

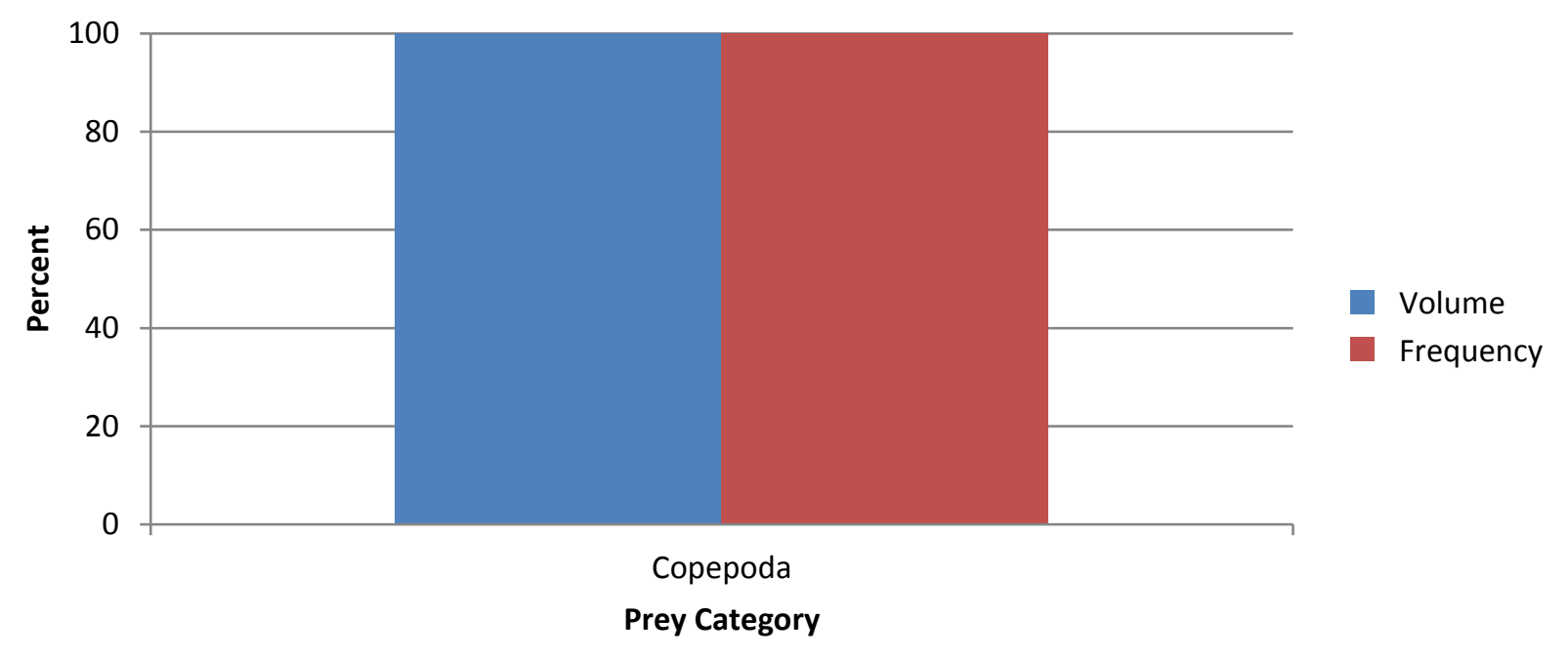

Figure 7.22 Percent volume and percent frequency of stomach contents for Cyclothone braueri $(\mathrm{N}=6)$ during the Lophelia II program.

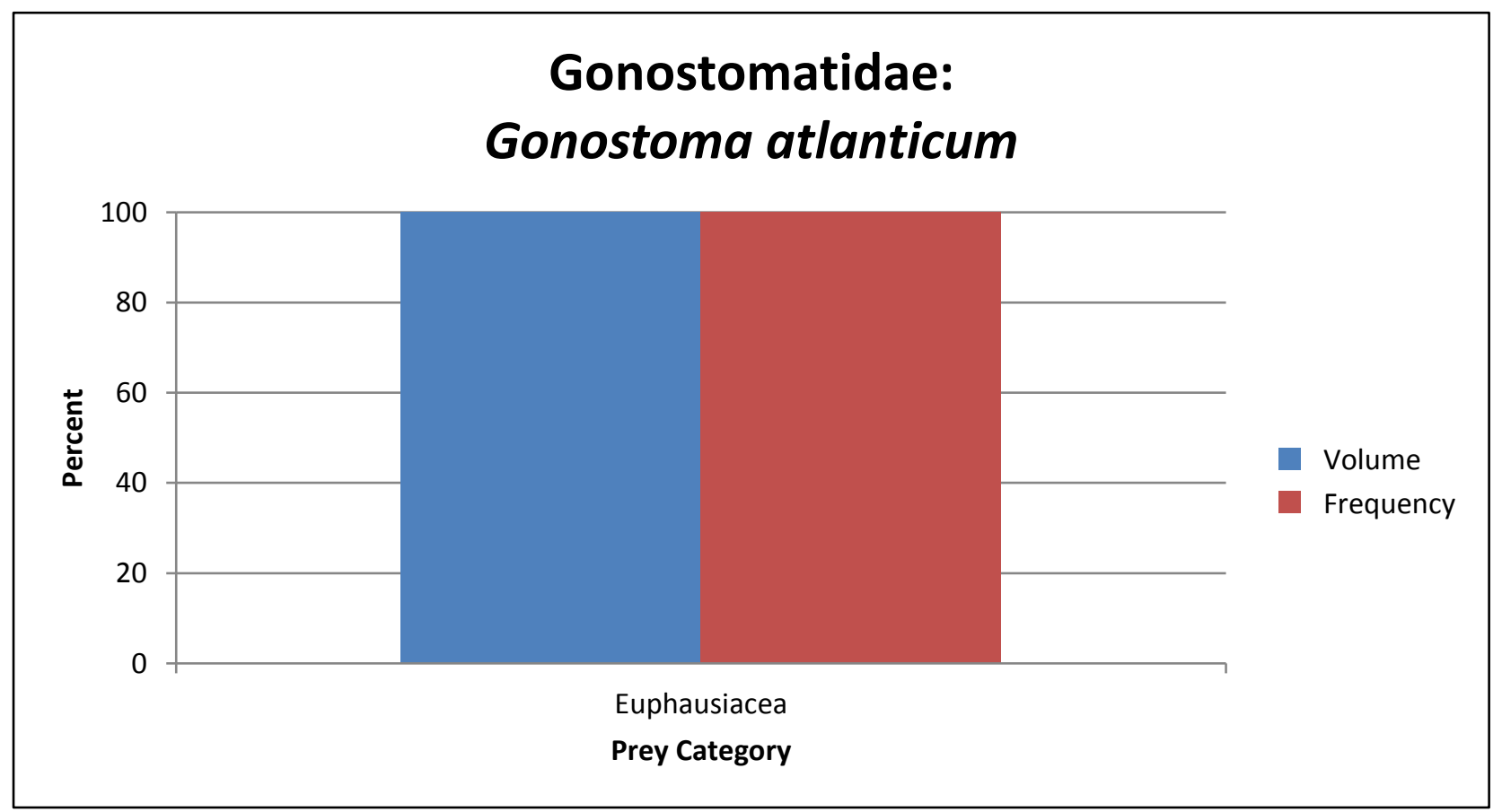

Figure 7.23 Percent volume and percent frequency of stomach contents for Gonostoma atlanticum ( $N=1)$ during the Lophelia II program. 


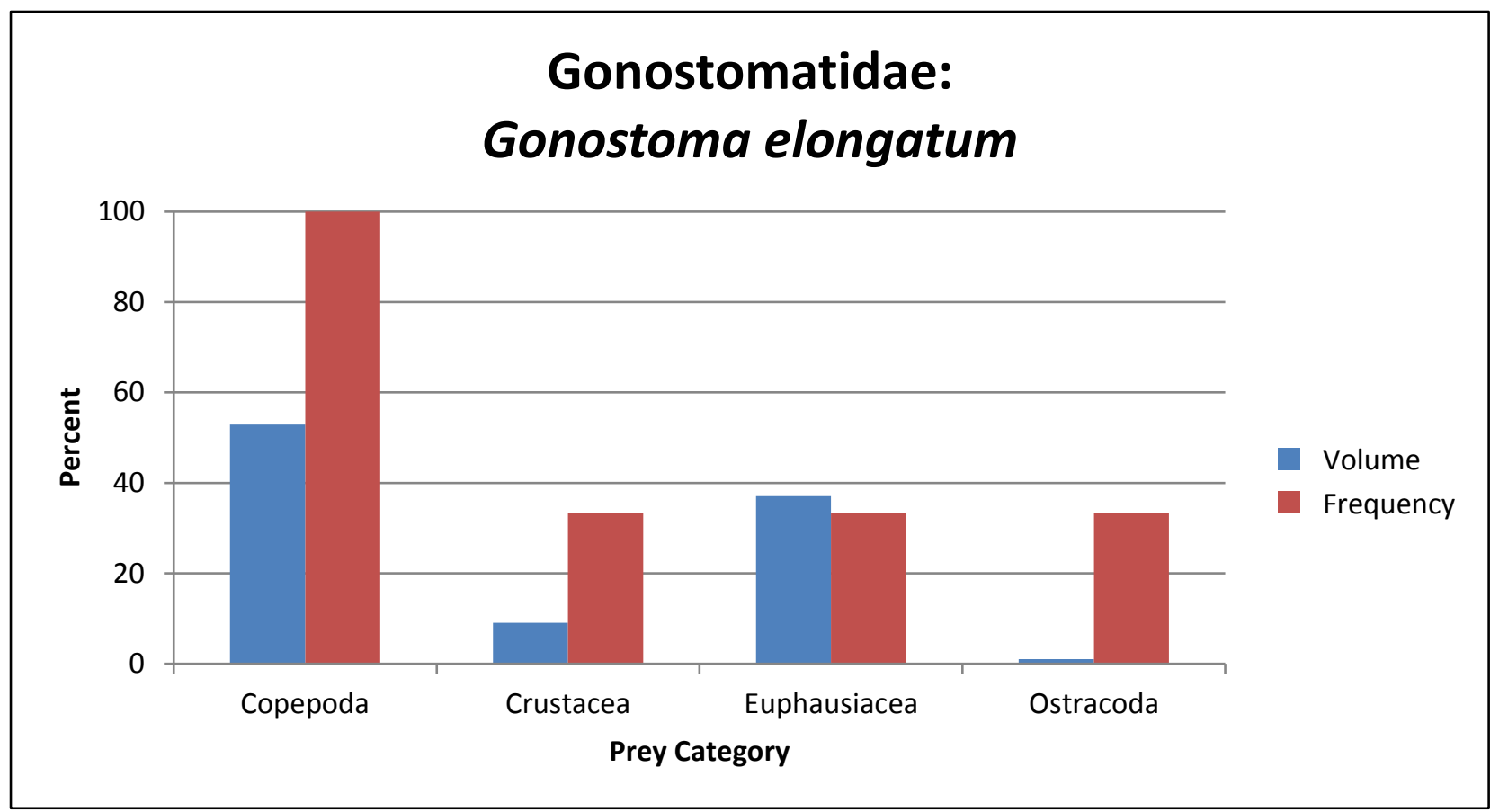

Figure 7.24 Percent volume and percent frequency of stomach contents for Gonostoma elongatum ( $N=3$ ) during the Lophelia II program.

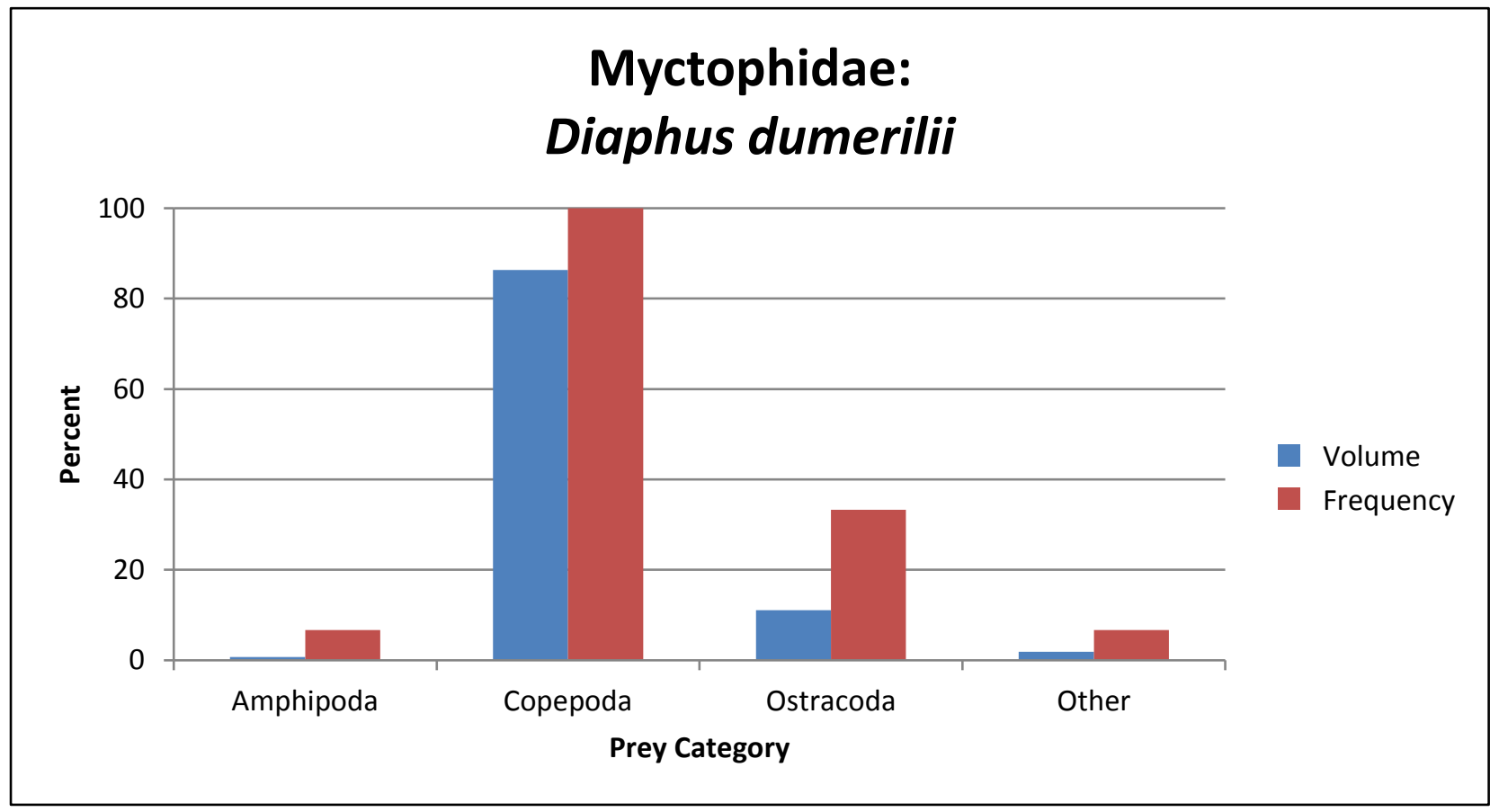

Figure 7.25 Percent volume and percent frequency of stomach contents for Diaphus dumerilii $(\mathrm{N}=15)$ during the Lophelia II program. 


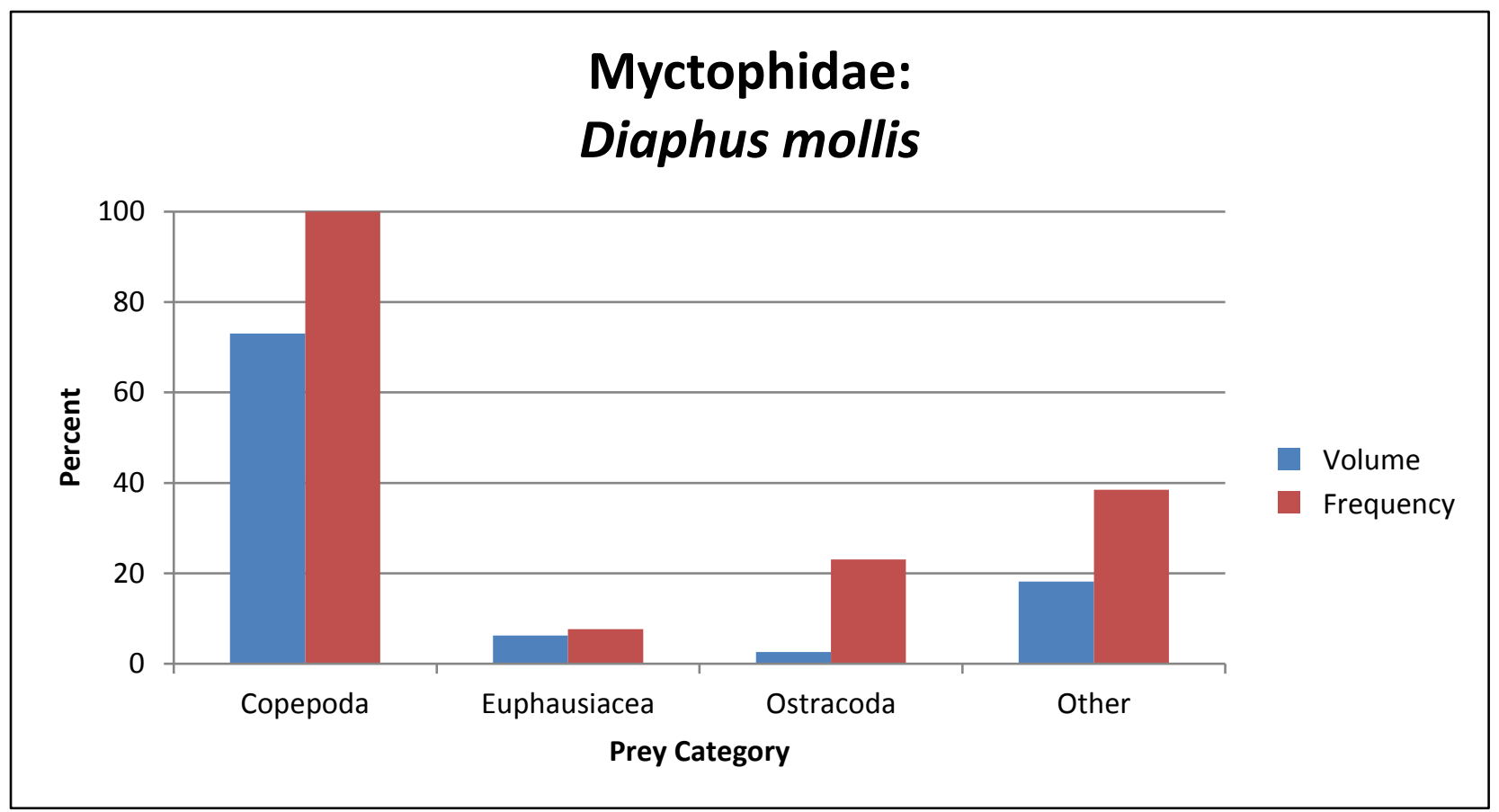

Figure 7.26 Percent volume and percent frequency of stomach contents for Diaphus mollis $(\mathrm{N}=13)$ during the Lophelia Il program.

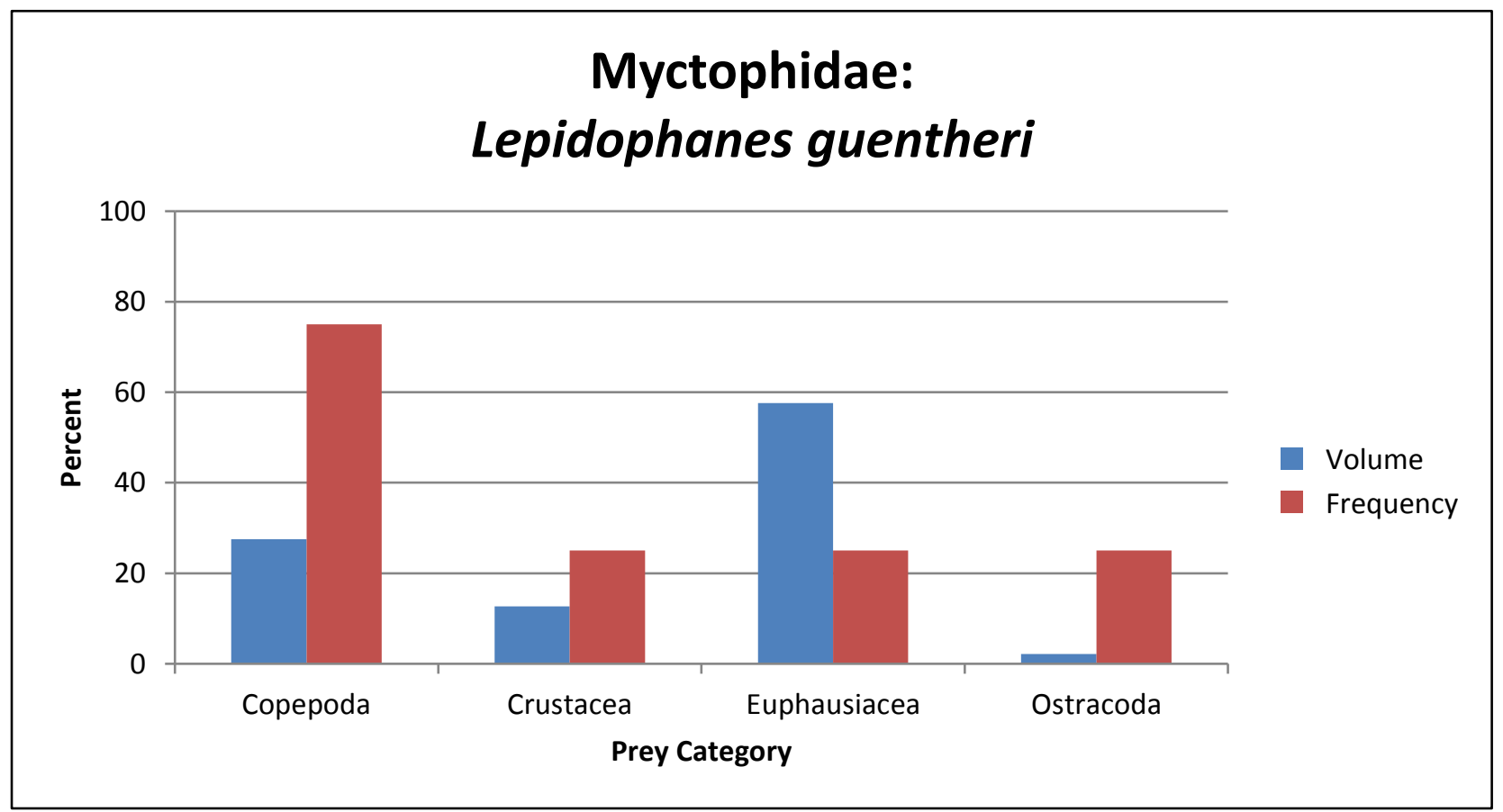

Figure 7.27 Percent volume and percent frequency of stomach contents for Lepidophanes guentheri $(\mathrm{N}=4)$ during the Lophelia II program. 


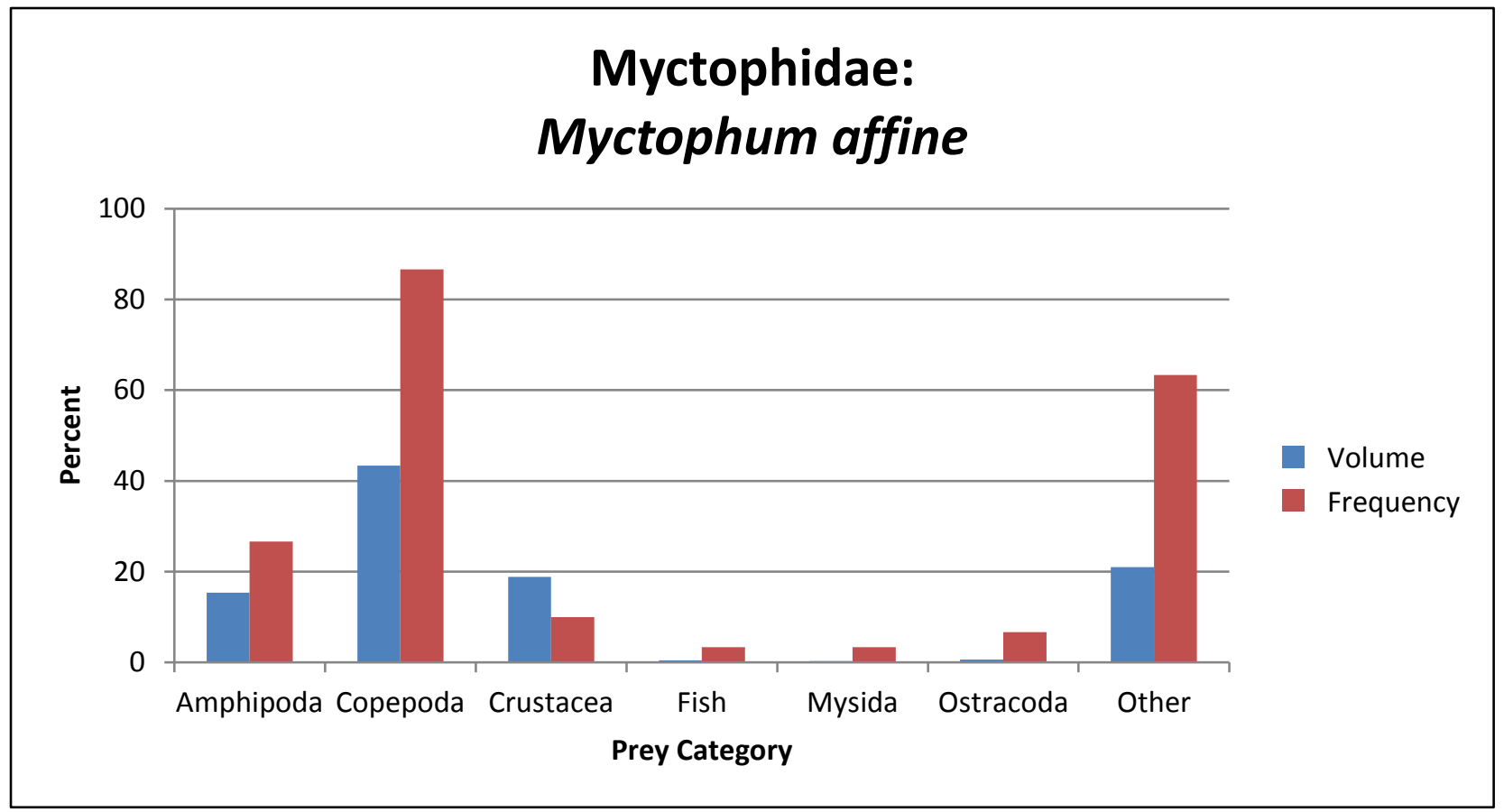

Figure 7.28 Percent volume and percent frequency of stomach contents for Myctophum affine $(\mathrm{N}=30)$ during the Lophelia II program.

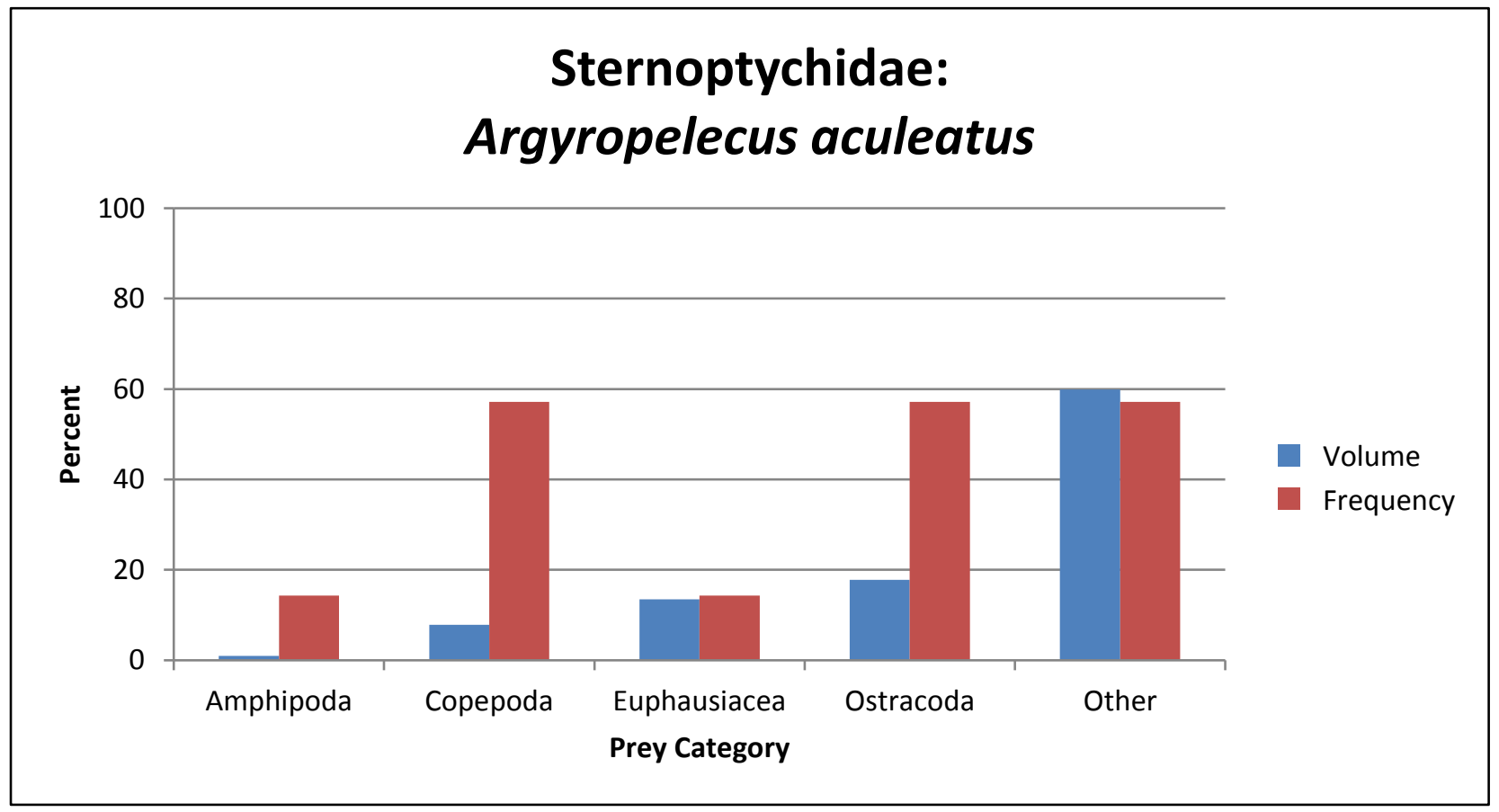

Figure 7.29 Percent volume and percent frequency of stomach contents for Argyropelecus aculeatus ( $\mathrm{N}=7$ ) during the Lophelia II program. 


\section{Sternoptychidae: Argyropelecus hemigymnus}

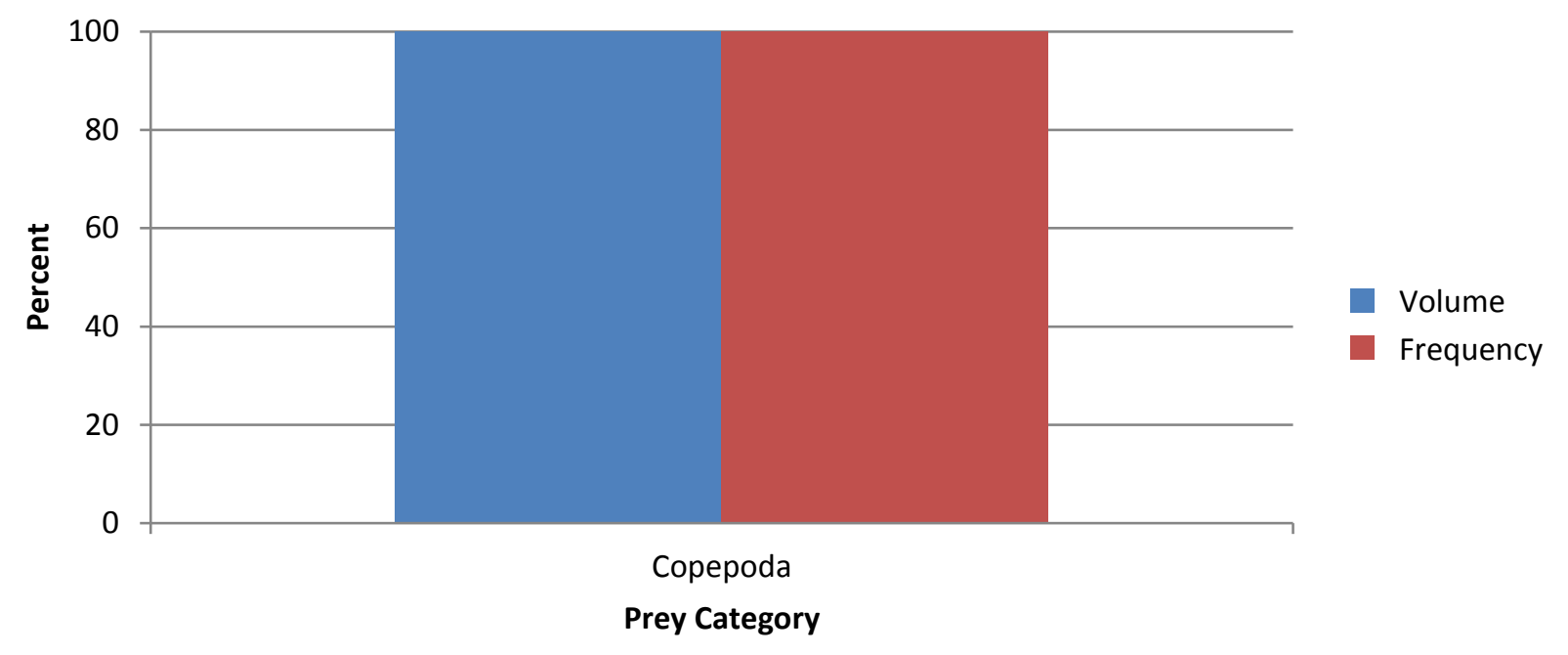

Figure 7.30 Percent volume and percent frequency of stomach contents for Argyropelecus hemigymnus ( $\mathrm{N}=2$ ) during the Lophelia II program.

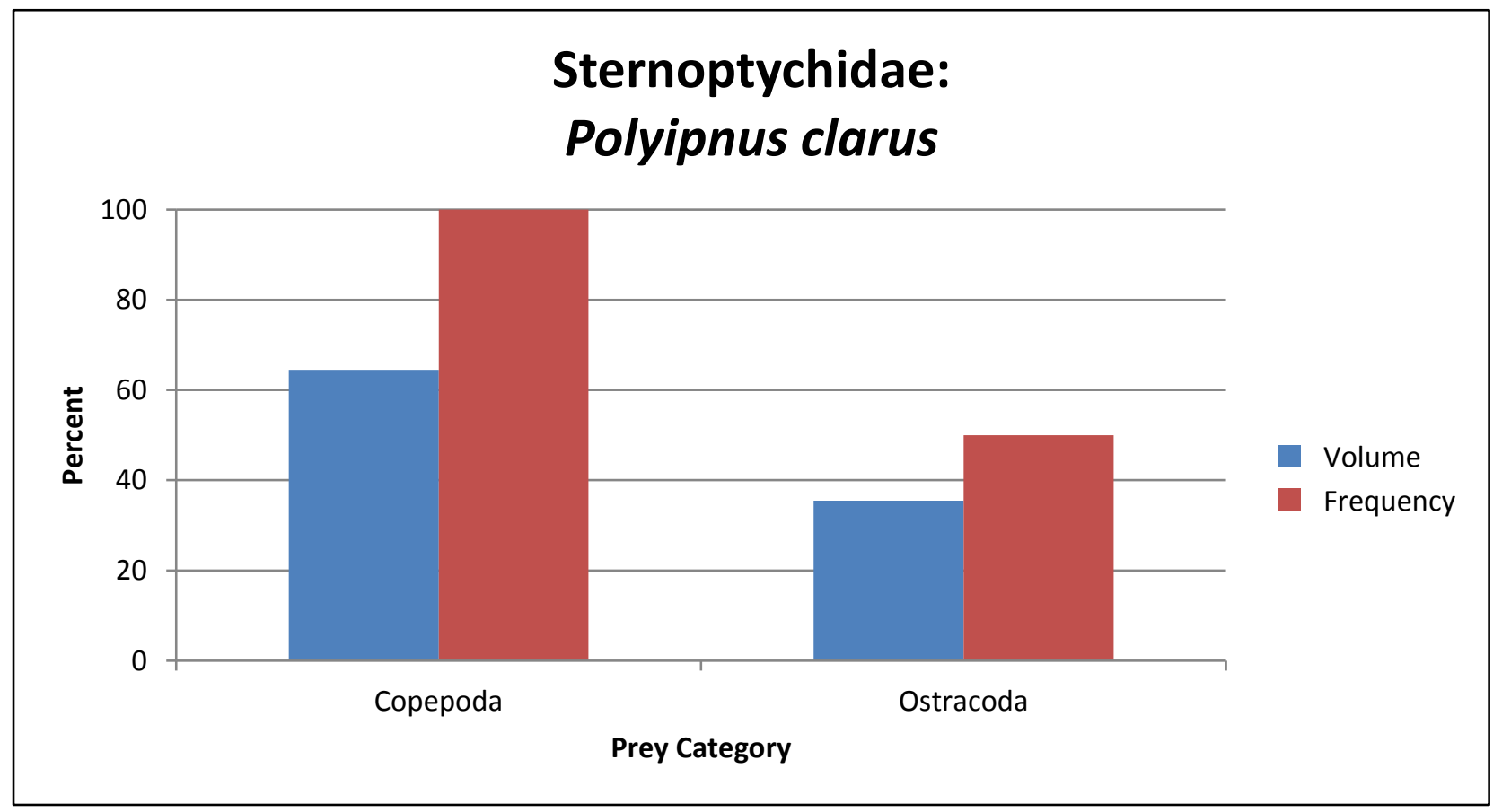

Figure 7.31 Percent volume and percent frequency of stomach contents for Polyipnus clarus ( $\mathrm{N}=2$ ) during the Lophelia II program. 


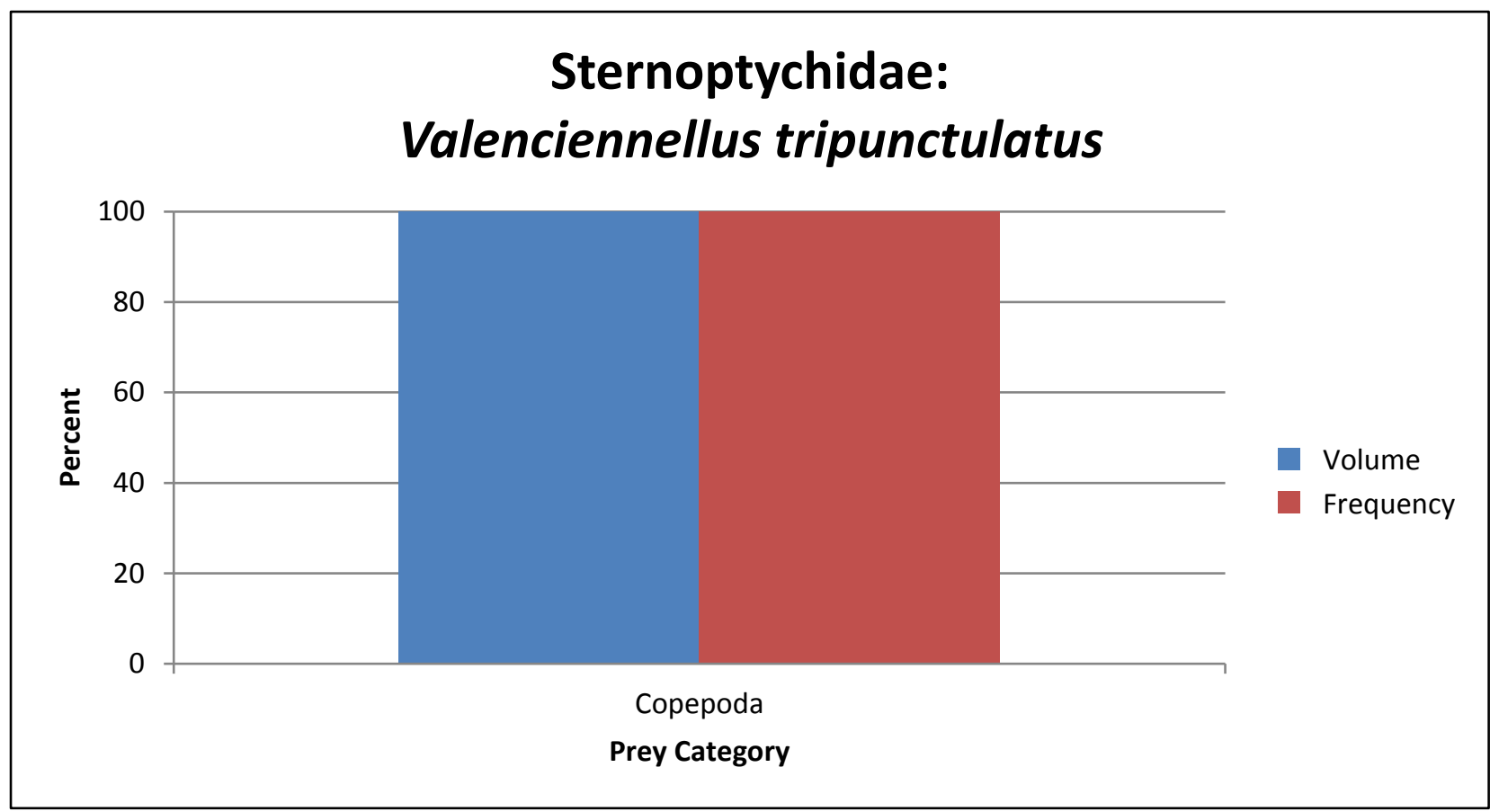

Figure 7.32 Percent volume and percent frequency of stomach contents for Valenciennellus tripunctulatus $(\mathrm{N}=28)$ during the Lophelia II program.

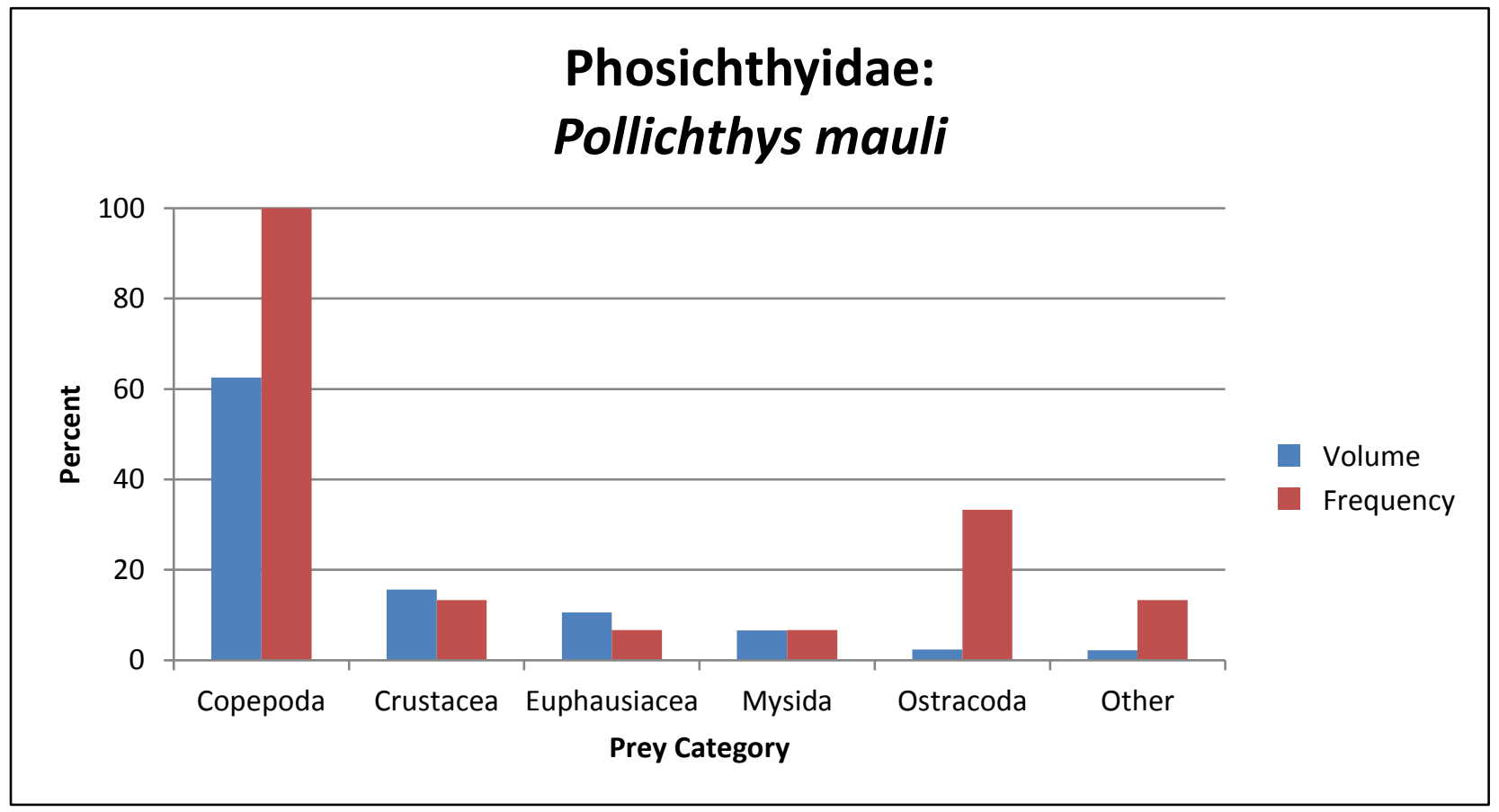

Figure 7.33 Percent volume and percent frequency of stomach contents for Pollichthys mauli $(\mathrm{N}=16)$ during the Lophelia II program. 


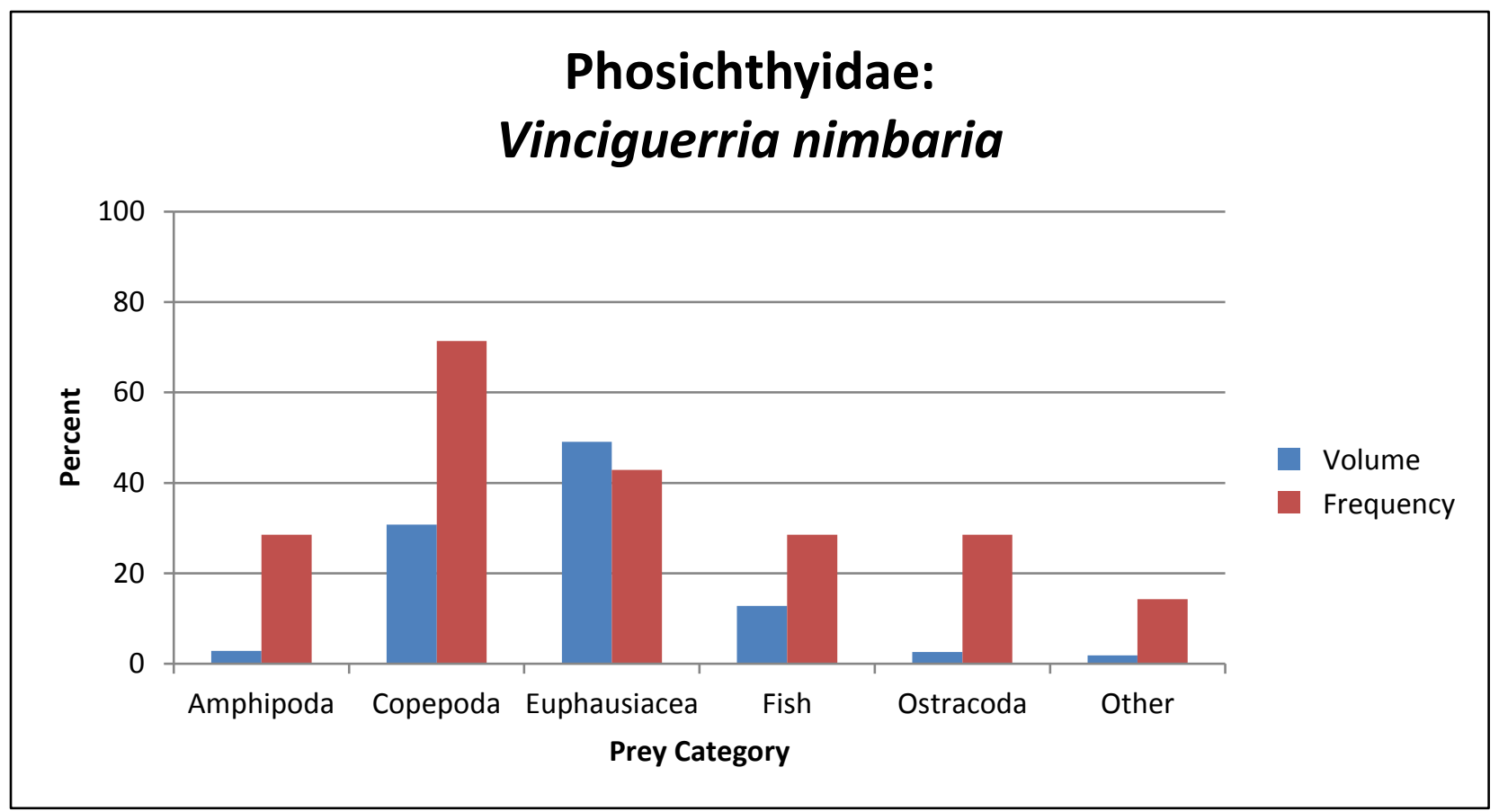

Figure 7.34 Percent volume and percent frequency of stomach contents for Vinciguerria nimbaria $(\mathrm{N}=7)$ during the Lophelia II program.

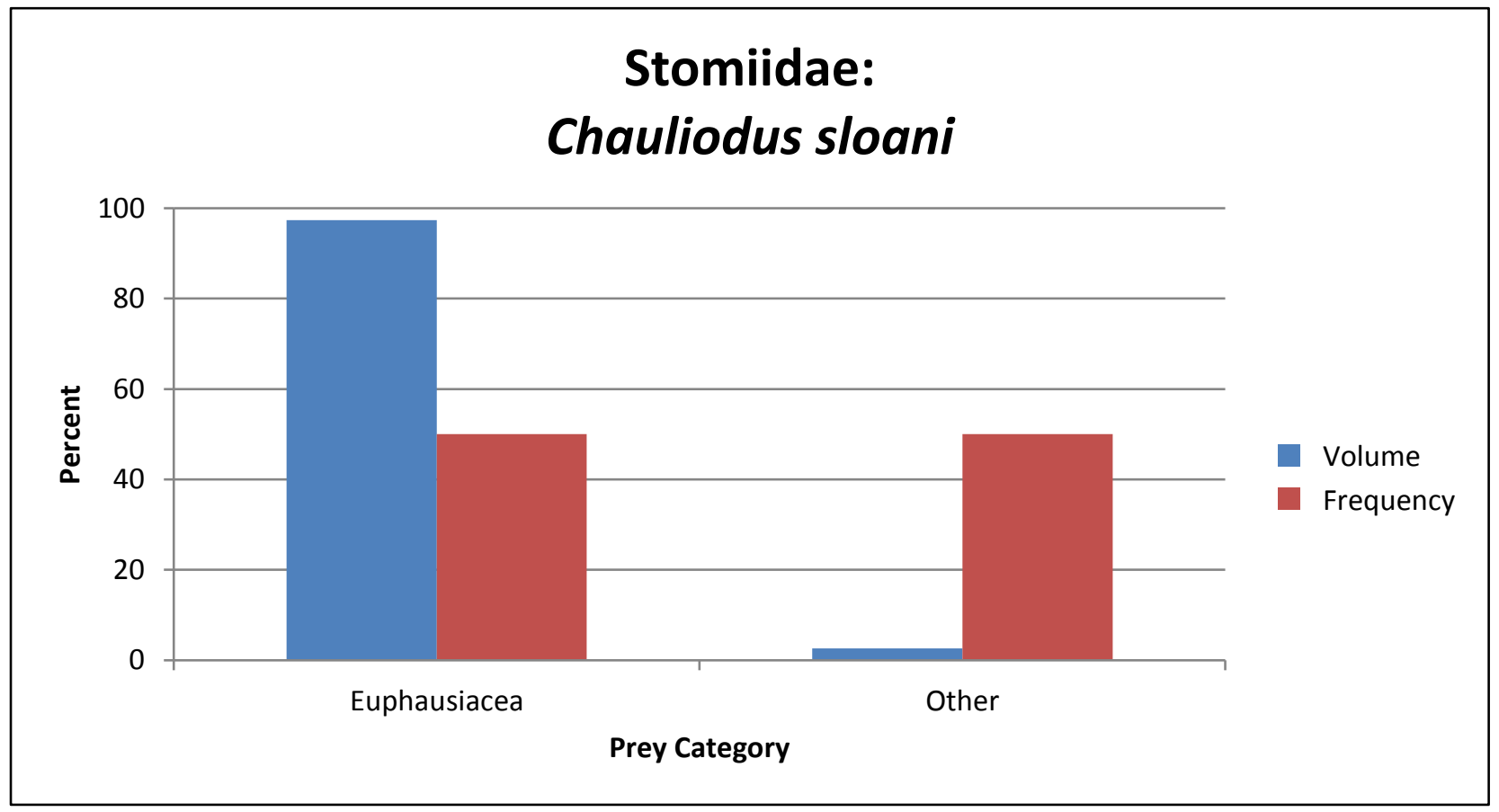

Figure 7.35 Percent volume and percent frequency of stomach contents for Chauliodus sloani $(\mathrm{N}=2)$ during the Lophelia II program. 


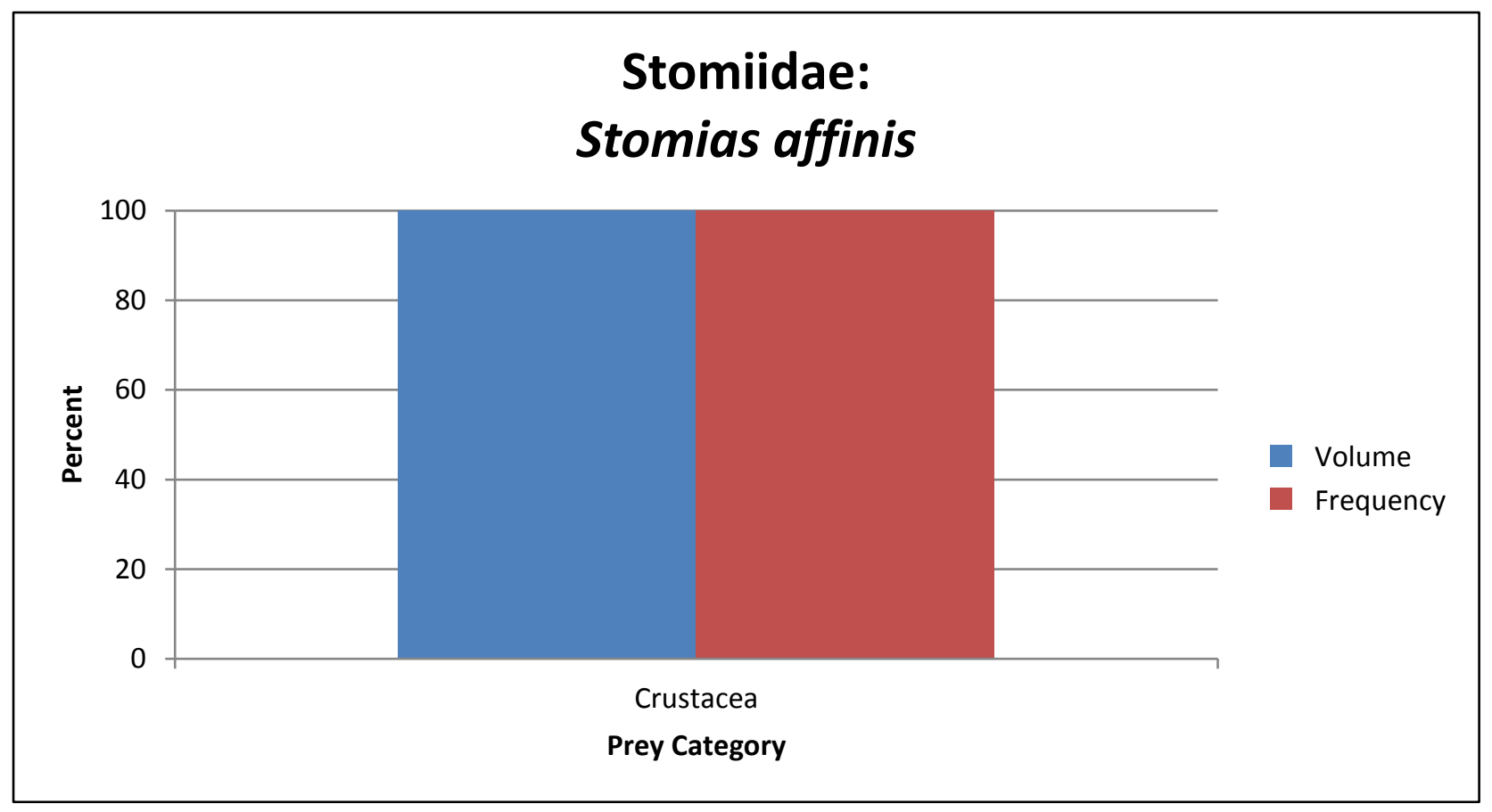

Figure 7.36 Percent volume and percent frequency of stomach contents for Stomias affinis $(\mathrm{N}=1)$ during the Lophelia II program. 


\title{
8 FISHES ASSOCIATED WITH DEEP-SEA CORAL HABITATS IN THE GULF OF MEXICO
}

\author{
By Steve W. Ross, ${ }^{1}$ Andrea Quattrini, ${ }^{2}$ and Mike Rhode ${ }^{1}$
}

${ }^{1}$ University of North Carolina Wilmington, Center for Marine Science, Wilmington, NC

${ }^{2}$ Harvey Mudd College, Claremont, CA

\subsection{Introduction}

Deep-sea or cold-water corals support extensive and important ecosystems along continental slopes. There is growing appreciation of their functions as fish habitat (Costello and others, 2005; Ross and Quattrini, 2007; Söffker and others, 2011) and as hotspots of biodiversity (Henry and others, 2010). These unique, diverse habitats occurring along the continental slope in the Gulf of Mexico (GOM) and off the southeastern United States (SEUS) have escaped detailed examination because of their depths, rugged bottom topography, and strong currents. The scleractinian coral Lophelia pertusa is the dominant habitat forming coral in these regions, but significant structure is also provided by other hard corals and black and bamboo corals, as well as sponges and nonliving rocky substrata (Brooke and Schroeder, 2007; Ross and Nizinski, 2007). The SEUS and GOM may have the most extensive deep coral populations in U.S. waters (Hain and Corcoran, 2004); however, the deeper parts of these large regions remain poorly explored.

It has been assumed that deep-sea fishes generally lack habitat specificity or are opportunistic in terms of habitat selection, but this assumption lacks explicit data. Deep-sea fishes are usually grouped by depth; however, such classification may often be inaccurate (Haedrich and Merrett, 1990). In contrast, shallow-water marine and estuarine fishes have often demonstrated close or obligate habitat associations (Lubbers and others, 1990; Stein and others, 1992; Chittaro, 2004; Quattrini and Ross, 2006). In the deep sea, fish and habitat relationships have been obscured by the indirect methods, such as sampling by dredges and trawls, used to collect most data. Replicated, direct observation is the preferred method for gathering explicit data on faunal relationships to habitat in marine environments (Starr and others, 1995; Connell and others, 1998; Cailliet and others, 1999), but due to expense such methods are less frequently used in the deep sea. Visual censusing methods were emphasized in this study of GOM deep-reef ecosystems.

Although data are still limited, quantitative information on fish relationships with deep-reef habitats is increasing. In the cool temperate to boreal northwestern (north of Cape Hatteras, Auster, 2005) and northeastern (Mortensen and others, 1995; Costello and others, 2005; Söffker and others, 2011) North Atlantic Ocean, deep reefs seemed to be important to some fishes, but as yet, an obligate reef habitat and fish relationship has not been demonstrated in those regions. However, off the SEUS, where the deep-reef fish communities are fairly well described, there does appear to be a suite of fishes which are very closely tied to complex reef habitats (Ross and Quattrini, 2007, 2009). To date there are very few publications describing fish communities on deep reefs in the GOM, and these did not explicitly report habitat data or only reported a few species (Reed and others, 2006; Sulak and others, 2007). Although recreational fishermen are beginning to fish on some of the deep reefs in the GOM (S.W. Ross, personal observation), there are no known commercial exploitations on these deep reefs (Brooke and Schroeder, 2007). Data presented here represent the most extensive listing of fishes on 
deep coral and hardground slope habitats of the GOM. Our overall goals were to document species occurrences and relative abundances and to describe the degree of general habitat specificity of fishes on and around deep-reef habitats to elucidate which fishes were predictably associated with these continental slope habitats in the GOM.

\subsection{Methods}

Fish data were collected during five GOM cruises: National Oceanic and Atmospheric Administration (NOAA) ship Nancy Foster (Leg 1, 5-13 October 2008), research vessel (R/V) Seward Johnson (14-25 September 2009), R/V Cape Hatteras (20 September-3 October 2010), R/V Arctic Sunrise (12-22 October 2010), and NOAA ship Ronald H. Brown (10 November 2010). The undersea vehicles used to collect video and photographic data as well as specimens that were associated with these cruises were the Saab Falcon remotely operated vehicle (ROV), Johnson Sea Link II (JSL) submersible, Kraken II ROV, Dual DeepWorker submersible, and Jason II ROV. See Chapter 1 of this report for detailed station tables (table 1.1) and maps of the station locations. The 2008 cruise only sampled in the Viosca Knoll (VK) 826 (VK826) and VK862/906 study areas. The 2009 and Cape Hatteras cruises sampled both of the VK sites as well as the West Florida Slope (WFS) study area and collected limited data at the Mississippi Canyon 751 (MC751) site. The Arctic Sunrise cruise only sampled in the VK862/906 study area, and the WFS was the only site sampled by ROV in the GOM during the Ronald H. Brown cruise.

Fish occurrence and bottom habitat data were largely acquired by video and still cameras mounted to the various underwater vehicles. Because the video data were of marginal quality on the 2008 Nancy Foster cruise, those data are not incorporated here except as ancillary data (for example compiling species lists or examining behaviors). Regardless of the vehicle, video methods were standardized to the extent possible. Paired scaling lasers were available on all cameras. When the ROVs or submersibles were moving, the video camera was moved to predetermined pan/tilt positions, set on wide angle, and the vehicle moved at slow speeds as near to bottom as possible similar to previous methods (Ross and Quattrini, 2007). This was modified as needed in heavy coral/rock areas and as lighting needs changed. Transects were run across all habitat types, including reef and non-reef areas. At times during most dives, specimens were collected by using manipulator arms or suction devices mounted on the vehicles.

On the Nancy Foster and Cape Hatteras cruises, bottom fishes were also collected by using a small otter trawl. Trawl sample sites were selected by using multibeam sonar maps with the objective being to tow near, but not on, deep coral habitats. The otter trawl (4.9-meter [m] head rope, 38.1millimeter [mm] mesh) was deployed from the stern and towed for 30 minutes (min) at about 2-knot (3.7 kilometers per hour [km/h]) ground speed. On the Nancy Foster, Seward Johnson, and Cape Hatteras cruises, a few traps sets (usually 24 hours [h]) were made to supplement the fish collections.

Fish specimens from all collections were preserved at sea in 10-percent formalin in seawater solution. After the cruises, fish were rinsed in water and stored in 50 percent isopropanol. Fishes were identified to the lowest taxonomic level possible.

In the lab, the underwater videos from the vehicle cameras were viewed multiple times to document habitat and identify (to species if possible) and count fishes. For this report, one of three general habitat categories was assigned to each fish observation as in Ross and Quattrini (2007): prime reef (variable, but rugged profile of $\geq 1 \mathrm{~m}$, which could be rocky formations or dense stands of coral), transition reef (mostly dead coral rubble and (or) scattered small rocks with profiles $<1 \mathrm{~m}$, often with sandy bottom showing in places), off-reef (relatively flat bottom composed mostly of soft substrata). Species occurrence was compared among the three general habitat types. 


\subsection{Results}

\subsubsection{ROV and Submersible Data}

Twenty-three submersible dives and 18 ROV dives covered an overall depth range of 296 to 734 $\mathrm{m}$ and resulted in about $175 \mathrm{~h}$ of bottom video data (table 8.1). In total, 12 dives (60 h of video) were accomplished at VK826, 18 dives (55 h of video) at VK862/906, 9 dives (55 h of video) at WFS, and 2 dives (6 h of video) at MC751 (table 8.1). Site information can be found in Chapter 1 of this report. Video data from the Falcon ROV were of marginal value and are not included here.

During these dives, 66 taxa of fishes, representing 42 families, were documented (table 8.2). See Appendix D (fig. D.1) for in situ photographs of some of the more common benthic fishes observed during this project. Prime reef habitat yielded the most fish species $(n=42)$, followed closely by transition reef $(n=38)$ and off-reef $(n=39)$. Together, prime and transition reef habitats accounted for 52 fish species, 27 of which were unique to these habitats. The dominant benthic species that consistently characterized the two reef habitats were: Conger oceanicus, Nezumia spp., Laemonema barbatulum, Benthocometes robustus, Lophiodes beroe, Hoplostethus occidentalis, Beryx decadactylus, Grammicolepis brachiusculus, Helicolenus dactylopterus, and Hyperoglyphe perciformis. Thirteen species were unique to off-reef habitat (table 8.2). The dominant off-reef benthic species included Fenestraja plutonia (or F. sinusmexicanus), Chlorophthalmus agassizi, Polymixia lowei, Nezumia sp., Laemonema goodebeanorum, Merluccius albidus, Dibranchus cf. atlanticus, Synagrops spp., Epigonus oligolepis, and Symphurus marginatus.

Mesopelagic fishes were encountered frequently on or just over the bottom and often interacted with reef habitat and biota (table 8.2). The dominant families involved were Myctophidae, Sternoptychidae, and Paralepedidae.

\subsubsection{Trawl and Trap Data}

Underwater vehicle video data were supplemented by trawl and trap collections. While trawls and traps were sited (see Chapter 1 of this report) so as not to impact fragile reefs, these gears were operated as close to reefs as possible. We did not associate habitats with these catches because of the uncertainty of what habitats the gear encountered, but we assumed most of the habitats sampled by trawl or trap were off-reef, with a small amount of transition reef.

Overall, 69 species of fishes (including mesopelagic species) were collected by otter trawl, and 7 species were collected by trap (table 8.3). Trawling and trapping added 44 species to the overall numbers of taxa sampled around the reefs, including several benthic families not observed by submersible or ROV (for example, Etmopteridae, Notacanthidae, Peristediidae, and Paralichthyidae). Trawling produced a number of typically mesopelagic fishes (for example, myctophids, gonostomatids, and sternoptychids), and while it is possible these were captured in the water column as the net was deployed or retrieved, it is also likely that many of them were captured on or near the bottom, matching our in situ observations of midwater fishes on the bottom. The most abundant bottom fishes collected were in the families Macrouridae (Nezumia aequalis, Coelorinchus caribbaeus, C. coelorhincus, Malacocephalus occidentalis), Moridae (Laemonema goodebeanorum), and Ogcocephalidae (Dibranchus atlanticus) (table 8.3). Many of the bottom fishes collected by trawl are species typical of soft-sediment bottoms, such as Fenestraja sinusmexicanus, Urophycis spp., Chlorophthalmus agassizi, Peristedion greyae, and Symphurus marginatus. Several fish taxa that were common to the reef habitats (such as N. sclerorhynchus, Lophiodes spp., Beryx spp., Benthocometes robustus) were not collected by trawl or trap, suggesting that they did not stray far from reef areas. 
Traps were set opportunistically and usually yielded low numbers of species and individuals. Three species, Conger oceanicus, Urophycis floridana, and Pontinus rathbuni, were collected by trap but were not collected by trawl (table 8.3). The large amount of time required to set and retrieve the traps, coupled with small catches, generally made this gear inefficient to use. However, traps could be set closer to reef areas compared with trawling, but this also made traps vulnerable to snagging in reefs with consequent gear loss and habitat damage.

\subsection{Discussion}

The fish fauna of the GOM, including deep-sea fishes, is well known compared with that of many regions of the world (McEachran and Fechhelm, 1998, 2005; Powell and others, 2003). Reasons for this include a diversity of commercial and recreational fisheries, a number of government and academic marine laboratories available to conduct studies, and a long history of environmental studies related to hydrocarbon exploration. However, to date, only one publication (Sulak and others, 2007) documents in detail the fishes near or on deep-sea reefs ( $\geq 300-\mathrm{m}$ depths) in the GOM, but this study was restricted to the Viosca Knoll sites, and only the dominant species were examined for habitat specificity. Reed and others (2006) reported 10 taxa of fishes from the WFS, but about half were not identified to species, and no habitat associations were noted.

Similar to findings off the SEUS (Ross and Quattrini, 2007, 2009), we observed a diverse ichthyofauna (66 taxa) on and near these GOM deep reefs. However, considering that there were more hours of video data $(175 \mathrm{~h})$ in this GOM project compared with the SEUS (116 h, Ross and Quattrini, 2007), we expected a higher species richness in the GOM. Nevertheless, species richness observed in this study was substantially higher than the 36 fish taxa previously observed during JSL dives at Viosca Knoll (Sulak and others, 2007). Partial explanations for this difference are that our surveys visited more sites (for example, WFS) and had longer video time ( $<45 \mathrm{~h}$ in Sulak and others, 2007), and the wider geographic coverage and increased time provided additional opportunities to record more species. The range of depths covered in both regions was similar (296-734 $\mathrm{m}$ in GOM and 366-783 $\mathrm{m}$ off SEUS), and the shallower depths in the GOM yielded only one species (Epinephelus niveatus) that is near its maximum known depth.

Similar to observations off the SEUS (Ross and Quattrini, 2007, 2009), in the GOM there appears to be a strong association between some fish species and complex reef-like habitats (whether composed of deep-sea corals or rocky substrata). In agreement with Caruso and others (2007), we also observed a close tie to deep-reef habitats for most Lophiiform fishes in the region. Sulak and others (2007) noted that Grammicolepis brachiusculus could be an obligate associate of L. pertusa reefs, an observation supported in this study. In addition, we suggest that such species as Conger oceanicus, Dysommina rugosa, Nezumia sclerorhynchus, Physiculus kerrerae, Benthocometes robustus, Hoplostethus occidentalis, Beryx spp., Idiastion kyphos, and Anthias woodsi are obligate associates of deep-reef habitat. Of the dominant fishes noted by Sulak and others (2007), the only two species not observed in this study were Polyprion americanus and Lopholatilus chamaeleonticeps. The former species was only recently reported from the GOM (Sulak and others, 2007; Shipp and Sedberry, 2008) and may be rare in this region.

The strength and consistency of the fish association with deep reefs off the SEUS and in the GOM differs from that in the northeastern and northwestern (north of Cape Hatteras) North Atlantic Ocean, where deep-sea fish association with corals and other hard substrata are more opportunistic than obligate (Auster, 2005; Costello and others, 2005). The reasons for this difference are unclear, but may be a function of very different types of fishes in these areas. Some fish families that are common among the reefs of the SEUS and GOM (such as Congridae, Trachichthyidae, Berycidae, Grammicolepidae, 
and Serranidae) were less common or missing from more northerly deep-sea reefs (Auster, 2005; Costello and others, 2005; Söffker and others, 2011). Although the SEUS and GOM deep reefs share a number of fish families (such as Scyliorhinidae, Synaphobranchidae, Macrouridae, Moridae, Phycidae, Lophiidae, and Scorpaenidae) with more northern parts of the North Atlantic, the species composition within these families differs between regions. No species recorded here from the GOM were shared with those reported by Auster (2005) from the Gulf of Maine, and only a few genera or species were shared with reef habitats from the northeastern Atlantic (Costello and others, 2005; Söffker and others, 2011). The two scorpaenids Helicolenus dactylopterus and Trachyscorpia cristulata appear to occur on reef habitats universally throughout a large part of the North Atlantic (Auster, 2005; Costello and others, 2005; Söffker and others, 2011).

During the time frame of this study, one new species of fish, Bellotia robusta (family Bythitidae), was described from these deep-sea coral habitats (Nielsen and others, 2009). Because this small fish is cryptic and occurs deep within the coral matrix, it was not observed during our submersible or ROV dives and was not collected by trawl or trap. Several other fish species that were observed or collected during this study (for example, $N$. exoria, N. sclerorhynchus, I. kyphos, A. woodsi, and $C$. acanthopoma) appear to be rare or are new records for the GOM.

\subsection{Acknowledgments}

See the Acknowledgments section in Chapter 1 of this report. For assistance in identifying fishes, we thank J. Caruso (Tulane University), T. Casazza (University of North Carolina, Wilmington), J. McClain-Counts (USGS), J. Nielsen (University of Copenhagen), M. Rhode (University of North Carolina, Wilmington), and A. Roa-Varon (University of North Carolina, Wilmington).

\subsection{References}

Auster, P.J., 2005, Are deep-water corals important habitats for fishes?, in Freiwald, A., and Roberts, J.M., eds., Cold-water corals and ecosystems: Berlin, Springer-Verlag, p. 747-760.

Brooke, S., and Schroeder, W.W., 2007, State of deep coral ecosystems in the Gulf of Mexico region: Texas to the Florida Straits, in Lumsden, S.E., Hourigan, T.F., Bruckner, A.W., and Dorr, G., eds., The state of deep coral ecosystems of the United States: Silver Spring, Md., National Oceanic and Atmospheric Administration Technical Memorandum CRCP-3, p. 271-306.

Cailliet, G.M., Andrews, A.H., Wakefield, W., Moreno, G., and Rohdes, K.L., 1999, Fish faunal and habitat analyses using trawls, camera sleds and submersibles in benthic deep-sea habitats off central California: Oceanologica Acta, v. 22, p. 579-592.

Caruso, J.H., Ross, S.W., Sulak, K.J., and Sedberry, G.R., 2007, Deep-water chaunacid and lophiid anglerfishes (Pisces: Lophiiformes) off the Southeastern United States: Journal of Fish Biology, v. 70, p. 1015-1026.

Chittaro, P.M., 2004, Fish-habitat associations across multiple spatial scales: Coral Reefs, v. 23, p. 235244.

Connell, S.D., Samoilys, M.A., Lincoln Smith, M.P., and Leqata, J., 1998, Comparisons of abundance of coral-reef fish: catch and effort vs visual census: Australian Journal of Ecology v. 23, p. 579-586.

Costello, M.J., McCrea, M., Freiwald, A., Lundälv, T., Jonsson, L., Brett, B.J., van Weering, T.C.E., de Haas, H., Roberts, J.M., and Allen, D., 2005, Role of cold-water Lophelia pertusa coral reefs as fish habitat in the NE Atlantic, in Freiwald, A., and Roberts, J.M., eds., Cold-water corals and ecosystems: Berlin, Springer-Verlag, p. 771-805. 
Hain, S., and Corcoran, E., eds., 2004, Chapter 3. The status of the cold-water coral reefs of the world, in Wilkinson, C., ed., Status of coral reefs of the world: 2004: Perth, Western Australia, Australian Institute of Marine Science, v. 1, p. 115-135.

Haedrich, R.L., and Merrett, N.R., 1990, Little evidence for faunal zonation or communities in deep sea demersal fish faunas: Progress in Oceanography, v. 24, p. 239-250.

Henry, L-A., Davies, A.J., and Roberts, J.M., 2010, Beta diversity of cold-water coral reef communities off western Scotland: Coral Reefs, v. 29, p. 427-436.

Lubbers, L., Boynton, W.R., and Kemp, W.M., 1990, Variations in structure of estuarine fish communities in relation to abundance of submersed vascular plants: Marine Ecology Progress Series, v. 65 , p. 1-14.

McEachran, J.D., and Fechhelm, J.D., 1998, Fishes of the Gulf of Mexico: Austin, Tex., University of Texas Press, v. 1, 112 p.

McEachran, J.D., and Fechhelm, J.D., 2005, Fishes of the Gulf of Mexico: Austin, Tex., University of Texas Press, v. 2, 1004 p.

Mortensen, P.B., Hovland, M., Brattegard, T., and Farestveit, R., 1995, Deep water bioherms of the scleractinian coral Lophelia pertusa (L.) at $64^{\circ} \mathrm{N}$ on the Norwegian shelf: structure and associated megafauna: Sarsia, v. 80, p. 145-158.

Nielsen, J.G., Ross, S.W., and Cohen, D.M., 2009, Atlantic occurrence of the genus Bellottia (Teleostei, Bythitidae) with two new species from the Western North Atlantic: Zootaxa, v. 2018, p. 45-57.

Powell, S.M., Haedrich, R.L., and McEachran, J.D., 2003, The deep-sea demersal fish fauna of the northern Gulf of Mexico: Journal of Northwestern Atlantic Fisheries Science, v. 31, p. 19-33.

Quattrini, A.M., and Ross, S.W., 2006, Fishes associated with North Carolina shelf-edge hardbottoms and initial assessment of a proposed marine protected area: Bulletin of Marine Science, v. 79, p. 137163.

Reed, J.K., Weaver, D.C., and Pomponi, S.A., 2006, Habitat and fauna of deep-water Lophelia pertusa coral reefs off the southeastern U.S.: Blake Plateau, Straits of Florida, and Gulf of Mexico: Bulletin of Marine Science, v. 78, p. 343-375.

Ross, S.W., and Nizinski, M.S., 2007, State of deep coral ecosystems in the U.S. southeast region: Cape Hatteras to southeastern Florida, in Lumsden, S.E., Hourigan, T.F., Bruckner, A.W., and Dorr, G., eds., The state of deep coral ecosystems of the United States: Silver Spring, Md., National Oceanic and Atmospheric Administration Technical Memorandum CRCP-3, p. 233-270.

Ross, S.W., and Quattrini, A.M., 2007, The fish fauna associated with deep coral banks off the southeastern United States: Deep-Sea Research Part I, v. 54, p. 975-1007.

Ross, S.W., and Quattrini, A.M., 2009, Deep-sea reef fish assemblage patterns on the Blake Plateau (Western North Atlantic Ocean): Marine Ecology, v. 30, p. 74-92.

Shipp, R.L., and Sedberry, G., 2008, First capture of a wreckfish, Polyprion americanus, from the Gulf of Mexico: Gulf of Mexico Science, v. 26, p. 133-135.

Söffker, M., Sloman, K.A., and Hall-Spencer, J.M., 2011, In situ observations of fish associated with coral reefs off Ireland: Deep-Sea Research Part I, v. 58, p. 818-825.

Starr, R.M., Fox, D.S., Hixon, M.A., Tissot, B.N., Johnson, G.E., and Barss, W.H., 1995, Comparison of submersible-survey and hydroacoustic-survey estimates of fish density on a rocky bank: Fishery Bulletin, v. 94, p. 113-123.

Stein, D.L., Tissot, B.N., Hixon, M.A., and Barss, W., 1992, Fish-habitat associations on a deep reef at the edge of the Oregon continental shelf: Fishery Bulletin, v. 90, p. 540-551.

Sulak, K.J., Brooks, R.A., Luke, K.E., Norem, A.D., Randall, M.T., Quaid, A.J., Yeargin, G.E., Miller, J.M., Harden, W.M., Caruso, J.H., and Ross, S.W., 2007, Demersal fishes associated with Lophelia 
pertusa coral and hard-substrate biotopes on the continental slope, northern Gulf of Mexico, in George, R.Y., and Cairns, S.D., eds., Conservation and adaptive management of seamount and deepsea coral ecosystems: University of Miami, p. 65-92.

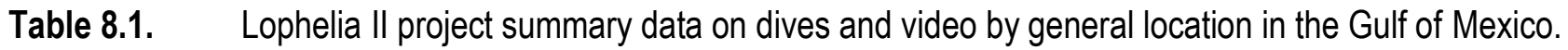

[see fig. 9.1 for locations; h, hour; m, meter; WFS, West Florida Slope; Falcon, Kraken II, and Jason II are remotely operated vehicles; JSL, Johnson Sea Link II submersible; DDW, Dual DeepWorker submersible]

\begin{tabular}{lccccc}
\hline Location & Dates & Vehicle & $\begin{array}{c}\text { Number } \\
\text { of dives }\end{array}$ & $\begin{array}{c}\text { Total video time } \\
(\mathbf{h})\end{array}$ & $\begin{array}{c}\text { Depth range } \\
(\mathbf{m})\end{array}$ \\
\hline VK826 & 7-8, 10-11 Oct 08 & Falcon & 5 & 25.03 & $415-485$ \\
VK862/906 & 9 Oct 08 & Falcon & 1 & 6.23 & $296-330$ \\
WFS & 16-17 Sep 09 & JSL & 3 & 7.93 & $414-536$ \\
VK826 & 22-24 Sep 09 & JSL & 4 & 11.18 & $463-484$ \\
VK862/906 & 18, 20-22 Sep 09 & JSL & 6 & 16.37 & $312-436$ \\
MC751 & 19 Sep 09 & JSL & 2 & 5.65 & $434-458$ \\
VK826 & 21-23 Sep 10 & Kraken II & 3 & 23.9 & $454-526$ \\
VK862 & 24-26 Sep 10 & Kraken II & 3 & 21.53 & $317-435$ \\
WFS & 28 Sep-2 Oct 10 & Kraken II & 5 & 41.6 & $485-584$ \\
VK862/906 & 15-16, 18-20 Oct 10 & DDW & 8 & 10.45 & $309-430$ \\
WFS & 10 Nov 10 & Jason II & 1 & 5.38 & $495-734$ \\
\hline
\end{tabular}


Table 8.2. Fishes documented from video analysis from Johnson Sea Link II (2009) and Dual DeepWorker (2010) submersibles and Kraken II remotely operated vehicle (2010) dives in the Gulf of Mexico by major habitat type during the Lophelia II program.

[PR, prime reef; TR, transition reef; OR, off-reef; X, documented; ROV, remotely operated vehicle]

\begin{tabular}{|c|c|c|c|}
\hline Taxa & PR & TR & OR \\
\hline \multicolumn{4}{|l|}{ Myxinidae } \\
\hline Eptatretus minor & & & $\mathrm{X}$ \\
\hline \multicolumn{4}{|l|}{ Scyliorhinidae } \\
\hline Scyliorhinus retifer & $\mathrm{X}$ & $\mathrm{X}$ & \\
\hline Scyliorhinidae (unidentified) & & & $\mathrm{X}$ \\
\hline \multicolumn{4}{|l|}{ Odontaspididae } \\
\hline Odontaspis ferox & $\mathrm{X}$ & & \\
\hline \multicolumn{4}{|l|}{ Squalidae } \\
\hline Cirrhigaleus asper & $\mathrm{X}$ & $\mathrm{X}$ & \\
\hline Squalidae (unidentified) & & & $\mathrm{X}$ \\
\hline \multicolumn{4}{|l|}{ Rajidae } \\
\hline Fenestraja plutonia/sinusmexicanus & & $\mathrm{X}$ & $\mathrm{X}$ \\
\hline \multicolumn{4}{|l|}{ Synaphobranchidae } \\
\hline Dysommina rugosa & $\mathrm{X}$ & $\mathrm{X}$ & \\
\hline Synaphobranchidae (unidentified) & $\mathrm{X}$ & $\mathrm{X}$ & \\
\hline \multicolumn{4}{|l|}{ Congridae } \\
\hline Conger oceanicus & $\mathrm{X}$ & $\mathrm{X}$ & $\mathrm{X}$ \\
\hline \multicolumn{4}{|l|}{ Nettastomatidae } \\
\hline Nettenchelys exoria & & $\mathrm{X}$ & \\
\hline Argentinidae (unidentified) & $\mathrm{X}$ & $\mathrm{X}$ & $\mathrm{X}$ \\
\hline Gonostomatidae (unidentified) & & & $\mathrm{X}$ \\
\hline \multicolumn{4}{|l|}{ Sternoptychidae } \\
\hline Maurolicus weitzmani & $\mathrm{X}$ & $\mathrm{X}$ & $\mathrm{X}$ \\
\hline Polyipnus sp. & $\mathrm{X}$ & $\mathrm{X}$ & $\mathrm{X}$ \\
\hline \multicolumn{4}{|l|}{ Stomiidae } \\
\hline Chauliodus sloani & & $\mathrm{X}$ & $\mathrm{X}$ \\
\hline \multicolumn{4}{|l|}{ Chlorophthalmidae } \\
\hline Chlorophthalmus agassizi & $\mathrm{X}$ & $\mathrm{X}$ & $\mathrm{X}$ \\
\hline \multicolumn{4}{|l|}{ Paralepididae } \\
\hline Sudis hyalina & $\mathrm{X}$ & & \\
\hline Paralepididae (unidentified) & & & $\mathrm{X}$ \\
\hline \multicolumn{4}{|l|}{ Myctophidae } \\
\hline Ceratoscopelus warmingii & & & $\mathrm{X}$ \\
\hline Myctophidae (unidentified) & $\mathrm{X}$ & & $\mathrm{X}$ \\
\hline \multicolumn{4}{|l|}{ Polymixiidae } \\
\hline Polymixia cf. lowei & $\mathrm{X}$ & $\mathrm{X}$ & $\mathrm{X}$ \\
\hline \multicolumn{4}{|l|}{ Ophidiidae } \\
\hline Benthocometes robustus & $\mathrm{X}$ & & \\
\hline
\end{tabular}


Table 8.2. Fishes documented from video analysis from Johnson Sea Link II (2009) and Dual DeepWorker (2010) submersibles and Kraken II remotely operated vehicle (2010) dives in the Gulf of Mexico by major habitat type during the Lophelia II program - continued.

[PR, prime reef; TR, transition reef; OR, off-reef; X, documented; ROV, remotely operated vehicle]

\begin{tabular}{lccc}
\hline Taxa & PR & TR & OR \\
\hline Bythitidae (unidentified) & X & X & \\
\hline Macrouridae & & & \\
$\quad$ Coelorinchus sp. & & X & X \\
$\quad$ Malacocephalus cf. occidentalis & & & X \\
$\quad$ Nezumia aequalis & $\mathrm{X}$ & $\mathrm{X}$ & $\mathrm{X}$ \\
Nezumia sclerorhynchus & $\mathrm{X}$ & $\mathrm{X}$ & $\mathrm{X}$ \\
Nezumia sp. & $\mathrm{X}$ & $\mathrm{X}$ & $\mathrm{X}$ \\
Macrouridae (unidentified) & $\mathrm{X}$ & $\mathrm{X}$ & $\mathrm{X}$ \\
\hline Moridae & & & \\
Laemonema barbatulum & $\mathrm{X}$ & $\mathrm{X}$ & \\
Laemonema goodebeanorum & $\mathrm{X}$ & $\mathrm{X}$ & $\mathrm{X}$ \\
Laemonema sp. & $\mathrm{X}$ & & \\
Physiculus fulvus/karrerae & $\mathrm{X}$ & $\mathrm{X}$ & \\
Moridae (unidentified) & $\mathrm{X}$ & $\mathrm{X}$ & $\mathrm{X}$ \\
\hline Phycidae & & & \\
Urophycis cf. cirrata & $\mathrm{X}$ & $\mathrm{X}$ & $\mathrm{X}$ \\
Urophycis earlii & $\mathrm{X}$ & & $\mathrm{X}$ \\
Urophycis sp. & & $\mathrm{X}$ & $\mathrm{X}$ \\
\hline
\end{tabular}

\section{Merlucciidae}

Merluccius albidus

\begin{tabular}{lcc}
\hline Gadiiformes (unidentified) & $\mathrm{X}$ \\
\hline Lophiidae & & \\
Lophiodes beroe & $\mathrm{X}$ & $\mathrm{X}$ \\
Lophiodes monodi & $\mathrm{X}$ & \\
\hline
\end{tabular}

\section{Chaunacidae}

Chaunax pictus

$\mathrm{X}$

Chaunax suttkusi $\mathrm{X}$

\section{Ogcocephalidae}

Dibranchus cf. atlanticus

X

\section{Trachichthyidae}

Gephroberyx darwini

Hoplostethus occidentalis

$\begin{array}{lll}X & & \\ X & X & X\end{array}$

\section{Berycidae}

Beryx decadactylus

$\mathrm{X}$

Beryx splendens

$\mathrm{X} \quad \mathrm{X}$

\section{Parazenidae}

Cyttopsis rosea

X X 
Table 8.2. Fishes documented from video analysis from Johnson Sea Link II (2009) and Dual DeepWorker (2010) submersibles and Kraken II remotely operated vehicle (2010) dives in the Gulf of Mexico by major habitat type during the Lophelia II program - continued.

[PR, prime reef; TR, transition reef; OR, off-reef; X, documented; ROV, remotely operated vehicle]

\begin{tabular}{|c|c|c|c|}
\hline Taxa & PR & TR & OR \\
\hline \multicolumn{4}{|l|}{ Grammicolepididae } \\
\hline Grammicolepis brachiusculus & $\mathrm{X}$ & $\mathrm{X}$ & \\
\hline \multicolumn{4}{|l|}{ Scorpaenidae } \\
\hline Helicolenus dactylopterus & $\mathrm{X}$ & $\mathrm{X}$ & $\mathrm{X}$ \\
\hline Idiastion kyphos & $\mathrm{X}$ & $\mathrm{X}$ & \\
\hline Pontinus rathbuni & $\mathrm{X}$ & $\mathrm{X}$ & $\mathrm{X}$ \\
\hline Setarches guentheri & & & $\mathrm{X}$ \\
\hline Trachyscorpia cristulata & $\mathrm{X}$ & $\mathrm{X}$ & $\mathrm{X}$ \\
\hline Scorpaenidae (unidentified) & $\mathrm{X}$ & $\mathrm{X}$ & $\mathrm{X}$ \\
\hline \multicolumn{4}{|l|}{ Acropomatidae } \\
\hline Synagrops sp. A & & & $\mathrm{X}$ \\
\hline Synagrops sp. & & $\mathrm{X}$ & $\mathrm{X}$ \\
\hline \multicolumn{4}{|l|}{ Serranidae } \\
\hline Anthiinae (unidentified) & $\mathrm{X}$ & & \\
\hline Anthias woodsi & $\mathrm{X}$ & $\mathrm{X}$ & \\
\hline Epinephelus niveatus & $\mathrm{X}$ & $\mathrm{X}$ & \\
\hline \multicolumn{4}{|l|}{ Epigonidae } \\
\hline Epigonus oligolepis & $\mathrm{X}$ & $\mathrm{X}$ & $\mathrm{X}$ \\
\hline \multicolumn{4}{|l|}{ Carangidae } \\
\hline Decapterus sp. & & $\mathrm{X}$ & \\
\hline \multicolumn{4}{|l|}{ Percophidae } \\
\hline Bembrops sp. & & & $\mathrm{X}$ \\
\hline \multicolumn{4}{|l|}{ Draconettidae } \\
\hline Centrodraco acanthopoma & $\mathrm{X}$ & & \\
\hline \multicolumn{4}{|l|}{ Trichiuridae } \\
\hline Benthodesmus tenuis & & $\mathrm{X}$ & $\mathrm{X}$ \\
\hline Trichiuridae (unidentified) & & $\mathrm{X}$ & \\
\hline \multicolumn{4}{|l|}{ Xiphiidae } \\
\hline Xiphias gladius & $\mathrm{X}$ & & \\
\hline \multicolumn{4}{|l|}{ Centrolophidae } \\
\hline Hyperoglyphe perciformis & $\mathrm{X}$ & $\mathrm{X}$ & \\
\hline \multicolumn{4}{|l|}{ Cynoglossidae } \\
\hline Symphurus marginatus & & $\mathrm{X}$ & $\mathrm{X}$ \\
\hline \multicolumn{4}{|l|}{ Triacanthodidae } \\
\hline Hollardia sp. & $\mathrm{X}$ & & \\
\hline Midwater fish (unidentified) & $\mathrm{X}$ & $\mathrm{X}$ & $\mathrm{X}$ \\
\hline Unidentified fishes & $\mathrm{X}$ & $\mathrm{X}$ & $\mathrm{X}$ \\
\hline
\end{tabular}


Table 8.3. Fishes caught by bottom otter trawl (OT) and traps during three cruises in the Gulf of Mexico as part of the Lophelia II program.

[NF, National Oceanic and Atmospheric Adminstration ship Nancy Foster; OT, otter trawl; SJ, research vessel Seward Johnson; CH, research vessel Cape Hatteras; m, meter; Numbers of specimens caught are followed by length or range of lengths, in millimeters (mm), of specimens in parentheses. The $\mathrm{CH} 2010 \mathrm{OT}$ column also includes a few species caught by a Tucker trawl that touched bottom]

\begin{tabular}{|c|c|c|c|c|c|}
\hline Taxa & $\begin{array}{c}\text { NF } 2008 \text { OT } \\
n=17 \\
(335-744 \mathrm{~m})\end{array}$ & $\begin{array}{c}\text { NF } 2008 \text { Trap } \\
n=1 \\
(335 \mathrm{~m})\end{array}$ & $\begin{array}{c}\text { SJ } 2009 \text { Trap } \\
n=2 \\
(305-325 \mathrm{~m})\end{array}$ & $\begin{array}{c}\text { CH } 2010 \text { OT } \\
n=6 \\
(471-575 \mathrm{~m})\end{array}$ & $\begin{array}{c}\text { CH } 2010 \text { Trap } \\
n=1 \\
(351-358 \mathrm{~m})\end{array}$ \\
\hline \multicolumn{6}{|l|}{ Scyliorhinidae } \\
\hline Galeus arae & & & & $1(305)$ & \\
\hline \multicolumn{6}{|l|}{ Etmopteridae } \\
\hline Etmopterus bigelowi & $1(157)$ & & & & \\
\hline Etmopterus schultzi & $3(202-295)$ & & & & \\
\hline Etmopterus virens & $2(187-194)$ & & & & \\
\hline \multicolumn{6}{|l|}{ Rajidae } \\
\hline $\begin{array}{c}\text { Fenestraja } \\
\text { sinusmexicanus }\end{array}$ & $5(122-267)$ & & & & \\
\hline \multicolumn{6}{|l|}{ Notacanthidae } \\
\hline Notacanthus chemnitzii & $1(109)$ & & & $1(155)$ & \\
\hline \multicolumn{6}{|l|}{ Synaphobranchidae } \\
\hline Dysommina rugosa & & & & $1(205)$ & \\
\hline \multicolumn{6}{|l|}{ Ophichthidae } \\
\hline Ophichthus cruentifer & & & & $1(412)$ & \\
\hline Pseudomyrophis nimius & $1(251)$ & & & & \\
\hline \multicolumn{6}{|l|}{ Congridae } \\
\hline Bathycongrus dubius & $7(200-357)$ & & & & \\
\hline Conger oceanicus & & & $4(725-758)$ & & $4(610-1018)$ \\
\hline $\begin{array}{l}\text { Pseudophichthys } \\
\text { splendens }\end{array}$ & $1(280)$ & & & & \\
\hline \multicolumn{6}{|l|}{ Nettastomatidae } \\
\hline Facciolella sp. & $1(460)$ & & & & \\
\hline \multicolumn{6}{|l|}{ Gonostomatidae } \\
\hline Gonostoma elongatum & $1(195)$ & & & $5(49-130)$ & \\
\hline \multicolumn{6}{|l|}{ Sternoptychidae } \\
\hline Argyropelecus aculeatus & & & & $1(17)$ & \\
\hline Polyipnus clarus & & & & $1(39)$ & \\
\hline Polyipnus sp. & $1(39)$ & & & & \\
\hline Sternoptyx diaphana & $1(22)$ & & & & \\
\hline \multicolumn{6}{|l|}{ Phosichthyidae } \\
\hline Polymetme corythaeola & $2(50-75)$ & & & & \\
\hline Yarella blackfordi & $8(108-187)$ & & & & \\
\hline
\end{tabular}


Table 8.3. Fishes caught by bottom otter trawl (OT) and traps during three cruises in the Gulf of Mexico as part of the Lophelia II program - continued.

[NF, National Oceanic and Atmospheric Adminstration ship Nancy Foster; OT, otter trawl; SJ, research vessel Seward Johnson; CH, research vessel Cape Hatteras; m, meter; Numbers of specimens caught are followed by length or range of lengths, in millimeters (mm), of specimens in parentheses. The $\mathrm{CH} 2010$ OT column also includes a few species caught by a Tucker trawl that touched bottom]

\begin{tabular}{|c|c|c|c|c|c|}
\hline Taxa & $\begin{array}{c}\text { NF } 2008 \text { OT } \\
n=17 \\
(335-744 \mathrm{~m})\end{array}$ & $\begin{array}{c}\text { NF } 2008 \text { Trap } \\
n=1 \\
(335 \mathrm{~m})\end{array}$ & $\begin{array}{c}\text { SJ } 2009 \text { Trap } \\
n=2 \\
(305-325 \mathrm{~m})\end{array}$ & $\begin{array}{c}\mathrm{CH} 2010 \text { OT } \\
n=6 \\
(471-575 \mathrm{~m})\end{array}$ & $\begin{array}{c}\text { CH } 2010 \text { Trap } \\
n=1 \\
(351-358 \mathrm{~m})\end{array}$ \\
\hline \multicolumn{6}{|l|}{ Stomiidae } \\
\hline Bathophilus pawneei & $1(80)$ & & & & \\
\hline Chauliodus sloani & $2(75-127)$ & & & $2(39-152)$ & \\
\hline Photostomias guernei & & & & $1(105)$ & \\
\hline \multicolumn{6}{|l|}{ Chlorophthalmidae } \\
\hline Chlorophthalmus agassizi & $1(120)$ & & & & \\
\hline Parasudis truculenta & $1(144)$ & & & & \\
\hline \multicolumn{6}{|l|}{ Myctophidae } \\
\hline Benthosema suborbitale & & & & $1(29)$ & \\
\hline Bolinichthys sp. & & & & $1(41)$ & \\
\hline Bolinichthys supralateralis & & & & $1(40)$ & \\
\hline Ceratoscopelus warmingii & & & & $1(47)$ & \\
\hline Diaphus dumerilii & $1(32)$ & & & $2(23)$ & \\
\hline Diaphus rafinesquii & $1(59)$ & & & & \\
\hline Hygophum higomii & $1(54)$ & & & & \\
\hline Hygophum taaningi & & & & $1(37)$ & \\
\hline Lampanyctus alatus & $1(42)$ & & & $1(46)$ & \\
\hline Lampanyctus sp. & & & & $1(42)$ & \\
\hline Lepidophanes guentheri & & & & $3(38-55)$ & \\
\hline \multicolumn{6}{|l|}{ Bythitidae } \\
\hline Cataetyx laticeps & $1(197)$ & & & & \\
\hline $\begin{array}{l}\text { Diplacanthopoma } \\
\text { brachysoma }\end{array}$ & $5(54-210)$ & & & & \\
\hline \multicolumn{6}{|l|}{ Aphyonidae } \\
\hline Barathronus bicolor & $3(105-140)$ & & & & \\
\hline \multicolumn{6}{|l|}{ Macrouridae } \\
\hline Bathygadus macrops & $24(120-406)$ & & & & \\
\hline Coelorinchus caribbaeus & $72(167-309)$ & & & & \\
\hline Coelorinchus caelorhincus & $27(130-347)$ & & & & \\
\hline Coryphaenoides zaniophorus & $6(118-352)$ & & & & \\
\hline Hymenocephalus billsam & & & & 1 & \\
\hline Hymenocephalus italicus & $4(127)$ & & & & \\
\hline Malacocephalus occidentalis & $32(135-316)$ & & & & \\
\hline Nezumia aequalis & $74(166-277)$ & & & $4(262)$ & \\
\hline Nezumia atlantica & $2(243-245)$ & & & & \\
\hline Nezumia longebarbata & & & & $1(122)$ & \\
\hline
\end{tabular}


Table 8.3. Fishes caught by bottom otter trawl (OT) and traps during three cruises in the Gulf of Mexico as part of the Lophelia II program - continued.

[NF, National Oceanic and Atmospheric Adminstration ship Nancy Foster; OT, otter trawl; SJ, research vessel Seward Johnson; CH, research vessel Cape Hatteras; m, meter; Numbers of specimens caught are followed by length or range of lengths, in millimeters $(\mathrm{mm})$, of specimens in parentheses. The $\mathrm{CH} 2010$ OT column also includes a few species caught by a Tucker trawl that touched bottom]

\begin{tabular}{|c|c|c|c|c|c|}
\hline Taxa & $\begin{array}{c}\text { NF } 2008 \text { OT } \\
n=17 \\
(335-744 \mathrm{~m})\end{array}$ & $\begin{array}{c}\text { NF } 2008 \text { Trap } \\
n=1 \\
(335 \mathrm{~m})\end{array}$ & $\begin{array}{c}\text { SJ } 2009 \text { Trap } \\
n=2 \\
(305-325 \mathrm{~m})\end{array}$ & $\begin{array}{c}\text { CH } 2010 \text { OT } \\
n=6 \\
(471-575 \mathrm{~m})\end{array}$ & $\begin{array}{c}\text { CH } 2010 \text { Trap } \\
n=1 \\
(351-358 \mathrm{~m})\end{array}$ \\
\hline \multicolumn{6}{|l|}{ Macrouridae } \\
\hline Nezumia sp. & & & & $5(90)$ & \\
\hline Macrouridae & & & & 5 & \\
\hline Macrourinae & $3(80)$ & & & & \\
\hline \multicolumn{6}{|l|}{ Steindachneriidae } \\
\hline Steindachneria argentea & $4(235-267)$ & & & & \\
\hline \multicolumn{6}{|l|}{ Moridae } \\
\hline Gadella imberbis & $2(63-150)$ & & & $2(155)$ & \\
\hline Laemonema barbatulum & & & & $8(81-245)$ & \\
\hline $\begin{array}{c}\text { Laemonema } \\
\text { goodebeanorum }\end{array}$ & $127(80-285)$ & & & $\begin{array}{l}30(77- \\
249)\end{array}$ & \\
\hline Laemonema sp. & & & & 1 & \\
\hline Physiculus fluvus & & & & $1(140)$ & \\
\hline \multicolumn{6}{|l|}{ Phycidae } \\
\hline Urophycis cirrata & $12(265-445)$ & & $1(425)$ & $1(334)$ & $1(328)$ \\
\hline Urophycis floridana & & $3(340-370)$ & $1(310)$ & & $3(332-373)$ \\
\hline \multicolumn{6}{|l|}{ Merlucciidae } \\
\hline Merluccius albidus & $2(240-380)$ & & & & \\
\hline \multicolumn{6}{|l|}{ Lophiidae } \\
\hline Lophius gastrophysus & $1(440)$ & & & & \\
\hline \multicolumn{6}{|l|}{ Chaunacidae } \\
\hline Chaunax suttkusi & $23(40-160)$ & & & $\begin{array}{c}2(142- \\
147)\end{array}$ & \\
\hline \multicolumn{6}{|l|}{ Ogcocephalidae } \\
\hline Dibranchus atlanticus & $112(22-173)$ & & & $\begin{array}{c}30(32- \\
102) \\
\end{array}$ & \\
\hline \multicolumn{6}{|l|}{ Trachichthyidae } \\
\hline Hoplostethus occidentalis & $6(75-145)$ & & & & \\
\hline \multicolumn{6}{|l|}{ Scorpaenidae } \\
\hline Helicolenus dactylopterus & $2(225-340)$ & & $10(226-300)$ & $\begin{array}{c}3(188- \\
272)\end{array}$ & \\
\hline Pontinus longispinis & $2(145-160)$ & & & & \\
\hline Pontinus rathbuni & & & $4(181-200)$ & & \\
\hline \multicolumn{6}{|l|}{ Peristediidae } \\
\hline Peristedion greyae & $19(119-203)$ & & & & \\
\hline \multicolumn{6}{|l|}{ Epigonidae } \\
\hline Epigonus pandionis & $10(94-175)$ & & & & \\
\hline
\end{tabular}


Table 8.3. Fishes caught by bottom otter trawl (OT) and traps during three cruises in the Gulf of Mexico as part of the Lophelia II program - continued.

[NF, National Oceanic and Atmospheric Adminstration ship Nancy Foster; OT, otter trawl; SJ, research vessel Seward Johnson; CH, research vessel Cape Hatteras; m, meter; Numbers of specimens caught are followed by length or range of lengths, in millimeters $(\mathrm{mm})$, of specimens in parentheses. The CH 2010 OT column also includes a few species caught by a Tucker trawl that touched bottom]

\begin{tabular}{|c|c|c|c|c|c|}
\hline Taxa & $\begin{array}{c}\text { NF } 2008 \text { OT } \\
n=17 \\
(335-744 \mathrm{~m})\end{array}$ & $\begin{array}{c}\text { NF } 2008 \text { Trap } \\
n=1 \\
(335 \mathrm{~m})\end{array}$ & $\begin{array}{c}\text { SJ } 2009 \text { Trap } \\
n=2 \\
(305-325 \mathrm{~m})\end{array}$ & $\begin{array}{c}\text { CH } 2010 \text { OT } \\
n=6 \\
(471-575 \mathrm{~m})\end{array}$ & $\begin{array}{c}\text { CH } 2010 \text { Trap } \\
n=1 \\
(351-358 \mathrm{~m})\end{array}$ \\
\hline \multicolumn{6}{|l|}{ Percophidae } \\
\hline Bembrops anatirostris & $6(160-256)$ & & & & \\
\hline Bembrops gobioides & $46(95-230)$ & & & & \\
\hline \multicolumn{6}{|l|}{ Draconettidae } \\
\hline $\begin{array}{c}\text { Centrodraco } \\
\text { acanthopoma }\end{array}$ & & & & $2(55-62)$ & \\
\hline \multicolumn{6}{|l|}{ Trichiuridae } \\
\hline Benthodesmus tenuis & $1(440)$ & & & & \\
\hline \multicolumn{6}{|l|}{ Paralichthyidae } \\
\hline $\begin{array}{l}\text { Paralichthys } \\
\text { squamilentus }\end{array}$ & $1(400)$ & & & & \\
\hline \multicolumn{6}{|l|}{ Poecilopsettidae } \\
\hline Poecilopsetta beanii & $18(48-120)$ & & & & \\
\hline \multicolumn{6}{|l|}{ Cynoglossidae } \\
\hline Symphurus marginatus & $38(62-134)$ & & & $3(62-105)$ & \\
\hline Total & 729 & 3 & 20 & 125 & 8 \\
\hline
\end{tabular}




\title{
9 DISTRIBUTION, ABUNDANCE, AND SPECIES COMPOSITION OF THE MEGAFAUNAL INVERTEBRATE ASSEMBLAGES ASSOCIATED WITH DEEP. SEA CORAL HABITATS IN THE GULF OF MEXICO
}

\author{
By Martha S. Nizinski, ${ }^{1}$ and Cheryl L. Ames ${ }^{2}$
}

\footnotetext{
${ }^{1}$ National Marine Fisheries Service National Systematics Laboratory, Smithsonian Institution, Washington, DC

${ }^{2}$ University of North Carolina Wilmington, Center for Marine Science, Wilmington, NC
}

\subsection{Introduction}

Exploration and investigation of deep-sea coral habitats in the Gulf of Mexico (GOM) have recently become the focus of much interest. These deep reefs, composed mostly of Lophelia pertusa, are far more extensive than previously thought (Brooke and Schroeder, 2007). Structure-forming deep-sea corals provide a variety of microhabitats (Buhl-Mortensen and Mortensen, 2005; Mortensen and Fosså, 2006; Buhl-Mortensen and others, 2010) and therefore support a diverse faunal assemblage composed of recreationally, commercially, and ecologically important species. These habitats are hotspots of biodiversity (Rogers, 1999; Roberts and others, 2006; Roberts and others, 2008) with reported estimates of species diversity similar to or higher than those reported for shallow-water reefs (Jensen and Frederiksen, 1992; Rogers, 1999). Several studies have examined the composition of faunal assemblages associated with deep-sea coral habitats particularly in the eastern North Atlantic (Jensen and Fredriksen, 1992; Rogers, 1999; Mortensen and others, 1995; Jonsson and others, 2004; Ross and Quattrini, 2007; Henry and Roberts, 2007; Cordes and others, 2008; Roberts and others, 2008). Although our knowledge about species diversity and habitat associations of invertebrates associated with these deep-water reef environments continues to increase, the systematics, ecology, and species distributions of even the most conspicuous faunal groups remain largely unknown.

Several recent multi-disciplinary expeditions were conducted in the GOM, with the overall objectives to discover and explore deep-sea coral reefs in north-central and eastern GOM, to discover and quantify the extent of $L$. pertusa in these regions, to sample the fauna occurring in habitats adjacent to coral reefs, and to describe the faunal assemblage(s) associated with these deep-sea coral habitats. Specific objectives of the present study include (1) making accurate taxonomic identifications of associated megafaunal invertebrate assemblages observed on and around deep-sea coral habitats; (2) assessing levels of endemism at deep coral habitats; (3) examining patterns of species diversity and geographic distribution of the mega-invertebrate fauna between sites; (4) comparing patterns in megafaunal diversity and distribution observed in this study with those of megafauna from similar habitats in other locations; (5) assessing community structure, basic ecology, and population dynamics of the invertebrate fauna; and (6) examining phylogenetic and phylogeographic relationships of these species assemblages.

\subsection{Methods}

Samples were collected at 138 of 297 stations located at five deep-sea coral study sites (fig. 9.1) on the continental slope in the north-central to eastern GOM (MC751, VK826, VK862, VK906) and on the West Florida Slope (WFS). Samples were collected during three research cruises conducted over the 
course of 3 years (2008-2010). These included cruises on the National Oceanic and Atmospheric Administration (NOAA) ship Nancy Foster (October 2008), research vessel (R/V) Seward Johnson (Harbor Branch Oceanographic Institution; September 2009), and R/V Cape Hatteras (Duke-UNC Oceanographic Consortium; September-October 2010). One collection from the WFS, taken during the 2010 cruise on NOAA ship Ron Brown, was also included in this study. See Chapter 1 for a more detailed description of cruises and study sites.

A variety of sampling gear was used to collect material during this study. Abbreviated descriptions and protocols for each gear type are listed here.

\section{Bottom trawl}

The objective of employing this gear was to sample the bottom near, but not on, known deep-sea coral communities as well as sampling on soft substrata away from reef habitats. The otter trawl (4.9meter $[\mathrm{m}]$ head rope, 38.1-millimeter [mm] mesh) was deployed from the stern and towed for about 30 minutes $(\mathrm{min})$ at about 2-knot $(\mathrm{kn} ; 3.7$ kilometers per hour $[\mathrm{km} / \mathrm{h}])$ ground speed.

2. Tucker trawl

Discrete depth trawling was emphasized in the depth range of near surface to about $500 \mathrm{~m}$, in approximately 50-m bins. A 2x2-m (1.59-mm mesh) Tucker trawl (TT) was lowered off the stern to the target sampling depth in the open position, towed for $30 \mathrm{~min}$ at about 2-kn ground speed, usually against the current. At the end of the tow, a messenger was sent down the wire to trigger the net closed, and then the net was retrieved. A plankton net $(0.5$-m-diameter, 335-micrometer $[\mu \mathrm{m}] \mathrm{mesh})$ was sometimes suspended in the center mouth opening of the TT. Thus, two separate samples were obtained per deployment. A temperature-depth recorder (TDR, Sea-Bird SBE39) was attached to the net frame to record time, depth, and temperature about every 5 seconds (s) during each trawl deployment. Actual fishing depth was determined after each trawl from the TDR data, and this was used to adjust fishing methods to achieve desired sampling depths.

\section{MOCNESS trawl}

Discrete depth trawling was emphasized in the depth range of near surface to about $300 \mathrm{~m}$, in approximately 50-m bins. A Multiple opening/closing net and environmental sensing system (MOCNESS) (335- $\mu \mathrm{m}$ mesh), rigged with two nets, was deployed off the starboard side for night sampling. When the nets reached the target depth, the first net was opened and then towed for $30 \mathrm{~min}$ against the current at about 2-kn ground speed. After $30 \mathrm{~min}$, the first net was triggered to close and the second net to open. The second net was also towed for $30 \mathrm{~min}$. After triggering the second net to close, the MOCNESS was retrieved. This sampling method was repeated as many times as possible during a watch. A TDR (Sea-Bird) was attached to the net frame to record time, depth, and temperature during each trawl deployment.

4. Plankton nets

A 1-m-diameter plankton net $(505-\mu \mathrm{m}$ mesh) was towed for $30 \mathrm{~min}$ on the surface.

5. $\operatorname{ROV}($ Kraken II)

Each remotely operated vehicle (ROV) dive was scheduled for about 10 hours, 0800-1800 (deck to deck). Each ROV dive followed a similar pattern, emphasizing bottom transecting, collecting, and photographing specimens or habitats on or near the bottom. The ROV descended through the water column quickly and settled on the bottom. During descent, observations were made of distributions/behaviors of midwater fauna. Using a Trackpoint II system, position fixes were noted when the ROV landed on bottom, at each collection, at noteworthy observations, and when the ROV left bottom. Specimen collecting began soon after landing on the bottom, unless target animal abundance 
was low. Amount of time spent in this activity and amount of time spent moving the ROV to optimize collections varied by dive and current conditions. Specimen collecting occurred throughout most dives as target fauna were encountered to meet goals of trophic, genetic, microbiological, taxonomic, biological, and other objectives. Samples were placed in the biobox (12x36x10 inches, insulated polypropylene, divided into three chambers) or were often suctioned into one of eight suction sample buckets. Still photography (digital camera, Insite Pacific Scorpio) and video photography were conducted throughout each dive. Specimens were photographed on deck before they were preserved.

\section{Submersible (Johnson Sea Link II)}

Submersible dives followed a similar pattern to that of the ROV, emphasizing bottom video transecting, collecting, and photographing specimens on or near the bottom. Two dives of approximately 3-h duration were planned per day. Generally, the Johnson Sea Link II (JSL) descended through the water column quickly. The JSL was tracked from the surface support ship using a Trackpoint II system. A position fix was requested as soon as the sub was on bottom. Additional position fixes were requested and logged throughout the dive, especially where collections were made or transects started and stopped to aid in the georeferencing of collections. Specimen collecting (using submersible suction device into sampling canisters) usually began soon after landing on the bottom, unless target animal abundance was low. Amount of time spent in this activity and amount of time moving the sub to optimize collections depended on the scientists on board. Specimens were collected with either a suction tube or manipulator arm and housed in a variety of containers including a 12bucket rosette, front collecting basket, and other sampling buckets attached to the sub by the science crew. Still photography (digital camera) and video photography were conducted throughout the dive. Specimens were photographed on deck before they were preserved.

The majority of invertebrates were fixed and maintained in 75-percent ethanol. Cephalopods were fixed in a 10-percent formalin seawater solution and were transferred to 50-percent isopropanol after the cruise. Jellyfishes also were fixed in a 10-percent formalin seawater solution and were maintained in formalin for long-term storage. In the laboratory, all collections were sorted and curated. Individuals were identified to the lowest possible taxon by using historical literature, authoritative keys, and comparative material housed at the National Museum of Natural History, Smithsonian Institution, Washington, D.C. Some specimens were identified by taxonomic experts, or for others, their preliminary identifications were confirmed by taxonomic experts.

\subsection{Results}

Overall, 3,671 individuals, representing at least 9 phyla, 25 classes, 108 families, and 267 species of juvenile and adult invertebrates were collected at 138 of the 297 stations sampled (fig. 9.2; table 9.1). These specimens were taken in 12 surface, 55 midwater, and 71 bottom collections (fig. 9.3). The majority of species and specimens were taken on bottom, by submersible, by ROV, or by otter trawl. Among major taxa, only four (Cnidaria, Mollusca, Crustacea, Tunicata) had species that were collected in all three zones sampled.

A species accumulation curve (fig. 9.4), constructed for all taxa collected throughout the duration of this project, was calculated. Of interest is that this curve appears to be approaching an asymptote. However, this curve represents a conservative estimate of diversity as many specimens are still in the process of being identified. Thus, based on the samples collected during this study, overall (total) species diversity represented in the areas sampled has likely not yet been determined.

\section{$\underline{\text { Crustacea }}$}


The subphylum Crustacea (Phylum Arthropoda) was the dominant major invertebrate taxon in numbers of species and numbers of individuals. A total of 133 crustacean species and 2,167 individuals were represented in the samples (fig. 9.2). Crustaceans constituted 50 percent of the overall mega-faunal diversity and approximately 60 percent of overall abundance (fig. 9.5). Within the crustaceans, 91 percent of the abundance and 86 percent of the diversity was represented by the class Decapoda (114 species; $\mathrm{n}=1976$ individuals). Specimens belonging to six other crustacean taxa (Amphipoda, Cirripedia, Euphausiacea, Isopoda, Mysida, Ostracoda) were also collected (19 species and 191 individuals). A species accumulation curve illustrating overall diversity for only the Decapoda (fig. 9.6) appears to be approaching an asymptote, although the asymptote has not been reached for these species. Similar to the trend observed for total species diversity, total crustacean diversity in the areas sampled has not yet been determined. This curve represents a conservative estimate given that some more specimens need to be identified, and new species continue to be discovered in these collections.

Shrimps were the most speciose crustacean group (table 9.1), with caridean and dendrobranchian shrimps constituting about half (67/133 species) of the total crustacean diversity and 59 percent of the total decapod diversity. Carideans were slightly more diverse than dendrobranchians ( 37 versus 30 species, respectively). However, carideans were less abundant than the dendrobranchians (363 versus 491 individuals, respectively). One caridean species new to science was discovered and recently described from material collected during this project (Anker and Nizinski, 2011). Alpheus lentiginosus (Anker and Nizinski, 2011) is the deepest occurring alpheid shrimp in the GOM, and likely holds the deepest record for the family Alpheidae in the western Atlantic. The holotype, collected at MC751, was observed in close proximity to reef habitat, under the branches of L. pertusa. An additional individual was collected during the USGS-sponsored Lophelia I cruise in 2004 at VK862/906.

Anomurans were the next most speciose taxon with at least 20 species represented in collections. Anomurans were also the third most abundant taxon taken in the samples, following the dendrobrachian shrimps and brachyuran crabs in total number of individuals. Within anomurans, squat lobsters constituted the majority of individuals collected. At least 17 species of squat lobsters, from 4 families, represent the galatheoid assemblage taken during the collections. Families Munididae and Munidopsidae were most speciose, with at least six species from each family represented in the collections. Additionally, three species new to science were discovered. Species descriptions and diagnoses are currently in progress. Among the squat lobsters collected, Leiogalathea agassizii and Munida sanctipauli represent new records for the GOM.

Brachyurans (true crabs) were the second most abundant crustacean taxon ( $\mathrm{N}=468$ individuals), constituting 24 percent of the total decapods collected. High overall abundance among the samples was due primarily to large collections of Bathyplax typhla. This crab represented more than 50 percent of the brachyuran crabs collected. Brachyuran diversity was also high, with at least 19 species represented in the collections (fig. 9.5).

\section{Cnidaria}

Cnidaria was the second most diverse phylum collected (fig. 9.7; table 9.1). Samples of these organisms consisted of 52 species and 652 individuals, representing 3 of the 5 major classes of this phylum (only Cubozoa and Staurozoa were lacking). Anthozoa was the most abundant and diverse class of cnidarians (19 families, 41 species), constituting 79 percent of cnidarian diversity and 80 percent of the total cnidarian abundance. Of these, anemones (Order Actiniaria) were numerically dominant $(\mathrm{n}=$ 258 individuals). A single specimen of a tube-dwelling anemone (Order Cerianthiaria) was also collected. This specimen is the first cerianthid reported from deep waters of the GOM, and likely is an undescribed species. 
Actiniarians are poorly known from GOM deep water, therefore, information collected during this project adds significantly to the basic knowledge of these species. Multiple Halcurias pilatus specimens were collected at a depth range of 316-512 m, expanding the bathymetric range beyond the original estimate (342 m) for this species in the north-central GOM. Adamsia obvolva, an obligate symbiont of the hermit crab Parapagurus pictus, is the only actiniarian endemic to the GOM. Because of its obligate symbiotic relationship with its hermit crab host, its distribution depends on that of its host. Similar to other deepwater anemones, Actinoscyphia cf. saginata (Venus Flytrap Anemone) occurs over a broad depth range (310-744 m). Collections of this anemone were made at much shallower (400 $\mathrm{m}$ ) depths than those previously reported for this species (751-2,330 m; Ammons and Daly, 2008). The genus Actinoscyphia is diagnosed from other genera, in part, by using the basal disc to anchor to large glass sponge spicules. However, specimens of Actinoscyphia collected in this study were not attached to spicules, but were attached onto a variety of other long, narrow, cylindrical, biotic and abiotic objects. Given the abundance of $A$. cf. saginata specimens collected during this study, further taxonomic and systematic work is needed to determine if the venus flytrap anemones of the GOM represent a single species or a species complex.

Another anemone commonly observed in the GOM, Actinauge longicornis ("white sock" anemone) was observed on soft substrata. This species forms mudballs to anchor its pedal disc into the substratum, thus giving it the advantage over other epilithic species to colonize deep-sea sediments. Additionally, A. longicornis was also observed attached to various long cylindrical objects, similar to that observed for species of Actinoscyphia.

Subclass Octocorallia was the most diverse Anthozoan taxon, with at least 15 species represented in collections $(\mathrm{n}=97$ individuals). At least one rare octocoral specimen, Chelidonisis aurantiaca mexicana (Bayer and Stefani, 1987), collected at MC751, is among the identified material collected during this study. This species, described from a single individual nearly 25 years ago, has not been observed again until during this study. Antipatharians (black corals) and scleractinians (hard or reef-building corals) were equally abundant (73 and 71 individuals collected, respectively) and diverse (at least 6 species represented in each group). Based on collaborative work with D. Opresko (Smithsonian Institution), a taxonomic authority on this group, antipatharians are more speciose than had previously been estimated. Material previously identified as Leiopathes glaberrima represents a species complex, with at least four possible new species identified. Other cnidarians collected in this study include 10 species of Hydrozoa, including sytlasterids (lace corals), hydroids, and a single species of Schyphozoa (Pelagia noctiluca).

\section{Other Invertebrates}

For other groups of invertebrates, Phyla Mollusca (31 species and 412 individuals) and Echinodermata (34 species and 355 individuals) were also well represented in collections. Gastropods were the most diverse class of molluscs (16 species), followed by cephalopods (12 species). Gastropods were more abundant ( $\mathrm{n}=295$ individuals) in the samples than were cephalopods. Gastropod abundances were highly influenced by two species, Scaphander $\mathrm{sp} .(\mathrm{n}=110$ individuals $)$ and Gemmula periscelida ( $\mathrm{n}=64$ individuals), found on soft substrata. Two species of bivalves and a single polyplacophoran were also collected. All major taxa within the Echinodermata were represented. Samples consisted of 16 species of sea stars, 7 species of urchins, 5 species of brittle stars, 4 species of sea cucumber, and 2 species of crinoids.

Sponges (Porifera), comb jellies (Ctenophora), polycheate worms (Annelida), sea spiders (Chelicerata), pyrosomes, and sea squirts (Tunicata) composed the remainder of the major invertebrate taxa collected. With the exception of sponges ( 3 species, 33 individuals) and annelids ( 3 species, 36 
individuals), these groups were minor components of the overall abundance and diversity of megafaunal invertebrates taken during this study.

\section{All Invertebrates}

Among the invertebrates collected, the crab Bathyplax typhla (Decapoda: Brachyura: Goneplacidae) was the most abundant species, with 249 specimens collected, representing about 7 percent of the total number of individuals captured. The shrimp Pleoticus robustus $(\mathrm{n}=208)$ was also relatively abundant. The top 11 most abundant invertebrate taxa-Bathyplax typhla (249), Pleoticus robustus (208), Munida spp. (191), Scaphander sp. (110), Actinauge longicornis (86), Polycheles spp.(83), Plesionika acanthonotus (81), Brachyura spp. (80), Munidopsis spp.(77), Actiniaria spp. (73), and Eumunida picta (70) - account for 36 percent of the overall abundance of invertebrates taken across all cruises. Sixty species (23 percent) collected during this study are known only from a single individual, while another 35 species (13 percent) were represented by only 2 individuals.

Comparing the distribution of species among study sites revealed that about half of the species (134/267) were collected at only one of the five sites sampled. Fifty-three species, 28 species, and 14 species were collected at 2, 3, and 4 sites, respectively. Nine species were collected at all five sampling sites. These presumably more widespread taxa included demosponges, anemones, zoanthids, hydroids, corals (L. pertusa, Callogorgia sp.), squat lobsters (Eumunida picta, Munidopsis sp.), and the gastropod Coralliophila richardi.

\subsubsection{Species Composition and Relative Abundance Among Study Sites}

The most abundant taxa collected at all sites except VK862 were benthic species. Midwater shrimps (Sergestes spp.) were the most abundant taxon at VK862. Taxa from the orders Demospongiae, Actiniaria, Scleractinia, Zoantharia, and Decapoda were collected at all sites. More specifically, the corals L. pertusa and Callogorgia sp., the decapods Eumunida picta and Munidopsis sp., and the gastropod Coralliophila richardi were collected at all sites.

Species diversity and relative abundance of invertebrates was highest at VK826 (table 9.1; fig. 9.8). Collections from the 53 stations sampled at this site yielded 1,732 individuals, consisting of at least 147 species and representing all phyla collected, except Ctenophora, and represented nearly all class/orders identified in the study. Crustaceans were the most speciose taxa, with 74 species represented in collections. Crustaceans were also the numerical dominants of the VK826 megafaunal assemblage, where they constituted 69 percent of the total individuals collected at this site. Thirty-four percent of the crustacean catch collected here consisted of shrimps, followed in abundance by brachyurans (29 percent) and anomurans (18 percent). Cnidarians, echinoderms, and molluscs contributed 29, 21, and 14 species, respectively, to the overall diversity at this site.

Species richness (proportion of number of species to number of individuals) was much higher at MC751, despite the fact that only two stations were conducted in this area. Collections from the 2 stations sampled here yielded 95 individuals, representing at least 32 species and 6 of the 9 phyla collected in this study. No ctenophores, chelicerates, or tunicates were collected in these samples. Cnidarians and crustaceans were equally speciose with 11 species each. Cnidarians $(\mathrm{n}=36$ individuals $)$ were the numerically dominant group among invertebrates sampled here, and they accounted for 38 percent of the total individuals collected at this site. Crustaceans contributed 34 percent and echinoderms 21 percent of the total number of specimens caught at this site.

Relative abundance and numerical dominance of invertebrate species varied across sites. At MC751, the most abundant invertebrates collected were Eumunida picta (10), Callogorgia sp. (10), and Plexauridae (8). Bathyplax typhla (249), Munida spp. (119), and Polycheles spp. (72) were the most 
abundant taxa at VK826. Sergestid shrimps (Sergestes hansjacobi [39], Sergestes spp. [53]) were numerical dominants at VK862, while Pleoticus robustus (92), Scaphander sp. (110), and Munida spp. (68) were the taxa most abundant at VK862/VK906. Finally, actiniarians (37) and hydrozoans (36) were most abundant at WFS.

\subsubsection{Species Composition and Relative Abundance Among Vertical Zones}

Over half (51 percent) of the sampling effort was dedicated to bottom sampling, with 40 percent and 9 percent devoted to midwater and surface sampling, respectively. Not surprisingly, the majority of individuals ( 87 percent) were collected in bottom samples, with midwater samples accounting for 11 percent of individuals collected (fig. 9.9).

Invertebrates were collected at 12 of 18 stations where surface gear was deployed. Generally, catches were light and accounted for only 2 percent of the total individuals collected. For example, only one individual, a hydrozoan, was collected in the only tow made at VK826. Overall, 84 individuals representing 5 phyla, at least 17 families, and more than 19 species were collected in surface tows (table 9.2). Species richness is difficult to assess; many taxa are not identified below class or genus. Crustacea was the numerically dominant taxon, with 38 individuals (45 percent). Amphipods and euphausiids made up the majority of the crustacean catch. Surface sampling was most productive at WFS where 54 percent of the total number of individuals and 58 percent of total species collected in surface samples were taken.

Midwater invertebrates were collected at 54 of the 71 stations where midwater sampling occurred. No midwater invertebrates were collected at MC751. Overall, 389 individuals, representing 5 phyla, at least 26 families, and greater than 53 species were collected by midwater gear (table 9.3). Crustacea was the most speciose and numerically dominant taxon constituting almost half of the recognized diversity $(26$ species) and 57 percent $(n=220)$ of the individuals collected (fig. 9.10). Within Crustacea, shrimps dominated catches; they accounted for 80 percent of the individuals and 73 percent of the species captured. Across-site comparisons revealed that crustaceans were the numerically dominant taxon at all sites except WFS, where crustaceans, molluscs, and cnidarians were equally represented in the catches. Although sampling effort varied across the four study sites, the number of individuals captured was surprisingly consistent, with the singular exception of VK862/906 ( $\mathrm{n}=6$ stations). VK862/906 had considerably fewer individuals compared to those taken at other sites (38 individuals versus 124, 114, 113 individuals captured at VK826, VK862, and WFS, respectively).

By far, the majority of sampling effort was dedicated to bottom-tending gear, and this is reflected in the composition of the invertebrate fauna taken during this study. Benthic invertebrates accounted for 87 percent of total individuals collected. Overall, 3,201 individuals, representing 8 phyla, at least 85 families, and greater than 196 species were collected at 71 stations (table 9.4). The majority of stations were sampled at VK826 $(n=27)$ and VK862/906 $(n=23)$; therefore, the majority of individuals (84 percent) were collected at these two sites. Again, Crustacea was the most speciose and numerically dominant taxon constituting almost half of the recognized diversity (97 species) and 60 percent ( $\mathrm{n}=1908$ ) of the individuals collected (fig 9.11). Cnidarians, echinoderms, and molluscs were also well represented in these collections with 41 species $/ 547$ individuals, 33 species/354 individuals, and 16 species/314 individuals, respectively. Across-site comparisons revealed that crustaceans were the numerically dominant taxon at VK826 and VK 862/906. At VK862, abundances of cnidarians, crustaceans, and echinoderms were approximately the same, whereas at MC751 and WFS, abundances of crustaceans and cnidarians were approximately the same (fig. 9.11). 


\subsubsection{Species Composition and Habitat Associations}

Preliminary analysis, based primarily on crustacean taxa, revealed that faunal assemblages associated with coral habitats, including live and dead corals as well as coral rubble, differed from those assemblages found on or in soft substrata. Members of the hard substrata faunal assemblage include species in the genera Rochinia, Gastroptychus, Munidopsis, Chaceon, Bathynectes, Periclimenes, and Eugonatonotus. Associated fauna more likely found on soft substrata include species in the genera Nephropsis, Polycheles, Munida, Bathyplax, Heterocarpus, and Pleoticus. Additional analyses, including video analysis, are necessary to fully describe faunal assemblages, habitat associations, and relative abundances of species within these habitats. These analyses are currently ongoing.

\subsection{Discussion}

This study adds to the growing volume of data and literature describing the biodiversity of deepsea coral habitats in the GOM and represents one of the most comprehensive collections from this region. Diversity of the fauna associated with these deep reefs is comparable to that of their shallowwater counterparts (Rogers, 1999). Yet, based on species accumulation curves constructed for collections of megafauna taken during this study, we still have not accounted for the total diversity of these organisms at these deep-sea coral and adjacent habitats. This finding is significant given that the majority of invertebrate sampling during this study (84 percent of individuals collected; 72 percent of stations sampled) occurred at Viosca Knoll, one of the most well-developed and best documented $L$. pertusa communities in the GOM (Brooke and Schroeder, 2007).

Accurate identifications are fundamental and critical to all aspects of this investigation, as evidenced by collaborations with other co-principal investigators (for example, Kellogg and others, Chapter 2 this report; Coykendall and others, Chapter 4 this report). Without accurate identifications, it is difficult to adequately assess species composition, community structure, and biodiversity for faunal components associated with these habitats. Overall, taxonomy of deep-sea invertebrates is not well resolved. Many organisms are poorly known, and often little comparative material is available for study. Efforts to accurately identify material are hampered by a variety of factors. Taxonomic expertise for many taxa is lacking. Additionally, comprehensive taxonomic keys are lacking or in need of revision and updating. Recent discoveries of new species and relevant findings from recent taxonomic revisions need to be incorporated. Comparative material for many deep-sea organisms is limited. Until recently, taxonomists had to rely on historical collections, some made over 100 years ago, of which some are in bad shape due to poor preservation, missing limbs, broken pieces, and faded colors. Thus, two primary goals for processing material collected during our study has been incorporating contemporary material into museum collections and building a network of taxonomic experts. These collaborations have been instrumental to making significant progress in species identifications across diverse phyla.

With increased sampling and improved identifications using morphological and molecular approaches, knowledge of the invertebrate fauna associated with deep-sea coral habitats and off-reef areas continues to increase. To date, several species new to science have already been identified in our collection. These include one alpheid shrimp (Anker and Nizinski, 2011), at least four species of squat lobsters, one sea cucumber, two corals, one barnacle, and one or more species of zooanthids. More species discoveries are expected in a variety of taxa as taxonomic assessments of collected material proceeds. For example, actiniarians are poorly known from deep waters of the GOM, and few voucher specimens (for example a single individual per species currently recognized) were available for study. During our sampling, 30 additional actiniarian specimens were collected, among which new species are likely. Collections of contemporary, high-quality material, particularly those taken by submersible or ROV, provide the quality data necessary for species re-descriptions and evaluation of intra-specific 
variation among taxa. Based on these new collections, several possible species complexes are also hypothesized, including those for squat lobsters and anemones, where previously only a single species in each of these groups had been identified.

Not only are estimates of species diversity improving, but continued sampling also provides the data for better understanding of relative abundances, as well as improved estimates for geographic and bathymetric distributions of the species collected. For example, during this study our data document new records for species not previously reported from the GOM, and for several others, our data extend the known distributions of species that had previously been reported from other parts of the GOM. The squat lobsters, Leiogalathea agassizii and Munida sanctipauli, collected at a depth of $525 \mathrm{~m}$ at the WFS study site exemplify these findings. Leiogalathea agassizii was previously reported off northern Cuba, St. Lucia, Barbados, and St. Vincent at depths of 300-434 m (Baba and others, 2008), whereas Munida sanctipauli was reported to occur from the east-southeast region of the GOM (Felder and others, 2009), off St. Augustine, Fla., off Frederiksted, St. Croix, throughout the Antilles (485-575 m), and off Brazil (150-1,385 m) (Melo-Filho and Melo, 1992; Baba and others, 2008). Estimates of relative abundance (common versus rare) or simply species presence or absence of data in an area are difficult to determine for many deep-sea taxa when the taxonomy of these groups is poorly known. Similar to many other deep-sea taxa, numerous decapod species in the GOM are known from fewer than five specimens (Wicksten and Packard, 2005). Through continued sampling, using a variety of gears, species thought to be rare may actually be more common than once thought. Large sample sizes provide the data necessary to assess relative abundances and population dynamics of some species.

Based on available information, deep-sea species endemic to the GOM are few in number, and these species are rarely associated with deep-sea coral habitats. Although their lists consist of different species, Wicksten and Packard (2005) and Felder and others (2009) each report 11 species of deepwater decapods endemic to the GOM. Of these species, only one, Munidopsis glabra, has been collected at deep-sea coral habitats. The anemone, Adamsia obvolva, collected during our study, is another species endemic to the GOM. Neither of these two species is restricted to deep-sea coral habitats. The presence of relatively few species uniquely associated with deep-sea coral habitats corroborates the findings of other researchers studying these habitats. For example, the majority of fauna sampled on and adjacent to the coral framework (Cordes and others, 2008) and cold-seep sites (Carney, 1994; Martin and Haney, 2005) in the GOM were also representative of the background fauna found in other habitats on the continental slope of the GOM.

Lack of taxonomic resolution to species-level identifications has hindered our ability to accurately estimate species diversity at deep-sea coral habitats. Increased efforts to identify individuals have certainly increased the number of species known to associate with coral habitats, but further work is needed in this area to improve these diversity estimates. Based on results available to date, diversity estimates for faunal associates of deep-water coral habitats underestimate the total diversity likely occurring at these sites. For example, Cordes and others (2008) reported 68 taxa in the deep GOM, of which 9 percent, taken from 15 samples, were identified to species. By comparison, in our study, 267 taxa (48 percent identified to species) were collected from 138 stations. Even considering that some offreef organisms were included in our study and none of these were included in the study conducted by Cordes and others (2008), the diversity estimate in the present study appears to be much higher than that reported in the former study. If one assumes a linear relationship between number of taxa collected and number of samples made, however, then the number of taxa expected in our study should have been about 626 (68 taxa/15 samples extrapolated to 626 taxa/138 samples). The difference between the projected diversity estimate and our present estimate (267 taxa) likely indicates that the actual diversity for these habitats lies somewhere between these two estimates. While this projection has limitations 
related to underlying assumptions, this exercise does point out the fact that many samples are needed to understand and determine a reliable estimate of the diversity for faunal associates of a particular habitat. Additionally, it is important to recognize the difficulties encountered when attempting to compare results between most studies because levels of effort, types of gear used, taxonomic resolution of identifications, investigator bias (in other words, better resolution for taxa of interest), and sampling bias all vary between those studies. For example, collections made by Cordes and others (2008) had high proportions of polychaetes and relatively few echinoderms in contrast to collections from the present study where polychaetes were rarely collected and echinoderms were significantly represented among the collections. Some overlap in crustacean taxa were reported between the two studies. Type of sampling gear as well as taxonomic specialty and level of expertise may be driving some of the differences between studies.

Biological structures, such as deep-sea corals, highly influence species diversity at local and regional scales on deep continental margins (Buhl-Mortensen and others, 2010). Buhl-Mortensen and others (2010) suggested that increased habitat heterogeneity provided by habitat-forming organisms is related to depth because substrata and food resources become limited at deeper depths. Others (Soto, 1985; Pequegnat and others, 1990; Wicksten and Packard, 2005; Cordes and others, 2008) have also noted depth as a significant factor contributing to species composition and diversity, and ultimately, to similarities between community assemblages for deep-water fauna. Alternatively, other factors such as topography and hydrographic conditions (Soto, 1985), habitat complexity (Lessard-Pilon and others, 2010), and environmental factors relating to food, habitat, pollution, and location (Haedrich and others, 2008) have also been proposed as important predictors of diversity in deep-sea environments.

Crustaceans were the most speciose and abundant taxa sampled across all sites and vertical zones throughout this study. Crustaceans were also dominant components among taxa reported in association with deep-sea corals in other areas such as Atlantic Canada (Buhl-Mortensen and Mortensen, 2005) and in other studies involving deep-water fauna of select regions of the GOM (Soto, 1985; Pequegnat and others, 1990; Wicksten and Packard, 2005), as well as throughout the entire GOM (Felder and others, 2009). Although scope, scale, primary objectives, and focus vary between studies, diversity patterns are similar between these studies. Overall, abundances and diversity are high, species diversity is greatest in the eastern GOM, diversity tends to be highest at mid-range depths, species collected represent a subset of the total diversity of species reported for the GOM, and many species have widespread distributions and occur in a variety of habitats. A primary and important feature of this study is the analysis and re-evaluation of species composition and associations with deep-sea coral and adjacent habitats as we work to better understand local and regional differences between coral habitats.

\subsection{Future Directions}

Results presented here provide the first steps towards understanding species richness, biodiversity, and community structure of these deep-coral study sites. Taxonomic investigations continue on a variety of taxa. Species descriptions, re-descriptions, and generic revisions for several taxa are ongoing. Higher level systematic studies examining phylogenetic and phylogeographic relationships of various taxa, including crustaceans (squat lobsters and shrimps) and echinoderms, also are ongoing and nearing completion.

Taxonomic resolution to species-level identification not only refines our knowledge of biodiversity but also lays the foundation for continued investigations and analyses of species abundances, spatial and temporal distributions, and population dynamics of megafaunal invertebrate species associated with deep-sea coral habitats in the region. Additionally, accurate identifications are 
critical to all image analyses. Video and image analysis will provide additional data necessary to address ecological questions of habitat use and community structure at deep-reef sites and adjacent habitats.

\subsection{Acknowledgments}

We thank the captains and crews of the ships, ROVs, and submersible from all Lophelia II research cruises. The science crews from these expeditions are acknowledged for help with sample collecting, processing, and data requests. We appreciate the assistance and support of the Smithsonian Institution, National Museum of Natural History, Invertebrate Zoology Collection Management staff. O. Breedy (Universidad de Costa Rica), S. Cairns (Smithsonian Institution), T. Casazza (formally University of North Carolina, Wilmington), A. Collins (NOAA/NMFS), D. Felder (University of Louisiana, Lafayette), M. Harasewych (Smithsonian Institution), C. Mah (Smithsonian Institution), C. Messing (Nova Southeastern University), J. Norenburg (Smithsonian Institution), M. Okanishi (Kyoto University), D. Opresko (Smithsonian Institution affiliate), D. Pawson (Smithsonian Institution), A. Quattrini (Harvey Mudd College), F. Solis-Marin (Instituto de Ciencias del Mar y Limnología-UNAM), E. Strong (Smithsonian Institution), D. Tippett (former volunteer), R. Van Syoc (California Academy of Science), M. Vecchione (NOAA/NMFS), and A. Windsor (Smithsonian Institution affiliate) helped with species identifications. T. Munroe (NOAA/NMFS) read an earlier draft and provided helpful comments.

\subsection{References}

Ammons, A., and Daly, M., 2008, Distribution, habitat use and ecology of deepwater anemones (Actiniaria) in the Gulf of Mexico: Deep-Sea Research Part II, v. 55, p. 2657-2666.

Anker, A., and Nizinski, M., 2011, Description of a new deep-water species of Alpheus Fabricius, 1798 from the Gulf of Mexico (Crustacea, Decapoda, Alpheidae): Zootaxa, v. 2925, p. 49-56.

Baba, K., Macpherson, E., Poore, G.C.B., Ahyong, S., Bermudez, A., Cabezas, P., Lin, C.-W., Nizinski, M., Rodrigues, C., and Schnabel, K.E., 2008, Catalogue of squat lobsters of the world (Crustacea: Decapoda: Anomura - Families Chirostylidae, Galatheidae and Kiwaidae): Zootaxa, v. 1905, p. 1220.

Brooke, S., and Schroeder, W.W., 2007, State of deep coral ecosystems in the Gulf of Mexico region: Texas to the Florida straits, in Lumsden, S.E., Hourigan, T.F., Bruckner, A.W., and Dorr, G., eds., The state of deep coral ecosystems of the United States: Silver Spring, Md., National Oceanic and Atmospheric Administration Technical Memorandum CRCP-3, p. 271-306.

Buhl-Mortensen, L., and Mortensen, P.B., 2005, Distribution and diversity of species associated with deep-sea gorgonian corals off Atlantic Canada, in Freiwald, A., and Roberts, J.M., eds., Cold-water corals and ecosystems: Berlin, Springer-Verlag, p. 849-879.

Buhl-Mortensen, L., Vanreusel, A., Gooday, A.J., Levin, L.A., Priede, I.G., Buhl-Mortensen, P., Gheerardyn, G., King, N.J., and Raes, M., 2010, Biological structures as a source of habitat heterogeneity and biodiversity on the deep ocean margins: Marine Ecology, v. 31, p. 21-50.

Carney, R.S., 1994, Consideration of the oasis analogy for chemosynthetic communities at Gulf of Mexico hydrocarbon vents: Geo-Marine Letters, v. 14, p. 149-159.

Cordes, E.E., McGinley, M.P., Podowski, E.L., Becker, E.L., Lessard-Pilon, S., Viada, S.T., and Fisher, C.R., 2008, Coral communities of the deep Gulf of Mexico: Deep-Sea Research Part I, v. 55, p. 777787.

Felder, D.L, Álvarez, F., Goy, J.W., and Lemaitre, R., 2009, Decapoda (Crustacea) of the Gulf of Mexico, with comments on the Amphionidacea, in Felder, D.L., and Camp, D.K., eds., Gulf of Mexico origin, waters, and biota, v. 1, Biodiversity: College Station, Tex., Texas A\&M University Press, p. 1019-1104. 
Haedrich, R.L., Devine, J.A., and Kendall, V.J., 2008, Predictors of species richness in the deep-benthic fauna of the northern Gulf of Mexico: Deep-Sea Research Part II, v. 55, p. 2650-2656.

Henry, L.A., and Roberts, J.M., 2007, Biodiversity and ecological composition of macrobenthos on cold-water coral mounds and adjacent off-mound habitat in the bathyal Porcupine Seabight, NE Atlantic: Deep-Sea Research Part I, v. 54, p. 654-672.

Jensen, A., and Frederiksen, R., 1992, The fauna associated with the bank-forming deepwater coral Lophelia pertusa (Scleractinaria) on the Faroe shelf: Sarsia, v. 77, p. 53-69.

Jonsson, L.G., Nilsson, P.G., Floruta, F., and Lundälv, T., 2004, Distributional patterns of macro- and megafauna associated with a reef of the cold-water coral Lophelia pertusa on the Swedish west coast: Marine Ecology Progress Series, v. 284, p. 163-171.

Lessard-Pilon, S.A., Podowski, E.L., Cordes, E.E., and Fisher, C.R., 2010, Megafauna community composition associated with Lophelia pertusa colonies in the Gulf of Mexico: Deep-Sea Research Part II, v. 57, p. 1882-1890.

Martin, J.W., and Haney, T.A., 2005, Decapod crustaceans from hydrothermal vents and cold seeps: a review through 2005: Zoological Journal of the Linnean Society, v. 145, p. 445-522.

Melo-Filho, G.A.S., and Melo, G.A.S., 1992, Reexamination of the material of Munida (Crustacea: Anomura: Galatheidae) collected by the H.M.S. "Challenger" along the Brazilian coast: Proceedings of the Biological Society of Washington, v. 105, p. 760-774.

Mortensen, P.B., and Fosså, J.H., 2006, Species diversity and spatial distribution of invertebrates on Lophelia reefs in Norway: Proceedings of the $10^{\text {th }}$ International Coral Reef Symposium, Okinawa, $\mathrm{p}$. 1849-1868.

Mortensen, P.B., Hovland, M., Brattegard, T., and Farestveit, R., 1995, Deep water bioherms of the scleractinian coral Lophelia pertusa (L.) at $64^{\circ} \mathrm{N}$ on the Norwegian shelf: structure and associated megafauna: Sarsia, v. 80, p. 145-158.

Pequegnat, W.E., Gallaway, B.J., and Pequegnat, L.H., 1990, Aspects of the ecology of the deep-water fauna of the Gulf of Mexico: American Zoologist, v. 30, no. 1, p. 45-64.

Roberts, J.M., Henry, L.-A., Long, D., and Hartley, J.P., 2008, Cold-water coral reef frameworks, megafaunal communities and evidence for coral carbonate mounds on the Hatton Bank, north east Atlantic: Facies, v. 54, no. 3, p. 297-316.

Roberts, J.M., Wheeler, A.J., and Freiwald, A., 2006, Reefs of the deep: the biology and geology of cold-water ecosystems: Science, v. 312, p. 543-547.

Rogers, A.D., 1999, The biology of Lophelia pertusa (Linnaeus 1758) and other deep-water reefforming corals and impacts from human activities: International Review of Hydrobiology, v. 84, no. 4, p. 315-406.

Ross, S.W., and Quattrini, A.M., 2007, The fish fauna associated with deep coral banks off the southeastern United States: Deep-Sea Research Part I, v. 54, p. 975-1007.

Soto, L.A., 1985, Distributional patterns of deep-water brachyuran crabs in the Straits of Florida: Journal of Crustacean Biology, v. 5, no. 3, p. 480-499.

Wicksten, M.K., and Packard, J.M., 2005, A qualitative zoogeographic analysis of decapod crustaceans of the continental slopes and abyssal plain of the Gulf of Mexico: Deep-Sea Research Part I, v. 52, p. $1745-1765$. 
Table 9.1. Overall diversity and abundance of invertebrates collected (all sampling methods combined) at five deep coral study sites (MC751, VK826, VK862, VK862/VK906, West Florida Slope (WFS) in the north-central and eastern Gulf of Mexico, 2008-2010, as part of the Lophelia II program.

[n, number of stations where invertebrates were collected; see figure 9.1 for site locations]

\begin{tabular}{|c|c|c|c|c|c|c|c|c|c|}
\hline Phylum & Higher Classification & Family & Species & $\begin{array}{c}\text { MC751 } \\
n=2\end{array}$ & $\begin{array}{c}\text { VK826 } \\
n=53\end{array}$ & $\begin{array}{c}\text { VK862 } \\
n=13\end{array}$ & $\begin{array}{c}\text { VK862/VK906 } \\
n=34\end{array}$ & $\begin{array}{l}\text { WFS } \\
n=36\end{array}$ & $\begin{array}{l}\text { Totals } \\
\mathrm{n}=138 \\
\end{array}$ \\
\hline \multirow[t]{3}{*}{ Porifera } & Demospongiae & Stylocordylidae? & Stylocordyla sp.? & & 1 & 1 & 4 & & 6 \\
\hline & & & Demospongiae spp. & 1 & 5 & 1 & 5 & 2 & 14 \\
\hline & Hexactinellida & Aphrocallistidae & Aphrocallistes beatrix & & 4 & & & 9 & 13 \\
\hline \multirow[t]{25}{*}{ Cnidaria } & Anthozoa/ Actiniaria & Actinostolidae? & Actinostolidae sp.? & & 10 & 10 & 3 & & 23 \\
\hline & & Actinoscyphiidae & Actinoscyphia cf. saginata & 1 & 17 & 1 & 6 & 1 & 26 \\
\hline & & Halicuriidae & Halcurias pilatus & 6 & & 1 & 20 & & 27 \\
\hline & & Hormathiidae & Actinauge longicornis & & 28 & 3 & 53 & 2 & 86 \\
\hline & & & Adamsia obvolva & & 9 & & & & 9 \\
\hline & & & Hormathiidae sp. & & 19 & & 4 & & 23 \\
\hline & & Unknown & Actiniaria spp. & 2 & 17 & 1 & 33 & 37 & 90 \\
\hline & Anthozoa/ Cerianthiaria & Cerianthidae & Cerianthidae sp. & & 1 & & & & 1 \\
\hline & Anthozoa/Antipatharia & Cladopathidae & Sibopathes macrospina & & 4 & & & & 4 \\
\hline & & Leiopathidae & Leiopathes sp. A & & 29 & 3 & 4 & 1 & 37 \\
\hline & & & Leiopathes sp. B & & 6 & & & & 6 \\
\hline & & & Leiopathes sp. C & & 1 & & & & 1 \\
\hline & & & Leiopathes sp. D & & & & & 1 & 1 \\
\hline & & & Leiopathes spp. & & 13 & & 10 & 1 & 24 \\
\hline & Anthozoa/ Scleractinia & Caryophylliidae & Caryophyllia sp. & & 3 & & & & 3 \\
\hline & & & Lophelia pertusa & 2 & 23 & 1 & 5 & 23 & 54 \\
\hline & & Dendrophylliidae & Enallopsammia profunda & & & & & 1 & 1 \\
\hline & & & Thecopsammia socialis & & 6 & & & 1 & 7 \\
\hline & & Flabellidae & Javania cailleti & & 2 & 1 & & & 3 \\
\hline & & Oculinidae & Madrepora oculata & & & 1 & & 2 & 3 \\
\hline & Anthozoa/ Zoantharia & Parazoanthiidae & Parazoanthiidae sp. & & & & & 1 & 1 \\
\hline & & & Salvia sp.? & & & & & 1 & 1 \\
\hline & & Unknown & Zoantharia sp. & 2 & 11 & 2 & 4 & 2 & 21 \\
\hline & Anthozoa/ Octocorallia & Acanthogorgiidae & Acanthogorgia sp. & & 2 & & & & 2 \\
\hline & & Alcyoniidae & Anthomastus sp. & & 1 & & 5 & 2 & 8 \\
\hline
\end{tabular}


Table 9.1. Overall diversity and abundance of invertebrates collected (all sampling methods combined) at five deep coral study sites (MC751, VK826, VK862, VK862/906, West Florida Slope (WFS) in the north-central and eastern Gulf of Mexico, 2008-2010, as part of the Lophelia II program - continued.

[n, number of stations where invertebrates were collected; see figure 9.1 for site locations]

\begin{tabular}{|c|c|c|c|c|c|c|c|c|c|}
\hline Phylum & Higher Classification & Family & Species & $\begin{array}{c}M C 751 \\
\mathrm{n}=2\end{array}$ & $\begin{array}{c}\text { VK826 } \\
n=53\end{array}$ & $\begin{array}{c}\text { VK862 } \\
n=13\end{array}$ & $\begin{array}{c}\text { VK862/NK906 } \\
n=34\end{array}$ & $\begin{array}{l}\text { WFS } \\
\mathrm{n}=36\end{array}$ & $\begin{array}{l}\text { Totals } \\
\mathrm{n}=138\end{array}$ \\
\hline \multirow[t]{23}{*}{ Cnidaria } & Anthozoa/ Octocorallia & Alcyoniidae & Xeniidae sp.? & & & & 1 & & 1 \\
\hline & & Unknown & Alcyonacea sp. & 1 & & & 8 & 3 & 12 \\
\hline & & Anthotelidae & Anthothela sp. & & & & & 2 & 2 \\
\hline & & Clavulariidae & Clavularia sp. & & 1 & & & & 1 \\
\hline & & Isididae & $\begin{array}{l}\text { Chelidonisis aurantiaca } \\
\text { mexicana }\end{array}$ & 1 & & & & & 1 \\
\hline & & & Lepidisis sp. & & & & 5 & & 5 \\
\hline & & Nephtheidae & Nephtheidae sp. & & & & & 3 & 3 \\
\hline & & Primnoidae & Callogorgia americana & 1 & & & & & 1 \\
\hline & & & Callogorgia sp. & 10 & 15 & 1 & 1 & 1 & 28 \\
\hline & & & Plumarella dichotoma & 1 & & & & & 1 \\
\hline & & & Plumarella sp. & & 1 & & & 9 & 10 \\
\hline & & Plexauridae & Plexauridae sp. & 8 & 2 & & 2 & 10 & 22 \\
\hline & Hydrozoa/ Hydroidolina & Mitrocomidae & Earleria quadrata? & & & & & 1 & 1 \\
\hline & & Stylasteridae & Stylaster erubescens & & & & & 3 & 3 \\
\hline & & & Stylaster sp. & & & & & 2 & 2 \\
\hline & & Unknown & Leptothecata spp. & & 4 & & 3 & & 7 \\
\hline & & Unknown & Hydroidolina spp. & 2 & 22 & 2 & 6 & 45 & 77 \\
\hline & & Aequoreidae & Aequorea cf. macrodactyla & & 6 & & & 2 & 8 \\
\hline & & & Zygocanna vagans & & 4 & & 2 & & 6 \\
\hline & & Campanulariidae & Obelia sp.? & & & & 2 & & 2 \\
\hline & & Hippopodiidae & Hippopodius hippopus & & 5 & & 1 & 1 & 7 \\
\hline & & Unknown & Siphonophorae spp. & & 1 & 1 & & 2 & 4 \\
\hline & Scyphozoa & Pelagiidae & Pelagia noctiluca & & 12 & & & & 12 \\
\hline Ctenophora & Nuda/Beroida & Beroidae & Beroe sp. & & & & 1 & & 1 \\
\hline \multirow[t]{2}{*}{ Mollusca } & Bivalvia & Mytilidae & Bathymodiolus sp. & & 19 & & & & 19 \\
\hline & & Unknown & Bivalvia sp. & & 29 & 1 & 11 & & 41 \\
\hline
\end{tabular}


Table 9.1. Overall diversity and abundance of invertebrates collected (all sampling methods combined) at five deep coral study sites (MC751, VK826, VK862, VK862/906, West Florida Slope (WFS) in the north-central and eastern Gulf of Mexico, 2008-2010, as part of the Lophelia II program - continued.

[n, number of stations where invertebrates were collected; see figure 9.1 for site locations]

\begin{tabular}{|c|c|c|c|c|c|c|c|c|c|}
\hline Phylum & Higher Classification & Family & Species & $\begin{array}{c}M C 751 \\
n=2\end{array}$ & $\begin{array}{c}\text { VK826 } \\
n=53\end{array}$ & $\begin{array}{c}\text { VK862 } \\
n=13\end{array}$ & $\begin{array}{c}\text { VK862/VK906 } \\
n=34\end{array}$ & $\begin{array}{l}\text { WFS } \\
n=36\end{array}$ & $\begin{array}{l}\text { Totals } \\
n=138\end{array}$ \\
\hline \multirow[t]{25}{*}{ Mollusca } & \multirow[t]{10}{*}{$\begin{array}{l}\text { Cephalopoda/ } \\
\text { Decapodiformes }\end{array}$} & Chiroteuthidae & Chiroteuthis mega & & & & & 1 & 1 \\
\hline & & Cranchiidae & Cranchia cf. scabra & & & & & 5 & 5 \\
\hline & & & Cranchia sp. & & & & 1 & & 1 \\
\hline & & Enoploteuthidae & Enoploteuthidae sp. & & & & & 1 & 1 \\
\hline & & Lycoteuthidae & Lycoteuthidae sp. & & & & & 1 & 1 \\
\hline & & Pyroteuthidae & Pyroteuthis magaritifera & & & & 1 & & 1 \\
\hline & & & Pyroteuthidae sp. & & & & & 7 & 7 \\
\hline & & Sepiolidae & Heteroteuthis cf. dispar & & 1 & & & & 1 \\
\hline & & Tremoctopodidae & Tremoctopodidae sp. & & 9 & 3 & 1 & 6 & 19 \\
\hline & & Unknown & Decapodiformes spp. & & 4 & & 11 & 1 & 16 \\
\hline & \multirow[t]{2}{*}{$\begin{array}{l}\text { Cephalopoda/ } \\
\text { Octopodiformes }\end{array}$} & Argonautidae & Argonauta argo & & & & 1 & & 1 \\
\hline & & Unknown & Octopodiformes spp. & & 2 & & & 2 & 4 \\
\hline & \multirow[t]{5}{*}{ Gastropoda/ Neogastropoda } & Muricidae & Coralliophila richardi & 5 & 6 & 2 & 2 & 10 & 25 \\
\hline & & Muricidae & Muricidae sp? & & & 1 & & & 1 \\
\hline & & Turridae & Gemmula periscelida & & & & 64 & & 64 \\
\hline & & Volutidae & Scaphella dubia kieneri & & & & 2 & & 2 \\
\hline & & Unknown & Neogastropoda spp. & & 2 & & 10 & & 12 \\
\hline & $\begin{array}{l}\text { Gastropoda/ } \\
\text { Caenogastropoda }\end{array}$ & Xenophoridae & Onustus longleyi & & & & 2 & & 2 \\
\hline & \multirow[t]{2}{*}{ Gastropoda/ Vetigastropoda } & Calliostomatidae & Calliostoma sayanum & & & 1 & & & 1 \\
\hline & & Turbinidae & Gaza superba & & 16 & & 6 & & 22 \\
\hline & Gastropoda/ Gymnosomata & Unknown & Gymnosomata sp? & & & & & 8 & 8 \\
\hline & \multirow[t]{3}{*}{$\begin{array}{l}\text { Gastropoda/ } \\
\text { Opisthobranchia }\end{array}$} & Cymbuliidae & Cymbuliidae sp.? & & & & & 13 & 13 \\
\hline & & Phylliroidae & Phylliroe cf. atlantica & & & & & 1 & 1 \\
\hline & & Scaphandridae & Scaphander sp. & & & & 110 & & 110 \\
\hline & $\begin{array}{l}\text { Gastropoda/ } \\
\text { Pterotracheoidea }\end{array}$ & Cariiniidae & Carinaria cf. lamarki & & 6 & & 1 & 3 & 10 \\
\hline
\end{tabular}


Table 9.1. Overall diversity and abundance of invertebrates collected (all sampling methods combined) at five deep coral study sites (MC751, VK826, VK862, VK862/906, West Florida Slope (WFS) in the north-central and eastern Gulf of Mexico, 2008-2010, as part of the Lophelia II program - continued.

[n, number of stations where invertebrates were collected; see figure 9.1 for site locations]

\begin{tabular}{|c|c|c|c|c|c|c|c|c|c|}
\hline Phylum & Higher Classification & Family & Species & $\begin{array}{c}\mathrm{MC} 751 \\
\mathrm{n}=2\end{array}$ & $\begin{array}{c}\text { VK826 } \\
n=53\end{array}$ & $\begin{array}{c}\text { VK862 } \\
n=13\end{array}$ & $\begin{array}{c}\text { VK862/VK906 } \\
n=34\end{array}$ & $\begin{array}{l}\text { WFS } \\
n=36\end{array}$ & $\begin{array}{l}\text { Totals } \\
\mathrm{n}=138\end{array}$ \\
\hline \multirow[t]{4}{*}{ Mollusca } & Gastropoda/ Euthecosomata & Cavolinidae & Cavolinia cf. tridenata & & 6 & & & 8 & 14 \\
\hline & & & Clio cf. recurva & & 3 & & & & 3 \\
\hline & & & Diacria cf. trispinosa & & 7 & & & & 7 \\
\hline & Polyplacophora/ Chitonida & Unknown & Chitonida sp. & & 1 & & & & 1 \\
\hline \multirow[t]{3}{*}{ Annelida } & Polychaeta/ Eunicida & Eunicidae & Eunicidae spp. & & 8 & 1 & 14 & 9 & 32 \\
\hline & Polychaeta/ Sabellida & Serpulidae & Serpulidae sp. & 1 & 1 & & & & 2 \\
\hline & Polychaeta spp. & Unknown & Polychaeta spp. & & 1 & & & 1 & 2 \\
\hline Chelicerata & Pycnogonida & Unknown & Pycnogonida spp. & & 2 & & & 2 & 4 \\
\hline \multirow[t]{20}{*}{ Crustacea } & Decapoda/ Achelata & Polychelidae & Polycheles cf. perarmatus & & 2 & & & & 2 \\
\hline & & & Polycheles sculptus & & 3 & & & & 3 \\
\hline & & & Polycheles typhlops & & & & 1 & & 1 \\
\hline & & & Polycheles spp. & & 72 & & 11 & & 83 \\
\hline & & Scyllaridae & Scyllaridae spp. & & 1 & & & 9 & 10 \\
\hline & Decapoda/ Astacidea & Nephropidae & Nephropsis aculeata & & 28 & & & & 28 \\
\hline & & & Nephropsis spp. & & 56 & & 1 & & 57 \\
\hline & Decapoda/ Anomura & Chirostylidae & Gastroptychus salvadori & & 1 & & & & 1 \\
\hline & & & Gastroptychus sp. & & & & 5 & 1 & 6 \\
\hline & & & Uroptychus sp.? & 2 & & & & & 2 \\
\hline & & Eumunididae & Eumunida picta & 10 & 22 & 4 & 17 & 17 & 70 \\
\hline & & Munididae & Agononida longipes & & & & 14 & & 14 \\
\hline & & & Munida iris & & 1 & 2 & 1 & & 4 \\
\hline & & & Munida media & & 1 & & & & 1 \\
\hline & & & Munida sanctipauli & & 2 & 2 & & 7 & 11 \\
\hline & & & Munida spp. & 3 & 119 & 1 & 68 & & 191 \\
\hline & & & Munida valida & & 8 & & & & 8 \\
\hline & & Munidopsidae & Leiogalathea agassizii & & & & & 2 & 2 \\
\hline & & & Munidopsis expansa & & & & & 2 & 2 \\
\hline & & & Munidopsis glabra & & & 1 & & & 1 \\
\hline
\end{tabular}


Table 9.1. Overall diversity and abundance of invertebrates collected (all sampling methods combined) at five deep coral study sites (MC751, VK826, VK862, VK862/906, West Florida Slope (WFS) in the north-central and eastern Gulf of Mexico, 2008-2010, as part of the Lophelia II program - continued.

[n, number of stations where invertebrates were collected; see figure 9.1 for site locations]

\begin{tabular}{|c|c|c|c|c|c|c|c|c|c|}
\hline Phylum & Higher Classification & Family & Species & $\begin{array}{c}\mathrm{MC} 751 \\
\mathrm{n}=2\end{array}$ & $\begin{array}{c}\text { VK826 } \\
n=53\end{array}$ & $\begin{array}{c}\text { VK862 } \\
n=13\end{array}$ & $\begin{array}{c}\text { VK862/VK906 } \\
n=34\end{array}$ & $\begin{array}{l}\text { WFS } \\
\mathrm{n}=36\end{array}$ & $\begin{array}{l}\text { Totals } \\
\mathrm{n}=138\end{array}$ \\
\hline \multirow[t]{27}{*}{ Crustacea } & Decapoda/ Anomura & Munidopsidae & Munidopsis penescabra & & & & & 9 & 9 \\
\hline & & & Munidopsis robusta & & & & 9 & & 9 \\
\hline & & & Munidopsis spp. & 7 & 45 & 3 & 12 & 10 & 77 \\
\hline & & Unknown & Galatheoidea spp. & & 1 & & 30 & 1 & 32 \\
\hline & & Lithodidae & Paralomis cf. cubensis & & & & & 1 & 1 \\
\hline & & Parapaguridae & Parapagurus pictus & & 5 & & & & 5 \\
\hline & & Unknown & Paguroidea spp. & & 12 & & 3 & 3 & 18 \\
\hline & Decapoda/ Axiidea & Axiidae & Calocaris caribbaeus & & 6 & & & & 6 \\
\hline & Decapoda/ Brachyura & Gerionidae & Chaceon fenneri & & 2 & & & 1 & 3 \\
\hline & & & Chaceon quinquedens & & 2 & & & & 2 \\
\hline & & Goneplacidae & Bathyplax typhla & & 227 & & 22 & & 249 \\
\hline & & Inachidae & Dorhynchus thomsoni & & & & & 2 & 2 \\
\hline & & Inachoididae & Pyromaia arachna & & & & 8 & & 8 \\
\hline & & Majiidae & Rochinia crassa & 1 & 12 & & 4 & & 17 \\
\hline & & & Rochinia sp. & & 1 & & 1 & 1 & 3 \\
\hline & & & Rochinia tanneri & & 4 & & 11 & & 15 \\
\hline & & & Rochinia umbonata & & & & & 3 & 3 \\
\hline & & & Majidae spp. & 1 & 11 & & & 2 & 14 \\
\hline & & Polybiidae & Bathynectes longispina & 1 & 6 & 1 & 10 & & 18 \\
\hline & & & Raymanninus schmitti & & 23 & & 20 & & 43 \\
\hline & & Portunidae & Achelous floridanus & & & 1 & & & 1 \\
\hline & & Raninidae & Lyreidus sp.? & & & & 4 & & 4 \\
\hline & & Trichopeltariidae & Trichopeltarion nobile & & 2 & & & & 2 \\
\hline & & Unknown & Brachyura spp. & & 54 & 1 & 29 & & 84 \\
\hline & $\begin{array}{l}\text { Decapoda/ } \\
\text { Dendrobranchiata }\end{array}$ & Aristeidae & Aristaeopsis edwardsiana & & 3 & & & & 3 \\
\hline & & & Aristeaus antillensis & & 23 & & 15 & & 38 \\
\hline & & & Aristeidae spp. & & & & 19 & & 19 \\
\hline
\end{tabular}


Table 9.1. Overall diversity and abundance of invertebrates collected (all sampling methods combined) at five deep coral study sites (MC751, VK826, VK862, VK862/906, West Florida Slope (WFS) in the north-central and eastern Gulf of Mexico, 2008-2010, as part of the Lophelia II program - continued.

[n, number of stations where invertebrates were collected; see figure 9.1 for site locations]

\begin{tabular}{|c|c|c|c|c|c|c|c|c|c|}
\hline Phylum & Higher Classification & Family & Species & $\begin{array}{c}\mathrm{MC} 751 \\
\mathrm{n}=2\end{array}$ & $\begin{array}{c}\text { VK826 } \\
\mathrm{n}=53\end{array}$ & $\begin{array}{l}\text { VK862 } \\
\mathrm{n}=13\end{array}$ & $\begin{array}{c}\text { VK862/NK906 } \\
n=34\end{array}$ & $\begin{array}{l}\text { WFS } \\
n=36\end{array}$ & $\begin{array}{l}\text { Totals } \\
\mathrm{n}=138 \\
\end{array}$ \\
\hline \multirow[t]{27}{*}{ Crustacea } & $\begin{array}{l}\text { Decapoda/ } \\
\text { Dendrobranchiata }\end{array}$ & Benthesicymidae & Gennadas elegens & & 4 & & & & 4 \\
\hline & & & Gennadas sp. & & 2 & & & & 2 \\
\hline & & Penaeidae & Hepomadus glacialis? & 1 & & & & & 1 \\
\hline & & & Penaeopsis serrata & & & & 1 & & 1 \\
\hline & & & Parapeneus sp.? & & & & 18 & & 18 \\
\hline & & & Penaeidae spp. & & 30 & & & 1 & 31 \\
\hline & & Solenoceridae & Hymenopenaeus debilis & & & & 1 & & 1 \\
\hline & & & Pleoticus robustus & & 65 & & 143 & & 208 \\
\hline & & Sergestidae & Allosergestes sp. & & 4 & & & & 4 \\
\hline & & & Deosergestes sp. & & & & 1 & & 1 \\
\hline & & & Sergestes hansjacobi & & & 39 & & & 39 \\
\hline & & & Sergestes spp. & & 2 & 53 & 13 & 4 & 72 \\
\hline & & & Sergia cf. grandis & & 2 & & 1 & & 3 \\
\hline & & & Sergia hansjacobi & & 5 & & & & 5 \\
\hline & & & Sergia cf. laminata & & 1 & & & & 1 \\
\hline & & & Sergia cf. splendens & & & & 2 & & 2 \\
\hline & & & Sergia splendens & & 1 & & & & 1 \\
\hline & & & Sergia talismani & & 3 & & & & 3 \\
\hline & & & Sergia spp. & & 7 & 8 & & 1 & 16 \\
\hline & & & Sergestidae spp. & & 14 & & 2 & & 16 \\
\hline & & Unknown & Dendrobranchiata spp. & & 2 & & & & 2 \\
\hline & Decapoda/ Caridea & Alpheidae & Alpheus lentiginosus & 1 & & & & & 1 \\
\hline & & & Alpheidae sp. ? & & & & & 2 & 2 \\
\hline & & Bathypalaemonellidae & Bathypalaemonella sp. & & 5 & & & 2 & 7 \\
\hline & & Crangonidae & Metacrangon agassizii & & 1 & & & & 1 \\
\hline & & & Parapontophilus gracilis & & 1 & & 1 & & 2 \\
\hline & & Eugonatonotidae & Eugonatonotus crassus & & & & & 2 & 2 \\
\hline
\end{tabular}


Table 9.1. Overall diversity and abundance of invertebrates collected (all sampling methods combined) at five deep coral study sites (MC751, VK826, VK862, VK862/906, West Florida Slope (WFS) in the north-central and eastern Gulf of Mexico, 2008-2010, as part of the Lophelia II program - continued.

[n, number of stations where invertebrates were collected; see figure 9.1 for site locations]

\begin{tabular}{|c|c|c|c|c|c|c|c|c|c|}
\hline Phylum & Higher Classification & Family & Species & $\begin{array}{c}M C 751 \\
\mathrm{n}=2\end{array}$ & $\begin{array}{c}\text { VK826 } \\
n=53\end{array}$ & $\begin{array}{c}\text { VK862 } \\
n=13\end{array}$ & $\begin{array}{c}\text { VK862/VK906 } \\
n=34\end{array}$ & $\begin{array}{l}\text { WFS } \\
n=36\end{array}$ & $\begin{array}{l}\text { Totals } \\
\mathrm{n}=138\end{array}$ \\
\hline \multirow[t]{26}{*}{ Crustacea } & Decapoda/ Caridea & Hoippolytidae & Lysmata intermedia & & & & 1 & & 1 \\
\hline & & Nematocarcinidae & $\begin{array}{l}\text { Nematocarcinus cf. } \\
\text { rotundus }\end{array}$ & & 6 & & & & 6 \\
\hline & & & Nematocarcinidae sp. & & & & 3 & & 3 \\
\hline & & Oplophoridae & Acanthephyra armata & & 3 & & & & 3 \\
\hline & & & Oplophorus gracilirostris & & 6 & 3 & 3 & 2 & 14 \\
\hline & & & Systellaspis debillis & & 9 & & & & 9 \\
\hline & & Palaemonidae & Periclimenes pandionis & & 6 & & 4 & 12 & 22 \\
\hline & & & Periclimenes sp.? & 4 & 23 & & 1 & & 28 \\
\hline & & & Palaemonella sp.? & & 1 & & & & 1 \\
\hline & & & Pontoniinae sp.? & & 2 & & & & 2 \\
\hline & & Pandalidae & Heterocarpus ensifer & & & & & 19 & 19 \\
\hline & & & Pantomus parvulus & & & & 2 & & 2 \\
\hline & & & Plesionika acanthonotus & & 71 & & 10 & & 81 \\
\hline & & & Plesionika ensis & & 1 & & 1 & & 2 \\
\hline & & & Plesionika holthuisi & & 52 & & 1 & & 53 \\
\hline & & & Plesionika martia & & 2 & & & & 2 \\
\hline & & & $\begin{array}{l}\text { Plesionika } \\
\text { polyacanthomerus }\end{array}$ & & 1 & & & & 1 \\
\hline & & & Plesionika tenuipes & & & & 1 & & 1 \\
\hline & & & Plesionika willisi & & & 6 & & 5 & 11 \\
\hline & & & Plesionika spp. & & 4 & & & 2 & 6 \\
\hline & & & Stylopandalus richardi & & 3 & & 1 & & 4 \\
\hline & & & Pandalidae sp. & & 2 & & & & 2 \\
\hline & & Pasiphaedae & Pasiphaea merriami & & 1 & & & & 1 \\
\hline & & Unknown & Caridea spp. & & 43 & 5 & 23 & 3 & 74 \\
\hline & Euphausiacea & Euphausiidae & Euphausiidae spp. & & 4 & & & 9 & 13 \\
\hline & Isopoda & Cirolanidae & Bathynomus giganteus & & 2 & & 1 & & 3 \\
\hline
\end{tabular}


Table 9.1. Overall diversity and abundance of invertebrates collected (all sampling methods combined) at five deep coral study sites (MC751, VK826, VK862, VK862/906, West Florida Slope (WFS) in the north-central and eastern Gulf of Mexico, 2008-2010, as part of the Lophelia II program - continued.

[n, number of stations where invertebrates were collected; see figure 9.1 for site locations]

\begin{tabular}{|c|c|c|c|c|c|c|c|c|c|}
\hline Phylum & Higher Classification & Family & Species & $\begin{array}{c}\text { MC751 } \\
n=2\end{array}$ & $\begin{array}{c}\text { VK826 } \\
n=53\end{array}$ & $\begin{array}{c}\text { VK862 } \\
n=13\end{array}$ & $\begin{array}{c}\text { VK862/VK906 } \\
n=34\end{array}$ & $\begin{array}{l}\text { WFS } \\
n=36\end{array}$ & $\begin{array}{l}\text { Totals } \\
\mathrm{n}=138 \\
\end{array}$ \\
\hline \multirow[t]{13}{*}{ Crustacea } & Isopoda & Unknown & Isopoda sp. & & & & & \multicolumn{2}{|r|}{9} \\
\hline & Mysida & Unknown & Mysida sp.? & & & & & 4 & 4 \\
\hline & \multirow[t]{2}{*}{ Amphipoda } & Phronimidae & Phronima cf. sendentaria & & 9 & & & & 9 \\
\hline & & Unknown & Hyperiidea spp. & & 5 & & & 5 & 10 \\
\hline & \multirow[t]{2}{*}{ Copepoda } & Sapphirinidae & Sapphirina sp. & & 6 & & & & 6 \\
\hline & & Unknown & Copepoda spp. & & & & & 13 & 13 \\
\hline & \multirow[t]{5}{*}{ Cirripedia } & Heteralepadidae & Heteralepas sp. & & & & & 11 & 11 \\
\hline & & Poecilasmatidae & Poecilasma litum & 1 & 17 & & 45 & 4 & 67 \\
\hline & & Scalpellidae & Litoscalpellum regina & & 12 & 1 & 1 & & 14 \\
\hline & & & Scillaelepas superba & & & & & 4 & 4 \\
\hline & & Unknown & Thoracica spp. & & 1 & & 15 & & 16 \\
\hline & \multirow[t]{2}{*}{ Ostracoda } & Halocypridoidea & Alacia sp.? & & & & 4 & & 4 \\
\hline & & Unknown & Ostracoda spp. & & & & 4 & 4 & 8 \\
\hline \multirow[t]{14}{*}{ Echinodermata } & \multirow[t]{14}{*}{ Asterozoa/ Asteroidea } & Asteriidae & Sclerasterias contorta & 1 & 1 & & & & 2 \\
\hline & & & Stephanasterias albula & & & & & 1 & 1 \\
\hline & & Astropectinidae & $\begin{array}{l}\text { Astropecten } \mathrm{cf} . \\
\text { americanus }\end{array}$ & & & & 22 & & 22 \\
\hline & & & Psilaster andromeda & & 1 & & & & 1 \\
\hline & & & Psilaster cassiope & & 3 & & & & 3 \\
\hline & & Goniasteridae & Peltaster placenta & & & & & 1 & 1 \\
\hline & & & Goniasteridae sp. & & & & 1 & 1 & 2 \\
\hline & & Labidiasteridae & Coronaster briareus & & 2 & 1 & & & 3 \\
\hline & & Novodiniidae & Novodinia antillensis & & & 4 & 2 & & 6 \\
\hline & & Odontasteridae & Odontaster robustus & & 2 & 1 & & & 3 \\
\hline & & & Odontaster hispidus & & & & 3 & & 3 \\
\hline & & Ophidiasteridae & Hacelia superba & & & & 1 & & 1 \\
\hline & & Pseudarchasteridae & Pseudarchaster gracilis & & 1 & & & & 1 \\
\hline & & Zoroasteridae & Doraster constellatus & & 1 & & 1 & & 2 \\
\hline
\end{tabular}


Table 9.1. Overall diversity and abundance of invertebrates collected (all sampling methods combined) at five deep coral study sites (MC751, VK826, VK862, VK862/906, West Florida Slope (WFS) in the north-central and eastern Gulf of Mexico, 2008-2010, as part of the Lophelia II program - continued.

[n, number of stations where invertebrates were collected; see figure 9.1 for site locations]

\begin{tabular}{|c|c|c|c|c|c|c|c|c|c|}
\hline Phylum & Higher Classification & Family & Species & $\begin{array}{c}\mathrm{MC751} \\
\mathrm{n}=2\end{array}$ & $\begin{array}{c}\text { VK826 } \\
n=53\end{array}$ & $\begin{array}{c}\text { VK862 } \\
n=13\end{array}$ & $\begin{array}{c}\text { VK862/VK906 } \\
n=34\end{array}$ & $\begin{array}{l}\text { WFS } \\
n=36\end{array}$ & $\begin{array}{l}\text { Totals } \\
\mathrm{n}=138\end{array}$ \\
\hline \multirow[t]{20}{*}{ Echinodermata } & Asterozoa/ Asteroidea & Zoroasteridae & Zoroaster fulgens & & 10 & & & & 10 \\
\hline & & Unknown & Asteroidea spp. & & 3 & & 26 & & 29 \\
\hline & Asterozoa/ Ophiuroidea & Asteroschematidae & Ophiocreas glutinosum & 6 & & & 4 & & 10 \\
\hline & & Unknown & Ophiocreas spinulosus & 7 & 15 & & & & 22 \\
\hline & & Gorgonocephalidae & Asterogomphus vallatus & 3 & 10 & 2 & 5 & & 20 \\
\hline & & Ophiacanthidae & Ophiacantha cf. bidentata & & & & & 26 & 26 \\
\hline & & Unknown & Ophiuroidea spp. & & 17 & 1 & 18 & 16 & 52 \\
\hline & Crinoidea/ Comatulida & Antedonidae & Antedonidae spp. & & 4 & 1 & 1 & & 6 \\
\hline & & Antedonidae & Comatonia cristata & 1 & 2 & & 1 & & 4 \\
\hline & Echinoidea/ Cidaroida & Cidaridae & Cidaris abyssicola & & & & 6 & & 6 \\
\hline & & & Cidaris rugosa & 1 & 12 & 12 & 24 & & 49 \\
\hline & Echinoidea/ Euechinoidea & Brissidae & Brissopsis atlantica & & & & 1 & & 1 \\
\hline & & Echinidae & Gracilechinus alexandri & & 1 & 1 & & 1 & 3 \\
\hline & & & Gracilechinus gracilis & & & & 1 & 1 & 2 \\
\hline & & & Echinus tylodes & & 14 & & 4 & 2 & 20 \\
\hline & & Echinocyamidae & $\begin{array}{l}\text { Echinocyamus } \\
\text { grandiporus }\end{array}$ & & & & & 1 & 1 \\
\hline & Holothuroidea/ Molpadida & Molpadiidae & Molpadia cubana & & 3 & & & & 3 \\
\hline & & & Molpadia musculus & & 19 & & & & 19 \\
\hline & & & Molpadia parva & & 5 & & & & 5 \\
\hline & $\begin{array}{l}\text { Holothuroidea/ } \\
\text { Asphidochirotida }\end{array}$ & Synallactidae & Amphigymnas bahamensis & 1 & 13 & & 1 & & 15 \\
\hline \multirow[t]{4}{*}{ Tunicata } & Ascidiacea & Synallactidae & Ascidiacea sp. & & & & & 1 & 1 \\
\hline & Thaliacea/ Pyrosomatida & Pyrosomatidae & Pyrosoma atlanticum & & 4 & & 1 & & 5 \\
\hline & Thaliacea/ Salpida & Salpidae & Salpa spp. & & 2 & & & 2 & 4 \\
\hline & & & & 95 & 1,732 & 192 & 1,161 & 491 & 3,671 \\
\hline
\end{tabular}


Table 9.2. Invertebrate species and number of individuals collected at the surface over four deep coral study sites (VK826, VK862, VK862/VK906, and West Florida Slope) in the north-central and eastern Gulf of Mexico, 2008-2010, as part of the Lophelia II program.

[WFS, West Florida Slope; $\mathrm{n}=$ number of surface stations where invertebrate specimens were collected. No surface collections were made at MC751. See figure 9.1 for site locations]

\begin{tabular}{|c|c|c|c|c|c|c|c|c|}
\hline Phylum & Higher Classification & Family & Species & $\begin{array}{c}\text { VK826 } \\
n=2\end{array}$ & $\begin{array}{c}\text { VK862 } \\
n=1\end{array}$ & $\begin{array}{l}\text { VK862/VK906 } \\
n=5\end{array}$ & $\begin{array}{c}\text { WFS } \\
n=4\end{array}$ & $\begin{array}{c}\text { Totals } \\
\mathrm{n}=12\end{array}$ \\
\hline \multirow[t]{3}{*}{ Cnidaria } & Hydrozoa/ Hydroidolina & Aequoreidae & $\begin{array}{l}\text { Aequorea } \mathrm{cf} . \\
\text { macrodactyla }\end{array}$ & & & & 1 & 1 \\
\hline & & Campanulariidae & Obelia sp.? & & & 2 & & 2 \\
\hline & & Unknown & Hydrozoa spp. & 12 & 1 & 3 & 1 & 17 \\
\hline Ctenophora & Nuda/Beroida & Beroidae & Beroe sp. & & & 1 & & 1 \\
\hline \multirow[t]{4}{*}{ Mollusca } & Gastropoda/ Pterotracheoidea & Cariiniidae & Carinaria cf. lamarki & & & & 3 & 3 \\
\hline & Gastropoda/ Euthecosomata & Cavolinidae & Cavolinia cf. tridenata & & & & 8 & 8 \\
\hline & $\begin{array}{l}\text { Cephalopoda/ } \\
\text { Decapodiformes }\end{array}$ & Unknown & Decapodiformes spp. & & & 4 & & 4 \\
\hline & Gastropoda/ Gymnosomata & Unknown & Gymnosomata sp? & & & & 8 & 8 \\
\hline \multirow[t]{9}{*}{ Crustacea } & Decapoda/ Dendrobranchiata & Sergestidae & Sergestes spp. & & & & 1 & 1 \\
\hline & Decapoda/ Caridea & Unknown & Caridea spp. & & & 3 & & 3 \\
\hline & Decapoda/ Brachyura & Unknown & Brachyura spp. & & & 2 & & 2 \\
\hline & Euphausiacea & Euphausiidae & Euphausiidae spp. & & & & 9 & 9 \\
\hline & Mysida & Mysida sp.? & Mysida sp.? & & & & 4 & 4 \\
\hline & Peracarida/ Amphipoda & Phronimidae & Phronima cf. sendentaria & 3 & & & & 3 \\
\hline & & Unknown & Hyperiidea spp. & & & & 4 & 4 \\
\hline & Ostracoda & Halocyprididae & Alacia sp.? & & & 4 & & 4 \\
\hline & & Unknown & Ostracoda spp. & & & 4 & 4 & 8 \\
\hline \multirow[t]{2}{*}{ Tunicata } & Thaliacea/ Salpida & Salpidae & Salpa spp. & & & & 2 & 2 \\
\hline & & & & 15 & 1 & 23 & 45 & 84 \\
\hline
\end{tabular}


Table 9.3. Invertebrate species and number of individuals collected in midwater over four deep coral study sites (VK826, VK862,

VK862/VK906, and West Florida Slope) in the north-central and eastern Gulf of Mexico, 2008-2010, as part of the Lophelia II program.

[WFS, West Florida Slope; $\mathrm{n}=$ number of stations where invertebrate specimens were collected. No midwater collections were made at MC751. See figure 9.1 for site locations]

\begin{tabular}{|c|c|c|c|c|c|c|c|c|}
\hline Phylum & Higher Classification & Family & Species & $\begin{array}{c}\text { VK826 } \\
n=23\end{array}$ & $\begin{array}{c}\text { VK862 } \\
n=7\end{array}$ & $\begin{array}{c}\text { VK862/VK906 } \\
n=6\end{array}$ & $\begin{array}{l}\text { WFS } \\
n=18\end{array}$ & $\begin{array}{c}\text { Totals } \\
\mathrm{n}=54\end{array}$ \\
\hline \multirow[t]{7}{*}{ Cnidaria } & Hydrozoa/ Hydroidolina & Hydroidolina & Leptothecata spp. & 4 & & & & 4 \\
\hline & & Aequoreidae & Aequorea cf. macrodactyla & 6 & & & 1 & 7 \\
\hline & & & Zygocanna vagans & 4 & & 2 & & 6 \\
\hline & & Hippopodiidae & Hippopodius hippopus & 5 & & 1 & 1 & 7 \\
\hline & & Unknown & Siphonophorae spp. & 1 & 1 & & 2 & 4 \\
\hline & & Unknown & Hydrozoa spp. & & 1 & 6 & 36 & 43 \\
\hline & Scyphozoa & Pelagiidae & Pelagia noctiluca & 12 & & & & 12 \\
\hline \multirow[t]{16}{*}{ Mollusca } & $\begin{array}{l}\text { Cephalopoda/ } \\
\text { Decapodiformes }\end{array}$ & Chiroteuthidae & Chiroteuthis mega & & & & 1 & 1 \\
\hline & & Cranchiidae & Cranchia cf. scabra & & & & 5 & 5 \\
\hline & & & Cranchia sp. & & & 1 & & 1 \\
\hline & & Enoploteuthidae & Enoploteuthidae sp. & & & & 1 & 1 \\
\hline & & Lycoteuthidae & Lycoteuthidae sp. & & & & 1 & 1 \\
\hline & & & Pyroteuthidae sp. & & & & 7 & 7 \\
\hline & & Sepiolidae & Heteroteuthis cf. dispar & 1 & & & & 1 \\
\hline & & Tremoctopodidae & Tremoctopodidae sp. & 9 & 3 & 1 & 6 & 19 \\
\hline & & Unknown & Decapodiformes sp. & & & & 1 & 1 \\
\hline & $\begin{array}{l}\text { Cephalopoda/ } \\
\text { Octopodiformes }\end{array}$ & Unknown & Octopodiformes spp. & & & & 2 & 2 \\
\hline & $\begin{array}{l}\text { Gastropoda/ } \\
\text { Opisthobranchia }\end{array}$ & Cymbuliidae & Cymbuliidae sp.? & & & & 13 & 13 \\
\hline & & Phylliroidae & Phylliroe cf. atlantica & & & & 1 & 1 \\
\hline & $\begin{array}{l}\text { Gastropoda/ } \\
\text { Pterotracheoidea }\end{array}$ & Cariiniidae & Carinaria cf. lamarki & 6 & & 1 & & 7 \\
\hline & Gastropoda/ Euthecosomata & Cavolinidae & Cavolinia cf. tridenata & 6 & & & & 6 \\
\hline & & Cavolinidae & Clio cf. recurva & 3 & & & & 3 \\
\hline & & Cavolinidae & Diacria cf. trispinosa & 7 & & & & 7 \\
\hline
\end{tabular}


Table 9.3. Invertebrate species and numbers of individuals collected in midwater over four deep coral study sites (VK826, VK862, VK862/906, and West Florida Slope) in the north-central and eastern Gulf of Mexico, 2008-2010, as part of the Lophelia II program - continued.

[WFS, West Florida Slope; $n=$ number of stations where invertebrate specimens were collected. No midwater collections were made at MC751. See figure 9.1 for site locations]

\begin{tabular}{|c|c|c|c|c|c|c|c|c|}
\hline Phylum & Higher Classification & Family & Species & $\begin{array}{c}\text { VK826 } \\
n=23\end{array}$ & $\begin{array}{c}\text { VK862 } \\
n=7\end{array}$ & $\begin{array}{c}\text { VK862/VK906 } \\
n=6\end{array}$ & $\begin{array}{l}\text { WFS } \\
n=18\end{array}$ & $\begin{array}{c}\text { Totals } \\
n=54\end{array}$ \\
\hline \multirow[t]{25}{*}{ Crustacea } & Decapoda/ Achelata & Scyllaridae & Scyllaridae spp. & 1 & & & 9 & 10 \\
\hline & Decapoda/ Brachyura & Unknown & Brachyura spp. & & & 2 & & 2 \\
\hline & Decapoda/ Dendrobranchiata & Penaeidae & Penaeidae spp. & 2 & & & 1 & 3 \\
\hline & & Sergestidae & Allosergestes sp. & 4 & & & & 4 \\
\hline & & & Deosergestes sp. & & & 1 & & 1 \\
\hline & & & Sergestes hansjacobi & & 39 & & & 39 \\
\hline & & & Sergestes spp. & & 53 & 13 & 3 & 69 \\
\hline & & & Sergia cf. grandis & & & 1 & & 1 \\
\hline & & & Sergia hansjacobi & 5 & & & & 5 \\
\hline & & & Sergia cf. splendens & & & 2 & & 2 \\
\hline & & & Sergia splendens & 1 & & & & 1 \\
\hline & & & Sergia talismani & 3 & & & & 3 \\
\hline & & & Sergia spp. & 4 & 8 & & & 12 \\
\hline & Decapoda/ Caridea & Oplophoridae & Oplophorus gracilirostris & & 3 & 2 & 2 & 7 \\
\hline & & & Systellaspis debillis & & & 1 & & 1 \\
\hline & & Pandalidae & Plesionika willisi & & 6 & & 2 & 8 \\
\hline & & & Stylopandalus richardi & & & 1 & & 1 \\
\hline & & & Pandalidae sp. & 1 & & & & 1 \\
\hline & & Pasiphaedae & Pasiphaea merriami & 1 & & & & 1 \\
\hline & & Unknown & Caridea spp. & & & & 3 & 3 \\
\hline & Euphausiacea & Euphausiidae & Euphausiidae spp. & 4 & & & & 4 \\
\hline & Amphipoda & Phronimidae & Phronima cf. sendentaria & 6 & & & & 6 \\
\hline & & Unknown & Hyperiidea spp. & 4 & & & 1 & 5 \\
\hline & Copepoda & Sapphirinidae & Sapphirina sp. & 6 & & & & 6 \\
\hline & & Unknown & Copepoda spp. & & & & 13 & 13 \\
\hline Echinodermata & Echinoidea/ Euechinoidea & Echinocyamidae & Echinocyamus grandiporus & & & & 1 & 1 \\
\hline
\end{tabular}


Table 9.3. Invertebrate species and numbers of individuals collected in midwater over four deep coral study sites (VK826, VK862, VK862/906, and West Florida Slope) in the north-central and eastern Gulf of Mexico, 2008-2010, as part of the Lophelia II program - continued.

[WFS, West Florida Slope; $\mathrm{n}=$ number of stations where invertebrate specimens were collected. No midwater collections were made at MC751. See figure 9.1 for site locations]

\begin{tabular}{|c|c|c|c|c|c|c|c|c|}
\hline Phylum & Higher Classification & Family & Species & $\begin{array}{c}\text { VK826 } \\
n=23\end{array}$ & $\begin{array}{c}\text { VK862 } \\
n=7\end{array}$ & $\begin{array}{c}\text { VK862/VK906 } \\
n=6\end{array}$ & $\begin{array}{l}\text { WFS } \\
n=18\end{array}$ & $\begin{array}{c}\text { Totals } \\
\mathrm{n}=54\end{array}$ \\
\hline \multirow[t]{3}{*}{ Tunicata } & Thaliacea/ Pyrosomatida & Pyrosomatidae & Pyrosoma atlanticum & 4 & & & & 4 \\
\hline & Thaliacea/ Salpida & Salpidae & Salpa spp. & 3 & & & & 3 \\
\hline & & & & 124 & 114 & 38 & 113 & 389 \\
\hline
\end{tabular}


Table 9.4. Invertebrate species and number of individuals collected on the bottom at five deep coral study sites (MC751, VK826, VK862, VK862/VK906, and West Florida Slope) in the north-central and eastern Gulf of Mexico, 2008-2010, as part of the Lophelia II program.

[WFS, West Florida Slope; $\mathrm{n}=$ number of stations where invertebrate specimens were collected. See figure 9.1 for site locations]

\begin{tabular}{|c|c|c|c|c|c|c|c|c|c|}
\hline Phylum & Higher Classification & Family & Species & $\begin{array}{c}\text { MC751 } \\
n=2\end{array}$ & $\begin{array}{c}\text { VK826 } \\
n=27\end{array}$ & $\begin{array}{c}\text { VK862 } \\
n=5\end{array}$ & $\begin{array}{c}\text { VK862/VK906 } \\
n=23\end{array}$ & $\begin{array}{l}\text { WFS } \\
n=14\end{array}$ & $\begin{array}{c}\text { Totals } \\
\mathrm{n}=71\end{array}$ \\
\hline \multirow[t]{3}{*}{ Porifera } & Demospongiae & Stylocordylidae? & Stylocordyla sp.? & & 1 & 1 & 4 & & 6 \\
\hline & & Unknown & Demospongiae spp. & 1 & 5 & 1 & 5 & 2 & 14 \\
\hline & Hexactinellida & Aphrocallistidae & Aphrocallistes beatrix & & 4 & & & 9 & 13 \\
\hline \multirow[t]{26}{*}{ Cnidaria } & Anthozoa/ Actiniaria & Actinostolidae? & Actinostolidae sp. ? & & 10 & 10 & 3 & & 23 \\
\hline & & Actinoscyphiidae & Actinoscyphia cf. saginata & 1 & 17 & 1 & 6 & 1 & 26 \\
\hline & & Halicuriidae & Halcurias pilatus & 6 & & 1 & 20 & & 27 \\
\hline & & Hormathiidae & Actinauge longicornis & & 28 & 3 & 53 & 2 & 86 \\
\hline & & & Adamsia obvolva & & 9 & & & & 9 \\
\hline & & Unknown & Hormathiidae sp. & & 19 & & 4 & & 23 \\
\hline & & Unknown & Actiniaria spp. & 2 & 17 & 1 & 33 & 37 & 73 \\
\hline & Anthozoa/ Cerianthiaria & Cerianthidae & Cerianthidae sp. & & 1 & & & & 1 \\
\hline & Anthozoa/ Antipatharia & Cladopathidae & Sibopathes macrospina & & 4 & & & & 4 \\
\hline & & Leiopathidae & Leiopathes sp. A & & 29 & 3 & 4 & 1 & 37 \\
\hline & & & Leiopathes sp. B & & 6 & & & & 6 \\
\hline & & & Leiopathes sp. C & & 1 & & & & 1 \\
\hline & & & Leiopathes sp. D & & & & & 1 & 1 \\
\hline & & & Leiopathes spp. & & 13 & & 10 & 1 & 24 \\
\hline & Anthozoa/ Scleractinia & Caryophylliidae & Caryophyllia sp. & & 3 & & & & 3 \\
\hline & & & Lophelia pertusa & 2 & 23 & 1 & 5 & 23 & 54 \\
\hline & & & Enallopsammia profunda & & & & & 1 & 1 \\
\hline & & Dendrophylliidae & Thecopsammia socialis & & 6 & & & 1 & 7 \\
\hline & & Flabellidae & Javania cailleti & & 2 & 1 & & & 3 \\
\hline & & Oculinidae & Madrepora oculata & & & 1 & & 2 & 3 \\
\hline & Anthozoa/ Zooantidea & Parazoanthiidae & Parazoanthiidae sp. & & & & & 1 & 1 \\
\hline & & & Salvia sp.? & & & & & 1 & 1 \\
\hline & & Unknown & Zoanthidea sp. & 2 & 11 & 2 & 4 & 2 & 21 \\
\hline & Anthozoa/ Octocorallia & Acanthogorgiidae & Acanthogorgia sp. & & 2 & & & & 2 \\
\hline & & Alcyoniidae & Anthomastus sp. & & 1 & & 5 & 2 & 8 \\
\hline & & & Xeniidae sp.? & & & & 1 & & 1 \\
\hline
\end{tabular}


Table 9.4. Invertebrate species and numbers of individuals collected on the bottom at five deep coral study sites (MC751, VK826, VK862, VK862/906, and West Florida Slope) in the north-central and eastern Gulf of Mexico, 2008-2010, as part of the Lophelia II program continued.

[WFS, West Florida Slope; $\mathrm{n}=$ number of stations where invertebrate specimens were collected. See figure 9.1 for site locations]

\begin{tabular}{|c|c|c|c|c|c|c|c|c|c|}
\hline Phylum & Higher Classification & Family & Species & $\begin{array}{c}\mathrm{MC751} \\
\mathrm{n}=2\end{array}$ & $\begin{array}{c}\text { VK826 } \\
n=27\end{array}$ & $\begin{array}{c}\text { VK862 } \\
n=5\end{array}$ & $\begin{array}{c}\text { VK862/VK906 } \\
n=23\end{array}$ & $\begin{array}{l}\text { WFS } \\
n=14\end{array}$ & $\begin{array}{c}\text { Totals } \\
\mathrm{n}=71\end{array}$ \\
\hline \multirow[t]{15}{*}{ Cnidaria } & \multirow[t]{11}{*}{ Anthozoa/ Octocorallia } & Unknown & Alcyonacea sp. & 1 & & & 8 & 3 & 12 \\
\hline & & Anthotelidae & Anthothela sp. & & & & & 2 & 2 \\
\hline & & Clavulariidae & Clavularia sp. & & 1 & & & & 1 \\
\hline & & Isididae & $\begin{array}{l}\text { Chelidonisis aurantiaca } \\
\text { mexicana }\end{array}$ & 1 & & & & & 1 \\
\hline & & & Lepidisis sp. & & & & 5 & & 5 \\
\hline & & Nephtheidae & Nephtheidae sp. & & & & & 3 & 3 \\
\hline & & Primnoidae & Callogorgia americana & 1 & & & & & 1 \\
\hline & & & Callogorgia sp. & 10 & 15 & 1 & 1 & 1 & 28 \\
\hline & & & Plumarella dichotoma & 1 & & & & & 1 \\
\hline & & & Plumarella sp. & & 1 & & & 9 & 10 \\
\hline & & Plexauridae & Plexauridae sp. & 8 & 2 & & 2 & 10 & 22 \\
\hline & \multirow[t]{4}{*}{ Hydrozoa/ Hydroidolina } & Mitrocomidae & Earleria quadrata? & & & & & 1 & 1 \\
\hline & & Stylasteridae & Stylaster erubescens & & & & & 3 & 3 \\
\hline & & & Stylaster sp. & & & & & 2 & 2 \\
\hline & & Unknown & Hydroidolina spp. & 2 & 10 & & & 8 & 20 \\
\hline \multirow[t]{11}{*}{ Mollusca } & \multirow[t]{2}{*}{ Bivalvia } & Mytilidae & Bathymodiolus sp. & & 19 & & & & 19 \\
\hline & & Unknown & Bivalvia sp. & & 29 & 1 & 11 & & 41 \\
\hline & \multirow[t]{2}{*}{$\begin{array}{l}\text { Cephalopoda/ } \\
\text { Decapodiformes }\end{array}$} & Sepiolidae & Heteroteuthis cf. dispar & & & & 1 & & 1 \\
\hline & & Unknown & Decapodiformes spp. & & 4 & & 7 & & 11 \\
\hline & \multirow[t]{2}{*}{$\begin{array}{l}\text { Cephalopoda/ } \\
\text { Octopodiformes }\end{array}$} & Argonautidae & Argonauta argo & & & & 1 & & 1 \\
\hline & & Unknown & Octopodiformes spp. & & 2 & & & & 2 \\
\hline & \multirow[t]{5}{*}{ Gastropoda/ Neogastropoda } & Muricidae & Coralliophila richardi & 5 & 6 & 2 & 2 & 10 & 25 \\
\hline & & & Muricidae sp? & & & 1 & & & 1 \\
\hline & & Scaphellinae & Scaphella dubia kieneri & & & & 2 & & 2 \\
\hline & & Turridae & Gemmula periscelida & & & & 64 & & 64 \\
\hline & & Unknown & Neogastropoda spp. & & 2 & & 10 & & 12 \\
\hline
\end{tabular}


Table 9.4. Invertebrate species and numbers of individuals collected on the bottom at five deep coral study sites (MC751, VK826, VK862, VK862/906, and West Florida Slope) in the north-central and eastern Gulf of Mexico, 2008-2010, as part of the Lophelia II program continued.

[WFS, West Florida Slope; $\mathrm{n}=$ number of stations where invertebrate specimens were collected. See figure 9.1 for site locations]

\begin{tabular}{|c|c|c|c|c|c|c|c|c|c|}
\hline Phylum & Higher Classification & Family & Species & $\begin{array}{c}\mathrm{MC} 751 \\
\mathrm{n}=2\end{array}$ & $\begin{array}{c}\text { VK826 } \\
n=27\end{array}$ & $\begin{array}{c}\text { VK862 } \\
n=5\end{array}$ & $\begin{array}{c}\text { VK862/VK906 } \\
n=23\end{array}$ & $\begin{array}{l}\text { WFS } \\
n=14\end{array}$ & $\begin{array}{c}\text { Totals } \\
n=71\end{array}$ \\
\hline \multirow[t]{5}{*}{ Mollusca } & $\begin{array}{l}\text { Gastropoda/ } \\
\text { Caenogastropoda }\end{array}$ & Xenophoridae & Onustus longleyi & & & & 2 & & 2 \\
\hline & Gastropoda/ Vetigastropoda & Calliostomatidae & Calliostoma sayanum & & & 1 & & & 1 \\
\hline & & Turbinidae & Gaza superba & & 16 & & 6 & & 22 \\
\hline & Gastropoda/ Opisthobranchia & Scaphandridae & Scaphander sp. & & & & 110 & & 110 \\
\hline & Polyplacophora/ Chitonida & Unknown & Chitonida sp. & & 1 & & & & 1 \\
\hline \multirow[t]{3}{*}{ Annelida } & Polychaeta/ Eunicida & Eunicidae & Eunicidae spp. & & 8 & 1 & 14 & 9 & 32 \\
\hline & Polychaeta/ Sabellida & Serpulidae & Serpulidae sp. & 1 & 1 & & & & 2 \\
\hline & & Unknown & Polychaeta spp. & & 1 & & & 1 & 2 \\
\hline Chelicerata & Pycnogonida & Unknown & Pycnogonida spp. & & 2 & & & 2 & 4 \\
\hline \multirow[t]{18}{*}{ Crustacea } & Decapoda/ Achelata & Polychelidae & Polycheles cf. perarmatus & & 2 & & & & 2 \\
\hline & & & Polycheles sculptus & & 3 & & & & 3 \\
\hline & & & Polycheles typhlops & & & & 1 & & 1 \\
\hline & & & Polycheles spp. & & 72 & & 11 & & 83 \\
\hline & Decapoda/ Astacidea & Nephropidae & Nephropsis aculeata & & 28 & & & & 28 \\
\hline & & & Nephropsis spp. & & 56 & & 1 & & 57 \\
\hline & Decapoda/ Anomura & Chirostylidae & Gastroptychus salvadori & & 1 & & & & 1 \\
\hline & & & Gastroptychus sp. & & & & 5 & 1 & 6 \\
\hline & & & Uroptychus sp.? & 2 & & & & & 2 \\
\hline & & Eumunididae & Eumunida picta & 10 & 22 & 4 & 17 & 17 & 70 \\
\hline & & Munididae & Agononida longipes & & & & 14 & & 14 \\
\hline & & & Munida iris & & 1 & 2 & 1 & & 4 \\
\hline & & & Munida media & & 1 & & & & 1 \\
\hline & & & Munida sanctipauli & & 2 & 2 & & 7 & 11 \\
\hline & & & Munida spp & 3 & 119 & 1 & 68 & & 191 \\
\hline & & & Munida valida & & 8 & & & & 8 \\
\hline & & Munidopsidae & Leiogalathea agassizii & & & & & 2 & 2 \\
\hline & & & Munidopsis expansa & & & & & 2 & 2 \\
\hline
\end{tabular}


Table 9.4. Invertebrate species and numbers of individuals collected on the bottom at five deep coral study sites (MC751, VK826, VK862, VK862/906, and West Florida Slope) in the north-central and eastern Gulf of Mexico, 2008-2010, as part of the Lophelia II program continued.

[WFS, West Florida Slope; $\mathrm{n}=$ number of stations where invertebrate specimens were collected. See figure 9.1 for site locations]

\begin{tabular}{|c|c|c|c|c|c|c|c|c|c|}
\hline Phylum & Higher Classification & Family & Species & $\begin{array}{c}\mathrm{MC751} \\
\mathrm{n}=2\end{array}$ & $\begin{array}{c}\text { VK826 } \\
n=27\end{array}$ & $\begin{array}{c}\text { VK862 } \\
n=5\end{array}$ & $\begin{array}{c}\text { VK862/VK906 } \\
n=23\end{array}$ & $\begin{array}{l}\text { WFS } \\
n=14\end{array}$ & $\begin{array}{c}\text { Totals } \\
\mathrm{n}=71\end{array}$ \\
\hline \multirow[t]{28}{*}{ Crustacea } & Decapoda/ Anomura & Munidopsidae & Munidopsis glabra & & & 1 & & & 1 \\
\hline & & & Munidopsis penescabra & & & & & 9 & 9 \\
\hline & & & Munidopsis robusta & & & & 9 & & 9 \\
\hline & & & Munidopsis spp. & 7 & 45 & 3 & 12 & 10 & 77 \\
\hline & & Unknown & Galatheoidea spp. & & 1 & & 30 & 1 & 32 \\
\hline & & Lithodidae & Paralomis cf. cubensis & & & & & 1 & 1 \\
\hline & & Parapaguridae & Parapagurus pictus & & 5 & & & & 5 \\
\hline & & Unknown & Paguroidea spp. & & 12 & & 3 & 3 & 18 \\
\hline & Decapoda/ Axiidea & Axiidae & Calocaris caribbaeus & & 6 & & & & 6 \\
\hline & Decapoda/ Brachyura & Gerionidae & Chaceon fenneri & & 2 & & & 1 & 3 \\
\hline & & & Chaceon quinquedens & & 2 & & & & 2 \\
\hline & & Goneplacidae & Bathyplax typhla & & 227 & & 22 & & 249 \\
\hline & & Inachidae & Dorhynchus thomsoni & & & & & 2 & 2 \\
\hline & & Inachoididae & Pyromaia arachna & & & & 8 & & 8 \\
\hline & & Majiidae & Rochinia crassa & 1 & 12 & & 4 & & 17 \\
\hline & & & Rochinia sp. & & 1 & & 1 & 1 & 3 \\
\hline & & & Rochinia tanneri & & 4 & & 11 & & 15 \\
\hline & & & Rochinia umbonata & & & & & 3 & 3 \\
\hline & & & Majidae spp. & 1 & 11 & & & 2 & 14 \\
\hline & & Polybiidae & Bathynectes longispina & 1 & 6 & 1 & 10 & & 18 \\
\hline & & & Raymanninus schmitti & & 23 & & 20 & & 43 \\
\hline & & Portunidae & Achelous floridanus & & & 1 & & & 1 \\
\hline & & Raninidae & Lyreidus sp.? & & & & 4 & & 4 \\
\hline & & Trichopeltariidae & Trichopeltarion nobile & & 2 & & & & 2 \\
\hline & & Unknown & Brachyura spp. & & 54 & 1 & 25 & & 80 \\
\hline & Decapoda/ Dendrobranchiata & Aristeidae & Aristaeopsis edwardsiana & & 3 & & & & 3 \\
\hline & & & Aristeaus antillensis & & 23 & & 15 & & 38 \\
\hline & & & Aristeidae spp. & & & & 19 & & 19 \\
\hline
\end{tabular}


Table 9.4. Invertebrate species and numbers of individuals collected on the bottom at five deep coral study sites (MC751, VK826, VK862, VK862/906, and West Florida Slope) in the north-central and eastern Gulf of Mexico, 2008-2010, as part of the Lophelia II program continued.

[WFS, West Florida Slope; $\mathrm{n}=$ number of stations where invertebrate specimens were collected. See figure 9.1 for site locations]

\begin{tabular}{|c|c|c|c|c|c|c|c|c|c|}
\hline Phylum & Higher Classification & Family & Species & $\begin{array}{c}\text { MC751 } \\
n=2\end{array}$ & $\begin{array}{c}\text { VK826 } \\
n=27\end{array}$ & $\begin{array}{c}\text { VK862 } \\
n=5\end{array}$ & $\begin{array}{c}\text { VK862/VK906 } \\
n=23\end{array}$ & $\begin{array}{l}\text { WFS } \\
n=14\end{array}$ & $\begin{array}{c}\text { Totals } \\
\mathrm{n}=71\end{array}$ \\
\hline \multirow[t]{26}{*}{ Crustacea } & $\begin{array}{l}\text { Decapoda/ } \\
\text { Dendrobranchiata }\end{array}$ & Benthesicymidae & Gennadas elegens & & 4 & & & & 4 \\
\hline & & & Gennadas sp. & & 2 & & & & 2 \\
\hline & & Penaeidae & Hepomadus glacialis? & 1 & & & & & 1 \\
\hline & & & Penaeopsis serrata & & & & 1 & & 1 \\
\hline & & & Parapeneus sp.? & & & & 18 & & 18 \\
\hline & & & Penaeidae spp. & & 28 & & & & 28 \\
\hline & & Solenoceridae & Hymenopenaeus debilis & & & & 1 & & 1 \\
\hline & & & Pleoticus robustus & & 65 & & 143 & & 208 \\
\hline & & Sergestidae & Sergestes spp. & & 2 & & & & 2 \\
\hline & & & Sergia cf. grandis & & 2 & & & & 2 \\
\hline & & & Sergia cf. laminata & & 1 & & & & 1 \\
\hline & & & Sergia spp. & & 3 & & & 1 & 4 \\
\hline & & & Sergestidae spp. & & 3 & & & & 3 \\
\hline & & Unknown & Dendrobranchiata spp. & & 2 & & & & 2 \\
\hline & Decapoda/ Caridea & Alpheidae & Alpheus lentiginosus & 1 & & & & & 1 \\
\hline & & & Alpheidae sp. ? & & & & & 2 & 2 \\
\hline & & Bathypalaemonellidae & Bathypalaemonella sp. & & 5 & & & 2 & 7 \\
\hline & & Crangonidae & Metacrangon agassizii & & 1 & & & & 1 \\
\hline & & & Parapontophilus gracilis & & 1 & & 1 & & 2 \\
\hline & & Eugonatonotidae & Eugonatonotus crassus & & & & & 2 & 2 \\
\hline & & Hoippolytidae & Lysmata intermedia & & & & 1 & & 1 \\
\hline & & Nematocarcinidae & $\begin{array}{l}\text { Nematocarcinus cf. } \\
\text { rotundus }\end{array}$ & & 6 & & & & 6 \\
\hline & & & Nematocarcinidae sp. & & & & 3 & & 3 \\
\hline & & Oplophoridae & Acanthephyra armata & & 3 & & & & 3 \\
\hline & & & Oplophorus gracilirostris & & 6 & & & & 6 \\
\hline & & & Systellaspis debillis & & 9 & & & & 9 \\
\hline
\end{tabular}


Table 9.4. Invertebrate species and numbers of individuals collected on the bottom at five deep coral study sites (MC751, VK826, VK862, VK862/906, and West Florida Slope) in the north-central and eastern Gulf of Mexico, 2008-2010, as part of the Lophelia II program continued.

[WFS, West Florida Slope; $\mathrm{n}=$ number of stations where invertebrate specimens were collected. See figure 9.1 for site locations]

\begin{tabular}{|c|c|c|c|c|c|c|c|c|c|}
\hline Phylum & Higher Classification & Family & Species & $\begin{array}{c}\mathrm{MC751} \\
\mathrm{n}=2\end{array}$ & $\begin{array}{c}\text { VK826 } \\
n=27\end{array}$ & $\begin{array}{c}\text { VK862 } \\
n=5\end{array}$ & $\begin{array}{c}\text { VK862/VK906 } \\
n=23\end{array}$ & $\begin{array}{l}\text { WFS } \\
n=14\end{array}$ & $\begin{array}{c}\text { Totals } \\
\mathrm{n}=71\end{array}$ \\
\hline \multirow[t]{25}{*}{ Crustacea } & Decapoda/ Caridea & Palaemonidae & Periclimenes pandionis & & 6 & & 4 & 12 & 22 \\
\hline & & & Periclimenes sp.? & 4 & 23 & & 1 & & 28 \\
\hline & & & Palaemonella sp.? & & 1 & & & & 1 \\
\hline & & & Pontoniinae sp.? & & 2 & & & & 2 \\
\hline & & Pandalidae & Heterocarpus ensifer & & & & & 19 & 19 \\
\hline & & & Pantomus parvulus & & & & 2 & & 2 \\
\hline & & & Plesionika acanthonotus & & 71 & & 10 & & 81 \\
\hline & & & Plesionika ensis & & 1 & & 1 & & 2 \\
\hline & & & Plesionika holthuisi & & 52 & & 1 & & 53 \\
\hline & & & Plesionika martia & & 2 & & & & 2 \\
\hline & & & $\begin{array}{l}\text { Plesionika } \\
\text { polyacanthomerus }\end{array}$ & & 1 & & & & 1 \\
\hline & & & Plesionika tenuipes & & & & 1 & & 1 \\
\hline & & & Plesionika willisi & & & & & 3 & 3 \\
\hline & & & Plesionika spp. & & 4 & & & 2 & 6 \\
\hline & & & Stylopandalus richardi & & 3 & & & & 3 \\
\hline & & & Pandalidae sp. & & 1 & & & & 1 \\
\hline & & Unknown & Caridea spp. & & 43 & 5 & 20 & & 68 \\
\hline & Isopoda & Cirolanidae & Bathynomus giganteus & & 2 & & 1 & & 3 \\
\hline & & Unknown & Isopoda sp. & & & & & 9 & 9 \\
\hline & Amphipoda & Unknown & Hyperiidea spp. & & 1 & & & & 1 \\
\hline & Cirripedia & Heteralepadidae & Heteralepas sp. & & & & & 11 & 11 \\
\hline & & Poecilasmatidae & Poecilasma litum & 1 & 17 & & 45 & 4 & 67 \\
\hline & & Scalpellidae & Litoscalpellum regina & & 12 & 1 & 1 & & 14 \\
\hline & & & Scillaelepas superba & & & & & 4 & 4 \\
\hline & & Unknown & Thoracica spp. & & 1 & & 15 & & 16 \\
\hline \multirow[t]{2}{*}{ Echinodermata } & Asterozoa/ Asteroidea & Asteriidae & Sclerasterias contorta & 1 & 1 & & & & 2 \\
\hline & & & Stephanasterias albula & & & & & 1 & 1 \\
\hline
\end{tabular}


Table 9.4. Invertebrate species and numbers of individuals collected on the bottom at five deep coral study sites (MC751, VK826, VK862, VK862/906, and West Florida Slope) in the north-central and eastern Gulf of Mexico, 2008-2010, as part of the Lophelia II program continued.

[WFS, West Florida Slope; $\mathrm{n}=$ number of stations where invertebrate specimens were collected. See figure 9.1 for site locations]

\begin{tabular}{|c|c|c|c|c|c|c|c|c|c|}
\hline Phylum & Higher Classification & Family & Species & $\begin{array}{c}\text { MC751 } \\
n=2\end{array}$ & $\begin{array}{c}\text { VK826 } \\
n=27\end{array}$ & $\begin{array}{c}\text { VK862 } \\
n=5\end{array}$ & $\begin{array}{c}\text { VK862/VK906 } \\
n=23\end{array}$ & $\begin{array}{l}\text { WFS } \\
n=14\end{array}$ & $\begin{array}{c}\text { Totals } \\
n=71\end{array}$ \\
\hline \multirow[t]{27}{*}{ Echinodermata } & Asterozoa/ Asteroidea & Astropectinidae & $\begin{array}{l}\text { Astropecten cf. } \\
\text { americanus }\end{array}$ & & & & 22 & & 22 \\
\hline & & & Psilaster andromeda & & 1 & & & & 1 \\
\hline & & & Psilaster cassiope & & 3 & & & & 3 \\
\hline & & Goniasteridae & Peltaster placenta & & & & & 1 & 1 \\
\hline & & & Goniasteridae $s p$ & & & & 1 & 1 & 2 \\
\hline & & Labidiasteridae & Coronaster briareus & & 2 & 1 & & & 3 \\
\hline & & Novodiniidae & Novodinia antillensis & & & 4 & 2 & & 6 \\
\hline & & Odontasteridae & Odontaster robustus & & 2 & 1 & & & 3 \\
\hline & & & Odontaster hispidus & & & & 3 & & 3 \\
\hline & & Ophidiasteridae & Hacelia superba & & & & 1 & & 1 \\
\hline & & Pseudarchasteridae & Pseudarchaster gracilis & & 1 & & & & 1 \\
\hline & & Zoroasteridae & Doraster constellatus & & 1 & & 1 & & 2 \\
\hline & & & Zoroaster fulgens & & 10 & & & & 10 \\
\hline & & Unknown & Asteroidea spp. & & 3 & & 26 & & 29 \\
\hline & Asterozoa/ Ophiuroidea & Asteroschematidae & Ophiocreas glutinosum & 6 & & & 4 & & 10 \\
\hline & & & Ophiocreas spinulosus & 7 & 15 & & & & 22 \\
\hline & & Gorgonocephalidae & Asterogomphus vallatus & 3 & 10 & 2 & 5 & & 20 \\
\hline & & Ophiacanthidae & Ophiacantha cf. bidentata & & & & & 26 & 26 \\
\hline & & Unknown & Ophiuroidea spp. & & 17 & 1 & 19 & 16 & 53 \\
\hline & Crinoidea/ Comatulida & Antedonidae & Antedonidae spp. & & 4 & 1 & 1 & & 6 \\
\hline & & & Comatonia cristata & 1 & 2 & & 1 & & 4 \\
\hline & Echinoidea/ Cidaroida & Cidaridae & Cidaris abyssicola & & & & 6 & & 6 \\
\hline & & & Cidaris rugosa & 1 & 12 & 12 & 24 & & 49 \\
\hline & Echinoidea/ Euechinoidea & Brissidae & Brissopsis atlantica & & & & 1 & & 1 \\
\hline & & Echinidae & Gracilechinus alexandri & & 1 & 1 & & 1 & 3 \\
\hline & & & Gracilecinus gracilis & & & & 1 & 1 & 2 \\
\hline & & & Echinus tylodes & & 14 & & 4 & 2 & 20 \\
\hline
\end{tabular}


Table 9.4. Invertebrate species and numbers of individuals collected on the bottom at five deep coral study sites (MC751, VK826, VK862, VK862/906, and West Florida Slope) in the north-central and eastern Gulf of Mexico, 2008-2010, as part of the Lophelia II program continued.

[WFS, West Florida Slope; $n=$ number of stations where invertebrate specimens were collected. See figure 9.1 for site locations]

\begin{tabular}{|c|c|c|c|c|c|c|c|c|c|}
\hline Phylum & Higher Classification & Family & Species & $\begin{array}{c}M C 751 \\
n=2\end{array}$ & $\begin{array}{c}\text { VK826 } \\
\mathrm{n}=27\end{array}$ & $\begin{array}{c}\text { VK862 } \\
n=5\end{array}$ & $\begin{array}{c}\text { VK862/VK906 } \\
n=23\end{array}$ & $\begin{array}{l}\text { WFS } \\
n=14\end{array}$ & $\begin{array}{c}\text { Totals } \\
\mathrm{n}=71\end{array}$ \\
\hline \multirow[t]{4}{*}{ Echinodermata } & Holothuroidea/ Molpadida & Molpadiidae & Molpadia cubana & & 3 & & & & 3 \\
\hline & & & Molpadia musculus & & 19 & & & & 19 \\
\hline & & & Molpadia parva & & 5 & & & & 5 \\
\hline & $\begin{array}{l}\text { Holothuroidea/ } \\
\text { Asphidochirotida }\end{array}$ & Synallactidae & Amphigymnas bahamensis & 1 & 13 & & 1 & & 15 \\
\hline \multirow[t]{3}{*}{ Tunicata } & Ascidiacea & & Ascidiacea sp. & & & & & 1 & 1 \\
\hline & Thaliacea/ Pyrosomatida & Pyrosomatidae & Pyrosoma atlanticum & & & & 1 & & 1 \\
\hline & & & & 95 & 1,594 & 77 & 1,102 & 333 & 3,201 \\
\hline
\end{tabular}




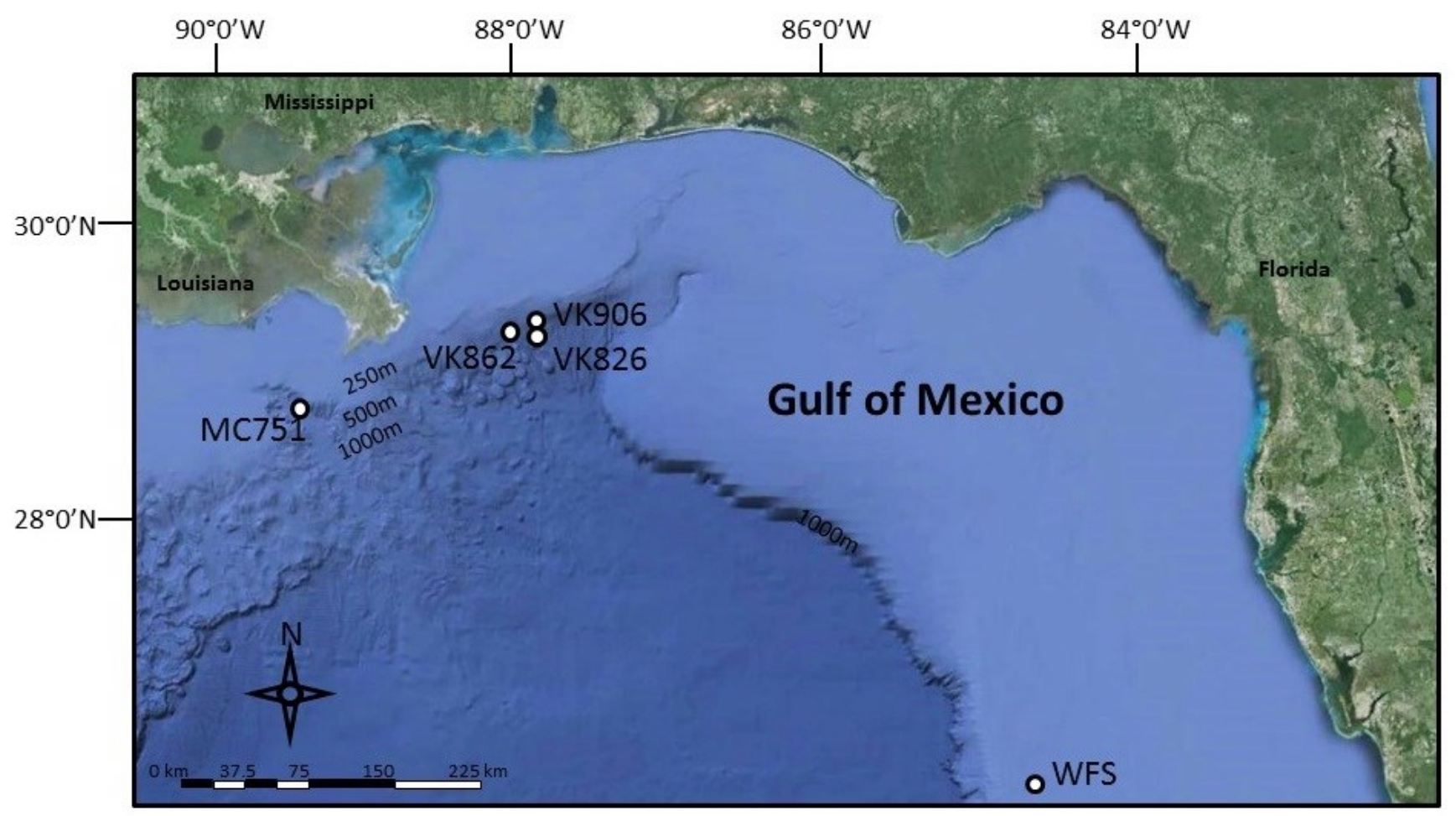

Figure 9.1 Map of study area in the North-central and eastern Gulf of Mexico showing location of sampling sites during the Lophelia II program. Base map data from Google, 2012. [VK906, VK862, VK826, and MC751 refer to Bureau of Ocean Energy Management lease blocks. WFS, West Florida Slope.] 


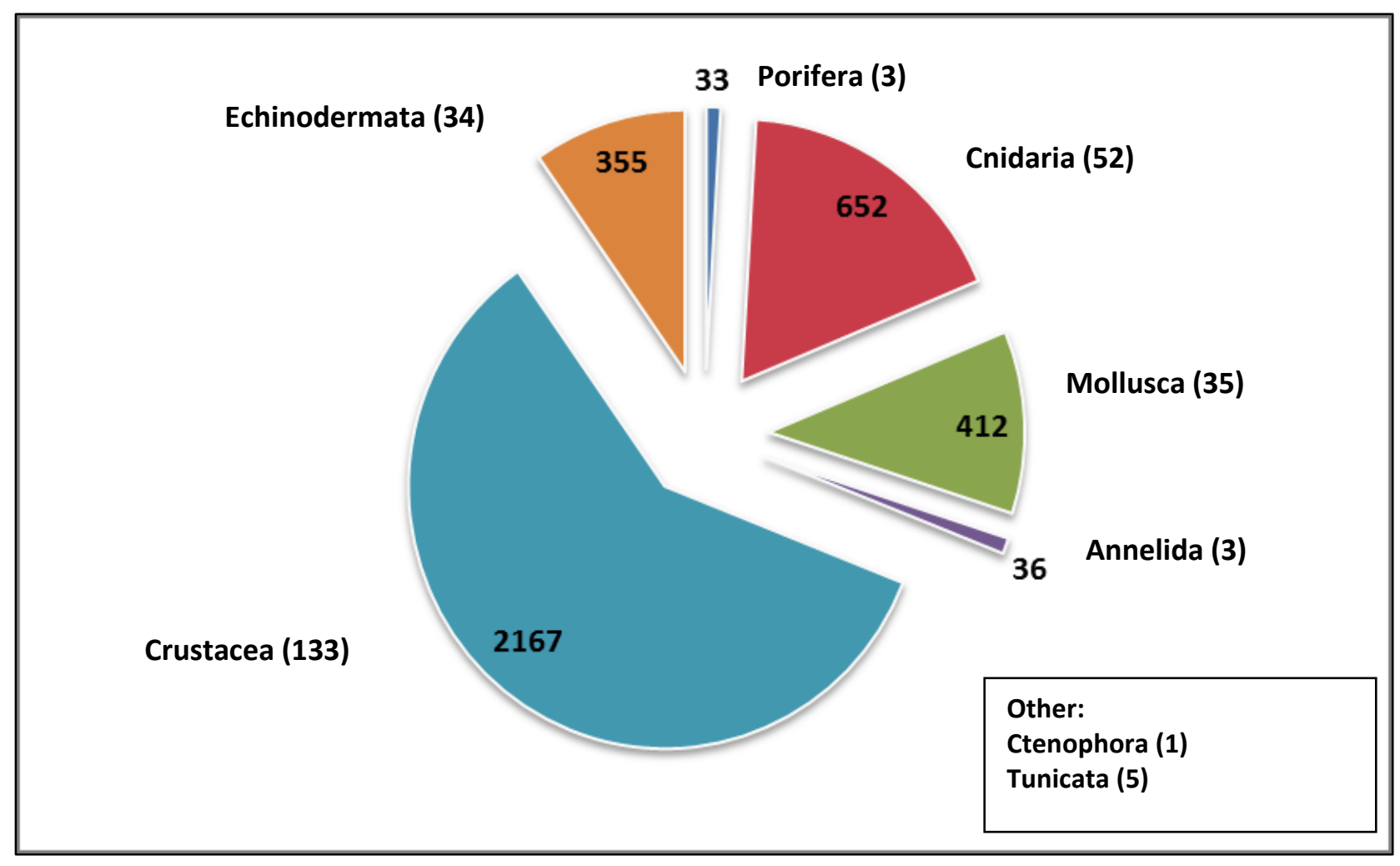

Figure 9.2 Overall (all sampling sites from all cruises combined) species richness and abundance of major invertebrate taxa during the Lophelia II program. [Number of species is shown in parentheses. Only abundance values greater than 30 are provided in figure.] 


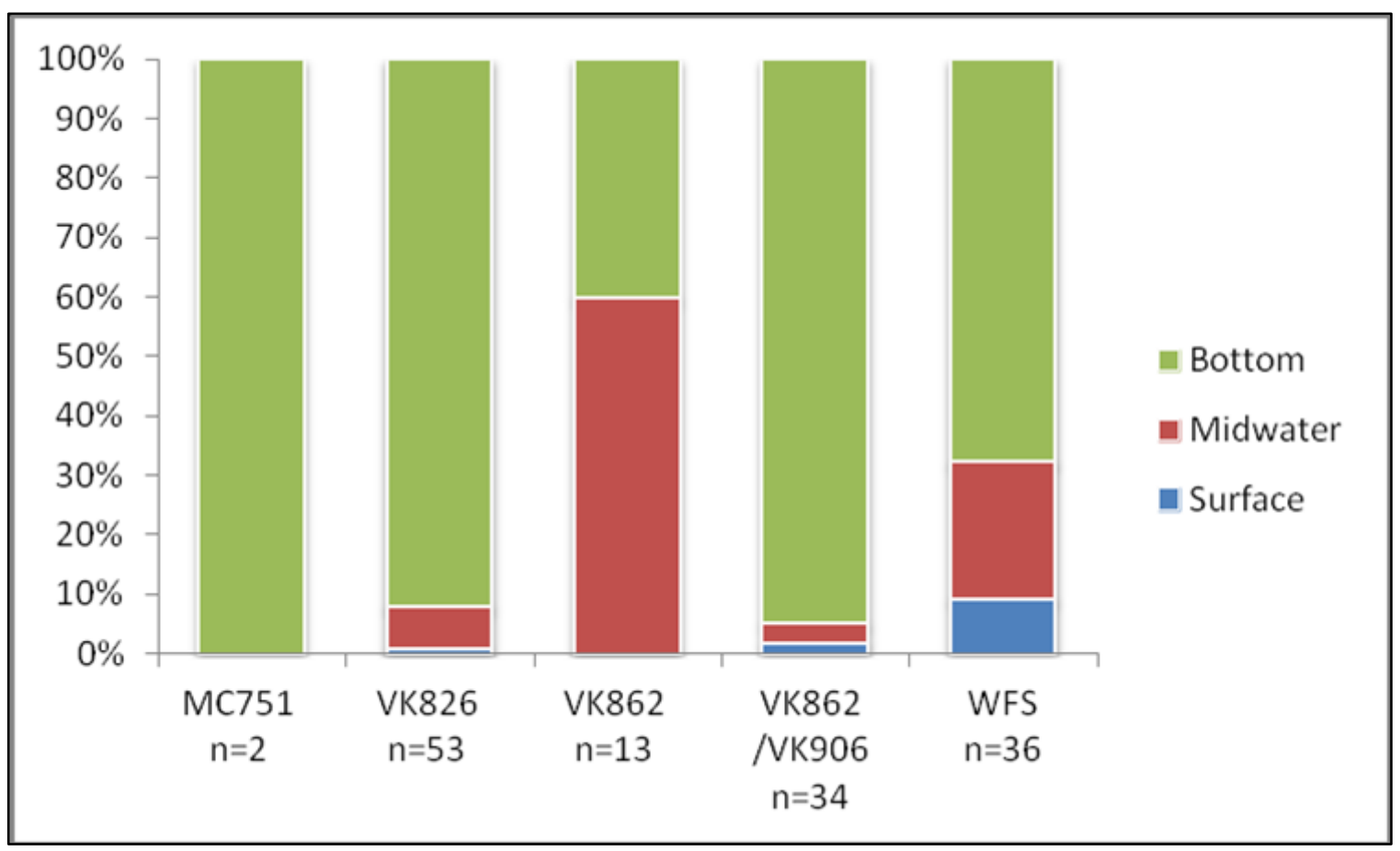

Figure 9.3 Percentage of collections represented in each vertical zone (all cruises combined) based on number of specimens collected at each site in surface, midwater, and bottom samples during the Lophelia II program. $[\mathrm{n}=$ number of stations where invertebrate specimens were collected.] 


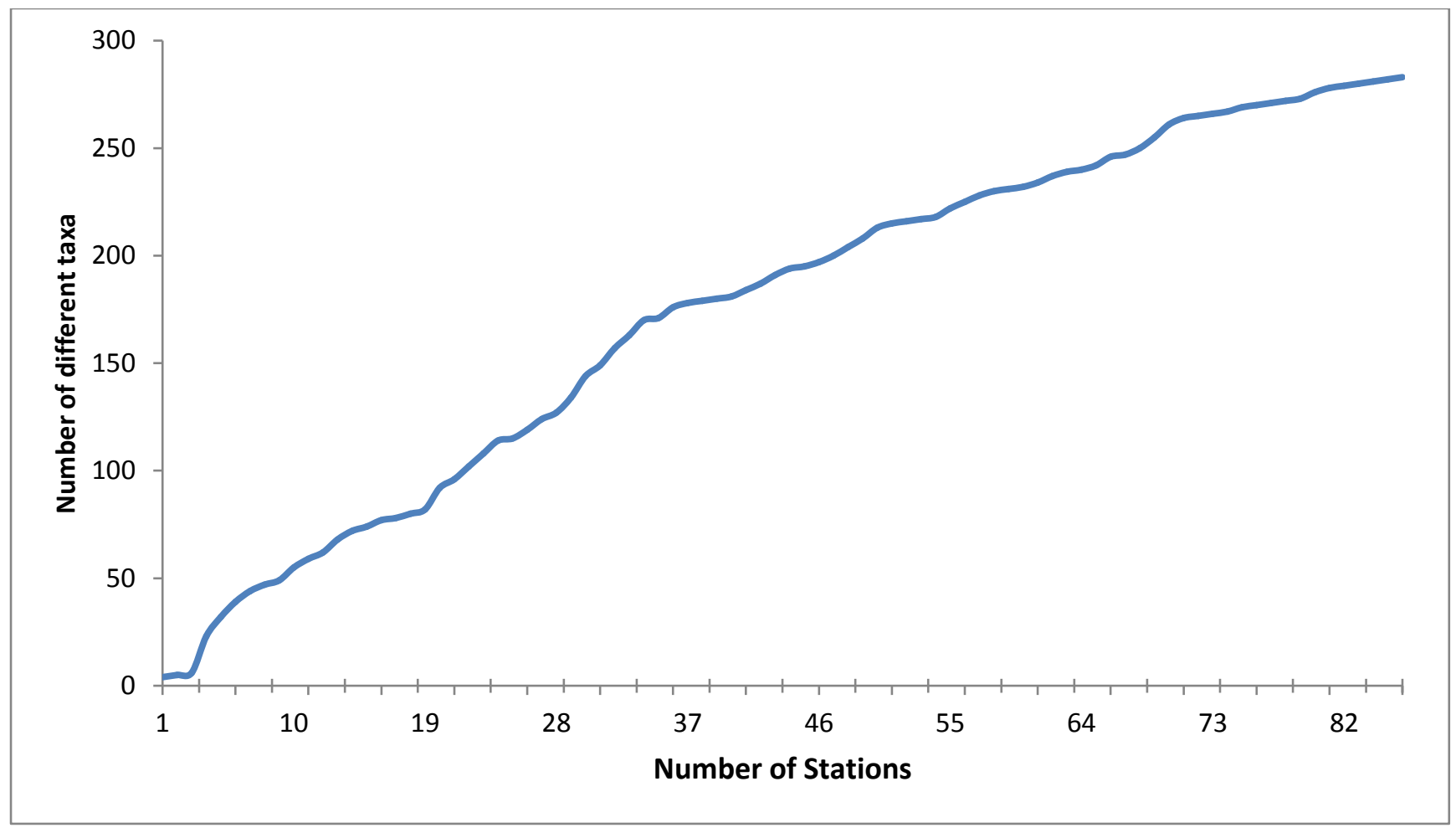

Figure 9.4. Species accumulation curve of the number of different megafaunal taxa collected over the duration of the project, at all sites during the Lophelia II program. Taxa were cumulatively collected at a total of 86 of the 204 stations sampled in 2008, 2009, and 2010. Many specimens are still unidentified; curve represents a conservative estimate of total number of species collected. 


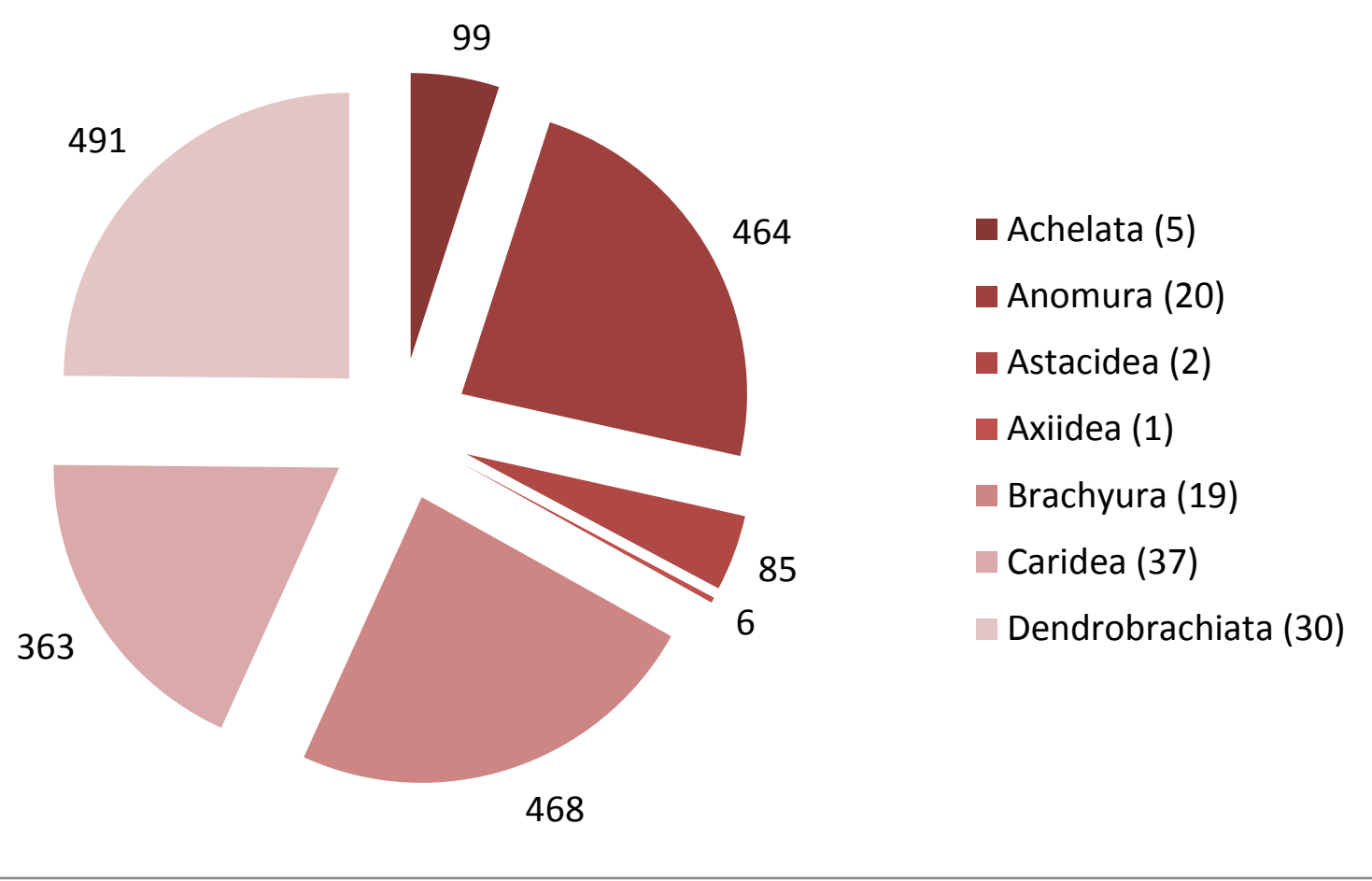

Figure 9.5 Overall species richness and abundance of Decapoda (Crustacea) collected from all sampling sites and cruises during the Lophelia II program. Number of Decapoda specimens collected at each site is shown in pie diagram. [Number of species represented in each taxon is given in parentheses]. 


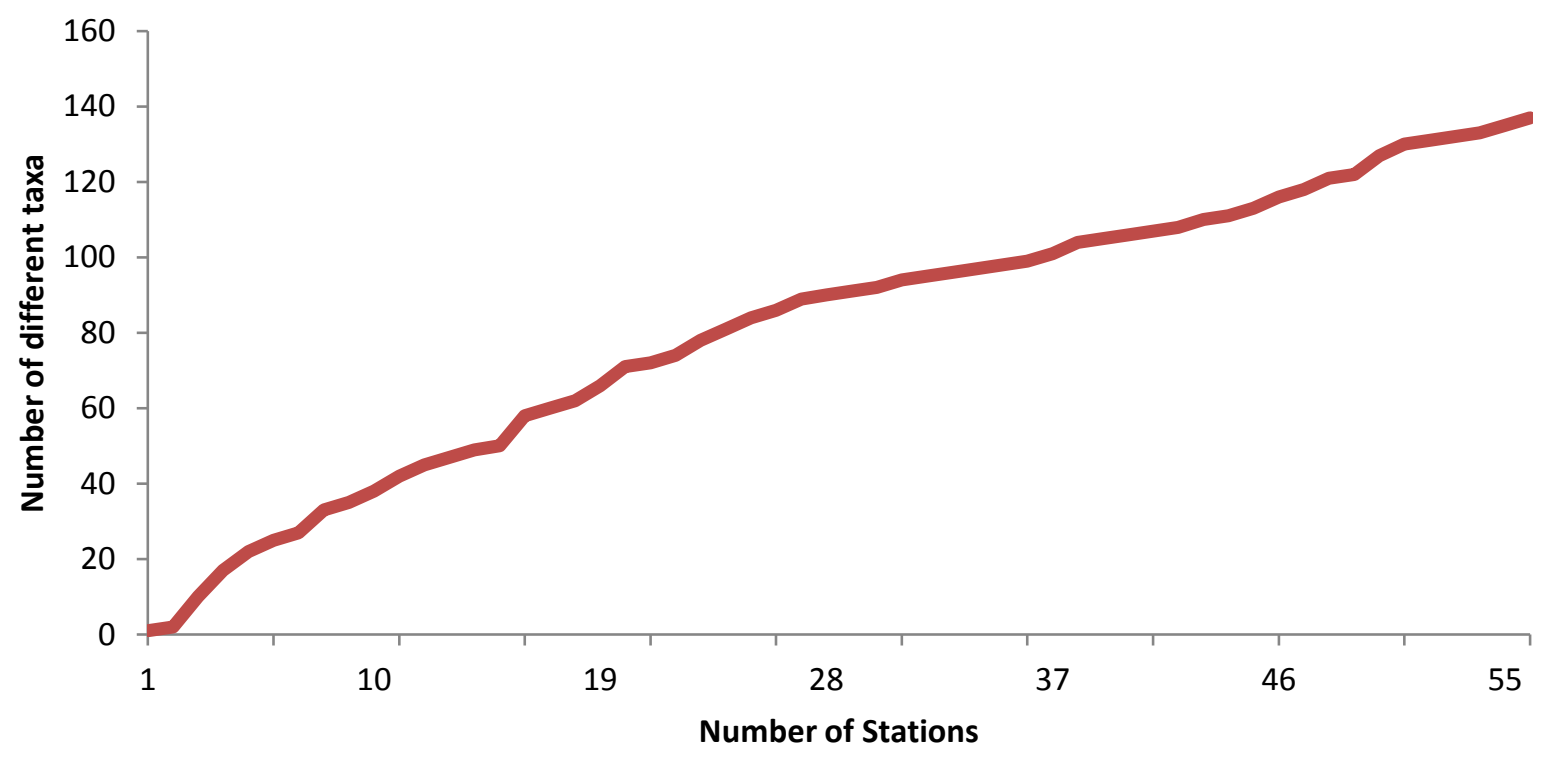

Figure 9.6 Species accumulation curve of the number of different crustacean taxa collected during all 3 years, at all sites during the Lophelia II program. [Taxa were cumulatively collected at 55 of the 204 stations sampled in 2008, 2009 and 2010. As many specimens are still unidentified, this curve represents a conservative estimate of total number of species collected.] 


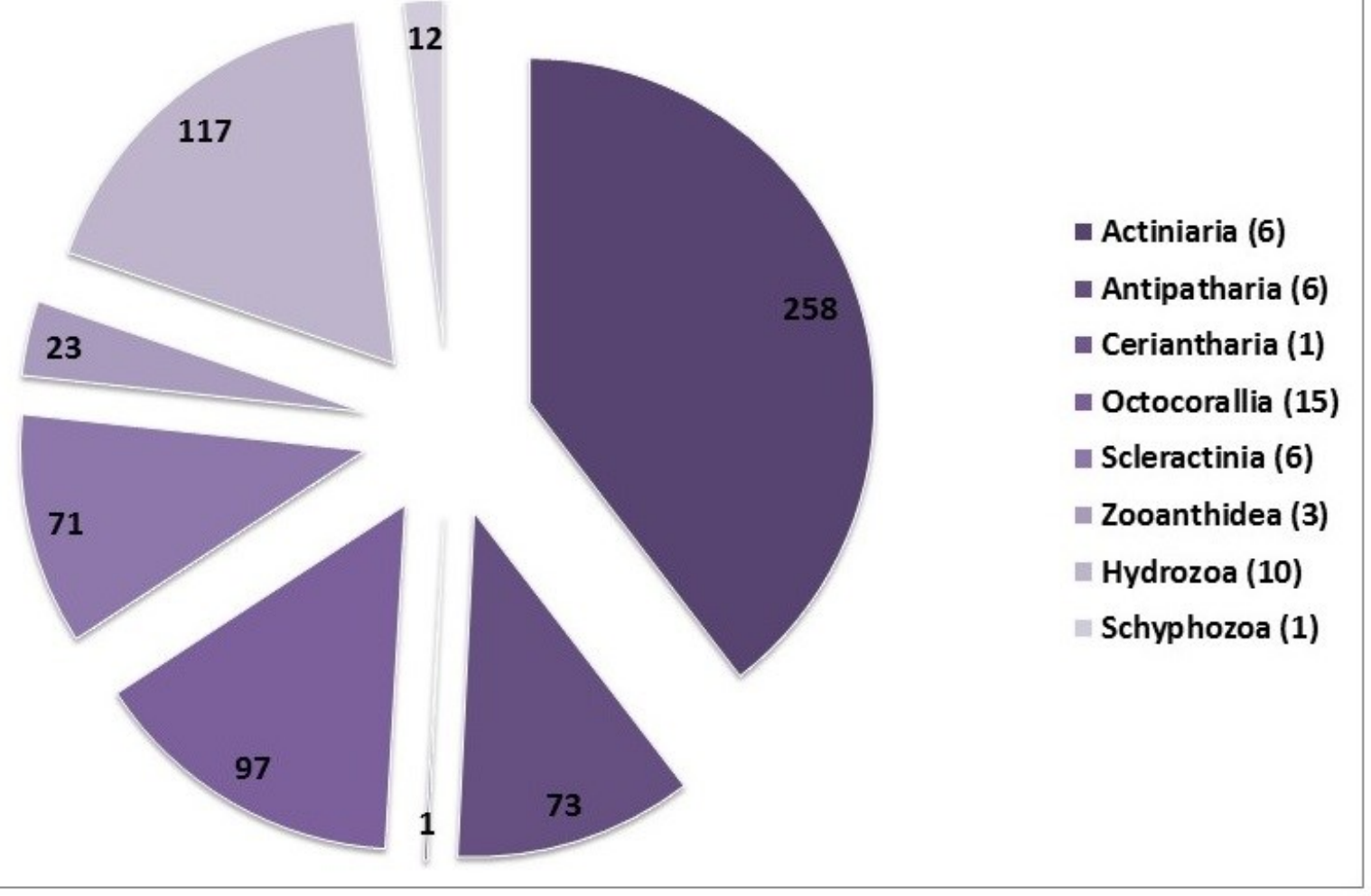

Figure 9.7 Abundance and diversity of Cnidaria specimens collected from all sampling sites and cruises during the Lophelia II program. [Number of Cnidaria specimens collected is shown in pie diagram. Number of species represented in each taxon is given in parentheses]. 


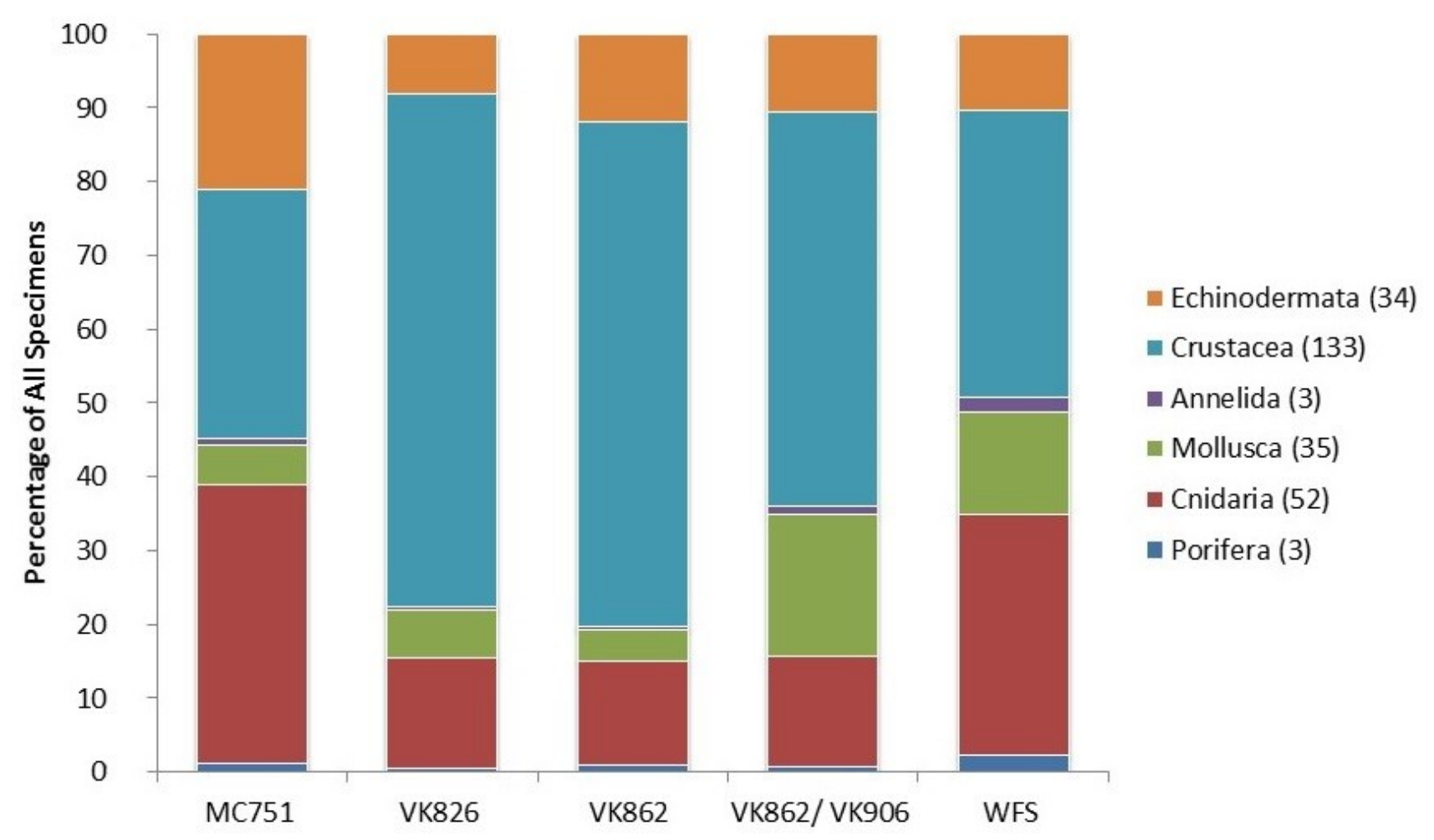

Figure 9.8 Percentage of the total invertebrate catch abundance represented by each major taxon by sampling site during the Lophelia Il program. [Number of species is shown in parentheses]. 


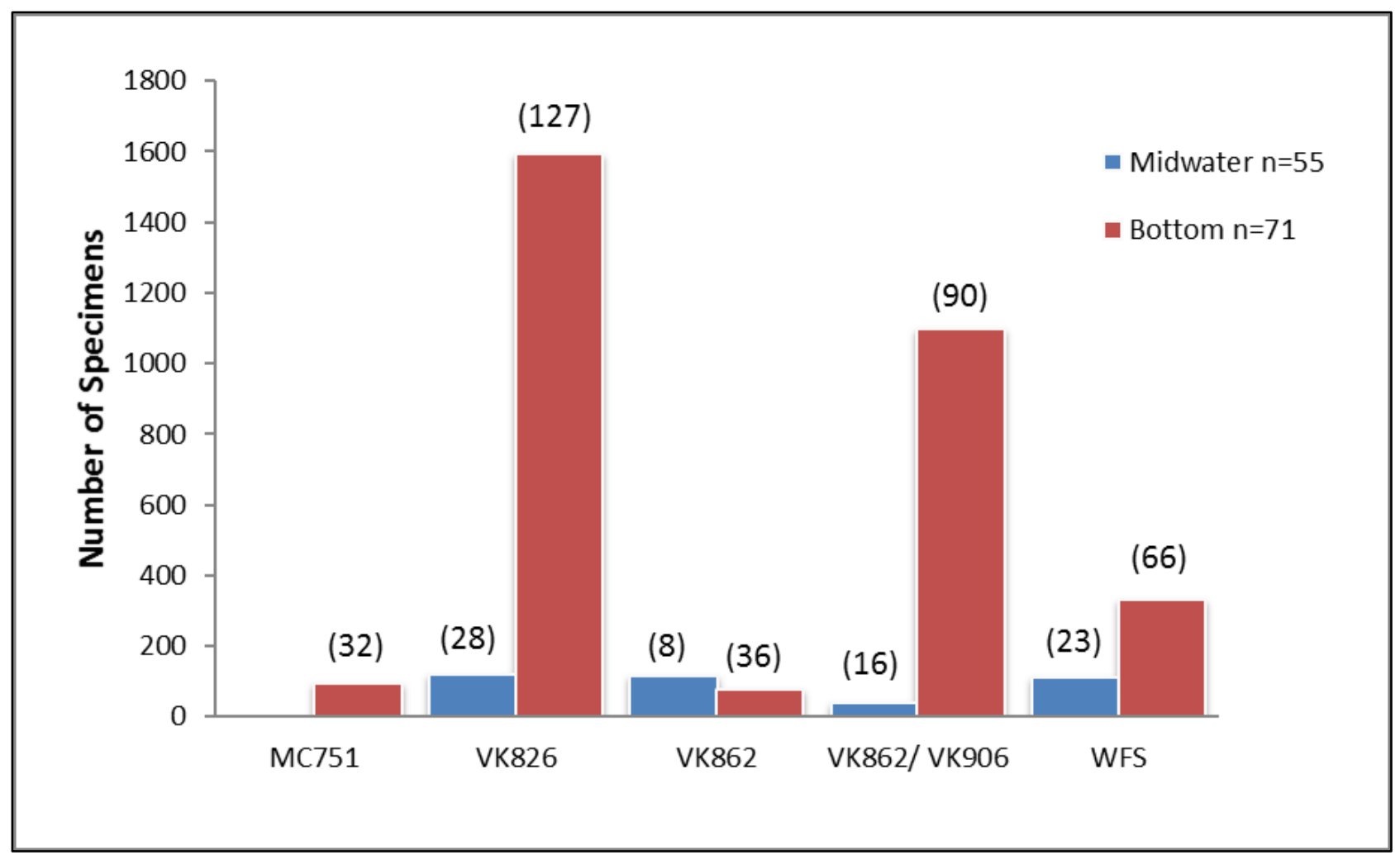

Figure 9.9 Total abundance of midwater and bottom invertebrates represented in each major taxon by site during the Lophelia II program. 


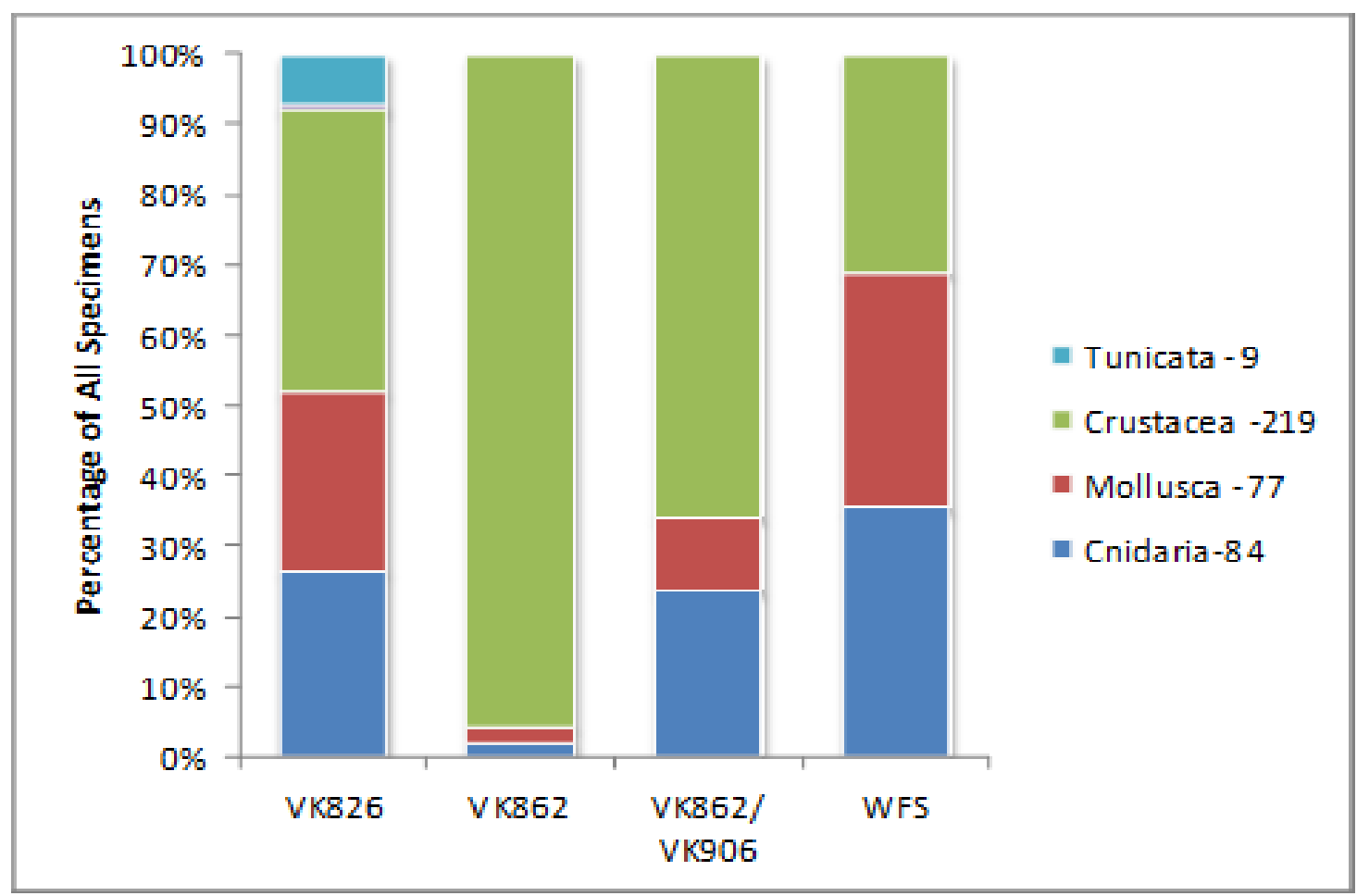

Figure 9.10 Percentage of total midwater invertebrate abundance represented in each major taxon by sampling site during the Lophelia II program. Corresponding abundance listed beside each taxon. Only abundance values greater than 30 are provided in figure. 


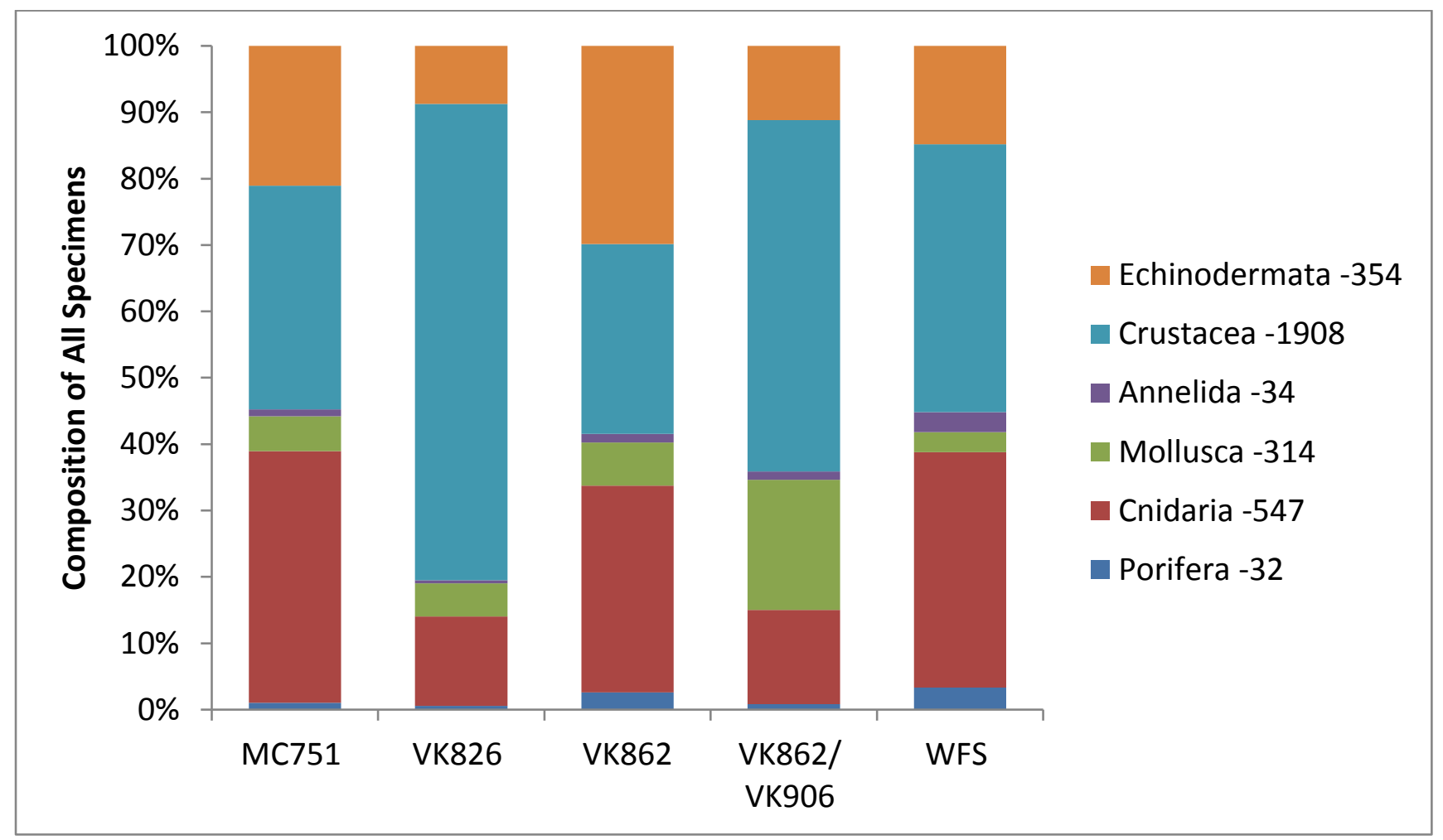

Figure 9.11 Percentage of total bottom invertebrate abundance represented in each major taxon by site during the Lophelia II program. Corresponding abundance listed beside each taxon. Only abundance values greater than 30 are provided in figure. 


\title{
10 REPRODUCTIVE BIOLOGY OF DEEP-SEA CORALS
}

\author{
By Sandra D. Brooke ${ }^{1}$ \\ ${ }^{1}$ Florida State University, Tallahassee, FL
}

\subsection{Introduction to the Reproductive Biology of Deep-sea Corals}

Lophelia pertusa is the most common structure-forming scleractinian coral in the Gulf of Mexico (GOM) region (Brooke and Schroeder, 2007). It has a wide distribution, which extends throughout the North Atlantic, South Atlantic, and Pacific Oceans and the Caribbean Sea at depths of 39-3,600 meters (m). This species forms massive bioherms along the northeastern (NE) Atlantic, particularly off Scandinavia, and in the western Atlantic from North Carolina to south Florida (Roberts and others, 2009).

Scleractinian corals can either be solitary polyps (cup corals), or colonial, comprising hundreds of individual polyps. Coral colonies grow via replication of polyps (asexual reproduction) in L. pertusa and other deep-water structure-forming species such as Enallopsammia sp., Oculina varicosa, and Solenosmillia variabilis; pieces of established colonies frequently break off through mechanical stress or disturbance and continue to grow, thereby establishing new colonies that are genetically identical to the parents (Wilson, 1979). Some uncommon modes of asexual reproduction such as polyp bail-out and parthenogenesis are known to occur in shallow-water corals (Sammarco, 1982; Krupp, 1983; Stoddart, 1983), but have not been reported for any deep-sea stony coral species. Asexual reproduction, however, does not account for the establishment of new, genetically different colonies, nor is it a mechanism for genetic exchange between established coral populations. These processes depend on sexual reproduction by means of dispersive planktonic larvae.

Most of the research on coral reproductive biology has focused on shallow, tropical, reefdwelling corals (Fadlallah, 1983; Baird and others, 2009; Harrison, 2011), whereas corals living in temperate, cold and deep-sea habitats have been neglected by comparison. Although scientific and conservation interests in deep-sea corals have expanded rapidly, information on their basic life histories is still lacking, partly because obtaining samples throughout the year from deep-sea coral ecosystems poses significant logistical challenges. Available evidence indicates that broadcast spawning of gametes is the dominant fertilization mechanism for deep-sea, structure-forming scleractinians (Brooke and Young, 2003; Burgess and Babcock, 2005; Waller, 2005; Waller and Tyler, 2005), followed by a dispersive larval stage. In contrast, deep-sea solitary scleractinians, such as Flabellum sp. (Waller and Tyler, 2011; Mercier and others, 2011), Caryophyllia sp. (Waller and others, 2005), and Fungiacyathus sp. (Waller and others, 2002; Flint and others, 2007), have various reproductive strategies, including hermaphroditism, gonochorism, brooding, and broadcast spawning (Harrison, 2011). Fertilization in corals may occur either internally or externally; in the former case, embryogenesis is completed within the polyps, and the larvae are released fully formed and often are competent to settle and begin a new colony. These species tend to have relatively short-distance dispersal. Larvae produced by external fertilization often disperse much greater distances than those that are brooded as their planktonic duration is longer, and they are subject to dispersal by currents (Harrison, 2011).

L. pertusa has a widespread distribution and can colonize many different types of habitats, including natural hard substrates of continental slopes, fjords, canyons, and seamounts (Roberts and 
others, 2009) and artificial substrates such as shipwrecks and oil rigs (Gass and Roberts, 2006). This coral can also tolerate a relatively wide range of temperatures $\left(4-15\right.$ degrees Celsius $\left.\left[{ }^{\circ} \mathrm{C}\right]\right)($ Brooke and others, 2013), sediment load (Brooke and others, 2009; Larsson and others, 2013) and oxygen levels (Dodds and others, 2007). Genetic analysis of this species has shown that populations in the North Atlantic are relatively isolated, indicating some limit to dispersal potential (Morrison and others, 2011). During a recent study on larval biology of this species, Larsson and others (2014) reported a long-lived planktonic phase that can last 3-5 weeks; however, incorporation of larval biology with physical oceanography models is needed to resolve apparent contradictions between the widespread distribution of this species and the relatively limited dispersal derived from population structure.

Information on the reproductive biology of L. pertusa was published recently using samples collected from the northeast Atlantic (Waller and Tyler, 2005) and the Trondheim Fjord in Norway (Brooke and Jarnegren, 2013). L. pertusa colonies were either male or female (gonochoristic), but not both (hermaphroditic), and had a seasonal reproductive cycle with one cohort per year, culminating in spawning during the winter (January and February). Eggs and sperm were released into the water to produce externally fertilized planktonic planular larvae. The larval biology and dispersal potential is not yet known for this species. In the GOM, samples of $L$. pertusa were collected during research cruises for the Lophelia I project, funded by the Minerals Management Service. Preliminary data on reproduction indicated that reproductive cycles in the GOM were on a different schedule from those of populations in the northeast Atlantic, but there were not sufficient samples to fully elucidate the differences. During this project, additional samples were collected to continue the reproductive work, and the results are presented in this chapter.

\subsection{Materials and Methods}

\subsubsection{USGS Study Sites}

\subsubsection{Viosca Knoll 826}

The most well-documented L. pertusa habitat in the GOM is on the southwest flank of a mound that lies in the southwest corner of Viosca Knoll lease block $826\left(29^{\circ} 09.5^{\prime} \mathrm{N} 88^{\circ} 01.0^{\prime} \mathrm{W}, 430-520 \mathrm{~m}\right)$ on the upper De Soto slope (fig. 1.4). Authigenic carbonate was abundant at this site and formed large plates, boulders, and rubble (Schroeder, 2002). This hard substrate occurred on the crest and flanks of the mound, where L. pertusa colonies were large and abundant. Coral colonies had a bushy morphology with highly anastomosed (fused together) and heavily calcified branches and large polyps. Individual colonies range from a few centimeters to over $1.5 \mathrm{~m}$ in diameter, with aggregations of colonies that appeared to be forming "thickets". Small colonies $(<25-50$ centimeter $[\mathrm{cm}]$ diameter) were usually completely live. Larger colonies and thickets usually had dead branches at the base with live terminal branches, and some colonies were completely dead. Colonies of the stony coral Madrepora oculata also occurred at this site, along with numerous gorgonians and antipatharians.

\subsubsection{Viosca Knoll 862}

This site is composed of a topographic high on the northern edge of a carbonate rock complex, which extends south for about 2 kilometers $(\mathrm{km})$ to the eastern rim of a submarine canyon in lease block VK862 (29ㅇ․ $06.4^{\prime} \mathrm{N}, 88^{\circ} 22.9^{\prime} \mathrm{W}, 300-500 \mathrm{~m}$; fig. 1.4). Diverse assemblages of corals including $L$. pertusa colonies encrusted the exposed hard substrate, along with dense aggregations of bamboo corals (Isididae), Callogorgia americana (Primnoidae), and large black corals (Antipathidiae). An unidentified species (or several species) of white anemone dominated the fauna at the northern end of this site, 
almost obscuring the substrate in places. The L. pertusa colonies at this site were smaller and less heavily calcified than those at VK826, and in the shallower northern end of the site, occurred only as isolated fairly small ( $<1-\mathrm{m}$ diameter) colonies. The deeper southern areas supported more abundant larger colonies on small mounds near the canyon's edge.

\subsubsection{West Florida Slope}

The western Florida shelf is composed of a gently sloping carbonate ramp, which increases in slope at the shelf edge, particularly at the West Florida Escarpment. Coral mounds occur along the 500$\mathrm{m}$ isobath for approximately $20 \mathrm{~km}$ between $26^{\circ} 20^{\prime} \mathrm{N} 84^{\circ} 45^{\prime} \mathrm{W}$ and $26^{\circ} 30 \mathrm{~N}, 84^{\circ} 50^{\prime} \mathrm{W}$. Coral mounds in this region were between 5 and $15 \mathrm{~m}$ tall and were dominated by L. pertusa, with less abundant colonies of M. oculata and Enallopsammia profunda. The cup coral Thecopsammia socialis often occurred in dense patches. Other cnidarians included stylasterine hydrocorals, gorgonians (including Bamboo corals), and antipatharians.

\subsubsection{Coral Collections}

Samples of deep-sea coral populations were collected by using a submersible or remotely operated vehicle (ROV) with manipulators on five separate cruises between August 2009 and November 2010. Twenty collections were made in total, from 5 different locations, between 334 and $536 \mathrm{~m} \mathrm{depth}$, resulting in 55 individual samples. Collections were made from large ( $\geq 1-\mathrm{m}$ diameter) apparently healthy colonies to avoid collecting immature samples. Collection details are outlined in table 10.1, which summarizes the research cruises, and table 10.2, which shows the sample metadata in more detail. During each collection, samples were taken from several (1-7) different healthy colonies and preserved immediately for histology in 10-percent buffered formalin.

\subsubsection{Histology}

Small polyps at the tips of each fragment were removed and not used for histological examination because they were potentially immature and non-reproductive (Rinkevich and Loya, 1987). The remainder of each fragment (about 8-10 polyps) was rinsed in distilled water and transferred to 5 percent hydrochloric acid for 12-24 hours (h) to decalcify the skeleton, leaving the polyp tissue intact for further processing. Polyps were rinsed again in distilled water then dehydrated through a series of ethanol concentrations (70 percent, 95 percent, 100 percent $\times 3$ changes) and transferred to a clearing agent (toluene). The polyps were then embedded in paraffin wax (3-5 per block), serially sectioned into 8-micrometer $(\mu \mathrm{m})$ slices, mounted on glass microscope slides, and stained using Mayer's Haematoxylin/Eosin B stains. Images of the microscope slides were taken by using an Optronics digital camera attached to an Olympus BX50 compound microscope. These images were used for the description of gametogenic status for each individual coral fragment.

For each female fragment, 100 oocytes were measured from 5 to 8 polyps. Only those oocytes with a visible nucleolus were measured. This ensured that the same egg was not measured more than

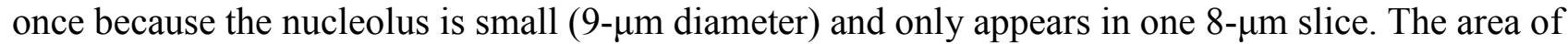
each oocyte was measured and recorded by using Image Tool (UTHSCSA) image analysis software. Oocyte feret diameter was calculated, which uses the measured area of the oocyte and estimates the diameter as if it were a circle (Brooke and Young, 2003; Waller and Tyler, 2005). Feret diameters were used to generate means and standard deviations of oocyte diameters and size-frequency distributions for each sampling date, which were then used to infer the timing of the female gametogenic cycle. The male 
reproductive periodicity was documented more qualitatively by developmental stage because the size of spermatocysts does not directly reflect their maturity.

\subsection{Results}

\subsubsection{Gametogenesis}

Of the 55 samples processed, 27 were male, 16 were female, and 12 were devoid of gametes. These were classified as indeterminate because their genders could not be established. The gender ratio within the collections was unequal $(\mathrm{M}: \mathrm{F}=1.68)$; however, a chi-squared analysis showed no significant difference between the numbers of each gender $\left(\chi^{2}=18.96, p=0.10\right)$, probably due to the small sample sizes (1-7 fragments) in each collection (table 10.2). There were insufficient samples from the same time period, site, and year to test for statistical differences in mean oocyte feret diameter among sites. For this study, discussion of gametogenesis and reproductive periodicity apply to data pooled across study sites and years.

\subsubsection{Female Gametogenesis}

During this project (2009-2010), female samples were collected only from July to November; therefore, a significant part of the gametogenic cycle is missing. Oogonia and pre-vitellogenic oocytes were not observed in these samples, but maturing vitellogenic oocytes were observed in July. Mature oocytes were present from mid-September to early November (fig. 10.1), with the largest oocytes occurring towards the end of that time period (late October-November). The largest average oocyte diameter from a single colony was $119.6 \mu \mathrm{m}$ (standard deviation [SD] 13.66; September 24, 2009), and the largest oocyte found was $153 \mu \mathrm{m}$ (October 24, 2010). The mean and standard deviation of oocyte diameters for September (fig. 10.1) show a greater range in size than those for October and November; however, the number of samples was small for the later months, so these data may not reflect true population variability. Monthly size-frequency histograms (fig. 10.2) provide a more detailed view of the variability in oocyte development during a particular time period. The frequency distributions show a wide range of egg sizes within an individual and population. Variation in egg size, especially in the more mature stages indicates protracted spawning periods rather than highly synchronous spawning where there would be a much smaller range of oocyte size classes (fig. 10.3).

\subsubsection{Male Gametogenesis}

The male gametogenic cycle was documented by stages (after Waller and Tyler, 2005) as follows: Stage I (early spermatogenesis), spermatocysts are lined with spermatocytes, but lumens are empty; Stage II (maturation phase), thick layer of spermatocytes with some spermatozoa present, but with mostly empty lumens; Stage III (mature) spermatocyst lumens are filled with spermatozoa; and Stage IV (post-spawn), spermatocysts are empty of spermatozoa, except for occasional remnants of spawning. Early spermatogenesis (Stage I) was not observed in these samples because there were no collections from early in the gametogenic cycle and the samples from July were either female or had no reproductive material. In September the spermatocysts were in the maturation phase (Stage II) with darkly stained spermatocytes and no spermatozoa present (fig. 10.4A-C). In November, the spermatocysts were mature (Stage III) and filled with spermatozoa (fig. 10.4D). As spermatogenesis progressed, the spermatocysts increased in size, often completely filling the mesoglea.

Twelve of the samples could not be assigned a gender because there were no visible gametes. This is a high number of individuals without reproductive material during the maturation phase of the 
gametogenic cycle. It is notable that 10 of these individuals were collected from the West Florida Slope and represent 66.6 percent of the total (15) samples from that region, compared with only 2 indeterminate samples out of a total of 40 (5.0 percent) from the northern GOM. There are too few samples to determine whether this is an artifact of low numbers, or reflects a real difference between the populations.

\subsection{Discussion}

This study augments earlier preliminary work on the gametogenic cycle of L. pertusa in the GOM (CSA International, Inc., 2007). The time period of these recent collections was similar (JulyNovember) to the earlier samples, so the gametogenic cycle is still incomplete; however the new data provide more complete information on the later part of the reproductive cycle, and are therefore a valuable contribution to our understanding of reproductive periodicity in this important species. These combined data show that populations of L. pertusa in the GOM begin to reach maturity in late September and are fully mature in late October to early November. The 2009-10 samples did not have the early stages of oocyte development (oogonia and pre-vitellogenic oocytes); however, samples collected on 16 November 2004 from the northern GOM show only pre-vitellogenic oocytes with no remnant mature stages (Continental Shelf Associates, 2007), indicating that spawning was completed before mid-November, with an almost immediate initiation of the next gametogenic cycle.

Previous research on reproduction in this species was published using samples from the NE Atlantic (Waller and Tyler, 2005) and the Trondheim Fjord in Norway (Brooke and Jarnegren, 2013). Both of these papers estimated (Waller and Tyler, 2005) and measured (Brooke and Jarnegren, 2013) that these populations had a protracted spawning period from late January to early March. Samples collected towards the end of the spawning period from the fjord encompassed initial gamete production and mature oocytes. These data clearly show that the Norwegian fjord populations have overlapping gametogenic cycles. In contrast, Waller and Tyler (2005) suggested that the NE Atlantic (785-980 m) populations had a resting phase after the spawning period, with initiation of gametogenesis in late summer. The GOM populations appear to spawn at least 3-4 months earlier than those from the NE Atlantic, and although there is no evidence of overlapping cycles, oogenesis seems to initiate shortly after spawning of the previous cycle is complete.

In summary, there are differences in female gametogenic cycles among populations of L. pertusa in the offshore NE Atlantic, Norwegian fjord and GOM populations. The reasons for these differences are unclear, but could be linked to external environmental variables such as temperature, currents, and timing of food supply; however, the exact mechanisms by which such environmental factors stimulate physiological responses are unknown (Roberts and others, 2009). In addition, while exogenous factors may influence invertebrate reproductive biology, there are also genetically constrained endogenous factors that may play a role in reproductive processes (Eckelbarger and Watling, 1995). Large regional differences in reproductive timing within a single species, as occurs with L. pertusa, provide a rare opportunity to study exogenous factors that may influence the drivers of reproduction and to identify which factors are constrained endogenously.

\subsection{References}

Baird, A.H., Guest, J.R., and Willis, B.L., 2009, Systematic and biogeographical patterns in the reproductive biology of scleractinian corals: Annual Review of Ecology and Evolutionary Systematics, v. 40, p. 551-571.

Brooke, S., Holmes, M., and Young, C.M., 2009, Effects of sediment on two morphotypes of Lophelia pertusa from the Gulf of Mexico: Marine Ecology Progress Series, v. 390, p. 137-144. 
Brooke, S., and Jarnegren, J., 2013, Reproductive periodicity of the deep-water scleractinian coral, Lophelia pertusa from the Trondheim Fjord, Norway: Marine Biology, v. 160, p. 139-153.

Brooke, S., Ross, S.W., Bane, J.M., Seim, H.E., and Young, C.M., 2013, Temperature tolerance of the deep-sea coral Lophelia pertusa from the southeastern United States: Deep Sea Research Part II: Topical Studies in Oceanography, v. 92, p. 240-248.

Brooke, S., and Schroeder, W.W., 2007, State of deep coral ecosystems in the Gulf of Mexico region: Texas to the Florida Straits, in Lumsden, S.E., Hourigan, T.F., Bruckner, A.W., and Dorr, G., eds., The state of deep coral ecosystems of the United States: Silver Spring, Md., National Oceanic and Atmospheric Administration Technical Memorandum CRCP-3, p. 271-306.

Brooke, S., and Young, C.M., 2003, Reproductive ecology of a deep-water scleractinian coral, Oculina varicosa: Continental Shelf Research, v. 23, p. 847-858.

Burgess, S., and Babcock, R.C., 2005, Reproductive ecology of three reef-forming, deep-sea corals in the New Zealand region, in Freiwald., A., and Roberts, J.M., eds., Cold-water corals and ecosystems: New York, Springer, p. 701-713.

CSA International, Inc., 2007, Characterization of northern Gulf of Mexico deepwater hard-bottom communities with emphasis on Lophelia coral: U.S. Department of Interior, Minerals Management Service, Gulf of Mexico OCS Region, OCS Study MMS 2007-044, 169 p.

Dodds, L.A., Roberts, J.M., Taylor, A.C., and Marubini, F., 2007, Metabolic tolerance of the cold-water coral Lophelia pertusa (Scleractinia) to temperature and dissolved oxygen change: Journal of Experimental Marine Biology and Ecology, v. 349, p. 205-214.

Eckelbarger, K.J., and Watling, L., 1995, Role of phylogenetic constraints in determining reproductive patterns in deep-sea invertebrates: Invertebrate Biology, v. 114, p. 256-269.

Fadlallah, Y.H., 1983, Sexual reproduction, development and larval biology of scleractinian corals. A review: Coral Reefs, v. 2, p. 129-150.

Flint, H., Waller, R., and Tyler, P., 2007, Reproductive ecology of Fungiacyathus marenzelleri from 4100 m depth in the Northeast Pacific Ocean: Marine Biology, v. 151, p. 843-849.

Gass, S.E., and Roberts, J.M., 2006, The occurrence of the coldwater coral Lophelia pertusa (Scleractinia) on oil and gas platforms in the North Sea: Colony growth, recruitment and environmental controls on distribution: Marine Pollution Bulletin, v. 52, p. 549-559.

Harrison, P.L., 2011, Sexual reproduction of scleractinian corals, in Dubinsky, Z., and Stambler, N., eds., Coral reefs: An ecosystem in transition: Springer, p. 59-85.

Krupp, D.A., 1983, Sexual reproduction and early development of the solitary coral Fungia scutaria (Anthozoa, scleractinia): Coral Reefs, v. 2, no. 3, p. 159-164.

Larsson, A.I., Järnegren, J., Strömberg, S.M., Dahl, M.P., Lundälv, T., and Brooke, S., 2014, Embryogenesis and larval biology of the cold water coral Lophelia pertusa: PLoS One, v. 9, no. 7, accessed May 8, 2017, at https://doi.org/10.1371/journal.pone.0102222.

Larsson, A.I., van Oevelen, D., Purser, A., and Thomsen, L., 2013, Tolerance to long-term exposure of suspended benthic sediments and drill cuttings in the cold-water coral Lophelia pertusa: Marine Pollution Bulletin, v. 70, p. 176-188.

Mercier, A., Sun, Z., and Hamel, J.F., 2011, Reproductive periodicity, spawning and development of the deep-sea scleractinian coral Flabellum angular: Marine Biology, v. 158, p. 371-380.

Morrison, C.L., Ross, S.W., Nizinski, M.S., Brooke, S., Järnegren, J., Waller, R.G., Johnson, R.L., and King, T.L., 2011, Genetic discontinuity among regional populations of Lophelia pertusa in the North Atlantic Ocean: Conservation Genetics, v. 12, p. 713-729. 
Rinkevich, B., and Loya, Y., 1987, Variability in the pattern of sexual reproduction of the coral Stylophora pistillata at Eilat, Red Sea: A long-term study: Biological Bulletin, v. 173, no. 2, p. 335344.

Roberts, J.M., Wheeler, A.J., Freiwald, A., and Cairns, S.D., 2009, Coldwater corals: The biology and geology of deep-sea coral habitats: Cambridge, Cambridge University Press, 352 p.

Sammarco, P.W., 1982, Polyp bail-out: An escape response to environmental stress and a new means of reproduction in corals: Marine Ecology Progress Series, v. 10, p. 57-65.

Schroeder, W.W., 2002, Observations of Lophelia pertusa and the surficial geology at a deep-water site in the northeastern Gulf of Mexico: Hydrobiologia, v. 471, p. 29-33.

Stoddart, J.A., 1983, Asexual production of planulae in the coral Pocillopora damicornis: Marine Biology, v. 76, p. 279-284.

Waller, R.G., 2005, Deep-water Scleractinia (Cnidaria: Anthozoa): Current knowledge of reproductive processes, in Freiwald, A., and Roberts, J.M., eds., Cold-water corals and ecosystems: New York, Springer, p. 691-700.

Waller, R.G., and Tyler, P.A., 2005, The reproductive biology of two deep-water, reef-building scleractinians from the NE Atlantic Ocean: Coral Reefs, v. 24, p. 514-522.

Waller, R.G., and Tyler, P.A., 2011, Reproductive patterns in two deep-water solitary corals from the north-east Atlantic_Flabellum alabastrum and F. angulare (Cnideria: Anthozoa: Scleractinia): Journal of the Marine Biological Association of the United Kingdom, v. 91, p. 669-675.

Waller, R.G., Tyler, P.A., and Gage, J.D., 2002, The reproductive ecology of the deep-sea scleractinian coral Fungiacyathus marenzelleri (Vaughan, 1906) in the northeast Atlantic Ocean: Coral Reefs, v. 21, p. 325-331.

Waller, R.G., Tyler, P.A., and Gage, J.D., 2005, Sexual reproduction in three hermaphroditic deep-sea Caryophyllia species (Anthozoa: Scleractinia) from the NE Atlantic Ocean: Coral Reefs, v. 24, p. 594-602.

Wilson, J.B., 1979, Patch development of the deep-water coral Lophelia pertusa (L.) on Rockall Bank: Journal of the Marine Biological Association of the United Kingdom, v. 59, p. 165-177. 
Table 10.1. Summary of cruises that provided samples for reproductive analysis during the Lophelia II program.

[JSL, Johnson Sea Link II submersible; NGOM, northern Gulf of Mexico; WFS, West Florida Slope;

VK, Viosca Knoll; MMS, Minerals Management Service; USGS, U.S. Geological Survey; NOAA,

National Oceanic and Atmospheric Administration; BOEMRE, Bureau of Ocean Energy Management,

Regulation, and Enforcement]

\begin{tabular}{ccccc}
\hline Date & Vessel/vehicle & Areas & $\begin{array}{c}\text { Funding } \\
\text { source }\end{array}$ & $\begin{array}{c}\text { Number of } \\
\text { samples }\end{array}$ \\
\hline 20 Aug-11 Sep 09 & Ronald Brown/Jason II & NGOM, WFS & MMS & 12 \\
14-25 Sep 09 & Seward Johnson/JSL & VK, WFS & USGS & 22 \\
17 Jul-4 Aug 10 & Seward Johnson/JSL & WFS & NOAA & 3 \\
20 Sep-3 Oct 10 & Cape Hatteras/Kraken II & VK, WFS & USGS & 11 \\
13 Oct-4 Nov 10 & Ronald Brown/Jason II & VK & BOEMRE & 7 \\
\hline
\end{tabular}

Table 10.2. Collection data for Lophelia pertusa samples from the Gulf of Mexico during 2009 and 2010 during the Lophelia II program.

[m, meter; Gender: M, male; F, female; I, indeterminate (no gametes); WFS, West Florida Slope; VK, Viosca Knoll; MC, Mississippi Canyon]

\begin{tabular}{|c|c|c|c|c|c|}
\hline Date & Site & Latitude & Longitude & Depth (m) & Gender \\
\hline 21 Aug 09 & WFS & $26^{\circ} 11.05^{\prime} \mathrm{N}$ & $83^{\circ} 17.56^{\prime} \mathrm{W}$ & 450 & $5 \mathrm{I}$ \\
\hline 4 Sep 09 & VK826 & $29^{\circ} 09.41^{\prime} \mathrm{N}$ & $87^{\circ} 59.36^{\prime} \mathrm{W}$ & 510 & $3 \mathrm{~F}, 4 \mathrm{M}$ \\
\hline 16 Sep 09 & WFS & $26^{\circ} 12.309^{\prime} \mathrm{N}$ & $84^{\circ} 43.621^{\prime} \mathrm{W}$ & $500-536$ & $1 \mathrm{~F}, 1 \mathrm{M}$ \\
\hline 16 Sep 09 & WFS & $26^{\circ} 12.710^{\prime} \mathrm{N}$ & $84^{\circ} 42.828^{\prime} \mathrm{W}$ & $414-428$ & $1 \mathrm{M}, 2 \mathrm{I}$ \\
\hline 17 Sep 09 & WFS & $26^{\circ} 12.473^{\prime} \mathrm{N}$ & $84^{\circ} 43.538^{\prime} \mathrm{W}$ & $477-531$ & $1 \mathrm{I}$ \\
\hline 19 Sep 09 & MC751 & $28^{\circ} 11.615^{\prime} \mathrm{N}$ & $89^{\circ} 48.088^{\prime} \mathrm{W}$ & $445-458$ & $3 \mathrm{~F}, 3 \mathrm{M}$ \\
\hline 20 Sep 09 & VK862/906 & $29^{\circ} 06.404^{\prime} \mathrm{N}$ & $88^{\circ} 23.101^{\prime} \mathrm{W}$ & $316-336$ & $1 \mathrm{M}$ \\
\hline 20 Sep 09 & VK862/906 & $29^{\circ} 04.155^{\prime} \mathrm{N}$ & $88^{\circ} 22.627^{\prime} \mathrm{W}$ & $389-436$ & $2 \mathrm{M}$ \\
\hline 21 Sep 09 & VK862/906 & $29^{\circ} 04.140^{\prime} \mathrm{N}$ & $88^{\circ} 22.671^{\prime} \mathrm{W}$ & $394-424$ & $1 \mathrm{~F}, 1 \mathrm{M}$ \\
\hline 22 Sep 09 & VK826 & $29^{\circ} 09.962^{\prime} \mathrm{N}$ & $88^{\circ} 00.708^{\prime} \mathrm{W}$ & $471-477$ & $3 \mathrm{M}$ \\
\hline 24 Sep 09 & VK826 & $29^{\circ} 10.187^{\prime} \mathrm{N}$ & $88^{\circ} 00.693^{\prime} \mathrm{W}$ & $476-477$ & $1 \mathrm{~F}, 1 \mathrm{M}$ \\
\hline 19 Jul 10 & WFS & $26^{\circ} 20.16^{\prime} \mathrm{N}$ & $84^{\circ} 45.36^{\prime} \mathrm{W}$ & $482-498$ & $1 \mathrm{~F}, 2 \mathrm{I}$ \\
\hline 22 Sep 10 & VK826 & $29^{\circ} 10.21^{\prime} \mathrm{N}$ & $88^{\circ} 00.82^{\prime} \mathrm{W}$ & $490-491$ & $3 \mathrm{M}, 2 \mathrm{I}$ \\
\hline 23 Sep 10 & VK826 & $29^{\circ} 10.2809^{\prime} \mathrm{N}$ & $88^{\circ} 00.8166^{\prime} \mathrm{W}$ & $487-489$ & $1 \mathrm{~F}, 2 \mathrm{M}$ \\
\hline 24 Sep 10 & VK862/906 & $29^{\circ} 06.2990^{\prime} \mathrm{N}$ & $88^{\circ} 23.0676^{\prime} \mathrm{W}$ & 334-339 & $1 \mathrm{~F}, 1 \mathrm{M}$ \\
\hline 28 Sep 10 & WFS & $26^{\circ} 12.4445^{\prime} \mathrm{N}$ & $84^{\circ} 43.6065^{\prime} \mathrm{W}$ & 504 & $1 \mathrm{~F}$ \\
\hline 17 Oct 10 & MC885 & $28^{\circ} 03.992^{\prime} \mathrm{N}$ & $89^{\circ} 42.822^{\prime} \mathrm{W}$ & No data & $1 \mathrm{M}$ \\
\hline 24 Oct 10 & VK906 & $29^{\circ} 04.134^{\prime} \mathrm{N}$ & $88^{\circ} 22.655^{\prime} \mathrm{W}$ & 470 & $1 \mathrm{~F}$ \\
\hline 27 Oct 10 & MC751 & $28^{\circ} 11.610^{\prime} \mathrm{N}$ & $89^{\circ} 47.918^{\prime} \mathrm{W}$ & 460 & $1 \mathrm{M}$ \\
\hline 1 Nov 10 & VK826 & $29^{\circ} 09.277^{\prime} \mathrm{N}$ & $88^{\circ} 01.355^{\prime} \mathrm{W}$ & 450 & $2 \mathrm{~F}, 2 \mathrm{M}$ \\
\hline
\end{tabular}




\section{L. pertusa oocyte diameter}

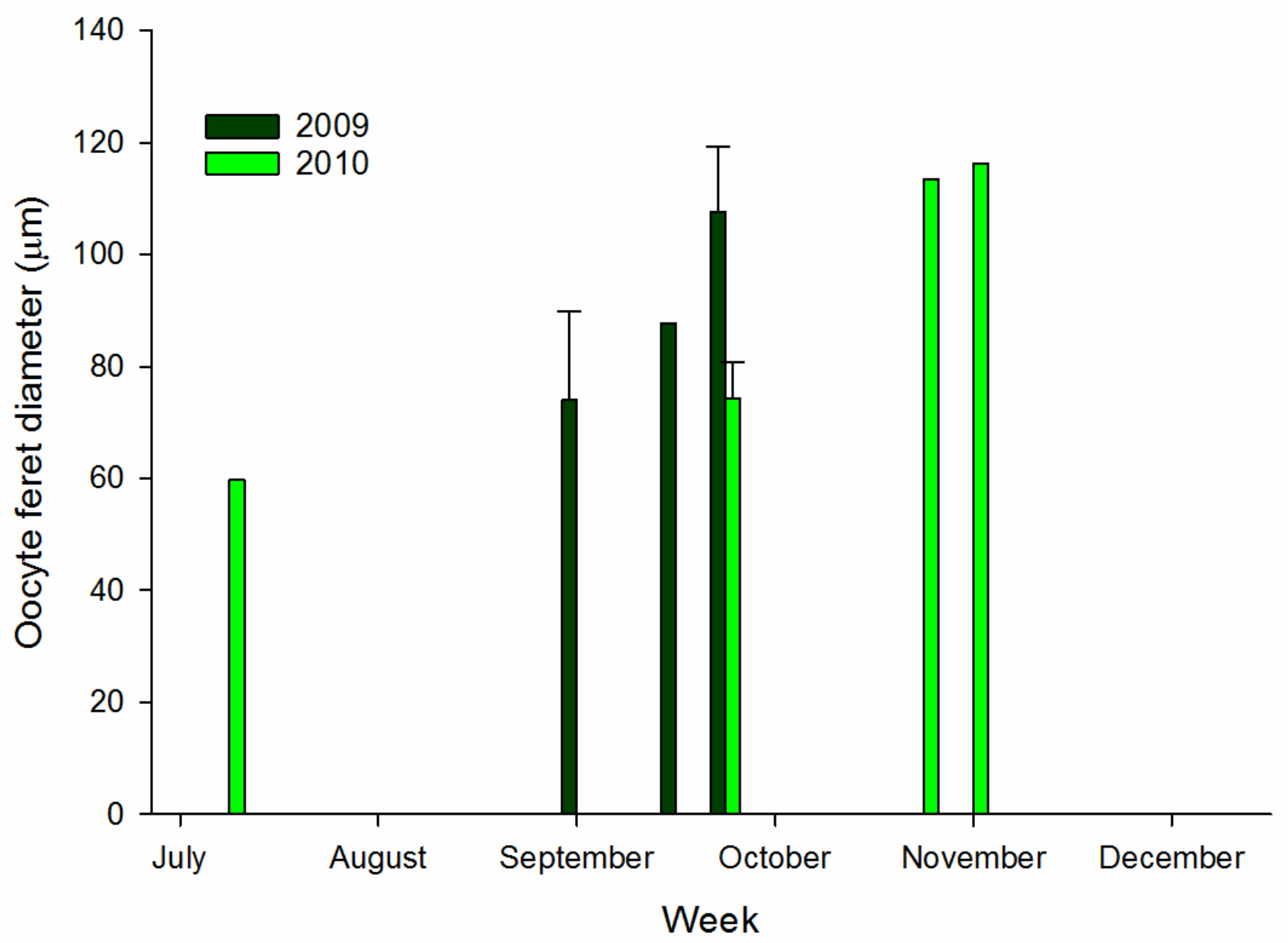

Figure 10.1 Mean oocyte diameters in micrometers $(\mu \mathrm{m})$ of Lophelia pertusa from different sampling periods showing the progression of the gametogenic cycle from early vitellogenic (July, week 29) to vitellogenic (September, week 44) and mature oocytes (October-November) during the Lophelia II program. Error bars represent 1 standard deviation from the mean. 


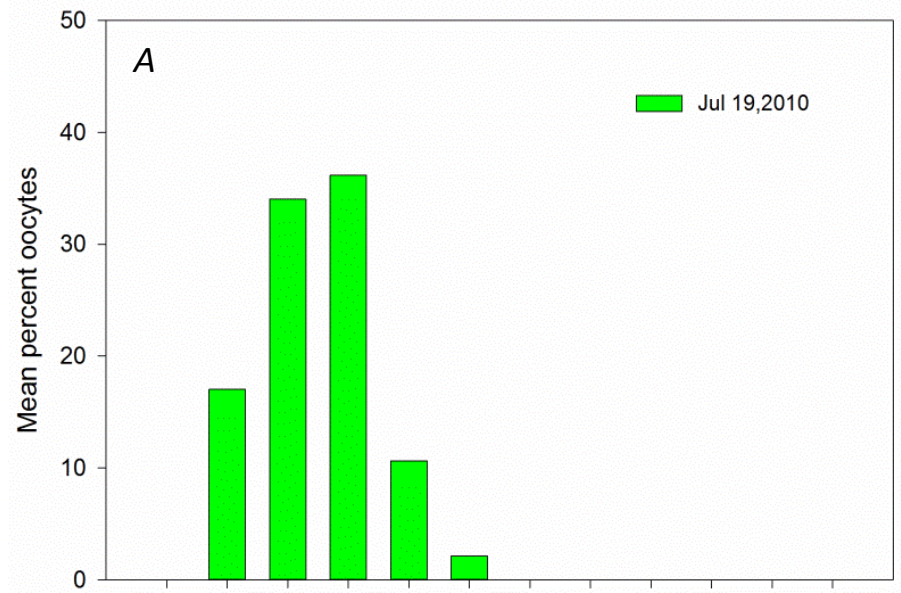

30- 40- 50- 60- 70- 80- 90- 100- 110- 120- 130- 140Oocyte size class $(\mu \mathrm{m})$

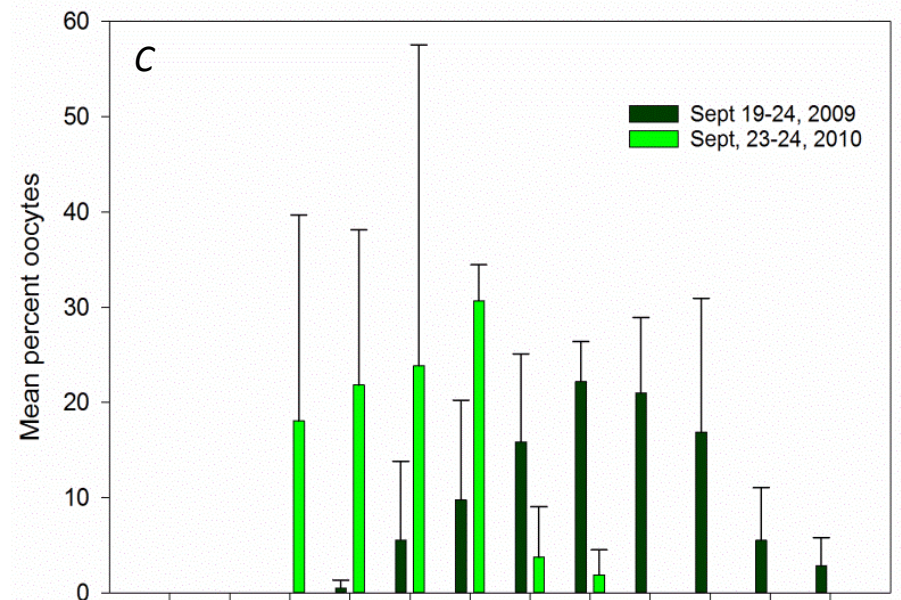

30- $40-50-60-70-80-90-100-110-120-130-140$

Oocyte size class $(\mu \mathrm{m})$

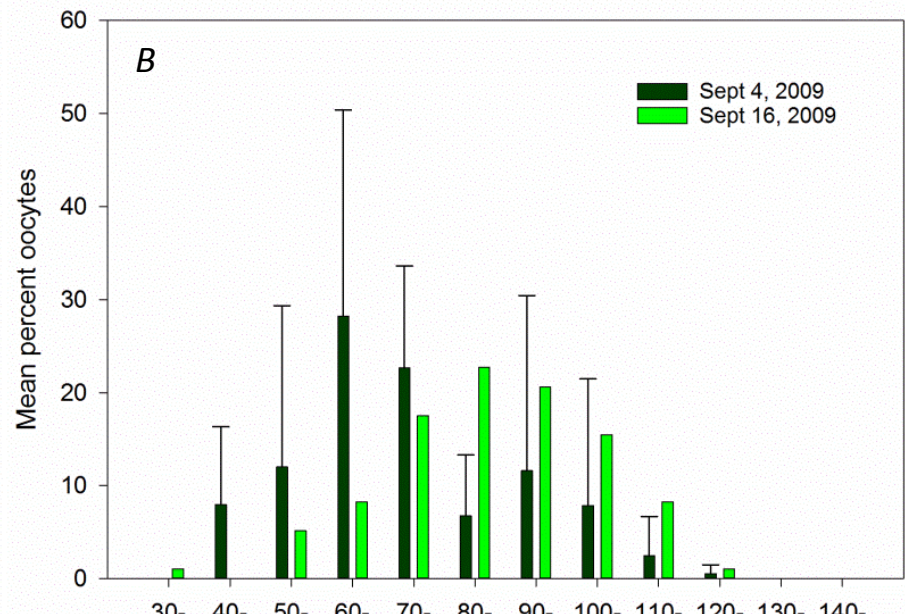

Oocyte size class $(\mu \mathrm{m})$

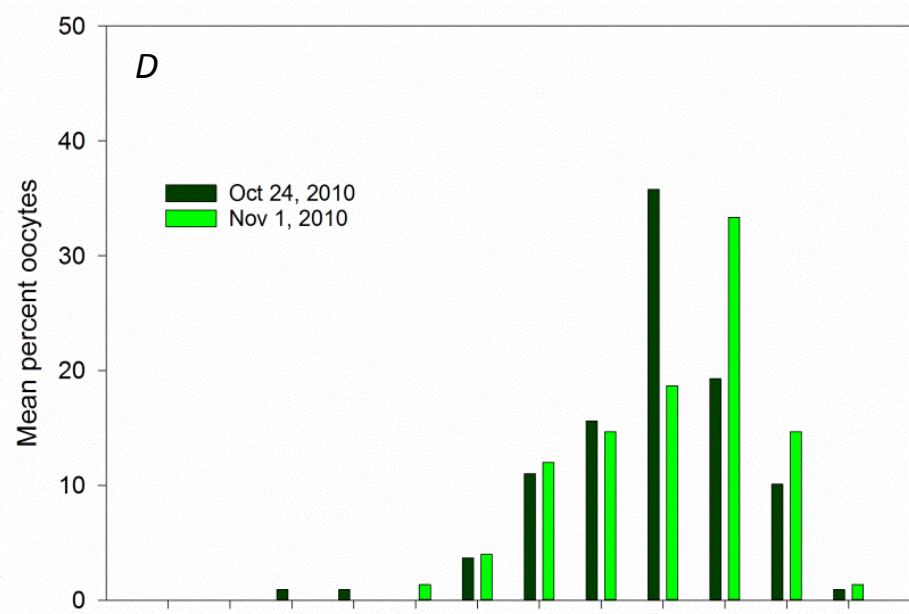

30- $40-50-60-70-80-90-100-110-120-130-140-$

Oocyte size class $(\mu \mathrm{m})$

Figure 10.2 Size-frequency distribution of Lophelia pertusa oocytes by sample period during the Lophelia II program. $A$, the distribution of early vitellogenic oocytes within a single female in July. In September, the median size increases from $B$, early September to $C$, late September as the oocytes mature. $D$, Large mature oocytes with a median size of 110 micrometers $(\mu \mathrm{m})$ and $120 \mu \mathrm{m}$ were measured in October and November, respectively. Error bars represent 1 standard deviation from the mean percent number of oocytes in each size category. 


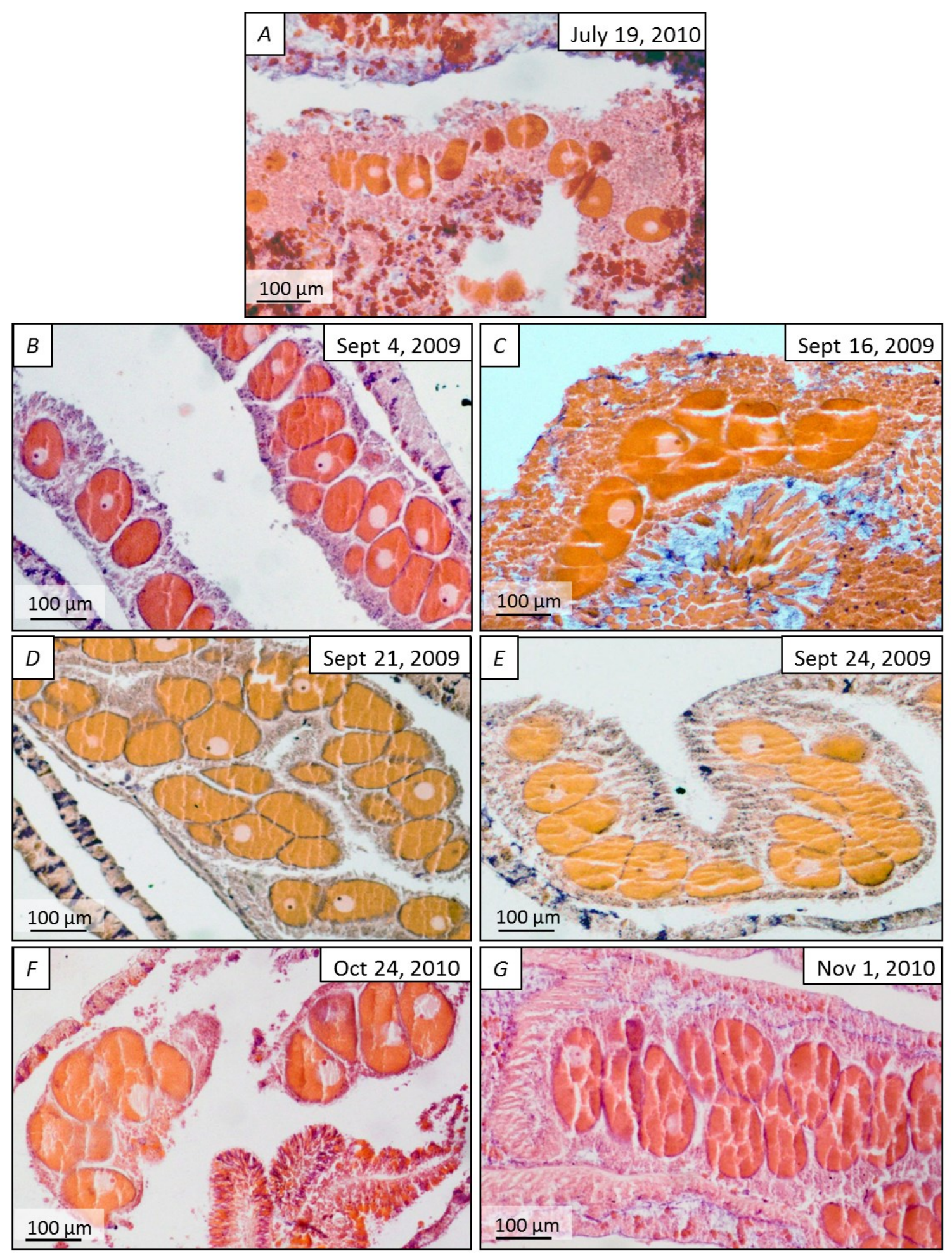

Figure 10.3 Plates showing stages of Lophelia pertusa oogenesis during the Lophelia II program. A, July: early vitellogenic oocytes; $B-E$, September: mature oocytes with nucleoli near periphery of nucleus; vitellogenic oocytes become more misshapen as they fill the mesenteries; $F-G$, October-November: large prespawning oocytes. 

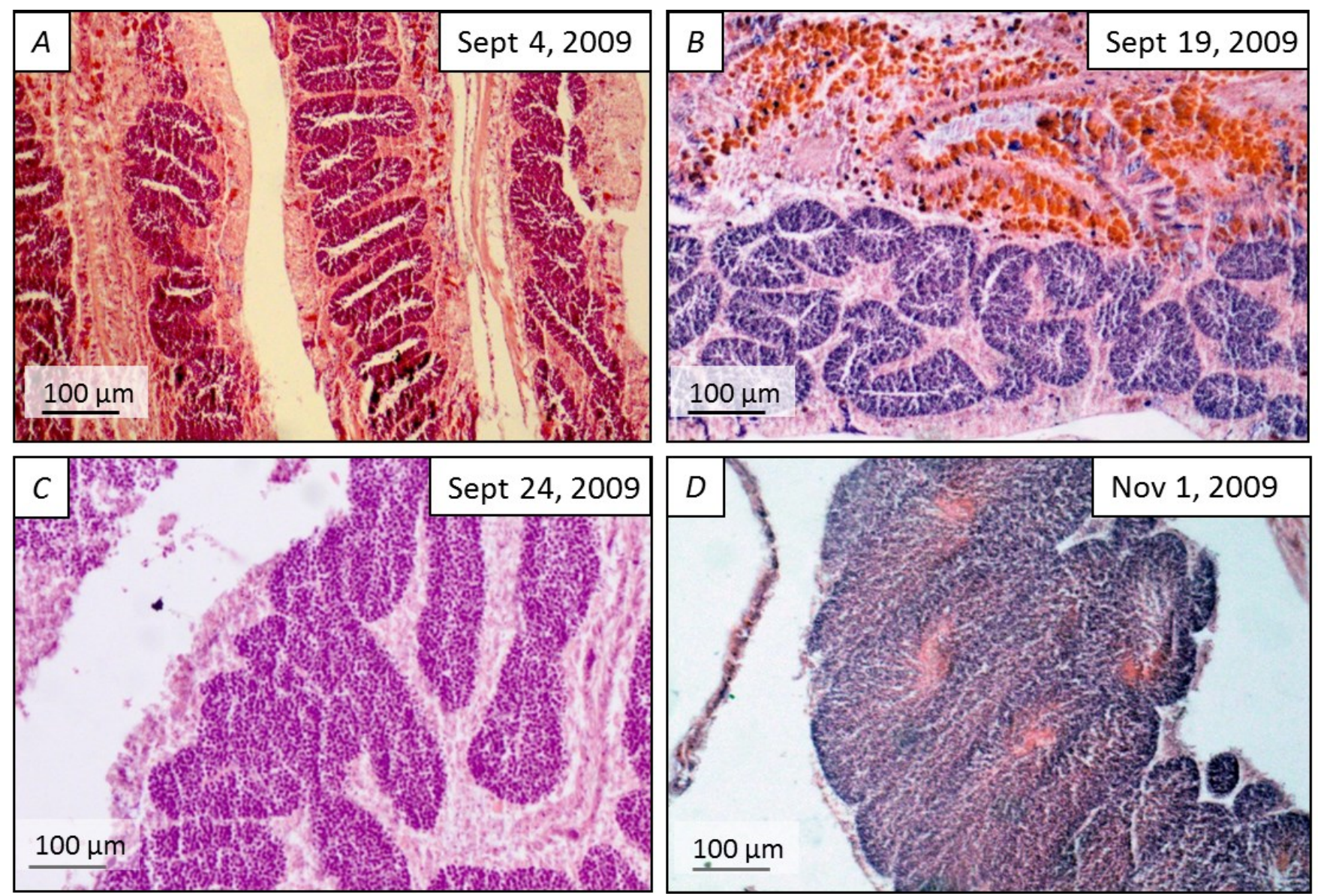

Figure 10.4 Spermatocysts in the mesenteries of male Lophelia pertusa colonies during the Lophelia II program: $A-C$, Stage II spermatocysts, from September samples, and $D$, samples collected in November showing mature spermatocysts showing primarily spermatozoa with some spermatocytes. 


\section{APPENDIX A. LIST OF MANUSCRIPTS PUBLISHED RELATED TO GULF OF MEXICO PROJECTS: LOPHELIA I, CHEMO III, LOPHELIA II}

\section{Deep Coral Banks (Lophelia I \& II)}

\section{$\underline{\text { Genetics }}$}

Morrison, C.L., Eackles, M.S., Johnson, R.L., and King, T.L., 2008, Characterization of 13 microsatellite loci for the deep-sea coral, Lophelia pertusa (Linnaeus 1758), from the western North Atlantic Ocean and Gulf of Mexico: Molecular Ecology Resources, v. 8, no. 5, p. 1037-1039.

Morrison, C.L., Ross, S.W., Nizinski, M.S., Brooke, S., Jarnegren, J., Waller, R.G., Johnson, R.L., and King, T.L., 2011, Genetic discontinuity among regional populations of Lophelia pertusa in the North Atlantic Ocean: Conservation Genetics, v. 12, p. 713-729.

$\underline{\text { Microbiology }}$

Galkiewicz, J.P., Pratte, Z.A., Gray, M.A., and Kellogg, C.A., 2011, Characterization of culturable bacteria isolated from the cold-water coral Lophelia pertusa: FEMS Microbiology Ecology, v. 77, no. 2, p. 333-346.

Galkiewicz, J.P., Stellick, S.H., Gray, M.A., and Kellogg, C.A., 2012, Cultured fungal associates from the deep-sea coral Lophelia pertusa: Deep-Sea Research Part I: Oceanographic Research Papers, v. 67 , p. $12-20$.

Gray, M.A., Stone, R.P., McLaughlin, M.R., and Kellogg, C.A., 2011, Microbial consortia of gorgonian corals from the Aleutian islands: FEMS Microbiology Ecology, v. 76, no. 1, p. 109-120.

Kellogg, C.A., Lisle, J.T., and Galkiewicz, J.P., 2009, Culture-independent characterization of bacterial communities associated with the cold-water coral Lophelia pertusa in the northeastern Gulf of Mexico: Applied and Environmental Microbiology, v. 75, no. 8, p. 2294-2303.

Olson, J.B., and Kellogg, C.A., 2010, Microbial ecology of corals, sponges, and algae in mesophotic coral environments: FEMS Microbiology Ecology, v. 73, no. 1, p. 17-30.

\section{Deep Coral Ecology}

Anker, A., and Nizinski, M., 2011, Description of a new deep-water species of Alpheus Fabricius, 1798 from the Gulf of Mexico (Crustacea, Decapoda, Alpheidae): Zootaxa, v. 2925, p. 49-56.

Brooke, S., Ross, S.W., Bane, J.M., Seim, H.E., and Young, C.M., 2013, Temperature tolerance of the deep-sea coral Lophelia pertusa from the southeastern United States: Deep-Sea Research Part II:

Topical Studies in Oceanography, v. 92, p. 240-248. 
Caruso, J.H., Ross, S.W., Sulak, K.J., and Sedberry, G.R., 2007, Deep-water chaunacid and lophiid anglerfishes (Pisces: Lophiiformes) off the south-eastern United States: Journal of Fish Biology, v. 70, no. 4, p. 1015-1026.

Demopoulos, A.W.J., Bourque, J.R., Cordes, E., and Stamler, K.M., 2016, Impacts of the Deepwater Horizon oil spill on deep-sea coral-associated sediment communities: Marine Ecology Progress Series, v. 561, p. 51-68.

Demopoulos, A.W.J., Bourque, J.R., and Frometa, J., 2014, Biodiversity and community composition of sediment macrofauna associated with deep-sea Lophelia pertusa habitats in the Gulf of Mexico: DeepSea Research Part I: Oceanographic Research Papers, v. 93, p. 91-103.

Fisher, C.R., Demopoulos, A.W.J., Cordes, E.E., Baums, I.B., White, H.K., and Bourque, J.R., 2014, Coral communities as indicators of ecosystem-level impacts of the Deepwater Horizon spill:

BioScience, v. 64, no. 9, p. 796-807.

Jaap, W.C., Ross, S.W., Brooke, S., and Arnold, W.S., 2014, Factors affecting coral reef fisheries in the eastern Gulf of Mexico, in Bortone, S., ed., Interrelationships between corals and fisheries: CRC Press, p. $83-112$.

Messing, C.G., Reed, J.K., Brooke, S.D., and Ross, S.W., 2008, Deep-water coral reefs of the United States, in Riegl, B.M., and Dodge, R.E., eds., Coral reefs of the USA: Netherlands, Springer, p. 767791.

Nielsen, J.G., Ross, S.W., and Cohen, D.M., 2009, Atlantic occurrence of the genus Bellottia (Teleostei, Bythitidae) with two new species from the Western North Atlantic: Zootaxa, v. 2018, p. 45-57.

Pietsch, T.W., Ross, S.W., Caruso, J.H., Saunders, M.G., and Fisher, C.R., 2013, In-situ observations of the deep-sea goosefish Sladenia shaefersi Caruso and Bullis (Lophiiformes: Lophiidae), with evidence of extreme sexual dimorphism: Copeia, v. 2013, no. 4, p. 660-665.

Prouty, N.G., Campbell, P.L., Mienis, F., Duineveld, G., Demopoulos, A.W.J., Ross, S.W., and Brooke, S., 2016a, Impact of Deepwater Horizon spill on food supply to deep-sea benthos communities: Estuarine, Coastal and Shelf Science, v. 169, p. 248-264.

Prouty, N.G., Fisher, C.R., Demopoulos, A.W.J., and Druffel, E.R.M., 2016b, Growth rates and ages of deep-sea corals impacted by the Deepwater Horizon oil spill: Deep-Sea Research Part II: Topical Studies in Oceanography, v. 129, p. 196-212.

Prouty, N., Roark, E., Buster, N., and Ross, S., 2011, Growth rate and age distribution of deep-sea black corals in the Gulf of Mexico: Marine Ecology Progress Series, v. 423, p. 101-115.

Prouty, N.G., Roark, E.B., Koenig, A.E., Demopoulos, A.W.J., Batista, F.C., Kocar, B.D., Selby, D., McCarthy, M.D., and Mienis, F., 2014, Deep-sea coral record of human impact on watershed quality in the Mississippi River Basin: Global Biogeochemical Cycles, v. 28, no. 1, p. 29-43. 
Schroeder, W.W., and Brooke, S., 2011, Habitat-forming deep-water scleractinian corals in the Gulf of Mexico, in Buster, N.A., and Holmes, C.W., eds., Gulf of Mexico origin, waters, and biota: Texas A\&M University Press, p. 355-366.

Sulak, K.J., Brooks, R.A., Luke, K.E., Norem, A.D., Randall, M., Quaid, A.J., Yeargin, G.E., Miller, J.M., Harden, W.M., Caruso, J.H., and Ross, S.W., 2007, Demersal fishes associated with Lophelia pertusa coral and hard-substrate biotopes on the continental slope, northern Gulf of Mexico: Bulletin of Marine Science, v. 81, supplement 1, p. 65-92.

White, H.K., Hsing, P.-Y., Cho, W., Shank, T.M., Cordes, E.E., Quattrini, A.M., Nelson, R.K., Camilli, R., Demopoulos, A.W.J., German, C.R., Brooks, J.M., Roberts, H.H., Shedd, W., Reddy, C.M., and Fisher, C.R., 2012, Impact of the Deepwater Horizon oil spill on a deep-water coral community in the Gulf of Mexico: Proceedings of the National Academy of Sciences, v. 109, no. 50, p. 20303-20308.

Williams, B., Risk, M.J., Ross, S.W., and Sulak, K.J., 2006, Deep-water antipatharians: Proxies of environmental change: Geology, v. 34, no. 9, p. 773-776.

Williams, B., Risk, M.J., Ross, S.W., and Sulak, K.J., 2007, Stable isotope data from deep-water antipatharians: 400-year records from the southeastern coast of the United States of America: Bulletin of Marine Science, v. 81, no. 3, p. 437-447.

\section{LANDER Related Lophelia II}

Davies, A.J., Duineveld, G.C.A., van Weering, T.C.E., Mienis, F., Quattrini, A.M., Seim, H.E., Bane, J.M., and Ross, S.W., 2010, Short-term environmental variability in cold-water coral habitat at Viosca Knoll, Gulf of Mexico: Deep-Sea Research Part I, Oceanographic Research Papers, v. 57, no. 2, p. 199212.

Mienis, F., Duineveld, G.C.A., Davies, A.J., Ross, S.W., Seim, H., Bane, J., and van Weering, T.C.E., 2012, The influence of near-bed hydrodynamic conditions on cold-water corals in the Viosca Knoll area, Gulf of Mexico: Deep-Sea Research Part I, Oceanographic Research Papers, v. 60, p. 32-45.

\section{Chemo III (project ended)}

Choy, C.A., Davison, P.C., Drazen, J.C., Flynn, A., Gier, E.J., Hoffman, J.C., McClain-Counts, J.P., Miller, T.W., Popp, B.N., Ross, S.W., and Sutton, T.T., 2012, Global trophic position comparison of two dominant mesopelagic fish families (Myctophidae, Stomiidae) using amino acid nitrogen isotopic analyses: PLoS ONE, v. 7, no. 11, p. e50133.

Demopoulos, A.W.J., Gualtieri, D., and Kovacs, K., 2010, Food-web structure of seep sediment macrobenthos from the Gulf of Mexico: Deep-Sea Research Part II: Topical Studies in Oceanography, v. 57, no. 21-23, p. 1972-1981.

Kellogg, C.A., 2010, Enumeration of viruses and prokaryotes in deep-sea sediments and cold seeps of the Gulf of Mexico: Deep-Sea Research Part II: Topical Studies in Oceanography, v. 57, no. 21-23, p. 2002-2007. 
McClain-Counts, J.P., 2011, Trophic structure of midwater fishes over cold seeps in the north central Gulf of Mexico: University of North Carolina Wilmington, M.S. Thesis, 126 p.

Ross, S.W., Quattrini, A.M., Roa-Varón, A.Y., and McClain, J.P., 2010, Species composition and distributions of mesopelagic fishes over the slope of the north-central Gulf of Mexico: Deep-Sea Research Part II: Topical Studies in Oceanography, v. 57, no. 21-23, p. 1926-1956.

Ross, S.W., Demopoulos, A.W.J., Kellogg, C.A., Morrison, C.L., Nizinkski, M.S., Ames, C.L., Casazza, T.L., Gualtieri, D., Kovacs, K.M., McClain, J.P., Quattrini, A.M., Roa-Varon, A.Y., and Thaler, A.D., 2012, Deepwater Program: Studies of Gulf of Mexico lower continental slope communities related to chemosynthetic and hard substrate habitats: U.S. Geological Survey Open-File Report 2012-1032, 301 p.

Thaler, A.D., Van Dover, C.L., and Vilgalys, R., 2012, Ascomycete phylotypes recovered from a Gulf of Mexico methane seep are identical to an uncultured deep-sea fungal clade from the Pacific: Fungal Ecology, v. 5, no. 2, p. 270-273.

\section{Broader Scope}

Casazza, T.L., and Ross, S.W., 2011, Pictorial guide to demersal DSC Continental Slope fishes off the SEUS: University of North Carolina Wilmington.

Norse, E.A., Brooke, S., Cheung, W.W.L., Clark, M.R., Ekeland, I., Froese, R., Gjerde, K.M., Haedrich, R.L., Heppell, S.S., and Morato, T., 2012, Sustainability of deep-sea fisheries: Marine Policy, v. 36, no. 2, p. 307-320.

This list only covers offshore/deep-sea projects in the GOM started in 2004 or after. Some papers may be more broadly based than the category under which they are listed. 


\section{APPENDIX B. STATIONS SAMPLED DURING FIVE LOPHELIA II CRUISES (2008-2010)}

Table B.1. Stations sampled at two deep coral study sites (VK826, VK862/906) in the Gulf of Mexico using the NOAA ship Nancy Foster,

October $6-13,2008$, as part of the Lophelia II program.

[min, minute; hr, hour; m, meter; CDT, central daylight time; D, day (0650 to $1830 \mathrm{hr}$ CDT); N, night (1830 to $0650 \mathrm{hr}$ CDT); D/N, samples covering both day and night; CTD, Sea-bird SBE 911+CTD; OT, otter trawl; PN, Plankton net (1.0-m diameter); TT, Tucker trawl with 0.5 m plankton net inserted; Phyto, phytoplankton sample; Traps, fish traps; S, surface; ROV, Saab Falcon remotely operated vehicle; Albex and Bobo, Royal Netherlands Institute for Sea Research benthic landers]

\begin{tabular}{|c|c|c|c|c|c|c|c|c|c|c|}
\hline Station number & Date & Location & Gear & $\begin{array}{c}\text { Sample } \\
\text { time } \\
\text { (min) }\end{array}$ & Time & Start latitude & $\begin{array}{c}\text { Start } \\
\text { longitude }\end{array}$ & End latitude & $\begin{array}{c}\text { End } \\
\text { longitude }\end{array}$ & $\begin{array}{l}\text { Depth range } \\
(\mathrm{m})\end{array}$ \\
\hline Lander-2008-001 & 06-11 Oct 08 & VK826 & Albex & 7,132 & $\mathrm{D} / \mathrm{N}$ & $29^{\circ} 09.661^{\prime} \mathrm{N}$ & $88^{\circ} 01.140^{\prime} \mathrm{W}$ & $29^{\circ} 09.661^{\prime} \mathrm{N}$ & $88^{\circ} 01.140^{\prime} \mathrm{W}$ & 451 \\
\hline Lander-2008-002 & 06-11 Oct 08 & VK826 & Bobo & 7,126 & $\mathrm{D} / \mathrm{N}$ & $29^{\circ} 10.007^{\prime} \mathrm{N}$ & $88^{\circ} 00.821^{\prime} \mathrm{W}$ & $29^{\circ} 10.007^{\prime} \mathrm{N}$ & $88^{\circ} 00.821^{\prime} \mathrm{W}$ & 476 \\
\hline Lander-2008-003 & 12 Oct 08 & VK826 & Bobo & $*$ & $\mathrm{D} / \mathrm{N}$ & $29^{\circ} 10.270^{\prime} \mathrm{N}$ & $88^{\circ} 00.558^{\prime} \mathrm{W}$ & $29^{\circ} 10.270^{\prime} \mathrm{N}$ & $88^{\circ} 00.558^{\prime} \mathrm{W}$ & 484 \\
\hline Lander-2008-004 & 12 Oct 08 & VK826 & Albex & $*$ & $\mathrm{D} / \mathrm{N}$ & $29^{\circ} 09.981^{\prime} \mathrm{N}$ & $88^{\circ} 00.775^{\prime} \mathrm{W}$ & $29^{\circ} 09.981^{\prime} \mathrm{N}$ & $88^{\circ} 00.775^{\prime} \mathrm{W}$ & 480 \\
\hline ROV-2008-001 & 7 Oct 08 & VK826 & ROV & 434 & $\mathrm{D}$ & $29^{\circ} 09.664^{\prime} \mathrm{N}$ & $88^{\circ} 01.095^{\prime} \mathrm{W}$ & $29^{\circ} 09.939^{\prime} \mathrm{N}$ & $88^{\circ} 01.009^{\prime} \mathrm{W}$ & $415-452$ \\
\hline ROV-2008-002 & 8 Oct 08 & VK826 & ROV & 317 & D & $29^{\circ} 09.951^{\prime} \mathrm{N}$ & $88^{\circ} 00.793^{\prime} \mathrm{W}$ & $29^{\circ} 09.386^{\prime} \mathrm{N}$ & $88^{\circ} 00.911^{\prime} \mathrm{W}$ & $434-485$ \\
\hline ROV-2008-003 & 9 Oct 08 & VK862/906 & ROV & 374 & D & $29^{\circ} 06.405^{\prime} \mathrm{N}$ & $88^{\circ} 23.032^{\prime} \mathrm{W}$ & $29^{\circ} 06.481^{\prime} \mathrm{N}$ & $88^{\circ} 22.985^{\prime} \mathrm{W}$ & $296-330$ \\
\hline ROV-2008-004 & 10 Oct 08 & VK826 & ROV & 361 & $\mathrm{D}$ & $29^{\circ} 10.185^{\prime} \mathrm{N}$ & $88^{\circ} 00.683^{\prime} \mathrm{W}$ & $29^{\circ} 10.289^{\prime} \mathrm{N}$ & $88^{\circ} 00.604^{\prime} \mathrm{W}$ & $456-481$ \\
\hline ROV-2008-005 & 11 Oct 08 & VK826 & ROV & 126 & $\mathrm{D}$ & $29^{\circ} 10.196^{\prime} \mathrm{N}$ & $88^{\circ} 00.101^{\prime} \mathrm{W}$ & $29^{\circ} 10.216^{\prime} \mathrm{N}$ & $88^{\circ} 00.730^{\prime} \mathrm{W}$ & $454-480$ \\
\hline ROV-2008-006 & 11 Oct 08 & VK826 & ROV & 264 & D & $29^{\circ} 10.221^{\prime} \mathrm{N}$ & $88^{\circ} 00.647^{\prime} \mathrm{W}$ & $29^{\circ} 10.215^{\prime} \mathrm{N}$ & $88^{\circ} 00.623^{\prime} \mathrm{W}$ & $467-479$ \\
\hline NF-2008-001 & 5 Oct 08 & VK826 & CTD & 7 & $\mathrm{~N}$ & $29^{\circ} 24.611^{\prime} \mathrm{N}$ & $87^{\circ} 59.264^{\prime} \mathrm{W}$ & $29^{\circ} 24.573^{\prime} \mathrm{N}$ & $87^{\circ} 59.499^{\prime} \mathrm{W}$ & $S-60$ \\
\hline NF-2008-002 & 5 Oct 08 & VK826 & CTD & 8 & $\mathrm{~N}$ & $29^{\circ} 19.381^{\prime} \mathrm{N}$ & $87^{\circ} 58.806^{\prime} \mathrm{W}$ & $29^{\circ} 19.348^{\prime} \mathrm{N}$ & $87^{\circ} 58.838^{\prime} \mathrm{W}$ & S-116 \\
\hline NF-2008-003 & 6 Oct 08 & VK826 & CTD & 18 & $\mathrm{~N}$ & $29^{\circ} 14.623^{\prime} \mathrm{N}$ & $88^{\circ} 00.726^{\prime} \mathrm{W}$ & $29^{\circ} 14.694^{\prime} \mathrm{N}$ & $88^{\circ} 00.837^{\prime} \mathrm{W}$ & S-246 \\
\hline NF-2008-004 & 6 Oct 08 & VK826 & CTD & 30 & $\mathrm{~N}$ & $29^{\circ} 09.619^{\prime} \mathrm{N}$ & $88^{\circ} 01.205^{\prime} \mathrm{W}$ & $29^{\circ} 09.724^{\prime} \mathrm{N}$ & $88^{\circ} 01.443^{\prime} \mathrm{W}$ & S-469 \\
\hline NF-2008-005 & 6 Oct 08 & VK826 & CTD & 76 & $\mathrm{~N}$ & $29^{\circ} 04.383^{\prime} \mathrm{N}$ & $88^{\circ} 01.973^{\prime} \mathrm{W}$ & $29^{\circ} 03.657^{\prime} \mathrm{N}$ & $88^{\circ} 01.473^{\prime} \mathrm{W}$ & S-1,084 \\
\hline NF-2008-006 & 6 Oct 08 & VK826 & CTD & 52 & $\mathrm{~N}$ & $29^{\circ} 06.155^{\prime} \mathrm{N}$ & $88^{\circ} 01.743^{\prime} \mathrm{W}$ & $29^{\circ} 06.108^{\prime} \mathrm{N}$ & $88^{\circ} 01.283^{\prime} \mathrm{W}$ & S-889 \\
\hline NF-2008-007 & 6 Oct 08 & VK826 & CTD & 42 & D & $29^{\circ} 08.618^{\prime} \mathrm{N}$ & $88^{\circ} 00.997^{\prime} \mathrm{W}$ & $29^{\circ} 08.722^{\prime} \mathrm{N}$ & $88^{\circ} 01.080^{\prime} \mathrm{W}$ & S-676 \\
\hline NF-2008-008 & 6 Oct 08 & VK826 & CTD & 36 & D & $29^{\circ} 08.880^{\prime} \mathrm{N}$ & $88^{\circ} 00.960^{\prime} \mathrm{W}$ & $29^{\circ} 08.883^{\prime} \mathrm{N}$ & $88^{\circ} 00.958^{\prime} \mathrm{W}$ & S-607 \\
\hline NF-2008-009 & 6 Oct 08 & VK826 & CTD & 32 & $\mathrm{D}$ & $29^{\circ} 09.145^{\prime} \mathrm{N}$ & $88^{\circ} 00.950^{\prime} \mathrm{W}$ & $29^{\circ} 09.150^{\prime} \mathrm{N}$ & $88^{\circ} 00.949^{\prime} \mathrm{W}$ & S-567 \\
\hline
\end{tabular}


Table B.1. Stations sampled at two deep coral study sites (VK826, VK862/906) in the Gulf of Mexico using the NOAA ship Nancy Foster, October 6-13, as part of the Lophelia II program - continued.

[min, minute; hr, hour; m, meter; CDT, central daylight time; D, day (0650 to $1830 \mathrm{hr} C D T)$; $\mathrm{N}$, night (1830 to $0650 \mathrm{hr}$ CDT); D/N, samples covering both day and night; CTD, Sea-bird SBE 911+CTD; OT, otter trawl; PN, Plankton net (1.0-m diameter); TT, Tucker trawl with 0.5 m plankton net inserted;

Phyto, phytoplankton sample; Traps, fish traps; S, surface; ROV, Saab Falcon remotely operated vehicle; Albex and Bobo, Royal Netherlands Institute for Sea Research benthic landers]

\begin{tabular}{|c|c|c|c|c|c|c|c|c|c|c|}
\hline Station number & Date & Location & Gear & $\begin{array}{c}\text { Sample } \\
\text { time } \\
\text { (min) }\end{array}$ & Time & Start latitude & $\begin{array}{c}\text { Start } \\
\text { longitude }\end{array}$ & End latitude & $\begin{array}{c}\text { End } \\
\text { longitude }\end{array}$ & $\begin{array}{c}\text { Depth } \\
\text { range }(\mathrm{m})\end{array}$ \\
\hline NF-2008-010 & 06-07 Oct 08 & VK826 & Traps & 899 & $\mathrm{D} / \mathrm{N}$ & $29^{\circ} 09.769^{\prime} \mathrm{N}$ & $88^{\circ} 00.956^{\prime} \mathrm{W}$ & $29^{\circ} 09.769^{\prime} \mathrm{N}$ & $88^{\circ} 00.956^{\prime} \mathrm{W}$ & 481 \\
\hline NF-2008-011 & 06-07 Oct 08 & VK826 & Traps & 1,579 & $\mathrm{D} / \mathrm{N}$ & $29^{\circ} 09.715^{\prime} \mathrm{N}$ & $88^{\circ} 01.394^{\prime} \mathrm{W}$ & $29^{\circ} 09.715^{\prime} \mathrm{N}$ & $88^{\circ} 01.394^{\prime} \mathrm{W}$ & 453 \\
\hline NF-2008-012 & 6 Oct 08 & VK826 & CTD & 41 & $\mathrm{D}$ & $29^{\circ} 09.159^{\prime} \mathrm{N}$ & $88^{\circ} 00.971^{\prime} \mathrm{W}$ & $29^{\circ} 09.159^{\prime} \mathrm{N}$ & $88^{\circ} 00.971^{\prime} \mathrm{W}$ & $\mathrm{S}-568$ \\
\hline NF-2008-013 & 6 Oct 08 & VK826 & CTD & 25 & $\mathrm{~N}$ & $29^{\circ} 09.439^{\prime} \mathrm{N}$ & $88^{\circ} 00.976^{\prime} \mathrm{W}$ & $29^{\circ} 09.439^{\prime} \mathrm{N}$ & $88^{\circ} 00.975^{\prime} \mathrm{W}$ & S-463 \\
\hline NF-2008-014 & 6 Oct 08 & VK826 & CTD & 30 & $\mathrm{~N}$ & $29^{\circ} 09.709^{\prime} \mathrm{N}$ & $88^{\circ} 00.975^{\prime} \mathrm{W}$ & $29^{\circ} 09.708^{\prime} \mathrm{N}$ & $88^{\circ} 00.975^{\prime} \mathrm{W}$ & $S-429$ \\
\hline NF-2008-015 & 6 Oct 08 & VK826 & CTD & 24 & $\mathrm{~N}$ & $29^{\circ} 09.970^{\prime} \mathrm{N}$ & $88^{\circ} 00.982^{\prime} \mathrm{W}$ & $29^{\circ} 09.972^{\prime} \mathrm{N}$ & $88^{\circ} 00.984^{\prime} \mathrm{W}$ & $S-460$ \\
\hline NF-2008-016 & 6 Oct 08 & VK826 & CTD & 25 & $\mathrm{~N}$ & $29^{\circ} 10.242^{\prime} \mathrm{N}$ & $88^{\circ} 00.984^{\prime} \mathrm{W}$ & $29^{\circ} 10.243{ }^{\prime} \mathrm{N}$ & $88^{\circ} 00.984^{\prime} \mathrm{W}$ & $\mathrm{S}-503$ \\
\hline NF-2008-017 & 6 Oct 08 & VK826 & Phyto & & $\mathrm{N}$ & $29^{\circ} 10.241^{\prime} \mathrm{N}$ & $88^{\circ} 00.984^{\prime} \mathrm{W}$ & $29^{\circ} 10.241^{\prime} \mathrm{N}$ & $88^{\circ} 00.984^{\prime} \mathrm{W}$ & S \\
\hline NF-2008-018 & 6 Oct 08 & VK826 & CTD & 23 & $\mathrm{~N}$ & $29^{\circ} 10.509^{\prime} \mathrm{N}$ & $88^{\circ} 00.987^{\prime} \mathrm{W}$ & $29^{\circ} 10.511^{\prime} \mathrm{N}$ & $88^{\circ} 00.985^{\prime} \mathrm{W}$ & $S-463$ \\
\hline NF-2008-019 & 6 Oct 08 & VK826 & Phyto & & $\mathrm{N}$ & $29^{\circ} 10.512^{\prime} \mathrm{N}$ & $88^{\circ} 00.985^{\prime} \mathrm{W}$ & $29^{\circ} 10.512^{\prime} \mathrm{N}$ & $88^{\circ} 00.985^{\prime} \mathrm{W}$ & S \\
\hline NF-2008-020 & 6 Oct 08 & VK826 & CTD & 24 & $\mathrm{~N}$ & $29^{\circ} 10.785^{\prime} \mathrm{N}$ & $88^{\circ} 00.986^{\prime} \mathrm{W}$ & $29^{\circ} 10.785^{\prime} \mathrm{N}$ & $88^{\circ} 00.985^{\prime} \mathrm{W}$ & S-457 \\
\hline NF-2008-021^ & 7 Oct 08 & VK826 & CTD & 316 & $\mathrm{~N}$ & $29^{\circ} 09.394^{\prime} \mathrm{N}$ & $88^{\circ} 01.209^{\prime} \mathrm{W}$ & $29^{\circ} 09.394^{\prime} \mathrm{N}$ & $88^{\circ} 01.209^{\prime} \mathrm{W}$ & $\mathrm{S}-511$ \\
\hline NF-2008-022 & 7 Oct 08 & VK826 & Phyto & & $\mathrm{N}$ & $29^{\circ} 09.397^{\prime} \mathrm{N}$ & $88^{\circ} 01.208^{\prime} \mathrm{W}$ & $29^{\circ} 09.397^{\prime} \mathrm{N}$ & $88^{\circ} 01.208^{\prime} \mathrm{W}$ & S \\
\hline NF-2008-023 & 7 Oct 08 & VK826 & Phyto & & $\mathrm{N}$ & $29^{\circ} 09.394^{\prime} \mathrm{N}$ & $88^{\circ} 01.209^{\prime} \mathrm{W}$ & $29^{\circ} 09.394^{\prime} \mathrm{N}$ & $88^{\circ} 01.209^{\prime} \mathrm{W}$ & S \\
\hline NF-2008-024 & 7 Oct 08 & VK826 & Phyto & & $\mathrm{N}$ & $29^{\circ} 09.393^{\prime} \mathrm{N}$ & $88^{\circ} 01.210^{\prime} \mathrm{W}$ & $29^{\circ} 09.393^{\prime} \mathrm{N}$ & $88^{\circ} 01.210^{\prime} \mathrm{W}$ & $\mathrm{S}$ \\
\hline NF-2008-027 & 8 Oct 08 & VK826 & $\mathrm{PN}$ & 31 & $\mathrm{~N}$ & $29^{\circ} 10.386^{\prime} \mathrm{N}$ & $88^{\circ} 00.502^{\prime} \mathrm{W}$ & $29^{\circ} 09.746^{\prime} \mathrm{N}$ & $88^{\circ} 01.279^{\prime} \mathrm{W}$ & S \\
\hline NF-2008-028 & 8 Oct 08 & VK826 & $\mathrm{PN}$ & 30 & $\mathrm{~N}$ & $29^{\circ} 10.313^{\prime} \mathrm{N}$ & $88^{\circ} 01.817^{\prime} \mathrm{W}$ & $29^{\circ} 09.548^{\prime} \mathrm{N}$ & $88^{\circ} 00.748^{\prime} \mathrm{W}$ & S \\
\hline NF-2008-029 & 8 Oct 08 & VK826 & $\mathrm{PN}$ & 32 & $\mathrm{~N}$ & $29^{\circ} 10.259^{\prime} \mathrm{N}$ & $88^{\circ} 01.154^{\prime} \mathrm{W}$ & $29^{\circ} 09.374^{\prime} \mathrm{N}$ & $88^{\circ} 01.154^{\prime} \mathrm{W}$ & $\mathrm{S}$ \\
\hline NF-2008-030 & 8 Oct 08 & VK826 & $\mathrm{PN}$ & 30 & $\mathrm{~N}$ & $29^{\circ} 10.379^{\prime} \mathrm{N}$ & $88^{\circ} 01.064^{\prime} \mathrm{W}$ & $29^{\circ} 09.605^{\prime} \mathrm{N}$ & $88^{\circ} 00.667^{\prime} \mathrm{W}$ & S \\
\hline NF-2008-031 & 8 Oct 08 & VK826 & $\mathrm{PN}$ & 31 & $\mathrm{~N}$ & $29^{\circ} 10.297^{\prime} \mathrm{N}$ & $88^{\circ} 00.842^{\prime} \mathrm{W}$ & $29^{\circ} 09.468^{\prime} \mathrm{N}$ & $88^{\circ} 00.637^{\prime} \mathrm{W}$ & S \\
\hline NF-2008-032 & 8 Oct 08 & VK826 & $\mathrm{PN}$ & 30 & $\mathrm{~N}$ & $29^{\circ} 10.226^{\prime} \mathrm{N}$ & $88^{\circ} 00.817^{\prime} \mathrm{W}$ & $29^{\circ} 09.257^{\prime} \mathrm{N}$ & $88^{\circ} 00.935^{\prime} \mathrm{W}$ & S \\
\hline NF-2008-033 & 8 Oct 08 & VK862/906 & OT & 30 & $\mathrm{~N}$ & $29^{\circ} 07.539^{\prime} \mathrm{N}$ & $88^{\circ} 22.134^{\prime} \mathrm{W}$ & $29^{\circ} 07.084^{\prime} \mathrm{N}$ & $88^{\circ} 23.129^{\prime} \mathrm{W}$ & $335-340$ \\
\hline NF-2008-034 & 8 Oct 08 & VK862/906 & OT & 30 & $\mathrm{~N}$ & $29^{\circ} 06.068^{\prime} \mathrm{N}$ & $88^{\circ} 24.966^{\prime} \mathrm{W}$ & $29^{\circ} 05.552^{\prime} \mathrm{N}$ & $88^{\circ} 25.873^{\prime} \mathrm{W}$ & $353-360$ \\
\hline NF-2008-035 & 9 Oct 08 & VK862/906 & OT & 30 & $\mathrm{~N}$ & $29^{\circ} 06.979^{\prime} \mathrm{N}$ & $88^{\circ} 21.174^{\prime} \mathrm{W}$ & $29^{\circ} 06.447^{\prime} \mathrm{N}$ & $88^{\circ} 22.155^{\prime} \mathrm{W}$ & 364-365 \\
\hline
\end{tabular}


Table B.1. Stations sampled at two deep coral study sites (VK826, VK862/906) in the Gulf of Mexico using the NOAA ship Nancy Foster, October $6-13$, as part of the Lophelia II program - continued.

[min, minute; hr, hour; m, meter; CDT, central daylight time; D, day (0650 to $1830 \mathrm{hr} C D T)$; $\mathrm{N}$, night (1830 to $0650 \mathrm{hr}$ CDT); D/N, samples covering both day and night; CTD, Sea-bird SBE 911+CTD; OT, otter trawl; PN, Plankton net (1.0-m diameter); TT, Tucker trawl with 0.5 m plankton net inserted;

Phyto, phytoplankton sample; Traps, fish traps; S, surface; ROV, Saab Falcon remotely operated vehicle; Albex and Bobo, Royal Netherlands Institute for Sea Research benthic landers]

\begin{tabular}{|c|c|c|c|c|c|c|c|c|c|c|}
\hline Station number & Date & Location & Gear & $\begin{array}{c}\text { Sample } \\
\text { time } \\
\text { (min) }\end{array}$ & Time & Start latitude & $\begin{array}{c}\text { Start } \\
\text { longitude }\end{array}$ & End latitude & $\begin{array}{c}\text { End } \\
\text { longitude }\end{array}$ & $\begin{array}{c}\text { Depth } \\
\text { range }(\mathrm{m})\end{array}$ \\
\hline NF-2008-036 & 9 Oct 08 & VK862/906 & OT & 30 & $\mathrm{~N}$ & $29^{\circ} 06.125^{\prime} \mathrm{N}$ & $88^{\circ} 20.995^{\prime} \mathrm{W}$ & $29^{\circ} 05.716^{\prime} \mathrm{N}$ & $88^{\circ} 21.938^{\prime} \mathrm{W}$ & $382-408$ \\
\hline NF-2008-037 & 9 Oct 08 & VK862/906 & OT & 30 & $\mathrm{~N}$ & $29^{\circ} 04.597^{\prime} \mathrm{N}$ & $88^{\circ} 24.402^{\prime} \mathrm{W}$ & $29^{\circ} 04.196^{\prime} \mathrm{N}$ & $88^{\circ} 25.280^{\prime} \mathrm{W}$ & $405-410$ \\
\hline NF-2008-038 & 09-10 Oct 08 & VK862/906 & Traps & 1,421 & $\mathrm{D} / \mathrm{N}$ & $29^{\circ} 06.359^{\prime} \mathrm{N}$ & $88^{\circ} 22.936^{\prime} \mathrm{W}$ & $29^{\circ} 06.359^{\prime} \mathrm{N}$ & $88^{\circ} 22.936^{\prime} \mathrm{W}$ & 366 \\
\hline NF-2008-039 & 09-10 Oct 08 & VK862/906 & Traps & 1,331 & $\mathrm{D} / \mathrm{N}$ & $29^{\circ} 06.302^{\prime} \mathrm{N}$ & $88^{\circ} 23.137^{\prime} \mathrm{W}$ & $29^{\circ} 06.302^{\prime} \mathrm{N}$ & $88^{\circ} 23.137^{\prime} \mathrm{W}$ & 335 \\
\hline NF-2008-040 & 9 Oct 08 & VK862/906 & OT & 30 & $\mathrm{~N}$ & $29^{\circ} 05.107^{\prime} \mathrm{N}$ & $88^{\circ} 21.614^{\prime} \mathrm{W}$ & $29^{\circ} 04.699^{\prime} \mathrm{N}$ & $88^{\circ} 22.620^{\prime} \mathrm{W}$ & $432-443$ \\
\hline NF-2008-041 & 9 Oct 08 & VK862/906 & OT & 30 & $\mathrm{~N}$ & $29^{\circ} 03.424^{\prime} \mathrm{N}$ & $88^{\circ} 24.753^{\prime} \mathrm{W}$ & $29^{\circ} 03.424^{\prime} \mathrm{N}$ & $88^{\circ} 25.858^{\prime} \mathrm{W}$ & $432-443$ \\
\hline NF-2008-042 & 10 Oct 08 & VK862/906 & OT & 30 & $\mathrm{~N}$ & $29^{\circ} 04.993^{\prime} \mathrm{N}$ & $88^{\circ} 20.954^{\prime} \mathrm{W}$ & $29^{\circ} 04.446{ }^{\prime} \mathrm{N}$ & $88^{\circ} 21.907^{\prime} \mathrm{W}$ & $432-464$ \\
\hline NF-2008-043 & 10 Oct 08 & VK862/906 & OT & 30 & $\mathrm{~N}$ & $29^{\circ} 03.530^{\prime} \mathrm{N}$ & $88^{\circ} 21.898^{\prime} \mathrm{W}$ & $29^{\circ} 03.530^{\prime} \mathrm{N}$ & $88^{\circ} 22.791^{\prime} \mathrm{W}$ & $512-532$ \\
\hline NF-2008-044 & 10 Oct 08 & VK862/906 & OT & 30 & $\mathrm{~N}$ & $29^{\circ} 06.441^{\prime} \mathrm{N}$ & $88^{\circ} 21.573^{\prime} \mathrm{W}$ & $29^{\circ} 05.945^{\prime} \mathrm{N}$ & $88^{\circ} 22.528^{\prime} \mathrm{W}$ & $360-376$ \\
\hline NF-2008-045 & 10 Oct 08 & VK826 & TT & 30 & $\mathrm{~N}$ & $29^{\circ} 10.310^{\prime} \mathrm{N}$ & $88^{\circ} 01.006^{\prime} \mathrm{W}$ & $29^{\circ} 09.326^{\prime} \mathrm{N}$ & $88^{\circ} 00.976^{\prime} \mathrm{W}$ & $104-140$ \\
\hline NF-2008-046 & 10 Oct 08 & VK826 & TT & 32 & $\mathrm{~N}$ & $29^{\circ} 10.482^{\prime} \mathrm{N}$ & $88^{\circ} 00.970^{\prime} \mathrm{W}$ & $29^{\circ} 09.529^{\prime} \mathrm{N}$ & $88^{\circ} 01.159^{\prime} \mathrm{W}$ & $46-63$ \\
\hline NF-2008-047 & 10 Oct 08 & VK826 & TT & 29 & $\mathrm{~N}$ & $29^{\circ} 10.564^{\prime} \mathrm{N}$ & $88^{\circ} 00.923^{\prime} \mathrm{W}$ & $29^{\circ} 09.702^{\prime} \mathrm{N}$ & $88^{\circ} 01.132^{\prime} \mathrm{W}$ & $51-80$ \\
\hline NF-2008-048 & 10 Oct 08 & VK826 & TT & 28 & $\mathrm{~N}$ & $29^{\circ} 10.036^{\prime} \mathrm{N}$ & $88^{\circ} 01.041^{\prime} \mathrm{W}$ & $29^{\circ} 09.199^{\prime} \mathrm{N}$ & $88^{\circ} 01.251^{\prime} \mathrm{W}$ & $23-40$ \\
\hline NF-2008-049 & 11 Oct 08 & VK826 & TT & 30 & $\mathrm{~N}$ & $29^{\circ} 10.117^{\prime} \mathrm{N}$ & $88^{\circ} 01.009^{\prime} \mathrm{W}$ & $29^{\circ} 09.181^{\prime} \mathrm{N}$ & $88^{\circ} 01.123^{\prime} \mathrm{W}$ & $278-305$ \\
\hline NF-2008-050 & 11 Oct 08 & VK826 & TT & 29 & $\mathrm{~N}$ & $29^{\circ} 10.083^{\prime} \mathrm{N}$ & $88^{\circ} 01.066^{\prime} \mathrm{W}$ & $29^{\circ} 09.217^{\prime} \mathrm{N}$ & $88^{\circ} 01.019^{\prime} \mathrm{W}$ & $13-21$ \\
\hline NF-2008-051 & 11 Oct 08 & VK826 & TT & 32 & $\mathrm{~N}$ & $29^{\circ} 09.864^{\prime} \mathrm{N}$ & $88^{\circ} 00.857^{\prime} \mathrm{W}$ & $29^{\circ} 08.913^{\prime} \mathrm{N}$ & $88^{\circ} 00.890^{\prime} \mathrm{W}$ & $361-381$ \\
\hline NF-2008-052 & 11 Oct 08 & VK826 & TT & 31 & $\mathrm{~N}$ & $29^{\circ} 09.932^{\prime} \mathrm{N}$ & $88^{\circ} 01.016^{\prime} \mathrm{W}$ & $29^{\circ} 09.329^{\prime} \mathrm{N}$ & $88^{\circ} 01.058^{\prime} \mathrm{W}$ & $72-90$ \\
\hline NF-2008-053 & 11 Oct 08 & VK826 & TT & 31 & $\mathrm{~N}$ & $29^{\circ} 10.088^{\prime} \mathrm{N}$ & $88^{\circ} 01.025^{\prime} \mathrm{W}$ & $29^{\circ} 09.193^{\prime} \mathrm{N}$ & $88^{\circ} 01.035^{\prime} \mathrm{W}$ & $13-18$ \\
\hline NF-2008-054 & 11 Oct 08 & VK826 & OT & 30 & $\mathrm{~N}$ & $29^{\circ} 10.424^{\prime} \mathrm{N}$ & $88^{\circ} 02.098^{\prime} \mathrm{W}$ & $29^{\circ} 11.177^{\prime} \mathrm{N}$ & $88^{\circ} 01.373^{\prime} \mathrm{W}$ & $457-502$ \\
\hline NF-2008-056 & 12 Oct 08 & VK826 & OT & 30 & $\mathrm{~N}$ & $29^{\circ} 10.439^{\prime} \mathrm{N}$ & $87^{\circ} 59.971^{\prime} \mathrm{W}$ & $29^{\circ} 11.077^{\prime} \mathrm{N}$ & $87^{\circ} 58.727^{\prime} \mathrm{W}$ & $497-525$ \\
\hline NF-2008-057 & 12 Oct 08 & VK826 & OT & 30 & $\mathrm{~N}$ & $29^{\circ} 10.574^{\prime} \mathrm{N}$ & $87^{\circ} 59.771^{\prime} \mathrm{W}$ & $29^{\circ} 11.189^{\prime} \mathrm{N}$ & $87^{\circ} 59.117^{\prime} \mathrm{W}$ & $481-507$ \\
\hline NF-2008-058 & 12 Oct 08 & VK826 & CTD & 24 & $\mathrm{D}$ & $29^{\circ} 10.425^{\prime} \mathrm{N}$ & $88^{\circ} 00.996^{\prime} \mathrm{W}$ & $29^{\circ} 10.424^{\prime} \mathrm{N}$ & $88^{\circ} 00.996^{\prime} \mathrm{W}$ & $\mathrm{S}-461$ \\
\hline NF-2008-059 & 12 Oct 08 & VK826 & CTD & 28 & $\mathrm{D}$ & $29^{\circ} 10.183^{\prime} \mathrm{N}$ & $88^{\circ} 00.877^{\prime} \mathrm{W}$ & $29^{\circ} 10.182^{\prime} \mathrm{N}$ & $88^{\circ} 00.877^{\prime} \mathrm{W}$ & $\mathrm{S}-500$ \\
\hline NF-2008-060 & 12 Oct 08 & VK826 & CTD & 15 & $\mathrm{D}$ & $29^{\circ} 09.910^{\prime} \mathrm{N}$ & $88^{\circ} 00.806^{\prime} \mathrm{W}$ & $29^{\circ} 09.923^{\prime} \mathrm{N}$ & $88^{\circ} 00.803^{\prime} \mathrm{W}$ & $\mathrm{S}-462$ \\
\hline
\end{tabular}


Table B.1. Stations sampled at two deep coral study sites (VK826, VK862/906) in the Gulf of Mexico using the NOAA ship Nancy Foster, October $6-13$, as part of the Lophelia II program - continued.

[min, minute; hr, hour; m, meter; CDT, central daylight time; D, day (0650 to $1830 \mathrm{hr}$ CDT); $\mathrm{N}$, night (1830 to $0650 \mathrm{hr} \mathrm{CDT}$ ); D/N, samples covering both day and night; CTD, Sea-bird SBE 911+CTD; OT, otter trawl; PN, Plankton net (1.0-m diameter); TT, Tucker trawl with $0.5 \mathrm{~m}$ plankton net inserted; Phyto, phytoplankton sample; Traps, fish traps; S, surface; ROV, Saab Falcon remotely operated vehicle; Albex and Bobo, Royal Netherlands Institute for Sea Research benthic landers]

\begin{tabular}{|c|c|c|c|c|c|c|c|c|c|c|}
\hline Station number & Date & Location & Gear & $\begin{array}{c}\text { Sample } \\
\text { time } \\
\text { (min) }\end{array}$ & Time & Start latitude & $\begin{array}{c}\text { Start } \\
\text { longitude }\end{array}$ & End latitude & $\begin{array}{c}\text { End } \\
\text { longitude }\end{array}$ & $\begin{array}{l}\text { Depth } \\
\text { range }(m)\end{array}$ \\
\hline NF-2008-061 & 12 Oct 08 & VK826 & CTD & 16 & $\mathrm{D}$ & $29^{\circ} 09.664^{\prime} \mathrm{N}$ & $88^{\circ} 00.720^{\prime} \mathrm{W}$ & $29^{\circ} 09.663^{\prime} \mathrm{N}$ & $88^{\circ} 00.718^{\prime} \mathrm{W}$ & $S-473$ \\
\hline NF-2008-062 & 12 Oct 08 & VK826 & CTD & 16 & $\mathrm{D}$ & $29^{\circ} 09.399^{\prime} \mathrm{N}$ & $88^{\circ} 00.647^{\prime} \mathrm{W}$ & $29^{\circ} 09.399^{\prime} \mathrm{N}$ & $88^{\circ} 00.647^{\prime} \mathrm{W}$ & $\mathrm{S}-502$ \\
\hline NF-2008-063A & 12 Oct 08 & VK826 & CTD & 18 & $\mathrm{D}$ & $29^{\circ} 09.139^{\prime} \mathrm{N}$ & $88^{\circ} 00.579^{\prime} \mathrm{W}$ & $29^{\circ} 09.138^{\prime} \mathrm{N}$ & $88^{\circ} 00.578^{\prime} \mathrm{W}$ & S-569 \\
\hline NF-2008-063B & 12 Oct 08 & VK826 & CTD & 22 & D & $29^{\circ} 08.885^{\prime} \mathrm{N}$ & $88^{\circ} 00.486^{\prime} \mathrm{W}$ & $29^{\circ} 08.884^{\prime} \mathrm{N}$ & $88^{\circ} 00.487^{\prime} \mathrm{W}$ & S-667 \\
\hline NF-2008-064 & 12 Oct 08 & VK826 & CTD & 22 & $\mathrm{D}$ & $29^{\circ} 08.607^{\prime} \mathrm{N}$ & $88^{\circ} 00.401^{\prime} \mathrm{W}$ & $29^{\circ} 08.609^{\prime} \mathrm{N}$ & $88^{\circ} 00.402^{\prime} \mathrm{W}$ & S-681 \\
\hline NF-2008-065 & 12 Oct 08 & VK826 & CTD & 22 & $\mathrm{D}$ & $29^{\circ} 08.619^{\prime} \mathrm{N}$ & $88^{\circ} 00.963^{\prime} \mathrm{W}$ & $29^{\circ} 08.619^{\prime} \mathrm{N}$ & $88^{\circ} 00.964^{\prime} \mathrm{W}$ & S-675 \\
\hline NF-2008-066 & 12 Oct 08 & VK826 & CTD & 33 & $\mathrm{~N}$ & $29^{\circ} 08.899^{\prime} \mathrm{N}$ & $88^{\circ} 00.975^{\prime} \mathrm{W}$ & $29^{\circ} 08.899^{\prime} \mathrm{N}$ & $88^{\circ} 00.977^{\prime} \mathrm{W}$ & S-618 \\
\hline NF-2008-067 & 12 Oct 08 & VK826 & CTD & 28 & $\mathrm{~N}$ & $29^{\circ} 09.164^{\prime} \mathrm{N}$ & $88^{\circ} 00.980^{\prime} \mathrm{W}$ & $29^{\circ} 09.164^{\prime} \mathrm{N}$ & $88^{\circ} 00.980^{\prime} \mathrm{W}$ & S-562 \\
\hline NF-2008-068 & 12 Oct 08 & VK826 & CTD & 25 & $\mathrm{~N}$ & $29^{\circ} 09.431^{\prime} \mathrm{N}$ & $88^{\circ} 00.973^{\prime} \mathrm{W}$ & $29^{\circ} 09.430^{\prime} \mathrm{N}$ & $88^{\circ} 00.976^{\prime} \mathrm{W}$ & S-463 \\
\hline NF-2008-069 & 12 Oct 08 & VK826 & CTD & 22 & $\mathrm{~N}$ & $29^{\circ} 09.702^{\prime} \mathrm{N}$ & $88^{\circ} 00.768^{\prime} \mathrm{W}$ & $29^{\circ} 09.701^{\prime} \mathrm{N}$ & $88^{\circ} 00.973^{\prime} \mathrm{W}$ & $S-436$ \\
\hline NF-2008-070 & 12 Oct 08 & VK826 & CTD & 24 & $\mathrm{~N}$ & $29^{\circ} 09.981^{\prime} \mathrm{N}$ & $88^{\circ} 00.987^{\prime} \mathrm{W}$ & $29^{\circ} 09.9711^{\prime} \mathrm{N}$ & $88^{\circ} 00.979^{\prime} \mathrm{W}$ & S-465 \\
\hline NF-2008-071 & 12 Oct 08 & VK826 & CTD & 26 & $\mathrm{~N}$ & $29^{\circ} 10.246^{\prime} \mathrm{N}$ & $88^{\circ} 00.979^{\prime} \mathrm{W}$ & $29^{\circ} 10.243^{\prime} \mathrm{N}$ & $88^{\circ} 00.978^{\prime} \mathrm{W}$ & S-506 \\
\hline NF-2008-072 & 13 Oct 08 & VK826 & OT & 30 & $\mathrm{~N}$ & $29^{\circ} 08.844^{\prime} \mathrm{N}$ & $88^{\circ} 02.976^{\prime} \mathrm{W}$ & $29^{\circ} 09.456^{\prime} \mathrm{N}$ & $88^{\circ} 02.089^{\prime} \mathrm{W}$ & $534-557$ \\
\hline NF-2008-073 & 13 Oct 08 & VK826 & OT & 28 & $\mathrm{~N}$ & $29^{\circ} 08.292^{\prime} \mathrm{N}$ & $88^{\circ} 01.722^{\prime} \mathrm{W}$ & $29^{\circ} 08.965^{\prime} \mathrm{N}$ & $88^{\circ} 01.007^{\prime} \mathrm{W}$ & $610-674$ \\
\hline NF-2008-074 & 13 Oct 08 & VK826 & OT & 30 & $\mathrm{~N}$ & $29^{\circ} 09.255^{\prime} \mathrm{N}$ & $87^{\circ} 59.606^{\prime} \mathrm{W}$ & $29^{\circ} 08.928^{\prime} \mathrm{N}$ & $87^{\circ} 58.669^{\prime} \mathrm{W}$ & $645-744$ \\
\hline NF-2008-077 & 13 Oct 08 & VK826 & $\mathrm{TT}$ & 30 & $\mathrm{D}$ & $29^{\circ} 10.469^{\prime} \mathrm{N}$ & $88^{\circ} 01.041^{\prime} \mathrm{W}$ & $29^{\circ} 10.482^{\prime} \mathrm{N}$ & $88^{\circ} 00.028^{\prime} \mathrm{W}$ & $94-119$ \\
\hline NF-2008-078 & 13 Oct 08 & VK826 & TT & 30 & $\mathrm{D}$ & $29^{\circ} 09.850^{\prime} \mathrm{N}$ & $88^{\circ} 01.315^{\prime} \mathrm{W}$ & $29^{\circ} 09.903^{\prime} \mathrm{N}$ & $88^{\circ} 00.241^{\prime} \mathrm{W}$ & $173-215$ \\
\hline NF-2008-079 & 13 Oct 08 & VK826 & TT & 30 & D & $29^{\circ} 09.876^{\prime} \mathrm{N}$ & $88^{\circ} 01.025^{\prime} \mathrm{W}$ & $29^{\circ} 09.826^{\prime} \mathrm{N}$ & $88^{\circ} 02.741^{\prime} \mathrm{W}$ & 244-306 \\
\hline NF-2008-080 & 13 Oct 08 & VK826 & TT & 30 & D & $29^{\circ} 09.754^{\prime} \mathrm{N}$ & $88^{\circ} 00.701^{\prime} \mathrm{W}$ & $29^{\circ} 09.656^{\prime} \mathrm{N}$ & $87^{\circ} 59.796^{\prime} \mathrm{W}$ & 309-358 \\
\hline NF-2008-081 & 13 Oct 08 & VK826 & TT & 27 & D & $29^{\circ} 09.683^{\prime} \mathrm{N}$ & $88^{\circ} 00.938^{\prime} \mathrm{W}$ & $29^{\circ} 09.607^{\prime} \mathrm{N}$ & $87^{\circ} 59.942^{\prime} \mathrm{W}$ & $416-438$ \\
\hline NF-2008-082 & 13 Oct 08 & VK826 & TT & 30 & $\mathrm{D}$ & $29^{\circ} 09.816^{\prime} \mathrm{N}$ & $88^{\circ} 00.630^{\prime} \mathrm{W}$ & $29^{\circ} 09.195^{\prime} \mathrm{N}$ & $87^{\circ} 59.974^{\prime} \mathrm{W}$ & $43-68$ \\
\hline NF-2008-083 & 13 Oct 08 & VK826 & TT & 30 & $\mathrm{D}$ & $29^{\circ} 09.729^{\prime} \mathrm{N}$ & $88^{\circ} 01.406^{\prime} \mathrm{W}$ & $29^{\circ} 10.147^{\prime} \mathrm{N}$ & $88^{\circ} 00.298^{\prime} \mathrm{W}$ & $29-46$ \\
\hline
\end{tabular}


Table B.2. Stations sampled on deep coral areas in the eastern and the north-central Gulf of Mexico using the research vessel (R/V) Seward Johnson, September 16-24, 2009, as part of the Lophelia II program.

[min, minute; hr, hour; m, meter; EDT, Eastern Daylight Time; WFS, West Florida Slope; D, Day (0700 to $1830 \mathrm{hr}$ EDT); N, Night (1830 to 0700 hr EDT); M, Morning (0700 to $1200 \mathrm{hr}$ EDT); A, Afternoon (1200 to $1830 \mathrm{hr}$ EDT); BC, Box Core; CTD, Sea-bird SBE 911+ CTD; Moc, MOCNESS 1x1m (335 $\mu \mathrm{m}$ ); OD, on deck; Phyto, Phytoplankton sample; PN, Plankton net (1.0 m, 0.5 m, 0.25-m diameter); JSL, Johnson Sea Link II submersible. Submersible depth range data are only for the period when the $J S L$ was on the bottom. For MOCNESS trawls that fished discrete depths, depth ranges are the minimum and maximum of all mean depths calculated per tow. For nets that failed to close and for the CTD casts, depth ranges include the absolute maximum depth of the sample to the surface $(\mathrm{S})]$

\begin{tabular}{|c|c|c|c|c|c|c|c|c|c|c|}
\hline Station number & Date & Location & Gear & $\begin{array}{c}\text { Sample } \\
\text { time } \\
\text { (min) }\end{array}$ & Time & Start latitude & $\begin{array}{c}\text { Start } \\
\text { longitude }\end{array}$ & End latitude & $\begin{array}{l}\text { End } \\
\text { longitude }\end{array}$ & $\begin{array}{c}\text { Depth range } \\
(\mathrm{m})\end{array}$ \\
\hline $\begin{array}{l}\text { JSLII-2009-GOM- } \\
3722\end{array}$ & 16 Sep 09 & WFS & $J S L$ & 158 & M & $26^{\circ} 12.189^{\prime} \mathrm{N}$ & $84^{\circ} 43.637^{\prime} \mathrm{W}$ & $26^{\circ} 12.309^{\prime} \mathrm{N}$ & $84^{\circ} 43.621^{\prime} \mathrm{W}$ & $536-500$ \\
\hline $\begin{array}{l}\text { JSLII-2009-GOM- } \\
3723\end{array}$ & 16 Sep 09 & WFS & $J S L$ & 157 & A & $26^{\circ} 12.427^{\prime} \mathrm{N}$ & $84^{\circ} 42.726^{\prime} \mathrm{W}$ & $26^{\circ} 12.710^{\prime} \mathrm{N}$ & $84^{\circ} 42.828^{\prime} \mathrm{W}$ & $428-414$ \\
\hline $\begin{array}{l}\text { JSLII-2009-GOM- } \\
3724\end{array}$ & 17 Sep 09 & WFS & $J S L$ & 161 & M & $26^{\circ} 12.213^{\prime} \mathrm{N}$ & $84^{\circ} 43.746^{\prime} \mathrm{W}$ & $26^{\circ} 12.473^{\prime} \mathrm{N}$ & $84^{\circ} 43.538^{\prime} \mathrm{W}$ & $531-477$ \\
\hline $\begin{array}{l}\text { JSLII-2009-GOM- } \\
3725\end{array}$ & 18 Sep 09 & VK862/906 & $J S L$ & 176 & A & $29^{\circ} 04.146^{\prime} \mathrm{N}$ & $88^{\circ} 22.567^{\prime} \mathrm{W}$ & $29^{\circ} 04.167^{\prime} \mathrm{N}$ & $88^{\circ} 22.637^{\prime} \mathrm{W}$ & $416-388$ \\
\hline $\begin{array}{l}\text { JSLII-2009-GOM- } \\
3726\end{array}$ & 19 Sep 09 & MC751 & $J S L$ & 165 & M & $28^{\circ} 11.623^{\prime} \mathrm{N}$ & $89^{\circ} 47.940^{\prime} \mathrm{W}$ & $28^{\circ} 11.744^{\prime} \mathrm{N}$ & $89^{\circ} 48.050^{\prime} \mathrm{W}$ & $438-434$ \\
\hline $\begin{array}{l}\text { JSLII-2009-GOM- } \\
3727\end{array}$ & 19 Sep 09 & MC751 & $J S L$ & 174 & A & $28^{\circ} 11.427^{\prime} \mathrm{N}$ & $89^{\circ} 47.922^{\prime} \mathrm{W}$ & $28^{\circ} 11.615^{\prime} \mathrm{N}$ & $89^{\circ} 48.088^{\prime} \mathrm{W}$ & $458-445$ \\
\hline $\begin{array}{l}\text { JSLII-2009-GOM- } \\
3728\end{array}$ & 20 Sep 09 & VK862/906 & $J S L$ & 159 & M & $29^{\circ} 06.376^{\prime} \mathrm{N}$ & $88^{\circ} 22.982^{\prime} \mathrm{W}$ & $29^{\circ} 06.404^{\prime} \mathrm{N}$ & $88^{\circ} 23.101^{\prime} \mathrm{W}$ & $336-316$ \\
\hline $\begin{array}{l}\text { JSLII-2009-GOM- } \\
3729\end{array}$ & 20 Sep 09 & VK862/906 & $J S L$ & 160 & A & $29^{\circ} 04.152^{\prime} \mathrm{N}$ & $88^{\circ} 22.747^{\prime} \mathrm{W}$ & $29^{\circ} 04.155^{\prime} \mathrm{N}$ & $88^{\circ} 22.627^{\prime} \mathrm{W}$ & $436-389$ \\
\hline $\begin{array}{l}\text { JSLII-2009-GOM- } \\
3730\end{array}$ & 21 Sep 09 & VK862/906 & $J S L$ & 168 & M & $29^{\circ} 05.040^{\prime} \mathrm{N}$ & $88^{\circ} 23.820^{\prime} \mathrm{W}$ & $29^{\circ} 05.273^{\prime} \mathrm{N}$ & $88^{\circ} 23.872^{\prime} \mathrm{W}$ & 419-396 \\
\hline $\begin{array}{l}\text { JSLII-2009-GOM- } \\
3731\end{array}$ & 21 Sep 09 & VK862/906 & $J S L$ & 161 & A & $29^{\circ} 04.098^{\prime} \mathrm{N}$ & $88^{\circ} 22.714^{\prime} \mathrm{W}$ & $29^{\circ} 04.140^{\prime} \mathrm{N}$ & $88^{\circ} 22.671^{\prime} \mathrm{W}$ & 424-394 \\
\hline $\begin{array}{l}\text { JSLII-2009-GOM- } \\
3732\end{array}$ & 22 Sep 09 & VK862/906 & $J S L$ & 158 & M & $29^{\circ} 06.549^{\prime} \mathrm{N}$ & $88^{\circ} 23.250^{\prime} \mathrm{W}$ & $29^{\circ} 06.477^{\prime} \mathrm{N}$ & $88^{\circ} 23.118^{\prime} \mathrm{W}$ & $354-312$ \\
\hline $\begin{array}{l}\text { JSLII-2009-GOM- } \\
3733\end{array}$ & 22 Sep 09 & VK826 & $J S L$ & 168 & A & $29^{\circ} 09.769^{\prime} \mathrm{N}$ & $88^{\circ} 00.586^{\prime} \mathrm{W}$ & $29^{\circ} 09.962^{\prime} \mathrm{N}$ & $88^{\circ} 00.708^{\prime} \mathrm{W}$ & $477-471$ \\
\hline $\begin{array}{l}\text { JSLII-2009-GOM- } \\
3734\end{array}$ & 23 Sep 09 & VK826 & $J S L$ & 166 & M & $29^{\circ} 10.178^{\prime} \mathrm{N}$ & $88^{\circ} 00.766^{\prime} \mathrm{W}$ & $29^{\circ} 10.217^{\prime} \mathrm{N}$ & $88^{\circ} 00.713^{\prime} \mathrm{W}$ & $481-463$ \\
\hline $\begin{array}{l}\text { JSLII-2009-GOM- } \\
3735\end{array}$ & 23 Sep 09 & VK826 & $J S L$ & 171 & A & $29^{\circ} 10.259^{\prime} \mathrm{N}$ & $88^{\circ} 00.803^{\prime} \mathrm{W}$ & $29^{\circ} 10.249^{\prime} \mathrm{N}$ & $88^{\circ} 00.686^{\prime} \mathrm{W}$ & $484-464$ \\
\hline
\end{tabular}


Table B.2. Stations sampled on deep coral areas in the eastern and north-central Gulf of Mexico using the research vessel (R/V) Seward Johnson, September 16-24, 2009, as part of the Lophelia II program - continued.

[min, minute; hr, hour; m, meter; EDT, Eastern Daylight Time; WFS, West Florida Slope; D, Day (0700 to $1830 \mathrm{hr}$ EDT); N, Night (1830 to $0700 \mathrm{hr}$ EDT); M, Morning (0700 to $1200 \mathrm{hr}$ EDT); A, Afternoon (1200 to $1830 \mathrm{hr}$ EDT); BC, Box Core; CTD, Sea-bird SBE 911+ CTD; Moc, MOCNESS 1x1m (335 $\mu \mathrm{m})$; OD, on deck; Phyto, Phytoplankton sample; PN, Plankton net (1.0 m, 0.5 m, 0.25-m diameter); JSL, Johnson Sea Link II submersible. Submersible depth range data are only for the period when the $J S L$ was on the bottom. For MOCNESS trawls that fished discrete depths, depth ranges are the minimum and maximum of all mean depths calculated per tow. For nets that failed to close and for the CTD casts, depth ranges include the absolute maximum depth of the sample to the surface $(\mathrm{S})]$

\begin{tabular}{|c|c|c|c|c|c|c|c|c|c|c|}
\hline Station number & Date & Location & Gear & $\begin{array}{c}\text { Sample } \\
\text { time } \\
\text { (min) }\end{array}$ & Time & Start latitude & $\begin{array}{c}\text { Start } \\
\text { longitude }\end{array}$ & End latitude & $\begin{array}{l}\text { End } \\
\text { longitude }\end{array}$ & $\begin{array}{c}\text { Depth range } \\
(\mathrm{m})\end{array}$ \\
\hline $\begin{array}{l}\text { JSLII-2009-GOM- } \\
3736\end{array}$ & 24 Sep 09 & VK826 & $J S L$ & 166 & M & $29^{\circ} 10.254^{\prime} \mathrm{N}$ & $88^{\circ} 00.668^{\prime} \mathrm{W}$ & $29^{\circ} 10.187^{\prime} \mathrm{N}$ & $88^{\circ} 00.693^{\prime} \mathrm{W}$ & $477-476$ \\
\hline SJ-2009-GOM-002 & 16 Sep 09 & WFS & CTD & 21 & $\mathrm{~N}$ & $26^{\circ} 12.304^{\prime} \mathrm{N}$ & $84^{\circ} 43.657^{\prime} \mathrm{W}$ & $26^{\circ} 12.387^{\prime} \mathrm{N}$ & $84^{\circ} 43.399^{\prime} \mathrm{W}$ & S-439 \\
\hline SJ-2009-GOM-003 & 16 Sep 09 & WFS & OD & & $\mathrm{N}$ & $26^{\circ} 12.465^{\prime} \mathrm{N}$ & $84^{\circ} 43.208^{\prime} \mathrm{W}$ & $26^{\circ} 12.465^{\prime} \mathrm{N}$ & $84^{\circ} 43.208^{\prime} \mathrm{W}$ & $\mathrm{S}$ \\
\hline SJ-2009-GOM-004 & 16 Sep 09 & WFS & $\mathrm{BC}$ & & $\mathrm{N}$ & $26^{\circ} 12.289^{\prime} \mathrm{N}$ & $84^{\circ} 43.763^{\prime} \mathrm{W}$ & $26^{\circ} 12.289^{\prime} \mathrm{N}$ & $84^{\circ} 43.763^{\prime} \mathrm{W}$ & 500 \\
\hline SJ-2009-GOM-005 & 16 Sep 09 & WFS & $\mathrm{BC}$ & & $\mathrm{N}$ & $26^{\circ} 12.388^{\prime} \mathrm{N}$ & $84^{\circ} 43.520^{\prime} \mathrm{W}$ & $26^{\circ} 12.388^{\prime} \mathrm{N}$ & $84^{\circ} 43.520^{\prime} \mathrm{W}$ & 498 \\
\hline SJ-2009-GOM-006 & 16 Sep 09 & WFS & $\mathrm{BC}$ & & $\mathrm{N}$ & $26^{\circ} 12.453^{\prime} \mathrm{N}$ & $84^{\circ} 43.531^{\prime} \mathrm{W}$ & $26^{\circ} 12.453^{\prime} \mathrm{N}$ & $84^{\circ} 43.531^{\prime} \mathrm{W}$ & 488 \\
\hline SJ-2009-GOM-007 & 16 Sep 09 & WFS & Phyto & & $\mathrm{N}$ & $26^{\circ} 12.535^{\prime} \mathrm{N}$ & $84^{\circ} 43.316^{\prime} \mathrm{W}$ & $26^{\circ} 12.535^{\prime} \mathrm{N}$ & $84^{\circ} 43.316^{\prime} \mathrm{W}$ & 481 \\
\hline SJ-2009-GOM-008 & 16 Sep 09 & WFS & CTD & 21 & $\mathrm{~N}$ & $26^{\circ} 12.361 ' \mathrm{~N}$ & $84^{\circ} 43.679^{\prime} \mathrm{W}$ & $26^{\circ} 12.336^{\prime} \mathrm{N}$ & $84^{\circ} 43.611^{\prime} \mathrm{W}$ & $0-459$ \\
\hline SJ-2009-GOM-009 & 16 Sep 09 & WFS & $\mathrm{PN} 1.0 \mathrm{~m}$ & 13 & $\mathrm{~N}$ & $26^{\circ} 12.530^{\prime} \mathrm{N}$ & $84^{\circ} 43.848^{\prime} \mathrm{W}$ & $26^{\circ} 12.608^{\prime} \mathrm{N}$ & $84^{\circ} 43.588^{\prime} \mathrm{W}$ & $\mathrm{S}$ \\
\hline SJ-2009-GOM-010 & 17 Sep 09 & WFS & $\mathrm{PN} 1.0 \mathrm{~m}$ & 15 & $\mathrm{~N}$ & $26^{\circ} 12.808^{\prime} \mathrm{N}$ & $84^{\circ} 43.248^{\prime} \mathrm{W}$ & $26^{\circ} 13.009^{\prime} \mathrm{N}$ & $84^{\circ} 43.040^{\prime} \mathrm{W}$ & $\mathrm{S}-4$ \\
\hline SJ-2009-GOM-011 & 17 Sep 09 & WFS & Phyto & & $\mathrm{N}$ & $26^{\circ} 13.009^{\prime} \mathrm{N}$ & $84^{\circ} 43.016^{\prime} \mathrm{W}$ & $26^{\circ} 13.009^{\prime} \mathrm{N}$ & $84^{\circ} 43.016^{\prime} \mathrm{W}$ & 410 \\
\hline SJ-2009-GOM-012 & 17 Sep 09 & WFS & CTD & 15 & $\mathrm{~N}$ & $26^{\circ} 12.291 \mathrm{~N}$ & $84^{\circ} 43.652^{\prime} \mathrm{W}$ & $26^{\circ} 12.339^{\prime} \mathrm{N}$ & $84^{\circ} 43.584^{\prime} \mathrm{W}$ & $S-437$ \\
\hline SJ-2009-GOM-013 & 17 Sep 09 & WFS & $\mathrm{PN} 1.0 \mathrm{~m}$ & 15 & $\mathrm{~N}$ & $26^{\circ} 12.411^{\prime} \mathrm{N}$ & $84^{\circ} 43.623^{\prime} \mathrm{W}$ & $26^{\circ} 12.583^{\prime} \mathrm{N}$ & $84^{\circ} 43.254^{\prime} \mathrm{W}$ & $\mathrm{S}-25$ \\
\hline SJ-2009-GOM-014 & 17 Sep 09 & WFS & CTD & 16 & $\mathrm{~N}$ & $26^{\circ} 12.270^{\prime} \mathrm{N}$ & $84^{\circ} 43.672^{\prime} \mathrm{W}$ & $26^{\circ} 12.256^{\prime} \mathrm{N}$ & $84^{\circ} 43.682^{\prime} \mathrm{W}$ & $\mathrm{S}-438$ \\
\hline SJ-2009-GOM-015 & 17 Sep 09 & WFS & PN $1.0 \mathrm{~m}$ & 17 & $\mathrm{~N}$ & $26^{\circ} 12.369^{\prime} \mathrm{N}$ & $84^{\circ} 43.276^{\prime} \mathrm{W}$ & $26^{\circ} 12.102^{\prime} \mathrm{N}$ & $84^{\circ} 43.561^{\prime} \mathrm{W}$ & $0-8$ \\
\hline SJ-2009-GOM-016 & 18 Sep 09 & VK862/906 & Phyto & & $\mathrm{N}$ & $29^{\circ} 04.023^{\prime} \mathrm{N}$ & $88^{\circ} 22.645^{\prime} \mathrm{W}$ & $29^{\circ} 04.023^{\prime} \mathrm{N}$ & $88^{\circ} 22.645^{\prime} \mathrm{W}$ & 461 \\
\hline SJ-2009-GOM-017 & 18 Sep 09 & VK862/906 & Phyto & & $\mathrm{N}$ & $29^{\circ} 04.054^{\prime} \mathrm{N}$ & $88^{\circ} 22.617^{\prime} \mathrm{W}$ & $29^{\circ} 04.054^{\prime} \mathrm{N}$ & $88^{\circ} 22.617^{\prime} \mathrm{W}$ & 412 \\
\hline SJ-2009-GOM-018 & 18 Sep 09 & VK862/906 & Phyto & & $\mathrm{N}$ & $29^{\circ} 04.026^{\prime} \mathrm{N}$ & $88^{\circ} 22.750^{\prime} \mathrm{W}$ & $29^{\circ} 04.026^{\prime} \mathrm{N}$ & $88^{\circ} 22.750^{\prime} \mathrm{W}$ & 424 \\
\hline SJ-2009-GOM-019 & 19 Sep 09 & MC751 & CTD & 17 & $\mathrm{~N}$ & $28^{\circ} 11.616^{\prime} \mathrm{N}$ & $89^{\circ} 47.949^{\prime} \mathrm{W}$ & $28^{\circ} 11.521^{\prime} \mathrm{N}$ & $89^{\circ} 48.136^{\prime} \mathrm{W}$ & $\mathrm{S}-423$ \\
\hline SJ-2009-GOM-020 & 19 Sep 09 & MC751 & Phyto & & $\mathrm{N}$ & $28^{\circ} 11.489^{\prime} \mathrm{N}$ & $89^{\circ} 48.231^{\prime} \mathrm{W}$ & $28^{\circ} 11.489^{\prime} \mathrm{N}$ & $89^{\circ} 48.231^{\prime} \mathrm{W}$ & 451 \\
\hline SJ-2009-GOM-021 & 19 Sep 09 & MC751 & Phyto & & $\mathrm{N}$ & $28^{\circ} 11.487^{\prime} \mathrm{N}$ & $89^{\circ} 48.249^{\prime} \mathrm{W}$ & $28^{\circ} 11.487^{\prime} \mathrm{N}$ & $89^{\circ} 48.249^{\prime} \mathrm{W}$ & 450 \\
\hline SJ-2009-GOM-022 & 19 Sep 09 & MC751 & Phyto & & $\mathrm{N}$ & $28^{\circ} 11.397^{\prime} \mathrm{N}$ & $89^{\circ} 48.335^{\prime} \mathrm{W}$ & $28^{\circ} 11.397^{\prime} \mathrm{N}$ & $89^{\circ} 48.335^{\prime} \mathrm{W}$ & 455 \\
\hline SJ-2009-GOM-023 & 19 Sep 09 & MC751 & Phyto & & $\mathrm{N}$ & $28^{\circ} 11.641 ' \mathrm{~N}$ & $89^{\circ} 47.842^{\prime} \mathrm{W}$ & $28^{\circ} 11.641^{\prime} \mathrm{N}$ & $89^{\circ} 47.842^{\prime} \mathrm{W}$ & 441 \\
\hline
\end{tabular}


Table B.2. Stations sampled on deep coral areas in the eastern and north-central Gulf of Mexico using the research vessel (R/V) Seward Johnson, September 16-24, 2009, as part of the Lophelia II program - continued.

[min, minute; hr, hour; m, meter; EDT, Eastern Daylight Time; WFS, West Florida Slope; D, Day (0700 to $1830 \mathrm{hr}$ EDT); N, Night (1830 to $0700 \mathrm{hr}$ EDT); M, Morning (0700 to $1200 \mathrm{hr}$ EDT); A, Afternoon (1200 to $1830 \mathrm{hr}$ EDT); BC, Box Core; CTD, Sea-bird SBE 911+ CTD; Moc, MOCNESS 1x1m (335 $\mu \mathrm{m})$; OD, on deck; Phyto, Phytoplankton sample; PN, Plankton net (1.0 m, 0.5 m, 0.25 -m diameter); JSL, Johnson Sea Link II submersible. Submersible depth range data are only for the period when the $J S L$ was on the bottom. For MOCNESS trawls that fished discrete depths, depth ranges are the minimum and maximum of all mean depths calculated per tow. For nets that failed to close and for the CTD casts, depth ranges include the absolute maximum depth of the sample to the surface $(\mathrm{S})]$

\begin{tabular}{|c|c|c|c|c|c|c|c|c|c|c|}
\hline Station number & Date & Location & Gear & $\begin{array}{c}\text { Sample } \\
\text { time } \\
\text { (min) }\end{array}$ & Time & Start latitude & $\begin{array}{c}\text { Start } \\
\text { longitude }\end{array}$ & End latitude & $\begin{array}{c}\text { End } \\
\text { longitude }\end{array}$ & $\begin{array}{c}\text { Depth } \\
\text { range }(m)\end{array}$ \\
\hline SJ-2009-GOM-024 & 19 Sep 09 & MC751 & Phyto & & $\mathrm{N}$ & $28^{\circ} 11.844^{\prime} \mathrm{N}$ & $89^{\circ} 47.599^{\prime} \mathrm{W}$ & $28^{\circ} 11.844^{\prime} \mathrm{N}$ & $89^{\circ} 47.599^{\prime} \mathrm{W}$ & 453 \\
\hline SJ-2009-GOM-025 & 19 Sep 09 & MC751 & $\mathrm{BC}$ & & $\mathrm{D}$ & $28^{\circ} 11.831^{\prime} \mathrm{N}$ & $89^{\circ} 47.910^{\prime} \mathrm{W}$ & $28^{\circ} 11.831^{\prime} \mathrm{N}$ & $89^{\circ} 47.910^{\prime} \mathrm{W}$ & 431 \\
\hline SJ-2009-GOM-026 & 19 Sep 09 & MC751 & $\mathrm{BC}$ & & $\mathrm{D}$ & $28^{\circ} 11.839^{\prime} \mathrm{N}$ & $89^{\circ} 47.936^{\prime} \mathrm{W}$ & $28^{\circ} 11.839^{\prime} \mathrm{N}$ & $89^{\circ} 47.936^{\prime} \mathrm{W}$ & 427 \\
\hline SJ-2009-GOM-027 & 19 Sep 09 & MC751 & $\mathrm{BC}$ & & $\mathrm{D}$ & $28^{\circ} 11.813^{\prime} \mathrm{N}$ & $89^{\circ} 47.926^{\prime} \mathrm{W}$ & $28^{\circ} 11.813^{\prime} \mathrm{N}$ & $89^{\circ} 47.926^{\prime} \mathrm{W}$ & 429 \\
\hline SJ-2009-GOM-028 & 19 Sep 09 & MC751 & $\mathrm{BC}$ & & $\mathrm{D}$ & $28^{\circ} 11.805^{\prime} \mathrm{N}$ & $89^{\circ} 47.991^{\prime} \mathrm{W}$ & $28^{\circ} 11.805^{\prime} \mathrm{N}$ & $89^{\circ} 47.991^{\prime} \mathrm{W}$ & 428 \\
\hline SJ-2009-GOM-029 & 19 Sep 09 & MC751 & CTD & 16 & $\mathrm{D}$ & $28^{\circ} 11.470^{\prime} \mathrm{N}$ & $89^{\circ} 47.915^{\prime} \mathrm{W}$ & $28^{\circ} 11.438^{\prime} \mathrm{N}$ & $89^{\circ} 47.835^{\prime} \mathrm{W}$ & S-428 \\
\hline SJ-2009-GOM-030 & 20 Sep 09 & VK862/906 & Traps & 2,877 & $\mathrm{D}$ & $29^{\circ} 06.283^{\prime} \mathrm{N}$ & $88^{\circ} 23.091^{\prime} \mathrm{W}$ & $29^{\circ} 06.394^{\prime} \mathrm{N}$ & $88^{\circ} 23.282^{\prime} \mathrm{W}$ & 305 \\
\hline SJ-2009-GOM-031 & 20 Sep 09 & VK862/906 & Traps & 1,483 & $\mathrm{D}$ & $29^{\circ} 06.390^{\prime} \mathrm{N}$ & $88^{\circ} 23.140^{\prime} \mathrm{W}$ & $29^{\circ} 06.523^{\prime} \mathrm{N}$ & $88^{\circ} 23.482^{\prime} \mathrm{W}$ & 325 \\
\hline SJ-2009-GOM-032 & 20 Sep 09 & VK862/906 & $\mathrm{BC}$ & & $\mathrm{D}$ & $29^{\circ} 04.335^{\prime} \mathrm{N}$ & $88^{\circ} 22.463^{\prime} \mathrm{W}$ & $29^{\circ} 04.335^{\prime} \mathrm{N}$ & $88^{\circ} 22.463^{\prime} \mathrm{W}$ & 401 \\
\hline SJ-2009-GOM-033 & 20 Sep 09 & VK862/906 & $\mathrm{BC}$ & & $\mathrm{D}$ & $29^{\circ} 04.362^{\prime} \mathrm{N}$ & $88^{\circ} 22.685^{\prime} \mathrm{W}$ & $29^{\circ} 04.362^{\prime} \mathrm{N}$ & $88^{\circ} 22.685^{\prime} \mathrm{W}$ & 418 \\
\hline SJ-2009-GOM-034 & 20 Sep 09 & VK862/906 & CTD & 15 & $\mathrm{D}$ & $29^{\circ} 04.128^{\prime} \mathrm{N}$ & $88^{\circ} 22.734^{\prime} \mathrm{W}$ & $29^{\circ} 04.139^{\prime} \mathrm{N}$ & $88^{\circ} 22.686^{\prime} \mathrm{W}$ & S-374 \\
\hline SJ-2009-GOM-035 & 20 Sep 09 & VK862/906 & $\mathrm{BC}$ & & $\mathrm{D}$ & $29^{\circ} 04.347^{\prime} \mathrm{N}$ & $88^{\circ} 22.506^{\prime} \mathrm{W}$ & $29^{\circ} 04.347^{\prime} \mathrm{N}$ & $88^{\circ} 22.506^{\prime} \mathrm{W}$ & 397 \\
\hline SJ-2009-GOM-036 & 20 Sep 09 & VK862/906 & $\mathrm{BC}$ & 15 & $\mathrm{~N}$ & $29^{\circ} 04.115^{\prime} \mathrm{N}$ & $88^{\circ} 22.720^{\prime} \mathrm{W}$ & $29^{\circ} 04.183^{\prime} \mathrm{N}$ & $88^{\circ} 22.774^{\prime} \mathrm{W}$ & S-371 \\
\hline SJ-2009-GOM-037 & 20 Sep 09 & VK862/906 & $\mathrm{BC}$ & & $\mathrm{N}$ & $29^{\circ} 04.146^{\prime} \mathrm{N}$ & $88^{\circ} 22.724^{\prime} \mathrm{W}$ & $29^{\circ} 04.146^{\prime} \mathrm{N}$ & $88^{\circ} 22.724^{\prime} \mathrm{W}$ & 417 \\
\hline SJ-2009-GOM-038 & 20 Sep 09 & VK862/906 & Phyto & & $\mathrm{N}$ & $29^{\circ} 04.174^{\prime} \mathrm{N}$ & $88^{\circ} 22.758^{\prime} \mathrm{W}$ & $29^{\circ} 04.174^{\prime} \mathrm{N}$ & $88^{\circ} 22.758^{\prime} \mathrm{W}$ & 427 \\
\hline SJ-2009-GOM-039 & 20 Sep 09 & VK862/906 & Phyto & & $\mathrm{N}$ & $29^{\circ} 04.228^{\prime} \mathrm{N}$ & $88^{\circ} 22.826^{\prime} \mathrm{W}$ & $29^{\circ} 04.228^{\prime} \mathrm{N}$ & $88^{\circ} 22.826^{\prime} \mathrm{W}$ & 418 \\
\hline SJ-2009-GOM-040 & 20 Sep 09 & VK862/906 & Phyto & & $\mathrm{N}$ & $29^{\circ} 04.117^{\prime} \mathrm{N}$ & $88^{\circ} 22.718^{\prime} \mathrm{W}$ & $29^{\circ} 04.117^{\prime} \mathrm{N}$ & $88^{\circ} 22.718^{\prime} \mathrm{W}$ & 400 \\
\hline SJ-2009-GOM-041 & 20 Sep 09 & VK862/906 & Phyto & & $\mathrm{N}$ & $29^{\circ} 04.055^{\prime} \mathrm{N}$ & $88^{\circ} 22.606^{\prime} \mathrm{W}$ & $29^{\circ} 04.055^{\prime} \mathrm{N}$ & $88^{\circ} 22.606^{\prime} \mathrm{W}$ & 415 \\
\hline SJ-2009-GOM-042 & 20 Sep 09 & VK862/906 & PN $1.0 \mathrm{~m}$ & 30 & $\mathrm{~N}$ & $29^{\circ} 04.184^{\prime} \mathrm{N}$ & $88^{\circ} 22.924^{\prime} \mathrm{W}$ & $29^{\circ} 04.083^{\prime} \mathrm{N}$ & $88^{\circ} 23.152^{\prime} \mathrm{W}$ & S-38 \\
\hline SJ-2009-GOM-043 & 21 Sep 09 & VK862/906 & CTD & 13 & $\mathrm{~N}$ & $29^{\circ} 04.148^{\prime} \mathrm{N}$ & $88^{\circ} 22.708^{\prime} \mathrm{W}$ & $29^{\circ} 04.192^{\prime} \mathrm{N}$ & $88^{\circ} 22.803^{\prime} \mathrm{W}$ & S-398 \\
\hline SJ-2009-GOM-044 & 21 Sep 09 & VK862/906 & $\mathrm{PN} 0.5 \mathrm{~m}$ & 30 & $\mathrm{~N}$ & $29^{\circ} 04.112^{\prime} \mathrm{N}$ & $88^{\circ} 22.805^{\prime} \mathrm{W}$ & $29^{\circ} 03.873^{\prime} \mathrm{N}$ & $88^{\circ} 22.603^{\prime} \mathrm{W}$ & S \\
\hline SJ-2009-GOM-045 & 21 Sep 09 & VK862/906 & Dip Net & & $\mathrm{N}$ & $29^{\circ} 03.895^{\prime} \mathrm{N}$ & $88^{\circ} 22.620^{\prime} \mathrm{W}$ & $29^{\circ} 03.895^{\prime} \mathrm{N}$ & $88^{\circ} 22.620^{\prime} \mathrm{W}$ & 487 \\
\hline SJ-2009-GOM-046 & 21 Sep 09 & VK862/906 & OD & & $\mathrm{N}$ & $29^{\circ} 04.144^{\prime} \mathrm{N}$ & $88^{\circ} 22.750^{\prime} \mathrm{W}$ & $29^{\circ} 04.144^{\prime} \mathrm{N}$ & $88^{\circ} 22.750^{\prime} \mathrm{W}$ & S \\
\hline SJ-2009-GOM-047 & 21 Sep 09 & VK862/906 & $\mathrm{PN} 0.5 \mathrm{~m}$ & 30 & $\mathrm{~N}$ & $29^{\circ} 04.228^{\prime} \mathrm{N}$ & $88^{\circ} 22.834^{\prime} \mathrm{W}$ & $29^{\circ} 04.366^{\prime} \mathrm{N}$ & $88^{\circ} 22.716^{\prime} \mathrm{W}$ & $\mathrm{S}$ \\
\hline
\end{tabular}


Table B.2. Stations sampled on deep coral areas in the eastern and north-central Gulf of Mexico using the research vessel (R/V) Seward Johnson, September 16-24, 2009, as part of the Lophelia II program - continued.

[min, minute; hr, hour; m, meter; EDT, Eastern Daylight Time; WFS, West Florida Slope; D, Day (0700 to $1830 \mathrm{hr}$ EDT); N, Night (1830 to $0700 \mathrm{hr}$ EDT); M, Morning (0700 to $1200 \mathrm{hr}$ EDT); A, Afternoon (1200 to $1830 \mathrm{hr}$ EDT); BC, Box Core; CTD, Sea-bird SBE 911+ CTD; Moc, MOCNESS 1x1m (335 $\mu \mathrm{m})$; OD, on deck; Phyto, Phytoplankton sample; PN, Plankton net (1.0 m, 0.5 m, 0.25 -m diameter); JSL, Johnson Sea Link II submersible. Submersible depth range data are only for the period when the $J S L$ was on the bottom. For MOCNESS trawls that fished discrete depths, depth ranges are the minimum and maximum of all mean depths calculated per tow. For nets that failed to close and for the CTD casts, depth ranges include the absolute maximum depth of the sample to the surface $(\mathrm{S})]$

\begin{tabular}{|c|c|c|c|c|c|c|c|c|c|c|}
\hline Station number & Date & Location & Gear & $\begin{array}{c}\text { Sample } \\
\text { time } \\
\text { (min) }\end{array}$ & Time & Start latitude & $\begin{array}{c}\text { Start } \\
\text { longitude }\end{array}$ & End latitude & $\begin{array}{c}\text { End } \\
\text { longitude }\end{array}$ & $\begin{array}{c}\text { Depth } \\
\text { range }(m)\end{array}$ \\
\hline SJ-2009-GOM-048 & 21 Sep 09 & VK862/906 & CTD & 14 & $\mathrm{~N}$ & $29^{\circ} 04.135^{\prime} \mathrm{N}$ & $88^{\circ} 22.777^{\prime} \mathrm{W}$ & $29^{\circ} 04.114^{\prime} \mathrm{N}$ & $88^{\circ} 22.834^{\prime} \mathrm{W}$ & S-398 \\
\hline SJ-2009-GOM-049 & 21 Sep 09 & VK862/906 & $\mathrm{PN} 0.5 \mathrm{~m}$ & 35 & $\mathrm{~N}$ & $29^{\circ} 04.094^{\prime} \mathrm{N}$ & $88^{\circ} 22.868^{\prime} \mathrm{W}$ & $29^{\circ} 04.078^{\prime} \mathrm{N}$ & $88^{\circ} 22.792^{\prime} \mathrm{W}$ & $\mathrm{S}$ \\
\hline SJ-2009-GOM-050 & 21 Sep 09 & VK862/906 & $\mathrm{PN} 0.5 \mathrm{~m}$ & 30 & $\mathrm{~N}$ & $29^{\circ} 04.076^{\prime} \mathrm{N}$ & $88^{\circ} 22.909^{\prime} \mathrm{W}$ & $29^{\circ} 04.165^{\prime} \mathrm{N}$ & $88^{\circ} 22.901^{\prime} \mathrm{W}$ & $\mathrm{S}$ \\
\hline SJ-2009-GOM-051 & 21 Sep 09 & VK862/906 & $\mathrm{BC}$ & & $\mathrm{D}$ & $29^{\circ} 06.672^{\prime} \mathrm{N}$ & $88^{\circ} 23.050^{\prime} \mathrm{W}$ & $29^{\circ} 06.672^{\prime} \mathrm{N}$ & $88^{\circ} 23.050^{\prime} \mathrm{W}$ & 369 \\
\hline SJ-2009-GOM-052 & 21 Sep 09 & VK862/906 & $\mathrm{BC}$ & & $\mathrm{D}$ & $29^{\circ} 06.397^{\prime} \mathrm{N}$ & $88^{\circ} 23.063^{\prime} \mathrm{W}$ & $29^{\circ} 06.397^{\prime} \mathrm{N}$ & $88^{\circ} 23.063^{\prime} \mathrm{W}$ & 314 \\
\hline SJ-2009-GOM-053 & 21 Sep 09 & VK862/906 & $\mathrm{BC}$ & & $\mathrm{D}$ & $29^{\circ} 04.106^{\prime} \mathrm{N}$ & $88^{\circ} 22.641^{\prime} \mathrm{W}$ & $29^{\circ} 04.106^{\prime} \mathrm{N}$ & $88^{\circ} 22.641^{\prime} \mathrm{W}$ & 392 \\
\hline SJ-2009-GOM-054 & 21 Sep 09 & VK862/906 & Moc & 30 & $\mathrm{~N}$ & $29^{\circ} 02.401^{\prime} \mathrm{N}$ & $88^{\circ} 22.341^{\prime} \mathrm{W}$ & $29^{\circ} 01.530^{\prime} \mathrm{N}$ & $88^{\circ} 22.868^{\prime} \mathrm{W}$ & $252-332$ \\
\hline SJ-2009-GOM-055 & 21 Sep 09 & VK862/906 & Moc & 30 & $\mathrm{~N}$ & $29^{\circ} 01.530^{\prime} \mathrm{N}$ & $88^{\circ} 22.868^{\prime} \mathrm{W}$ & $29^{\circ} 01.354^{\prime} \mathrm{N}$ & $88^{\circ} 23.722^{\prime} \mathrm{W}$ & $277-317$ \\
\hline SJ-2009-GOM-056 & 22 Sep 09 & VK862/906 & Moc & 30 & $\mathrm{~N}$ & $29^{\circ} 00.829^{\prime} \mathrm{N}$ & $88^{\circ} 23.846^{\prime} \mathrm{W}$ & $29^{\circ} 01.247^{\prime} \mathrm{N}$ & $88^{\circ} 24.227^{\prime} \mathrm{W}$ & $217-261$ \\
\hline SJ-2009-GOM-057 & 22 Sep 09 & VK862/906 & Moc & 30 & $\mathrm{~N}$ & $29^{\circ} 01.247^{\prime} \mathrm{N}$ & $88^{\circ} 24.227^{\prime} \mathrm{W}$ & $29^{\circ} 01.550^{\prime} \mathrm{N}$ & $88^{\circ} 23.127^{\prime} \mathrm{W}$ & $217-221$ \\
\hline SJ-2009-GOM-058 & 22 Sep 09 & VK862/906 & Moc & 30 & $\mathrm{~N}$ & $29^{\circ} 04.257^{\prime} \mathrm{N}$ & $88^{\circ} 22.945^{\prime} \mathrm{W}$ & $29^{\circ} 04.353^{\prime} \mathrm{N}$ & $88^{\circ} 21.899^{\prime} \mathrm{W}$ & $168-196$ \\
\hline SJ-2009-GOM-059 & 22 Sep 09 & VK862/906 & Moc & 30 & $\mathrm{~N}$ & $29^{\circ} 04.353^{\prime} \mathrm{N}$ & $88^{\circ} 21.899^{\prime} \mathrm{W}$ & $29^{\circ} 04.042^{\prime} \mathrm{N}$ & $88^{\circ} 20.809^{\prime} \mathrm{W}$ & $143-181$ \\
\hline SJ-2009-GOM-060 & 22 Sep 09 & VK862/906 & Moc & 30 & $\mathrm{~N}$ & $29^{\circ} 04.330^{\prime} \mathrm{N}$ & $88^{\circ} 23.009^{\prime} \mathrm{W}$ & $29^{\circ} 04.931^{\prime} \mathrm{N}$ & $88^{\circ} 21.861^{\prime} \mathrm{W}$ & $111-117$ \\
\hline SJ-2009-GOM-061 & 22 Sep 09 & VK862/906 & Moc & 30 & $\mathrm{~N}$ & $29^{\circ} 04.931 ' \mathrm{~N}$ & $88^{\circ} 21.861^{\prime} \mathrm{W}$ & $29^{\circ} 05.475^{\prime} \mathrm{N}$ & $88^{\circ} 20.688^{\prime} \mathrm{W}$ & $73-112$ \\
\hline SJ-2009-GOM-062 & 22 Sep 09 & VK826 & $\mathrm{BC}$ & & $\mathrm{D}$ & $29^{\circ} 10.207^{\prime} \mathrm{N}$ & $88^{\circ} 00.798^{\prime} \mathrm{W}$ & $29^{\circ} 10.207^{\prime} \mathrm{N}$ & $88^{\circ} 00.798^{\prime} \mathrm{W}$ & 470 \\
\hline SJ-2009-GOM-063 & 22 Sep 09 & VK826 & $\mathrm{BC}$ & & $\mathrm{D}$ & $29^{\circ} 10.241^{\prime} \mathrm{N}$ & $88^{\circ} 00.740^{\prime} \mathrm{W}$ & $29^{\circ} 10.241^{\prime} \mathrm{N}$ & $88^{\circ} 00.740^{\prime} \mathrm{W}$ & 461 \\
\hline SJ-2009-GOM-064 & 22 Sep 09 & VK826 & CTD & 16 & $\mathrm{~N}$ & $29^{\circ} 09.805^{\prime} \mathrm{N}$ & $88^{\circ} 00.574^{\prime} \mathrm{W}$ & $29^{\circ} 09.729^{\prime} \mathrm{N}$ & $88^{\circ} 00.674^{\prime} \mathrm{W}$ & $\mathrm{S}-424$ \\
\hline SJ-2009-GOM-065 & 22 Sep 09 & VK826 & Moc & 30 & $\mathrm{~N}$ & $29^{\circ} 08.436^{\prime} \mathrm{N}$ & $88^{\circ} 01.588^{\prime} \mathrm{W}$ & $29^{\circ} 08.328^{\prime} \mathrm{N}$ & $88^{\circ} 00.980^{\prime} \mathrm{W}$ & $280-325$ \\
\hline SJ-2009-GOM-066 & 23 Sep 09 & VK826 & Moc & 29 & $\mathrm{~N}$ & $29^{\circ} 08.328^{\prime} \mathrm{N}$ & $88^{\circ} 09.980^{\prime} \mathrm{W}$ & $29^{\circ} 08.148^{\prime} \mathrm{N}$ & $88^{\circ} 00.438^{\prime} \mathrm{W}$ & $238-303$ \\
\hline SJ-2009-GOM-067 & 23 Sep 09 & VK826 & Moc & 30 & $\mathrm{~N}$ & $29^{\circ} 07.649^{\prime} \mathrm{N}$ & $88^{\circ} 02.522^{\prime} \mathrm{W}$ & $29^{\circ} 07.536^{\prime} \mathrm{N}$ & $88^{\circ} 02.944^{\prime} \mathrm{W}$ & $176-224$ \\
\hline SJ-2009-GOM-068 & 23 Sep 09 & VK826 & Moc & 30 & $\mathrm{~N}$ & $29^{\circ} 07.536^{\prime} \mathrm{N}$ & $88^{\circ} 02.944^{\prime} \mathrm{W}$ & $29^{\circ} 07.671 \mathrm{~N}$ & $88^{\circ} 02.282^{\prime} \mathrm{W}$ & $145-220$ \\
\hline SJ-2009-GOM-069 & 23 Sep 09 & VK826 & Moc & 30 & $\mathrm{~N}$ & $29^{\circ} 07.412^{\prime} \mathrm{N}$ & $88^{\circ} 03.097^{\prime} \mathrm{W}$ & $29^{\circ} 07.786^{\prime} \mathrm{N}$ & $88^{\circ} 02.592^{\prime} \mathrm{W}$ & $160-264$ \\
\hline SJ-2009-GOM-070 & 23 Sep 09 & VK826 & Moc & 30 & $\mathrm{~N}$ & $29^{\circ} 07.786^{\prime} \mathrm{N}$ & $88^{\circ} 02.592^{\prime} \mathrm{W}$ & $29^{\circ} 08.305^{\prime} \mathrm{N}$ & $88^{\circ} 01.943^{\prime} \mathrm{W}$ & $123-161$ \\
\hline SJ-2009-GOM-071 & 23 Sep 09 & VK826 & OD & & $\mathrm{D}$ & $29^{\circ} 10.222^{\prime} \mathrm{N}$ & $88^{\circ} 00.817^{\prime} \mathrm{W}$ & $29^{\circ} 10.222^{\prime} \mathrm{N}$ & $88^{\circ} 00.817^{\prime} \mathrm{W}$ & $\mathrm{S}$ \\
\hline
\end{tabular}


Table B.2. Stations sampled on deep coral areas in the eastern and north-central Gulf of Mexico using the research vessel (R/V) Seward Johnson, September 16-24, 2009, as part of the Lophelia II program - continued.

[min, minute; hr, hour; m, meter; EDT, Eastern Daylight Time; WFS, West Florida Slope; D, Day (0700 to $1830 \mathrm{hr}$ EDT); N, Night (1830 to $0700 \mathrm{hr}$ EDT); M, Morning (0700 to $1200 \mathrm{hr}$ EDT); A, Afternoon (1200 to $1830 \mathrm{hr}$ EDT); BC, Box Core; CTD, Sea-bird SBE 911+ CTD; Moc, MOCNESS 1x1m (335 $\mu \mathrm{m})$; OD, on deck; Phyto, Phytoplankton sample; PN, Plankton net (1.0 m, 0.5 m, 0.25 -m diameter); JSL, Johnson Sea Link II submersible. Submersible depth range data are only for the period when the $J S L$ was on the bottom. For MOCNESS trawls that fished discrete depths, depth ranges are the minimum and maximum of all mean depths calculated per tow. For nets that failed to close and for the CTD casts, depth ranges include the absolute maximum depth of the sample to the surface (S)]

\begin{tabular}{|c|c|c|c|c|c|c|c|c|c|c|}
\hline Station number & Date & Location & Gear & $\begin{array}{c}\text { Sample } \\
\text { time } \\
\text { (min) }\end{array}$ & Time & Start latitude & $\begin{array}{c}\text { Start } \\
\text { longitude }\end{array}$ & End latitude & $\begin{array}{c}\text { End } \\
\text { longitude }\end{array}$ & $\begin{array}{c}\text { Depth } \\
\text { range }(m)\end{array}$ \\
\hline SJ-2009-GOM-072 & 23 Sep 09 & VK826 & $\mathrm{BC}$ & & $\mathrm{D}$ & $29^{\circ} 10.063^{\prime} \mathrm{N}$ & $88^{\circ} 00.789^{\prime} \mathrm{W}$ & $29^{\circ} 10.063^{\prime} \mathrm{N}$ & $88^{\circ} 00.789^{\prime} \mathrm{W}$ & 472 \\
\hline SJ-2009-GOM-073 & 23 Sep 09 & VK826 & $\mathrm{BC}$ & & $\mathrm{D}$ & $29^{\circ} 10.258^{\prime} \mathrm{N}$ & $88^{\circ} 00.853^{\prime} \mathrm{W}$ & $29^{\circ} 10.258^{\prime} \mathrm{N}$ & $88^{\circ} 00.853^{\prime} \mathrm{W}$ & 484 \\
\hline SJ-2009-GOM-074 & 23 Sep 09 & VK826 & $\mathrm{BC}$ & & $\mathrm{D}$ & $29^{\circ} 10.246^{\prime} \mathrm{N}$ & $88^{\circ} 00.680^{\prime} \mathrm{W}$ & $29^{\circ} 10.246^{\prime} \mathrm{N}$ & $88^{\circ} 00.680^{\prime} \mathrm{W}$ & 458 \\
\hline SJ-2009-GOM-075 & 23 Sep 09 & VK826 & CTD & 16 & $\mathrm{~N}$ & $29^{\circ} 10.203^{\prime} \mathrm{N}$ & $88^{\circ} 00.547^{\prime} \mathrm{W}$ & $29^{\circ} 10.191^{\prime} \mathrm{N}$ & $88^{\circ} 00.676^{\prime} \mathrm{W}$ & S-428 \\
\hline SJ-2009-GOM-076 & 23 Sep 09 & VK826 & Moc & 30 & $\mathrm{~N}$ & $29^{\circ} 09.052^{\prime} \mathrm{N}$ & $88^{\circ} 02.020^{\prime} \mathrm{W}$ & $29^{\circ} 08.032^{\prime} \mathrm{N}$ & $88^{\circ} 01.390^{\prime} \mathrm{W}$ & $244-300$ \\
\hline SJ-2009-GOM-077 & 23 Sep 09 & VK826 & Moc & 30 & $\mathrm{~N}$ & $29^{\circ} 08.302^{\prime} \mathrm{N}$ & $88^{\circ} 01.390^{\prime} \mathrm{W}$ & $29^{\circ} 07.273^{\prime} \mathrm{N}$ & $88^{\circ} 01.642^{\prime} \mathrm{W}$ & $255-285$ \\
\hline SJ-2009-GOM-078 & 24 Sep 09 & VK826 & Moc & 30 & $\mathrm{~N}$ & $29^{\circ} 07.490^{\prime} \mathrm{N}$ & $88^{\circ} 02.749^{\prime} \mathrm{W}$ & $29^{\circ} 07.790^{\prime} \mathrm{N}$ & $88^{\circ} 01.903^{\prime} \mathrm{W}$ & $189-222$ \\
\hline SJ-2009-GOM-079 & 24 Sep 09 & VK826 & Moc & 30 & $\mathrm{~N}$ & $29^{\circ} 07.790^{\prime} \mathrm{N}$ & $88^{\circ} 01.903^{\prime} \mathrm{W}$ & $29^{\circ} 07.479^{\prime} \mathrm{N}$ & $88^{\circ} 01.025^{\prime} \mathrm{W}$ & 169-189 \\
\hline SJ-2009-GOM-080 & 24 Sep 09 & VK826 & Moc & 30 & $\mathrm{~N}$ & $29^{\circ} 07.525^{\prime} \mathrm{N}$ & $88^{\circ} 02.276^{\prime} \mathrm{W}$ & $29^{\circ} 08.021^{\prime} \mathrm{N}$ & $88^{\circ} 01.951^{\prime} \mathrm{W}$ & $121-144$ \\
\hline SJ-2009-GOM-081 & 24 Sep 09 & VK826 & Moc & 30 & $\mathrm{~N}$ & $29^{\circ} 08.021^{\prime} \mathrm{N}$ & $88^{\circ} 01.951^{\prime} \mathrm{W}$ & $29^{\circ} 08.429^{\prime} \mathrm{N}$ & $88^{\circ} 01.526^{\prime} \mathrm{W}$ & $89-123$ \\
\hline SJ-2009-GOM-082 & 24 Sep 09 & VK826 & Moc & 30 & $\mathrm{~N}$ & $29^{\circ} 07.248^{\prime} \mathrm{N}$ & $88^{\circ} 03.833^{\prime} \mathrm{W}$ & $29^{\circ} 08.135^{\prime} \mathrm{N}$ & $88^{\circ} 03.437^{\prime} \mathrm{W}$ & $77-105$ \\
\hline SJ-2009-GOM-083 & 24 Sep 09 & VK826 & Moc & 30 & $\mathrm{~N}$ & $29^{\circ} 08.135^{\prime} \mathrm{N}$ & $88^{\circ} 03.437^{\prime} \mathrm{W}$ & $29^{\circ} 09.089^{\prime} \mathrm{N}$ & $88^{\circ} 03.015^{\prime} \mathrm{W}$ & $53-78$ \\
\hline SJ-2009-GOM-084 & 24 Sep 09 & VK826 & OD & & $\mathrm{N}$ & $29^{\circ} 09.792^{\prime} \mathrm{N}$ & $88^{\circ} 01.739^{\prime} \mathrm{W}$ & $29^{\circ} 09.792^{\prime} \mathrm{N}$ & $88^{\circ} 01.739^{\prime} \mathrm{W}$ & $\mathrm{S}$ \\
\hline
\end{tabular}


Table B.3. Stations sampled in the north-central and eastern Gulf of Mexico using the research vessel (R/V) Cape Hatteras,September 21October 2, 2010, as part of the Lophelia II program.

[min, minute; hr, hour; CDT, Central Daylight Time; m, meter; D, Day (0700 to $1900 \mathrm{hr}$ CDT); N, Night (1900 to $0700 \mathrm{hr}$ CDT); S, surface; CTD, Sea-bird SBE 911+CTD; MC, multi-core; OT=3.5-m otter trawl; TT=2 x 2 m Tucker trawl; PN in TT, 0.5-m plankton net embedded in Tucker trawl; OD, on deck; PN, 1.0-m plankton net; Phyto, phytoplankton sample; ROV, remotely operated vehicle Kraken II; ROV depth range data are only for the period when the ROV was on the bottom]

\begin{tabular}{|c|c|c|c|c|c|c|c|c|c|c|}
\hline Station number & Date & Location & Gear & $\begin{array}{c}\text { Sample } \\
\text { time } \\
\text { (min) }\end{array}$ & Time & Start latitude & $\begin{array}{c}\text { Start } \\
\text { longitude }\end{array}$ & End latitude & $\begin{array}{l}\text { End } \\
\text { longitude }\end{array}$ & $\begin{array}{l}\text { Depth } \\
\text { range } \\
\text { (m) }\end{array}$ \\
\hline ROV-2010-CH-001 & 21 Sep 10 & VK826 & ROV & 339 & $\mathrm{D}$ & $29^{\circ} 09.710^{\prime} \mathrm{N}$ & $88^{\circ} 00.618^{\prime} \mathrm{W}$ & $29^{\circ} 09.720^{\prime} \mathrm{N}$ & $88^{\circ} 01.269^{\prime} \mathrm{W}$ & $454-520$ \\
\hline ROV-2010-CH-002 & 22 Sep 10 & VK826 & ROV & 445 & $\mathrm{D}$ & $29^{\circ} 10.191^{\prime} \mathrm{N}$ & $88^{\circ} 00.873^{\prime} \mathrm{W}$ & $29^{\circ} 10.179^{\prime} \mathrm{N}$ & $88^{\circ} 00.757^{\prime} \mathrm{W}$ & $488-526$ \\
\hline ROV-2010-CH-003 & 23 Sep 10 & VK826 & ROV & 650 & $\mathrm{D}$ & $29^{\circ} 10.259^{\prime} \mathrm{N}$ & $88^{\circ} 00.817^{\prime} \mathrm{W}$ & $29^{\circ} 10.324^{\prime} \mathrm{N}$ & $88^{\circ} 00.718^{\prime} \mathrm{W}$ & $475-499$ \\
\hline ROV-2010-CH-004 & 24 Sep 10 & VK862 & ROV & 526 & $\mathrm{D}$ & $29^{\circ} 06.444^{\prime} \mathrm{N}$ & $88^{\circ} 23.456^{\prime} \mathrm{W}$ & $29^{\circ} 10.324^{\prime} \mathrm{N}$ & $88^{\circ} 00.718^{\prime} \mathrm{W}$ & $317-373$ \\
\hline ROV-2010-CH-005 & 25 Sep 10 & VK862 & ROV & 536 & $\mathrm{D}$ & $29^{\circ} 04.350^{\prime} \mathrm{N}$ & $88^{\circ} 22.975^{\prime} \mathrm{W}$ & $29^{\circ} 04.496^{\prime} \mathrm{N}$ & $88^{\circ} 22.699^{\prime} \mathrm{W}$ & $401-435$ \\
\hline ROV-2010-CH-006 & 26 Sep 10 & VK862 & ROV & 230 & $\mathrm{D}$ & $29^{\circ} 04.330^{\prime} \mathrm{N}$ & $88^{\circ} 22.588^{\prime} \mathrm{W}$ & $29^{\circ} 04.338^{\prime} \mathrm{N}$ & $88^{\circ} 22.465^{\prime} \mathrm{W}$ & $408-430$ \\
\hline ROV-2010-CH-007 & 28 Sep 10 & WFS & ROV & 572 & $\mathrm{D}$ & $26^{\circ} 12.506^{\prime} \mathrm{N}$ & $84^{\circ} 43.583^{\prime} \mathrm{W}$ & $26^{\circ} 12.439^{\prime} \mathrm{N}$ & $84^{\circ} 43.515^{\prime} \mathrm{W}$ & $485-522$ \\
\hline ROV-2010-CH-008 & 29 Sep 10 & WFS & ROV & 562 & $\mathrm{D}$ & $26^{\circ} 11.754^{\prime} \mathrm{N}$ & $84^{\circ} 44.381^{\prime} \mathrm{W}$ & $26^{\circ} 11.882^{\prime} \mathrm{N}$ & $84^{\circ} 43.914^{\prime} \mathrm{W}$ & $530-584$ \\
\hline ROV-2010-CH-009 & 30 Sep 10 & WFS & ROV & 497 & $\mathrm{D}$ & $26^{\circ} 12.260^{\prime} \mathrm{N}$ & $84^{\circ} 43.942^{\prime} \mathrm{W}$ & $26^{\circ} 12.264^{\prime} \mathrm{N}$ & $84^{\circ} 43.772^{\prime} \mathrm{W}$ & $519-549$ \\
\hline ROV-2010-CH-010 & 1 Oct 10 & WFS & ROV & 555 & $\mathrm{D}$ & $26^{\circ} 20.154^{\prime} \mathrm{N}$ & $84^{\circ} 45.677^{\prime} \mathrm{W}$ & $26^{\circ} 20.082^{\prime} \mathrm{N}$ & $84^{\circ} 43.335^{\prime} \mathrm{W}$ & $497-531$ \\
\hline ROV-2010-CH-011 & 2 Oct 10 & WFS & ROV & 310 & $\mathrm{D}$ & $26^{\circ} 24.463^{\prime} \mathrm{N}$ & $84^{\circ} 46.707^{\prime} \mathrm{W}$ & $26^{\circ} 24.395^{\prime} \mathrm{N}$ & $84^{\circ} 46.622^{\prime} \mathrm{W}$ & $494-522$ \\
\hline CH-2010-001 & 21 Sep 10 & VK826 & Z-trap & & $\mathrm{N}$ & $29^{\circ} 10.250^{\prime} \mathrm{N}$ & $88^{\circ} 00.660^{\prime} \mathrm{W}$ & LOST & LOST & 478 \\
\hline CH-2010-002 & 21 Sep 10 & VK826 & CTD & 10 & $\mathrm{~N}$ & $29^{\circ} 09.418^{\prime} \mathrm{N}$ & $88^{\circ} 00.633^{\prime} \mathrm{W}$ & $29^{\circ} 09.425^{\prime} \mathrm{N}$ & $88^{\circ} 00.636^{\prime} \mathrm{W}$ & S-507 \\
\hline CH-2010-003 & 21 Sep 10 & VK826 & Z-trap & 2,924 & $\mathrm{~N}$ & $29^{\circ} 09.725^{\prime} \mathrm{N}$ & $88^{\circ} 01.063^{\prime} \mathrm{W}$ & $29^{\circ} 09.725^{\prime} \mathrm{N}$ & $88^{\circ} 01.063^{\prime} \mathrm{W}$ & 526 \\
\hline CH-2010-004 & 21 Sep 10 & VK826 & OT & 30 & $\mathrm{~N}$ & $29^{\circ} 10.053^{\prime} \mathrm{N}$ & $88^{\circ} 01.020^{\prime} \mathrm{W}$ & $29^{\circ} 10.054^{\prime} \mathrm{N}$ & $87^{\circ} 59.798^{\prime} \mathrm{W}$ & $481-575$ \\
\hline CH-2010-005 & 21 Sep 10 & VK826 & OD & & $\mathrm{N}$ & $29^{\circ} 09.212^{\prime} \mathrm{N}$ & $87^{\circ} 59.197^{\prime} \mathrm{W}$ & $29^{\circ} 09.212^{\prime} \mathrm{N}$ & $87^{\circ} 59.197^{\prime} \mathrm{W}$ & $\mathrm{S}$ \\
\hline CH-2010-006 & 22 Sep 10 & VK826 & OT & 30 & $\mathrm{~N}$ & $29^{\circ} 10.103^{\prime} \mathrm{N}$ & $88^{\circ} 01.232^{\prime} \mathrm{W}$ & $29^{\circ} 09.883^{\prime} \mathrm{N}$ & $88^{\circ} 00.159^{\prime} \mathrm{W}$ & $489-500$ \\
\hline CH-2010-007 & 22 Sep 10 & VK826 & OT & 30 & $\mathrm{~N}$ & $29^{\circ} 09.962^{\prime} \mathrm{N}$ & $88^{\circ} 01.049^{\prime} \mathrm{W}$ & $29^{\circ} 09.749^{\prime} \mathrm{N}$ & $88^{\circ} 01.193^{\prime} \mathrm{W}$ & $478-500$ \\
\hline CH-2010-008 & 22 Sep 10 & VK826 & $\mathrm{MC}$ & & $\mathrm{N}$ & $29^{\circ} 10.064^{\prime} \mathrm{N}$ & $88^{\circ} 00.842^{\prime} \mathrm{W}$ & $29^{\circ} 10.064^{\prime} \mathrm{N}$ & $88^{\circ} 00.842^{\prime} \mathrm{W}$ & 475 \\
\hline CH-2010-009 & 22 Sep 10 & VK826 & $\mathrm{MC}$ & & $\mathrm{N}$ & $29^{\circ} 10.048^{\prime} \mathrm{N}$ & $88^{\circ} 00.926^{\prime} \mathrm{W}$ & $29^{\circ} 10.048^{\prime} \mathrm{N}$ & $88^{\circ} 00.926^{\prime} \mathrm{W}$ & 473 \\
\hline CH-2010-010 & 22 Sep 10 & VK826 & $\mathrm{MC}$ & & $\mathrm{N}$ & $29^{\circ} 10.086^{\prime} \mathrm{N}$ & $88^{\circ} 01.371^{\prime} \mathrm{W}$ & $29^{\circ} 10.086^{\prime} \mathrm{N}$ & $88^{\circ} 01.371^{\prime} \mathrm{W}$ & 494 \\
\hline CH-2010-011 & 23 Sep 10 & VK826 & OT & 30 & $\mathrm{~N}$ & $29^{\circ} 10.084^{\prime} \mathrm{N}$ & $88^{\circ} 01.908^{\prime} \mathrm{W}$ & $29^{\circ} 10.083^{\prime} \mathrm{N}$ & $88^{\circ} 00.682^{\prime} \mathrm{W}$ & $471-515$ \\
\hline CH-2010-012 & 23 Sep 10 & VK826 & OT & 30 & $\mathrm{~N}$ & $29^{\circ} 10.099^{\prime} \mathrm{N}$ & $88^{\circ} 01.722^{\prime} \mathrm{W}$ & $29^{\circ} 10.071^{\prime} \mathrm{N}$ & $88^{\circ} 00.550^{\prime} \mathrm{W}$ & $478-517$ \\
\hline CH-2010-013 & 23 Sep 10 & VK826 & CTD & 20 & $\mathrm{~N}$ & $29^{\circ} 10.242^{\prime} \mathrm{N}$ & $88^{\circ} 00.675^{\prime} \mathrm{W}$ & $29^{\circ} 10.228^{\prime} \mathrm{N}$ & $88^{\circ} 00.613^{\prime} \mathrm{W}$ & S-465 \\
\hline CH-2010-014 & 23 Sep 10 & VK826 & CTD & 11 & $\mathrm{~N}$ & $29^{\circ} 10.279^{\prime} \mathrm{N}$ & $88^{\circ} 00.750^{\prime} \mathrm{W}$ & $29^{\circ} 10.295^{\prime} \mathrm{N}$ & $88^{\circ} 00.661^{\prime} \mathrm{W}$ & $\mathrm{S}-451$ \\
\hline
\end{tabular}


Table B.3. Stations sampled in the north-central and eastern Gulf of Mexico using the research vessel (R/V) Cape Hatteras, September 21October 2, 2010, as part of the Lophelia II program - continued.

[min, minute; hr, hour; CDT, Central Daylight Time; m, meter; D, Day (0700 to $1900 \mathrm{hr}$ CDT); N, Night (1900 to $0700 \mathrm{hr}$ CDT); S, surface; CTD, Sea-bird SBE 911+ CTD; MC, multi-core; OT=3.5-m otter trawl; TT=2 x 2 m Tucker trawl; PN in TT, 0.5-m plankton net embedded in Tucker trawl; OD, on deck; PN, 1.0-m plankton net; Phyto, phytoplankton sample; ROV, remotely operated vehicle Kraken II; ROV depth range data are only for the period when the ROV was on the bottom]

\begin{tabular}{|c|c|c|c|c|c|c|c|c|c|c|}
\hline Station number & Date & Location & Gear & $\begin{array}{c}\text { Sample } \\
\text { time } \\
\text { (min) }\end{array}$ & Time & Start latitude & $\begin{array}{c}\text { Start } \\
\text { longitude }\end{array}$ & End latitude & $\begin{array}{c}\text { End } \\
\text { longitude }\end{array}$ & $\begin{array}{l}\text { Depth } \\
\text { range } \\
\text { (m) }\end{array}$ \\
\hline CH-2010-015 & $23 \mathrm{Sep} 10$ & VK826 & Phyto & & $\mathrm{D}$ & $29^{\circ} 10.230^{\prime} \mathrm{N}$ & $88^{\circ} 00.739^{\prime} \mathrm{W}$ & $29^{\circ} 10.230^{\prime} \mathrm{N}$ & $88^{\circ} 00.739^{\prime} \mathrm{W}$ & $\mathrm{S}$ \\
\hline CH-2010-016 & 23 Sep 10 & VK826 & Phyto & & $\mathrm{D}$ & $29^{\circ} 10.264^{\prime} \mathrm{N}$ & $88^{\circ} 00.746^{\prime} \mathrm{W}$ & $29^{\circ} 10.264^{\prime} \mathrm{N}$ & $88^{\circ} 00.746^{\prime} \mathrm{W}$ & S \\
\hline CH-2010-017 & 23 Sep 10 & VK826 & Phyto & & $\mathrm{D}$ & $29^{\circ} 10.246^{\prime} \mathrm{N}$ & $88^{\circ} 00.739^{\prime} \mathrm{W}$ & $29^{\circ} 10.246^{\prime} \mathrm{N}$ & $88^{\circ} 00.739^{\prime} \mathrm{W}$ & S \\
\hline CH-2010-018 & 23 Sep 10 & VK826 & Phyto & & $\mathrm{D}$ & $29^{\circ} 10.222^{\prime} \mathrm{N}$ & $88^{\circ} 00.744^{\prime} \mathrm{W}$ & $29^{\circ} 10.222^{\prime} \mathrm{N}$ & $88^{\circ} 00.744^{\prime} \mathrm{W}$ & S \\
\hline CH-2010-019 & 23 Sep 10 & VK826 & Phyto & & $\mathrm{D}$ & $29^{\circ} 10.211^{\prime} \mathrm{N}$ & $88^{\circ} 00.724^{\prime} \mathrm{W}$ & $29^{\circ} 10.211^{\prime} \mathrm{N}$ & $88^{\circ} 00.724^{\prime} \mathrm{W}$ & S \\
\hline CH-2010-020 & 23 Sep 10 & VK826 & CTD & 11 & $\mathrm{~N}$ & $29^{\circ} 10.246^{\prime} \mathrm{N}$ & $88^{\circ} 00.830^{\prime} \mathrm{W}$ & $29^{\circ} 10.238^{\prime} \mathrm{N}$ & $88^{\circ} 00.725^{\prime} \mathrm{W}$ & S-477 \\
\hline CH-2010-021 & 24 Sep 10 & VK826 & CTD & 10 & $\mathrm{~N}$ & $29^{\circ} 09.940^{\prime} \mathrm{N}$ & $88^{\circ} 00.762^{\prime} \mathrm{W}$ & $29^{\circ} 10.011^{\prime} \mathrm{N}$ & $88^{\circ} 00.718^{\prime} \mathrm{W}$ & S-459 \\
\hline CH-2010-022 & 24 Sep 10 & VK826 & CTD & 18 & $\mathrm{~N}$ & $29^{\circ} 09.680^{\prime} \mathrm{N}$ & $88^{\circ} 00.700^{\prime} \mathrm{W}$ & $29^{\circ} 09.786^{\prime} \mathrm{N}$ & $88^{\circ} 00.623^{\prime} \mathrm{W}$ & S-446 \\
\hline CH-2010-023 & $24 \mathrm{Sep} 10$ & VK826 & CTD & 20 & $\mathrm{~N}$ & $29^{\circ} 09.316^{\prime} \mathrm{N}$ & $88^{\circ} 00.698^{\prime} \mathrm{W}$ & $29^{\circ} 09.400^{\prime} \mathrm{N}$ & $88^{\circ} 00.614^{\prime} \mathrm{W}$ & $\mathrm{S}-523$ \\
\hline CH-2010-024 & 24 Sep 10 & VK826 & CTD & 14 & $\mathrm{~N}$ & $29^{\circ} 08.793^{\prime} \mathrm{N}$ & $88^{\circ} 00.523^{\prime} \mathrm{W}$ & $29^{\circ} 08.833^{\prime} \mathrm{N}$ & $88^{\circ} 00.483^{\prime} \mathrm{W}$ & $S-685$ \\
\hline CH-2010-025 & 24 Sep 10 & VK826 & OD & & $\mathrm{N}$ & $29^{\circ} 08.792^{\prime} \mathrm{N}$ & $88^{\circ} 00.522^{\prime} \mathrm{W}$ & $29^{\circ} 08.792^{\prime} \mathrm{N}$ & $88^{\circ} 00.522^{\prime} \mathrm{W}$ & S \\
\hline CH-2010-026 & 24 Sep 10 & VK826 & PN & 15 & $\mathrm{~N}$ & $29^{\circ} 09.741^{\prime} \mathrm{N}$ & $88^{\circ} 01.146^{\prime} \mathrm{W}$ & $29^{\circ} 09.612^{\prime} \mathrm{N}$ & $88^{\circ} 01.197^{\prime} \mathrm{W}$ & S \\
\hline CH-2010-027 & 24 Sep 10 & VK826 & PN & 10 & $\mathrm{~N}$ & $29^{\circ} 09.139^{\prime} \mathrm{N}$ & $88^{\circ} 01.225^{\prime} \mathrm{W}$ & $29^{\circ} 09.270^{\prime} \mathrm{N}$ & $88^{\circ} 01.278^{\prime} \mathrm{W}$ & S \\
\hline CH-2010-028 & 24 Sep 10 & VK826 & PN & 14 & $\mathrm{~N}$ & $29^{\circ} 09.086^{\prime} \mathrm{N}$ & $88^{\circ} 01.420^{\prime} \mathrm{W}$ & $29^{\circ} 08.865^{\prime} \mathrm{N}$ & $88^{\circ} 01.534^{\prime} \mathrm{W}$ & S \\
\hline CH-2010-029 & 24 Sep 10 & VK826 & CTD & 25 & $\mathrm{~N}$ & $29^{\circ} 09.091^{\prime} \mathrm{N}$ & $88^{\circ} 00.584^{\prime} \mathrm{W}$ & $29^{\circ} 09.238^{\prime} \mathrm{N}$ & $88^{\circ} 00.497^{\prime} \mathrm{W}$ & S-577 \\
\hline CH-2010-030 & 24 Sep 10 & VK862 & Z-trap & 2,506 & $\mathrm{~N}$ & $29^{\circ} 06.532^{\prime} \mathrm{N}$ & $88^{\circ} 22.887^{\prime} \mathrm{W}$ & $29^{\circ} 06.5322^{\prime} \mathrm{N}$ & $88^{\circ} 22.887^{\prime} \mathrm{W}$ & 351 \\
\hline CH-2010-031 & 24 Sep 10 & VK862 & $\mathrm{MC}$ & & $\mathrm{N}$ & $29^{\circ} 06.438^{\prime} \mathrm{N}$ & $88^{\circ} 23.414^{\prime} \mathrm{W}$ & $29^{\circ} 06.438^{\prime} \mathrm{N}$ & $88^{\circ} 23.414^{\prime} \mathrm{W}$ & 359 \\
\hline CH-2010-032 & $25 \mathrm{Sep} 10$ & VK862 & TT & 32 & $\mathrm{~N}$ & $29^{\circ} 06.075^{\prime} \mathrm{N}$ & $88^{\circ} 23.873^{\prime} \mathrm{W}$ & $29^{\circ} 06.913^{\prime} \mathrm{N}$ & $88^{\circ} 23.054^{\prime} \mathrm{W}$ & $187-225$ \\
\hline CH-2010-032PN & 25 Sep 10 & VK862 & $\mathrm{PN}$ in $\mathrm{TT}$ & 32 & $\mathrm{~N}$ & $29^{\circ} 06.075^{\prime} \mathrm{N}$ & $88^{\circ} 23.873^{\prime} \mathrm{W}$ & $29^{\circ} 06.913^{\prime} \mathrm{N}$ & $88^{\circ} 23.054^{\prime} \mathrm{W}$ & $187-225$ \\
\hline CH-2010-033 & $25 \mathrm{Sep} 10$ & VK862 & TT & 30 & $\mathrm{~N}$ & $29^{\circ} 04.671^{\prime} \mathrm{N}$ & $88^{\circ} 23.822^{\prime} \mathrm{W}$ & $29^{\circ} 05.290^{\prime} \mathrm{N}$ & $88^{\circ} 23.178^{\prime} \mathrm{W}$ & $211-249$ \\
\hline CH-2010-033PN & 25 Sep 10 & VK862 & $\mathrm{PN}$ in $\mathrm{TT}$ & 30 & $\mathrm{~N}$ & $29^{\circ} 04.671^{\prime} \mathrm{N}$ & $88^{\circ} 23.822^{\prime} \mathrm{W}$ & $29^{\circ} 05.290^{\prime} \mathrm{N}$ & $88^{\circ} 23.178^{\prime} \mathrm{W}$ & $211-249$ \\
\hline CH-2010-034 & 25 Sep 10 & VK862 & TT & 31 & $\mathrm{~N}$ & $29^{\circ} 04.482^{\prime} \mathrm{N}$ & $88^{\circ} 23.959^{\prime} \mathrm{W}$ & $29^{\circ} 05.209^{\prime} \mathrm{N}$ & $88^{\circ} 23.193^{\prime} \mathrm{W}$ & $91-124$ \\
\hline CH-2010-034PN & $25 \mathrm{Sep} 10$ & VK862 & $\mathrm{PN}$ in $\mathrm{TT}$ & 31 & $\mathrm{~N}$ & $29^{\circ} 04.482^{\prime} \mathrm{N}$ & $88^{\circ} 23.959^{\prime} \mathrm{W}$ & $29^{\circ} 05.209^{\prime} \mathrm{N}$ & $88^{\circ} 23.193^{\prime} \mathrm{W}$ & $91-124$ \\
\hline CH-2010-035 & 25 Sep 10 & VK862 & CTD & 10 & $\mathrm{~N}$ & $29^{\circ} 04.103^{\prime} \mathrm{N}$ & $88^{\circ} 22.687^{\prime} \mathrm{W}$ & $29^{\circ} 04.116^{\prime} \mathrm{N}$ & $88^{\circ} 22.666^{\prime} \mathrm{W}$ & S-407 \\
\hline CH-2010-036 & $25 \mathrm{Sep} 10$ & VK862 & CTD & 14 & $\mathrm{D}$ & $29^{\circ} 04.120^{\prime} \mathrm{N}$ & $88^{\circ} 22.670^{\prime} \mathrm{W}$ & $29^{\circ} 04.125^{\prime} \mathrm{N}$ & $88^{\circ} 22.667^{\prime} \mathrm{W}$ & $\mathrm{S}-252$ \\
\hline
\end{tabular}


Table B.3. Stations sampled in the north-central and eastern Gulf of Mexico using the research vessel (R/V) Cape Hatteras, September 21October 2, 2010, as part of the Lophelia II program - continued.

[min, minute; hr, hour; CDT, Central Daylight Time; m, meter; D, Day (0700 to $1900 \mathrm{hr}$ CDT); N, Night (1900 to $0700 \mathrm{hr}$ CDT); S, surface; CTD, Sea-bird SBE 911+ CTD; MC, multi-core; OT=3.5-m otter trawl; TT=2 x 2 m Tucker trawl; PN in TT, 0.5-m plankton net embedded in Tucker trawl; OD, on deck; PN, 1.0-m plankton net; Phyto, phytoplankton sample; ROV, remotely operated vehicle Kraken II; ROV depth range data are only for the period when the ROV was on the bottom]

\begin{tabular}{|c|c|c|c|c|c|c|c|c|c|c|}
\hline Station number & Date & Location & Gear & $\begin{array}{c}\text { Sample } \\
\text { time } \\
\text { (min) }\end{array}$ & Time & Start latitude & $\begin{array}{c}\text { Start } \\
\text { longitude }\end{array}$ & End latitude & $\begin{array}{l}\text { End } \\
\text { longitude }\end{array}$ & $\begin{array}{c}\text { Depth } \\
\text { range }(m)\end{array}$ \\
\hline CH-2010-037 & $25 \mathrm{Sep} 10$ & VK862 & $\mathrm{MC}$ & & $\mathrm{N}$ & $29^{\circ} 04.458^{\prime} \mathrm{N}$ & $88^{\circ} 22.895^{\prime} \mathrm{W}$ & $29^{\circ} 04.458^{\prime} \mathrm{N}$ & $88^{\circ} 22.895^{\prime} \mathrm{W}$ & 411 \\
\hline CH-2010-038 & 25 Sep 10 & VK862 & CTD & 7 & $\mathrm{~N}$ & $29^{\circ} 04.488^{\prime} \mathrm{N}$ & $88^{\circ} 22.489^{\prime} \mathrm{W}$ & $29^{\circ} 04.510^{\prime} \mathrm{N}$ & $88^{\circ} 22.939^{\prime} \mathrm{W}$ & $S-403$ \\
\hline CH-2010-039 & 25 Sep 10 & VK862 & TT & 32 & $\mathrm{~N}$ & $29^{\circ} 04.126^{\prime} \mathrm{N}$ & $88^{\circ} 23.375^{\prime} \mathrm{W}$ & $29^{\circ} 04.130^{\prime} \mathrm{N}$ & $88^{\circ} 23.249^{\prime} \mathrm{W}$ & 210-239 \\
\hline CH-2010-039PN & $25 \mathrm{Sep} 10$ & VK862 & $\mathrm{PN}$ in $\mathrm{TT}$ & 32 & $\mathrm{~N}$ & $29^{\circ} 04.126^{\prime} \mathrm{N}$ & $88^{\circ} 23.375^{\prime} \mathrm{W}$ & $29^{\circ} 04.130^{\prime} \mathrm{N}$ & $88^{\circ} 23.249^{\prime} \mathrm{W}$ & 210-239 \\
\hline CH-2010-040 & 26 Sep 10 & VK862 & TT & 31 & $\mathrm{~N}$ & $29^{\circ} 04.182^{\prime} \mathrm{N}$ & $88^{\circ} 23.382^{\prime} \mathrm{W}$ & $29^{\circ} 04.312^{\prime} \mathrm{N}$ & $88^{\circ} 22.230^{\prime} \mathrm{W}$ & 95-141 \\
\hline CH-2010-040PN & 26 Sep 10 & VK862 & $\mathrm{PN}$ in $\mathrm{TT}$ & 31 & $\mathrm{~N}$ & $29^{\circ} 04.182^{\prime} \mathrm{N}$ & $88^{\circ} 23.382^{\prime} \mathrm{W}$ & $29^{\circ} 04.312^{\prime} \mathrm{N}$ & $88^{\circ} 22.230^{\prime} \mathrm{W}$ & 95-141 \\
\hline CH-2010-041 & 26 Sep 10 & VK862 & TT & 30 & $\mathrm{~N}$ & $29^{\circ} 04.198^{\prime} \mathrm{N}$ & $88^{\circ} 23.291^{\prime} \mathrm{W}$ & $29^{\circ} 04.308^{\prime} \mathrm{N}$ & $88^{\circ} 22.346^{\prime} \mathrm{W}$ & $120-264$ \\
\hline CH-2010-041PN & 26 Sep 10 & VK862 & $\mathrm{PN}$ in $\mathrm{TT}$ & 30 & $\mathrm{~N}$ & $29^{\circ} 04.198^{\prime} \mathrm{N}$ & $88^{\circ} 23.291^{\prime} \mathrm{W}$ & $29^{\circ} 04.308^{\prime} \mathrm{N}$ & $88^{\circ} 22.346^{\prime} \mathrm{W}$ & $120-264$ \\
\hline CH-2010-042 & 26 Sep 10 & VK862 & TT & 30 & $\mathrm{~N}$ & $29^{\circ} 04.169^{\prime} \mathrm{N}$ & $88^{\circ} 23.329^{\prime} \mathrm{W}$ & $29^{\circ} 04.605^{\prime} \mathrm{N}$ & $88^{\circ} 22.273^{\prime} \mathrm{W}$ & $283-355$ \\
\hline CH-2010-042PN & 26 Sep 10 & VK862 & $\mathrm{PN}$ in $\mathrm{TT}$ & 30 & $\mathrm{~N}$ & $29^{\circ} 04.169^{\prime} \mathrm{N}$ & $88^{\circ} 23.329^{\prime} \mathrm{W}$ & $29^{\circ} 04.605^{\prime} \mathrm{N}$ & $88^{\circ} 22.273^{\prime} \mathrm{W}$ & $283-355$ \\
\hline CH-2010-043 & 26 Sep 10 & VK862 & TT & 30 & $\mathrm{~N}$ & $29^{\circ} 04.081^{\prime} \mathrm{N}$ & $88^{\circ} 23.473^{\prime} \mathrm{W}$ & $29^{\circ} 04.415^{\prime} \mathrm{N}$ & $88^{\circ} 22.466^{\prime} \mathrm{W}$ & S-108 \\
\hline CH-2010-043PN & 26 Sep 10 & VK862 & $\mathrm{PN}$ in $\mathrm{TT}$ & 30 & $\mathrm{~N}$ & $29^{\circ} 04.081^{\prime} \mathrm{N}$ & $88^{\circ} 23.473^{\prime} \mathrm{W}$ & $29^{\circ} 04.415^{\prime} \mathrm{N}$ & $88^{\circ} 22.466^{\prime} \mathrm{W}$ & S-108 \\
\hline CH-2010-044 & 26 Sep 10 & VK862 & CTD & 10 & $\mathrm{~N}$ & $29^{\circ} 03.805^{\prime} \mathrm{N}$ & $88^{\circ} 22.602^{\prime} \mathrm{W}$ & $29^{\circ} 03.796^{\prime} \mathrm{N}$ & $88^{\circ} 22.493^{\prime} \mathrm{W}$ & S-480 \\
\hline CH-2010-045 & 26 Sep 10 & VK862 & CTD & 17 & $\mathrm{D}$ & $29^{\circ} 04.485^{\prime} \mathrm{N}$ & $88^{\circ} 23.209^{\prime} \mathrm{W}$ & $29^{\circ} 04.620^{\prime} \mathrm{N}$ & $88^{\circ} 23.257^{\prime} \mathrm{W}$ & S-394 \\
\hline CH-2010-046 & 27 Sep 10 & WFS & Z-trap & 4,220 & $\mathrm{~N}$ & $26^{\circ} 12.164^{\prime} \mathrm{N}$ & $84^{\circ} 42.299^{\prime} \mathrm{W}$ & $26^{\circ} 12.164^{\prime} \mathrm{N}$ & $84^{\circ} 42.299^{\prime} \mathrm{W}$ & $S-419$ \\
\hline CH-2010-047 & 27 Sep 10 & WFS & $\mathrm{MC}$ & & $\mathrm{N}$ & $26^{\circ} 12.595^{\prime} \mathrm{N}$ & $84^{\circ} 43.574^{\prime} \mathrm{W}$ & $26^{\circ} 12.595^{\prime} \mathrm{N}$ & $84^{\circ} 43.574^{\prime} \mathrm{W}$ & 488 \\
\hline CH-2010-048 & 27 Sep 10 & WFS & $\mathrm{TT}$ & 31 & $\mathrm{~N}$ & $26^{\circ} 12.343^{\prime} \mathrm{N}$ & $84^{\circ} 43.907^{\prime} \mathrm{W}$ & $26^{\circ} 12.291^{\prime} \mathrm{N}$ & $84^{\circ} 42.600^{\prime} \mathrm{W}$ & S-111 \\
\hline CH-2010-048PN & 27 Sep 10 & WFS & $\mathrm{PN}$ in $\mathrm{TT}$ & 31 & $\mathrm{~N}$ & $26^{\circ} 12.343^{\prime} \mathrm{N}$ & $84^{\circ} 43.907^{\prime} \mathrm{W}$ & $26^{\circ} 12.291^{\prime} \mathrm{N}$ & $84^{\circ} 42.600^{\prime} \mathrm{W}$ & S-111 \\
\hline CH-2010-049 & 28 Sep 10 & WFS & TT & 30 & $\mathrm{~N}$ & $26^{\circ} 12.346^{\prime} \mathrm{N}$ & $84^{\circ} 43.861^{\prime} \mathrm{W}$ & $26^{\circ} 12.731^{\prime} \mathrm{N}$ & $84^{\circ} 42.784^{\prime} \mathrm{W}$ & $81-123$ \\
\hline CH-2010-049PN & 28 Sep 10 & WFS & $\mathrm{PN}$ in $\mathrm{TT}$ & 30 & $\mathrm{~N}$ & $26^{\circ} 12.346^{\prime} \mathrm{N}$ & $84^{\circ} 43.861^{\prime} \mathrm{W}$ & $26^{\circ} 12.731^{\prime} \mathrm{N}$ & $84^{\circ} 42.784^{\prime} \mathrm{W}$ & $81-123$ \\
\hline CH-2010-050 & 28 Sep 10 & WFS & TT & 30 & $\mathrm{~N}$ & $26^{\circ} 12.336^{\prime} \mathrm{N}$ & $84^{\circ} 43.883^{\prime} \mathrm{W}$ & $26^{\circ} 12.796^{\prime} \mathrm{N}$ & $84^{\circ} 42.740^{\prime} \mathrm{W}$ & S-242 \\
\hline CH-2010-050PN & 28 Sep 10 & WFS & $\mathrm{PN}$ in $\mathrm{TT}$ & 30 & $\mathrm{~N}$ & $26^{\circ} 12.336^{\prime} \mathrm{N}$ & $84^{\circ} 43.883^{\prime} \mathrm{W}$ & $26^{\circ} 12.796^{\prime} \mathrm{N}$ & $84^{\circ} 42.740^{\prime} \mathrm{W}$ & S-242 \\
\hline CH-2010-051 & 28 Sep 10 & WFS & TT & 30 & $\mathrm{~N}$ & $26^{\circ} 12.373^{\prime} \mathrm{N}$ & $84^{\circ} 43.724^{\prime} \mathrm{W}$ & $26^{\circ} 12.737^{\prime} \mathrm{N}$ & $84^{\circ} 42.644^{\prime} \mathrm{W}$ & 293-349 \\
\hline CH-2010-051PN & 28 Sep 10 & WFS & $\mathrm{PN}$ in $\mathrm{TT}$ & 30 & $\mathrm{~N}$ & $26^{\circ} 12.373^{\prime} \mathrm{N}$ & $84^{\circ} 43.724^{\prime} \mathrm{W}$ & $26^{\circ} 12.737^{\prime} \mathrm{N}$ & $84^{\circ} 42.644^{\prime} \mathrm{W}$ & 293-349 \\
\hline CH-2010-052 & 28 Sep 10 & WFS & TT & 30 & $\mathrm{~N}$ & $26^{\circ} 12.331^{\prime} \mathrm{N}$ & $84^{\circ} 43.677^{\prime} \mathrm{W}$ & $26^{\circ} 13.031^{\prime} \mathrm{N}$ & $84^{\circ} 41.994^{\prime} \mathrm{W}$ & S-366 \\
\hline
\end{tabular}


Table B.3. Stations sampled in the north-central and eastern Gulf of Mexico using the research vessel (R/V) Cape Hatteras, September 21October 2, 2010, as part of the Lophelia II program - continued.

[min, minute; hr, hour; CDT, Central Daylight Time; m, meter; D, Day (0700 to $1900 \mathrm{hr}$ CDT); N, Night (1900 to $0700 \mathrm{hr}$ CDT); S, surface; CTD, Sea-bird SBE 911+ CTD; MC, multi-core; OT=3.5-m otter trawl; TT=2 x 2 m Tucker trawl; PN in TT, 0.5-m plankton net embedded in Tucker trawl; OD, on deck; PN, 1.0-m plankton net; Phyto, phytoplankton sample; ROV, remotely operated vehicle Kraken II; ROV depth range data are only for the period when the ROV was on the bottom]

\begin{tabular}{|c|c|c|c|c|c|c|c|c|c|c|}
\hline Station number & Date & Location & Gear & $\begin{array}{c}\text { Sample } \\
\text { time } \\
\text { (min) }\end{array}$ & Time & Start latitude & $\begin{array}{c}\text { Start } \\
\text { longitude }\end{array}$ & End latitude & $\begin{array}{l}\text { End } \\
\text { longitude }\end{array}$ & $\begin{array}{l}\text { Depth } \\
\text { range }(m)\end{array}$ \\
\hline CH-2010-052PN & $28 \mathrm{Sep} 10$ & WFS & $\mathrm{PN}$ in $\mathrm{TT}$ & 30 & $\mathrm{~N}$ & $26^{\circ} 12.331 ' \mathrm{~N}$ & $84^{\circ} 43.677^{\prime} \mathrm{W}$ & $26^{\circ} 13.031 ' \mathrm{~N}$ & $84^{\circ} 41.994^{\prime} \mathrm{W}$ & S-366 \\
\hline CH-2010-053 & 28 Sep 10 & WFS & TT & 30 & $\mathrm{~N}$ & $26^{\circ} 12.387^{\prime} \mathrm{N}$ & $84^{\circ} 43.745^{\prime} \mathrm{W}$ & $26^{\circ} 13.580^{\prime} \mathrm{N}$ & $84^{\circ} 42.814^{\prime} \mathrm{W}$ & $41-60$ \\
\hline CH-2010-053PN & 28 Sep 10 & WFS & $\mathrm{PN}$ in $\mathrm{TT}$ & 30 & $\mathrm{~N}$ & $26^{\circ} 12.387^{\prime} \mathrm{N}$ & $84^{\circ} 43.745^{\prime} \mathrm{W}$ & $26^{\circ} 13.580^{\prime} \mathrm{N}$ & $84^{\circ} 42.814^{\prime} \mathrm{W}$ & $41-60$ \\
\hline CH-2010-054 & $28 \mathrm{Sep} 10$ & WFS & CTD & 12 & $\mathrm{D}$ & $26^{\circ} 12.351^{\prime} \mathrm{N}$ & $84^{\circ} 43.666^{\prime} \mathrm{W}$ & $26^{\circ} 12.559^{\prime} \mathrm{N}$ & $84^{\circ} 43.717^{\prime} \mathrm{W}$ & $S-493$ \\
\hline CH-2010-055 & 28 Sep 10 & WFS & CTD & 8 & $\mathrm{~N}$ & $26^{\circ} 12.098^{\prime} \mathrm{N}$ & $84^{\circ} 42.016^{\prime} \mathrm{W}$ & $26^{\circ} 12.081^{\prime} \mathrm{N}$ & $84^{\circ} 42.016^{\prime} \mathrm{W}$ & S-368 \\
\hline CH-2010-056 & 28 Sep 10 & WFS & CTD & 9 & $\mathrm{~N}$ & $26^{\circ} 12.031^{\prime} \mathrm{N}$ & $84^{\circ} 42.362^{\prime} \mathrm{W}$ & $26^{\circ} 12.020^{\prime} \mathrm{N}$ & $84^{\circ} 42.297^{\prime} \mathrm{W}$ & $\mathrm{S}-404$ \\
\hline CH-2010-057 & 28 Sep 10 & WFS & CTD & 10 & $\mathrm{~N}$ & $26^{\circ} 12.018^{\prime} \mathrm{N}$ & $84^{\circ} 42.652^{\prime} \mathrm{W}$ & $26^{\circ} 12.030^{\prime} \mathrm{N}$ & $84^{\circ} 42.557^{\prime} \mathrm{W}$ & $S-430$ \\
\hline CH-2010-058 & 28 Sep 10 & WFS & CTD & 10 & $\mathrm{~N}$ & $26^{\circ} 12.044^{\prime} \mathrm{N}$ & $84^{\circ} 42.949^{\prime} \mathrm{W}$ & $26^{\circ} 12.059^{\prime} \mathrm{N}$ & $84^{\circ} 42.839^{\prime} \mathrm{W}$ & $S-441$ \\
\hline CH-2010-059 & $28 \mathrm{Sep} 10$ & WFS & CTD & 12 & $\mathrm{~N}$ & $26^{\circ} 12.018^{\prime} \mathrm{N}$ & $84^{\circ} 43.285^{\prime} \mathrm{W}$ & $26^{\circ} 12.063^{\prime} \mathrm{N}$ & $84^{\circ} 43.156^{\prime} \mathrm{W}$ & $S-489$ \\
\hline CH-2010-060 & $28 \mathrm{Sep} 10$ & WFS & CTD & 12 & $\mathrm{~N}$ & $26^{\circ} 12.026^{\prime} \mathrm{N}$ & $84^{\circ} 43.531^{\prime} \mathrm{W}$ & $26^{\circ} 12.074^{\prime} \mathrm{N}$ & $84^{\circ} 43.524^{\prime} \mathrm{W}$ & $\mathrm{S}-519$ \\
\hline CH-2010-061 & 28 Sep 10 & WFS & CTD & 13 & $\mathrm{~N}$ & $26^{\circ} 11.993^{\prime} \mathrm{N}$ & $84^{\circ} 43.878^{\prime} \mathrm{W}$ & $26^{\circ} 12.093^{\prime} \mathrm{N}$ & $84^{\circ} 43.759^{\prime} \mathrm{W}$ & $\mathrm{S}-527$ \\
\hline CH-2010-062 & 29 Sep 10 & WFS & CTD & 14 & $\mathrm{~N}$ & $26^{\circ} 12.060^{\prime} \mathrm{N}$ & $84^{\circ} 44.325^{\prime} \mathrm{W}$ & $26^{\circ} 12.138^{\prime} \mathrm{N}$ & $84^{\circ} 44.434^{\prime} \mathrm{W}$ & S-600 \\
\hline CH-2010-063 & 29 Sep 10 & WFS & CTD & 11 & $\mathrm{~N}$ & $26^{\circ} 12.067^{\prime} \mathrm{N}$ & $84^{\circ} 44.455^{\prime} \mathrm{W}$ & $26^{\circ} 12.061^{\prime} \mathrm{N}$ & $84^{\circ} 44.507^{\prime} \mathrm{W}$ & S-602 \\
\hline CH-2010-064 & 29 Sep 10 & WFS & CTD & 12 & $\mathrm{~N}$ & $26^{\circ} 12.022^{\prime} \mathrm{N}$ & $84^{\circ} 44.699^{\prime} \mathrm{W}$ & $26^{\circ} 12.111^{\prime} \mathrm{N}$ & $84^{\circ} 44.735^{\prime} \mathrm{W}$ & S-632 \\
\hline CH-2010-065 & 29 Sep 10 & WFS & TT & 31 & $\mathrm{~N}$ & $26^{\circ} 12.250^{\prime} \mathrm{N}$ & $84^{\circ} 43.900^{\prime} \mathrm{W}$ & $26^{\circ} 12.397{ }^{\prime} \mathrm{N}$ & $84^{\circ} 42.771^{\prime} \mathrm{W}$ & $69-154$ \\
\hline CH-2010-065PN & 29 Sep 10 & WFS & $\mathrm{PN}$ in $\mathrm{TT}$ & 31 & $\mathrm{~N}$ & $26^{\circ} 12.250^{\prime} \mathrm{N}$ & $84^{\circ} 43.900^{\prime} \mathrm{W}$ & $26^{\circ} 12.397^{\prime} \mathrm{N}$ & $84^{\circ} 42.771^{\prime} \mathrm{W}$ & $69-154$ \\
\hline CH-2010-066 & 29 Sep 10 & WFS & TT & 31 & $\mathrm{~N}$ & $26^{\circ} 12.262^{\prime} \mathrm{N}$ & $84^{\circ} 43.871^{\prime} \mathrm{W}$ & $26^{\circ} 11.870^{\prime} \mathrm{N}$ & $84^{\circ} 42.982^{\prime} \mathrm{W}$ & $229-315$ \\
\hline CH-2010-067 & 29 Sep 10 & WFS & $\mathrm{TT}$ & 30 & $\mathrm{~N}$ & $26^{\circ} 12.217^{\prime} \mathrm{N}$ & $84^{\circ} 43.757^{\prime} \mathrm{W}$ & $26^{\circ} 11.965^{\prime} \mathrm{N}$ & $84^{\circ} 42.833^{\prime} \mathrm{W}$ & $325-426$ \\
\hline CH-2010-068 & 29 Sep 10 & WFS & TT & 30 & $\mathrm{~N}$ & $26^{\circ} 12.281^{\prime} \mathrm{N}$ & $84^{\circ} 43.905^{\prime} \mathrm{W}$ & $26^{\circ} 12.126^{\prime} \mathrm{N}$ & $84^{\circ} 42.937^{\prime} \mathrm{W}$ & $397-467$ \\
\hline CH-2010-069 & 29 Sep 10 & WFS & Phyto & & $\mathrm{D}$ & $26^{\circ} 11.891^{\prime} \mathrm{N}$ & $84^{\circ} 44.075^{\prime} \mathrm{W}$ & $26^{\circ} 11.891^{\prime} \mathrm{N}$ & $84^{\circ} 44.075^{\prime} \mathrm{W}$ & S \\
\hline CH-2010-070 & 29 Sep 10 & WFS & Phyto & & D & $26^{\circ} 11.898^{\prime} \mathrm{N}$ & $84^{\circ} 44.092^{\prime} \mathrm{W}$ & $26^{\circ} 11.898^{\prime} \mathrm{N}$ & $84^{\circ} 44.092^{\prime} \mathrm{W}$ & S \\
\hline CH-2010-071 & 29 Sep 10 & WFS & Phyto & & D & $26^{\circ} 11.899^{\prime} \mathrm{N}$ & $84^{\circ} 44.094^{\prime} \mathrm{W}$ & $26^{\circ} 11.899^{\prime} \mathrm{N}$ & $84^{\circ} 44.094^{\prime} \mathrm{W}$ & S \\
\hline CH-2010-072 & 29 Sep 10 & WFS & Phyto & & $\mathrm{D}$ & $26^{\circ} 11.895^{\prime} \mathrm{N}$ & $84^{\circ} 44.096^{\prime} \mathrm{W}$ & $26^{\circ} 11.895^{\prime} \mathrm{N}$ & $84^{\circ} 44.096^{\prime} \mathrm{W}$ & S \\
\hline CH-2010-073 & 29 Sep 10 & WFS & Phyto & & D & $26^{\circ} 11.900^{\prime} \mathrm{N}$ & $84^{\circ} 44.091^{\prime} \mathrm{W}$ & $26^{\circ} 11.900^{\prime} \mathrm{N}$ & $84^{\circ} 44.091^{\prime} \mathrm{W}$ & $\mathrm{S}$ \\
\hline CH-2010-074 & 29 Sep 10 & WFS & CTD & 18 & $\mathrm{~N}$ & $26^{\circ} 12.370^{\prime} \mathrm{N}$ & $84^{\circ} 44.705^{\prime} \mathrm{W}$ & $26^{\circ} 12.630^{\prime} \mathrm{N}$ & $84^{\circ} 44.029^{\prime} \mathrm{W}$ & S-647 \\
\hline
\end{tabular}


Table B.3. Stations sampled in the north-central and eastern Gulf of Mexico using the research vessel (R/V) Cape Hatteras, September 21October 2, 2010, as part of the Lophelia II program - continued.

[min, minute; hr, hour; CDT, Central Daylight Time; m, meter; D, Day (0700 to $1900 \mathrm{hr}$ CDT); N, Night (1900 to $0700 \mathrm{hr}$ CDT); S, surface; CTD, Sea-bird SBE 911+ CTD; MC, multi-core; OT=3.5-m otter trawl; TT=2 x 2 m Tucker trawl; PN in TT, 0.5-m plankton net embedded in Tucker trawl; OD, on deck; PN, 1.0-m plankton net; Phyto, phytoplankton sample; ROV, remotely operated vehicle Kraken II; ROV depth range data are only for the period when the ROV was on the bottom]

\begin{tabular}{|c|c|c|c|c|c|c|c|c|c|c|}
\hline Station number & Date & Location & Gear & $\begin{array}{c}\text { Sample } \\
\text { time } \\
\text { (min) }\end{array}$ & Time & Start latitude & $\begin{array}{c}\text { Start } \\
\text { longitude }\end{array}$ & End latitude & $\begin{array}{l}\text { End } \\
\text { longitude }\end{array}$ & $\begin{array}{l}\text { Depth } \\
\text { range }(m)\end{array}$ \\
\hline CH-2010-075 & 29 Sep 10 & WFS & TT & 31 & $\mathrm{~N}$ & $26^{\circ} 12.011^{\prime} \mathrm{N}$ & $84^{\circ} 43.605^{\prime} \mathrm{W}$ & $26^{\circ} 12.943^{\prime} \mathrm{N}$ & $84^{\circ} 44.053^{\prime} \mathrm{W}$ & $\mathrm{N} / \mathrm{A}$ \\
\hline CH-2010-075PN & 29 Sep 10 & WFS & $\mathrm{PN}$ in $\mathrm{TT}$ & 31 & $\mathrm{~N}$ & $26^{\circ} 12.011^{\prime} \mathrm{N}$ & $84^{\circ} 43.605^{\prime} \mathrm{W}$ & $26^{\circ} 12.943^{\prime} \mathrm{N}$ & $84^{\circ} 44.053^{\prime} \mathrm{W}$ & N/A \\
\hline CH-2010-076 & 29 Sep 10 & WFS & TT & 33 & $\mathrm{~N}$ & $26^{\circ} 12.147^{\prime} \mathrm{N}$ & $84^{\circ} 43.624^{\prime} \mathrm{W}$ & $26^{\circ} 12.770^{\prime} \mathrm{N}$ & $84^{\circ} 44.603^{\prime} \mathrm{W}$ & $242-277$ \\
\hline CH-2010-077 & 30 Sep 10 & WFS & TT & 31 & $\mathrm{~N}$ & $26^{\circ} 12.110^{\prime} \mathrm{N}$ & $84^{\circ} 43.538^{\prime} \mathrm{W}$ & $26^{\circ} 12.075^{\prime} \mathrm{N}$ & $84^{\circ} 43.495^{\prime} \mathrm{W}$ & S-144 \\
\hline CH-2010-078 & 30 Sep 10 & WFS & TT & 29 & $\mathrm{~N}$ & $26^{\circ} 12.169^{\prime} \mathrm{N}$ & $84^{\circ} 43.907^{\prime} \mathrm{W}$ & $26^{\circ} 12.943^{\prime} \mathrm{N}$ & $84^{\circ} 44.753^{\prime} \mathrm{W}$ & $310-370$ \\
\hline CH-2010-079 & 30 Sep 10 & WFS & TT & 30 & $\mathrm{~N}$ & $26^{\circ} 12.216^{\prime} \mathrm{N}$ & $84^{\circ} 43.697^{\prime} \mathrm{W}$ & $26^{\circ} 12.570^{\prime} \mathrm{N}$ & $84^{\circ} 44.849^{\prime} \mathrm{W}$ & S-266 \\
\hline CH-2010-080 & 30 Sep 10 & WFS & TT & 30 & $\mathrm{~N}$ & $26^{\circ} 12.534^{\prime} \mathrm{N}$ & $84^{\circ} 43.841^{\prime} \mathrm{W}$ & $26^{\circ} 13.360^{\prime} \mathrm{N}$ & $84^{\circ} 44.797^{\prime} \mathrm{W}$ & $340-364$ \\
\hline CH-2010-081 & 30 Sep 10 & WFS & TT & 34 & $\mathrm{~N}$ & $26^{\circ} 12.472^{\prime} \mathrm{N}$ & $84^{\circ} 43.016^{\prime} \mathrm{W}$ & $26^{\circ} 13.161^{\prime} \mathrm{N}$ & $84^{\circ} 44.042^{\prime} \mathrm{W}$ & $\mathrm{S}-525$ \\
\hline CH-2010-082 & 30 Sep 10 & WFS & TT & 31 & $\mathrm{~N}$ & $26^{\circ} 12.338^{\prime} \mathrm{N}$ & $84^{\circ} 43.014^{\prime} \mathrm{W}$ & $26^{\circ} 12.004^{\prime} \mathrm{N}$ & $84^{\circ} 44.975^{\prime} \mathrm{W}$ & $315-364$ \\
\hline CH-2010-083 & 1 Oct 10 & WFS & TT & 29 & $\mathrm{~N}$ & $26^{\circ} 12.577^{\prime} \mathrm{N}$ & $84^{\circ} 43.418^{\prime} \mathrm{W}$ & $26^{\circ} 13.222^{\prime} \mathrm{N}$ & $84^{\circ} 44.438^{\prime} \mathrm{W}$ & $\mathrm{S}-231$ \\
\hline CH-2010-084 & 1 Oct 10 & WFS & TT & 30 & $\mathrm{~N}$ & $26^{\circ} 12.166^{\prime} \mathrm{N}$ & $84^{\circ} 43.788^{\prime} \mathrm{W}$ & $26^{\circ} 13.077^{\prime} \mathrm{N}$ & $84^{\circ} 44.728^{\prime} \mathrm{W}$ & $50-76$ \\
\hline CH-2010-085 & 1 Oct 10 & WFS & CTD & 10 & $\mathrm{~N}$ & $26^{\circ} 20.204^{\prime} \mathrm{N}$ & $84^{\circ} 45.616^{\prime} \mathrm{W}$ & $26^{\circ} 20.210^{\prime} \mathrm{N}$ & $84^{\circ} 45.561^{\prime} \mathrm{W}$ & S-509 \\
\hline CH-2010-086 & 1 Oct 10 & WFS & CTD & 8 & $\mathrm{~N}$ & $26^{\circ} 20.174^{\prime} \mathrm{N}$ & $84^{\circ} 44.558^{\prime} \mathrm{W}$ & $26^{\circ} 20.186^{\prime} \mathrm{N}$ & $84^{\circ} 44.524^{\prime} \mathrm{W}$ & S-389 \\
\hline CH-2010-087 & 1 Oct 10 & WFS & CTD & 9 & $\mathrm{~N}$ & $26^{\circ} 20.161^{\prime} \mathrm{N}$ & $84^{\circ} 44.773^{\prime} \mathrm{W}$ & $26^{\circ} 20.174^{\prime} \mathrm{N}$ & $84^{\circ} 44.801^{\prime} \mathrm{W}$ & $\mathrm{S}-453$ \\
\hline CH-2010-088 & 1 Oct 10 & WFS & CTD & 10 & $\mathrm{~N}$ & $26^{\circ} 20.136^{\prime} \mathrm{N}$ & $84^{\circ} 45.104^{\prime} \mathrm{W}$ & $26^{\circ} 20.145^{\prime} \mathrm{N}$ & $84^{\circ} 45.096^{\prime} \mathrm{W}$ & $S-465$ \\
\hline CH-2010-089 & 1 Oct 10 & WFS & CTD & 11 & $\mathrm{~N}$ & $26^{\circ} 20.145^{\prime} \mathrm{N}$ & $84^{\circ} 45.413^{\prime} \mathrm{W}$ & $26^{\circ} 20.162^{\prime} \mathrm{N}$ & $84^{\circ} 45.394^{\prime} \mathrm{W}$ & S-478 \\
\hline CH-2010-090 & 1 Oct 10 & WFS & CTD & 12 & $\mathrm{~N}$ & $26^{\circ} 20.122^{\prime} \mathrm{N}$ & $84^{\circ} 45.741^{\prime} \mathrm{W}$ & $26^{\circ} 20.152^{\prime} \mathrm{N}$ & $84^{\circ} 45.702^{\prime} \mathrm{W}$ & S-507 \\
\hline CH-2010-091 & 1 Oct 10 & WFS & CTD & 15 & $\mathrm{~N}$ & $26^{\circ} 20.140^{\prime} \mathrm{N}$ & $84^{\circ} 46.060^{\prime} \mathrm{W}$ & $26^{\circ} 20.173^{\prime} \mathrm{N}$ & $84^{\circ} 45.987^{\prime} \mathrm{W}$ & $\mathrm{S}-550$ \\
\hline CH-2010-092 & 1 Oct 10 & WFS & CTD & 14 & $\mathrm{~N}$ & $26^{\circ} 20.144^{\prime} \mathrm{N}$ & $84^{\circ} 46.346^{\prime} \mathrm{W}$ & $26^{\circ} 20.160^{\prime} \mathrm{N}$ & $84^{\circ} 46.286^{\prime} \mathrm{W}$ & S-588 \\
\hline CH-2010-093 & 2 Oct 10 & WFS & TT & 34 & $\mathrm{~N}$ & $26^{\circ} 24.432^{\prime} \mathrm{N}$ & $84^{\circ} 46.572^{\prime} \mathrm{W}$ & $26^{\circ} 24.398^{\prime} \mathrm{N}$ & $84^{\circ} 46.365^{\prime} \mathrm{W}$ & 199-330 \\
\hline CH-2010-094 & 2 Oct 10 & WFS & TT & 31 & $\mathrm{~N}$ & $26^{\circ} 24.450^{\prime} \mathrm{N}$ & $84^{\circ} 47.869^{\prime} \mathrm{W}$ & $26^{\circ} 24.462^{\prime} \mathrm{N}$ & $84^{\circ} 47.777^{\prime} \mathrm{W}$ & S-149 \\
\hline CH-2010-095 & 2 Oct 10 & WFS & TT & 30 & $\mathrm{~N}$ & $26^{\circ} 24.488^{\prime} \mathrm{N}$ & $84^{\circ} 46.777^{\prime} \mathrm{W}$ & $26^{\circ} 24.446^{\prime} \mathrm{N}$ & $84^{\circ} 45.568^{\prime} \mathrm{W}$ & $17-55$ \\
\hline CH-2010-096 & 2 Oct 10 & WFS & TT & 30 & $\mathrm{~N}$ & $26^{\circ} 24.457^{\prime} \mathrm{N}$ & $84^{\circ} 46.736^{\prime} \mathrm{W}$ & $26^{\circ} 24.462^{\prime} \mathrm{N}$ & $84^{\circ} 45.600^{\prime} \mathrm{W}$ & 219-270 \\
\hline CH-2010-097 & 2 Oct 10 & WFS & TT & 31 & $\mathrm{~N}$ & $26^{\circ} 24.450^{\prime} \mathrm{N}$ & $84^{\circ} 46.821^{\prime} \mathrm{W}$ & $26^{\circ} 24.521^{\prime} \mathrm{N}$ & $84^{\circ} 46.758^{\prime} \mathrm{W}$ & $7-26$ \\
\hline CH-2010-098 & 2 Oct 10 & WFS & CTD & 10 & $\mathrm{~N}$ & $26^{\circ} 24.486^{\prime} \mathrm{N}$ & $84^{\circ} 46.744^{\prime} \mathrm{W}$ & $26^{\circ} 24.506^{\prime} \mathrm{N}$ & $84^{\circ} 46.689^{\prime} \mathrm{W}$ & S-498 \\
\hline
\end{tabular}


Table B.4. Stations sampled on deep coral areas in the north-central Gulf of Mexico using the research vessel (R/V) Arctic Sunrise, October 12-22, 2010, as part of the Lophelia II program.

[min, minute; m, meter; $D D W$, Dual DeepWorker submersible. Submersible depth range data are only for the period when the $D D W$ was on the bottom]

\begin{tabular}{|c|c|c|c|c|c|c|c|c|c|c|}
\hline Station number & Date & Location & Gear & $\begin{array}{c}\text { Sample } \\
\text { time } \\
\text { (min) }\end{array}$ & Time & Start latitude & $\begin{array}{c}\text { Start } \\
\text { longitude }\end{array}$ & End latitude & $\begin{array}{l}\text { End } \\
\text { longitude }\end{array}$ & $\begin{array}{c}\text { Depth } \\
\text { range }(\mathrm{m})\end{array}$ \\
\hline AS-2010-001 & 15 Oct 10 & VK862/906 & Lander & & $\mathrm{D}$ & $29^{\circ} 04.290^{\prime} \mathrm{N}$ & $88^{\circ} 22.630^{\prime} \mathrm{W}$ & & & 420 \\
\hline DDW-2010-001 & 15 Oct 10 & VK862/906 & $D D W$ & 58 & $\mathrm{D}$ & $29^{\circ} 04.319^{\prime} \mathrm{N}$ & $88^{\circ} 22.515^{\prime} \mathrm{W}$ & $29^{\circ} 04.194^{\prime} \mathrm{N}$ & $88^{\circ} 22.542^{\prime} \mathrm{W}$ & $395-429$ \\
\hline DDW-2010-002 & 16 Oct 10 & VK862/906 & $D D W$ & 84 & $\mathrm{D}$ & $29^{\circ} 04.221^{\prime} \mathrm{N}$ & $88^{\circ} 22.642^{\prime} \mathrm{W}$ & $29^{\circ} 04.248^{\prime} \mathrm{N}$ & $88^{\circ} 22.653^{\prime} \mathrm{W}$ & $314-426$ \\
\hline DDW-2010-003 & 16 Oct 10 & VK862/906 & $D D W$ & & D & $29^{\circ} 04.268^{\prime} \mathrm{N}$ & $88^{\circ} 22.803^{\prime} \mathrm{W}$ & & & \\
\hline DDW-2010-004 & 17 Oct 10 & Alabama Alps & $D D W$ & 86 & $\mathrm{D}$ & $29^{\circ} 15.262^{\prime} \mathrm{N}$ & $88^{\circ} 20.384^{\prime} \mathrm{W}$ & $29^{\circ} 15.126^{\prime} \mathrm{N}$ & $88^{\circ} 20.255^{\prime} \mathrm{W}$ & $63-89$ \\
\hline DDW-2010-005 & 17 Oct 10 & Alabama Alps & $D D W$ & 100 & D & $29^{\circ} 15.097^{\prime} \mathrm{N}$ & $88^{\circ} 20.203^{\prime} \mathrm{W}$ & $29^{\circ} 15.085^{\prime} \mathrm{N}$ & $88^{\circ} 20.408^{\prime} \mathrm{W}$ & 64-95 \\
\hline DDW-2010-006 & 18 Oct 10 & VK862/906 & $D D W$ & 163 & D & $29^{\circ} 06.277^{\prime} \mathrm{N}$ & $88^{\circ} 23.249^{\prime} \mathrm{W}$ & $29^{\circ} 06.432^{\prime} \mathrm{N}$ & $88^{\circ} 23.129^{\prime} \mathrm{W}$ & $309-355$ \\
\hline DDW-2010-007 & 19 Oct 10 & VK862/906 & $D D W$ & 48 & $\mathrm{D}$ & $29^{\circ} 04.250^{\prime} \mathrm{N}$ & $88^{\circ} 22.668^{\prime} \mathrm{W}$ & $29^{\circ} 04.255^{\prime} \mathrm{N}$ & $88^{\circ} 22.704^{\prime} \mathrm{W}$ & $391-430$ \\
\hline DDW-2010-008 & 19 Oct 10 & VK862/906 & $D D W$ & 51 & D & $29^{\circ} 04.189^{\prime} \mathrm{N}$ & $88^{\circ} 22.711^{\prime} \mathrm{W}$ & $29^{\circ} 04.158^{\prime} \mathrm{N}$ & $88^{\circ} 22.651^{\prime} \mathrm{W}$ & $394-401$ \\
\hline DDW-2010-009 & 19 Oct 10 & VK862/906 & $D D W$ & 122 & $\mathrm{D}$ & $29^{\circ} 04.209^{\prime} \mathrm{N}$ & $88^{\circ} 22.637^{\prime} \mathrm{W}$ & $29^{\circ} 04.171^{\prime} \mathrm{N}$ & $88^{\circ} 22.634^{\prime} \mathrm{W}$ & $379-413$ \\
\hline DDW-2010-010 & 20 Oct 10 & VK862/906 & $D D W$ & 101 & D & $29^{\circ} 04.436{ }^{\prime} \mathrm{N}$ & $88^{\circ} 22.752^{\prime} \mathrm{W}$ & $29^{\circ} 04.442^{\prime} \mathrm{N}$ & $88^{\circ} 22.776^{\prime} \mathrm{W}$ & $390-411$ \\
\hline
\end{tabular}


Table B.5. Stations sampled in the eastern Gulf of Mexico using the NOAA ship Ronald H. Brown November 9-22, 2010, as part of the Lophelia II program.

[min, minute; m, meter; hr, hour; EST, Eastern Standard Time; N, Night (2000 to $0800 \mathrm{hr}$ EST); ROV, remotely operated vehicle Jason II. ROV depth range data are only for the period when the ROV was on the bottom]

\begin{tabular}{|c|c|c|c|c|c|c|c|c|c|c|}
\hline Station number & Date & Location & Gear & $\begin{array}{c}\text { Sample } \\
\text { time } \\
\text { (min) }\end{array}$ & Time & Start latitude & $\begin{array}{c}\text { Start } \\
\text { longitude }\end{array}$ & End latitude & $\begin{array}{c}\text { End } \\
\text { longitude }\end{array}$ & $\begin{array}{l}\text { Depth } \\
\text { range }(\mathrm{m})\end{array}$ \\
\hline ROV-2010-RB-542 & 10 Nov 10 & WFS & ROV & 323 & $\mathrm{D}$ & $26^{\circ} 12.228^{\prime} \mathrm{N}$ & $84^{\circ} 45.295^{\prime} \mathrm{W}$ & $26^{\circ} 12.312^{\prime} \mathrm{N}$ & $84^{\circ} 43.712^{\prime} \mathrm{W}$ & $495-734$ \\
\hline
\end{tabular}




\section{APPENDIX C. RADIOCARBON RESULTS}

Table C.1. Radiocarbon results from radial transects and radiocarbon calibration results from the Lophelia II program.

[All calibrations were done using a $\Delta \mathrm{R}$ of $-30 \pm 26{ }^{14} \mathrm{C}$ years (Wagner and others, 2009), the CALIB 6.0 (Stuiver and Reimer, 1993 ) computer calibration program, and the marine09 calibration dataset (Hughen and others, 2009). $\mu \mathrm{m}$, micrometer; cal yr BP, calendar years before present; Lab, the lab where the samples were processed; KCCAMS, the Keck Carbon Cycle AMS Laboratory at the University of California Irvine; BetaAnalytic, Beta Analytic in Miami Florida; NOSAMS, the National Ocean Sciences Accelerator Mass Spectrometry Facility at Woods Hole Oceanographic Institution; LLNL-CAMS, the Center for Accelerator Mass Spectrometry at the Lawrence Livermore National Laboratory. Distance is from outer edge into the center of the specimen. Radiocarbon concentration is given as fraction Modern, $\Delta 14 \mathrm{C}$, and conventional radiocarbon age. $\Delta{ }^{14} \mathrm{C}$ values are not age corrected (Stiuver and Polloch, 1977), nor are the conventional radiocarbon ages reservoir corrected. See Chapter 5 for references]

\begin{tabular}{|c|c|c|c|c|c|c|c|c|c|c|c|c|}
\hline Sample ID & Lab & $\begin{array}{c}\text { Distance } \\
(\mu \mathrm{m})^{1}\end{array}$ & $\begin{array}{l}\text { Fraction } \\
\text { modern }\end{array}$ & \pm & $\Delta^{14} \mathrm{C}$ & ${ }^{14} \mathrm{C}$ age & \pm & $\begin{array}{c}\text { Number } \\
\text { of age } \\
\text { ranges }\end{array}$ & $\begin{array}{c}\text { Minimum } \\
\text { cal age } \\
\text { range } \\
\text { (1s) cal } \\
\text { BP }\end{array}$ & $\begin{array}{l}\text { Maximum } \\
\text { cal age } \\
\text { range } \\
\text { (1s) cal } \\
\text { BP }\end{array}$ & $\begin{array}{c}\text { Relative } \\
\text { area under } \\
\text { distribution }\end{array}$ & $\begin{array}{c}\text { Median } \\
\text { probability } \\
\text { age }\end{array}$ \\
\hline \multicolumn{13}{|c|}{ GOM-JSL05-4876-BC1 } \\
\hline 48761.1 & KCCAMS & 47 & 1.0772 & 0.0036 & 69.42 & Modern & & & & & & \\
\hline 48761.2 & KCCAMS & 95 & 1.0763 & 0.0035 & 68.49 & Modern & & & & & & \\
\hline 48761.3 & KCCAMS & 130 & 1.0829 & 0.0038 & 75.04 & Modern & & & & & & \\
\hline 48761.4 & KCCAMS & 165 & 1.0828 & 0.0038 & 74.94 & Modern & & & & & & \\
\hline 48761.5 & KCCAMS & 206 & 1.0720 & 0.0035 & 64.25 & Modern & & & & & & \\
\hline 48762.3 & KCCAMS & 3,383 & 0.9323 & 0.0031 & -77.27 & 590 & 30 & 2 & $\begin{array}{l}150 \\
225 \\
\end{array}$ & $\begin{array}{l}160 \\
315 \\
\end{array}$ & $\begin{array}{l}0.04 \\
0.96 \\
\end{array}$ & 265 \\
\hline 48763.1 & KCCAMS & 3,418 & 0.9323 & 0.0031 & -74.45 & 565 & 30 & 3 & $\begin{array}{l}145 \\
190 \\
220 \\
\end{array}$ & $\begin{array}{l}165 \\
215 \\
285 \\
\end{array}$ & $\begin{array}{l}0.11 \\
0.17 \\
0.72 \\
\end{array}$ & 235 \\
\hline 48764.1 & KCCAMS & 7,025 & 0.8891 & 0.0030 & -117.30 & 945 & 30 & 1 & 530 & 605 & 1.00 & 570 \\
\hline 48765.1 & KCCAMS & 9,353 & 0.8675 & 0.0029 & -138.75 & 1,140 & 30 & 1 & 670 & 745 & 1.00 & 715 \\
\hline 48765.21 & KCCAMS & 14,258 & 0.8551 & 0.0028 & -151.08 & 1,260 & 30 & 1 & 785 & 885 & 1.00 & 830 \\
\hline \multicolumn{13}{|c|}{ GOM-JSL09-3728_BC1 } \\
\hline 37281.1 & KCCAMS & 45 & 1.0610 & 0.0035 & 53.35 & Modern & & & & & & \\
\hline 37281.2 & KCCAMS & 72 & 1.0753 & 0.0036 & 67.50 & Modern & & & & & & \\
\hline 37281.3 & KCCAMS & 95 & 1.0773 & 0.0039 & 69.50 & Modern & & & & & & \\
\hline 37281.4 & KCCAMS & 112 & 1.0803 & 0.0037 & 72.45 & Modern & & & & & & \\
\hline 37281.5 & KCCAMS & 151 & 1.1036 & 0.0036 & 95.62 & Modern & & & & & & \\
\hline
\end{tabular}


Table C.1. Radiocarbon results from radial transects and radiocarbon calibration results from the Lophelia II program - continued.

[All calibrations were done using a $\Delta \mathrm{R}$ of $-30 \pm 26{ }^{14} \mathrm{C}$ years (Wagner and others, 2009), the CALIB 6.0 (Stuiver and Reimer, 1993) computer calibration program, and the marine09 calibration dataset (Hughen and others, 2009). $\mu \mathrm{m}$, micrometer; cal yr BP, calendar years before present; Lab, the lab where the samples were processed; KCCAMS, the Keck Carbon Cycle AMS Laboratory at the University of California Irvine; BetaAnalytic, Beta Analytic in Miami Florida; NOSAMS, the National Ocean Sciences Accelerator Mass Spectrometry Facility at Woods Hole Oceanographic Institution; LLNL-CAMS, the Center for Accelerator Mass Spectrometry at the Lawrence Livermore National Laboratory. Distance is from outer edge into the center of the specimen. Radiocarbon concentration is given as fraction Modern, $\Delta 14 \mathrm{C}$, and conventional radiocarbon age. $\Delta{ }^{14} \mathrm{C}$ values are not age corrected (Stiuver and Polloch, 1977), nor are the conventional radiocarbon ages reservoir corrected. See Chapter 5 for references]

\begin{tabular}{|c|c|c|c|c|c|c|c|c|c|c|c|c|}
\hline Sample ID & Lab & $\begin{array}{c}\text { Distance } \\
(\mu \mathrm{m})^{1}\end{array}$ & $\begin{array}{l}\text { Fraction } \\
\text { modern }\end{array}$ & \pm & $\Delta^{14} \mathrm{C}$ & ${ }^{14} \mathrm{C}$ age & \pm & $\begin{array}{l}\text { Number } \\
\text { of age } \\
\text { ranges }\end{array}$ & $\begin{array}{c}\text { Minimum } \\
\text { cal age } \\
\text { range } \\
\text { (1s) cal } \\
\text { BP }\end{array}$ & $\begin{array}{l}\text { Maximum } \\
\text { cal age } \\
\text { range } \\
\text { (1s) cal } \\
\text { BP } \\
\end{array}$ & $\begin{array}{l}\text { Relative } \\
\text { area under } \\
\text { distribution }\end{array}$ & $\begin{array}{l}\text { Median } \\
\text { probability } \\
\text { age }\end{array}$ \\
\hline \multicolumn{13}{|c|}{ GOM-JSL09-3728_BC1 } \\
\hline 37282.1 & KCCAMS & 1,418 & 0.9036 & 0.0032 & -102.98 & 815 & 30 & 1 & 450 & 505 & 1.00 & 475 \\
\hline 37283.1 & KCCAMS & 3,589 & 0.8221 & 0.0081 & -183.89 & 1,570 & 80 & 1 & 1,070 & 1,245 & 1.00 & 1,150 \\
\hline $\begin{array}{l}32783.1 \\
\text { duplicate }\end{array}$ & KCCAMS & 3,589 & 0.8188 & 0.0089 & -187.09 & 1,610 & 90 & 1 & 1,100 & 1,285 & 1.00 & 1,190 \\
\hline 37284.1 & KCCAMS & 4,382 & 0.8220 & 0.0029 & -183.94 & 1,580 & 30 & 1 & 1,130 & 1,230 & 1.00 & 1,170 \\
\hline 37285.1 & KCCAMS & 5,404 & 0.8126 & 0.0028 & -193.30 & 1,670 & 30 & 1 & 1,220 & 1,295 & 1.00 & 1,250 \\
\hline 37286.1 & KCCAMS & 7,144 & 0.7955 & 0.0027 & -210.21 & 1,840 & 30 & 1 & 1,350 & 1,465 & 1.00 & 1,410 \\
\hline 37287.1 & KCCAMS & 11,838 & 0.7724 & 0.0026 & -233.18 & 2,080 & 30 & 1 & 1,615 & 1,740 & 1.00 & 1,690 \\
\hline 37287.19 & KCCAMS & 18,090 & 0.7439 & 0.0025 & -261.44 & 2,380 & 30 & 1 & 1,985 & 2,100 & 1.00 & 2,040 \\
\hline \multicolumn{13}{|c|}{ GOM-TOW_BC1 (delaminated transect) } \\
\hline GOMBC 1-2 & BetaAnalytic & 17 & 0.9338 & & -72.59 & 550 & 60 & 1 & 130 & 285 & 1.00 & 205 \\
\hline GOMBC 1-6 & BetaAnalytic & 68 & 0.9526 & & -53.94 & 390 & 40 & & & & & \\
\hline GOMBC 1_14a & NOSAMS & 102 & 0.9351 & 0.0031 & -71.30 & 540 & 25 & 2 & $\begin{array}{l}145 \\
180 \\
\end{array}$ & $\begin{array}{l}165 \\
265 \\
\end{array}$ & $\begin{array}{l}0.18 \\
0.82 \\
\end{array}$ & 205 \\
\hline GOMBC 1-13 & BetaAnalytic & 145 & 0.9315 & & -74.90 & 570 & 40 & 2 & $\begin{array}{l}145 \\
185 \\
\end{array}$ & $\begin{array}{l}165 \\
295 \\
\end{array}$ & $\begin{array}{l}0.12 \\
0.88 \\
\end{array}$ & 240 \\
\hline GOMBC 1_1a & NOSAMS & 170 & 0.9362 & 0.0039 & -70.26 & 530 & 35 & 1 & 140 & 260 & 1.00 & 190 \\
\hline GOMBC 1_25a & NOSAMS & 238 & 0.9503 & 0.0033 & -56.27 & 1410 & 30 & & & & & \\
\hline GOMBC 1-23 & BetaAnalytic & 306 & 0.9188 & & -87.48 & 680 & 40 & 1 & 300 & 405 & 1.00 & 360 \\
\hline GOMBC 1_2a & NOSAMS & 357 & 0.9390 & 0.0032 & -67.45 & 505 & 25 & 1 & 125 & 245 & 1.00 & 170 \\
\hline GOMBC 1_10a & NOSAMS & 425 & 0.9233 & 0.0032 & -83.00 & 640 & 25 & 1 & 270 & 360 & 1.00 & 320 \\
\hline
\end{tabular}

${ }^{1}{ }^{14} \mathrm{C}$ age beyond the range of the marine 09 calibration dataset (Hughen and others, 2009). 
Table C.1. Radiocarbon results from radial transects and radiocarbon calibration results from the Lophelia II program - continued.

[All calibrations were done using a $\Delta \mathrm{R}$ of $-30 \pm 26{ }^{14} \mathrm{C}$ years (Wagner and others, 2009), the CALIB 6.0 (Stuiver and Reimer, 1993) computer calibration program, and the marine 09 calibration dataset (Hughen and others, 2009). $\mu \mathrm{m}$, micrometer; cal yr BP, calendar years before present; Lab, the lab where the samples were processed; KCCAMS, the Keck Carbon Cycle AMS Laboratory at the University of California Irvine; BetaAnalytic, Beta Analytic in Miami Florida; NOSAMS, the National Ocean Sciences Accelerator Mass Spectrometry Facility at Woods Hole Oceanographic Institution; LLNL-CAMS, the Center for Accelerator Mass Spectrometry at the Lawrence Livermore National Laboratory. Distance is from outer edge into the center of the specimen. Radiocarbon concentration is given as fraction Modern, $\Delta 14 \mathrm{C}$, and conventional radiocarbon age. $\Delta^{14} \mathrm{C}$ values are not age corrected (Stiuver and Polloch, 1977), nor are the conventional radiocarbon ages reservoir corrected. See Chapter 5 for references]

\begin{tabular}{|c|c|c|c|c|c|c|c|c|c|c|c|c|}
\hline Sample ID & Lab & $\begin{array}{c}\text { Distance } \\
(\mu \mathrm{m})^{1}\end{array}$ & $\begin{array}{l}\text { Fraction } \\
\text { modern }\end{array}$ & \pm & $\Delta^{14} \mathrm{C}$ & ${ }^{14} \mathrm{C}$ age & \pm & $\begin{array}{l}\text { Number } \\
\text { of age } \\
\text { ranges }\end{array}$ & $\begin{array}{c}\text { Minimum } \\
\text { cal age } \\
\text { range } \\
\text { (1s) cal } \\
\text { BP } \\
\end{array}$ & $\begin{array}{c}\text { Maximum } \\
\text { cal age } \\
\text { range } \\
\text { (1s) cal } \\
\text { BP } \\
\end{array}$ & $\begin{array}{l}\text { Relative } \\
\text { area under } \\
\text { distribution }\end{array}$ & $\begin{array}{l}\text { Median } \\
\text { probability } \\
\text { age }\end{array}$ \\
\hline \multicolumn{13}{|c|}{ GOM-TOW_BC1 (delaminated transect) } \\
\hline GOMBC 1-31 & BetaAnalytic & 485 & 0.9455 & & -60.98 & 450 & 40 & 1 & 0 & 140 & 1.00 & 100 \\
\hline GOMBC 1_15a & NOSAMS & 578 & 0.9240 & 0.0033 & -82.36 & 635 & 30 & 1 & 265 & 360 & 1.00 & 315 \\
\hline $\begin{array}{l}\text { GOM-TOW-BC1 } \\
117\end{array}$ & KCCAMS & 578 & 0.9287 & 0.0031 & -77.99 & 595 & 30 & 2 & $\begin{array}{l}155 \\
230 \\
\end{array}$ & $\begin{array}{l}155 \\
325 \\
\end{array}$ & $\begin{array}{l}0.00 \\
1.00 \\
\end{array}$ & 270 \\
\hline GOMBC 1_8a & NOSAMS & 833 & 0.9373 & 0.0036 & -69.17 & 520 & 30 & 1 & 135 & 250 & 1.00 & 185 \\
\hline GOMBC 1_27a & NOSAMS & 944 & 0.9411 & 0.0036 & -65.36 & 485 & 30 & 1 & 90 & 235 & 1.00 & 145 \\
\hline GOMBC 1_28a & NOSAMS & 1,148 & 0.9493 & 0.0036 & -57.21 & ${ }^{1} 415$ & 30 & & & & & \\
\hline GOMBC 1_13a & NOSAMS & 1,233 & 0.9373 & 0.0029 & -69.13 & 520 & 25 & 1 & 140 & 250 & 1.00 & 185 \\
\hline GOMBC 1_4a & NOSAMS & 1,420 & 0.9342 & 0.0028 & -72.17 & 545 & 25 & 2 & $\begin{array}{l}145 \\
185 \\
\end{array}$ & $\begin{array}{l}165 \\
265 \\
\end{array}$ & $\begin{array}{l}0.17 \\
0.83 \\
\end{array}$ & 210 \\
\hline GOMBC 1_26a & NOSAMS & 1,573 & 0.9396 & 0.0032 & -66.87 & 500 & 25 & 1 & 120 & 245 & 1.00 & 165 \\
\hline GOMBC 1_11a & NOSAMS & 1,717 & 0.9231 & 0.0035 & -83.28 & 645 & 30 & 1 & 275 & 370 & 1.00 & 325 \\
\hline GOMBC 1_5a & NOSAMS & 1,811 & 0.9400 & 0.0035 & -66.43 & 495 & 30 & 1 & 105 & 240 & 1.00 & 160 \\
\hline GOMBC 1_16a & NOSAMS & 1,981 & 0.9125 & 0.0031 & -93.76 & 735 & 25 & 1 & 365 & 460 & 1.00 & 410 \\
\hline GOMBC 1_30a & NOSAMS & 2,130 & 0.9453 & 0.0043 & -61.16 & 450 & 35 & 1 & 0 & 130 & 1.00 & 100 \\
\hline GOMBC 1_29a & NOSAMS & 2,419 & 0.9429 & 0.0032 & -63.60 & 470 & 25 & 4 & $\begin{array}{c}0 \\
55 \\
160 \\
210 \\
\end{array}$ & $\begin{array}{c}10 \\
150 \\
195 \\
225 \\
\end{array}$ & $\begin{array}{l}0.05 \\
0.68 \\
0.19 \\
0.08 \\
\end{array}$ & 120 \\
\hline GOMBC 1_17a & NOSAMS & 2,597 & 0.9196 & 0.0031 & -86.74 & 675 & 25 & 1 & 300 & 395 & 1.00 & 355 \\
\hline
\end{tabular}

\footnotetext{
${ }^{1}{ }^{14} \mathrm{C}$ age beyond the range of the marine 09 calibration dataset (Hughen and others, 2009).
} 
Table C.1. Radiocarbon results from radial transects and radiocarbon calibration results from the Lophelia II program - continued.

[All calibrations were done using a $\Delta \mathrm{R}$ of $-30 \pm 26{ }^{14} \mathrm{C}$ years (Wagner and others, 2009), the CALIB 6.0 (Stuiver and Reimer, 1993) computer calibration program, and the marine 09 calibration dataset (Hughen and others, 2009). $\mu \mathrm{m}$, micrometer; cal yr BP, calendar years before pre sent; Lab, the lab where the samples were processed; KCCAMS, the Keck Carbon Cycle AMS Laboratory at the University of California Irvine; BetaAnalytic, Beta Analytic in Miami Florida; NOSAMS, the National Ocean Sciences Accelerator Mass Spectrometry Facility at Woods Hole Oceanographic Institution; LLNL-CAMS, the Center for Accelerator Mass Spectrometry at the Lawrence Livermore National Laboratory. Distance is from outer edge into the center of the specimen. Radiocarbon concentration is given as fraction Modern, $\Delta 14 \mathrm{C}$, and conventional radiocarbon age. $\Delta^{14} \mathrm{C}$ values are not age corrected (Stiuver and Polloch, 1977), nor are the conventional radiocarbon ages reservoir corrected. See Chapter 5 for references]

\begin{tabular}{|c|c|c|c|c|c|c|c|c|c|c|c|c|}
\hline Sample ID & Lab & $\begin{array}{c}\text { Distance } \\
(\mu \mathrm{m})^{1}\end{array}$ & $\begin{array}{l}\text { Fraction } \\
\text { modern }\end{array}$ & \pm & $\Delta^{14 C}$ & ${ }^{14} \mathrm{C}$ age & \pm & $\begin{array}{l}\text { Number } \\
\text { of age } \\
\text { ranges }\end{array}$ & $\begin{array}{c}\text { Minimum } \\
\text { cal age } \\
\text { range } \\
\text { (1s) cal } \\
\text { BP }\end{array}$ & $\begin{array}{c}\text { Maximum } \\
\text { cal age } \\
\text { range } \\
\text { (1s) cal } \\
\text { BP }\end{array}$ & $\begin{array}{c}\text { Relative } \\
\text { area under } \\
\text { distribution }\end{array}$ & $\begin{array}{l}\text { Median } \\
\text { probability } \\
\text { age }\end{array}$ \\
\hline \multicolumn{13}{|c|}{ GOM-TOW_BC1 (delaminated transect) } \\
\hline GOMBC 1_18a & NOSAMS & 3,048 & 0.9419 & 0.0035 & -64.55 & 480 & 30 & 2 & $\begin{array}{c}80 \\
155\end{array}$ & $\begin{array}{l}155 \\
230\end{array}$ & $\begin{array}{l}0.53 \\
0.47\end{array}$ & 135 \\
\hline $\begin{array}{l}\text { GOM-TOW-BC1 } \\
34\end{array}$ & KCCAMS & 3,048 & 0.9421 & 0.0031 & -64.70 & 480 & 30 & 2 & $\begin{array}{r}80 \\
155 \\
\end{array}$ & $\begin{array}{l}155 \\
230 \\
\end{array}$ & $\begin{array}{l}0.53 \\
0.47 \\
\end{array}$ & 135 \\
\hline GOMBC 1_3a & NOSAMS & 3,226 & 0.9424 & 0.0035 & -64.11 & 475 & 30 & 3 & $\begin{array}{c}65 \\
160 \\
205 \\
\end{array}$ & $\begin{array}{l}150 \\
200 \\
225\end{array}$ & $\begin{array}{l}0.62 \\
0.25 \\
0.13 \\
\end{array}$ & 130 \\
\hline GOMBC 1_7a & NOSAMS & 3,422 & 0.9281 & 0.0039 & -78.26 & 600 & 35 & 2 & $\begin{array}{l}230 \\
345 \\
\end{array}$ & $\begin{array}{l}335 \\
355\end{array}$ & $\begin{array}{l}0.97 \\
0.03\end{array}$ & 275 \\
\hline GOMBC 1_19a & NOSAMS & 3,915 & 0.9213 & 0.0035 & -85.07 & 660 & 30 & 1 & 285 & 385 & 1.00 & 340 \\
\hline GOMBC $16 a$ & NOSAMS & 3,991 & 0.9223 & 0.0035 & -84.05 & 650 & 30 & 1 & 280 & 375 & 1.00 & 330 \\
\hline GOMBC 1_20a & NOSAMS & 4,340 & 0.8971 & 0.0028 & -109.11 & 870 & 25 & 1 & 485 & 535 & 1.00 & 510 \\
\hline GOMBC 1_21a & NOSAMS & 4,722 & 0.9068 & 0.0040 & -99.41 & 785 & 35 & 1 & 420 & 495 & 1.00 & 450 \\
\hline GOMBC 1_22a & NOSAMS & 5,173 & 0.9081 & 0.0029 & -98.16 & 775 & 25 & 1 & 420 & 485 & 1.00 & 450 \\
\hline GOMBC 1_9a & NOSAMS & 5,743 & 0.9349 & 0.0050 & -71.49 & 540 & 40 & 1 & 140 & 265 & 1.00 & 200 \\
\hline GOMBC 1_23a & NOSAMS & 6,457 & 0.9031 & 0.0034 & -103.10 & 820 & 30 & 1 & 450 & 510 & 1.00 & 480 \\
\hline GOMBC $124 a$ & NOSAMS & 6,763 & 0.9044 & 0.0032 & -101.82 & 805 & 30 & 1 & 440 & 500 & 1.00 & 470 \\
\hline GOMBC 1_12a & NOSAMS & 7,604 & 0.8743 & 0.0027 & -131.70 & 1080 & 25 & 1 & 635 & 695 & 1.00 & 670 \\
\hline \multicolumn{13}{|c|}{ GOM-TOW_BC1 (milled transect) } \\
\hline 145279 & LLNL-CAMS & 0 & 0.9335 & 0.0039 & -73.10 & 550 & 35 & 2 & $\begin{array}{l}145 \\
180\end{array}$ & $\begin{array}{l}165 \\
275 \\
\end{array}$ & $\begin{array}{l}0.16 \\
0.84 \\
\end{array}$ & 210 \\
\hline 145280 & LLNL-CAMS & 5,100 & 0.9028 & 0.0031 & -103.66 & 820 & 30 & 1 & 450 & 510 & 1.00 & 480 \\
\hline
\end{tabular}


Table C.1. Radiocarbon results from radial transects and radiocarbon calibration results from the Lophelia II program - continued.

[All calibrations were done using a $\Delta \mathrm{R}$ of $-30 \pm 26{ }^{14} \mathrm{C}$ years (Wagner and others, 2009), the CALIB 6.0 (Stuiver and Reimer, 1993) computer calibration program, and the marine09 calibration dataset (Hughen and others, 2009). $\mu \mathrm{m}$, micrometer; cal yr BP, calendar years before present; Lab, the lab where the samples were processed; KCCAMS, the Keck Carbon Cycle AMS Laboratory at the University of California Irvine; BetaAnalytic, Beta Analytic in Miami Florida; NOSAMS, the National Ocean Sciences Accelerator Mass Spectrometry Facility at Woods Hole Oceanographic Institution; LLNL-

CAMS, the Center for Accelerator Mass Spectrometry at the Lawrence Livermore National Laboratory. Distance is from outer edge into the center of the specimen. Radiocarbon concentration is given as fraction Modern, $\Delta 14 \mathrm{C}$, and conventional radiocarbon age. $\Delta{ }^{14} \mathrm{C}$ values are not age corrected (Stiuver and Polloch, 1977), nor are the conventional radiocarbon ages reservoir corrected. See Chapter 5 for references]

\begin{tabular}{|c|c|c|c|c|c|c|c|c|c|c|c|c|}
\hline Sample ID & Lab & $\begin{array}{c}\text { Distance } \\
(\mu \mathrm{m})^{1}\end{array}$ & $\begin{array}{l}\text { Fraction } \\
\text { modern }\end{array}$ & \pm & $\Delta^{14} \mathrm{C}$ & ${ }^{14} \mathrm{C}$ age & \pm & $\begin{array}{l}\text { Number } \\
\text { of age } \\
\text { ranges }\end{array}$ & $\begin{array}{c}\text { Minimum } \\
\text { cal age } \\
\text { range } \\
\text { (1s) cal } \\
\text { BP }\end{array}$ & $\begin{array}{c}\text { Maximum } \\
\text { cal age } \\
\text { range } \\
\text { (1s) cal } \\
\text { BP } \\
\end{array}$ & $\begin{array}{c}\text { Relative } \\
\text { area under } \\
\text { distribution }\end{array}$ & $\begin{array}{l}\text { Median } \\
\text { probability } \\
\text { age }\end{array}$ \\
\hline \multicolumn{13}{|c|}{ GOM-TOW_BC1 (milled transect) } \\
\hline 145281 & LLNL-CAMS & 9,750 & 0.8737 & 0.0034 & -132.53 & 1,090 & 35 & 1 & 635 & 705 & 1.00 & 670 \\
\hline \multicolumn{13}{|c|}{ GOM-TOW-BC2 (delaminated transect) } \\
\hline 1.1 & KCCAMS & 191 & 1.0549 & 0.0018 & 47.26 & Modern & & & & & & \\
\hline 1.2 & KCCAMS & 383 & 0.9516 & 0.0017 & -55.29 & ${ }^{1} 400$ & 15 & & & & & \\
\hline 1.3 & KCCAMS & 574 & 0.9474 & 0.0016 & -59.47 & 435 & 15 & 1 & 0 & 95 & 1.00 & 70 \\
\hline 1.4 & KCCAMS & 766 & 0.9413 & 0.0016 & -65.54 & 485 & 15 & 1 & 100 & 230 & 1.00 & 145 \\
\hline $2 \mathrm{a} .1$ & KCCAMS & 1,539 & 0.9395 & 0.0018 & -67.29 & 500 & 20 & 1 & 125 & 240 & 1.00 & 170 \\
\hline $3 a .1$ & KCCAMS & 3,021 & 0.9172 & 0.0017 & -89.40 & 695 & 15 & 1 & 320 & 410 & 1.00 & 370 \\
\hline 4.1 & KCCAMS & 4,194 & 0.8658 & 0.0018 & -140.45 & 1,160 & 20 & 1 & 680 & 755 & 1.00 & 725 \\
\hline 7.1 & KCCAMS & 6,153 & 0.8480 & 0.0014 & -158.15 & 1,330 & 15 & 1 & 870 & 945 & 1.00 & 905 \\
\hline 8.1 & KCCAMS & 7,562 & 0.7576 & 0.0013 & -247.88 & & 15 & & & & & \\
\hline 9.1 & KCCAMS & 9,027 & 0.8273 & 0.0014 & -178.68 & 1,530 & 15 & 1 & 1,070 & 1,155 & 1.00 & 1,110 \\
\hline 10.1 & KCCAMS & 10,035 & 0.8235 & 0.0015 & -182.47 & 1,560 & 15 & 1 & 1,110 & 1,210 & 1.00 & 1,150 \\
\hline 11.5 & KCCAMS & 11,379 & 0.7765 & 0.0013 & -229.10 & 2,030 & 15 & 1 & 1,590 & 1,685 & 1.00 & 1,630 \\
\hline \multicolumn{13}{|c|}{ GOM-TOW-BC2 (milled transect) } \\
\hline 145276 & LLNL-CAMS & 0 & 0.9580 & 0.0033 & -48.81 & 345 & 30 & & & & & \\
\hline 145277 & LLNL-CAMS & 8,500 & 0.8184 & 0.0037 & -187.38 & 1,610 & 40 & 1 & 1,160 & 1,260 & 1.00 & 1,200 \\
\hline 145278 & LLNL-CAMS & 15,000 & 0.7706 & 0.0035 & -234.85 & 2,100 & 40 & 1 & 1,650 & 1,790 & 1.00 & 1,710 \\
\hline \multicolumn{13}{|c|}{ GOM-JSL04-4734_BC1 } \\
\hline VK 1 & NOSAMS & 125 & 1.0793 & 0.0036 & 71.86 & Modern & & & & & & \\
\hline VK 2 & NOSAMS & 313 & 1.1061 & 0.0042 & 98.50 & Modern & & & & & & \\
\hline
\end{tabular}

${ }^{1}{ }^{14} \mathrm{C}$ age beyond the range of the marine 09 calibration dataset (Hughen and others, 2009). 
Table C.1. Radiocarbon results from radial transects and radiocarbon calibration results from the Lophelia II program - continued.

[All calibrations were done using a $\Delta \mathrm{R}$ of $-30 \pm 26{ }^{14} \mathrm{C}$ years (Wagner and others, 2009), the CALIB 6.0 (Stuiver and Reimer, 1993) computer calibration program, and the marine09 calibration dataset (Hughen and others, 2009). $\mu \mathrm{m}$, micrometer; cal yr BP, calendar years before present; Lab, the lab where the samples were processed; KCCAMS, the Keck Carbon Cycle AMS Laboratory at the University of California Irvine; BetaAnalytic, Beta Analytic in Miami Florida; NOSAMS, the National Ocean Sciences Accelerator Mass Spectrometry Facility at Woods Hole Oceanographic Institution; LLNL-

CAMS, the Center for Accelerator Mass Spectrometry at the Lawrence Livermore National Laboratory. Distance is from outer edge into the center of the specimen. Radiocarbon concentration is given as fraction Modern, $\Delta 14 \mathrm{C}$, and conventional radiocarbon age. $\Delta{ }^{14} \mathrm{C}$ values are not age corrected (Stiuver and Polloch, 1977), nor are the conventional radiocarbon ages reservoir corrected. See Chapter 5 for references]

\begin{tabular}{|c|c|c|c|c|c|c|c|c|c|c|c|c|}
\hline Sample ID & Lab & $\begin{array}{c}\text { Distance } \\
(\mu \mathrm{m})^{1}\end{array}$ & $\begin{array}{l}\text { Fraction } \\
\text { modern }\end{array}$ & \pm & $\Delta^{14} \mathrm{C}$ & ${ }^{14} \mathrm{C}$ age & \pm & $\begin{array}{l}\text { Number } \\
\text { of age } \\
\text { ranges }\end{array}$ & $\begin{array}{c}\text { Minimum } \\
\text { cal age } \\
\text { range } \\
\text { (1s) cal } \\
\text { BP }\end{array}$ & $\begin{array}{c}\text { Maximum } \\
\text { cal age } \\
\text { range } \\
\text { (1s) cal } \\
\text { BP } \\
\end{array}$ & $\begin{array}{c}\text { Relative } \\
\text { area under } \\
\text { distribution }\end{array}$ & $\begin{array}{l}\text { Median } \\
\text { probability } \\
\text { age }\end{array}$ \\
\hline \multicolumn{13}{|c|}{ GOM-JSL04-4734_BC1 } \\
\hline VK 1a & NOSAMS & 313 & 1.1045 & 0.0042 & 96.91 & Modern & & & & & & \\
\hline VK 3a & BetaAnalytic & 414 & 1.1090 & & 101.38 & Modern & & & & & & \\
\hline VK 4 & NOSAMS & 464 & 1.1109 & 0.0039 & 103.31 & Modern & & & & & & \\
\hline VK 6 & NOSAMS & 614 & 1.1200 & 0.0042 & 112.28 & Modern & & & & & & \\
\hline VK 8 & NOSAMS & 827 & 1.1267 & 0.0059 & 118.93 & Modern & & & & & & \\
\hline VK 8a & NOSAMS & 1,090 & 1.1222 & 0.0043 & 114.52 & Modern & & & & & & \\
\hline VK 1a & BetaAnalytic & 1,153 & 1.1120 & & 104.36 & Modern & & & & & & \\
\hline VK 34a & NOSAMS & 1,266 & 1.0754 & 0.0039 & 68.02 & Modern & & & & & & \\
\hline VK 16 & NOSAMS & 1,504 & 0.9662 & 0.0041 & -40.44 & ${ }^{1275}$ & 35 & & & & & \\
\hline VK 10a & NOSAMS & 1,567 & 0.9555 & 0.0030 & -51.10 & ${ }^{1365}$ & 25 & & & & & \\
\hline VK 5a & NOSAMS & 1,767 & 0.9332 & 0.0095 & -73.18 & 555 & 80 & 1 & 100 & 305 & 1.00 & 210 \\
\hline VK 23a & NOSAMS & 1,968 & 0.9616 & 0.0037 & -45.00 & ${ }^{1} 315$ & 30 & & & & & \\
\hline VK 33a & NOSAMS & 2,106 & 0.9530 & 0.0035 & -53.56 & ${ }^{1385}$ & 30 & & & & & \\
\hline VK 2a & NOSAMS & 2,319 & 0.9432 & 0.0035 & -63.27 & 470 & 30 & 4 & $\begin{array}{c}0 \\
55 \\
160 \\
210 \\
\end{array}$ & $\begin{array}{c}10 \\
150 \\
195 \\
225 \\
\end{array}$ & $\begin{array}{l}0.05 \\
0.67 \\
0.19 \\
0.08 \\
\end{array}$ & 120 \\
\hline VK 14a & NOSAMS & 2,582 & 0.9510 & 0.0037 & -55.53 & ${ }^{1} 405$ & 30 & & & & & \\
\hline VK 11a & NOSAMS & 2,732 & 0.9485 & 0.0036 & -57.99 & 425 & 30 & 1 & 0 & 100 & 1.00 & 70 \\
\hline $\mathrm{VK} 7 \mathrm{a}$ & NOSAMS & 2,870 & 0.9401 & 0.0037 & -66.41 & 495 & 30 & 1 & 105 & 240 & 1.00 & 160 \\
\hline
\end{tabular}

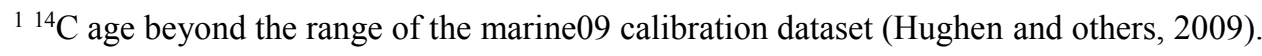


Table C.1. Radiocarbon results from radial transects and radiocarbon calibration results from the Lophelia II program - continued.

[All calibrations were done using a $\Delta \mathrm{R}$ of $-30 \pm 26{ }^{14} \mathrm{C}$ years (Wagner and others, 2009), the CALIB 6.0 (Stuiver and Reimer, 1993) computer calibration program, and the marine 09 calibration dataset (Hughen and others, 2009). $\mu \mathrm{m}$, micrometer; cal yr BP, calendar years before present; Lab, the lab where the samples were processed; KCCAMS, the Keck Carbon Cycle AMS Laboratory at the University of California Irvine; BetaAnalytic, Beta Analytic in Miami Florida; NOSAMS, the National Ocean Sciences Accelerator Mass Spectrometry Facility at Woods Hole Oceanographic Institution; LLNL-

CAMS, the Center for Accelerator Mass Spectrometry at the Lawrence Livermore National Laboratory. Distance is from outer edge into the center of the specimen. Radiocarbon concentration is given as fraction Modern, $\Delta 14 \mathrm{C}$, and conventional radiocarbon age. $\Delta{ }^{14} \mathrm{C}$ values are not age corrected (Stiuver and Polloch, 1977), nor are the conventional radiocarbon ages reservoir corrected. See Chapter 5 for references]

\begin{tabular}{|c|c|c|c|c|c|c|c|c|c|c|c|c|}
\hline Sample ID & Lab & $\begin{array}{c}\text { Distance } \\
(\mu \mathrm{m})^{1}\end{array}$ & $\begin{array}{l}\text { Fraction } \\
\text { modern }\end{array}$ & \pm & $\Delta^{14} \mathrm{C}$ & ${ }^{14} \mathrm{C}$ age & \pm & $\begin{array}{l}\text { Number } \\
\text { of age } \\
\text { ranges }\end{array}$ & $\begin{array}{c}\text { Minimum } \\
\text { cal age } \\
\text { range } \\
\text { (1s) cal } \\
\text { BP }\end{array}$ & $\begin{array}{c}\text { Maximum } \\
\text { cal age } \\
\text { range } \\
\text { (1s) cal } \\
\text { BP }\end{array}$ & $\begin{array}{c}\text { Relative } \\
\text { area under } \\
\text { distribution }\end{array}$ & $\begin{array}{l}\text { Median } \\
\text { probability } \\
\text { age }\end{array}$ \\
\hline \multicolumn{13}{|c|}{ GOM-JSL04-4734_BC1 } \\
\hline VK 20a & NOSAMS & 2,971 & 0.9316 & 0.0037 & -74.80 & 570 & 30 & 3 & $\begin{array}{l}150 \\
190 \\
220\end{array}$ & $\begin{array}{l}160 \\
215 \\
290\end{array}$ & $\begin{array}{l}0.10 \\
0.14 \\
0.76\end{array}$ & 240 \\
\hline VK 16a & NOSAMS & 3,171 & 0.9419 & 0.0036 & -64.53 & 480 & 30 & 2 & $\begin{array}{c}80 \\
155\end{array}$ & $\begin{array}{l}155 \\
230\end{array}$ & $\begin{array}{l}0.53 \\
0.47\end{array}$ & 135 \\
\hline VK 4a & NOSAMS & 3,322 & 0.9417 & 0.0036 & -64.76 & 480 & 30 & 2 & $\begin{array}{c}80 \\
155\end{array}$ & $\begin{array}{l}155 \\
230\end{array}$ & $\begin{array}{l}0.53 \\
0.47\end{array}$ & 135 \\
\hline VK 38a & NOSAMS & 3,484 & 0.9537 & 0.0037 & -52.84 & 380 & 30 & & & & & \\
\hline VK 6a & NOSAMS & 3,635 & 0.9356 & 0.0037 & -70.87 & 535 & 30 & 2 & $\begin{array}{l}145 \\
170\end{array}$ & $\begin{array}{l}170 \\
260\end{array}$ & $\begin{array}{l}0.21 \\
0.79\end{array}$ & 195 \\
\hline VK 26a & NOSAMS & 3,886 & 0.9399 & 0.0036 & -66.55 & 495 & 30 & 1 & 105 & 240 & 1.00 & 160 \\
\hline VK 24a & NOSAMS & 4,086 & 0.9454 & 0.0032 & -61.13 & 450 & 25 & 2 & $\begin{array}{c}0 \\
40\end{array}$ & $\begin{array}{c}35 \\
130\end{array}$ & $\begin{array}{l}0.25 \\
0.75\end{array}$ & 95 \\
\hline VK 12a & NOSAMS & 4,324 & 0.9370 & 0.0029 & -69.43 & 520 & 25 & 1 & 140 & 250 & 1.00 & 185 \\
\hline VK 28a & NOSAMS & 4,550 & 0.9249 & 0.0040 & -81.50 & 625 & 35 & 1 & 260 & 360 & 1.00 & 305 \\
\hline VK 35a & NOSAMS & 4,675 & 0.9462 & 0.0036 & -60.33 & 445 & 30 & 2 & $\begin{array}{c}0 \\
40\end{array}$ & $\begin{array}{c}35 \\
125 \\
\end{array}$ & $\begin{array}{l}0.28 \\
0.72\end{array}$ & 90 \\
\hline VK 27a & NOSAMS & 4,788 & 0.9381 & 0.0032 & -68.31 & 510 & 25 & 1 & 130 & 245 & 1.00 & 175 \\
\hline VK 22a & NOSAMS & 4,989 & 0.9358 & 0.0031 & -70.60 & 530 & 25 & 2 & $\begin{array}{l}145 \\
170\end{array}$ & $\begin{array}{l}170 \\
255\end{array}$ & $\begin{array}{l}0.22 \\
0.78\end{array}$ & 195 \\
\hline VK 3a & NOSAMS & 5,189 & 0.9148 & 0.0037 & -91.51 & 715 & 30 & 1 & 330 & 435 & 1.00 & 385 \\
\hline VK 25a & NOSAMS & 5,502 & 0.9269 & 0.0036 & -79.50 & 610 & 30 & 1 & 245 & 335 & 1.00 & 290 \\
\hline
\end{tabular}


Table C.1. Radiocarbon results from radial transects and radiocarbon calibration results from the Lophelia II program - continued.

[All calibrations were done using a $\Delta \mathrm{R}$ of $-30 \pm 26{ }^{14} \mathrm{C}$ years (Wagner and others, 2009), the CALIB 6.0 (Stuiver and Reimer, 1993) computer calibration program, and the marine 09 calibration dataset (Hughen and others, 2009). $\mu \mathrm{m}$, micrometer; cal yr BP, calendar years before present; Lab, the lab where the samples were processed; KCCAMS, the Keck Carbon Cycle AMS Laboratory at the University of California Irvine; BetaAnalytic, Beta Analytic in Miami Florida; NOSAMS, the National Ocean Sciences Accelerator Mass Spectrometry Facility at Woods Hole Oceanographic Institution; LLNL-

CAMS, the Center for Accelerator Mass Spectrometry at the Lawrence Livermore National Laboratory. Distance is from outer edge into the center of the specimen. Radiocarbon concentration is given as fraction Modern, $\Delta 14 \mathrm{C}$, and conventional radiocarbon age. $\Delta{ }^{14} \mathrm{C}$ values are not age corrected (Stiuver and Polloch, 1977), nor are the conventional radiocarbon ages reservoir corrected. See Chapter 5 for references]

\begin{tabular}{|c|c|c|c|c|c|c|c|c|c|c|c|c|}
\hline Sample ID & Lab & $\begin{array}{c}\text { Distance } \\
(\mu \mathrm{m})^{1}\end{array}$ & $\begin{array}{l}\text { Fraction } \\
\text { modern }\end{array}$ & \pm & $\Delta^{14} \mathrm{C}$ & ${ }^{14} \mathrm{C}$ age & \pm & $\begin{array}{l}\text { Number } \\
\text { of age } \\
\text { ranges }\end{array}$ & $\begin{array}{c}\text { Minimum } \\
\text { cal age } \\
\text { range } \\
\text { (1s) cal } \\
\text { BP }\end{array}$ & $\begin{array}{c}\text { Maximum } \\
\text { cal age } \\
\text { range } \\
\text { (1s) cal } \\
\text { BP }\end{array}$ & $\begin{array}{c}\text { Relative } \\
\text { area under } \\
\text { distribution }\end{array}$ & $\begin{array}{l}\text { Median } \\
\text { probability } \\
\text { age }\end{array}$ \\
\hline \multicolumn{13}{|c|}{ GOM-JSL04-4734_BC1 } \\
\hline VK 32a & NOSAMS & 5,628 & 0.9325 & 0.0037 & -73.93 & 560 & 30 & 2 & $\begin{array}{l}145 \\
190 \\
\end{array}$ & $\begin{array}{l}165 \\
280 \\
\end{array}$ & $\begin{array}{l}0.13 \\
0.87 \\
\end{array}$ & 225 \\
\hline VK 5a & BetaAnalytic & 5,966 & 0.9315 & & -74.90 & 570 & 40 & 2 & $\begin{array}{l}145 \\
185 \\
\end{array}$ & $\begin{array}{l}165 \\
295 \\
\end{array}$ & $\begin{array}{l}0.12 \\
0.88 \\
\end{array}$ & 240 \\
\hline VK 36a & NOSAMS & 6,217 & 0.9351 & 0.0036 & -71.33 & 540 & 30 & 2 & $\begin{array}{l}145 \\
175 \\
\end{array}$ & $\begin{array}{l}165 \\
265 \\
\end{array}$ & $\begin{array}{l}0.19 \\
0.81 \\
\end{array}$ & 200 \\
\hline VK 13a & NOSAMS & 6,468 & 0.9220 & 0.0036 & -84.36 & 650 & 30 & 1 & 280 & 375 & 1.00 & 330 \\
\hline VK 31a & NOSAMS & 6,768 & 0.9173 & 0.0030 & -88.97 & 695 & 25 & 1 & 320 & 425 & 1.00 & 370 \\
\hline VK 29a & NOSAMS & 7,069 & 0.9249 & 0.0040 & -81.43 & 625 & 35 & 1 & 260 & 360 & 1.00 & 305 \\
\hline VK 39a & NOSAMS & 7,270 & 0.9376 & 0.0034 & -68.84 & 515 & 30 & 1 & 130 & 250 & 1.00 & 180 \\
\hline VK 19a & NOSAMS & 7,596 & 0.9134 & 0.0039 & -92.90 & 725 & 35 & 2 & $\begin{array}{l}335 \\
350\end{array}$ & $\begin{array}{l}345 \\
450\end{array}$ & $\begin{array}{l}0.09 \\
0.91\end{array}$ & 395 \\
\hline VK 17a & NOSAMS & 7,784 & 0.9072 & 0.0033 & -99.04 & 780 & 30 & 1 & 420 & 490 & 1.00 & 450 \\
\hline VK $37 \mathrm{a}$ & NOSAMS & 8,047 & 0.9217 & 0.0036 & -84.68 & 655 & 30 & 1 & 285 & 380 & 1.00 & 335 \\
\hline VK 30a & NOSAMS & 8,273 & 0.8926 & 0.0040 & -113.54 & 910 & 35 & 2 & $\begin{array}{l}500 \\
580 \\
\end{array}$ & $\begin{array}{l}565 \\
595 \\
\end{array}$ & $\begin{array}{l}0.90 \\
0.10 \\
\end{array}$ & 540 \\
\hline VK 4a & BetaAnalytic & 8,398 & 0.8974 & & -108.81 & 870 & 40 & 1 & 475 & 545 & 1.00 & 515 \\
\hline VK 9a & NOSAMS & 8,824 & 0.8803 & 0.0031 & -125.78 & 1,020 & 30 & 2 & $\begin{array}{l}565 \\
595\end{array}$ & $\begin{array}{l}580 \\
655\end{array}$ & $\begin{array}{l}0.13 \\
0.87\end{array}$ & 620 \\
\hline
\end{tabular}




\section{APPENDIX D. PHOTOGRAPHS OF SELECTED BENTHIC FISHES OBSERVED DURING THE LOPHELIA II PROJECT IN THE GULF OF MEXICO}

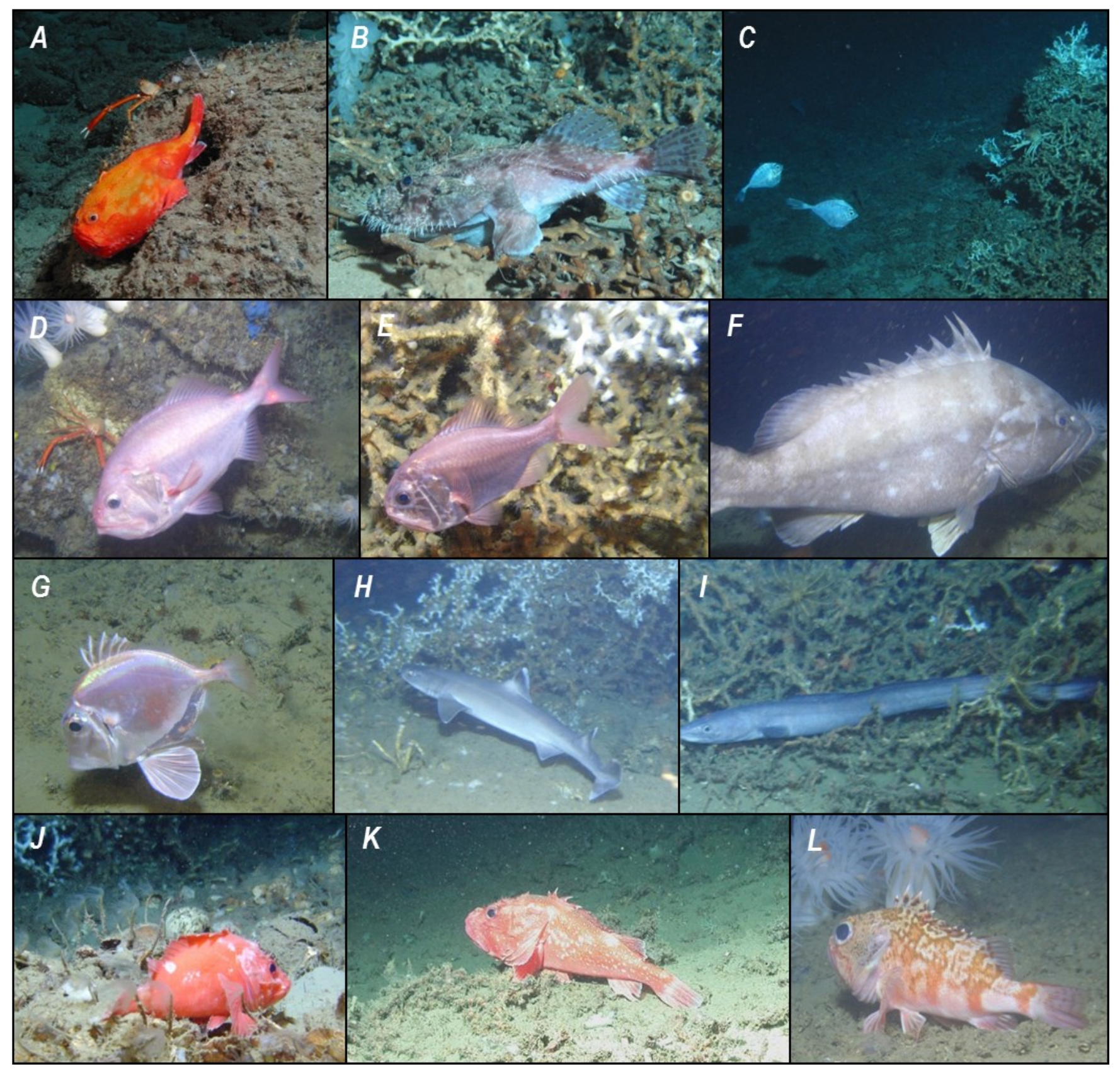

Figure D.1 Photographs of selected benthic fishes observed during the Lophelia II project in the Gulf of Mexico. A, Chaunax pictus at West Florida Slope (WFS); B, Lophiodes beroe at WFS; C, Grammicolepis brachiusculus at WFS; D, Gephryoberyx darwini at VK862; E, Hoplostethus occidentalis at VK826; $F$, Epinephelus niveatus at VK862; G, Cyttopsis rosea at VK862; H, Cirrhigaleus asper at VK826; I, Conger oceanicus at VK826; J, Idiastion kyphos at VK826; K, Trachyscorpia cristulata at VK826; and L, Helicolenus dactylopterus at VK862. Photo credits: Steve Ross, University of North Carolina Wilmington. 